Pacific Northwest

National Laboratory

Operated by Battelle for the

U.S. Department of Energy

\title{
Hanford Site National Environmental Policy Act (NEPA) Characterization
}

\author{
D. A. Neitzel, Editor \\ E. J. Antonio D. G. Horton \\ T. O. Eschbach G. V. Last \\ R. A. Fowler T. M. Poston \\ S. M. Goodwin $\quad$ E. L. Prendergast \\ D. W. Harvey A. C. Rohay \\ P. L. Hendrickson P. D. Thorne \\ D. J. Hoitink
}

September 2001

Prepared for the U.S. Department of Energy under Contract DE-AC06-76RL01830 


\title{
DISCLAIMER
}

This report was prepared as an account of work sponsored by an agency of the United States Government. Neither the United States Government nor any agency thereof, nor Battelle Memorial Institute, nor any of their employees, makes any warranty, express or implied, or assumes any legal liability or responsibility for the accuracy, completeness, or usefulness of any information, apparatus, product, or process disclosed, or represents that its use would not infringe privately owned rights. Reference herein to any specific commercial product, process, or service by trade name, trademark, manufacturer, or otherwise does not necessarily constitute or imply its endorsement, recommendation, or favoring by the United States Government or any agency thereof, or Battelle Memorial Institute. The views and opinions of authors expressed herein do not necessarily state or reflect those of the United States Government or any agency thereof.

\author{
PACIFIC NORTHWEST NATIONAL LABORATORY \\ operated by \\ BATTELLE \\ for the \\ UNITED STATES DEPARTMENT OF ENERGY \\ under Contract DE-AC06-76RL01830
}

\author{
Printed in the United States of America \\ Available to DOE and DOE contractors from the \\ Office of Scientific and Technical Information, P.O. Box 62, Oak Ridge, TN 37831; \\ prices available from (615) 576-8401. \\ Available to the public from the National Technical Information Service, \\ U.S. Department of Commerce, 5285 Port Royal Rd., Springfield, VA 22161
}




\title{
Hanford Site National Environmental Policy Act (NEPA) Characterization
}

\author{
D. A. Neitzel, Editor \\ E. J. Antonio \\ T. O. Eschbach \\ R. A. Fowler \\ S. M. Goodwin \\ D. W. Harvey \\ P. L. Hendrickson \\ D. J. Hoitink \\ D. G. Horton \\ G. V. Last \\ T. M. Poston \\ E. L. Prendergast \\ A. C. Rohay \\ P. D. Thorne
}

September 2001

Prepared for

the U.S. Department of Energy

under Contract DE-AC06-76RL01830

Pacific Northwest National Laboratory

Richland, Washington 99352 


\section{Preface}

This document describes the U.S. Department of Energy's (DOE) Hanford Site environment. It is updated each year and is intended to provide a consistent description of the Hanford Site environment for the many National Environmental Policy Act (NEPA) documents being prepared by DOE contractors. No statements of significance or environmental consequences are provided. This year's report is the thirteenth revision of the original document published in 1988 and is (until replaced by the fourteenth revision) the only version that is relevant for use in the preparation of Hanford NEPA, State Environmental Policy Act (SEPA), and Comprehensive Environmental Response, Compensation, and Liability Act (CERCLA) documents.

The two chapters included in this document (Chapters 4 and 6) are numbered to correspond to the chapters where such information is typically presented in environmental impact statements (EISs) and other Hanford Site NEPA or CERCLA documentation. Chapter 4.0 (Affected Environment) describes Hanford Site climate and meteorology, geology, hydrology, ecology, cultural, archaeological, and historical resources, socioeconomics, occupational safety, and noise. Sources for extensive tabular data related to these topics are provided in the chapter. Most subjects are divided into a general description of the characteristics of the Hanford Site, followed by site-specific information, where available, of the 100, 200, 300, and other areas. This division allows the reader to go directly to those sections of particular interest. When specific information on each of these separate areas is not complete or available, the general Hanford Site description should be used.

Chapter 6.0 (Statutory and Regulatory Requirements) describes federal and state laws and regulations, DOE directives and permits, and presidential executive orders that are applicable to the NEPA documents prepared for Hanford Site activities. Information in Chapter 6 of this document can be adapted and supplemented with specific information for a chapter covering statutory and regulatory requirements in an environmental assessment or environmental impact statement.

People preparing environmental assessments and EISs should also be cognizant of the document titled Recommendations for the Preparation of Environmental Assessments and Environmental Impact Statements published by the DOE Office of NEPA Oversight. ${ }^{\text {(a) }}$

PNNL staff prepared individual sections of this document, with input from other Site contractors. More detailed data are available from reference sources cited or from the authors. Sections were updated with the best available information through June 2001.

Any interested individual seeking baseline data on the Hanford Site and its past activities may also use this information by which to evaluate projected activities and their impacts. The following personnel are responsible for the various sections of this document and can be contacted with questions:
Document Editor
D. A. Neitzel
(509) 376-0602
duane.neitzel@pnl.gov
Climate/Meteorology
D. J. Hoitink
(509) 372-6414
Air Quality
P. L. Hendrickson
(509) 372-4294
dana.j.hoitink@pnl.gov
paul.hendrickson@pnl.gov

(a) U.S. Department of Energy (DOE). 1993. Recommendations for the Preparation of Environmental Assessments and Environmental Impact Statements. U.S. Department of Energy, Office of NEPA Oversight, Washington, D.C. 


\begin{tabular}{|c|c|c|c|}
\hline Geology & S. M. Goodwin & (509) 376-0915 & shannon.goodwin@pnl.gov \\
\hline & A. C. Rohay & (509) 376-6925 & alan.rohay@pnl.gov \\
\hline Hydrology & P. D. Thorne & (509) 372-4482 & paul.thorne@pnl.gov \\
\hline & D. G. Horton & (509) 376-6868 & dg.horton@pnl.gov \\
\hline & G. V. Last & (509) 376-3961 & george.last@pnl.gov \\
\hline Ecology & T. M. Poston & (509) 376-5678 & ted.poston@pnl.gov \\
\hline Cultural, Archaeological, & D. W. Harvey & (509) 373-2945 & david.harvey@pnl.gov \\
\hline and Historical Resources & E. L. Prendergast & (509) 376-4626 & Ellen.Prendergast@.pnl.gov \\
\hline & T. O. Eschbach & (509) 375-6734 & tara.eschbach@pnl.gov \\
\hline Socioeconomics & R. A. Fowler & (509) 372-4332 & richard.fowler@pnl.gov \\
\hline Noise & T. M. Poston & (509) 376-5678 & ted.poston@pnl.gov \\
\hline Occupational Safety & E. J. Antonio & (509) 375-3809 & E.Antonio@pnl.gov \\
\hline $\begin{array}{l}\text { Statutory and Regulatory } \\
\text { Requirements }\end{array}$ & P. L. Hendrickson & (509) 372-4294 & paul.hendrickson@pnl.gov \\
\hline
\end{tabular}

The suggested citation for this document is Neitzel, D. A., ed. 2001. Hanford Site National Environmental Policy Act (NEPA) Characterization. PNL-6415, Rev. 13. Pacific Northwest National Laboratory, Richland, Washington.

A copy of the document is available, upon request, from Duane A. Neitzel at (509) 376-0602. The document is also available electronically at http://www.pnl.gov/nepa 


\section{Acknowledgments}

As the editor of Revision 13, I hope you find this document useful in preparing Hanford Site NEPA documents or as a useful source of information about the Hanford environment. Many people are responsible for the years of work that went into preparing this description of the Hanford Site. The authors of the individual sections took great care to accurately describe their work. As the editor I thank them. Most importantly, I thank J. P. Duncan and A. L. Bunn for all their work in making sure Revision 13 was completed on time and presented in a way that is useful to everyone needing an up-to-date description of the Hanford environment. J. P. Duncan also maintains the electronic copy of the document throughout the year.

D. A. Neitzel 


\title{
Acronyms, Abbreviations, and Symbols
}

\author{
AAS \\ Associate in applied science \\ AEA \\ Atomic Energy Act \\ ALE \\ ARPA \\ BCAA \\ BCRFD \\ BHI \\ BNI \\ BNSF \\ Fitzner/Eberhardt Arid Lands Ecology Reserve \\ Archaeological Resources Protection Act \\ Benton Clean Air Authority \\ Benton County Rural Fire Department \\ Bechtel Hanford, Inc. \\ Bechtel National, Inc. \\ BPA \\ BWIP \\ CAA \\ CBC \\ CCP \\ CEQ \\ CERCLA \\ CFR \\ $\mathrm{CHG}$ \\ Corps \\ COS \\ CRBG \\ CWA \\ DART \\ dB \\ dBA \\ DOC \\ DOE \\ DOE-RL \\ $\mathrm{DOH}$ \\ DOI \\ DOL \\ DWS \\ E/Q \\ EA \\ EC \\ Burlington Northern Santa Fe Railroad \\ Bonneville Power Administration \\ Basalt Waste Isolation Project \\ Clean Air Act \\ Columbia Basin College \\ Comprehensive Conservation Plan \\ Council on Environmental Quality \\ Comprehensive Environmental Response, Compensation, and Liability Act \\ Code of Federal Regulations \\ CH2M Hill Hanford Group, Inc. \\ U.S. Army Corps of Engineers \\ Characterization of systems \\ Columbia River Basalt Group \\ Clean Water Act \\ Data acquisition in real time \\ Decibels \\ A-weighted sound level \\ U.S. Department of Commerce \\ U.S. Department of Energy \\ U.S. Department of Energy, Richland Operations Office \\ Washington State Department of Health \\ U.S. Department of the Interior \\ U.S. Department of Labor \\ Drinking water standards \\ Atmospheric dispersion estimates \\ Environmental assessment \\ Environmental concern \\ Ecology \\ EDNA \\ Washington State Department of Ecology \\ EIS \\ EJ \\ Environmental designation for noise abatement \\ Environmental Impact Statement \\ Environmental justice \\ EMT \\ EO \\ Emergency medical technician \\ Environmental Objections \\ E.O. \\ Executive Order \\ EPA \\ U.S. Environmental Protection Agency \\ ESU \\ Evolutionary significant units
}




\section{Acronyms, Abbreviations, and Symbols (contd.)}

\begin{tabular}{|c|c|}
\hline ERDF & Environmental Restoration Disposal Facility \\
\hline EU & Environmentally unsatisfactory \\
\hline ExHPIF & Expanded Historic Property Inventory Form \\
\hline FEMA & Federal Emergency Management Agency \\
\hline FEP & Features, events, and processes \\
\hline FFCA & Federal Facilities Compliance Act \\
\hline FFTF & Fast Flux Test Facility \\
\hline FR & Federal Register \\
\hline FY & Fiscal Year \\
\hline GENII & Generation II Model For Environmental Dose Calculations \\
\hline HAMMER & Hazardous Materials Management and Emergency Response \\
\hline HCP-EIS & Hanford Comprehensive Land-Use Plan Environmental Impact Statement \\
\hline HCRL & Hanford Cultural Resources Laboratory \\
\hline HEHF & Hanford Environmental Health Foundation \\
\hline HMS & Hanford Meteorology Station \\
\hline $\mathrm{H} / \mathrm{PP}$ & Hanford Plio-Pleistocene \\
\hline $\mathrm{Hz}$ & Hertz \\
\hline $\mathrm{kWh}$ & Kilowatt-hour \\
\hline $\mathrm{L}_{\mathrm{eq}}$ & Equivalent sound level \\
\hline LIGO & Laser Interferometer Gravitational Wave Observatory \\
\hline LLWPA & Low-Level Radioactive Waste Policy Act \\
\hline $\mathrm{LO}$ & Lack of objections \\
\hline LWC & Lost workday cases \\
\hline LWD & Lost workdays \\
\hline $\mathrm{Ma}$ & Million years \\
\hline MMI & Modified Mercalli intensity \\
\hline MOU & Memorandum of Understanding \\
\hline National Register & National Register of Historic Places \\
\hline NEPA & National Environmental Policy Act \\
\hline NESHAP & National Emission Standards for Hazardous Air Pollutants \\
\hline NHPA & National Historic Preservation Act \\
\hline NMFS & National Marine Fisheries Service \\
\hline NPDES & National Pollutant Discharge Elimination System \\
\hline NPL & National Priorities List \\
\hline NPPC & Northwest Power Planning Council \\
\hline NPR & New production reactor \\
\hline NPS & National Park Service \\
\hline NRC & U.S. Nuclear Regulatory Commission \\
\hline NWPA & Nuclear Waste Policy Act \\
\hline OFM & Office of Financial Management \\
\hline ORP & U.S. Department of Energy Office of River Protection \\
\hline OSHA & Occupational Safety and Health Administration \\
\hline OSPI & Office of Superintendent of Public Instruction \\
\hline PCB & Polychlorinated biphenyls \\
\hline PHMC & Project Hanford Management Contract \\
\hline PL & Public Law \\
\hline $\mathrm{PM}_{2.5}$ & Particulate matter (2.5 micrometers or less) \\
\hline $\mathrm{PM}_{10}$ & Particulate matter (10 micrometers or less) \\
\hline
\end{tabular}




\section{Acronyms, Abbreviations, and Symbols (cont'd.)}

PNNL

PSD

PUREX

RCRA

RCW

REIS

$\mathrm{RM}$

ROD

$\mathrm{S}$

SARA

SCR

SDWA

SEPA

SHPO

SIP

SNF

SR

SSW

SW

TCP

TEDF

TRC

Tri-Cities

TSCA

TSD

TSP

$\mathrm{UO}_{3}$

USC

USFWS

USGS

WAC

WDFW

WMA

WSR

WSU-TC

$\overline{\chi^{\prime}} \mathrm{Q}^{\prime}$
Pacific Northwest National Laboratory

Prevention of significant deterioration

Plutonium-Uranium Extraction

Resource Conservation and Recovery Act

Revised Code of Washington

Regional Economic Information System

River Mile

Record of Decision

South

Superfund Amendments and Reauthorization Act

Software change request

Safe Drinking Water Act

State Environmental Policy Act

State Historic Preservation Officer

State Implementation Plan

Spent nuclear fuel

State route

South-southwest

Southwest

Traditional Cultural Place

Treated Effluent Disposal Facility

Total recordable cases

Kennewick, Pasco, and Richland

Toxic Substances Control Act

Treatment, Storage, and/or Disposal

Total Suspended Particulates

Uranium trioxide

United States Code

U.S. Fish and Wildlife Service

U.S. Geological Survey

Washington Administrative Code

Washington State Department of Fish and Wildlife

Waste management area

Washington State Register

Washington State University, Tri Cities

Atmospheric dispersion coefficient 


\section{Acronyms, Abbreviations, and Symbols (cont'd.)}

Names And Symbols For Units Of Measure, Radioactivity, Time, and Mathematical Terms

\begin{tabular}{|c|c|c|c|}
\hline \begin{tabular}{ll}
\multicolumn{2}{l}{ Length } \\
$\mathrm{cm}$ & centimeter \\
$\mathrm{ft}$ & foot \\
$\mathrm{in}$. & inch \\
$\mathrm{km}$ & kilometer \\
$\mathrm{m}$ & meter \\
$\mathrm{mi}$ & mile \\
$\mathrm{mm}$ & millimeter \\
\end{tabular} & $\begin{array}{l}\text { Area } \\
\mathrm{km}^{2} \text { square kilometer } \\
\mathrm{mi}^{2} \text { square mile } \\
\mathrm{ac} \quad \text { acre } \\
\text { ha hectare }\end{array}$ & \begin{tabular}{ll}
\multicolumn{2}{l}{ Volume } \\
$\mathrm{m}^{3}$ & cubic meter \\
gal & gallon \\
$\mathrm{L}$ & liter
\end{tabular} & \begin{tabular}{ll}
\multicolumn{2}{l}{ Time/Speed } \\
$\mathrm{s} \quad$ second \\
$\mathrm{hr}$ & hour \\
$\mathrm{yr}$ & year \\
$\mathrm{mph}$ & miles per hour \\
$\mathrm{m} / \mathrm{s}$ & meters per second \\
$\mathrm{g}$ & acceleration of \\
gravity \\
\end{tabular} \\
\hline \multirow[t]{2}{*}{ 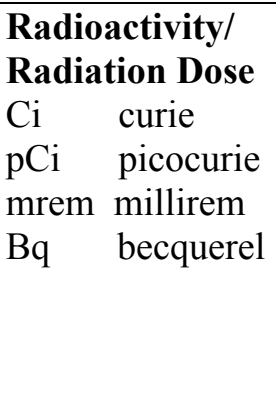 } & $\begin{array}{l}\text { Temperature } \\
{ }^{\circ} \mathrm{C} \text { degrees Celsius } \\
{ }^{\circ} \mathrm{F} \text { degrees Fahrenheit }\end{array}$ & \multirow{2}{*}{\begin{tabular}{ll} 
& \multicolumn{2}{l}{ Mathematical } \\
$>\quad$ & greater than \\
$\geq$ & greater than or equal to \\
$<$ & less than \\
$\leq$ & less than or equal to \\
$\sim$ & approximately \\
avg & average \\
max & maximum \\
min & minimum
\end{tabular}} & \begin{tabular}{ll}
\multicolumn{2}{l}{ Mass } \\
$\mathrm{g}$ & gram \\
$\mathrm{kg}$ & kilogram \\
$\mathrm{mg}$ & milligram \\
$\mu \mathrm{g}$ & microgram
\end{tabular} \\
\hline & $\begin{array}{l}\text { Chemical } \\
\mathrm{M} \text { molar }\end{array}$ & & $\begin{array}{l}\text { Concentration } \\
\text { ppm parts per million }\end{array}$ \\
\hline
\end{tabular}




\section{Contents}

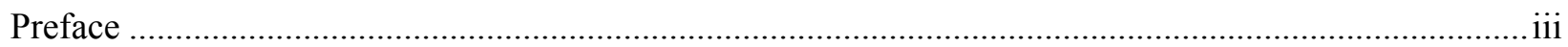

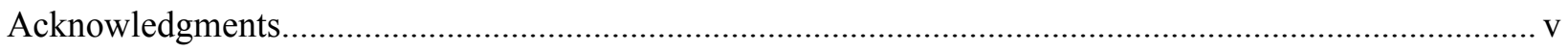

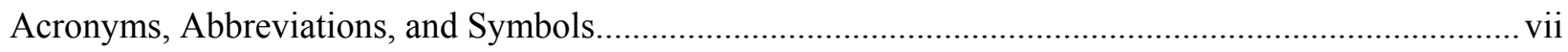

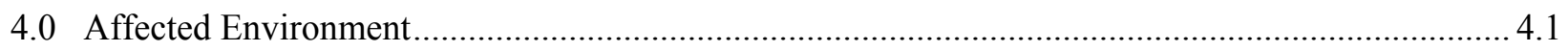

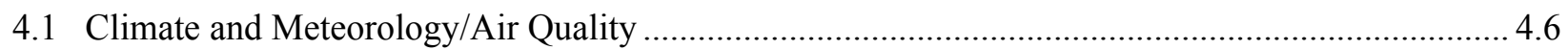

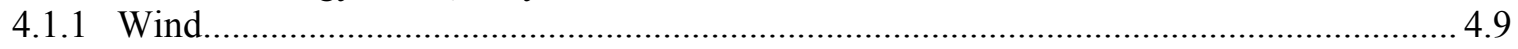

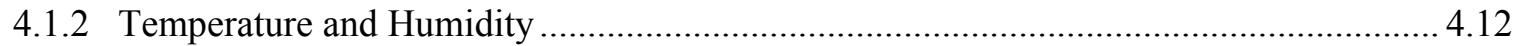

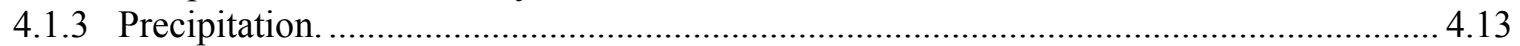

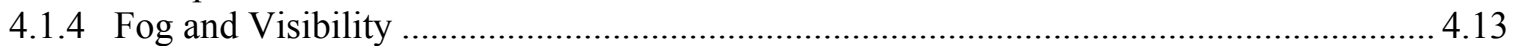

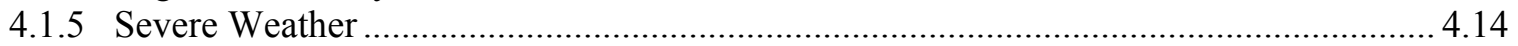

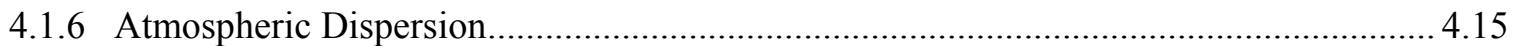

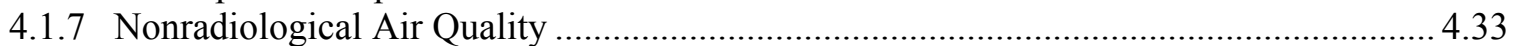

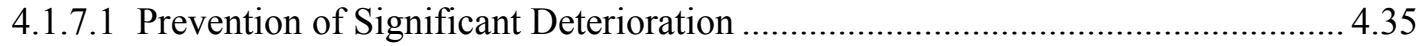

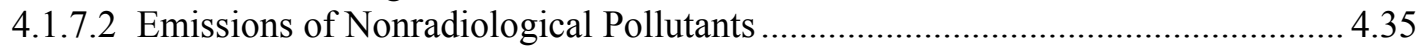

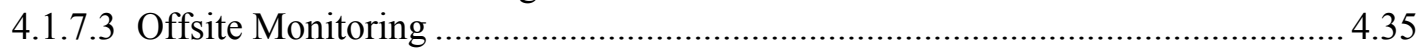

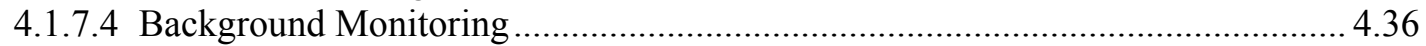

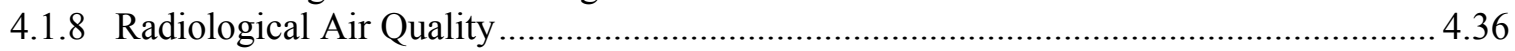

4.2 Geology

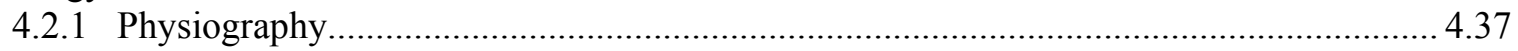

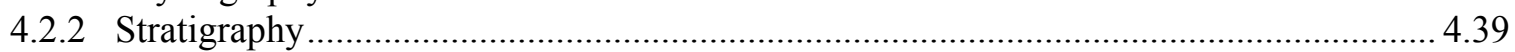

4.2.2.1 Columbia River Basalt Group ................................................................. 4.39

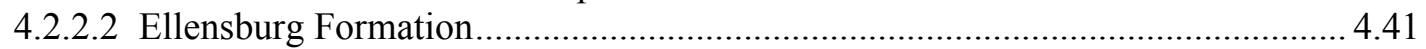

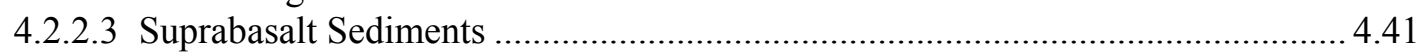

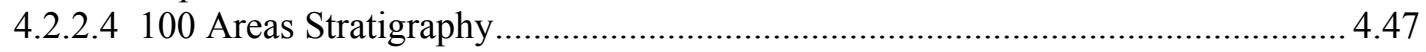

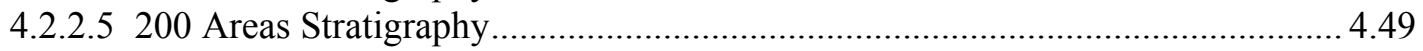

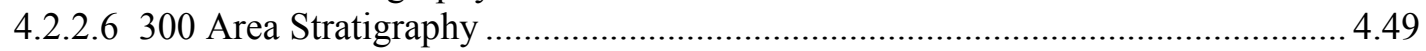

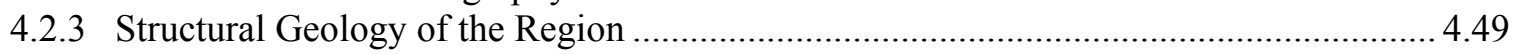

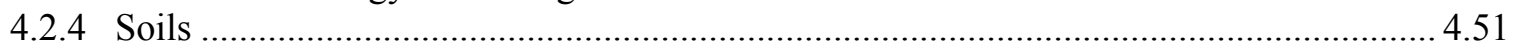

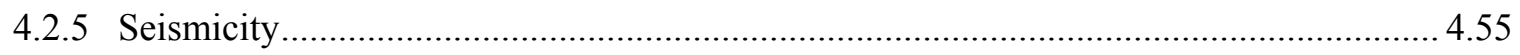

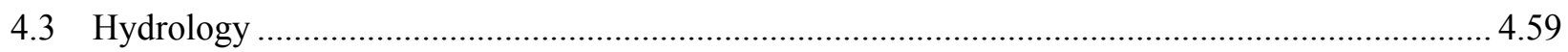

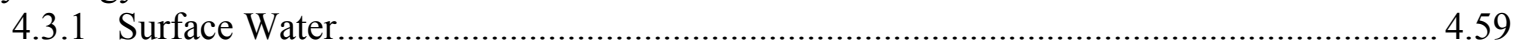

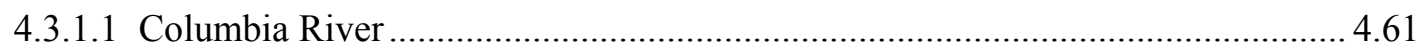

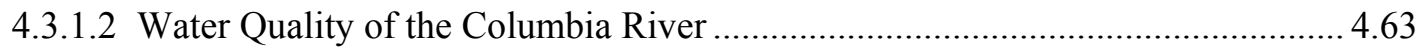

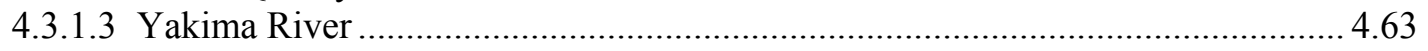

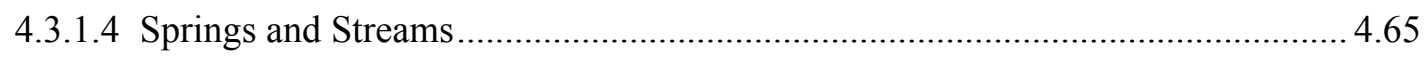

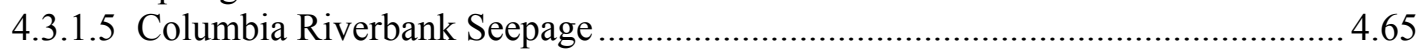

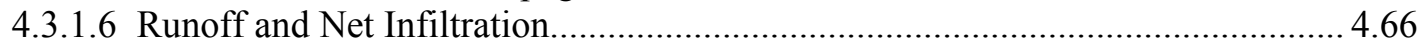

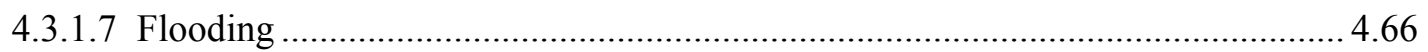

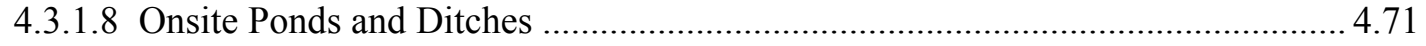

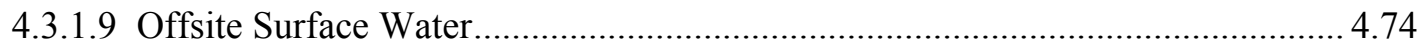

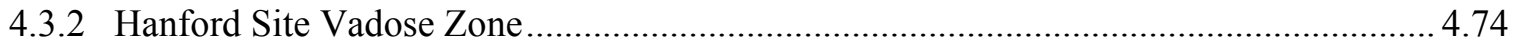

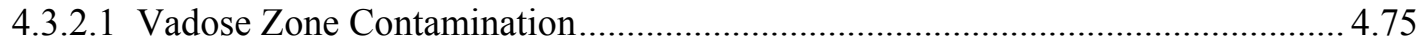

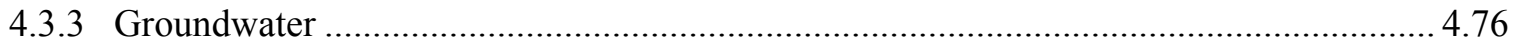




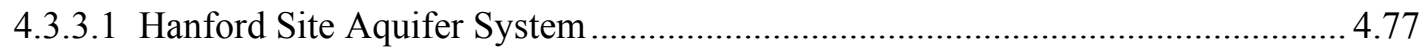

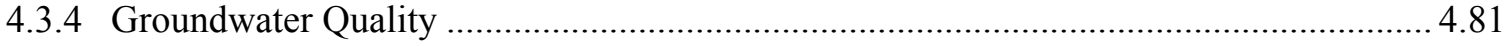

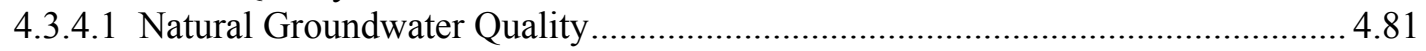

4.3.4.2 Groundwater Contamination and Monitoring ................................................. 4.81

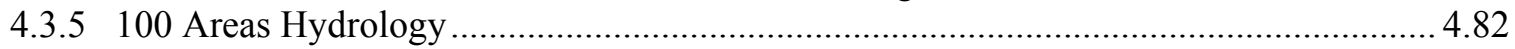

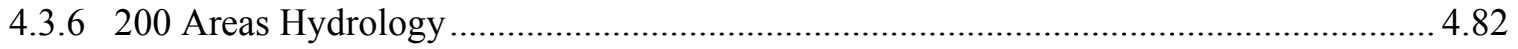

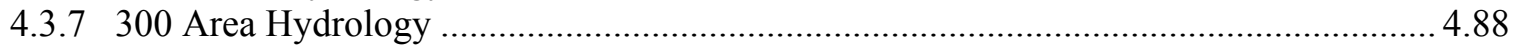

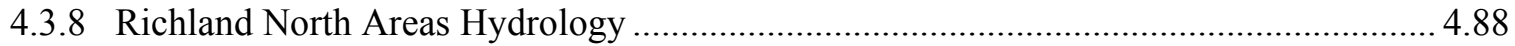

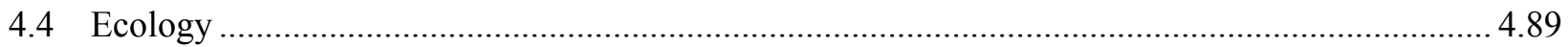

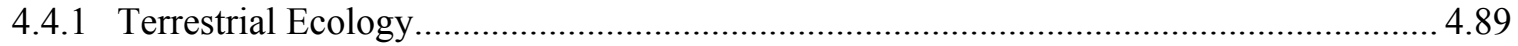

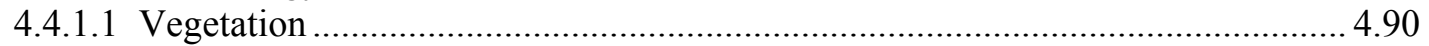

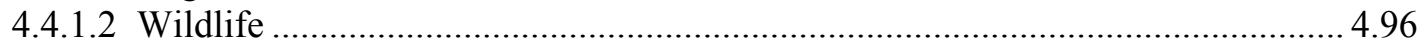

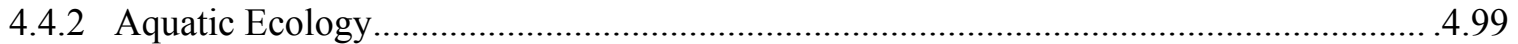

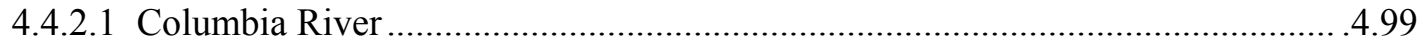

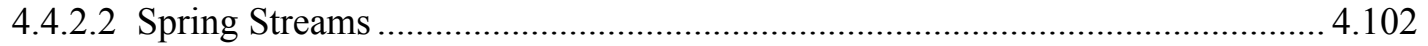

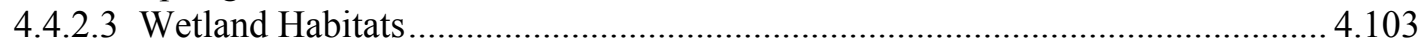

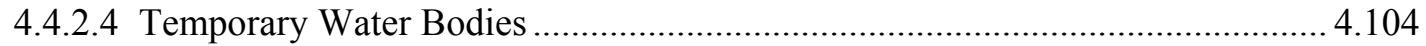

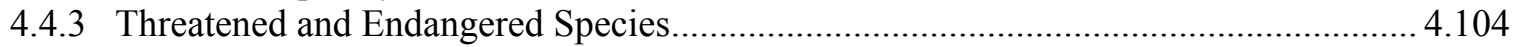

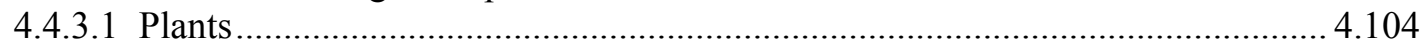

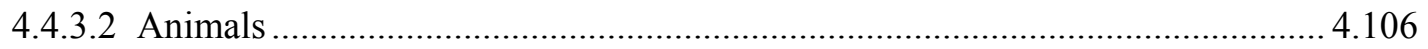

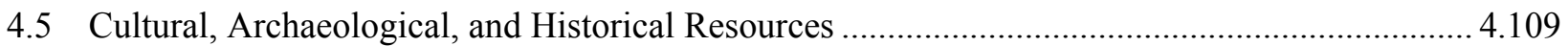

4.5.1 Native American Cultural Landscape .............................................................................. 4.113

4.5.1.1 Traditional Cultural Places and Traditional Use Areas.................................... 4.113

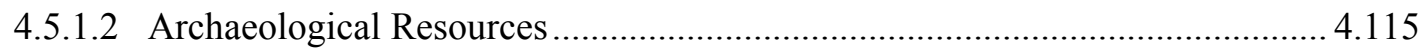

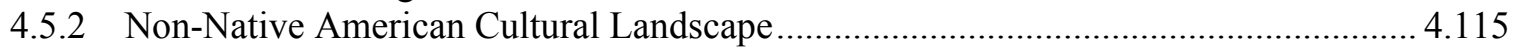

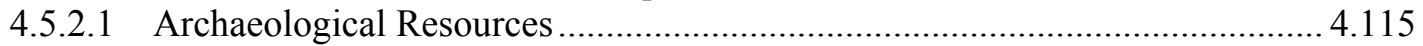

4.5.2.2 Traditional Cultural Places................................................................... 4.116

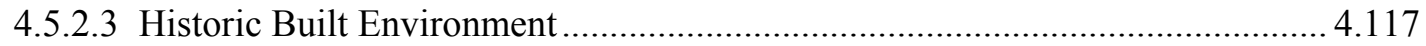

4.5.3 Cultural, Archaeological, and Historical Resources Impacts from the

24 Command Wildland Fire............................................................................. 4.117

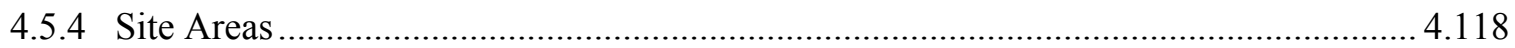

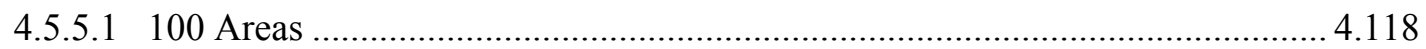

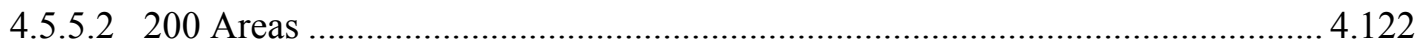

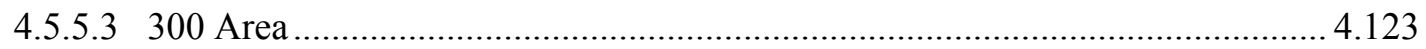

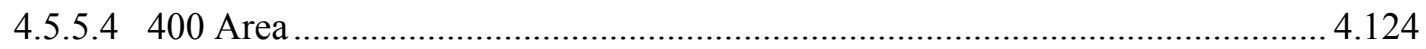

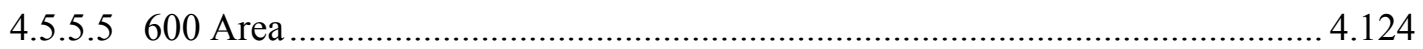

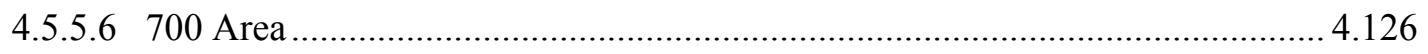

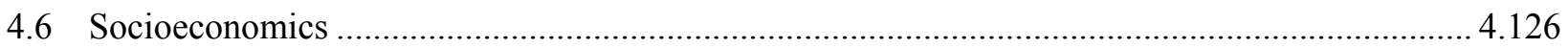

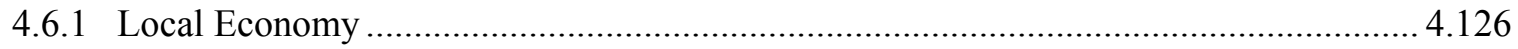

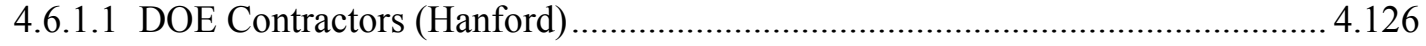

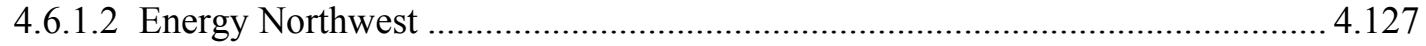

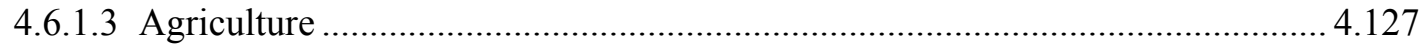

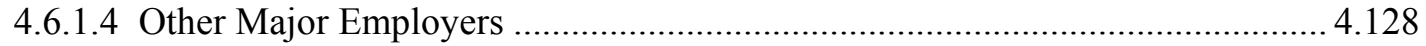

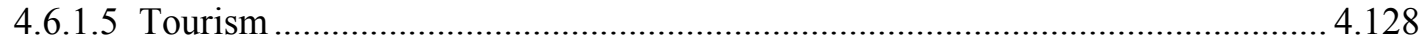

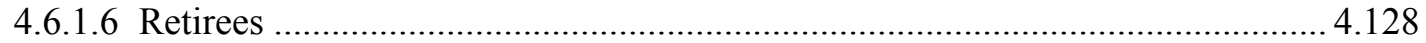

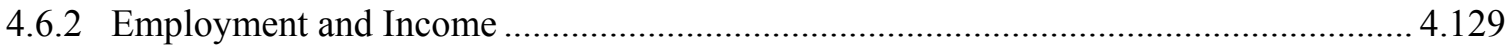

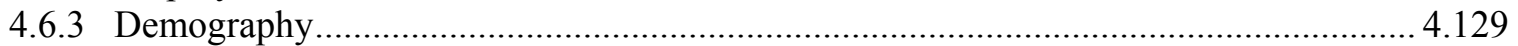

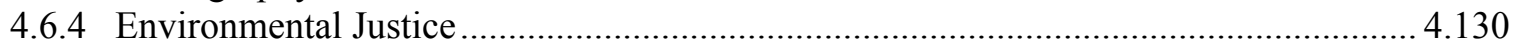




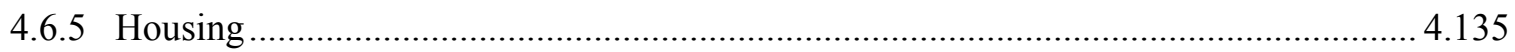

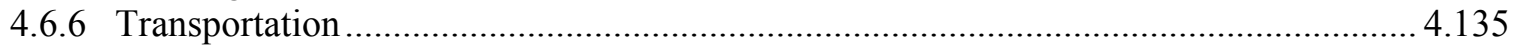

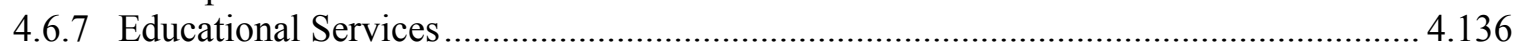

4.6.8 Health Care and Human Services .......................................................................... 4.137

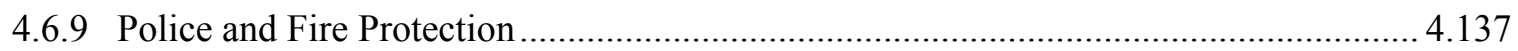

4.6.10 Parks and Recreation...................................................................................... 4.139

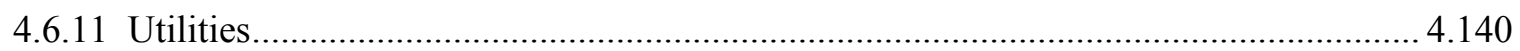

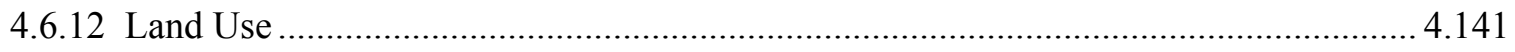

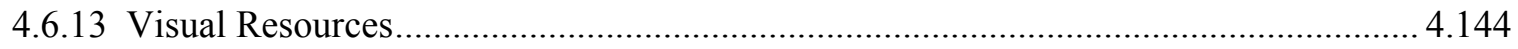

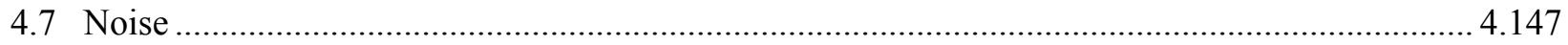

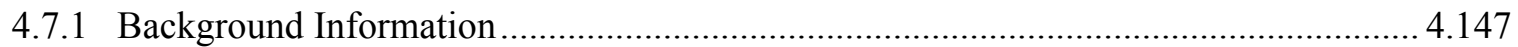

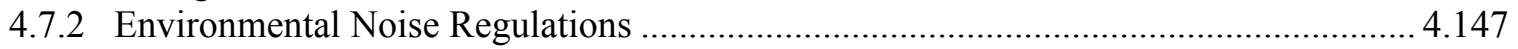

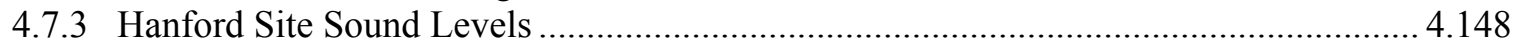

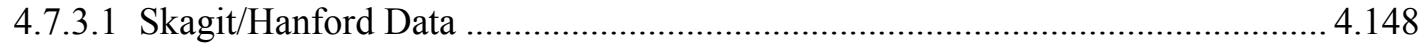

4.7.3.2 Basalt Waste Isolation Project (BWIP) Data ............................................... 4.148

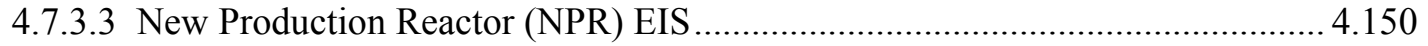

4.7.3.4 Noise Levels of Hanford Field Activities ........................................................ 4.151

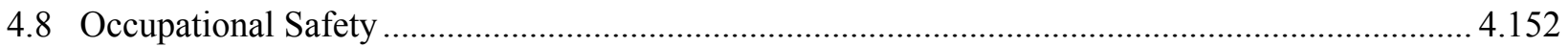

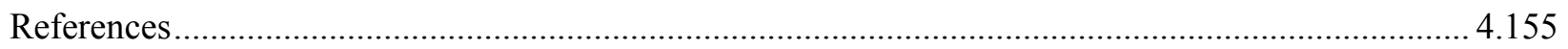

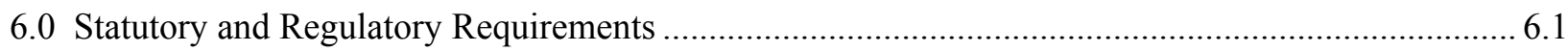

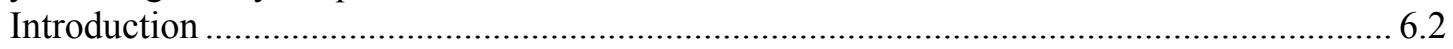

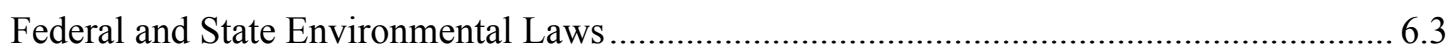

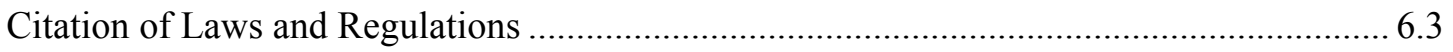

Specific Federal Laws Cited in the CEQ Regulations.................................................... 6.3

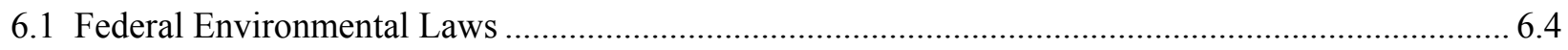

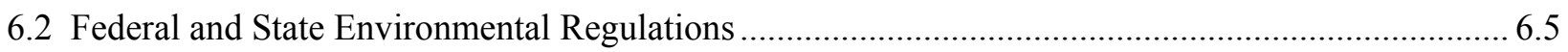

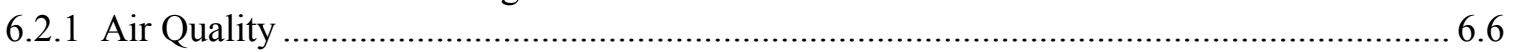

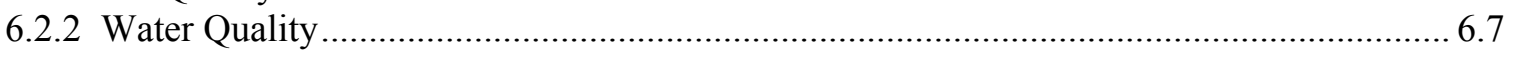

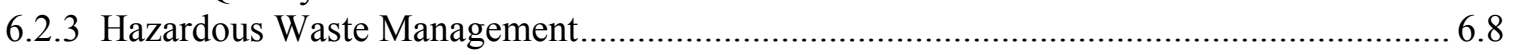

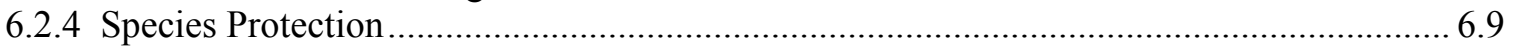

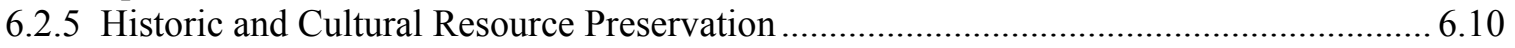

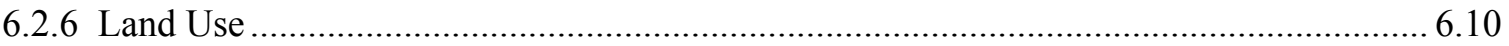

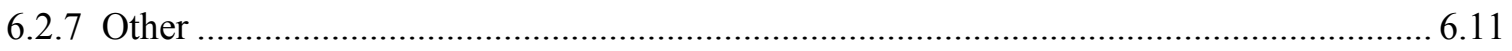

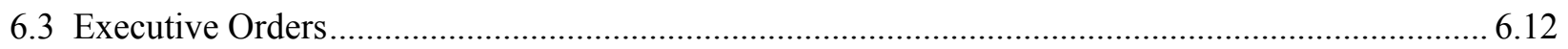

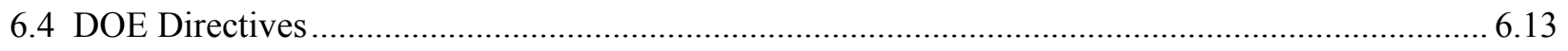

6.5 Treaties of the United States with American Indian Tribes of the Hanford Region........................ 6.14

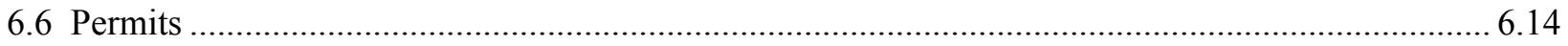

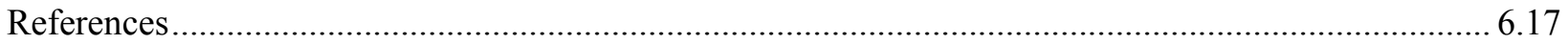

Appendix A, Hanford Site Species Lists .......................................................................................... A.1 


\section{Figures}

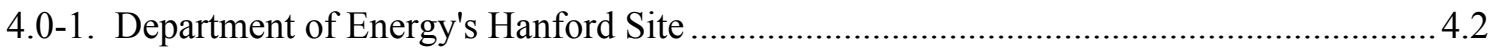

4.0-2. Hanford Reach National Monument as of June 2000 ..................................................... 4.3

4.0-3. Area of Hanford Site Burned as a Result of the June 27 - July 2, 2000

Wildfire.

4.1-1. Hanford Meteorological Monitoring Network

4.1-2. Wind Roses at the $9.1 \mathrm{~m}$ (30 ft) Level of the Hanford Meteorological

Monitoring Network, 1982 to 2000

4.1-3. Wind Roses at the $60 \mathrm{~m}(197 \mathrm{ft})$ Level of the Hanford Meteorological

Monitoring Network, 1986 to 2000

4.2-1. Physiographic Provinces of the Pacific Northwest, with Columbia Intermontane Province Shown in White

4.2-2. Paleoflow Directions and Landforms Associated with Cataclysmic Flooding in the Central Columbia Plateau

4.2-3. Stratigraphic Column for the Pasco Basin....

4.2-4. Stratigraphic Column for the Hanford Site Showing Correlations Among Various Authors.

4.2-5. Location of Surficial Features

4.2-6. Location of Structural Features

4.2-7. Soil Map of the Hanford Site

4.2-8. Historical Seismicity of the Columbia Plateau and Surrounding Areas 4.56

4.2-9. Seismicity of the Columbia Plateau and Surrounding Areas as Measured by Seismographs

4.3-1. Surface Water Features Including Rivers, Ponds, Major Springs, and Ephemeral Streams on the Hanford Site.....

4.3-2. Average daily flow for the Columbia River below Priest Rapids Dam from 1990 through April 2001

4.3-3. Average daily flow for the Columbia River During 2000 4.62

4.3-4. Columbia River Monitoring Locations 4.64

4.3-5. Average daily flow for the Yakima River from 1991 through April 2001 4.65 
4.3-7. Locations of Principal Dams Within the Columbia Plateau .......................................... 4.68

4.3-8. Flood Area for the Probable Maximum Flood .............................................................. 4.70

4.3-9. Flood Area from a 100-Year Flood of the Yakima River near the Hanford Site

4.3-10. Extent of Probable Maximum Flood in Cold Creek Area

4.3-11. Water Table Elevations for the Unconfined Aquifer at Hanford, March/April 2000

4.3-12. Distribution of Major Radionuclides in Groundwater at Concentrations Above the Maximum Contaminant Level or Interim Drinking Water Standard During Fiscal Year 2000

4.3-13. Distribution of Major Hazardous Chemicals in Groundwater at Concentrations Above the Maximum Contaminant Level or Interim Drinking Water Standard During Fiscal Year 2000

4.3-14. Geologic Units Intersected by the Water Table in the 100 Areas

4.4-1. Distribution of Vegetation Types and Land Use Areas on the Hanford Site

4.4-2. Columbia River Islands

4.5-1. Cultural Resources Areas Surveyed on the Hanford Site

4.6-1. Location of Minority Populations Near the Hanford Site

4.6-2. Location of Low-Income Populations Near the Hanford Site

4.6-3. DOE's Preferred Alternative for Land Use on the Hanford Site

4.6-4. Viewshed From Gable Mountain

4.7-1. Location of Background Noise Measurements

4.8-1. Occupational Injury and Illness Total Recordable Case Rates at the Hanford Site 


\section{Tables}

4.1-1. Station Numbers, Names, and Instrumentation for each Hanford Meteorological Monitoring Network Site

4.1-2. Number of Days with Peak Gusts Above Specific Thresholds at $15 \mathrm{~m}(50 \mathrm{ft})$

Level, 1945 through 2000

4.1-3. Number of Days with Fog by Season.

4.1-4. Estimate of the Probability of Extreme Winds Associated with Tornadoes

Striking a Point at Hanford

4.1-5. Estimates of Extreme Winds at the Hanford Site

4.1-6. Percent Probabilities for Extended Periods of Surface-Based Inversions

4.1-7. Joint Frequency Distributions of Atmospheric Stability, Wind Speed and

Transport Direction for the 100 Area at $9 \mathrm{~m}$ Above Ground Level

4.1-8. Joint Frequency Distributions of Atmospheric Stability, Wind Speed and

Transport Direction for the 100 Area at $60 \mathrm{~m}$ Above Ground Level

4.1-9. Joint Frequency Distributions of Atmospheric Stability, Wind Speed and

Transport Direction for the 200 Areas at $9 \mathrm{~m}$ Above Ground Level.....

4.1-10. Joint Frequency Distributions of Atmospheric Stability, Wind Speed and

Transport Direction for the 200 Areas at $61 \mathrm{~m}$ Above Ground Level

4.1-11. Joint Frequency Distributions of Atmospheric Stability, Wind Speed and

Transport Direction for the 300 Area at $9 \mathrm{~m}$ Above Ground Level

4.1-12. Joint Frequency Distributions of Atmospheric Stability, Wind Speed and

Transport Direction for the 300 Area at $60 \mathrm{~m}$ Above Ground Level

4.1-13. Joint Frequency Distributions of Atmospheric Stability, Wind Speed and Transport Direction for the 400 Area at $9 \mathrm{~m}$ Above Ground Level.

4.1-14. Joint Frequency Distributions of Atmospheric Stability, Wind Speed and Transport Direction for the 400 Area at $60 \mathrm{~m}$ Above Ground Level

4.1-15. $\quad \chi / \mathrm{Q}^{\prime}$ Values $\left(\mathrm{sec} \mathrm{m}^{-3}\right)$ for Chronic Ground-Level Releases from 100-N Area

4.1-16. $\quad \chi / \mathrm{Q}^{\prime}$ Values $\left(\mathrm{sec}^{-3}\right)$ for Chronic 61-m Stack Releases from 100-N Area ...................... 4.25

4.1-17. $\chi / \mathrm{Q}^{\prime}$ Values $\left(\mathrm{sec}^{-3}\right)$ for Chronic Ground-Level Releases from 200 Areas...................... 4.26

4.1-18. $\quad \chi / \mathrm{Q}^{\prime}$ Values $\left(\mathrm{sec}^{-3}\right)$ for Chronic 61-m Stack Releases from 200 Areas ........................ 4.26 
4.1-19. $\chi / \mathrm{Q}^{\prime}$ Values $\left(\mathrm{sec} \mathrm{m}^{-3}\right)$ for Chronic Ground-Level Releases from 300 Area

4.1-20. $\quad \chi / \mathrm{Q}^{\prime}$ Values $\left(\mathrm{sec} \mathrm{m}^{-3}\right)$ for Chronic 61-m Stack Releases from 300 Area

4.1-21. $\quad \chi / \mathrm{Q}^{\prime}$ Values $\left(\mathrm{sec}^{-3}\right)$ for Chronic Ground-Level Releases from 400 Area 4.28

4.1-22. $\chi / \mathrm{Q}^{\prime}$ Values $\left(\mathrm{sec} \mathrm{m}^{-3}\right)$ for Chronic 30-m Stack Releases from 400 Area 4.28

4.1-23. 95th Percentile E/Q Values $\left(\mathrm{sec}^{-3}\right.$ ) for Acute Ground Level Releases from 100-N Area

4.1-24. 95th Percentile E/Q Values $\left(\mathrm{sec}^{-3}\right.$ ) for Acute 61-m Stack Releases from 100-N Area

4.1-25. 95th Percentile E/Q Values $\left(\mathrm{sec}^{-3}\right.$ ) for Acute Ground-Level Releases from 200 Areas

4.1-26. 95th Percentile E/Q Values $\left(\mathrm{sec}^{-3}\right.$ ) for Acute 61-m Stack Releases from 200 Areas.

4.1-27. 95th Percentile E/Q Values $\left(\mathrm{sec}^{-3}\right.$ ) for Acute Ground-Level Releases from 300 Area

4.1-28. 95th Percentile E/Q Values $\left(\mathrm{sec}^{-3}\right.$ ) for Acute 61-m Stack Releases from 300 Area

4.1-29. 95th Percentile E/Q Values $\left(\mathrm{sec}^{-3}\right.$ ) for Acute Ground-Level Releases from 400 Area

4.1-30. 95th Percentile E/Q Values $\left(\mathrm{sec} \mathrm{m}^{-3}\right.$ ) for Acute 30-m Stack Releases from 400 Area

4.1-31. National and Washington State Ambient Air Quality Standards...................................... 4.34

4.1-32. Non-radioactive Constituents Discharged to the Atmosphere, 1999 ............................... 4.35

4.1-33. Radionuclides Discharged to the Atmosphere at the Hanford Site, 1999 .......................... 4.37

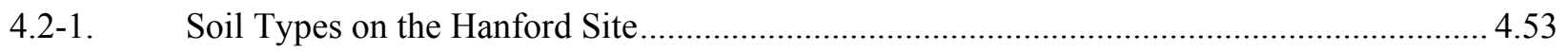

4.3-1. Maximum Concentrations of Groundwater Contaminants in Fiscal Year 2000................. 4.83

4.4-1. Federal- or Washington State-Listed Threatened (T) and Endangered (E) Species Occurring on the Hanford Site

4.4-2. Washington State Plant Species of Concern Occurring on the Hanford Site

4.4-3. Washington State Candidate Animal Species Occurring on the Hanford Site 
4.5-1. Historic Buildings, Archaeological Sites and Districts Listed in the National Register of Historic Places

4.5-2. Archaeological Sites and Historic Districts Determined Eligible for Listing in the National Register of Historic Places

4.5-3. Archaeological Sites and Districts Listed in the Washington Heritage

Register.....

4.6-1. Federal Government Transfer Payments in Benton and Franklin Counties, 1999

4.6-2. Nonagricultural Wage and Salary Workers in Benton and Franklin Counties, 1999 and 2000

4.6-3. Population Estimates and Percentages by Race and Hispanic Origin, 2000

4.6.4. Number and Percentages of Persons Living in Low-Income Households in Counties Near the Hanford Site, 1997 and 1989

4.6-5. Police Personnel in the Tri-Cities, 2001

4.6-6. Fire Protection Personnel in the Tri-Cities, 2001

4.6-7. Examples of Physical Recreational Facilities Available in the Tri-Cities

4.6-8. Areas of the Hanford Site Outgranted/Released to Date

4.7-1. Applicable State Noise Limitations for the Hanford Site Based on Source and Receptor EDNA Designation

4.7-2. Background Noise Levels Measured at Isolated Areas

4.7-3. Modeled Noise Resulting from Automobile Traffic at Hanford in Association with the New Production Reactor Environmental Impact Statement

4.7-4. Monitored Levels of Noise Propagated from Outdoor Activities at the Hanford Site

4.8-1. Occupational Injury, Illness, and Fatality Incidence Rates for Department of Energy Facilities and Private Industries, 1996-2000 Averages.

A-1. Common Vascular Plants on the Hanford Site …............................................................ A.3

A-2. $\quad$ Mammals That Have Been Observed on the Hanford Site .................................................. A.7

A-3. Reptiles and Amphibians found on the Hanford Site. ….............................................. A.8

A-4. Common bird species known to occur annually on the Hanford Site ................................ A.9

A-5. Fish Species in the Hanford Reach of the Columbia River. .............................................. A.13 


\subsection{Affected Environment}

The U.S. Department of Energy (DOE) Hanford Site lies within the semiarid Pasco Basin of the Columbia Plateau in southeastern Washington State (Figure 4.0-1). The Site occupies an area of about $1517 \mathrm{~km}^{2}$ (about $586 \mathrm{mi}^{2}$ ) ${ }^{(a)}$ north of the confluence of the Yakima River with the Columbia River. The Hanford Site is about $50 \mathrm{~km}(30 \mathrm{mi})$ north to south and $40 \mathrm{~km}(24 \mathrm{mi})$ east to west. This land, with restricted public access, provides a buffer for the smaller areas currently used for storage of nuclear materials, waste storage, and waste disposal. The Columbia River flows through the northern part of the Hanford Site and, turning south, forms part of the Site's eastern boundary. The Yakima River runs near the southern boundary of the Hanford Site and joins the Columbia River at the city of Richland, which bounds the Hanford Site on the southeast. Rattlesnake Mountain, Yakima Ridge, and Umtanum Ridge form the southwestern and western boundaries. The Saddle Mountains form the northern boundary of the Hanford Site. Two small east-west ridges, Gable Butte and Gable Mountain, rise above the plateau of the central part of the Hanford Site. Adjoining lands to the west, north, and east are principally range and agricultural land. The cities of Kennewick, Pasco, and Richland (the Tri-Cities) constitute the nearest population centers and are located southeast of the Hanford Site.

On June 9, 2000, William J. Clinton, by Presidential Proclamation, created the Hanford Reach National Monument under the authority of the 1906 Antiquities Act (65 FR 37253). As established, the Monument totals $792.6 \mathrm{~km}^{2}\left(306 \mathrm{mi}^{2}\right)$ and includes Fitzner/Eberhardt Arid Lands Ecology Reserve (ALE), Saddle Mountain Wildlife Refuge, McGee Ranch/Riverlands Area, and land 1/4 mile inland from the mean high-water mark on the south and west shores of the 51-mi long Hanford Reach, the last freeflowing, non-tidal stretch of the Columbia River. It also includes Wahluke Slope, federally owned islands in the Hanford Reach, White Bluffs, and the sand dune area northwest of the Energy Northwest Site (Figure 4.0-2). This designation establishes the protection and management of the lands within the region of the monument. By memorandum, the president also directed the Secretary of Energy to consult with the Secretary of the Interior regarding the incorporation of additional Hanford Site lands into the Monument as the land is remediated.

On June 14, 2001, the DOE Richland Operations Office (DOE-RL) and the U.S. Fish and Wildlife Service (USFWS) signed an amended Memorandum of Understanding (MOU) delegating management responsibilities for the Hanford Reach National Monument. The MOU spells out the roles and responsibilities of each agency for the Wahluke Slope and ALE Reserve. Objectives of the MOU are to ensure:

- the preservation of natural and cultural resources while maintaining current use of Saddle Mountain Wildlife Refuge as a research natural area and safety buffer for ongoing missions on the Hanford Site

- the portions of the Monument managed by USFWS are managed in accordance with the Presidential Proclamation

- that the integrity of the Refuge as an intact ecological unit is maintained

- that the Refuge is managed as a resource that provides an opportunity for Native Americans to exercise traditional religious and cultural activities consistent with the foregoing objectives

(a) This figure is based on the newest GIS interpolation of the Hanford Site legal boundary. Historically, the Site area was reported as $1450 \mathrm{~km}^{2}\left(560 \mathrm{mi}^{2}\right)$, calculated by the addition of sections and subunits based on surveys from the $1800 \mathrm{~s}$. Included in the Site is $36.42 \mathrm{~km}^{2}\left(14.1 \mathrm{mi}^{2}\right)$ of Columbia River surface water and $1 \mathrm{mi}^{2}$ of Washington State land (DOE 1999a). 


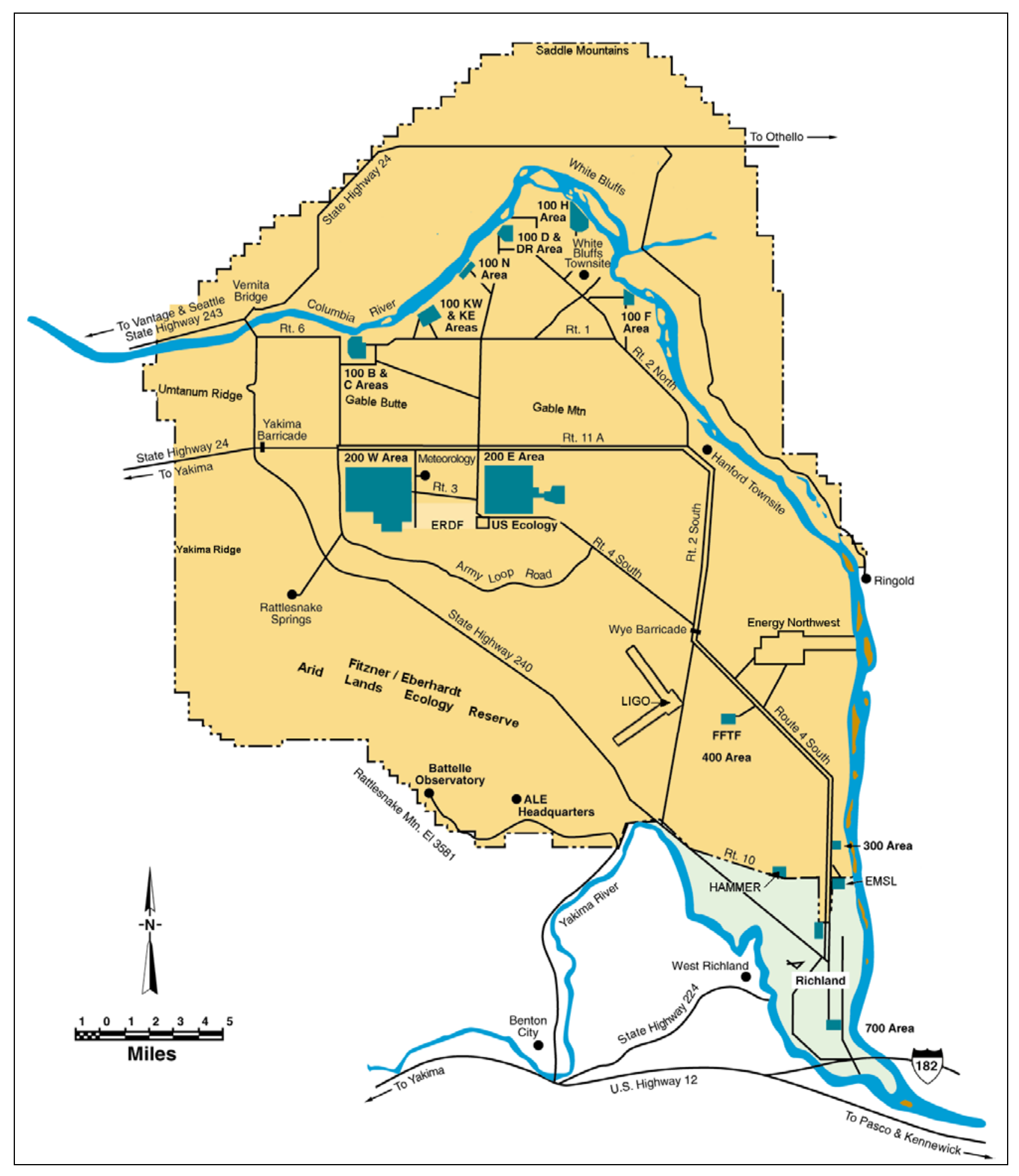

Figure 4.0-1. Department of Energy's Hanford Site 


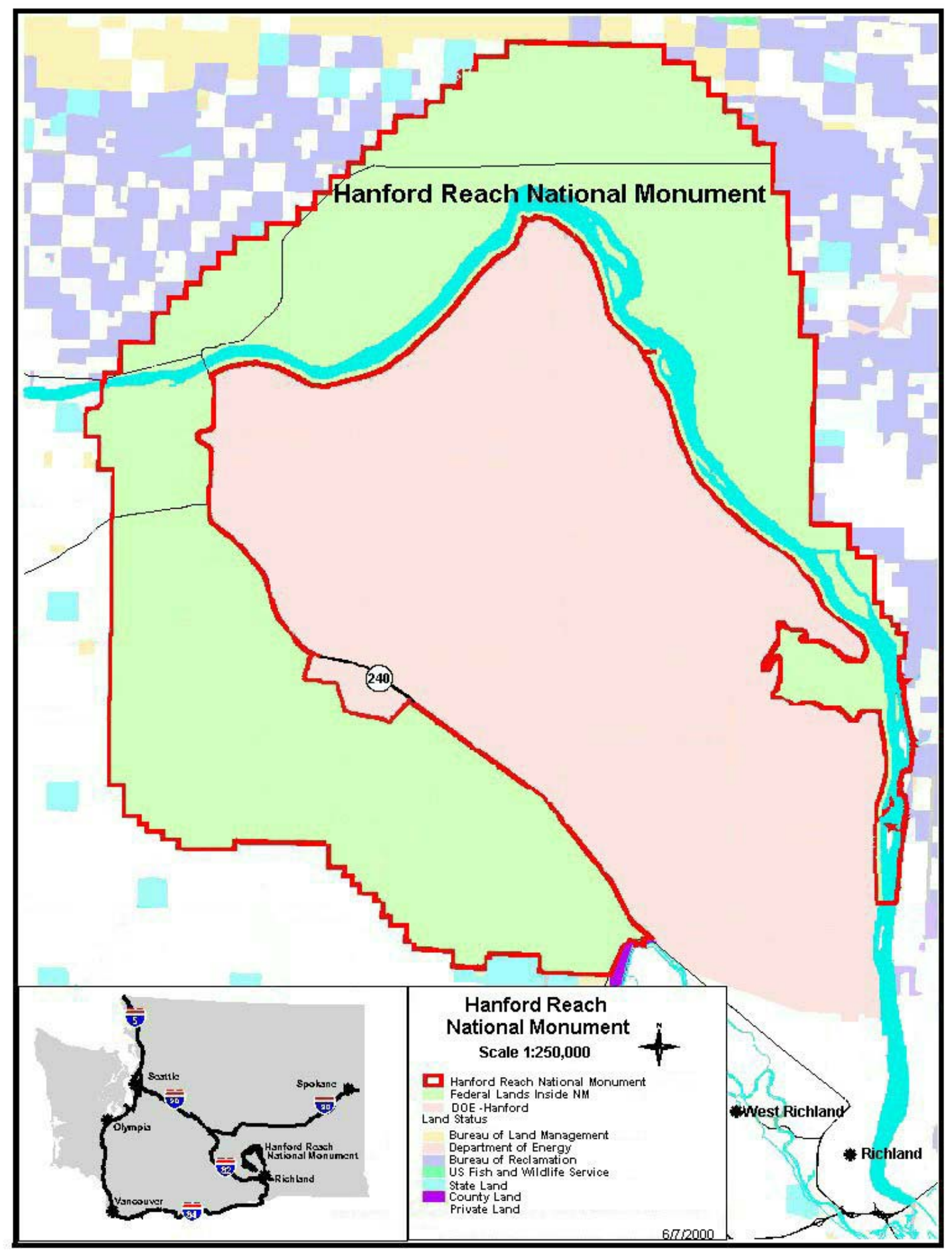

Figure 4.0-2. Hanford Reach National Monument as of June 2000 (modified from 65 FR 37253) 
- that access to the Refuge is available for the educational, scientific, and recreational benefit of the public to the extent this access and use is consistent with the foregoing objectives and compatible with Refuge purposes

- that worker safety and public protection are maintained

- protection and preservation and continued monitoring of nationally significant cultural resources including archeological and historic resources and traditional cultural places.

As a result of the MOU, the USFWS is the lead agency in producing a Comprehensive Conservation Plan (CCP) for management of the Hanford Reach National Monument and the overlay areas, Saddle Mountain National Wildlife Refuge, Wahluke Slope, and ALE. Development of the CCP will be a public process, including input from local governments, affected Native American Tribes, stakeholders, and others, including the recently initiated Federal Advisory Committee for the Hanford Reach National Monument. The DOE will participate in writing the CCP, and in cooperation with the USFWS, approve the plan. Under the MOU, which is intended to remain in effect for 25 years, DOE and USFWS will produce other agreements for such actions as site access, security, emergency preparedness, mutual assistance, wildland fire response, and cultural and biological resource management.

Between June 27 and July 2, 2000, a large wildfire swept through the Hanford Site. Designated the 24 Command Fire, it burned approximately $655 \mathrm{~km}^{2}\left(250 \mathrm{mi}^{2}\right)$, including virtually all of the ALE Reserve (BAER 2000) (Figure 4.0-3). As a result, approximately $178.1 \mathrm{~km}^{2}\left(68.8 \mathrm{mi}^{2}\right)$ of shrub-steppe community and $196.3 \mathrm{~km}^{2}\left(75.8 \mathrm{mi}^{2}\right)$ of communities containing sparse shrub overstories were lost. The fire extended across the southwestern half of the Hanford Site, bordered the 200 Area, and followed major roads to the 300 Area. The fire crossed State Route (SR) 240, Army Loop Road, and the SR 240 Access Road and burned east to Route 10 and northeast to Route 4. With the exception of minor structures in the vicinity of ALE, no facilities were burned on Site. In addition to the fire spreading across the Hanford Site, the fire burned south into Benton City. The fire destroyed 11 residences in Benton City, as well as other structures and outbuildings. The fire had no impact on the waste storage areas or underground tanks on the Hanford Site.

The Hanford Site encompasses more than 1500 waste management units and groundwater contamination plumes that have been grouped into 62 operable units. Each unit has complementary characteristics of such parameters as geography, waste content, type of facility, and relationship of contaminant plumes. This grouping into operable units allows for economies of scale to reduce the cost and number of characterization investigations and remedial actions that will be required for the Hanford Site to complete environmental cleanup efforts (WHC 1989). The 62 operable units have been aggregated into four areas: 22 in the 100 Area (17 source, 5 groundwater), 33 in the 200 Areas ( 29 source and 4 groundwater), 3 in the 300 Area ( 2 source, 1 groundwater), and 4 in the former 1100 Area. ${ }^{(a)}$ Those persons contemplating NEPA-related activities on the Hanford Site should be aware of the existence and location of the various operable units. Detailed information concerning the operable units may be found online at URL: http://www.bhi-erc.com/projects/p m/eis/wids/wids.htm. Current maps showing the locations of the operable units can be obtained from the environmental restoration contractor.

(a) Source: Personal communication with L. Dietz, Bechtel Hanford Inc. (BHI), August 1999. 


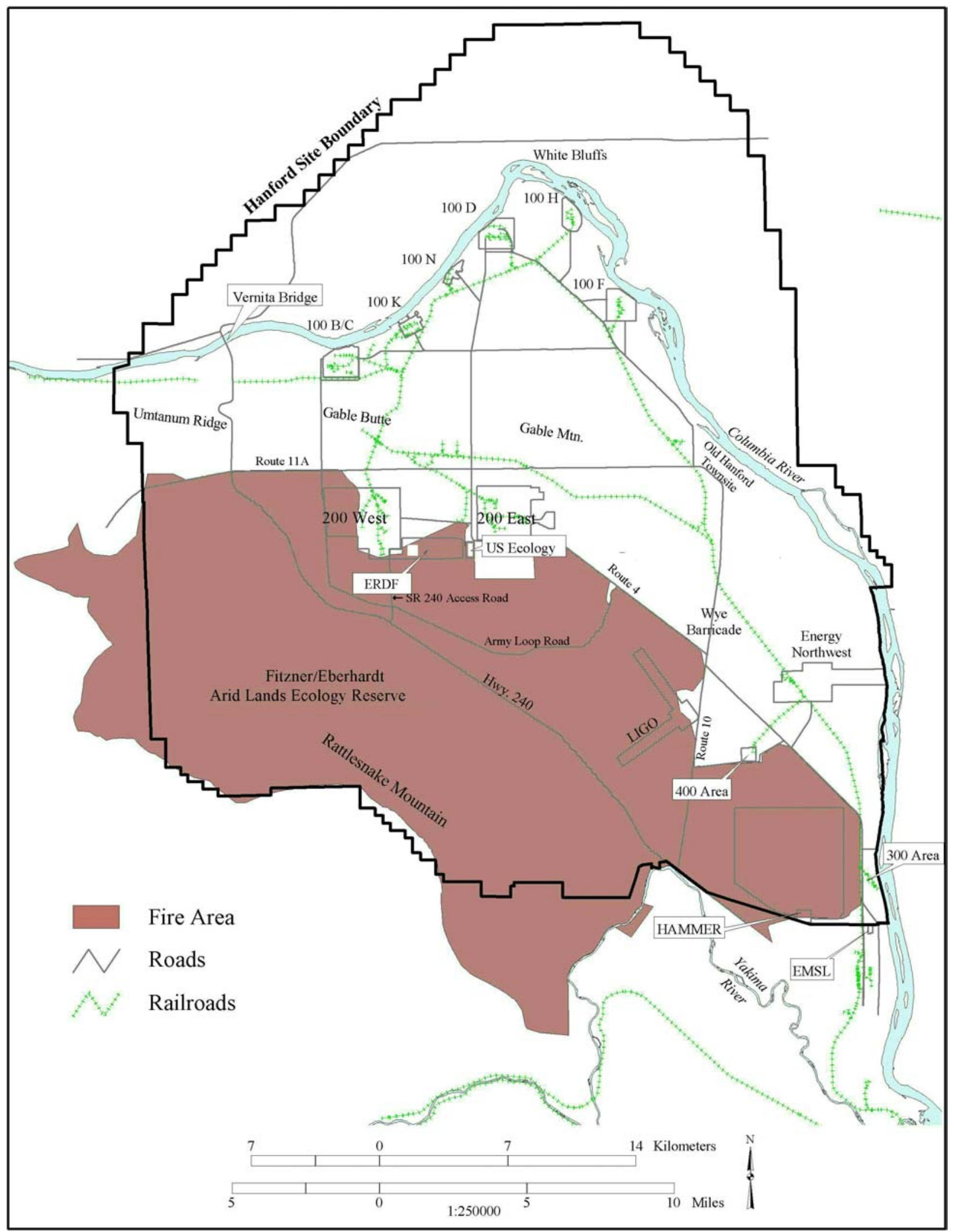

Figure 4.0-3. Area of Hanford Site Burned as a Result of the June 27 - July 2, 2000, Wildfire 


\subsection{Climate and Meteorology/Air Quality}

\section{J. Hoitink and P. L. Hendrickson}

The Hanford Site is located in a semiarid region of southeastern Washington State. The region's climate is greatly influenced by the Pacific Ocean, the Cascade Mountain Range to the west, and other mountain ranges located to the north and east. The Pacific Ocean moderates temperatures throughout the Pacific Northwest and the Cascade Range generates a rain shadow that limits rain and snowfall in the eastern half of Washington State. The Cascade Range also serves as a source of cold air drainage, which has a considerable effect on the wind regime on the Hanford Site. Mountain ranges to the north and east of the region shield the area from the severe winter storms and frigid air masses that move southward across Canada.

Climatological data for the Hanford Site are compiled at the Hanford Meteorology Station (HMS). The HMS is located on Hanford's 200 Central Plateau, just outside the northeast corner of 200 West Area

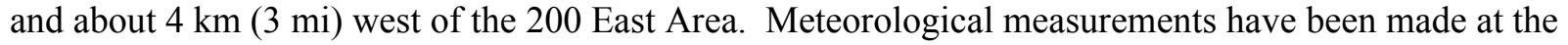
HMS since late 1944. Prior to the establishment of the HMS, local meteorological observations were made at the Old Hanford Townsite (1912 through late 1943) and in Richland (1943-1944). A climatological summary for Hanford is provided in Hoitink et al. (2001). ${ }^{(a)}$

Data from the HMS capture the general climatic conditions for the region and describe the specific climate of the Central Plateau. The large size of the Hanford Site and its complex topography can give rise to substantial spatial variations in wind, precipitation, temperature, and other meteorological parameters. For example, this is seen in the marked differences in the annual distribution of wind directions and speeds measured at the HMS on the Central Plateau and at the 300 Area near the southeastern corner of the Hanford Site. To accurately characterize meteorological differences across the Hanford Site, the HMS operates a network of automated monitoring stations. These stations, which currently number 30, are located throughout the Site and in neighboring areas (Figure 4.1-1). A 124-m (408-ft) instrumented meteorological tower operates at the HMS. A 61-m (200-ft) instrumented tower operates at each of the 100-N, 300, and 400 Area meteorology-monitoring sites. Most of the other network stations use short-instrumented towers with heights of about $9 \mathrm{~m}(30 \mathrm{ft})$. Instrumentation on each tower is described in Table 4.1-1. Data are collected and processed at each monitoring site, and key information is transmitted to the HMS every $15 \mathrm{~min}$. This monitoring network has been in full operation since the early 1980 s.

For reporting purposes throughout this section the seasons are defined as follows:

$$
\begin{aligned}
& \text { Winter - December through February } \\
& \text { Spring - March through May } \\
& \text { Summer - June through August } \\
& \text { Autumn - September through November. }
\end{aligned}
$$

\footnotetext{
(a) Hanford climatological data summaries have been updated annually since 1995. Earlier climatological reports that have been extensively cited include Glantz et al. (1990) and Stone et al. (1983). A detailed report on Hanford's meteorological monitoring instrumentation is provided in Glantz and Islam (1988).
} 


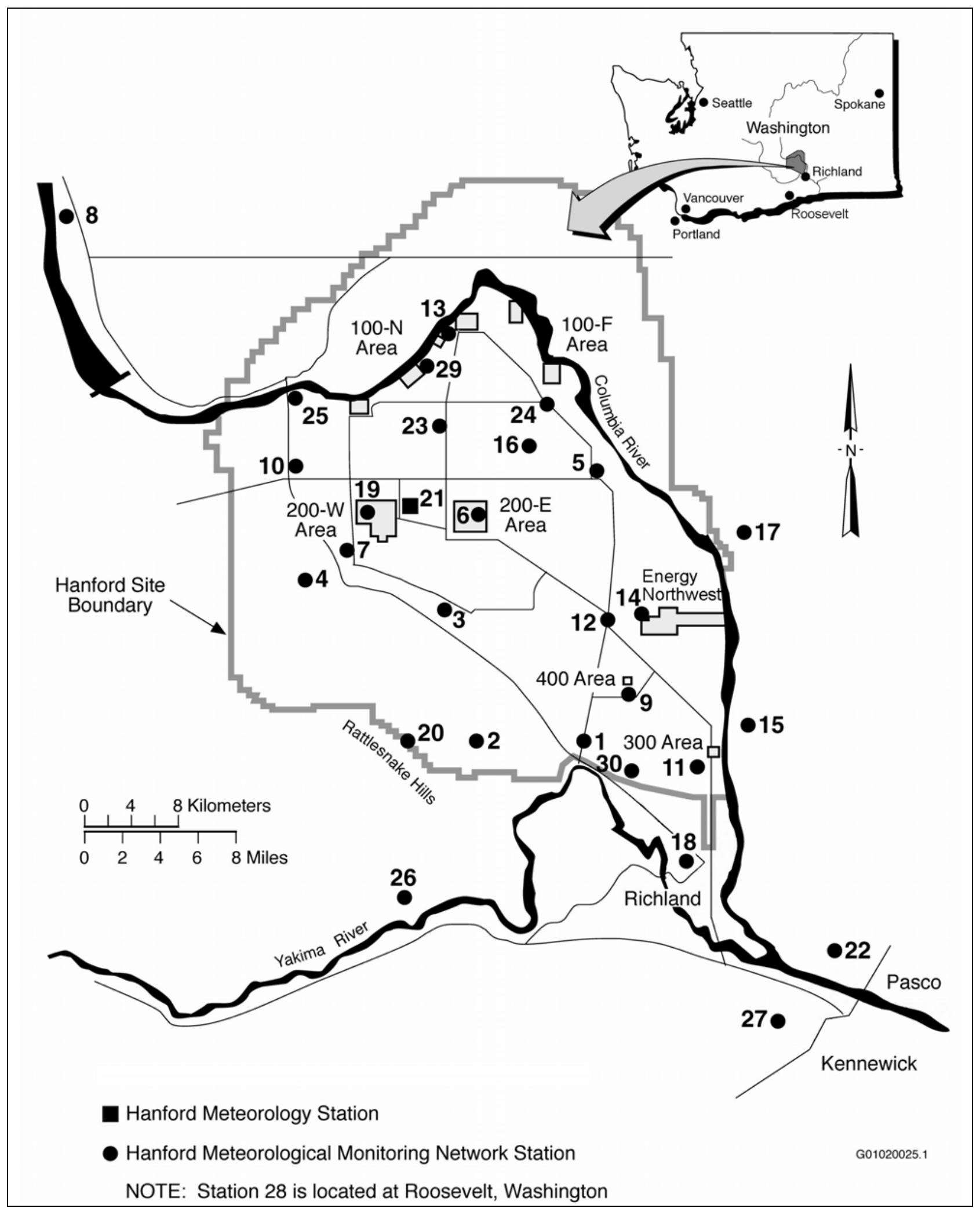

Figure 4.1-1. Hanford Meteorological Monitoring Network 
Table 4.1-1. Station Numbers, Names, and Instrumentation for each Hanford Meteorological Monitoring Network Site

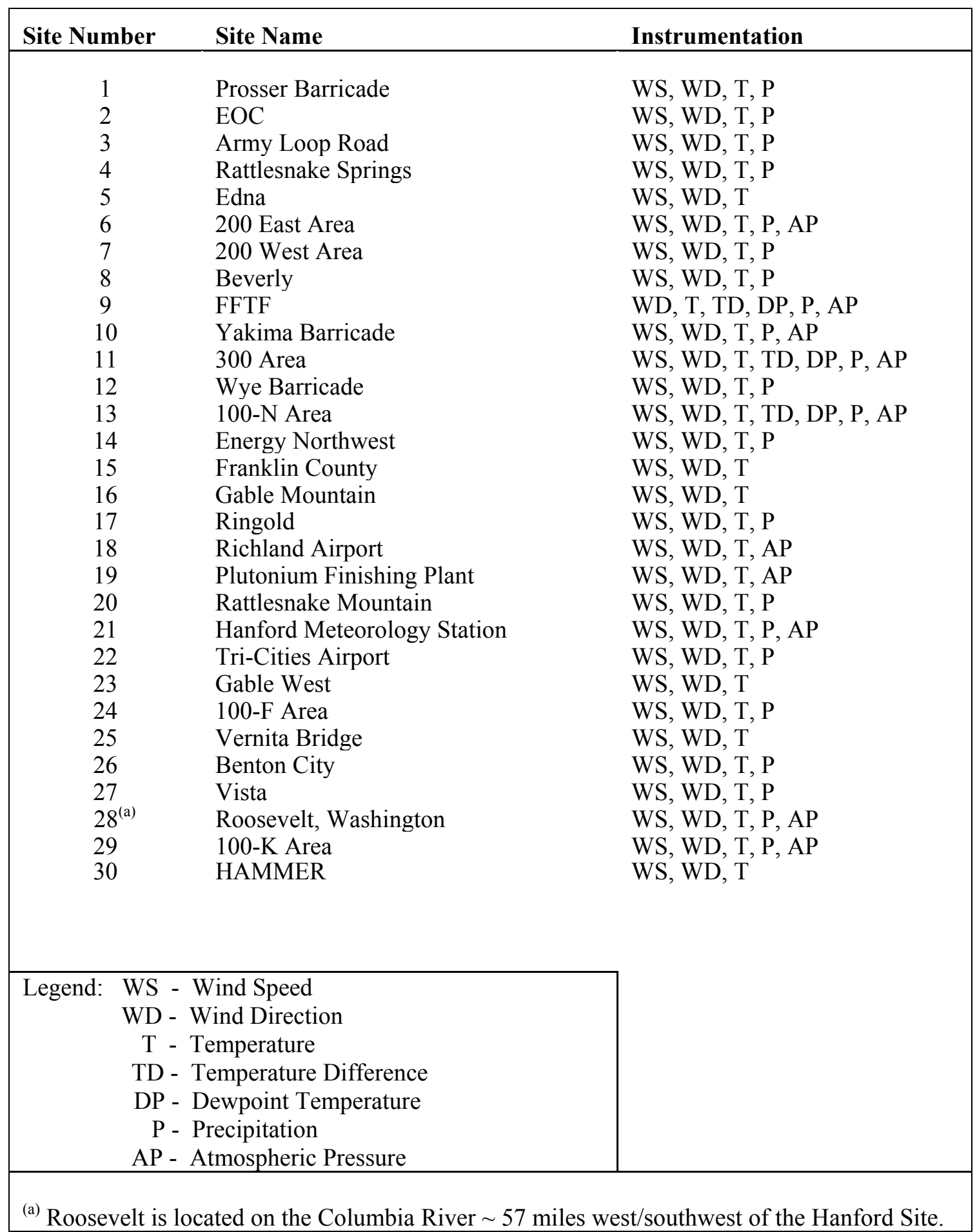




\subsubsection{Wind}

Wind data at the HMS are collected at $2.1 \mathrm{~m}(7 \mathrm{ft})$ above the ground and at the 15.2-, 61.0-, and 121.9-m $(50-, 200-$, and 400-ft) levels on the 124-m (408-ft) tower. Each of the three $61-\mathrm{m}(200-\mathrm{ft})$ towers has wind-measuring instrumentation at the 10,25 , and $60 \mathrm{~m}(33,82$, and $197 \mathrm{ft})$ levels. The short towers measure winds at $9.1 \mathrm{~m}(30 \mathrm{ft})$ above ground level.

Prevailing wind directions near the surface on Hanford's Central Plateau are from the northwest in all months of the year (Figure 4.1-2). Winds from the northwest occur most frequently during the winter and summer. Winds from the southwest also have a high frequency of occurrence on the Central Plateau. During the spring and fall, there is an increase in the frequency of winds from the southwest and a corresponding decrease in winds from the northwest.

In the southeastern portion of the Hanford Site (including the 300 and 400 Areas), the prevailing wind direction near the surface is from the southwest during most months; winds from the northwest are much less common (Figure 4.1-2). In the 100 Area and along the Columbia River, local winds are strongly influenced by the topography near the river. At the $100-\mathrm{K}$ and $100-\mathrm{N}$ facilities, the prevailing wind direction is from the west. At the 100-F facility and near the Old Hanford Townsite (EDNA station), winds often have a northwesterly or southeasterly component.

Stations that are relatively close together can exhibit significant differences in wind patterns. For

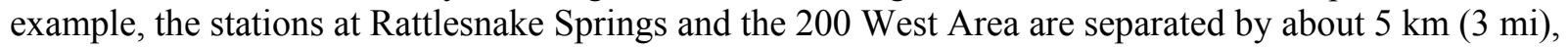
yet the wind patterns at the two stations are very different (see Figure 4.1-2). Care should be taken when assessing the appropriateness of the wind data used in estimating environmental impacts. When possible, wind data from the closest representative station should be used for assessing local dispersion conditions.

Monthly and annual joint-frequency distributions of wind direction versus wind speed for the HMS

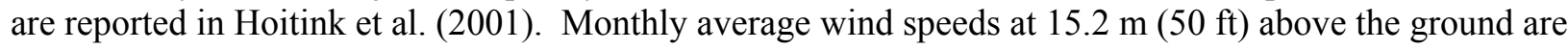
lower during the winter months, averaging 2.7 to $3.1 \mathrm{~m} / \mathrm{s}$ (6 to $7 \mathrm{mph}$ ), and faster during the summer, averaging 3.6 to $4.0 \mathrm{~m} / \mathrm{s}$ ( 8 to $9 \mathrm{mph})$. The fastest wind speeds at the HMS are usually associated with flow from the southwest. However, the summertime drainage winds from the northwest frequently exceed speeds of $13 \mathrm{~m} / \mathrm{s}(30 \mathrm{mph})$. The maximum speed of the drainage winds (and their frequency of occurrence) tends to decrease as one moves toward the southeast across the Hanford Site.

Table 4.1-2 presents information on number of days, by month and annually, with wind gusts $\geq 11$ $\mathrm{m} / \mathrm{s}(25 \mathrm{mph})$ and $16 \mathrm{~m} / \mathrm{s}(35 \mathrm{mph})$ for the HMS. The table also includes record high and low values.

Surface features have less influence on winds aloft than winds near the surface. However, there are still substantial spatial variations in the wind distributions across Hanford at $61 \mathrm{~m}(200 \mathrm{ft})$ above ground level (Figure 4.1-3). For elevated releases, the most representative data may come from the closest representative $61-\mathrm{m}(200-\mathrm{ft})$ tower rather than the nearest $9.1-\mathrm{m}(30-\mathrm{ft})$ tower. 


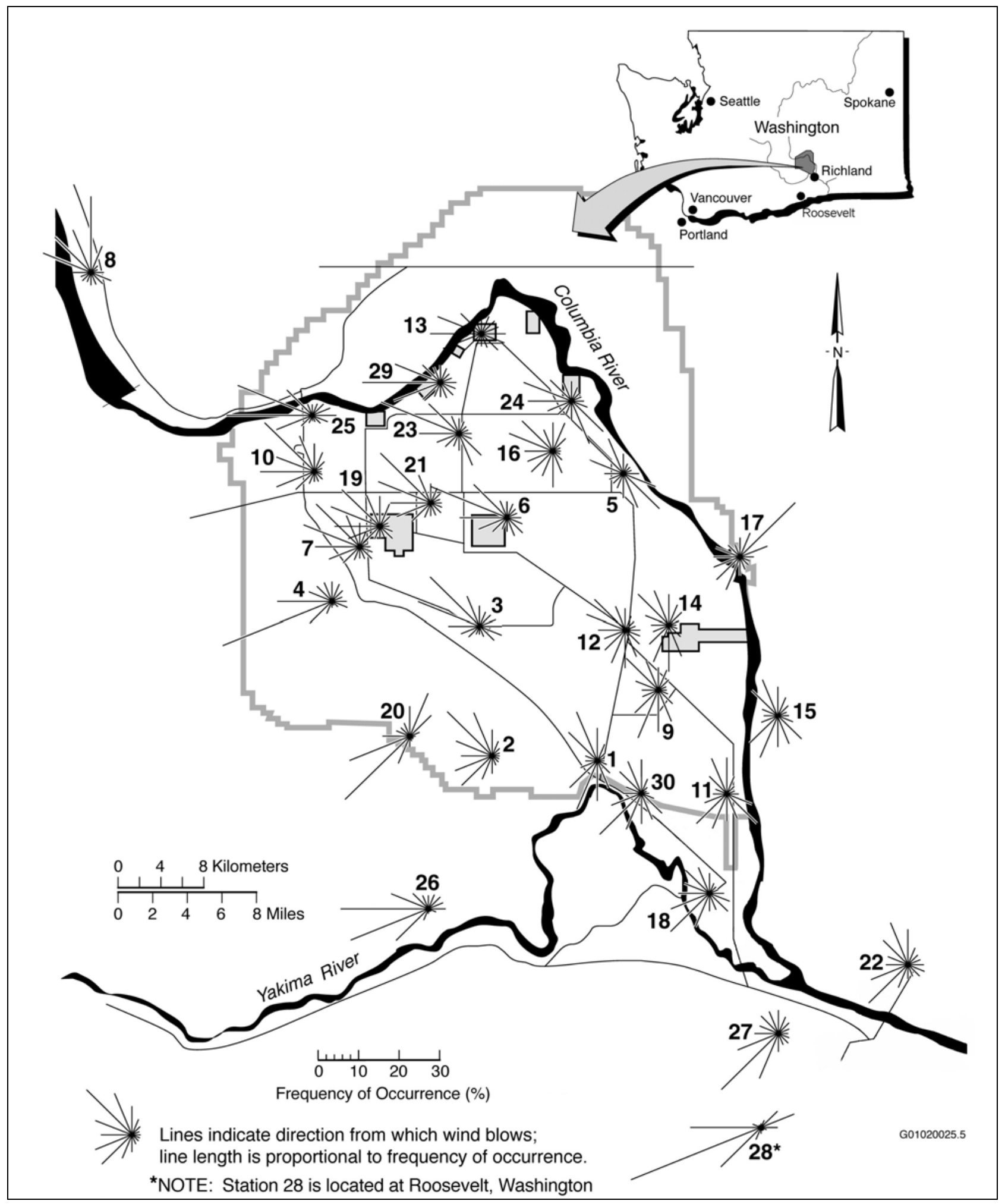

Figure 4.1-2. Wind Roses at the $9.1 \mathrm{~m}$ (30 ft) Level of the Hanford Meteorological Monitoring Network, 1982 to 2000 


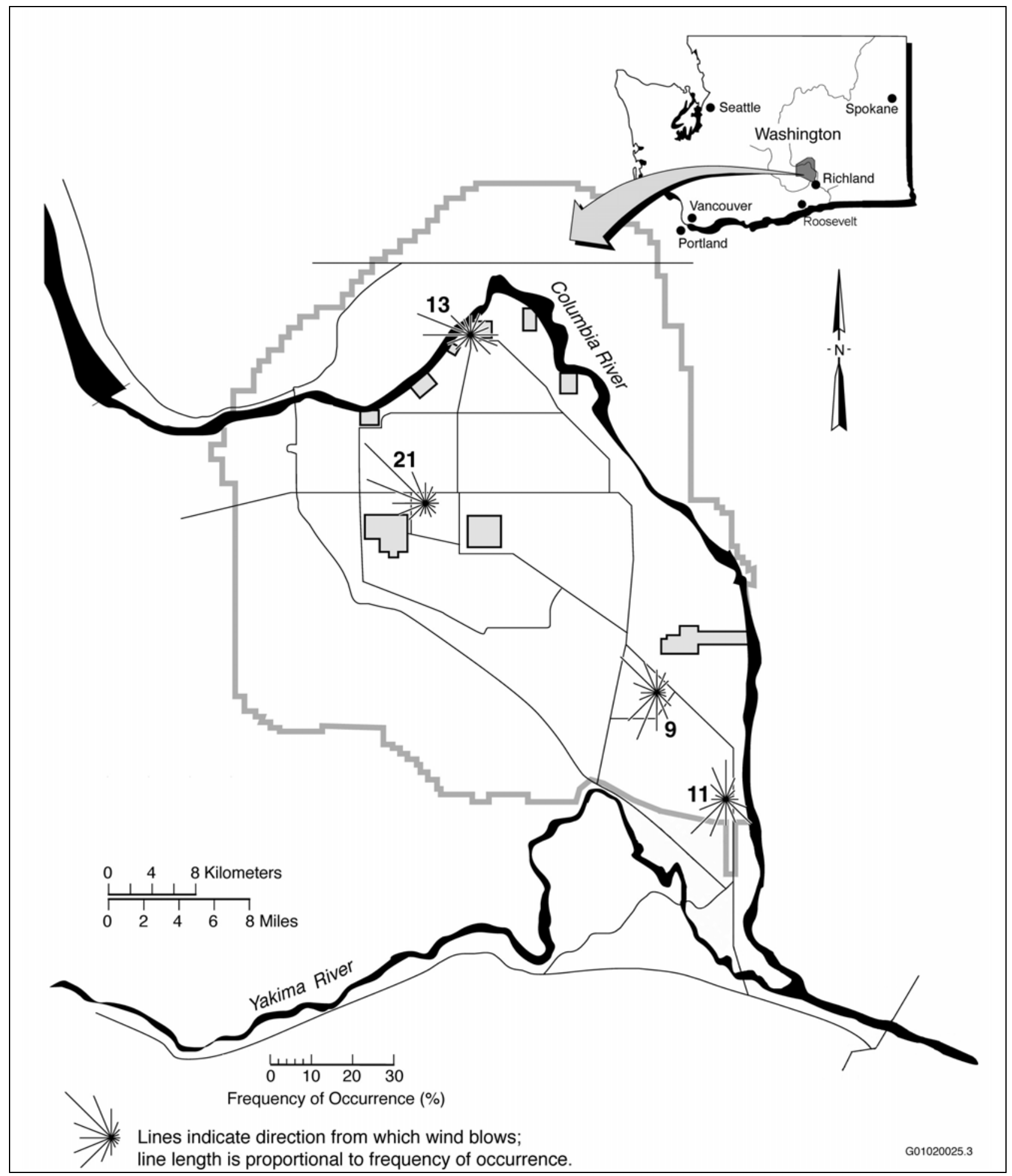

Figure 4.1-3. Wind Roses at the $60 \mathrm{~m}(197 \mathrm{ft})$ Level of the Hanford Meteorological Monitoring Network, 1986 to 2000 
Table 4.1-2. Number of Days with Peak Gusts Above Specific Thresholds at 15 m (50 ft) Level, 1945 through 2000

\begin{tabular}{|c|c|c|c|c|c|c|c|c|c|c|}
\hline \multicolumn{6}{|c|}{ Days with Peak Gusts $\geq 11 \mathrm{~m} / \mathrm{s}(25 \mathrm{mph})$} & \multicolumn{5}{|c|}{ Days with Peak Gusts $\geq 16 \mathrm{~m} / \mathrm{s}(35 \mathrm{mph})$} \\
\hline Month & Avg & Max & Year & Min & Year & Avg & Max & Year & Min & Year \\
\hline January & 7.6 & 21 & 1953 & 0 & $1985^{\text {(a) }}$ & 4.0 & 14 & 1953 & 0 & $1985^{(\mathrm{a})}$ \\
\hline February & 8.7 & 17 & $1976^{(\mathrm{a})}$ & 2 & $1952^{(\mathrm{a})}$ & 3.8 & 14 & 1976 & 0 & $2000^{(a)}$ \\
\hline March & 12.9 & 21 & 1977 & 4 & 1992 & 5.4 & 14 & 1997 & 0 & 1992 \\
\hline April & 16.9 & 26 & 1954 & 8 & 1946 & 6.3 & 12 & 1972 & 1 & 1967 \\
\hline May & 18.7 & 26 & 1978 & 9 & 1945 & 6.1 & 10 & $2000^{(a)}$ & 0 & 1957 \\
\hline June & 19.6 & 26 & 1963 & 11 & $1950^{(\mathrm{a})}$ & 6.2 & 12 & 1973 & 1 & 1982 \\
\hline July & 19.4 & 26 & 1995 & 11 & 1955 & 5.5 & 11 & $1994^{(\mathrm{a})}$ & 1 & $1982^{(\mathrm{a})}$ \\
\hline August & 15.8 & 24 & 2000 & 7 & 1945 & 4.1 & 12 & 1996 & 0 & $1978^{(a)}$ \\
\hline September & 11.2 & 17 & 1971 & 7 & $1975^{(\mathrm{a})}$ & 3.3 & 7 & $1992^{(a)}$ & 0 & 1975 \\
\hline October & 8.8 & 17 & $1985^{(a)}$ & 3 & $1987^{(\mathrm{a})}$ & 3.1 & 11 & 1997 & 0 & $1993^{(a)}$ \\
\hline November & 8.3 & 16 & 1990 & 0 & 1979 & 3.8 & 10 & 1998 & 0 & $1997^{(\mathrm{a})}$ \\
\hline December & 7.5 & 15 & 1968 & 0 & 1985 & 4.2 & 11 & 1957 & 0 & $1985^{(\mathrm{a})}$ \\
\hline Annual & 155.7 & 192 & 1999 & 123 & 1952 & 55.9 & 83 & $1999^{(a)}$ & 31 & 1978 \\
\hline
\end{tabular}

\subsubsection{Temperature and Humidity}

Temperature measurements are made at the $0.9,9.1,15.2,30.5,61.0,76.2,91.4$, and $121.9 \mathrm{~m}(3,30$, $50,100,200,250,300$, and $400 \mathrm{ft})$ levels of the 124-m (408-ft) tower at the HMS. The three 61-m (200$\mathrm{ft})$ towers have temperature-measuring instrumentation at the 2,10 , and $60 \mathrm{~m}(\sim 6.5,33$, and $197 \mathrm{ft})$ levels. Temperatures are measured at the $2 \mathrm{~m}(\sim 6.5 \mathrm{ft})$ level on the $9-\mathrm{m}(30-\mathrm{ft})$ towers. Relative humidity/dew point temperature measurements are made at the HMS and at the three $61-\mathrm{m}(200-\mathrm{ft})$ tower locations.

Monthly averages and extremes of temperature, dew point, and humidity are presented in Hoitink et al. (2001). Based on data collected from 1946 through 2000, the average monthly temperatures at the HMS range from a low of $-0.7^{\circ} \mathrm{C}\left(31^{\circ} \mathrm{F}\right)$ in January to a high of $24.7^{\circ} \mathrm{C}\left(76^{\circ} \mathrm{F}\right)$ in July. The highest winter monthly average temperatures were $6.9^{\circ} \mathrm{C}\left(44^{\circ} \mathrm{F}\right)$ in February 1958 and February 1991, and the lowest average monthly temperature was $-11.1^{\circ} \mathrm{C}\left(12^{\circ} \mathrm{F}\right)$ in January 1950 . The highest monthly average temperature was $27.9^{\circ} \mathrm{C}\left(82^{\circ} \mathrm{F}\right)$ in July 1985 , and lowest summer monthly average temperature was $17.2^{\circ} \mathrm{C}\left(63^{\circ} \mathrm{F}\right)$ in June 1953 .

Daily maximum temperatures at the HMS vary from an average of $2^{\circ} \mathrm{C}\left(35^{\circ} \mathrm{F}\right)$ in late December and early January to $36^{\circ} \mathrm{C}\left(96^{\circ} \mathrm{F}\right)$ in late July. There are, on average, 52 days during the summer months with maximum temperatures $\geq 32^{\circ} \mathrm{C}\left(90^{\circ} \mathrm{F}\right)$ and 12 days with maxima greater than or equal to $38^{\circ} \mathrm{C}\left(100^{\circ} \mathrm{F}\right)$. The greatest number of consecutive days with maximum daily temperatures of $\geq 32^{\circ} \mathrm{C}\left(90^{\circ} \mathrm{F}\right)$ is 32 days. The $45^{\circ} \mathrm{C}\left(113^{\circ} \mathrm{F}\right)$ record maximum temperature occurred at the HMS on August 4, 1961. 
From mid-November through early March, the average daily minimum temperature is below freezing; the daily minimum in late December and early January is $-6^{\circ} \mathrm{C}\left(21^{\circ} \mathrm{F}\right)$. On average, a daily minimum temperature of $\leq-18^{\circ} \mathrm{C}\left(\sim 0^{\circ} \mathrm{F}\right)$ occurs only 3 days per year; however, only about one winter in two experiences such low temperatures. The greatest number of consecutive days with minimum daily temperatures of $\leq-18^{\circ} \mathrm{C}\left(\sim 0^{\circ} \mathrm{F}\right)$ is 11 days. The $-31^{\circ} \mathrm{C}\left(-23^{\circ} \mathrm{F}\right)$ record minimum temperature occurred on both February 1 and 3, 1950.

The annual average relative humidity at the HMS is 55\%. It is highest during the winter months, averaging about $76 \%$, and lowest during the summer, averaging about $36 \%$. The annual average dewpoint temperature at the HMS is $1^{\circ} \mathrm{C}\left(34^{\circ} \mathrm{F}\right)$. In the winter, the dewpoint temperature averages about $-3^{\circ} \mathrm{C}\left(27^{\circ} \mathrm{F}\right)$, and in the summer it averages about $6^{\circ} \mathrm{C}\left(43^{\circ} \mathrm{F}\right)$.

\subsubsection{Precipitation}

Average annual precipitation at the HMS is $17 \mathrm{~cm}$ (6.8 in.). In 1995, the wettest year on record 31.3 $\mathrm{cm}$ (12.3 in.) of precipitation was measured; in 1976, the driest year, only $7.6 \mathrm{~cm}$ (3 in.) was measured. The wettest season on record was the winter of 1996-1997 with $14.1 \mathrm{~cm}$ (5.4 in.) of precipitation; the driest season was the summer of 1973 when only $0.1 \mathrm{~cm}$ (0.03 in.) of precipitation was measured. Most precipitation occurs during the late autumn and winter, with more than half of the annual amount occurring from November through February. Days with $>1.3 \mathrm{~cm}(0.50$ in.) precipitation occur on average less than one time each year.

Average snowfall ranges from $0.25 \mathrm{~cm}(0.1 \mathrm{in}$.$) in October to a maximum of 13.2 \mathrm{~cm}(5.2 \mathrm{in}$.$) in$ December and decreases to $0.8 \mathrm{~cm}$ (0.3 in.) in March. The record monthly snowfall of $59.4 \mathrm{~cm}(23.4 \mathrm{in}$.) occurred in January 1950. The seasonal record snowfall of $142.5 \mathrm{~cm}$ (56.1 in.) occurred during the winter of 1992-1993. Snowfall accounts for about 38\% of all precipitation from December through February.

\subsubsection{Fog and Visibility}

Fog has been recorded during every month of the year at the HMS; however, $89 \%$ of the occurrences are from November through February, with less than 3\% from April through September (Table 4.1-3). The average number of days per year with fog (visibility $\leq 9.6 \mathrm{~km}[6 \mathrm{mi}]$ ) is 47 , while those with dense fog (visibility $\leq 0.4 \mathrm{~km}[0.25 \mathrm{mi}]$ ), is 25 . The greatest number of days with fog was 84 days in 1985-1986, and the least was 22 in 1948-1949. The greatest number of days with dense fog was 42 days in 19501951, and the least was 9 days in 1948-1949. The greatest persistence of fog was $114 \mathrm{hr}$ (in December 1985), and the greatest persistence of dense fog was $47 \mathrm{hr}$ (in December 1957).

Other phenomena causing restrictions to visibility (i.e., visibility $\leq 9.6 \mathrm{~km}[6 \mathrm{mi}]$ ) include dust, blowing dust, and smoke from field burning. There are few such days; an average of 5 days per year have dust or blowing dust, and less than 1 day per year, on average, has reduced visibility from smoke.

Table 4.1-3. Number of Days with Fog by Season

\begin{tabular}{|lccccc|}
\hline Category & Winter & Spring & Summer & Autumn & Total \\
\hline Fog & 32 & 3 & $\leq 2$ & 12 & 47 \\
Dense fog & 17 & 1 & $\leq 2$ & 7 & 25 \\
\hline
\end{tabular}




\subsubsection{Severe Weather}

Concerns about severe weather usually center on hurricanes, tornadoes, and thunderstorms. Fortunately, Washington does not experience hurricanes. In addition, tornadoes are infrequent and generally small in the northwestern portion of the United States. The National Climatic Data Center maintains a database that provides information on the incidence of tornados reported in each county in the United States. (This database can be accessed via the Internet at URL:

http://www.ncdc.noaa.gov/ol/climate/severeweather/extremes.html). This database reports that in the ten counties closest to the Hanford Site (Benton, Franklin, Grant, Adams, Yakima, Klickitat, Kittitas, and Walla Walla counties in Washington and Umatilla and Morrow counties in Oregon), there have been only 18 tornadoes recorded from 1950 through March 2001. Of these, 12 tornadoes had maximum wind speeds estimated to be in the range of 18 to $32 \mathrm{~m} / \mathrm{s}$ ( 40 to $72 \mathrm{mph}$ ), three had maximum wind speeds in the range of 33 to $50 \mathrm{~m} / \mathrm{s}$ (73 to $112 \mathrm{mph}$ ), and three had maximum wind speeds in the range of 51 to 71 $\mathrm{m} / \mathrm{s}(113$ to $157 \mathrm{mph}$ ). There were no deaths or substantial property damage (in excess of $\$ 50,000$ ) associated with any of these tornadoes.

Ramsdell and Andrews (1986) report that for the $5^{\circ}$ block centered at $117.5^{\circ}$ west longitude and $47.5^{\circ}$ north latitude (the area in which the Hanford Site is located), the expected path length of a tornado is 7.6 $\mathrm{km}(5 \mathrm{mi})$, the expected width is $95 \mathrm{~m}(312 \mathrm{ft})$, and the expected area is about $1.5 \mathrm{~km}^{2}\left(1 \mathrm{mi}^{2}\right)$. The estimated probability of a tornado striking a point on the Hanford Site, also from Ramsdell and Andrews (1986), is $9.6 \times 10^{-6} / \mathrm{yr}$. The probabilities of extreme winds associated with tornadoes striking a point can be estimated using the distribution of tornado intensities for the region. These probability estimates are given in Table 4.1-4.

The average occurrence of thunderstorms in the vicinity of the HMS is 10 per year. They are most frequent during the summer; however, they have occurred in every month. Thunderstorms can generate high-speed winds and hail. Using the National Weather Service criteria for classifying a thunderstorm as "severe" (i.e., hail with a diameter $\geq 19 \mathrm{~mm}[3 / 4 \mathrm{in}$.] or wind gusts of $\geq 25.9 \mathrm{~m} / \mathrm{s}[58 \mathrm{mph}]$ ), only $1.9 \%$ of all thunderstorm events surveyed at the HMS have been "severe" storms, and all met the criteria based on their wind gusts. High-speed winds at Hanford are more commonly associated with strong cold frontal passages. In rare cases, intense low-pressure systems can generate winds of near hurricane force. Estimates of the extreme winds, based on peak gusts, are given in Hoitink et al. (2001) and are shown in Table 4.1-5.

Table 4.1-4. Estimate of the Probability of Extreme Winds Associated with Tornadoes Striking a Point at Hanford (Based on information presented in Ramsdell and Andrews 1986)

\begin{tabular}{|ccc|}
\hline \multicolumn{2}{|c|}{ Wind Speed } & Probability Per Year \\
$(\mathbf{m} / \mathbf{s})$ & $(\mathbf{m p h})$ & \\
\hline 28 & 62 & $2.6 \times 10^{-6}$ \\
56 & 124 & $6.5 \times 10^{-7}$ \\
83 & 186 & $1.6 \times 10^{-7}$ \\
111 & 249 & $3.9 \times 10^{-8}$ \\
\hline
\end{tabular}


Table 4.1-5. Estimates of Extreme Winds at the Hanford Site

\begin{tabular}{|ccccc|}
\hline & \multicolumn{5}{c|}{ Peak Gusts } \\
Return & $\begin{array}{c}\mathbf{1 5 . 2} \mathbf{m} \text { (50 ft) } \\
\text { Period (yr) }\end{array}$ & $\begin{array}{c}\text { 6bove } \mathbf{~ m} \text { (200 ft) } \\
\text { Abound } \\
\text { (m/s) }\end{array}$ & $\begin{array}{c}\text { Above Ground } \\
\text { (m/s) }\end{array}$ & (mph) \\
\hline & & & & \\
2 & 27 & 60 & 30 & 68 \\
10 & 32 & 71 & 36 & 81 \\
100 & 38 & 85 & 43 & 97 \\
1000 & 44 & 98 & 50 & 112 \\
\hline
\end{tabular}

\subsubsection{Atmospheric Dispersion}

Atmospheric dispersion (the transport and diffusion of gases and particles within the atmosphere) is a function of wind speed, duration and direction of wind, intensity of atmospheric turbulence, and mixing depth. Atmospheric turbulence is not directly measured at the Hanford Site; instead, the impact of turbulence on atmospheric dispersion is characterized using atmospheric stability. Atmospheric stability describes the thermal stratification or vertical temperature structure of the atmosphere. Generally, six or seven different classes of atmospheric stability are used to describe the atmosphere. These classes range from extremely unstable (when atmospheric turbulence is greatest) to extremely stable (when atmospheric mixing is at a minimum and wind speeds are low). When the atmosphere is unstable, pollutants can rapidly diffuse through a wide volume of the atmosphere. When the atmosphere is stable, pollutants will diffuse much more slowly in a vertical direction. Horizontal dispersion may be limited during stable conditions; however, plumes may also fan out horizontally during stable conditions, particularly when the wind speed is low. Most major pollutant incidents are associated with stable conditions when inversions can trap pollutants near the ground.

Favorable dispersion conditions are most common in the summer when neutral and unstable stratification exists, about $56 \%$ of the time (Stone et al. 1983). Less favorable dispersion conditions are most common during the winter when moderately to extremely stable stratification exists, about $66 \%$ of the time (Stone et al. 1983). Less favorable conditions also occur periodically for surface and low-level releases in all seasons from about sunset to about an hour after sunrise as a result of ground-based temperature inversions and shallow mixing layers. Occasionally, there are extended periods of poor dispersion conditions associated with stagnant air in stationary high-pressure systems. These instances tend to occur during the winter months (Stone et al. 1983).

Stone et al. (1972) estimated the probability of extended periods of poor dispersion conditions. The probability of an inversion once established persisting more than $12 \mathrm{hr}$ varies from a low of about $10 \%$ in May and June to a high of about $64 \%$ in September and October. These probabilities decrease rapidly for durations of $>12 \mathrm{hr}$. Table 4.1-6 summarizes the probabilities associated with extended surface-based inversions. 
Table 4.1-6. Percent Probabilities for Extended Periods of Surface-Based Inversions (Based on data from Stone et al. 1972)

\begin{tabular}{|lccc|}
\hline & \multicolumn{3}{c|}{ Inversion duration } \\
\cline { 2 - 4 } Months & 12-hr & 24-hr & 48-hr \\
\hline & & & \\
January-February & 54.0 & 2.5 & 0.28 \\
March-April & 50.0 & $<0.1$ & $<0.1$ \\
May-June & 10.0 & $<0.1$ & $<0.1$ \\
July-August & 18.0 & $<0.1$ & $<0.1$ \\
September-October & 64.0 & 0.11 & $<0.1$ \\
November-December & 50.0 & 1.2 & 0.13 \\
\hline
\end{tabular}

Many simple dispersion models use the joint frequency distribution of atmospheric stability, wind speed, and wind direction to compute diffusion factors for both chronic and acute releases. Tables 4.1-7 through 4.1-14 present joint frequency distributions of atmospheric stability, wind speed, and transport

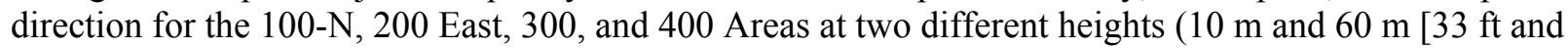
$197 \mathrm{ft}])$. The values in the joint frequency distributions represent the percentage of the time that pollutants would initially be transported toward the direction listed ${ }^{\text {(a) }}$ (e.g., S, SSW, SW). For each station, the joint frequency distributions were determined using local wind data measured at $9.1 \mathrm{~m}(30 \mathrm{ft})$ above ground level and atmospheric stability measurements at the HMS. For the $60 \mathrm{~m}(197 \mathrm{ft})$ joint frequency distributions, wind speed was estimated assuming a power law represented the wind speed profile. A more detailed description of the procedures used to develop the joint frequency distributions is found in Appendix H.1 of the Recommended Environmental Dose Calculation Methods and HanfordSpecific Parameters (Schreckhise et al. 1993).

Tables 4.1-15 through 4.1-22 present the annual sector-average atmospheric dispersion coefficient $\left(\bar{\chi} / \mathrm{Q}^{\prime}\right)$ where $\chi$, is the air concentration and $\mathrm{Q}$ is the emission rate. Tables 4.1-23 through 4.1-30 present the $95 \%$ centerline atmospheric dispersion estimates (E/Q) for the four major Hanford operating areas $(100,200,300$, and 400 Areas). For each area, with the exception of the 400 Area, atmospheric diffusion factors are for a ground-level release and a release at $60 \mathrm{~m}(197 \mathrm{ft})$. For the 400 Area, the diffusion factors are for a ground-level release and a release at $30 \mathrm{~m}(98 \mathrm{ft})$. These diffusion factors are presented as a function of direction and distance from the release point and were calculated using the Generation II Model For Environmental Dose Calculations (GENII) code (Napier et al. 1988) based on meteorological measurements averaged over the years 1983 through 1991.

(a) The "transport direction" and the "wind direction" are different methods of reporting the same basic information. Wind direction and transport direction are always out of phase by $180^{\circ}$. 
Table 4.1-7. Joint Frequency Distributions of Atmospheric Stability, Wind Speed, and Transport Direction for the 100 Area at $9 \mathrm{~m}$ Above Ground Level. Based on 1983-1996 data from the $100-\mathrm{N}$ instrumented tower.

\begin{tabular}{|c|c|c|c|c|c|c|c|c|c|c|c|c|c|c|c|c|c|}
\hline \multirow{2}{*}{$\begin{array}{c}\text { Average } \\
\text { Wind Speed } \\
\mathbf{m} / \mathbf{s}\end{array}$} & \multirow[t]{2}{*}{$\begin{array}{l}\text { Atmospheric } \\
\text { Stability Class }\end{array}$} & \multicolumn{16}{|c|}{ Percentage of Time Wind Blows from the $100 \mathrm{~N}$ Area Toward the Direction Indicated } \\
\hline & & $\mathbf{S}$ & SSW & SW & WSW & W & WNW & NW & NNW & $\mathbf{N}$ & NNE & NE & ENE & $\mathbf{E}$ & ESE & SE & SSE \\
\hline \multirow[t]{7}{*}{0.89} & A & 0.28 & 0.19 & 0.17 & 0.16 & 0.22 & 0.2 & 0.17 & 0.12 & 0.12 & 0.12 & 0.15 & 0.19 & 0.26 & 0.31 & 0.37 & 0.33 \\
\hline & B & 0.11 & 0.08 & 0.1 & 0.08 & 0.11 & 0.14 & 0.11 & 0.07 & 0.06 & 0.06 & 0.08 & 0.1 & 0.12 & 0.13 & 0.17 & 0.14 \\
\hline & $\mathrm{C}$ & 0.11 & 0.09 & 0.09 & 0.1 & 0.1 & 0.13 & 0.11 & 0.08 & 0.08 & 0.07 & 0.08 & 0.1 & 0.11 & 0.13 & 0.13 & 0.13 \\
\hline & D & 0.51 & 0.42 & 0.45 & 0.54 & 0.82 & 1 & 0.82 & 0.65 & 0.59 & 0.55 & 0.6 & 0.66 & 0.75 & 0.73 & 0.69 & 0.59 \\
\hline & E & 0.48 & 0.43 & 0.51 & 0.61 & 0.84 & 0.86 & 0.71 & 0.54 & 0.5 & 0.47 & 0.58 & 0.68 & 0.75 & 0.77 & 0.67 & 0.55 \\
\hline & $\mathrm{F}$ & 0.45 & 0.4 & 0.54 & 0.61 & 0.77 & 0.66 & 0.51 & 0.34 & 0.31 & 0.33 & 0.48 & 0.69 & 0.79 & 0.83 & 0.7 & 0.57 \\
\hline & $\mathrm{G}$ & 0.21 & 0.19 & 0.23 & 0.28 & 0.31 & 0.23 & 0.18 & 0.13 & 0.12 & 0.13 & 0.24 & 0.37 & 0.51 & 0.47 & 0.4 & 0.29 \\
\hline \multirow[t]{7}{*}{2.65} & $\bar{A}$ & 0.45 & 0.48 & 0.36 & 0.15 & 0.23 & 0.31 & 0.27 & 0.17 & 0.13 & 0.14 & 0.32 & 0.47 & 0.51 & 0.47 & 0.45 & 0.45 \\
\hline & B & 0.14 & 0.16 & 0.11 & 0.06 & 0.11 & 0.13 & 0.13 & 0.09 & 0.05 & 0.04 & 0.12 & 0.18 & 0.2 & 0.14 & 0.15 & 0.12 \\
\hline & $\mathrm{C}$ & 0.1 & 0.12 & 0.1 & 0.06 & 0.09 & 0.12 & 0.11 & 0.07 & 0.04 & 0.04 & 0.1 & 0.15 & 0.17 & 0.12 & 0.11 & 0.12 \\
\hline & D & 0.4 & 0.46 & 0.4 & 0.38 & 0.53 & 0.7 & 0.75 & 0.41 & 0.3 & 0.33 & 0.56 & 1.01 & 0.98 & 0.76 & 0.52 & 0.42 \\
\hline & $\mathrm{E}$ & 0.22 & 0.23 & 0.31 & 0.51 & 0.7 & 0.72 & 0.64 & 0.36 & 0.26 & 0.28 & 0.64 & 1.39 & 1.54 & 0.9 & 0.48 & 0.25 \\
\hline & $\mathrm{F}$ & 0.13 & 0.14 & 0.2 & 0.51 & 0.71 & 0.49 & 0.3 & 0.16 & 0.11 & 0.15 & 0.34 & 0.8 & 0.92 & 0.56 & 0.31 & 0.15 \\
\hline & G & 0.03 & 0.05 & 0.09 & 0.23 & 0.27 & 0.16 & 0.09 & 0.04 & 0.04 & 0.05 & 0.15 & 0.36 & 0.46 & 0.23 & 0.08 & 0.04 \\
\hline \multirow[t]{7}{*}{4.7} & $\mathrm{~A}$ & 0.09 & 0.27 & 0.18 & 0.04 & 0.06 & 0.08 & 0.11 & 0.05 & 0.05 & 0.08 & 0.28 & 0.3 & 0.35 & 0.36 & 0.17 & 0.08 \\
\hline & B & 0.02 & 0.07 & 0.06 & 0.02 & 0.02 & 0.02 & 0.04 & 0.02 & 0.02 & 0.02 & 0.07 & 0.08 & 0.11 & 0.1 & 0.04 & 0.03 \\
\hline & $\mathrm{C}$ & 0.03 & 0.05 & 0.04 & 0.02 & 0.02 & 0.03 & 0.05 & 0.03 & 0.01 & 0.03 & 0.07 & 0.05 & 0.09 & 0.08 & 0.04 & 0.02 \\
\hline & D & 0.14 & 0.21 & 0.16 & 0.07 & 0.07 & 0.14 & 0.3 & 0.15 & 0.09 & 0.17 & 0.34 & 0.53 & 0.83 & 0.64 & 0.22 & 0.14 \\
\hline & E & 0.09 & 0.12 & 0.08 & 0.06 & 0.06 & 0.07 & 0.25 & 0.14 & 0.1 & 0.13 & 0.29 & 0.82 & 1.47 & 0.95 & 0.2 & 0.08 \\
\hline & $\mathrm{F}$ & 0.06 & 0.07 & 0.04 & 0.05 & 0.04 & 0.04 & 0.08 & 0.04 & 0.02 & 0.02 & 0.07 & 0.27 & 0.24 & 0.14 & 0.05 & 0.04 \\
\hline & G & 0.01 & 0.01 & 0.01 & 0.01 & 0.02 & 0.01 & 0.01 & 0.01 & 0 & 0 & 0.02 & 0.06 & 0.03 & 0.02 & 0.01 & 0.01 \\
\hline \multirow[t]{7}{*}{7.15} & $\mathrm{~A}$ & 0.04 & 0.1 & 0.08 & 0.01 & 0 & 0.01 & 0.03 & 0.02 & 0.01 & 0.04 & 0.21 & 0.15 & 0.23 & 0.36 & 0.18 & 0.03 \\
\hline & B & 0.02 & 0.04 & 0.02 & 0 & 0 & 0 & 0.01 & 0.01 & 0.01 & 0.01 & 0.07 & 0.03 & 0.06 & 0.1 & 0.05 & 0 \\
\hline & $\mathrm{C}$ & 0.01 & 0.02 & 0.02 & 0 & 0 & 0 & 0.01 & 0.01 & 0.01 & 0.01 & 0.05 & 0.03 & 0.05 & 0.07 & 0.03 & 0.01 \\
\hline & D & 0.05 & 0.1 & 0.06 & 0.02 & 0.01 & 0.01 & 0.05 & 0.05 & 0.05 & 0.1 & 0.28 & 0.19 & 0.38 & 0.7 & 0.26 & 0.05 \\
\hline & E & 0.04 & 0.09 & 0.05 & 0.02 & 0 & 0.01 & 0.02 & 0.02 & 0.02 & 0.06 & 0.13 & 0.15 & 0.47 & 0.67 & 0.15 & 0.03 \\
\hline & $\mathrm{F}$ & 0.01 & 0.04 & 0.01 & 0 & 0 & 0 & 0 & 0 & 0 & 0 & 0.01 & 0.03 & 0.04 & 0.03 & 0.01 & 0.02 \\
\hline & G & 0 & 0 & 0 & 0 & 0 & 0 & 0 & 0 & 0 & 0 & 0 & 0.01 & 0 & 0 & 0 & 0 \\
\hline \multirow[t]{7}{*}{9.8} & $\overline{\mathrm{A}}$ & $\overline{0}$ & 0.02 & 0.03 & 0.01 & $\overline{0}$ & $\overline{0}$ & $\overline{0}$ & $\overline{0}$ & 0 & 0.01 & 0.07 & 0.05 & 0.07 & 0.17 & 0.11 & 0 \\
\hline & B & 0 & 0.01 & 0.01 & 0 & 0 & 0 & 0 & 0 & 0 & 0.01 & 0.03 & 0.02 & 0.02 & 0.05 & 0.03 & 0 \\
\hline & $\mathrm{C}$ & 0 & 0.01 & 0.01 & 0 & 0 & 0 & 0 & 0 & 0 & 0.01 & 0.02 & 0.01 & 0.01 & 0.05 & 0.03 & 0 \\
\hline & D & 0.02 & 0.04 & 0.03 & 0.01 & 0 & 0 & 0 & 0.01 & 0.01 & 0.06 & 0.11 & 0.06 & 0.08 & 0.25 & 0.15 & 0.01 \\
\hline & E & 0.02 & 0.02 & 0.03 & 0.01 & 0 & 0 & 0 & 0 & 0.01 & 0.03 & 0.05 & 0.02 & 0.04 & 0.15 & 0.07 & 0.01 \\
\hline & $\mathrm{F}$ & 0 & 0.01 & 0 & 0 & 0 & 0 & 0 & 0 & 0 & 0 & 0 & 0 & 0 & 0 & 0 & 0 \\
\hline & $\mathrm{G}$ & 0 & 0 & 0 & 0 & 0 & 0 & 0 & 0 & 0 & 0 & 0 & 0 & 0 & 0 & 0 & 0 \\
\hline \multirow[t]{7}{*}{12.7} & $\mathrm{~A}$ & 0 & 0 & 0 & 0 & 0 & 0 & 0 & 0 & 0 & 0 & 0.02 & 0.01 & 0.01 & 0.05 & 0.04 & 0 \\
\hline & B & 0 & 0 & 0 & 0 & 0 & 0 & 0 & 0 & 0 & 0 & 0.01 & 0 & 0 & 0.02 & 0.01 & 0 \\
\hline & $\mathrm{C}$ & 0 & 0 & 0 & 0 & 0 & 0 & 0 & 0 & 0 & 0 & 0.01 & 0 & 0 & 0.01 & 0 & 0 \\
\hline & D & 0 & 0.02 & 0.01 & 0 & 0 & 0 & 0 & 0 & 0 & 0.03 & 0.03 & 0.02 & 0.02 & 0.06 & 0.02 & 0.01 \\
\hline & E & 0 & 0.01 & 0.03 & 0 & 0 & 0 & 0 & 0 & 0 & 0.02 & 0.02 & 0 & 0 & 0.02 & 0.02 & 0 \\
\hline & $\mathrm{F}$ & 0 & 0 & 0 & 0 & 0 & 0 & 0 & 0 & 0 & 0 & 0 & 0 & 0 & 0 & 0 & 0 \\
\hline & G & 0 & 0 & 0 & 0 & 0 & 0 & 0 & 0 & 0 & 0 & 0 & 0 & 0 & 0 & 0 & 0 \\
\hline \multirow[t]{7}{*}{15.6} & $\mathrm{~A}$ & 0 & 0 & 0 & 0 & 0 & 0 & 0 & 0 & 0 & 0 & 0 & 0 & 0 & 0 & 0 & 0 \\
\hline & B & 0 & 0 & 0 & 0 & 0 & 0 & 0 & 0 & 0 & 0 & 0 & 0 & 0 & 0 & 0 & 0 \\
\hline & $\mathrm{C}$ & 0 & 0 & 0 & 0 & 0 & 0 & 0 & 0 & 0 & 0 & 0 & 0 & 0 & 0 & 0 & 0 \\
\hline & D & 0 & 0 & 0 & 0 & 0 & 0 & 0 & 0 & 0 & 0.01 & 0 & 0 & 0 & 0 & 0 & 0 \\
\hline & E & 0 & 0 & 0 & 0 & 0 & 0 & 0 & 0 & 0 & 0 & 0 & 0 & 0 & 0 & 0 & 0 \\
\hline & $\mathrm{F}$ & 0 & 0 & 0 & 0 & 0 & 0 & 0 & 0 & 0 & 0 & 0 & 0 & 0 & 0 & 0 & 0 \\
\hline & G & 0 & 0 & 0 & 0 & 0 & 0 & 0 & 0 & 0 & 0 & 0 & 0 & 0 & 0 & 0 & 0 \\
\hline \multirow[t]{7}{*}{19} & $\mathrm{~A}$ & 0 & 0 & 0 & 0 & 0 & 0 & 0 & 0 & 0 & 0 & 0 & 0 & 0 & 0 & 0 & 0 \\
\hline & B & 0 & 0 & 0 & 0 & 0 & 0 & 0 & 0 & 0 & 0 & 0 & 0 & 0 & 0 & 0 & 0 \\
\hline & $\mathrm{C}$ & 0 & 0 & 0 & 0 & 0 & 0 & 0 & 0 & 0 & 0 & 0 & 0 & 0 & 0 & 0 & 0 \\
\hline & D & 0 & 0 & 0 & 0 & 0 & 0 & 0 & 0 & 0 & 0 & 0 & 0 & 0 & 0 & 0 & 0 \\
\hline & E & 0 & 0 & 0 & 0 & 0 & 0 & 0 & 0 & 0 & 0 & 0 & 0 & 0 & 0 & 0.01 & 0 \\
\hline & $\mathrm{F}$ & 0 & 0 & 0 & 0 & 0 & 0 & 0 & 0 & 0 & 0 & 0 & 0 & 0 & 0 & 0 & 0 \\
\hline & $\mathrm{G}$ & 0 & 0 & 0 & 0 & 0 & 0 & 0 & 0 & 0 & 0 & 0 & 0 & 0 & 0 & 0 & 0 \\
\hline
\end{tabular}


Table 4.1-8. Joint Frequency Distributions of Atmospheric Stability, Wind Speed, and Transport Direction for the 100 Area at $60 \mathrm{~m}$ Above Ground Level. Based on 1983-1996 data from the $100-\mathrm{N}$ instrumented tower.

\begin{tabular}{|c|c|c|c|c|c|c|c|c|c|c|c|c|c|c|c|c|c|}
\hline \multirow{2}{*}{$\begin{array}{c}\text { Average } \\
\text { Wind Speed } \\
\text { m/s }\end{array}$} & \multirow[t]{2}{*}{$\begin{array}{c}\text { Atmospheric } \\
\text { Stability Class }\end{array}$} & \multicolumn{16}{|c|}{ Percentage of Time Wind Blows from the 100N Area Toward the Direction Indicated } \\
\hline & & $\mathbf{S}$ & SSW & SW & WSW & W & WNW & NW & NNW & $\mathbf{N}$ & NNE & NE & ENE & $\mathbf{E}$ & ESE & SE & SSE \\
\hline \multirow[t]{7}{*}{0.89} & A & 0.23 & 0.22 & 0.17 & 0.16 & 0.2 & 0.21 & 0.18 & 0.14 & 0.11 & 0.1 & 0.12 & 0.12 & 0.17 & 0.22 & 0.24 & 0.26 \\
\hline & B & 0.13 & 0.1 & 0.09 & 0.08 & 0.1 & 0.14 & 0.09 & 0.06 & 0.06 & 0.06 & 0.06 & 0.08 & 0.1 & 0.09 & 0.12 & 0.11 \\
\hline & C & 0.11 & 0.1 & 0.07 & 0.07 & 0.11 & 0.11 & 0.11 & 0.07 & 0.06 & 0.06 & 0.07 & 0.08 & 0.1 & 0.1 & 0.09 & 0.11 \\
\hline & D & 0.51 & 0.43 & 0.41 & 0.45 & 0.66 & 0.76 & 0.65 & 0.5 & 0.43 & 0.37 & 0.36 & 0.47 & 0.52 & 0.54 & 0.5 & 0.48 \\
\hline & $\mathrm{E}$ & 0.4 & 0.36 & 0.43 & 0.52 & 0.67 & 0.62 & 0.56 & 0.42 & 0.33 & 0.29 & 0.33 & 0.36 & 0.48 & 0.43 & 0.37 & 0.34 \\
\hline & $\mathrm{F}$ & 0.32 & 0.34 & 0.47 & 0.61 & 0.82 & 0.69 & 0.55 & 0.36 & 0.25 & 0.23 & 0.24 & 0.24 & 0.29 & 0.35 & 0.35 & 0.3 \\
\hline & G & 0.17 & 0.16 & 0.24 & 0.35 & 0.49 & 0.38 & 0.21 & 0.15 & 0.12 & 0.1 & 0.1 & 0.11 & 0.15 & 0.18 & 0.2 & 0.17 \\
\hline \multirow[t]{7}{*}{2.65} & $\bar{A}$ & 0.44 & 0.5 & 0.3 & 0.13 & 0.19 & 0.29 & 0.25 & 0.19 & 0.12 & 0.11 & 0.21 & 0.37 & 0.39 & 0.28 & 0.27 & 0.29 \\
\hline & B & 0.15 & 0.19 & 0.1 & 0.06 & 0.12 & 0.12 & 0.15 & 0.09 & 0.05 & 0.05 & 0.1 & 0.14 & 0.17 & 0.13 & 0.11 & 0.09 \\
\hline & $\mathrm{C}$ & 0.11 & 0.15 & 0.11 & 0.05 & 0.11 & 0.13 & 0.11 & 0.08 & 0.05 & 0.05 & 0.08 & 0.13 & 0.15 & 0.12 & 0.09 & 0.09 \\
\hline & D & 0.5 & 0.51 & 0.42 & 0.34 & 0.53 & 0.65 & 0.79 & 0.43 & 0.31 & 0.23 & 0.37 & 0.54 & 0.75 & 0.65 & 0.46 & 0.35 \\
\hline & E & 0.29 & 0.33 & 0.3 & 0.42 & 0.68 & 0.73 & 0.63 & 0.38 & 0.25 & 0.19 & 0.26 & 0.43 & 0.76 & 0.9 & 0.59 & 0.29 \\
\hline & $\mathrm{F}$ & 0.26 & 0.24 & 0.22 & 0.46 & 0.89 & 0.77 & 0.49 & 0.24 & 0.14 & 0.1 & 0.14 & 0.22 & 0.49 & 0.7 & 0.45 & 0.23 \\
\hline & G & 0.11 & 0.09 & 0.13 & 0.23 & 0.43 & 0.35 & 0.18 & 0.07 & 0.05 & 0.04 & 0.06 & 0.06 & 0.16 & 0.28 & 0.23 & 0.14 \\
\hline \multirow[t]{7}{*}{4.7} & $\bar{A}$ & 0.12 & 0.29 & 0.18 & 0.05 & 0.06 & 0.1 & 0.11 & 0.06 & 0.05 & 0.06 & 0.19 & 0.22 & 0.31 & 0.24 & 0.13 & 0.08 \\
\hline & B & 0.05 & 0.1 & 0.05 & 0.02 & 0.02 & 0.03 & 0.05 & 0.04 & 0.03 & 0.03 & 0.08 & 0.08 & 0.12 & 0.1 & 0.04 & 0.03 \\
\hline & $\mathrm{C}$ & 0.03 & 0.08 & 0.05 & 0.03 & 0.02 & 0.04 & 0.05 & 0.03 & 0.01 & 0.02 & 0.06 & 0.06 & 0.1 & 0.07 & 0.03 & 0.04 \\
\hline & D & 0.22 & 0.29 & 0.19 & 0.1 & 0.1 & 0.18 & 0.37 & 0.17 & 0.12 & 0.12 & 0.23 & 0.29 & 0.51 & 0.41 & 0.2 & 0.18 \\
\hline & E & 0.18 & 0.2 & 0.17 & 0.14 & 0.17 & 0.21 & 0.4 & 0.2 & 0.15 & 0.15 & 0.24 & 0.37 & 1.04 & 1.03 & 0.32 & 0.17 \\
\hline & $\mathrm{F}$ & 0.13 & 0.12 & 0.08 & 0.11 & 0.14 & 0.16 & 0.22 & 0.12 & 0.05 & 0.05 & 0.05 & 0.14 & 0.42 & 0.57 & 0.19 & 0.1 \\
\hline & G & 0.03 & 0.04 & 0.03 & 0.03 & 0.05 & 0.06 & 0.06 & 0.02 & 0.02 & 0.02 & 0.02 & 0.04 & 0.08 & 0.14 & 0.09 & 0.04 \\
\hline \multirow[t]{7}{*}{7.15} & $\bar{A}$ & 0.06 & 0.17 & 0.09 & 0.03 & 0.02 & 0.03 & 0.05 & 0.03 & 0.02 & 0.04 & 0.2 & 0.17 & 0.26 & 0.31 & 0.1 & 0.03 \\
\hline & B & 0.03 & 0.05 & 0.02 & 0.01 & 0.01 & 0.01 & 0.02 & 0.01 & 0.01 & 0.02 & 0.05 & 0.05 & 0.06 & 0.08 & 0.04 & 0.02 \\
\hline & $\mathrm{C}$ & 0.03 & 0.02 & 0.01 & 0.01 & 0 & 0.02 & 0.03 & 0.02 & 0.01 & 0.02 & 0.05 & 0.03 & 0.06 & 0.08 & 0.02 & 0.01 \\
\hline & D & 0.12 & 0.15 & 0.09 & 0.05 & 0.05 & 0.05 & 0.14 & 0.11 & 0.08 & 0.12 & 0.23 & 0.2 & 0.5 & 0.7 & 0.24 & 0.07 \\
\hline & E & 0.1 & 0.14 & 0.1 & 0.07 & 0.04 & 0.06 & 0.16 & 0.13 & 0.08 & 0.1 & 0.2 & 0.26 & 1.26 & 1.67 & 0.24 & 0.06 \\
\hline & $\mathrm{F}$ & 0.08 & 0.1 & 0.05 & 0.05 & 0.04 & 0.04 & 0.09 & 0.05 & 0.03 & 0.02 & 0.03 & 0.07 & 0.31 & 0.28 & 0.06 & 0.03 \\
\hline & $\mathrm{G}$ & 0.02 & 0.02 & 0 & 0.01 & 0.03 & 0.02 & 0.02 & 0.01 & 0.01 & 0.01 & 0.01 & 0.01 & 0.04 & 0.05 & 0.02 & 0.01 \\
\hline \multirow[t]{7}{*}{9.8} & $\bar{A}$ & 0.06 & 0.08 & 0.05 & 0.01 & 0.01 & 0.01 & 0.02 & 0.01 & 0.01 & 0.01 & 0.11 & 0.09 & 0.15 & 0.29 & 0.12 & 0.02 \\
\hline & B & 0.03 & 0.02 & 0.01 & 0 & 0 & 0.01 & 0.01 & 0 & 0.01 & 0.01 & 0.05 & 0.02 & 0.04 & 0.08 & 0.05 & 0.01 \\
\hline & $\mathrm{C}$ & 0.01 & 0.02 & 0.01 & 0 & 0.01 & 0.01 & 0 & 0 & 0.01 & 0.01 & 0.03 & 0.04 & 0.02 & 0.06 & 0.03 & 0.01 \\
\hline & D & 0.09 & 0.1 & 0.07 & 0.03 & 0.03 & 0.03 & 0.06 & 0.05 & 0.04 & 0.07 & 0.16 & 0.14 & 0.31 & 0.68 & 0.25 & 0.03 \\
\hline & $\mathrm{E}$ & 0.05 & 0.08 & 0.07 & 0.03 & 0.03 & 0.03 & 0.04 & 0.04 & 0.03 & 0.06 & 0.1 & 0.1 & 0.47 & 0.97 & 0.17 & 0.03 \\
\hline & $\mathrm{F}$ & 0.04 & 0.05 & 0.03 & 0.02 & 0.03 & 0.02 & 0.02 & 0.01 & 0.01 & 0 & 0.01 & 0.01 & 0.09 & 0.08 & 0.02 & 0.01 \\
\hline & $\mathrm{G}$ & 0 & 0 & 0 & 0 & 0.01 & 0.01 & 0 & 0 & 0 & 0 & 0 & 0 & 0.01 & 0.02 & 0 & 0 \\
\hline \multirow[t]{7}{*}{12.7} & $\mathrm{~A}$ & 0.01 & 0.03 & 0.03 & 0 & 0 & 0 & 0 & 0 & 0 & 0.01 & 0.04 & 0.04 & 0.05 & 0.12 & 0.1 & 0 \\
\hline & B & 0.01 & 0.01 & 0.01 & 0 & 0 & 0 & 0 & 0 & 0 & 0 & 0.02 & 0.01 & 0.02 & 0.04 & 0.02 & 0 \\
\hline & $\mathrm{C}$ & 0.01 & 0.01 & 0.01 & 0 & 0 & 0 & 0 & 0 & 0 & 0.01 & 0.02 & 0.01 & 0.01 & 0.03 & 0.02 & 0 \\
\hline & D & 0.04 & 0.06 & 0.04 & 0.02 & 0.01 & 0.01 & 0.02 & 0.02 & 0.01 & 0.06 & 0.1 & 0.07 & 0.11 & 0.32 & 0.14 & 0.02 \\
\hline & $\mathrm{E}$ & 0.03 & 0.06 & 0.06 & 0.01 & 0.01 & 0.01 & 0.01 & 0.02 & 0.01 & 0.03 & 0.05 & 0.04 & 0.12 & 0.29 & 0.09 & 0.02 \\
\hline & $\mathrm{F}$ & 0.01 & 0.02 & 0 & 0 & 0.01 & 0.01 & 0 & 0 & 0 & 0 & 0 & 0 & 0.02 & 0.02 & 0 & 0 \\
\hline & G & 0 & 0 & 0 & 0 & 0 & 0 & 0 & 0 & 0 & 0 & 0 & 0 & 0 & 0 & 0 & 0 \\
\hline \multirow[t]{7}{*}{15.6} & $\mathrm{~A}$ & 0 & 0 & 0 & 0 & 0 & 0 & 0 & 0 & 0 & 0 & 0.01 & 0.01 & 0.01 & 0.04 & 0.01 & 0 \\
\hline & B & 0 & 0 & 0 & 0 & 0 & 0 & 0 & 0 & 0 & 0 & 0 & 0 & 0 & 0.01 & 0.01 & 0 \\
\hline & $\mathrm{C}$ & 0 & 0 & 0 & 0 & 0 & 0 & 0 & 0 & 0 & 0 & 0.01 & 0 & 0 & 0.01 & 0 & 0 \\
\hline & D & 0.01 & 0.01 & 0.01 & 0 & 0 & 0 & 0 & 0 & 0 & 0.03 & 0.05 & 0.03 & 0.03 & 0.06 & 0.02 & 0 \\
\hline & E & 0.01 & 0.02 & 0.02 & 0 & 0 & 0 & 0 & 0 & 0 & 0.02 & 0.02 & 0.01 & 0.01 & 0.05 & 0.03 & 0.01 \\
\hline & $\mathrm{F}$ & 0 & 0 & 0 & 0 & 0 & 0 & 0 & 0 & 0 & 0 & 0 & 0 & 0 & 0 & 0 & 0 \\
\hline & $\mathrm{G}$ & 0 & 0 & 0 & 0 & 0 & 0 & 0 & 0 & 0 & 0 & 0 & 0 & 0 & 0 & 0 & 0 \\
\hline \multirow[t]{7}{*}{19} & $\mathrm{~A}$ & 0 & 0 & 0 & 0 & 0 & 0 & 0 & 0 & 0 & 0 & 0 & 0 & 0 & 0.01 & 0 & 0 \\
\hline & B & 0 & 0 & 0 & 0 & 0 & 0 & 0 & 0 & 0 & 0 & 0 & 0 & 0 & 0 & 0 & 0 \\
\hline & C & 0 & 0 & 0 & 0 & 0 & 0 & 0 & 0 & 0 & 0 & 0 & 0 & 0 & 0 & 0 & 0 \\
\hline & D & 0 & 0 & 0 & 0 & 0 & 0 & 0 & 0 & 0 & 0.03 & 0.01 & 0 & 0.01 & 0.01 & 0 & 0 \\
\hline & E & 0 & 0 & 0 & 0 & 0 & 0 & 0 & 0 & 0 & 0.01 & 0 & 0 & 0 & 0.02 & 0.01 & 0 \\
\hline & F & 0 & 0 & 0 & 0 & 0 & 0 & 0 & 0 & 0 & 0 & 0 & 0 & 0 & 0 & 0 & 0 \\
\hline & $\mathrm{G}$ & 0 & 0 & 0 & 0 & 0 & 0 & 0 & 0 & 0 & 0 & 0 & 0 & 0 & 0 & 0 & 0 \\
\hline
\end{tabular}


Table 4.1-9. Joint Frequency Distributions of Atmospheric Stability, Wind Speed, and Transport Direction for the 200 Areas at 9 m Above Ground Level. Based on 1983-1996 data from the HMS instrumented tower.

\begin{tabular}{|c|c|c|c|c|c|c|c|c|c|c|c|c|c|c|c|c|c|}
\hline \multirow{2}{*}{$\begin{array}{c}\text { Average } \\
\text { Wind Speed } \\
\mathbf{m} / \mathbf{s}\end{array}$} & \multirow{2}{*}{$\begin{array}{c}\text { Atmospheric } \\
\text { Stability Class }\end{array}$} & \multicolumn{16}{|c|}{ Percentage of Time Wind Blows from the 200 Area Toward the Direction Indicated } \\
\hline & & $\mathbf{S}$ & SSW & SW & WSW & $\mathbf{W}$ & WNW & NW & NNW & $\mathbf{N}$ & NNE & NE & ENE & $\mathbf{E}$ & ESE & SE & SSE \\
\hline \multirow[t]{7}{*}{\begin{tabular}{l|l}
0.89 &
\end{tabular}} & $\mathrm{~A}$ & 0.28 & 0.31 & 0.34 & 0.25 & 0.23 & 0.22 & 0.18 & 0.13 & 0.11 & 0.1 & 0.08 & 0.07 & 0.1 & 0.1 & 0.16 & 0.21 \\
\hline & B & 0.14 & 0.15 & 0.16 & 0.11 & 0.11 & 0.09 & 0.08 & 0.05 & 0.05 & 0.04 & 0.04 & 0.04 & 0.05 & 0.07 & 0.09 & 0.12 \\
\hline & C & 0.15 & 0.15 & 0.14 & 0.1 & 0.09 & 0.09 & 0.09 & 0.06 & 0.04 & 0.04 & 0.05 & 0.05 & 0.06 & 0.07 & 0.1 & 0.13 \\
\hline & $\mathrm{D}$ & 0.87 & 0.76 & 0.72 & 0.55 & 0.6 & 0.65 & 0.64 & 0.42 & 0.36 & 0.31 & 0.35 & 0.38 & 0.49 & 0.59 & 0.77 & 0.83 \\
\hline & $\mathrm{E}$ & 0.4 & 0.29 & 0.27 & 0.26 & 0.3 & 0.35 & 0.46 & 0.41 & 0.36 & 0.35 & 0.44 & 0.49 & 0.55 & 0.66 & 0.65 & 0.57 \\
\hline & $\mathrm{F}$ & 0.25 & 0.16 & 0.15 & 0.15 & 0.15 & 0.2 & 0.25 & 0.24 & 0.26 & 0.29 & 0.35 & 0.36 & 0.43 & 0.45 & 0.42 & 0.33 \\
\hline & $\mathrm{G}$ & 0.1 & 0.09 & 0.1 & 0.07 & 0.08 & 0.08 & 0.1 & 0.1 & 0.1 & 0.1 & 0.14 & 0.11 & 0.14 & 0.15 & 0.17 & 0.15 \\
\hline \multirow[t]{7}{*}{2.65} & A & 0.64 & 0.45 & 0.35 & 0.32 & 0.35 & 0.37 & 0.34 & 0.23 & 0.17 & 0.2 & 0.27 & 0.2 & 0.17 & 0.26 & 0.6 & 0.7 \\
\hline & B & 0.26 & 0.17 & 0.11 & 0.1 & 0.1 & 0.12 & 0.1 & 0.07 & 0.06 & 0.06 & 0.09 & 0.07 & 0.07 & 0.14 & 0.29 & 0.31 \\
\hline & $\mathrm{C}$ & 0.22 & 0.13 & 0.1 & 0.08 & 0.08 & 0.09 & 0.1 & 0.05 & 0.05 & 0.05 & 0.06 & 0.06 & 0.06 & 0.1 & 0.25 & 0.28 \\
\hline & D & 0.64 & 0.46 & 0.3 & 0.27 & 0.31 & 0.36 & 0.43 & 0.29 & 0.23 & 0.24 & 0.3 & 0.39 & 0.55 & 1.05 & 1.72 & 1.12 \\
\hline & $\mathrm{E}$ & 0.29 & 0.16 & 0.11 & 0.1 & 0.21 & 0.28 & 0.35 & 0.41 & 0.31 & 0.29 & 0.53 & 0.98 & 1.68 & 2.09 & 1.71 & 0.77 \\
\hline & F & 0.15 & 0.07 & 0.05 & 0.06 & 0.09 & 0.11 & 0.3 & 0.33 & 0.31 & 0.37 & 0.65 & 1.23 & 1.74 & 1.89 & 1.57 & 0.59 \\
\hline & $\mathrm{G}$ & 0.04 & 0.03 & 0.02 & 0.02 & 0.04 & 0.04 & 0.13 & 0.18 & 0.19 & 0.2 & 0.32 & 0.65 & 0.68 & 0.78 & 0.69 & 0.19 \\
\hline \multirow[t]{7}{*}{4.7} & A & 0.19 & 0.22 & 0.11 & 0.04 & 0.04 & 0.03 & 0.04 & 0.04 & 0.05 & 0.13 & 0.31 & 0.36 & 0.21 & 0.23 & 0.61 & 0.3 \\
\hline & B & 0.04 & 0.04 & 0.03 & 0.02 & 0.01 & 0.01 & 0.01 & 0.01 & 0.01 & 0.04 & 0.06 & 0.1 & 0.08 & 0.09 & 0.22 & 0.09 \\
\hline & $\mathrm{C}$ & 0.04 & 0.03 & 0.02 & 0.01 & 0 & 0 & 0.01 & 0.01 & 0.02 & 0.04 & 0.05 & 0.08 & 0.07 & 0.08 & 0.2 & 0.09 \\
\hline & D & 0.14 & 0.13 & 0.06 & 0.04 & 0.05 & 0.04 & 0.07 & 0.09 & 0.11 & 0.19 & 0.34 & 0.52 & 0.57 & 1.11 & 1.45 & 0.37 \\
\hline & $\mathrm{E}$ & 0.07 & 0.06 & 0.04 & 0.02 & 0.02 & 0.02 & 0.06 & 0.1 & 0.11 & 0.15 & 0.37 & 0.66 & 1.09 & 1.95 & 1.78 & 0.25 \\
\hline & F & 0.02 & 0.01 & 0.01 & 0.01 & 0.01 & 0.01 & 0.04 & 0.09 & 0.04 & 0.03 & 0.08 & 0.3 & 0.33 & 0.53 & 0.72 & 0.11 \\
\hline & $\mathrm{G}$ & 0 & 0 & 0 & 0 & 0 & 0 & 0.02 & 0.04 & 0.01 & 0.02 & 0.04 & 0.18 & 0.1 & 0.16 & 0.32 & 0.03 \\
\hline \multirow[t]{7}{*}{7.15} & A & 0.03 & 0.06 & 0.04 & 0.01 & 0 & 0 & 0.01 & 0.01 & 0.02 & 0.06 & 0.23 & 0.33 & 0.15 & 0.17 & 0.44 & 0.11 \\
\hline & B & 0.01 & 0.01 & 0.01 & 0 & 0 & 0 & 0 & 0 & 0.01 & 0.03 & 0.06 & 0.08 & 0.03 & 0.05 & 0.12 & 0.02 \\
\hline & $\mathrm{C}$ & 0.01 & 0.01 & 0.01 & 0 & 0 & 0 & 0 & 0 & 0.01 & 0.02 & 0.04 & 0.07 & 0.03 & 0.03 & 0.08 & 0.02 \\
\hline & D & 0.03 & 0.05 & 0.03 & 0.01 & 0 & 0 & 0.01 & 0.03 & 0.06 & 0.16 & 0.38 & 0.35 & 0.24 & 0.6 & 0.85 & 0.11 \\
\hline & E & 0.01 & 0.05 & 0.02 & 0 & 0 & 0 & 0 & 0.02 & 0.05 & 0.11 & 0.25 & 0.23 & 0.15 & 0.47 & 0.93 & 0.06 \\
\hline & $\mathrm{F}$ & 0 & 0 & 0 & 0 & 0 & 0 & 0 & 0.01 & 0 & 0 & 0.01 & 0.02 & 0.01 & 0.01 & 0.02 & 0 \\
\hline & $\mathrm{G}$ & 0 & 0 & 0 & 0 & 0 & 0 & 0 & 0 & 0 & 0 & 0 & 0 & 0 & 0 & 0 & 0 \\
\hline \multirow[t]{7}{*}{9.8} & $\bar{A}$ & 0 & 0.01 & 0.01 & 0 & 0 & 0 & 0 & 0 & 0 & 0.02 & 0.08 & 0.11 & 0.04 & 0.02 & 0.15 & 0.02 \\
\hline & B & 0 & 0.01 & 0 & 0 & 0 & 0 & 0 & 0 & 0 & 0.01 & 0.03 & 0.03 & 0.01 & 0.01 & 0.04 & 0 \\
\hline & $\mathrm{C}$ & 0 & 0 & 0 & 0 & 0 & 0 & 0 & 0 & 0 & 0.01 & 0.02 & 0.01 & 0.01 & 0.01 & 0.03 & 0 \\
\hline & D & 0.01 & 0.02 & 0 & 0.01 & 0 & 0 & 0 & 0 & 0.01 & 0.08 & 0.16 & 0.09 & 0.03 & 0.11 & 0.26 & 0.02 \\
\hline & E & 0 & 0.02 & 0.01 & 0 & 0 & 0 & 0 & 0 & 0.01 & 0.05 & 0.07 & 0.04 & 0.01 & 0.05 & 0.18 & 0.01 \\
\hline & F & 0 & 0 & 0 & 0 & 0 & 0 & 0 & 0 & 0 & 0 & 0 & 0.01 & 0 & 0 & 0 & 0 \\
\hline & $\mathrm{G}$ & 0 & 0 & 0 & 0 & 0 & 0 & 0 & 0 & 0 & 0 & 0 & 0 & 0 & 0 & 0 & 0 \\
\hline \multirow[t]{7}{*}{12.7} & A & 0 & 0 & 0 & 0 & 0 & 0 & 0 & 0 & 0 & 0 & 0.01 & 0.02 & 0 & 0 & 0.01 & 0 \\
\hline & B & 0 & 0 & 0 & 0 & 0 & 0 & 0 & 0 & 0 & 0 & 0.01 & 0 & 0 & 0 & 0 & 0 \\
\hline & $\mathrm{C}$ & 0 & 0 & 0 & 0 & 0 & 0 & 0 & 0 & 0 & 0 & 0.01 & 0 & 0 & 0 & 0 & 0 \\
\hline & D & 0 & 0 & 0 & 0 & 0 & 0 & 0 & 0 & 0 & 0.03 & 0.04 & 0.02 & 0.01 & 0.01 & 0.01 & 0 \\
\hline & $\mathrm{E}$ & 0 & 0 & 0 & 0 & 0 & 0 & 0 & 0 & 0 & 0.02 & 0.01 & 0.01 & 0 & 0 & 0.01 & 0 \\
\hline & $\mathrm{F}$ & 0 & 0 & 0 & 0 & 0 & 0 & 0 & 0 & 0 & 0 & 0 & 0 & 0 & 0 & 0 & 0 \\
\hline & $\mathrm{G}$ & 0 & 0 & 0 & 0 & 0 & 0 & 0 & 0 & 0 & 0 & 0 & 0 & 0 & 0 & 0 & 0 \\
\hline \multirow[t]{7}{*}{15.6} & $\mathrm{~A}$ & 0 & 0 & 0 & 0 & 0 & 0 & 0 & 0 & 0 & 0 & 0 & 0 & 0 & 0 & 0 & 0 \\
\hline & B & 0 & 0 & 0 & 0 & 0 & 0 & 0 & 0 & 0 & 0 & 0 & 0 & 0 & 0 & 0 & 0 \\
\hline & $\mathrm{C}$ & 0 & 0 & 0 & 0 & 0 & 0 & 0 & 0 & 0 & 0 & 0 & 0 & 0 & 0 & 0 & 0 \\
\hline & D & 0 & 0 & 0 & 0 & 0 & 0 & 0 & 0 & 0 & 0 & 0 & 0 & 0 & 0 & 0 & 0 \\
\hline & E & 0 & 0 & 0 & 0 & 0 & 0 & 0 & 0 & 0 & 0 & 0 & 0 & 0 & 0 & 0 & 0 \\
\hline & $\mathrm{F}$ & 0 & 0 & 0 & 0 & 0 & 0 & 0 & 0 & 0 & 0 & 0 & 0 & 0 & 0 & 0 & 0 \\
\hline & $\mathrm{G}$ & 0 & 0 & 0 & 0 & 0 & 0 & 0 & 0 & 0 & 0 & 0 & 0 & 0 & 0 & 0 & 0 \\
\hline \multirow[t]{7}{*}{19} & $\mathrm{~A}$ & 0 & 0 & 0 & 0 & 0 & 0 & 0 & 0 & 0 & 0 & 0 & 0 & 0 & 0 & 0 & 0 \\
\hline & B & 0 & 0 & 0 & 0 & 0 & 0 & 0 & 0 & 0 & 0 & 0 & 0 & 0 & 0 & 0 & 0 \\
\hline & $\mathrm{C}$ & 0 & 0 & 0 & 0 & 0 & 0 & 0 & 0 & 0 & 0 & 0 & 0 & 0 & 0 & 0 & 0 \\
\hline & $\mathrm{D}$ & 0 & 0 & 0 & 0 & 0 & 0 & 0 & 0 & 0 & 0 & 0 & 0 & 0 & 0 & 0 & 0 \\
\hline & E & 0 & 0 & 0 & 0 & 0 & 0 & 0 & 0 & 0 & 0 & 0 & 0 & 0 & 0 & 0 & 0 \\
\hline & $\mathrm{F}$ & 0 & 0 & 0 & 0 & 0 & 0 & 0 & 0 & 0 & 0 & 0 & 0 & 0 & 0 & 0 & 0 \\
\hline & $\mathrm{G}$ & 0 & 0 & 0 & 0 & 0 & 0 & 0 & 0 & 0 & 0 & 0 & 0 & 0 & 0 & 0 & 0 \\
\hline
\end{tabular}


Table 4.1-10. Joint Frequency Distributions of Atmospheric Stability, Wind Speed, and Transport Direction for the 200 Areas at $61 \mathrm{~m}$ Above Ground Level. Based on 1983-1996 data from the HMS instrumented tower.

\begin{tabular}{|c|c|c|c|c|c|c|c|c|c|c|c|c|c|c|c|c|c|}
\hline \multirow{2}{*}{$\begin{array}{c}\text { Average } \\
\text { Wind Speed } \\
\mathbf{m} / \mathbf{s} \\
\end{array}$} & \multirow{2}{*}{$\begin{array}{c}\text { Atmospheric } \\
\text { Stability Class } \\
\end{array}$} & \multicolumn{16}{|c|}{ Percentage of Time Wind Blows from the 200 Area Toward the Direction Indicated } \\
\hline & & $\mathbf{S}$ & SSW & SW & WSW & W & WNW & NW & NNW & $\mathbf{N}$ & NNE & NE & ENE & E & ESE & SE & SSE \\
\hline \multirow[t]{7}{*}{0.89} & $\mathrm{~A}$ & 0.11 & 0.13 & 0.15 & 0.11 & 0.11 & 0.12 & 0.07 & 0.05 & 0.03 & 0.02 & 0.04 & 0.03 & 0.05 & 0.03 & 0.05 & 0.07 \\
\hline & B & 0.09 & 0.09 & 0.08 & 0.07 & 0.07 & 0.06 & 0.06 & 0.03 & 0.02 & 0.03 & 0.02 & 0.02 & 0.02 & 0.03 & 0.05 & 0.07 \\
\hline & C & 0.09 & 0.08 & 0.1 & 0.08 & 0.07 & 0.06 & 0.06 & 0.04 & 0.02 & 0.02 & 0.02 & 0.02 & 0.03 & 0.04 & 0.04 & 0.08 \\
\hline & D & 0.58 & 0.53 & 0.51 & 0.43 & 0.45 & 0.49 & 0.52 & 0.35 & 0.24 & 0.22 & 0.22 & 0.2 & 0.27 & 0.35 & 0.44 & 0.54 \\
\hline & E & 0.29 & 0.22 & 0.2 & 0.18 & 0.22 & 0.28 & 0.32 & 0.25 & 0.18 & 0.17 & 0.17 & 0.17 & 0.23 & 0.25 & 0.31 & 0.32 \\
\hline & $\mathrm{F}$ & 0.2 & 0.13 & 0.12 & 0.11 & 0.14 & 0.14 & 0.19 & 0.14 & 0.13 & 0.12 & 0.13 & 0.12 & 0.17 & 0.19 & 0.23 & 0.21 \\
\hline & G & 0.07 & 0.05 & 0.05 & 0.05 & 0.06 & 0.07 & 0.1 & 0.07 & 0.07 & 0.06 & 0.08 & 0.09 & 0.09 & 0.11 & 0.12 & 0.1 \\
\hline \multirow[t]{7}{*}{2.65} & A & 0.61 & 0.5 & 0.46 & 0.41 & 0.43 & 0.41 & 0.43 & 0.3 & 0.2 & 0.18 & 0.18 & 0.17 & 0.12 & 0.16 & 0.43 & 0.58 \\
\hline & B & 0.25 & 0.2 & 0.16 & 0.12 & 0.14 & 0.13 & 0.12 & 0.1 & 0.07 & 0.06 & 0.07 & 0.05 & 0.06 & 0.09 & 0.22 & 0.27 \\
\hline & C & 0.23 & 0.16 & 0.13 & 0.09 & 0.1 & 0.1 & 0.12 & 0.07 & 0.05 & 0.06 & 0.06 & 0.05 & 0.04 & 0.08 & 0.21 & 0.28 \\
\hline & D & 0.79 & 0.56 & 0.39 & 0.32 & 0.39 & 0.37 & 0.5 & 0.34 & 0.22 & 0.23 & 0.24 & 0.25 & 0.35 & 0.63 & 1.29 & 1.1 \\
\hline & E & 0.37 & 0.23 & 0.18 & 0.16 & 0.22 & 0.23 & 0.34 & 0.34 & 0.18 & 0.18 & 0.25 & 0.34 & 0.5 & 0.8 & 0.95 & 0.66 \\
\hline & $\mathrm{F}$ & 0.28 & 0.13 & 0.11 & 0.08 & 0.1 & 0.12 & 0.22 & 0.23 & 0.18 & 0.17 & 0.23 & 0.3 & 0.53 & 0.79 & 0.81 & 0.6 \\
\hline & $\mathrm{G}$ & 0.09 & 0.05 & 0.04 & 0.03 & 0.04 & 0.03 & 0.08 & 0.11 & 0.1 & 0.1 & 0.13 & 0.19 & 0.33 & 0.41 & 0.32 & 0.23 \\
\hline \multirow[t]{7}{*}{4.7} & A & 0.32 & 0.29 & 0.18 & 0.08 & 0.08 & 0.06 & 0.09 & 0.09 & 0.09 & 0.15 & 0.28 & 0.27 & 0.14 & 0.19 & 0.64 & 0.41 \\
\hline & B & 0.09 & 0.08 & 0.04 & 0.03 & 0.03 & 0.02 & 0.02 & 0.03 & 0.03 & 0.04 & 0.08 & 0.09 & 0.05 & 0.09 & 0.28 & 0.15 \\
\hline & C & 0.06 & 0.05 & 0.03 & 0.02 & 0.02 & 0.01 & 0.02 & 0.02 & 0.02 & 0.04 & 0.05 & 0.07 & 0.05 & 0.07 & 0.21 & 0.13 \\
\hline & D & 0.2 & 0.16 & 0.09 & 0.06 & 0.08 & 0.08 & 0.13 & 0.14 & 0.12 & 0.16 & 0.26 & 0.31 & 0.31 & 0.83 & 1.55 & 0.48 \\
\hline & E & 0.21 & 0.1 & 0.09 & 0.06 & 0.09 & 0.08 & 0.15 & 0.21 & 0.13 & 0.15 & 0.27 & 0.54 & 0.95 & 1.72 & 1.52 & 0.45 \\
\hline & $\mathrm{F}$ & 0.14 & 0.06 & 0.04 & 0.02 & 0.04 & 0.03 & 0.09 & 0.2 & 0.08 & 0.06 & 0.15 & 0.35 & 0.78 & 1.34 & 1.41 & 0.49 \\
\hline & $\mathrm{G}$ & 0.04 & 0.01 & 0 & 0 & 0 & 0 & 0.03 & 0.05 & 0.03 & 0.03 & 0.06 & 0.15 & 0.33 & 0.47 & 0.64 & 0.27 \\
\hline \multirow[t]{7}{*}{7.15} & A & 0.05 & 0.11 & 0.07 & 0.02 & 0.01 & 0 & 0.01 & 0.02 & 0.02 & 0.09 & 0.29 & 0.37 & 0.15 & 0.16 & 0.48 & 0.11 \\
\hline & B & 0.02 & 0.02 & 0.02 & 0.01 & 0 & 0 & 0 & 0.01 & 0.01 & 0.03 & 0.05 & 0.09 & 0.04 & 0.06 & 0.14 & 0.03 \\
\hline & C & 0.01 & 0.01 & 0.01 & 0 & 0 & 0 & 0 & 0.01 & 0.01 & 0.03 & 0.05 & 0.07 & 0.04 & 0.05 & 0.12 & 0.02 \\
\hline & D & 0.06 & 0.08 & 0.04 & 0.02 & 0.01 & 0.01 & 0.04 & 0.08 & 0.08 & 0.17 & 0.34 & 0.46 & 0.39 & 0.85 & 1.18 & 0.15 \\
\hline & E & 0.07 & 0.05 & 0.04 & 0.02 & 0.02 & 0.01 & 0.05 & 0.1 & 0.09 & 0.14 & 0.31 & 0.64 & 0.9 & 2.11 & 1.71 & 0.15 \\
\hline & $\mathrm{F}$ & 0.04 & 0.03 & 0.03 & 0.01 & 0.01 & 0 & 0.03 & 0.08 & 0.03 & 0.03 & 0.06 & 0.23 & 0.39 & 0.88 & 1.3 & 0.15 \\
\hline & $\mathrm{G}$ & 0 & 0 & 0 & 0 & 0 & 0 & 0.02 & 0.04 & 0.01 & 0 & 0.01 & 0.05 & 0.08 & 0.2 & 0.61 & 0.1 \\
\hline \multirow[t]{7}{*}{9.8} & A & 0.01 & 0.03 & 0.04 & 0.01 & 0 & 0 & 0 & 0 & 0.01 & 0.03 & 0.16 & 0.21 & 0.06 & 0.1 & 0.31 & 0.03 \\
\hline & B & 0 & 0.01 & 0 & 0 & 0 & 0 & 0 & 0 & 0 & 0.01 & 0.05 & 0.05 & 0.01 & 0.03 & 0.08 & 0.01 \\
\hline & C & 0 & 0 & 0 & 0 & 0 & 0 & 0 & 0 & 0 & 0.01 & 0.04 & 0.04 & 0.02 & 0.03 & 0.05 & 0.01 \\
\hline & D & 0.02 & 0.03 & 0.02 & 0.01 & 0 & 0 & 0 & 0.02 & 0.04 & 0.11 & 0.29 & 0.28 & 0.15 & 0.51 & 0.68 & 0.04 \\
\hline & E & 0.02 & 0.04 & 0.02 & 0 & 0 & 0 & 0.01 & 0.02 & 0.04 & 0.09 & 0.24 & 0.28 & 0.2 & 0.78 & 1.04 & 0.03 \\
\hline & $\mathrm{F}$ & 0 & 0 & 0 & 0 & 0 & 0 & 0 & 0.01 & 0.01 & 0 & 0.02 & 0.03 & 0.04 & 0.08 & 0.19 & 0.01 \\
\hline & $\mathrm{G}$ & 0 & 0 & 0 & 0 & 0 & 0 & 0 & 0 & 0 & 0 & 0 & 0 & 0.01 & 0.03 & 0.08 & 0 \\
\hline \multirow[t]{7}{*}{12.7} & A & 0 & 0 & 0.01 & 0 & 0 & 0 & 0 & 0 & 0 & 0.02 & 0.09 & 0.1 & 0.02 & 0.02 & 0.16 & 0.01 \\
\hline & B & 0 & 0.01 & 0 & 0 & 0 & 0 & 0 & 0 & 0 & 0.01 & 0.04 & 0.03 & 0.01 & 0.01 & 0.05 & 0 \\
\hline & C & 0 & 0 & 0 & 0 & 0 & 0 & 0 & 0 & 0 & 0.01 & 0.02 & 0.02 & 0 & 0.01 & 0.04 & 0 \\
\hline & D & 0.01 & 0.02 & 0.01 & 0.01 & 0 & 0 & 0 & 0.01 & 0.01 & 0.1 & 0.23 & 0.12 & 0.04 & 0.24 & 0.48 & 0.01 \\
\hline & E & 0 & 0.02 & 0.01 & 0 & 0 & 0 & 0 & 0 & 0.02 & 0.07 & 0.13 & 0.08 & 0.04 & 0.19 & 0.39 & 0 \\
\hline & $\mathrm{F}$ & 0 & 0 & 0 & 0 & 0 & 0 & 0 & 0 & 0 & 0 & 0 & 0.01 & 0 & 0 & 0 & 0 \\
\hline & $\mathrm{G}$ & 0 & 0 & 0 & 0 & 0 & 0 & 0 & 0 & 0 & 0 & 0 & 0 & 0 & 0 & 0 & 0 \\
\hline \multirow[t]{7}{*}{15.6} & A & 0 & 0 & 0 & 0 & 0 & 0 & 0 & 0 & 0 & 0 & 0.02 & 0.02 & 0 & 0 & 0.02 & 0 \\
\hline & B & 0 & 0 & 0 & 0 & 0 & 0 & 0 & 0 & 0 & 0 & 0.01 & 0.01 & 0 & 0 & 0.01 & 0 \\
\hline & C & 0 & 0 & 0 & 0 & 0 & 0 & 0 & 0 & 0 & 0 & 0.01 & 0.01 & 0 & 0 & 0.01 & 0 \\
\hline & D & 0 & 0 & 0 & 0 & 0 & 0 & 0 & 0 & 0 & 0.04 & 0.08 & 0.03 & 0.01 & 0.03 & 0.06 & 0 \\
\hline & E & 0 & 0 & 0 & 0 & 0 & 0 & 0 & 0 & 0 & 0.03 & 0.04 & 0.01 & 0.01 & 0.03 & 0.05 & 0 \\
\hline & $\mathrm{F}$ & 0 & 0 & 0 & 0 & 0 & 0 & 0 & 0 & 0 & 0 & 0 & 0 & 0 & 0 & 0 & 0 \\
\hline & $\mathrm{G}$ & 0 & 0 & 0 & 0 & 0 & 0 & 0 & 0 & 0 & 0 & 0 & 0 & 0 & 0 & 0 & 0 \\
\hline \multirow[t]{7}{*}{19} & A & 0 & 0 & 0 & 0 & 0 & 0 & 0 & 0 & 0 & 0 & 0 & 0 & 0 & 0 & 0 & 0 \\
\hline & B & 0 & 0 & 0 & 0 & 0 & 0 & 0 & 0 & 0 & 0 & 0 & 0 & 0 & 0 & 0 & 0 \\
\hline & C & 0 & 0 & 0 & 0 & 0 & 0 & 0 & 0 & 0 & 0 & 0 & 0 & 0 & 0 & 0 & 0 \\
\hline & D & 0 & 0 & 0 & 0 & 0 & 0 & 0 & 0 & 0 & 0.01 & 0.03 & 0.01 & 0 & 0 & 0 & 0 \\
\hline & E & 0 & 0 & 0 & 0 & 0 & 0 & 0 & 0 & 0 & 0.01 & 0.01 & 0 & 0 & 0 & 0 & 0 \\
\hline & $\mathrm{F}$ & 0 & 0 & 0 & 0 & 0 & 0 & 0 & 0 & 0 & 0 & 0 & 0 & 0 & 0 & 0 & 0 \\
\hline & $\mathrm{G}$ & 0 & 0 & 0 & 0 & 0 & 0 & 0 & 0 & 0 & 0 & 0 & 0 & 0 & 0 & 0 & 0 \\
\hline
\end{tabular}


Table 4.1-11. Joint Frequency Distributions of Atmospheric Stability, Wind Speed, and Transport Direction for the 300 Area at $9 \mathrm{~m}$ Above Ground Level. Based on 1983-1996 data from the 300 Area instrumented tower.

\begin{tabular}{|c|c|c|c|c|c|c|c|c|c|c|c|c|c|c|c|c|c|}
\hline \multirow{2}{*}{$\begin{array}{c}\text { Average } \\
\text { Wind Speed } \\
\mathbf{m} / \mathbf{s}\end{array}$} & \multirow{2}{*}{$\begin{array}{c}\text { Atmospheric } \\
\text { Stability Class }\end{array}$} & \multicolumn{16}{|c|}{ Percentage of Time Wind Blows from the 300 Area Toward the Direction Indicated } \\
\hline & & $\mathbf{S}$ & SSW & SW & WSW & W & WNW & NW & NNW & $\mathbf{N}$ & NNE & NE & ENE & $\mathbf{E}$ & ESE & SE & SSE \\
\hline \multirow[t]{7}{*}{0.89} & $\mathrm{~A}$ & 0.08 & 0.06 & 0.08 & 0.1 & 0.11 & 0.11 & 0.12 & 0.11 & 0.08 & 0.06 & 0.05 & 0.04 & 0.03 & 0.03 & 0.06 & 0.07 \\
\hline & B & 0.06 & 0.05 & 0.03 & 0.04 & 0.06 & 0.05 & 0.07 & 0.05 & 0.04 & 0.03 & 0.04 & 0.04 & 0.03 & 0.03 & 0.04 & 0.06 \\
\hline & $\mathrm{C}$ & 0.05 & 0.03 & 0.04 & 0.04 & 0.05 & 0.06 & 0.07 & 0.06 & 0.04 & 0.03 & 0.02 & 0.03 & 0.03 & 0.02 & 0.04 & 0.05 \\
\hline & D & 0.35 & 0.21 & 0.17 & 0.17 & 0.22 & 0.37 & 0.4 & 0.43 & 0.38 & 0.36 & 0.37 & 0.3 & 0.3 & 0.28 & 0.42 & 0.49 \\
\hline & E & 0.34 & 0.18 & 0.13 & 0.13 & 0.19 & 0.34 & 0.51 & 0.56 & 0.57 & 0.46 & 0.46 & 0.4 & 0.44 & 0.47 & 0.54 & 0.49 \\
\hline & $\mathrm{F}$ & 0.26 & 0.16 & 0.12 & 0.08 & 0.15 & 0.26 & 0.48 & 0.51 & 0.51 & 0.37 & 0.37 & 0.32 & 0.32 & 0.4 & 0.48 & 0.45 \\
\hline & G & 0.17 & 0.08 & 0.04 & 0.04 & 0.08 & 0.11 & 0.2 & 0.22 & 0.21 & 0.16 & 0.17 & 0.14 & 0.15 & 0.18 & 0.25 & 0.23 \\
\hline \multirow[t]{7}{*}{2.65} & $\mathrm{~A}$ & 0.23 & 0.3 & 0.39 & 0.41 & 0.55 & 0.56 & 0.53 & 0.27 & 0.21 & 0.26 & 0.26 & 0.16 & 0.08 & 0.05 & 0.08 & 0.19 \\
\hline & B & 0.13 & 0.15 & 0.13 & 0.15 & 0.19 & 0.21 & 0.26 & 0.15 & 0.11 & 0.13 & 0.11 & 0.06 & 0.03 & 0.03 & 0.04 & 0.11 \\
\hline & $\mathrm{C}$ & 0.13 & 0.13 & 0.11 & 0.12 & 0.15 & 0.19 & 0.24 & 0.13 & 0.11 & 0.12 & 0.11 & 0.05 & 0.02 & 0.03 & 0.06 & 0.12 \\
\hline & D & 0.99 & 0.53 & 0.32 & 0.34 & 0.57 & 1 & 1.34 & 0.73 & 0.66 & 0.67 & 0.56 & 0.37 & 0.23 & 0.24 & 0.61 & 1.2 \\
\hline & $\mathrm{E}$ & 1.07 & 0.34 & 0.09 & 0.1 & 0.25 & 1.07 & 1.77 & 1.06 & 1.06 & 0.76 & 0.61 & 0.45 & 0.35 & 0.42 & 0.69 & 1.22 \\
\hline & $\mathrm{F}$ & 0.65 & 0.15 & 0.03 & 0.02 & 0.1 & 0.92 & 1.82 & 0.97 & 0.66 & 0.42 & 0.25 & 0.14 & 0.14 & 0.18 & 0.42 & 0.81 \\
\hline & $\mathrm{G}$ & 0.29 & 0.05 & 0.01 & 0 & 0.03 & 0.33 & 0.8 & 0.4 & 0.22 & 0.12 & 0.07 & 0.04 & 0.04 & 0.06 & 0.19 & 0.37 \\
\hline \multirow[t]{7}{*}{4.7} & $\bar{A}$ & 0.27 & 0.52 & 0.35 & 0.09 & 0.11 & 0.21 & 0.27 & 0.13 & 0.19 & 0.47 & 0.58 & 0.29 & 0.08 & 0.06 & 0.09 & 0.14 \\
\hline & B & 0.11 & 0.16 & 0.08 & 0.03 & 0.03 & 0.08 & 0.09 & 0.05 & 0.09 & 0.22 & 0.23 & 0.11 & 0.04 & 0.02 & 0.04 & 0.08 \\
\hline & $\mathrm{C}$ & 0.11 & 0.14 & 0.08 & 0.03 & 0.02 & 0.06 & 0.1 & 0.05 & 0.07 & 0.16 & 0.2 & 0.09 & 0.02 & 0.01 & 0.05 & 0.09 \\
\hline & D & 0.75 & 0.46 & 0.24 & 0.09 & 0.1 & 0.21 & 0.4 & 0.25 & 0.4 & 0.87 & 0.92 & 0.5 & 0.2 & 0.14 & 0.45 & 0.9 \\
\hline & $\mathrm{E}$ & 1.03 & 0.34 & 0.06 & 0.04 & 0.05 & 0.25 & 0.34 & 0.22 & 0.49 & 0.8 & 0.92 & 0.52 & 0.21 & 0.17 & 0.44 & 0.79 \\
\hline & $\mathrm{F}$ & 0.77 & 0.22 & 0.02 & 0.02 & 0.03 & 0.24 & 0.26 & 0.1 & 0.23 & 0.36 & 0.33 & 0.13 & 0.04 & 0.03 & 0.08 & 0.39 \\
\hline & $\mathrm{G}$ & 0.42 & 0.12 & 0 & 0 & 0.01 & 0.12 & 0.15 & 0.04 & 0.07 & 0.11 & 0.09 & 0.03 & 0.01 & 0 & 0.02 & 0.16 \\
\hline \multirow[t]{7}{*}{7.15} & $\mathrm{~A}$ & 0.12 & 0.16 & 0.04 & 0 & 0 & 0 & 0.02 & 0.01 & 0.05 & 0.28 & 0.56 & 0.41 & 0.11 & 0.04 & 0.09 & 0.09 \\
\hline & B & 0.04 & 0.04 & 0.01 & 0 & 0 & 0 & 0.01 & 0 & 0.02 & 0.12 & 0.16 & 0.1 & 0.03 & 0.02 & 0.03 & 0.04 \\
\hline & C & 0.03 & 0.03 & 0.01 & 0 & 0 & 0 & 0.01 & 0.01 & 0.01 & 0.1 & 0.16 & 0.09 & 0.03 & 0.01 & 0.03 & 0.04 \\
\hline & D & 0.15 & 0.11 & 0.03 & 0.01 & 0.01 & 0.02 & 0.03 & 0.03 & 0.14 & 0.49 & 0.7 & 0.39 & 0.15 & 0.07 & 0.38 & 0.4 \\
\hline & E & 0.14 & 0.07 & 0.04 & 0.02 & 0.01 & 0.01 & 0.03 & 0.02 & 0.09 & 0.32 & 0.56 & 0.25 & 0.09 & 0.05 & 0.26 & 0.28 \\
\hline & $\mathrm{F}$ & 0.05 & 0.03 & 0.02 & 0.02 & 0 & 0 & 0 & 0 & 0.02 & 0.08 & 0.15 & 0.05 & 0.01 & 0 & 0.02 & 0.05 \\
\hline & $\mathrm{G}$ & 0.03 & 0.02 & 0 & 0 & 0 & 0 & 0 & 0 & 0.02 & 0.04 & 0.06 & 0.01 & 0 & 0 & 0 & 0.01 \\
\hline \multirow[t]{7}{*}{9.8} & $\mathrm{~A}$ & 0.01 & 0.03 & 0 & 0 & 0 & 0 & 0 & 0 & 0.01 & 0.09 & 0.17 & 0.15 & 0.07 & 0.01 & 0.03 & 0.02 \\
\hline & B & 0.01 & 0.01 & 0 & 0 & 0 & 0 & 0 & 0 & 0.01 & 0.03 & 0.05 & 0.04 & 0.02 & 0 & 0.02 & 0.01 \\
\hline & C & 0.01 & 0 & 0 & 0 & 0 & 0 & 0 & 0 & 0.01 & 0.02 & 0.04 & 0.04 & 0.01 & 0 & 0.01 & 0.01 \\
\hline & D & 0.02 & 0.01 & 0.01 & 0 & 0 & 0 & 0 & 0 & 0.02 & 0.15 & 0.28 & 0.14 & 0.07 & 0.01 & 0.16 & 0.09 \\
\hline & E & 0.02 & 0.03 & 0.02 & 0.01 & 0 & 0 & 0 & 0 & 0.01 & 0.09 & 0.24 & 0.05 & 0.02 & 0.01 & 0.08 & 0.04 \\
\hline & $\mathrm{F}$ & 0 & 0 & 0 & 0 & 0 & 0 & 0 & 0 & 0 & 0.01 & 0.04 & 0 & 0 & 0 & 0 & 0 \\
\hline & G & 0 & 0 & 0 & 0 & 0 & 0 & 0 & 0 & 0 & 0.01 & 0.02 & 0 & 0 & 0 & 0 & 0 \\
\hline \multirow[t]{7}{*}{12.7} & $\mathrm{~A}$ & 0 & 0 & 0 & 0 & 0 & 0 & 0 & 0 & 0 & 0.01 & 0.04 & 0.03 & 0.02 & 0 & 0 & 0 \\
\hline & B & 0 & 0 & 0 & 0 & 0 & 0 & 0 & 0 & 0 & 0 & 0.02 & 0.01 & 0 & 0 & 0 & 0 \\
\hline & $\mathrm{C}$ & 0 & 0 & 0 & 0 & 0 & 0 & 0 & 0 & 0 & 0.01 & 0.02 & 0 & 0 & 0 & 0 & 0 \\
\hline & D & 0.01 & 0.01 & 0 & 0 & 0 & 0 & 0 & 0 & 0.01 & 0.05 & 0.15 & 0.04 & 0.02 & 0.01 & 0.03 & 0.02 \\
\hline & E & 0 & 0.01 & 0 & 0 & 0 & 0 & 0 & 0 & 0 & 0.04 & 0.12 & 0.01 & 0 & 0 & 0.01 & 0 \\
\hline & F & 0 & 0 & 0 & 0 & 0 & 0 & 0 & 0 & 0 & 0 & 0.01 & 0 & 0 & 0 & 0 & 0 \\
\hline & $\mathrm{G}$ & 0 & 0 & 0 & 0 & 0 & 0 & 0 & 0 & 0 & 0 & 0.01 & 0 & 0 & 0 & 0 & 0 \\
\hline \multirow[t]{7}{*}{15.6} & $\mathrm{~A}$ & 0 & $\overline{0}$ & $\overline{0}$ & 0 & 0 & 0 & 0 & 0 & 0 & 0 & 0.01 & $\overline{0}$ & 0 & $\overline{0}$ & 0 & 0 \\
\hline & B & 0 & 0 & 0 & 0 & 0 & 0 & 0 & 0 & 0 & 0 & 0 & 0 & 0 & 0 & 0 & 0 \\
\hline & $\mathrm{C}$ & 0 & 0 & 0 & 0 & 0 & 0 & 0 & 0 & 0 & 0.01 & 0.01 & 0 & 0 & 0 & 0 & 0 \\
\hline & D & 0 & 0 & 0 & 0 & 0 & 0 & 0 & 0 & 0 & 0.02 & 0.05 & 0.01 & 0.01 & 0 & 0 & 0 \\
\hline & E & 0 & 0 & 0 & 0 & 0 & 0 & 0 & 0 & 0 & 0.01 & 0.03 & 0 & 0 & 0 & 0 & 0 \\
\hline & $\mathrm{F}$ & 0 & 0 & 0 & 0 & 0 & 0 & 0 & 0 & 0 & 0 & 0 & 0 & 0 & 0 & 0 & 0 \\
\hline & $\mathrm{G}$ & 0 & 0 & 0 & 0 & 0 & 0 & 0 & 0 & 0 & 0 & 0 & 0 & 0 & 0 & 0 & 0 \\
\hline \multirow[t]{7}{*}{19} & $\bar{A}$ & 0 & 0 & 0 & 0 & 0 & 0 & 0 & 0 & 0 & 0 & 0 & 0 & 0 & 0 & 0 & 0 \\
\hline & B & 0 & 0 & 0 & 0 & 0 & 0 & 0 & 0 & 0 & 0 & 0 & 0 & 0 & 0 & 0 & 0 \\
\hline & $\mathrm{C}$ & 0 & 0 & 0 & 0 & 0 & 0 & 0 & 0 & 0 & 0 & 0 & 0 & 0 & 0 & 0 & 0 \\
\hline & D & 0 & 0 & 0 & 0 & 0 & 0 & 0 & 0 & 0 & 0.01 & 0.01 & 0 & 0 & 0 & 0 & 0 \\
\hline & E & 0 & 0 & 0 & 0 & 0 & 0 & 0 & 0 & 0 & 0.01 & 0 & 0 & 0 & 0 & 0 & 0 \\
\hline & $\mathrm{F}$ & 0 & 0 & 0 & 0 & 0 & 0 & 0 & 0 & 0 & 0 & 0 & 0 & 0 & 0 & 0 & 0 \\
\hline & $\mathrm{G}$ & 0 & 0 & 0 & 0 & 0 & 0 & 0 & 0 & 0 & 0 & 0 & 0 & 0 & 0 & 0 & 0 \\
\hline
\end{tabular}


Table 4.1-12. Joint Frequency Distributions of Atmospheric Stability, Wind Speed, and Transport Direction for the 300 Area at $60 \mathrm{~m}$ Above Ground Level. Based on 1983-1996 data from the 300 Area instrumented tower.

\begin{tabular}{|c|c|c|c|c|c|c|c|c|c|c|c|c|c|c|c|c|c|}
\hline \multirow{2}{*}{$\begin{array}{c}\text { Average } \\
\text { Wind Speed } \\
\mathbf{m} / \mathbf{s}\end{array}$} & \multirow[t]{2}{*}{$\begin{array}{c}\text { Atmospheric } \\
\text { Stability Class }\end{array}$} & \multicolumn{16}{|c|}{ Percentage of Time Wind Blows from the 300 Area Toward the Direction Indicated } \\
\hline & & $\mathbf{S}$ & SSW & SW & WSW & W & WNW & NW & NNW & $\mathbf{N}$ & NNE & NE & ENE & E & ESE & SE & SSE \\
\hline \multirow[t]{7}{*}{0.89} & A & 0.08 & 0.07 & 0.07 & 0.09 & 0.1 & 0.11 & 0.13 & 0.11 & 0.09 & 0.05 & 0.05 & 0.04 & 0.04 & 0.03 & 0.05 & 0.06 \\
\hline & B & 0.06 & 0.05 & 0.03 & 0.04 & 0.04 & 0.06 & 0.07 & 0.05 & 0.04 & 0.03 & 0.02 & 0.03 & 0.03 & 0.03 & 0.03 & 0.04 \\
\hline & $\mathrm{C}$ & 0.04 & 0.04 & 0.04 & 0.03 & 0.05 & 0.05 & 0.05 & 0.06 & 0.04 & 0.03 & 0.02 & 0.02 & 0.02 & 0.03 & 0.03 & 0.04 \\
\hline & D & 0.3 & 0.23 & 0.18 & 0.17 & 0.24 & 0.31 & 0.3 & 0.34 & 0.28 & 0.18 & 0.2 & 0.17 & 0.18 & 0.17 & 0.27 & 0.31 \\
\hline & $\mathrm{E}$ & 0.3 & 0.22 & 0.17 & 0.15 & 0.2 & 0.25 & 0.3 & 0.34 & 0.35 & 0.27 & 0.23 & 0.19 & 0.2 & 0.22 & 0.25 & 0.31 \\
\hline & $\mathrm{F}$ & 0.25 & 0.19 & 0.18 & 0.14 & 0.16 & 0.23 & 0.33 & 0.3 & 0.28 & 0.26 & 0.21 & 0.16 & 0.17 & 0.2 & 0.21 & 0.24 \\
\hline & G & 0.1 & 0.08 & 0.06 & 0.04 & 0.07 & 0.1 & 0.15 & 0.13 & 0.14 & 0.11 & 0.1 & 0.08 & 0.1 & 0.1 & 0.12 & 0.14 \\
\hline \multirow[t]{7}{*}{2.65} & $\mathrm{~A}$ & 0.25 & 0.27 & 0.36 & 0.39 & 0.52 & 0.54 & 0.49 & 0.29 & 0.19 & 0.23 & 0.22 & 0.13 & 0.06 & 0.04 & 0.06 & 0.15 \\
\hline & B & 0.15 & 0.13 & 0.13 & 0.14 & 0.18 & 0.19 & 0.24 & 0.16 & 0.12 & 0.11 & 0.1 & 0.07 & 0.03 & 0.03 & 0.03 & 0.09 \\
\hline & $\mathrm{C}$ & 0.14 & 0.12 & 0.1 & 0.11 & 0.16 & 0.19 & 0.22 & 0.14 & 0.1 & 0.11 & 0.09 & 0.06 & 0.02 & 0.02 & 0.03 & 0.09 \\
\hline & $\mathrm{D}$ & 0.89 & 0.57 & 0.36 & 0.36 & 0.51 & 0.71 & 1.06 & 0.7 & 0.52 & 0.53 & 0.46 & 0.29 & 0.19 & 0.17 & 0.34 & 0.75 \\
\hline & E & 0.83 & 0.44 & 0.15 & 0.1 & 0.22 & 0.45 & 0.86 & 0.81 & 0.78 & 0.7 & 0.62 & 0.43 & 0.34 & 0.36 & 0.4 & 0.64 \\
\hline & $\mathrm{F}$ & 0.56 & 0.3 & 0.08 & 0.04 & 0.13 & 0.46 & 0.87 & 0.82 & 0.74 & 0.52 & 0.34 & 0.26 & 0.2 & 0.11 & 0.23 & 0.43 \\
\hline & $\mathrm{G}$ & 0.28 & 0.11 & 0.03 & 0.01 & 0.03 & 0.23 & 0.46 & 0.34 & 0.27 & 0.16 & 0.1 & 0.07 & 0.06 & 0.06 & 0.1 & 0.23 \\
\hline \multirow[t]{7}{*}{4.7} & $\mathrm{~A}$ & 0.25 & 0.56 & 0.37 & 0.12 & 0.11 & 0.21 & 0.34 & 0.17 & 0.2 & 0.44 & 0.57 & 0.25 & 0.08 & 0.04 & 0.07 & 0.11 \\
\hline & B & 0.12 & 0.19 & 0.1 & 0.04 & 0.04 & 0.08 & 0.12 & 0.06 & 0.07 & 0.21 & 0.21 & 0.12 & 0.03 & 0.02 & 0.03 & 0.07 \\
\hline & $\mathrm{C}$ & 0.12 & 0.17 & 0.11 & 0.04 & 0.02 & 0.07 & 0.11 & 0.06 & 0.07 & 0.16 & 0.19 & 0.08 & 0.02 & 0.01 & 0.04 & 0.07 \\
\hline & D & 0.83 & 0.55 & 0.25 & 0.13 & 0.13 & 0.27 & 0.55 & 0.3 & 0.34 & 0.76 & 0.79 & 0.45 & 0.2 & 0.15 & 0.3 & 0.71 \\
\hline & E & 1.01 & 0.35 & 0.08 & 0.07 & 0.08 & 0.27 & 0.59 & 0.42 & 0.6 & 0.93 & 0.87 & 0.6 & 0.35 & 0.27 & 0.43 & 0.85 \\
\hline & $\mathrm{F}$ & 0.8 & 0.27 & 0.02 & 0.02 & 0.04 & 0.25 & 0.66 & 0.32 & 0.4 & 0.53 & 0.47 & 0.25 & 0.09 & 0.04 & 0.08 & 0.39 \\
\hline & G & 0.41 & 0.13 & 0 & 0 & 0.01 & 0.12 & 0.34 & 0.12 & 0.14 & 0.16 & 0.12 & 0.05 & 0.02 & 0.01 & 0.02 & 0.17 \\
\hline \multirow[t]{7}{*}{7.15} & $\mathrm{~A}$ & 0.16 & 0.27 & 0.07 & 0.01 & 0 & 0.01 & 0.03 & 0.02 & 0.04 & 0.32 & 0.61 & 0.45 & 0.11 & 0.05 & 0.07 & 0.08 \\
\hline & B & 0.06 & 0.07 & 0.01 & 0 & 0 & 0.01 & 0.01 & 0.01 & 0.02 & 0.14 & 0.19 & 0.1 & 0.04 & 0.02 & 0.03 & 0.05 \\
\hline & $\mathrm{C}$ & 0.04 & 0.05 & 0.01 & 0 & 0 & 0 & 0.02 & 0.01 & 0.02 & 0.11 & 0.17 & 0.1 & 0.02 & 0.01 & 0.02 & 0.04 \\
\hline & D & 0.36 & 0.19 & 0.04 & 0.01 & 0.01 & 0.03 & 0.08 & 0.07 & 0.18 & 0.58 & 0.79 & 0.47 & 0.18 & 0.12 & 0.36 & 0.51 \\
\hline & E & 0.6 & 0.17 & 0.05 & 0.02 & 0.02 & 0.08 & 0.18 & 0.11 & 0.18 & 0.5 & 0.97 & 0.65 & 0.25 & 0.16 & 0.44 & 0.63 \\
\hline & $\mathrm{F}$ & 0.48 & 0.15 & 0.03 & 0.02 & 0.01 & 0.05 & 0.11 & 0.04 & 0.06 & 0.22 & 0.37 & 0.2 & 0.04 & 0.01 & 0.06 & 0.3 \\
\hline & G & 0.31 & 0.07 & 0 & 0 & 0 & 0.01 & 0.03 & 0.01 & 0.02 & 0.06 & 0.1 & 0.04 & 0.01 & 0 & 0.02 & 0.14 \\
\hline \multirow[t]{7}{*}{9.8} & $\mathrm{~A}$ & 0.04 & 0.05 & 0.01 & 0 & 0 & 0 & 0 & 0 & 0.01 & 0.11 & 0.22 & 0.25 & 0.1 & 0.02 & 0.05 & 0.03 \\
\hline & B & 0.02 & 0.01 & 0 & 0 & 0 & 0 & 0 & 0 & 0.01 & 0.03 & 0.06 & 0.05 & 0.02 & 0.01 & 0.02 & 0.01 \\
\hline & $\mathrm{C}$ & 0.01 & 0.01 & 0 & 0 & 0 & 0 & 0 & 0 & 0 & 0.03 & 0.06 & 0.05 & 0.02 & 0.01 & 0.02 & 0.01 \\
\hline & D & 0.07 & 0.04 & 0.02 & 0.01 & 0 & 0.01 & 0.02 & 0.01 & 0.03 & 0.22 & 0.37 & 0.28 & 0.12 & 0.05 & 0.29 & 0.19 \\
\hline & $\mathrm{E}$ & 0.08 & 0.05 & 0.04 & 0.01 & 0.01 & 0.01 & 0.01 & 0.02 & 0.03 & 0.17 & 0.5 & 0.25 & 0.09 & 0.04 & 0.29 & 0.19 \\
\hline & $\mathrm{F}$ & 0.05 & 0.01 & 0.02 & 0.02 & 0 & 0 & 0 & 0 & 0.01 & 0.05 & 0.14 & 0.06 & 0.01 & 0 & 0.01 & 0.04 \\
\hline & $\mathrm{G}$ & 0.02 & 0.01 & 0 & 0 & 0 & 0 & 0 & 0 & 0 & 0.02 & 0.05 & 0.02 & 0 & 0 & 0 & 0.01 \\
\hline \multirow[t]{7}{*}{12.7} & $\mathrm{~A}$ & 0 & 0.02 & 0.01 & 0 & 0 & 0 & 0 & 0 & 0 & 0.03 & 0.09 & 0.07 & 0.06 & 0 & 0.01 & 0.01 \\
\hline & B & 0 & 0.01 & 0 & 0 & 0 & 0 & 0 & 0 & 0 & 0.01 & 0.04 & 0.03 & 0.02 & 0 & 0.01 & 0 \\
\hline & $\mathrm{C}$ & 0 & 0 & 0 & 0 & 0 & 0 & 0 & 0 & 0 & 0 & 0.04 & 0.03 & 0.01 & 0 & 0.01 & 0.01 \\
\hline & D & 0.01 & 0.02 & 0.01 & 0 & 0 & 0 & 0 & 0 & 0.01 & 0.1 & 0.26 & 0.11 & 0.06 & 0.01 & 0.13 & 0.04 \\
\hline & $\mathrm{E}$ & 0.01 & 0.02 & 0.02 & 0.01 & 0 & 0 & 0 & 0 & 0.01 & 0.07 & 0.24 & 0.06 & 0.02 & 0.02 & 0.09 & 0.02 \\
\hline & $\mathrm{F}$ & 0 & 0 & 0.01 & 0.01 & 0 & 0 & 0 & 0 & 0 & 0.01 & 0.05 & 0.01 & 0 & 0 & 0 & 0 \\
\hline & $\mathrm{G}$ & 0 & 0 & 0 & 0 & 0 & 0 & 0 & 0 & 0 & 0 & 0.03 & 0 & 0 & 0 & 0 & 0 \\
\hline \multirow[t]{7}{*}{15.6} & $\mathrm{~A}$ & 0 & 0 & 0 & 0 & 0 & 0 & 0 & 0 & 0 & 0 & 0.02 & 0.01 & 0.01 & 0 & 0 & 0 \\
\hline & B & 0 & 0 & 0 & 0 & 0 & 0 & 0 & 0 & 0 & 0 & 0.01 & 0 & 0 & 0 & 0.01 & 0 \\
\hline & $\mathrm{C}$ & 0 & 0 & 0 & 0 & 0 & 0 & 0 & 0 & 0 & 0 & 0.02 & 0.01 & 0 & 0 & 0 & 0 \\
\hline & D & 0 & 0.01 & 0 & 0 & 0 & 0 & 0 & 0 & 0 & 0.02 & 0.13 & 0.02 & 0.01 & 0 & 0.01 & 0.01 \\
\hline & $\mathrm{E}$ & 0 & 0.01 & 0.01 & 0 & 0 & 0 & 0 & 0 & 0 & 0.03 & 0.1 & 0.01 & 0 & 0 & 0.01 & 0 \\
\hline & $\mathrm{F}$ & 0 & 0 & 0 & 0 & 0 & 0 & 0 & 0 & 0 & 0 & 0 & 0 & 0 & 0 & 0 & 0 \\
\hline & $\mathrm{G}$ & 0 & 0 & 0 & 0 & 0 & 0 & 0 & 0 & 0 & 0 & 0.01 & 0 & 0 & 0 & 0 & 0 \\
\hline \multirow[t]{7}{*}{19} & $\mathrm{~A}$ & 0 & 0 & 0 & 0 & 0 & 0 & 0 & 0 & 0 & 0 & 0.02 & 0 & 0 & 0 & 0 & 0 \\
\hline & B & 0 & 0 & 0 & 0 & 0 & 0 & 0 & 0 & 0 & 0 & 0 & 0 & 0 & 0 & 0 & 0 \\
\hline & $\mathrm{C}$ & 0 & 0 & 0 & 0 & 0 & 0 & 0 & 0 & 0 & 0 & 0.01 & 0 & 0 & 0 & 0 & 0 \\
\hline & D & 0.01 & 0 & 0 & 0 & 0 & 0 & 0 & 0 & 0 & 0.01 & 0.09 & 0.01 & 0.01 & 0 & 0 & 0 \\
\hline & E & 0 & 0.01 & 0.01 & 0 & 0 & 0 & 0 & 0 & 0 & 0.01 & 0.06 & 0 & 0 & 0 & 0 & 0 \\
\hline & $\mathrm{F}$ & 0 & 0 & 0 & 0 & 0 & 0 & 0 & 0 & 0 & 0 & 0 & 0 & 0 & 0 & 0 & 0 \\
\hline & G & 0 & 0 & 0 & 0 & 0 & 0 & 0 & 0 & 0 & 0 & 0.01 & 0 & 0 & 0 & 0 & 0 \\
\hline
\end{tabular}


Table 4.1-13. Joint Frequency Distributions of Atmospheric Stability, Wind Speed, and Transport Direction for the 400 Area at $9 \mathrm{~m}$ Above Ground Level. Based on 1983-1996 data from the 400 Area instrumented tower.

\begin{tabular}{|c|c|c|c|c|c|c|c|c|c|c|c|c|c|c|c|c|c|}
\hline \multirow{2}{*}{$\begin{array}{c}\text { Average } \\
\text { Wind Speed } \\
\mathbf{m} / \mathbf{s}\end{array}$} & \multirow[t]{2}{*}{$\begin{array}{c}\text { Atmospheric } \\
\text { Stability Class }\end{array}$} & \multicolumn{16}{|c|}{ Percentage of Time Wind Blows from the 400 Area Toward the Direction Indicated } \\
\hline & & $\mathbf{S}$ & SSW & SW & WSW & W & WNW & NW & NNW & $\mathbf{N}$ & NNE & NE & ENE & $\mathbf{E}$ & ESE & SE & SSE \\
\hline \multirow[t]{7}{*}{0.89} & A & 0.1 & 0.12 & 0.1 & 0.12 & 0.12 & 0.15 & 0.12 & 0.09 & 0.11 & 0.09 & 0.08 & 0.06 & 0.05 & 0.06 & 0.07 & 0.1 \\
\hline & B & 0.05 & 0.06 & 0.06 & 0.06 & 0.06 & 0.06 & 0.07 & 0.05 & 0.05 & 0.05 & 0.04 & 0.03 & 0.03 & 0.03 & 0.05 & 0.05 \\
\hline & C & 0.04 & 0.05 & 0.06 & 0.05 & 0.05 & 0.06 & 0.07 & 0.05 & 0.04 & 0.04 & 0.04 & 0.03 & 0.03 & 0.03 & 0.04 & 0.06 \\
\hline & $\mathrm{D}$ & 0.35 & 0.33 & 0.3 & 0.25 & 0.26 & 0.33 & 0.37 & 0.36 & 0.34 & 0.32 & 0.31 & 0.23 & 0.24 & 0.31 & 0.4 & 0.39 \\
\hline & $\mathrm{E}$ & 0.29 & 0.25 & 0.22 & 0.17 & 0.22 & 0.24 & 0.26 & 0.27 & 0.35 & 0.38 & 0.38 & 0.32 & 0.32 & 0.34 & 0.39 & 0.38 \\
\hline & $\mathrm{F}$ & 0.28 & 0.24 & 0.18 & 0.14 & 0.15 & 0.15 & 0.18 & 0.2 & 0.3 & 0.29 & 0.29 & 0.24 & 0.23 & 0.27 & 0.28 & 0.27 \\
\hline & $\mathrm{G}$ & 0.15 & 0.11 & 0.07 & 0.06 & 0.06 & 0.07 & 0.08 & 0.09 & 0.12 & 0.13 & 0.11 & 0.09 & 0.11 & 0.11 & 0.14 & 0.12 \\
\hline \multirow[t]{7}{*}{2.65} & $\mathrm{~A}$ & 0.35 & 0.41 & 0.4 & 0.3 & 0.3 & 0.39 & 0.46 & 0.42 & 0.5 & 0.39 & 0.2 & 0.13 & 0.15 & 0.16 & 0.19 & 0.23 \\
\hline & B & 0.16 & 0.15 & 0.13 & 0.09 & 0.11 & 0.11 & 0.16 & 0.18 & 0.18 & 0.16 & 0.08 & 0.05 & 0.05 & 0.05 & 0.09 & 0.13 \\
\hline & $\mathrm{C}$ & 0.14 & 0.13 & 0.12 & 0.07 & 0.08 & 0.09 & 0.14 & 0.16 & 0.17 & 0.12 & 0.06 & 0.04 & 0.05 & 0.06 & 0.1 & 0.13 \\
\hline & D & 0.67 & 0.59 & 0.54 & 0.33 & 0.32 & 0.37 & 0.73 & 0.99 & 0.87 & 0.74 & 0.4 & 0.26 & 0.33 & 0.54 & 0.97 & 0.91 \\
\hline & $\mathrm{E}$ & 0.6 & 0.49 & 0.36 & 0.2 & 0.17 & 0.25 & 0.62 & 1 & 1.12 & 1.11 & 0.68 & 0.46 & 0.54 & 0.72 & 1.1 & 0.84 \\
\hline & $\mathrm{F}$ & 0.57 & 0.56 & 0.32 & 0.12 & 0.1 & 0.15 & 0.42 & 0.76 & 0.91 & 0.79 & 0.46 & 0.25 & 0.22 & 0.35 & 0.7 & 0.64 \\
\hline & $\mathrm{G}$ & 0.31 & 0.29 & 0.14 & 0.05 & 0.04 & 0.05 & 0.14 & 0.31 & 0.34 & 0.26 & 0.16 & 0.08 & 0.08 & 0.16 & 0.33 & 0.31 \\
\hline \multirow[t]{7}{*}{4.7} & A & 0.35 & 0.39 & 0.21 & 0.07 & 0.07 & 0.07 & 0.13 & 0.18 & 0.53 & 0.68 & 0.29 & 0.18 & 0.17 & 0.17 & 0.24 & 0.23 \\
\hline & B & 0.12 & 0.11 & 0.06 & 0.02 & 0.02 & 0.03 & 0.05 & 0.08 & 0.2 & 0.28 & 0.1 & 0.05 & 0.04 & 0.03 & 0.09 & 0.1 \\
\hline & C & 0.09 & 0.11 & 0.06 & 0.01 & 0.02 & 0.02 & 0.05 & 0.07 & 0.16 & 0.22 & 0.08 & 0.04 & 0.04 & 0.04 & 0.09 & 0.1 \\
\hline & D & 0.35 & 0.31 & 0.22 & 0.08 & 0.05 & 0.08 & 0.28 & 0.54 & 0.86 & 1.14 & 0.44 & 0.21 & 0.25 & 0.56 & 1.08 & 0.7 \\
\hline & $\mathrm{E}$ & 0.22 & 0.2 & 0.1 & 0.03 & 0.02 & 0.03 & 0.29 & 0.9 & 0.98 & 1.13 & 0.55 & 0.25 & 0.31 & 0.8 & 1.54 & 0.68 \\
\hline & $\mathrm{F}$ & 0.17 & 0.17 & 0.07 & 0.02 & 0 & 0.01 & 0.22 & 0.91 & 0.75 & 0.63 & 0.21 & 0.06 & 0.06 & 0.18 & 0.73 & 0.51 \\
\hline & $\mathrm{G}$ & 0.08 & 0.08 & 0.02 & 0 & 0 & 0.01 & 0.1 & 0.46 & 0.29 & 0.2 & 0.06 & 0.01 & 0.01 & 0.05 & 0.32 & 0.22 \\
\hline \multirow[t]{7}{*}{7.15} & $\mathrm{~A}$ & 0.08 & 0.1 & 0.07 & 0.01 & 0 & 0.01 & 0.01 & 0.02 & 0.13 & 0.59 & 0.41 & 0.21 & 0.16 & 0.12 & 0.19 & 0.11 \\
\hline & B & 0.03 & 0.03 & 0.01 & 0 & 0 & 0 & 0.01 & 0.01 & 0.04 & 0.22 & 0.1 & 0.07 & 0.04 & 0.04 & 0.06 & 0.03 \\
\hline & $\mathrm{C}$ & 0.02 & 0.02 & 0.01 & 0 & 0 & 0 & 0.01 & 0.01 & 0.04 & 0.19 & 0.1 & 0.05 & 0.03 & 0.04 & 0.05 & 0.03 \\
\hline & D & 0.09 & 0.09 & 0.04 & 0.01 & 0 & 0.01 & 0.05 & 0.06 & 0.27 & 0.89 & 0.51 & 0.22 & 0.16 & 0.32 & 0.67 & 0.18 \\
\hline & E & 0.03 & 0.06 & 0.03 & 0 & 0 & 0.01 & 0.02 & 0.08 & 0.2 & 0.67 & 0.45 & 0.16 & 0.09 & 0.3 & 0.6 & 0.13 \\
\hline & $\mathrm{F}$ & 0.01 & 0.01 & 0.01 & 0 & 0 & 0 & 0.01 & 0.06 & 0.11 & 0.28 & 0.11 & 0.02 & 0.01 & 0.01 & 0.05 & 0.03 \\
\hline & $\mathrm{G}$ & 0 & 0 & 0 & 0 & 0 & 0 & 0.01 & 0.05 & 0.06 & 0.11 & 0.04 & 0.01 & 0 & 0 & 0.02 & 0.01 \\
\hline \multirow[t]{7}{*}{9.8} & A & 0.01 & 0.03 & 0.02 & 0 & 0 & 0 & 0 & 0 & 0.01 & 0.11 & 0.18 & 0.13 & 0.07 & 0.04 & 0.06 & 0.01 \\
\hline & B & 0 & 0.01 & 0 & 0 & 0 & 0 & 0 & 0 & 0.01 & 0.03 & 0.05 & 0.03 & 0.02 & 0.01 & 0.03 & 0 \\
\hline & $\mathrm{C}$ & 0 & 0 & 0 & 0 & 0 & 0 & 0 & 0 & 0 & 0.02 & 0.04 & 0.02 & 0.01 & 0.01 & 0.03 & 0.01 \\
\hline & D & 0.01 & 0.03 & 0.01 & 0.01 & 0 & 0 & 0 & 0 & 0.02 & 0.19 & 0.27 & 0.12 & 0.05 & 0.1 & 0.26 & 0.02 \\
\hline & $\mathrm{E}$ & 0 & 0.04 & 0.01 & 0 & 0 & 0 & 0 & 0 & 0.01 & 0.16 & 0.21 & 0.05 & 0.02 & 0.05 & 0.11 & 0.01 \\
\hline & $\mathrm{F}$ & 0 & 0 & 0 & 0 & 0 & 0 & 0 & 0 & 0 & 0.02 & 0.03 & 0 & 0 & 0 & 0 & 0 \\
\hline & $\mathrm{G}$ & 0 & 0 & 0 & 0 & 0 & 0 & 0 & 0 & 0.01 & 0.02 & 0.02 & 0 & 0 & 0 & 0 & 0 \\
\hline \multirow[t]{7}{*}{12.7} & $\mathrm{~A}$ & 0 & 0 & 0 & 0 & 0 & 0 & 0 & 0 & 0 & 0.01 & 0.05 & 0.03 & 0.01 & 0.01 & 0.01 & 0 \\
\hline & B & 0 & 0 & 0 & 0 & 0 & 0 & 0 & 0 & 0 & 0.01 & 0.02 & 0 & 0 & 0 & 0 & 0 \\
\hline & $\mathrm{C}$ & 0 & 0 & 0 & 0 & 0 & 0 & 0 & 0 & 0 & 0 & 0.02 & 0.01 & 0 & 0 & 0.01 & 0 \\
\hline & D & 0 & 0.01 & 0 & 0 & 0 & 0 & 0 & 0 & 0 & 0.05 & 0.17 & 0.04 & 0.01 & 0.01 & 0.04 & 0 \\
\hline & $\mathrm{E}$ & 0 & 0.01 & 0.01 & 0 & 0 & 0 & 0 & 0 & 0 & 0.05 & 0.07 & 0.01 & 0 & 0.01 & 0.02 & 0 \\
\hline & $\mathrm{F}$ & 0 & 0 & 0 & 0 & 0 & 0 & 0 & 0 & 0 & 0 & 0.01 & 0 & 0 & 0 & 0 & 0 \\
\hline & $\mathrm{G}$ & 0 & 0 & 0 & 0 & 0 & 0 & 0 & 0 & 0 & 0 & 0 & 0 & 0 & 0 & 0 & 0 \\
\hline \multirow[t]{7}{*}{15.6} & $\mathrm{~A}$ & 0 & 0 & 0 & 0 & 0 & 0 & 0 & 0 & 0 & 0 & 0.02 & 0 & 0 & 0 & 0 & 0 \\
\hline & B & 0 & 0 & 0 & 0 & 0 & 0 & 0 & 0 & 0 & 0 & 0 & 0 & 0 & 0 & 0 & 0 \\
\hline & $\mathrm{C}$ & 0 & 0 & 0 & 0 & 0 & 0 & 0 & 0 & 0 & 0 & 0.01 & 0 & 0 & 0 & 0 & 0 \\
\hline & D & 0 & 0 & 0 & 0 & 0 & 0 & 0 & 0 & 0 & 0.01 & 0.04 & 0.01 & 0.01 & 0 & 0 & 0 \\
\hline & $\mathrm{E}$ & 0 & 0 & 0.01 & 0 & 0 & 0 & 0 & 0 & 0 & 0.01 & 0.02 & 0 & 0 & 0 & 0 & 0 \\
\hline & $\mathrm{F}$ & 0 & 0 & 0 & 0 & 0 & 0 & 0 & 0 & 0 & 0 & 0 & 0 & 0 & 0 & 0 & 0 \\
\hline & $\mathrm{G}$ & 0 & 0 & 0 & 0 & 0 & 0 & 0 & 0 & 0 & 0 & 0 & 0 & 0 & 0 & 0 & 0 \\
\hline \multirow[t]{7}{*}{19} & $\mathrm{~A}$ & 0 & 0 & 0 & 0 & 0 & 0 & 0 & 0 & 0 & 0 & 0 & 0 & 0 & 0 & 0 & 0 \\
\hline & B & 0 & 0 & 0 & 0 & 0 & 0 & 0 & 0 & 0 & 0 & 0 & 0 & 0 & 0 & 0 & 0 \\
\hline & $\mathrm{C}$ & 0 & 0 & 0 & 0 & 0 & 0 & 0 & 0 & 0 & 0 & 0 & 0 & 0 & 0 & 0 & 0 \\
\hline & D & 0 & 0 & 0 & 0 & 0 & 0 & 0 & 0 & 0 & 0 & 0.01 & 0 & 0 & 0 & 0 & 0 \\
\hline & $\mathrm{E}$ & 0 & 0 & 0 & 0 & 0 & 0 & 0 & 0 & 0 & 0 & 0.01 & 0 & 0 & 0 & 0 & 0 \\
\hline & $\mathrm{F}$ & 0 & 0 & 0 & 0 & 0 & 0 & 0 & 0 & 0 & 0 & 0 & 0 & 0 & 0 & 0 & 0 \\
\hline & $\mathrm{G}$ & 0 & 0 & 0 & 0 & 0 & 0 & 0 & 0 & 0 & 0 & 0 & 0 & 0 & 0 & 0 & 0 \\
\hline
\end{tabular}


Table 4.1-14. Joint Frequency Distributions of Atmospheric Stability, Wind Speed, and Transport Direction for the 400 Area at $60 \mathrm{~m}$ Above Ground Level. Based on 1983-1996 data from the 400 Area instrumented tower.

\begin{tabular}{|c|c|c|c|c|c|c|c|c|c|c|c|c|c|c|c|c|c|}
\hline \multirow{2}{*}{$\begin{array}{c}\text { Average } \\
\text { Wind Speed } \\
\mathbf{m} / \mathbf{s}\end{array}$} & \multirow{2}{*}{$\begin{array}{c}\text { Atmospheric } \\
\text { Stability Class }\end{array}$} & \multicolumn{16}{|c|}{ Percentage of Time Wind Blows from the 400 Area Toward the Direction Indicated } \\
\hline & & $\mathbf{S}$ & SSW & SW & WSW & W & WNW & NW & NNW & $\mathbf{N}$ & NNE & NE & ENE & $\mathbf{E}$ & ESE & SE & SSE \\
\hline \multirow[t]{7}{*}{0.89} & A & 0.08 & 0.09 & 0.1 & 0.09 & 0.1 & 0.12 & 0.1 & 0.09 & 0.09 & 0.07 & 0.06 & 0.05 & 0.06 & 0.04 & 0.07 & 0.07 \\
\hline & B & 0.05 & 0.04 & 0.06 & 0.05 & 0.06 & 0.05 & 0.05 & 0.05 & 0.05 & 0.03 & 0.04 & 0.03 & 0.03 & 0.04 & 0.05 & 0.04 \\
\hline & $\mathrm{C}$ & 0.04 & 0.03 & 0.05 & 0.04 & 0.05 & 0.06 & 0.05 & 0.04 & 0.03 & 0.04 & 0.03 & 0.02 & 0.02 & 0.03 & 0.04 & 0.05 \\
\hline & D & 0.22 & 0.21 & 0.19 & 0.19 & 0.23 & 0.23 & 0.31 & 0.3 & 0.25 & 0.21 & 0.22 & 0.17 & 0.18 & 0.19 & 0.22 & 0.23 \\
\hline & E & 0.18 & 0.17 & 0.14 & 0.13 & 0.15 & 0.16 & 0.22 & 0.22 & 0.25 & 0.21 & 0.22 & 0.21 & 0.19 & 0.17 & 0.18 & 0.2 \\
\hline & $\mathrm{F}$ & 0.15 & 0.14 & 0.13 & 0.13 & 0.13 & 0.15 & 0.19 & 0.2 & 0.22 & 0.19 & 0.18 & 0.15 & 0.15 & 0.15 & 0.13 & 0.16 \\
\hline & G & 0.07 & 0.06 & 0.06 & 0.06 & 0.06 & 0.06 & 0.07 & 0.09 & 0.09 & 0.06 & 0.06 & 0.05 & 0.07 & 0.05 & 0.06 & 0.06 \\
\hline \multirow[t]{7}{*}{2.65} & A & 0.27 & 0.31 & 0.32 & 0.23 & 0.24 & 0.29 & 0.36 & 0.33 & 0.35 & 0.28 & 0.17 & 0.11 & 0.11 & 0.13 & 0.15 & 0.2 \\
\hline & B & 0.12 & 0.13 & 0.11 & 0.08 & 0.09 & 0.1 & 0.15 & 0.16 & 0.16 & 0.15 & 0.07 & 0.05 & 0.05 & 0.05 & 0.1 & 0.12 \\
\hline & $\mathrm{C}$ & 0.11 & 0.11 & 0.12 & 0.07 & 0.07 & 0.09 & 0.12 & 0.14 & 0.16 & 0.11 & 0.06 & 0.05 & 0.05 & 0.06 & 0.08 & 0.11 \\
\hline & D & 0.5 & 0.51 & 0.48 & 0.34 & 0.31 & 0.37 & 0.57 & 0.74 & 0.7 & 0.54 & 0.3 & 0.24 & 0.24 & 0.36 & 0.65 & 0.61 \\
\hline & E & 0.41 & 0.35 & 0.29 & 0.2 & 0.21 & 0.2 & 0.36 & 0.54 & 0.65 & 0.54 & 0.48 & 0.4 & 0.43 & 0.47 & 0.57 & 0.52 \\
\hline & $\mathrm{F}$ & 0.4 & 0.39 & 0.26 & 0.13 & 0.1 & 0.16 & 0.28 & 0.57 & 0.62 & 0.47 & 0.36 & 0.26 & 0.28 & 0.27 & 0.37 & 0.41 \\
\hline & $\mathrm{G}$ & 0.2 & 0.18 & 0.11 & 0.05 & 0.03 & 0.05 & 0.13 & 0.28 & 0.29 & 0.19 & 0.12 & 0.08 & 0.09 & 0.09 & 0.15 & 0.18 \\
\hline \multirow[t]{7}{*}{4.7} & $\mathrm{~A}$ & 0.34 & 0.4 & 0.25 & 0.09 & 0.06 & 0.06 & 0.14 & 0.2 & 0.47 & 0.61 & 0.23 & 0.14 & 0.12 & 0.17 & 0.2 & 0.21 \\
\hline & B & 0.13 & 0.14 & 0.09 & 0.03 & 0.02 & 0.02 & 0.07 & 0.09 & 0.2 & 0.23 & 0.11 & 0.05 & 0.03 & 0.04 & 0.08 & 0.1 \\
\hline & $\mathrm{C}$ & 0.09 & 0.12 & 0.09 & 0.02 & 0.02 & 0.03 & 0.06 & 0.09 & 0.17 & 0.21 & 0.07 & 0.03 & 0.03 & 0.04 & 0.08 & 0.1 \\
\hline & $\mathrm{D}$ & 0.42 & 0.44 & 0.36 & 0.13 & 0.1 & 0.1 & 0.33 & 0.46 & 0.73 & 0.87 & 0.43 & 0.16 & 0.21 & 0.33 & 0.85 & 0.65 \\
\hline & $\mathrm{E}$ & 0.34 & 0.3 & 0.21 & 0.09 & 0.06 & 0.07 & 0.35 & 0.61 & 0.79 & 0.8 & 0.68 & 0.33 & 0.35 & 0.61 & 1.04 & 0.63 \\
\hline & $\mathrm{F}$ & 0.3 & 0.25 & 0.14 & 0.05 & 0.03 & 0.03 & 0.22 & 0.54 & 0.64 & 0.6 & 0.44 & 0.13 & 0.11 & 0.19 & 0.54 & 0.55 \\
\hline & $\mathrm{G}$ & 0.18 & 0.15 & 0.07 & 0.01 & 0 & 0.01 & 0.1 & 0.29 & 0.27 & 0.22 & 0.13 & 0.03 & 0.03 & 0.06 & 0.24 & 0.33 \\
\hline \multirow[t]{7}{*}{7.15} & $\mathrm{~A}$ & 0.14 & 0.16 & 0.09 & 0.01 & 0 & 0.01 & 0.03 & 0.03 & 0.13 & 0.59 & 0.39 & 0.17 & 0.13 & 0.11 & 0.19 & 0.13 \\
\hline & B & 0.04 & 0.04 & 0.02 & 0.01 & 0 & 0 & 0.01 & 0.01 & 0.06 & 0.23 & 0.11 & 0.05 & 0.03 & 0.04 & 0.08 & 0.07 \\
\hline & $\mathrm{C}$ & 0.04 & 0.04 & 0.02 & 0 & 0 & 0 & 0.02 & 0.02 & 0.06 & 0.19 & 0.09 & 0.04 & 0.03 & 0.04 & 0.06 & 0.04 \\
\hline & D & 0.14 & 0.15 & 0.06 & 0.02 & 0.01 & 0.01 & 0.12 & 0.17 & 0.39 & 0.95 & 0.52 & 0.21 & 0.15 & 0.34 & 0.88 & 0.37 \\
\hline & E & 0.14 & 0.11 & 0.07 & 0.01 & 0.01 & 0.01 & 0.17 & 0.28 & 0.44 & 1 & 0.79 & 0.23 & 0.19 & 0.74 & 1.52 & 0.48 \\
\hline & $\mathrm{F}$ & 0.13 & 0.1 & 0.06 & 0.02 & 0.01 & 0 & 0.11 & 0.21 & 0.33 & 0.56 & 0.39 & 0.05 & 0.03 & 0.15 & 0.67 & 0.43 \\
\hline & $\mathrm{G}$ & 0.05 & 0.03 & 0.01 & 0 & 0 & 0 & 0.03 & 0.07 & 0.15 & 0.2 & 0.11 & 0.02 & 0.01 & 0.05 & 0.27 & 0.21 \\
\hline \multirow[t]{7}{*}{9.8} & $\bar{A}$ & 0.02 & 0.06 & 0.04 & 0.01 & 0 & 0 & 0 & 0 & 0.02 & 0.16 & 0.25 & 0.16 & 0.08 & 0.06 & 0.1 & 0.04 \\
\hline & B & 0.01 & 0.02 & 0.01 & 0 & 0 & 0 & 0.01 & 0.01 & 0.01 & 0.06 & 0.06 & 0.05 & 0.02 & 0.01 & 0.04 & 0.02 \\
\hline & $\mathrm{C}$ & 0.01 & 0.01 & 0.01 & 0.01 & 0 & 0 & 0 & 0 & 0.01 & 0.06 & 0.05 & 0.03 & 0.02 & 0.02 & 0.04 & 0.02 \\
\hline & D & 0.03 & 0.05 & 0.03 & 0.01 & 0.01 & 0.01 & 0.03 & 0.03 & 0.1 & 0.36 & 0.39 & 0.17 & 0.1 & 0.22 & 0.65 & 0.1 \\
\hline & E & 0.04 & 0.06 & 0.05 & 0.01 & 0.01 & 0.01 & 0.02 & 0.04 & 0.11 & 0.44 & 0.46 & 0.15 & 0.08 & 0.5 & 1.06 & 0.11 \\
\hline & $\mathrm{F}$ & 0.02 & 0.02 & 0.03 & 0.01 & 0.01 & 0.01 & 0.01 & 0.02 & 0.04 & 0.19 & 0.16 & 0.02 & 0.01 & 0.06 & 0.25 & 0.08 \\
\hline & G & 0 & 0 & 0 & 0 & 0 & 0 & 0 & 0.01 & 0.02 & 0.1 & 0.06 & 0.01 & 0 & 0.03 & 0.11 & 0.03 \\
\hline \multirow[t]{7}{*}{12.7} & $\mathrm{~A}$ & 0 & 0.02 & 0.02 & 0 & 0 & 0 & 0 & 0 & 0 & 0.04 & 0.08 & 0.09 & 0.04 & 0.01 & 0.04 & 0 \\
\hline & B & 0 & 0.02 & 0 & 0 & 0 & 0 & 0 & 0 & 0 & 0.02 & 0.04 & 0.02 & 0.01 & 0.01 & 0.02 & 0 \\
\hline & $\mathrm{C}$ & 0 & 0 & 0 & 0 & 0 & 0 & 0 & 0 & 0 & 0.02 & 0.03 & 0.02 & 0.01 & 0 & 0.02 & 0 \\
\hline & D & 0.01 & 0.02 & 0.01 & 0.01 & 0 & 0 & 0.01 & 0.01 & 0.01 & 0.12 & 0.26 & 0.09 & 0.04 & 0.09 & 0.32 & 0.05 \\
\hline & $\mathrm{E}$ & 0.01 & 0.03 & 0.01 & 0.01 & 0 & 0 & 0.01 & 0.02 & 0.02 & 0.16 & 0.27 & 0.08 & 0.02 & 0.08 & 0.28 & 0.03 \\
\hline & $\mathrm{F}$ & 0 & 0.01 & 0 & 0 & 0 & 0 & 0 & 0.01 & 0.01 & 0.03 & 0.06 & 0.01 & 0.01 & 0 & 0.01 & 0.01 \\
\hline & $\mathrm{G}$ & 0 & 0 & 0 & 0 & 0 & 0 & 0 & 0 & 0.01 & 0.02 & 0.02 & 0 & 0 & 0 & 0 & 0 \\
\hline \multirow[t]{7}{*}{15.6} & A & 0 & 0 & 0 & 0 & 0 & 0 & 0 & 0 & 0 & 0.01 & 0.02 & 0.02 & 0 & 0 & 0.01 & 0 \\
\hline & B & 0 & 0 & 0 & 0 & 0 & 0 & 0 & 0 & 0 & 0 & 0.01 & 0.01 & 0 & 0 & 0.01 & 0 \\
\hline & $\mathrm{C}$ & 0 & 0 & 0 & 0 & 0 & 0 & 0 & 0 & 0 & 0.01 & 0.02 & 0.01 & 0 & 0 & 0 & 0 \\
\hline & $\mathrm{D}$ & 0 & 0 & 0.01 & 0 & 0 & 0 & 0 & 0 & 0 & 0.06 & 0.14 & 0.04 & 0.01 & 0.01 & 0.04 & 0.01 \\
\hline & E & 0 & 0.01 & 0.01 & 0 & 0 & 0 & 0 & 0 & 0.01 & 0.06 & 0.1 & 0.03 & 0 & 0.01 & 0.05 & 0.02 \\
\hline & $\mathrm{F}$ & 0 & 0 & 0 & 0 & 0 & 0 & 0 & 0 & 0 & 0.01 & 0.01 & 0 & 0 & 0 & 0 & 0 \\
\hline & $\mathrm{G}$ & 0 & 0 & 0 & 0 & 0 & 0 & 0 & 0 & 0 & 0.01 & 0 & 0 & 0 & 0 & 0 & 0 \\
\hline \multirow[t]{7}{*}{19} & $\mathrm{~A}$ & 0 & 0 & 0 & 0 & 0 & 0 & 0 & 0 & 0 & 0.01 & 0.02 & 0 & 0 & 0 & 0 & 0 \\
\hline & B & 0 & 0 & 0 & 0 & 0 & 0 & 0 & 0 & 0 & 0 & 0.01 & 0 & 0 & 0 & 0 & 0 \\
\hline & $\mathrm{C}$ & 0 & 0 & 0 & 0 & 0 & 0 & 0 & 0 & 0 & 0.01 & 0.01 & 0 & 0 & 0 & 0 & 0 \\
\hline & D & 0 & 0 & 0 & 0 & 0 & 0 & 0 & 0 & 0 & 0.02 & 0.09 & 0.01 & 0.01 & 0 & 0 & 0 \\
\hline & E & 0 & 0 & 0.01 & 0 & 0 & 0 & 0 & 0 & 0 & 0.04 & 0.05 & 0.01 & 0 & 0 & 0.01 & 0 \\
\hline & $\mathrm{F}$ & 0 & 0 & 0 & 0 & 0 & 0 & 0 & 0 & 0 & 0 & 0 & 0 & 0 & 0 & 0 & 0 \\
\hline & G & 0 & 0 & 0 & 0 & 0 & 0 & 0 & 0 & 0 & 0 & 0 & 0 & 0 & 0 & 0 & 0 \\
\hline
\end{tabular}


Table 4.1-15. $\bar{\chi} / \mathrm{Q}^{\prime}$ Values $\left(\mathrm{sec} \mathrm{m}^{-3}\right)$ for Chronic Ground-Level Releases from 100-N Area Based on 1983 through 1991 Meteorological Information (Schreckhise et al. 1993)

\begin{tabular}{|c|c|c|c|c|c|c|c|c|c|c|c|c|c|c|c|c|c|}
\hline Distance & & & & & & & or (Wind & rom 100-1 & Toward D & rection In & icated) & & & Distance & & & \\
\hline (km) & $\mathrm{S}$ & SSW & SW & WSW & W & WNW & NW & NNW & $\mathrm{N}$ & NNE & $\mathrm{NE}$ & ENE & $\mathrm{E}$ & ESE & SE & SSE & $(\mathrm{km})$ \\
\hline 0.1 & $2 . \overline{\mathrm{BE}-04}$ & $1.5 \mathrm{E}-04$ & $1 \overline{6 \mathrm{E}-04}$ & $1.9 \mathrm{E}-04$ & $3 . \overline{6 \mathrm{E}-04}$ & $2.3 \mathrm{E}-04$ & $1 \overline{9 \mathrm{E}-04}$ & $1.3 \mathrm{E}-04$ & $1 . \overline{7 \mathrm{E}-04}$ & $1.4 \mathrm{E}-04$ & $2.0 \mathrm{E}-04$ & $3.1 \mathrm{E}-04$ & $5 . \overline{1 \mathrm{E}-04}$ & $3.2 \mathrm{E}-04$ & $2 . \overline{4 \mathrm{E}-04}$ & $2.0 \mathrm{E}-04$ & $\frac{11}{0.1}$ \\
\hline 0.2 & $6.3 \mathrm{E}-05$ & $4.1 \mathrm{E}-05$ & 4.3E-05 & $5.2 \mathrm{E}-05$ & $1.0 \mathrm{E}-04$ & 6.4E-05 & $5.2 \mathrm{E}-05$ & $3.7 \mathrm{E}-05$ & $4.7 \mathrm{E}-05$ & $3.8 \mathrm{E}-05$ & $5.5 \mathrm{E}-05$ & $8.6 \mathrm{E}-05$ & 1.4E-04 & $8.7 \mathrm{E}-05$ & $6.5 \mathrm{E}-05$ & $5.5 \mathrm{E}-05$ & 0.2 \\
\hline 0.3 & $3.0 \mathrm{E}-05$ & $1.9 \mathrm{E}-05$ & $2.1 \mathrm{E}-05$ & $2.5 \mathrm{E}-05$ & $4.8 \mathrm{E}-05$ & $3.1 \mathrm{E}-05$ & $2.5 \mathrm{E}-05$ & $1.8 \mathrm{E}-05$ & $2.2 \mathrm{E}-05$ & $1.8 \mathrm{E}-05$ & $2.6 \mathrm{E}-05$ & 4.1E-05 & $6.8 \mathrm{E}-05$ & 4.1E-05 & $3.1 \mathrm{E}-05$ & 2.6E-05 & 0.3 \\
\hline 0.4 & $1.8 \mathrm{E}-05$ & $1.2 \mathrm{E}-05$ & $1.2 \mathrm{E}-05$ & $1.5 \mathrm{E}-05$ & $2.9 \mathrm{E}-05$ & $1.8 \mathrm{E}-05$ & $1.5 \mathrm{E}-05$ & $1.0 \mathrm{E}-05$ & 1.3E-05 & $1.1 \mathrm{E}-05$ & $1.6 \mathrm{E}-05$ & $2.5 \mathrm{E}-05$ & 4.0E-05 & $2.5 \mathrm{E}-05$ & $1.8 \mathrm{E}-05$ & 1.6E-05 & 0.4 \\
\hline 0.5 & $1.2 \mathrm{E}-05$ & 7.7E-06 & $8.2 \mathrm{E}-06$ & $1.0 \mathrm{E}-05$ & $1.9 \mathrm{E}-05$ & $1.2 \mathrm{E}-05$ & $1.0 \mathrm{E}-05$ & 7.0E-06 & 8.9E-06 & 7.2E-06 & $1.1 \mathrm{E}-05$ & $1.7 \mathrm{E}-05$ & $2.7 \mathrm{E}-05$ & $1.7 \mathrm{E}-05$ & $1.2 \mathrm{E}-05$ & $1.1 \mathrm{E}-05$ & 0.5 \\
\hline 0.6 & 8.6E-06 & $5.6 \mathrm{E}-06$ & $5.9 \mathrm{E}-06$ & 7.3E-06 & $1.4 \mathrm{E}-05$ & $8.8 \mathrm{E}-06$ & 7.2E-06 & $5.1 \mathrm{E}-06$ & 6.4E-06 & $5.2 \mathrm{E}-06$ & 7.6E-06 & $1.2 \mathrm{E}-05$ & $2.0 \mathrm{E}-05$ & $1.2 \mathrm{E}-05$ & 8.9E-06 & 7.6E-06 & 0.6 \\
\hline 0.7 & $6.5 \mathrm{E}-06$ & $4.2 \mathrm{E}-06$ & $4.5 \mathrm{E}-06$ & $5.5 \mathrm{E}-06$ & $1.1 \mathrm{E}-05$ & $6.7 \mathrm{E}-06$ & $5.5 \mathrm{E}-06$ & $3.8 \mathrm{E}-06$ & $4.9 \mathrm{E}-06$ & $4.0 \mathrm{E}-06$ & $5.8 \mathrm{E}-06$ & $9.1 \mathrm{E}-06$ & $1.5 \mathrm{E}-05$ & $9.1 \mathrm{E}-06$ & $6.8 \mathrm{E}-06$ & $5.8 \mathrm{E}-06$ & 0.7 \\
\hline 0.8 & $5.1 \mathrm{E}-06$ & 3.3E-06 & $3.5 \mathrm{E}-06$ & 4.4E-06 & $8.4 \mathrm{E}-06$ & $5.3 \mathrm{E}-06$ & $4.3 \mathrm{E}-06$ & $3.0 \mathrm{E}-06$ & 3.9E-06 & $3.1 \mathrm{E}-06$ & $4.5 \mathrm{E}-06$ & $7.2 \mathrm{E}-06$ & $1.2 \mathrm{E}-05$ & 7.2E-06 & $5.3 \mathrm{E}-06$ & $4.5 \mathrm{E}-06$ & 0.8 \\
\hline 0.9 & 4.2E-06 & $2.7 \mathrm{E}-06$ & $2.9 \mathrm{E}-06$ & $3.5 \mathrm{E}-06$ & $6.8 \mathrm{E}-06$ & 4.3E-06 & $3.5 \mathrm{E}-06$ & $2.5 \mathrm{E}-06$ & $3.1 \mathrm{E}-06$ & $2.5 \mathrm{E}-06$ & 3.7E-06 & $5.8 \mathrm{E}-06$ & $9.5 \mathrm{E}-06$ & $5.8 \mathrm{E}-06$ & 4.3E-06 & $3.7 \mathrm{E}-06$ & 0.9 \\
\hline 1.0 & $3.5 \mathrm{E}-06$ & $2.2 \mathrm{E}-06$ & $2.4 \mathrm{E}-06$ & $2.9 \mathrm{E}-06$ & 5.6E-06 & $3.6 \mathrm{E}-06$ & $2.9 \mathrm{E}-06$ & 2.1E-06 & 2.6E-06 & 2.1E-06 & $3.1 \mathrm{E}-06$ & $4.8 \mathrm{E}-06$ & $7.9 \mathrm{E}-06$ & 4.8E-06 & 3.6E-06 & $3.1 \mathrm{E}-06$ & 1.0 \\
\hline 2.4 & $8.0 \mathrm{E}-07$ & $5.2 \mathrm{E}-07$ & $5.5 \mathrm{E}-07$ & $6.8 \mathrm{E}-07$ & 1.3E-06 & $8.3 \mathrm{E}-07$ & $6.7 \mathrm{E}-07$ & 4.7E-07 & $6.0 \mathrm{E}-07$ & $4.9 \mathrm{E}-07$ & $7.1 \mathrm{E}-07$ & $1.1 \mathrm{E}-06$ & 1.8E-06 & $1.1 \mathrm{E}-06$ & 8.3E-07 & $7.1 \mathrm{E}-07$ & 2.4 \\
\hline 4.0 & 3.6E-07 & $2.4 \mathrm{E}-07$ & $2.5 \mathrm{E}-07$ & $3.1 \mathrm{E}-07$ & $6.0 \mathrm{E}-07$ & $3.8 \mathrm{E}-07$ & $3.1 \mathrm{E}-07$ & 2.1E-07 & 2.7E-07 & 2.2E-07 & $3.2 \mathrm{E}-07$ & $5.2 \mathrm{E}-07$ & 8.4E-07 & $5.1 \mathrm{E}-07$ & $3.8 \mathrm{E}-07$ & 3.2E-07 & 4.0 \\
\hline 5.6 & $2.2 \mathrm{E}-07$ & $1.4 \mathrm{E}-07$ & $1.5 \mathrm{E}-07$ & $1.9 \mathrm{E}-07$ & 3.6E-07 & $2.3 \mathrm{E}-07$ & $1.9 \mathrm{E}-07$ & 1.3E-07 & $1.7 \mathrm{E}-07$ & 1.3E-07 & $2.0 \mathrm{E}-07$ & $3.1 \mathrm{E}-07$ & $5.1 \mathrm{E}-07$ & $3.1 \mathrm{E}-07$ & 2.3E-07 & $2.0 \mathrm{E}-07$ & 5.6 \\
\hline 7.2 & $1.5 \mathrm{E}-07$ & $9.9 \mathrm{E}-08$ & $1.1 \mathrm{E}-07$ & 1.3E-07 & $2.5 \mathrm{E}-07$ & $1.6 \mathrm{E}-07$ & 1.3E-07 & $9.0 \mathrm{E}-08$ & $1.1 \mathrm{E}-07$ & 9.3E-08 & 1.4E-07 & 2.2E-07 & 3.6E-07 & $2.2 \mathrm{E}-07$ & 1.6E-07 & 1.4E-07 & 7.2 \\
\hline 12.1 & 7.4E-08 & $4.8 \mathrm{E}-08$ & $5.2 \mathrm{E}-08$ & $6.4 \mathrm{E}-08$ & $1.2 \mathrm{E}-07$ & 7.6E-08 & $6.2 \mathrm{E}-08$ & 4.3E-08 & $5.5 \mathrm{E}-08$ & $4.5 \mathrm{E}-08$ & 6.6E-08 & $1.1 \mathrm{E}-07$ & $1.7 \mathrm{E}-07$ & $1.1 \mathrm{E}-07$ & $7.8 \mathrm{E}-08$ & $6.7 \mathrm{E}-08$ & 12.1 \\
\hline 24.1 & $2.9 \mathrm{E}-08$ & $1.8 \mathrm{E}-08$ & $2.0 \mathrm{E}-08$ & $2.5 \mathrm{E}-08$ & 4.7E-08 & $2.9 \mathrm{E}-08$ & 2.4E- 08 & $1.6 \mathrm{E}-08$ & 2.1E-08 & $1.7 \mathrm{E}-08$ & $2.5 \mathrm{E}-08$ & 4.1E-08 & $6.7 \mathrm{E}-08$ & 4.1E-08 & 3.0E-08 & 2.6E-08 & 24.1 \\
\hline 40.3 & 1.4E-08 & $9.3 \mathrm{E}-09$ & $1.0 \mathrm{E}-08$ & 1.3E-08 & 2.4E-08 & $1.4 \mathrm{E}-08$ & $1.2 \mathrm{E}-08$ & $8.1 \mathrm{E}-09$ & 1.0E-08 & $8.5 \mathrm{E}-09$ & 1.3E-08 & $2.0 \mathrm{E}-08$ & 3.4E-08 & 2.0E-08 & $1.5 \mathrm{E}-08$ & $1.3 \mathrm{E}-08$ & 40.3 \\
\hline 56.3 & $9.2 \mathrm{E}-09$ & $5.9 \mathrm{E}-09$ & $6.4 \mathrm{E}-09$ & $8.0 \mathrm{E}-09$ & $1.5 \mathrm{E}-08$ & $9.2 \mathrm{E}-09$ & $7.5 \mathrm{E}-09$ & $5.2 \mathrm{E}-09$ & 6.6E-09 & $5.4 \mathrm{E}-09$ & $8.0 \mathrm{E}-09$ & 1.3E-08 & $2.2 \mathrm{E}-08$ & $1.3 \mathrm{E}-08$ & $9.7 \mathrm{E}-09$ & 8.3E-09 & 56.3 \\
\hline 72.4 & 6.6E-09 & 4.3E-09 & 4.6E-09 & $5.8 \mathrm{E}-09$ & $1.1 \mathrm{E}-08$ & 6.6E-09 & 5.3E-09 & $3.7 \mathrm{E}-09$ & 4.7E-09 & $3.9 \mathrm{E}-09$ & $5.8 \mathrm{E}-09$ & $9.4 \mathrm{E}-09$ & $1.5 \mathrm{E}-08$ & $9.4 \mathrm{E}-09$ & $7.0 \mathrm{E}-09$ & $6.0 \mathrm{E}-09$ & 72.4 \\
\hline
\end{tabular}

Table 4.1-16. $\bar{\chi} / \mathrm{Q}^{\prime}$ Values $\left(\mathrm{sec} \mathrm{m}^{-3}\right)$ for Chronic 61-m Stack Releases from 100-N Area Based on 1983 through 1991 Meteorological Information (Schreckhise et al. 1993)

\begin{tabular}{|c|c|c|c|c|c|c|c|c|c|c|c|c|c|c|c|c|c|}
\hline Distance & & & & & & & or (Wind & com $100-\mathrm{N}$ & Toward D & ection Inc & ated) & & & & nce & & \\
\hline$(\mathrm{km})$ & $\mathrm{S}$ & SSW & SW & WSW & W & WNW & NW & NNW & $\mathrm{N}$ & NNE & NE & ENE & E & ESE & SE & SSE & $(\mathrm{km})$ \\
\hline 0.1 & $1 . \overline{4 \mathrm{E}-09}$ & $7.9 \mathrm{E}-10$ & $6 . \overline{5 \mathrm{E}-10}$ & $4.3 \mathrm{E}-10$ & $9 . \overline{4 \mathrm{E}-10}$ & $6.8 \mathrm{E}-10$ & $5 \overline{.6 \mathrm{E}-10}$ & $3.8 \mathrm{E}-10$ & $5 . \overline{5 \mathrm{E}-10}$ & $4.0 \mathrm{E}-10$ & $7 . \overline{0 \mathrm{E}-10}$ & $9.4 \mathrm{E}-10$ & $1 . \overline{7 \mathrm{E}-09}$ & $1 . \overline{4 \mathrm{E}-09}$ & $1 . \overline{3 \mathrm{E}-09}$ & $1 . \overline{1 \mathrm{E}-09}$ & 0.1 \\
\hline 0.2 & $2.6 \mathrm{E}-07$ & $1.5 \mathrm{E}-07$ & $1.3 \mathrm{E}-07$ & $8.3 \mathrm{E}-08$ & $1.8 \mathrm{E}-07$ & $1.4 \mathrm{E}-07$ & $1.1 \mathrm{E}-07$ & $7.8 \mathrm{E}-08$ & $1.1 \mathrm{E}-07$ & 7.8E-08 & $1.4 \mathrm{E}-07$ & $1.8 \mathrm{E}-07$ & 3.3E-07 & 2.7E-07 & $2.4 \mathrm{E}-07$ & $2.1 \mathrm{E}-07$ & 0.2 \\
\hline 0.3 & $9 \mathrm{E}-07$ & $9 \mathrm{E}-07$ & $5 \mathrm{E}-07$ & 7 & $3.6 \mathrm{E}-07$ & $.7 \mathrm{E}-07$ & 2.3E-07 & $1.6 \mathrm{E}-07$ & 2.1E-07 & & 2.7E-07 & $5 \mathrm{E}-07$ & $6.2 \mathrm{E}-07$ & $.0 \mathrm{E}-07$ & 4.5E-07 & $.9 \mathrm{E}-07$ & 0.3 \\
\hline 0.4 & IE-07 & $5 \mathrm{E}-07$ & $1 \mathrm{E}-07$ & $5 \mathrm{E}-07$ & $3.2 \mathrm{E}-07$ & $2.4 \mathrm{E}-07$ & $2.1 \mathrm{E}-07$ & & $1.9 \mathrm{E}-07$ & & 2.3E-07 & DE-07 & $5.1 \mathrm{E}-07$ & $.2 \mathrm{E}-07$ & & $2 \mathrm{E}-07$ & 0.4 \\
\hline 0.5 & $3.1 \mathrm{E}-07$ & $1.9 \mathrm{E}-07$ & $1.7 \mathrm{E}-07$ & $1.2 \mathrm{E}-07$ & 2.7E-07 & $2.0 \mathrm{E}-07$ & & & & & & & & & & & 0.5 \\
\hline 0.6 & $2.6 \mathrm{E}-07$ & $1.6 \mathrm{E}-07$ & $1.5 \mathrm{E}-07$ & $1.1 \mathrm{E}-07$ & $2.4 \mathrm{E}-07$ & $1.9 \mathrm{E}-07$ & $1.6 \mathrm{E}-07$ & & & & $1.7 \mathrm{E}-07$ & & & $2.7 \mathrm{E}-07$ & $2.4 \mathrm{E}-07$ & $2.0 \mathrm{E}-07$ & 0.6 \\
\hline 0.7 & & & & & & $1.8 \mathrm{E}$ & & & & & & & & -07 & -07 & 87 & 0.7 \\
\hline 0.8 & & $1.4 \mathrm{E}$ & $1.3 \mathrm{E}-\mathrm{C}$ & & & $1.9 \mathrm{E}-07$ & & & & & & & & & & & 0.8 \\
\hline 0.9 & $2.1 \mathrm{E}-07$ & $1.4 \mathrm{E}-07$ & $1.2 \mathrm{E}-07$ & $1.1 \mathrm{E}-07$ & 2.3E-07 & $1.9 \mathrm{E}-07$ & $1.7 \mathrm{E}-07$ & $1.2 \mathrm{E}-07$ & $1.5 \mathrm{E}-$ & & & $1.9 \mathrm{E}-07$ & & $2.2 \mathrm{E}-07$ & $1.9 \mathrm{E}-07$ & $1.6 \mathrm{E}-07$ & 0.9 \\
\hline 2.4 & & & & & & & & & & & & & & & & & 2.4 \\
\hline 4.0 & $0 \mathrm{E}-07$ & $7 \mathrm{E}$ & & & & $1.1 \mathrm{E}$ & $9.2 \mathrm{E}$ & & & & & & & -07 & -08 & -08 & 4.0 \\
\hline 5.6 & 7.4E-08 & $4.8 \mathrm{E}-\mathrm{C}$ & $4.8 \mathrm{E}-08$ & $5.7 \mathrm{E}$ & $1.1 \mathrm{E}-07$ & $8.0 \mathrm{E}-08$ & $6.6 \mathrm{E}-$ & & & & & & & -08 & -08 & $5.9 \mathrm{E}-08$ & 5.6 \\
\hline 7.2 & & & & & & & & & & & & & & & & & 7.2 \\
\hline 12.1 & & & & & & & & & & & & & & & & & 12.1 \\
\hline 24.1 & & & & & & & & & & & & & & & & & 24.1 \\
\hline 40.3 & 8.2E-09 & $5.5 \mathrm{E}-09$ & $5.6 \mathrm{E}-09$ & 7.3E-09 & $1.4 \mathrm{E}-08$ & $8.5 \mathrm{E}-09$ & $6.9 \mathrm{E}-09$ & 4.9E-09 & 6.4E-09 & $5.1 \mathrm{E}-09$ & 7.2E-09 & $1.1 \mathrm{E}-08$ & $1.8 \mathrm{E}-08$ & $1.1 \mathrm{E}-08$ & $8.5 \mathrm{E}-09$ & 7.2E-09 & 40.3 \\
\hline 56.3 & & & & & & $5.7 \mathrm{E}-09$ & & & & & & & & & & & 56.3 \\
\hline 72.4 & 4.1E-09 & $2.7 \mathrm{E}-09$ & $2.8 \mathrm{E}-09$ & 3.7E-09 & $6.9 \mathrm{E}-09$ & 4.2E-09 & 3.4E-09 & 2.4E-09 & 3.1E-09 & $2.5 \mathrm{E}-09$ & 3.6E-09 & $5.5 \mathrm{E}-09$ & 8.9E-09 & $5.5 \mathrm{E}-09$ & 4.3E-09 & 3.7E-09 & 72.4 \\
\hline
\end{tabular}


Table 4.1-17. $\bar{\chi} / \mathrm{Q}^{\prime}$ Values $\left(\mathrm{sec}^{-3}\right)$ for Chronic Ground-Level Releases from 200 Areas Based on 1983 through 1991 Meteorological Information (Schreckhise et al. 1993)

\begin{tabular}{|c|c|c|c|c|c|c|c|c|c|c|c|c|c|c|c|c|c|c|}
\hline \multirow{2}{*}{\multicolumn{2}{|c|}{$\begin{array}{l}\text { Distance } \\
(\mathrm{km})\end{array}$}} & \multicolumn{12}{|c|}{$\underline{\text { Sector (Wind from } 200 \text { Areas Toward Direction Indicated) }}$} & \multicolumn{5}{|c|}{ Distance } \\
\hline & & $\mathrm{S}$ & SSW & SW & WSW & $\mathrm{W}$ & WNW & NW & NNW & $\mathrm{N}$ & NNE & $\mathrm{NE}$ & ENE & $\mathrm{E}$ & ESE & SE & SSE & $(\mathrm{km})$ \\
\hline & 0.1 & $\overline{1.7 \mathrm{E}-04}$ & $\overline{1.0 \mathrm{E}-04}$ & $9 . \overline{9 \mathrm{E}-05}$ & $1.0 \mathrm{E}-04$ & $1 . \overline{7 \mathrm{E}-04}$ & $1.4 \mathrm{E}-04$ & $1 . \overline{6 \mathrm{E}-04}$ & $1.5 \mathrm{E}-04$ & $1 . \overline{6 \mathrm{E}-04}$ & $9 \overline{9.0 \mathrm{E}-05}$ & $1 . \overline{1 E-04}$ & $\overline{1.4 \mathrm{E}-04}$ & $3.8 \mathrm{E}-04$ & $4 . \overline{0 \mathrm{E}-04}$ & $2 . \overline{\mathrm{EE}-04}$ & $1 . \overline{5 \mathrm{E}-04}$ & 0.1 \\
\hline & 0.2 & 4.6E-05 & $2.8 \mathrm{E}-05$ & $2.7 \mathrm{E}-05$ & $2.7 \mathrm{E}-05$ & 4.7E-05 & $3.8 \mathrm{E}-05$ & $4.3 \mathrm{E}-05$ & $4.3 \mathrm{E}-05$ & $4.5 \mathrm{E}-05$ & $2.5 \mathrm{E}-05$ & $3.0 \mathrm{E}-05$ & $3.9 \mathrm{E}-05$ & $1.1 \mathrm{E}-04$ & $1.1 \mathrm{E}-04$ & $6.9 \mathrm{E}-05$ & $4.0 \mathrm{E}-05$ & 0.2 \\
\hline & 0.3 & $2.2 \mathrm{E}-05$ & $1.3 \mathrm{E}-05$ & $1.3 \mathrm{E}-05$ & $1.3 \mathrm{E}-05$ & $2.2 \mathrm{E}-05$ & $1.8 \mathrm{E}-05$ & $2.1 \mathrm{E}-05$ & $2.0 \mathrm{E}-05$ & $2.1 \mathrm{E}-05$ & $1.2 \mathrm{E}-05$ & $1.4 \mathrm{E}-05$ & $1.9 \mathrm{E}-05$ & $5.0 \mathrm{E}-05$ & $5.3 \mathrm{E}-05$ & $3.3 \mathrm{E}-05$ & $1.9 \mathrm{E}-05$ & 0.3 \\
\hline & 0.4 & $1.3 \mathrm{E}-05$ & $7.8 \mathrm{E}-06$ & $7.5 \mathrm{E}-06$ & $7.5 \mathrm{E}-06$ & $1.3 \mathrm{E}-05$ & $1.1 \mathrm{E}-05$ & $1.2 \mathrm{E}-05$ & $1.2 \mathrm{E}-05$ & $1.3 \mathrm{E}-05$ & 7.1E-06 & 8.4E-06 & $1.1 \mathrm{E}-05$ & $3.0 \mathrm{E}-05$ & $3.2 \mathrm{E}-05$ & $2.0 \mathrm{E}-05$ & $1.1 \mathrm{E}-05$ & 0.4 \\
\hline & 0.5 & $8.4 \mathrm{E}-06$ & $5.2 \mathrm{E}-06$ & $5.0 \mathrm{E}-06$ & $5.0 \mathrm{E}-06$ & $8.7 \mathrm{E}-06$ & 7.1E-06 & $8.2 \mathrm{E}-06$ & $8.1 \mathrm{E}-06$ & $8.6 \mathrm{E}-06$ & $4.8 \mathrm{E}-06$ & $5.7 \mathrm{E}-06$ & $7.5 \mathrm{E}-06$ & $2.0 \mathrm{E}-05$ & $2.2 \mathrm{E}-05$ & $1.3 \mathrm{E}-05$ & $7.5 \mathrm{E}-06$ & 0.5 \\
\hline & 0.6 & $6.1 \mathrm{E}-06$ & $3.7 \mathrm{E}-06$ & $3.6 \mathrm{E}-06$ & $3.6 \mathrm{E}-06$ & $6.3 \mathrm{E}-06$ & $5.1 \mathrm{E}-06$ & $5.9 \mathrm{E}-06$ & $5.9 \mathrm{E}-06$ & $6.2 \mathrm{E}-06$ & $3.5 \mathrm{E}-06$ & $4.1 \mathrm{E}-06$ & $5.4 \mathrm{E}-06$ & $1.5 \mathrm{E}-05$ & $1.6 \mathrm{E}-05$ & $9.5 \mathrm{E}-06$ & $5.4 \mathrm{E}-06$ & 0.6 \\
\hline & 0.7 & $4.6 \mathrm{E}-06$ & $2.8 \mathrm{E}-06$ & $2.7 \mathrm{E}-06$ & $2.7 \mathrm{E}-06$ & $4.8 \mathrm{E}-06$ & $3.9 \mathrm{E}-06$ & $4.5 \mathrm{E}-06$ & $4.5 \mathrm{E}-06$ & 4.7E-06 & $2.6 \mathrm{E}-06$ & $3.1 \mathrm{E}-06$ & $4.1 \mathrm{E}-06$ & $1.1 \mathrm{E}-05$ & $1.2 \mathrm{E}-05$ & 7.2E-06 & $4.1 \mathrm{E}-06$ & 0.7 \\
\hline & 0.8 & $3.6 \mathrm{E}-06$ & $2.2 \mathrm{E}-06$ & $2.1 \mathrm{E}-06$ & $2.1 \mathrm{E}-06$ & $3.8 \mathrm{E}-06$ & $3.1 \mathrm{E}-06$ & $3.5 \mathrm{E}-06$ & $3.5 \mathrm{E}-06$ & $3.7 \mathrm{E}-06$ & $2.1 \mathrm{E}-06$ & $2.5 \mathrm{E}-06$ & 3.3E-06 & $8.8 \mathrm{E}-06$ & $9.4 \mathrm{E}-06$ & $5.7 \mathrm{E}-06$ & $3.2 \mathrm{E}-06$ & 0.8 \\
\hline & 0.9 & $2.9 \mathrm{E}-06$ & $1.8 \mathrm{E}-06$ & $1.7 \mathrm{E}-06$ & $1.7 \mathrm{E}-06$ & $3.1 \mathrm{E}-06$ & $2.5 \mathrm{E}-06$ & $2.9 \mathrm{E}-06$ & $2.9 \mathrm{E}-06$ & $3.0 \mathrm{E}-06$ & $1.7 \mathrm{E}-06$ & $2.0 \mathrm{E}-06$ & $2.7 \mathrm{E}-06$ & $7.2 \mathrm{E}-06$ & 7.6E-06 & 4.6E-06 & $2.6 \mathrm{E}-06$ & 0.9 \\
\hline & 1.0 & $2.4 \mathrm{E}-06$ & $1.5 \mathrm{E}-06$ & $1.4 \mathrm{E}-06$ & $1.4 \mathrm{E}-06$ & $2.5 \mathrm{E}-06$ & $2.1 \mathrm{E}-06$ & $2.4 \mathrm{E}-06$ & $2.4 \mathrm{E}-06$ & $2.5 \mathrm{E}-06$ & $1.4 \mathrm{E}-06$ & $1.7 \mathrm{E}-06$ & $2.2 \mathrm{E}-06$ & $6.0 \mathrm{E}-06$ & $6.3 \mathrm{E}-06$ & $3.9 \mathrm{E}-06$ & $2.2 \mathrm{E}-06$ & 1.0 \\
\hline & 2.4 & $5.5 \mathrm{E}-07$ & $3.4 \mathrm{E}-07$ & $3.3 \mathrm{E}-07$ & $3.3 \mathrm{E}-07$ & $5.8 \mathrm{E}-07$ & $4.8 \mathrm{E}-07$ & $5.5 \mathrm{E}-07$ & $5.5 \mathrm{E}-07$ & $5.8 \mathrm{E}-07$ & $3.3 \mathrm{E}-07$ & $3.9 \mathrm{E}-07$ & $5.1 \mathrm{E}-07$ & $1.4 \mathrm{E}-06$ & $1.5 \mathrm{E}-06$ & $8.9 \mathrm{E}-07$ & $5.0 \mathrm{E}-07$ & 2.4 \\
\hline & 4.0 & $2.5 \mathrm{E}-07$ & $1.5 \mathrm{E}-07$ & $1.5 \mathrm{E}-07$ & $1.5 \mathrm{E}-07$ & $2.6 \mathrm{E}-07$ & $2.2 \mathrm{E}-07$ & $2.5 \mathrm{E}-07$ & $2.5 \mathrm{E}-07$ & $2.7 \mathrm{E}-07$ & $1.5 \mathrm{E}-07$ & $1.8 \mathrm{E}-07$ & $2.3 \mathrm{E}-07$ & $6.4 \mathrm{E}-07$ & $6.7 \mathrm{E}-07$ & $4.1 \mathrm{E}-07$ & $2.3 \mathrm{E}-07$ & 4.0 \\
\hline & 5.6 & $1.5 \mathrm{E}-07$ & $9.1 \mathrm{E}-08$ & $8.9 \mathrm{E}-08$ & $8.9 \mathrm{E}-08$ & $1.6 \mathrm{E}-07$ & $1.3 \mathrm{E}-07$ & $1.5 \mathrm{E}-07$ & $1.5 \mathrm{E}-07$ & $1.6 \mathrm{E}-07$ & $9.1 \mathrm{E}-08$ & $1.1 \mathrm{E}-07$ & $1.4 \mathrm{E}-07$ & $3.9 \mathrm{E}-07$ & $4.1 \mathrm{E}-07$ & $2.5 \mathrm{E}-07$ & $1.4 \mathrm{E}-07$ & 5.6 \\
\hline & 7.2 & $1.0 \mathrm{E}-07$ & $6.2 \mathrm{E}-08$ & $6.1 \mathrm{E}-08$ & $6.1 \mathrm{E}-08$ & $1.1 \mathrm{E}-07$ & $9.1 \mathrm{E}-08$ & $1.1 \mathrm{E}-07$ & $1.1 \mathrm{E}-07$ & $1.1 \mathrm{E}-07$ & $6.3 \mathrm{E}-08$ & $7.5 \mathrm{E}-08$ & $9.9 \mathrm{E}-08$ & $2.7 \mathrm{E}-07$ & $2.9 \mathrm{E}-07$ & $1.7 \mathrm{E}-07$ & $9.4 \mathrm{E}-08$ & 7.2 \\
\hline & 12.1 & $4.9 \mathrm{E}-08$ & $3.0 \mathrm{E}-08$ & $2.9 \mathrm{E}-08$ & $2.9 \mathrm{E}-08$ & $5.3 \mathrm{E}-08$ & 4.4E-08 & $5.1 \mathrm{E}-08$ & $5.2 \mathrm{E}-08$ & $5.5 \mathrm{E}-08$ & $3.1 \mathrm{E}-08$ & $3.6 \mathrm{E}-08$ & $4.8 \mathrm{E}-08$ & $1.3 \mathrm{E}-07$ & $1.4 \mathrm{E}-07$ & $8.2 \mathrm{E}-08$ & $4.5 \mathrm{E}-08$ & 12.1 \\
\hline & 24.1 & $1.9 \mathrm{E}-08$ & $1.1 \mathrm{E}-08$ & $1.1 \mathrm{E}-08$ & $1.1 \mathrm{E}-08$ & $2.0 \mathrm{E}-08$ & $1.7 \mathrm{E}-08$ & $2.0 \mathrm{E}-08$ & $2.0 \mathrm{E}-08$ & 2.1E-08 & $1.2 \mathrm{E}-08$ & $1.4 \mathrm{E}-08$ & $1.9 \mathrm{E}-08$ & $5.1 \mathrm{E}-08$ & $5.3 \mathrm{E}-08$ & $3.1 \mathrm{E}-08$ & $1.7 \mathrm{E}-08$ & 24.1 \\
\hline & 40.3 & $9.2 \mathrm{E}-09$ & $5.5 \mathrm{E}-09$ & $5.5 \mathrm{E}-09$ & $5.5 \mathrm{E}-09$ & $1.0 \mathrm{E}-08$ & 8.4E-09 & $9.8 \mathrm{E}-09$ & $1.0 \mathrm{E}-08$ & $1.1 \mathrm{E}-08$ & $6.0 \mathrm{E}-09$ & 7.0E-09 & $9.3 \mathrm{E}-09$ & $2.6 \mathrm{E}-08$ & $2.7 \mathrm{E}-08$ & $1.6 \mathrm{E}-08$ & 8.6E-09 & 40.3 \\
\hline & 56.3 & $5.9 \mathrm{E}-09$ & $3.5 \mathrm{E}-09$ & $3.5 \mathrm{E}-09$ & $3.5 \mathrm{E}-09$ & 6.4E-09 & $5.3 \mathrm{E}-09$ & $6.3 \mathrm{E}-09$ & 6.4E-09 & $6.9 \mathrm{E}-09$ & $3.8 \mathrm{E}-09$ & $4.5 \mathrm{E}-09$ & $6.0 \mathrm{E}-09$ & $1.7 \mathrm{E}-08$ & $1.7 \mathrm{E}-08$ & $1.0 \mathrm{E}-08$ & $5.5 \mathrm{E}-09$ & 56.3 \\
\hline & 72.4 & 4.2E-09 & $2.5 \mathrm{E}-09$ & $2.5 \mathrm{E}-09$ & $2.5 \mathrm{E}-09$ & 4.6E-09 & $3.8 \mathrm{E}-09$ & $4.5 \mathrm{E}-09$ & 4.6E-09 & $5.0 \mathrm{E}-09$ & 2.7E-09 & 3.2E-09 & 4.3E-09 & $1.2 \mathrm{E}-08$ & $1.2 \mathrm{E}-08$ & 7.1E-09 & $3.9 \mathrm{E}-09$ & 72.4 \\
\hline
\end{tabular}

Table 4.1-18. $\bar{\chi} / \mathrm{Q}^{\prime}$ Values $\left(\mathrm{sec} \mathrm{m}^{-3}\right)$ for Chronic 61-m Stack Releases from 200 Areas Based on 1983 through 1991 Meteorological Information (Schreckhise et al. 1993)

\begin{tabular}{|c|c|c|c|c|c|c|c|c|c|c|c|c|c|c|c|c|c|}
\hline Distance & & & & & & & (Wind fr & $\mathrm{m} 200 \mathrm{Ar}$ & is Toward & Direction & dicated) & & & & & & \\
\hline (km) & $\mathrm{S}$ & SSW & SW & WSW & W & WNW & NW & NNW & $\mathrm{N}$ & NNE & $\mathrm{NE}$ & ENE & E & ESE & SE & SSE & $(\mathrm{km})$ \\
\hline 0.1 & $1 . \overline{5 \mathrm{E}-09}$ & $9.3 \mathrm{E}-10$ & $7 \overline{9 \mathrm{E}-10}$ & $8.7 \mathrm{E}-10$ & $1 . \overline{4 \mathrm{E}-09}$ & $1.0 \mathrm{E}-09$ & $8 . \overline{2 \mathrm{E}-10}$ & $5.0 \mathrm{E}-10$ & $4 . \overline{9 \mathrm{E}-10}$ & $2.9 \mathrm{E}-10$ & $3 \overline{6 \mathrm{E}-10}$ & $5.3 \mathrm{E}-10$ & $6 . \overline{6 \mathrm{E}-10}$ & $\overline{7.0 \mathrm{E}-10}$ & $9 . \overline{1 \mathrm{E}-10}$ & $9 \overline{.0 \mathrm{E}-10}$ & 0.1 \\
\hline 0.2 & $3.0 \mathrm{E}-07$ & $1.8 \mathrm{E}-07$ & $1.5 \mathrm{E}-07$ & $1.7 \mathrm{E}-07$ & $2.8 \mathrm{E}-07$ & $2.0 \mathrm{E}-07$ & $1.6 \mathrm{E}-07$ & $9.6 \mathrm{E}-08$ & $9.5 \mathrm{E}-08$ & $5.5 \mathrm{E}-08$ & $6.8 \mathrm{E}-08$ & $1.0 \mathrm{E}-07$ & $1.3 \mathrm{E}-07$ & $1.4 \mathrm{E}-07$ & $1.8 \mathrm{E}-07$ & $1.8 \mathrm{E}-07$ & 0.2 \\
\hline 0.3 & $5.6 \mathrm{E}-07$ & $3.5 \mathrm{E}-07$ & $2.9 \mathrm{E}-07$ & $3.3 \mathrm{E}-07$ & $5.3 \mathrm{E}-07$ & $3.7 \mathrm{E}-07$ & $3.0 \mathrm{E}-07$ & $1.8 \mathrm{E}-07$ & $1.9 \mathrm{E}-07$ & $1.0 \mathrm{E}-07$ & $1.3 \mathrm{E}-07$ & $1.9 \mathrm{E}-07$ & $2.4 \mathrm{E}-07$ & $2.6 \mathrm{E}-07$ & $3.5 \mathrm{E}-07$ & $3.4 \mathrm{E}-07$ & 0.3 \\
\hline 0.4 & $4.7 \mathrm{E}-07$ & $3.1 \mathrm{E}-07$ & $2.5 \mathrm{E}-07$ & $2.8 \mathrm{E}-07$ & $4.4 \mathrm{E}-07$ & $3.1 \mathrm{E}-07$ & 2.6E-07 & $1.6 \mathrm{E}-07$ & $1.6 \mathrm{E}-07$ & 8.6E-08 & $1.0 \mathrm{E}-07$ & $1.6 \mathrm{E}-07$ & $2.0 \mathrm{E}-07$ & $2.2 \mathrm{E}-07$ & $3.0 \mathrm{E}-07$ & $2.9 \mathrm{E}-07$ & 0.4 \\
\hline 0.6 & $3.0 \mathrm{E}-07$ & 2.1E-07 & 1. $6 \mathrm{E}-07$ & $1.9 \mathrm{E}-07$ & $2.8 \mathrm{E}-07$ & $2.0 \mathrm{E}-07$ & $1.7 \mathrm{E}-07$ & $1.1 \mathrm{E}-07$ & $1.1 \mathrm{E}-07$ & $5.6 \mathrm{E}-08$ & $6.6 \mathrm{E}-08$ & $1.0 \mathrm{E}-07$ & & $1.6 \mathrm{E}-07$ & $2.2 \mathrm{E}-07$ & $2.0 \mathrm{E}-07$ & 0.6 \\
\hline 0.7 & $2.7 \mathrm{E}-07$ & $1.8 \mathrm{E}-07$ & $1.5 \mathrm{E}-07$ & $1.7 \mathrm{E}-07$ & $2.5 \mathrm{E}-07$ & $1.8 \mathrm{E}-07$ & $1.6 \mathrm{E}-07$ & $1.0 \mathrm{E}-07$ & $9.6 \mathrm{E}-08$ & $5.3 \mathrm{E}-08$ & $6.2 \mathrm{E}-08$ & $9.0 \mathrm{E}-08$ & $1.3 \mathrm{E}-07$ & $1.6 \mathrm{E}-07$ & $2.1 \mathrm{E}-07$ & $1.8 \mathrm{E}-07$ & 0.7 \\
\hline 0.8 & 2.6E-07 & $1.7 \mathrm{E}-07$ & $1.4 \mathrm{E}-07$ & $1.6 \mathrm{E}-07$ & $2.3 \mathrm{E}-07$ & $1.7 \mathrm{E}-07$ & $1.5 \mathrm{E}-07$ & $1.0 \mathrm{E}-07$ & 9.3E-08 & $5.2 \mathrm{E}-08$ & 6.3E-08 & 8.6E-08 & $1.4 \mathrm{E}-07$ & $1.7 \mathrm{E}-07$ & 2.1E-07 & $1.7 \mathrm{E}-07$ & 0.8 \\
\hline 0.9 & $2.5 \mathrm{E}-07$ & $1.7 \mathrm{E}-07$ & $1.3 \mathrm{E}-07$ & $1.5 \mathrm{E}-07$ & $2.2 \mathrm{E}-07$ & $1.6 \mathrm{E}-07$ & $1.5 \mathrm{E}-07$ & $1.0 \mathrm{E}-07$ & $9.2 \mathrm{E}-08$ & & $6.5 \mathrm{E}-08$ & $8.5 \mathrm{~F}$ & E-07 & $1.8 \mathrm{E}-07$ & E-07 & $1.7 \mathrm{E}-07$ & 0.9 \\
\hline 1.0 & $2.4 \mathrm{E}-07$ & $1.6 \mathrm{E}-07$ & $1.3 \mathrm{E}-07$ & $1.5 \mathrm{E}-07$ & $2.2 \mathrm{E}-07$ & $1.6 \mathrm{E}-07$ & $1.5 \mathrm{E}-07$ & $1.1 \mathrm{E}-07$ & $9.3 \mathrm{E}-08$ & 5.5 & $6.7 \mathrm{E}-08$ & 8.6 & E-07 & $2.0 \mathrm{E}-07$ & $2.1 \mathrm{E}-07$ & $1.7 \mathrm{E}-07$ & 1.0 \\
\hline 4.0 & $9.3 \mathrm{E}$ & & $5.2 \mathrm{E}-08$ & & & $7.0 \mathrm{E}-08$ & & & & & & & & $1.5 \mathrm{E}-07$ & & & 4.0 \\
\hline 5.6 & $6.4 \mathrm{E}-08$ & 4.1E-08 & $3.6 \mathrm{E}-08$ & $3.6 \mathrm{E}-08$ & $6.2 \mathrm{E}-08$ & $5.0 \mathrm{E}-08$ & $5.4 \mathrm{E}-08$ & $5.1 \mathrm{E}-08$ & $4.9 \mathrm{E}-08$ & $2.8 \mathrm{E}-08$ & 3.3E-08 & 4.3E-08 & -08 & $1.1 \mathrm{E}-07$ & $8.2 \mathrm{E}-08$ & $5.2 \mathrm{E}-08$ & 5.6 \\
\hline 7.2 & $4.8 \mathrm{E}$ & $3.0 \mathrm{E}-$ & $2.7 \mathrm{~F}$ & & & $3.8 \mathrm{E}-$ & & & & & & & & $8.8 \mathrm{E}-08$ & & & 7.2 \\
\hline 12.1 & $2.6 \mathrm{E}-$ & $1.6 \mathrm{E}-08$ & $1.4 \mathrm{E}-\mathrm{-}$ & $1.5 \mathrm{E}$ & & 2.1E-08 & & $2.4 \mathrm{E}$ & $2.4 \mathrm{E}$ & $1.3 \mathrm{E}$ & & $2.1 \mathrm{~F}$ & & $5.2 \mathrm{E}-08$ & -08 & 2.2E-08 & 12.1 \\
\hline 24.1 & $1.1 \mathrm{E}-08$ & $6.7 \mathrm{E}-09$ & $6.1 \mathrm{E}-09$ & $6.1 \mathrm{E}-09$ & $1.1 \mathrm{E}-08$ & $9.6 \mathrm{E}-09$ & & $1.2 \mathrm{E}-08$ & & $6.2 \mathrm{E}$ & & & & $2.4 \mathrm{E}-08$ & $1.6 \mathrm{E}-08$ & $9.5 \mathrm{E}-09$ & 24.1 \\
\hline 40.3 & $5.7 \mathrm{E}-09$ & $3.5 \mathrm{E}-09$ & $3.2 \mathrm{E}-09$ & $3.3 \mathrm{E}-09$ & $6.1 \mathrm{E}-09$ & $5.2 \mathrm{E}-09$ & $6.2 \mathrm{E}$ & 6.6E-09 & $6.6 \mathrm{E}-09$ & $3.5 \mathrm{E}-09$ & & $5.4 \mathrm{E}-09$ & & $1.3 \mathrm{E}-08$ & $8.8 \mathrm{E}-09$ & $5.1 \mathrm{E}-09$ & 40.3 \\
\hline 56.3 & $3.7 \mathrm{E}-$ & $2.3 \mathrm{E}-09$ & $2.1 \mathrm{E}-09$ & $2.1 \mathrm{E}-09$ & $4.1 \mathrm{E}-09$ & $3.5 \mathrm{E}-09$ & 4.2E-09 & $4.5 \mathrm{E}-09$ & & 2.4E-09 & & $3.7 \mathrm{E}-09$ & & $9.1 \mathrm{E}-09$ & $5.9 \mathrm{E}-09$ & 3.4E-09 & 56.3 \\
\hline 72.4 & $2.7 \mathrm{E}-09$ & $1.7 \mathrm{E}-09$ & 1.6E-09 & 1.6E-09 & $3.0 \mathrm{E}-09$ & $2.6 \mathrm{E}-09$ & 3.1E-09 & 3.4E-09 & $3.4 \mathrm{E}-09$ & $1.8 \mathrm{E}-09$ & $2.0 \mathrm{E}-09$ & $2.7 \mathrm{E}-09$ & $6.9 \mathrm{E}-09$ & $6.7 \mathrm{E}-09$ & 4.3E-09 & $2.5 \mathrm{E}-09$ & 72.4 \\
\hline
\end{tabular}


Table 4.1-19. $\bar{\chi} / \mathrm{Q}^{\prime}$ Values $\left(\mathrm{sec}^{-3}\right)$ for Chronic Ground-Level Releases from 300 Area Based on 1983 through 1991 Meteorological Information (Schreckhise et al. 1993)

\begin{tabular}{|c|c|c|c|c|c|c|c|c|c|c|c|c|c|c|c|c|c|}
\hline Distance & & & & & & Sect & $\mathrm{r}$ (Wind fr & $\mathrm{m} 300 \mathrm{Ar}$ & a Toward & irection I & dicated) & & & Distance & & & \\
\hline (km) & $\mathrm{S}$ & SSW & SW & WSW & W & WNW & NW & NNW & $\mathrm{N}$ & NNE & $\mathrm{NE}$ & ENE & E & ESE & SE & SSE & $(\mathrm{km})$ \\
\hline 0.1 & $2 . \overline{9 \mathrm{E}-04}$ & $9.0 \mathrm{E}-05$ & $5 . \overline{1 \mathrm{E}-05}$ & $4.4 \mathrm{E}-05$ & $1 . \overline{2 \mathrm{E}-04}$ & $2.0 \mathrm{E}-04$ & $2 \overline{8 \mathrm{E}-04}$ & $2.3 \mathrm{E}-04$ & $3 . \overline{0 \mathrm{E}-04}$ & $\overline{1.9 \mathrm{E}-04}$ & $1 . \overline{9 \mathrm{E}-04}$ & $\overline{1.4 \mathrm{E}-04}$ & $1 . \overline{7 \mathrm{E}-04}$ & $1 \overline{3 \mathrm{E}-04}$ & $1 . \overline{8 \mathrm{E}-04}$ & $2 \overline{4 \mathrm{E}-04}$ & 0.1 \\
\hline 0.2 & $7.8 \mathrm{E}-05$ & $2.5 \mathrm{E}-05$ & 1.4E-05 & $1.2 \mathrm{E}-05$ & $3.2 \mathrm{E}-05$ & $5.4 \mathrm{E}-05$ & 7.6E-05 & $6.2 \mathrm{E}-05$ & $8.3 \mathrm{E}-05$ & $5.2 \mathrm{E}-05$ & 5.3E-05 & $3.9 \mathrm{E}-05$ & 4.6E-05 & 3.7E-05 & $4.8 \mathrm{E}-05$ & $6.5 \mathrm{E}-05$ & 0.2 \\
\hline 0.3 & $3.8 \mathrm{E}-05$ & $1.2 \mathrm{E}-05$ & $6.5 \mathrm{E}-06$ & $5.6 \mathrm{E}-06$ & $1.5 \mathrm{E}-05$ & $2.6 \mathrm{E}-05$ & $3.7 \mathrm{E}-05$ & $3.0 \mathrm{E}-05$ & $4.0 \mathrm{E}-05$ & $2.5 \mathrm{E}-05$ & $2.5 \mathrm{E}-05$ & $1.8 \mathrm{E}-05$ & $2.2 \mathrm{E}-05$ & $1.8 \mathrm{E}-05$ & $2.3 \mathrm{E}-05$ & $3.1 \mathrm{E}-05$ & 0.3 \\
\hline 0.4 & $2.2 \mathrm{E}-05$ & $6.9 \mathrm{E}-06$ & $3.8 \mathrm{E}-06$ & $3.3 \mathrm{E}-06$ & $8.8 \mathrm{E}-06$ & $1.5 \mathrm{E}-05$ & $2.2 \mathrm{E}-05$ & $1.8 \mathrm{E}-05$ & $2.4 \mathrm{E}-05$ & $1.5 \mathrm{E}-05$ & $1.5 \mathrm{E}-05$ & $1.1 \mathrm{E}-05$ & $1.3 \mathrm{E}-05$ & $1.1 \mathrm{E}-05$ & $1.4 \mathrm{E}-05$ & $1.9 \mathrm{E}-05$ & 0.4 \\
\hline 0.5 & $1.5 \mathrm{E}-05$ & 4.6E-06 & $2.5 \mathrm{E}-06$ & $2.2 \mathrm{E}-06$ & $5.9 \mathrm{E}-06$ & $1.0 \mathrm{E}-05$ & $1.5 \mathrm{E}-05$ & $1.2 \mathrm{E}-05$ & 1.6E-05 & 1.0E-05 & 1.0E-05 & 7.3E-06 & $8.8 \mathrm{E}-06$ & & $9.3 \mathrm{E}-06$ & $1.3 \mathrm{E}-05$ & 0.5 \\
\hline 0.6 & $1.1 \mathrm{E}-05$ & 3.3E-06 & $1.8 \mathrm{E}-06$ & $1.6 \mathrm{E}-06$ & $4.2 \mathrm{E}-06$ & 7.4E-06 & $1.1 \mathrm{E}-05$ & $8.6 \mathrm{E}-06$ & $1.2 \mathrm{E}-05$ & $7.2 \mathrm{E}-06$ & $7.3 \mathrm{E}-06$ & $5.3 \mathrm{E}-06$ & $6.4 \mathrm{E}-06$ & & $6.7 \mathrm{E}-06$ & $9.1 \mathrm{E}-06$ & 0.6 \\
\hline 0.7 & $8.3 \mathrm{E}-06$ & $5 \mathrm{E}-06$ & $1.4 \mathrm{E}-06$ & $1.2 \mathrm{E}-06$ & $3.2 \mathrm{E}-06$ & $5.6 \mathrm{E}-06$ & $8.1 \mathrm{E}-06$ & $6.6 \mathrm{E}-06$ & $8.8 \mathrm{E}-06$ & $5.5 \mathrm{E}$ & $5.5 \mathrm{E}-06$ & $4.0 \mathrm{E}$ & $4.9 \mathrm{E}-06$ & & $5.1 \mathrm{E}-06$ & $6.9 \mathrm{E}-06$ & 0.7 \\
\hline 0.8 & $6.5 \mathrm{E}-06$ & $2.0 \mathrm{E}-06$ & $1.1 \mathrm{E}-06$ & $9.3 \mathrm{E}-$ & $2.5 \mathrm{E}-06$ & $4.5 \mathrm{E}-06$ & $6.4 \mathrm{E}-06$ & $5.2 \mathrm{E}-06$ & $6.9 \mathrm{E}-06$ & $4.3 \mathrm{E}$ & $4.4 \mathrm{E}-06$ & & $3.9 \mathrm{E}-06$ & & $4.1 \mathrm{E}-06$ & $5.5 \mathrm{E}-06$ & 0.8 \\
\hline 0.9 & $3 \mathrm{E}-06$ & $1.6 \mathrm{E}-\mathrm{C}$ & $8.7 \mathrm{E}-07$ & 7.6E- & $2.1 \mathrm{E}-06$ & $3.6 \mathrm{E}-06$ & $5.2 \mathrm{E}-06$ & 4.2E-06 & $5.6 \mathrm{E}-06$ & $3.5 \mathrm{E}-06$ & 3.6E-06 & 2.6E-06 & $3.1 \mathrm{E}-06$ & & $3.3 \mathrm{E}-06$ & 4.4E-06 & 0.9 \\
\hline 1.0 & $4.4 \mathrm{E}-06$ & $1.3 \mathrm{E}-06$ & 7.2E-07 & $6.3 \mathrm{E}$ & $1.7 \mathrm{E}-06$ & $3.0 \mathrm{E}-06$ & $4.3 \mathrm{E}-06$ & $3.5 \mathrm{E}-06$ & $4.7 \mathrm{E}-06$ & & & & & & $2.7 \mathrm{E}-06$ & E-06 & 1.0 \\
\hline 2.4 & $1.0 \mathrm{E}-06$ & $3.1 \mathrm{E}-07$ & $1.7 \mathrm{E}-07$ & $1.4 \mathrm{E}$ & $3.9 \mathrm{E}-07$ & $6.9 \mathrm{E}-07$ & $1.0 \mathrm{E}-06$ & $8.1 \mathrm{E}-07$ & $1.1 \mathrm{E}-06$ & $6.7 \mathrm{E}-07$ & $6.8 \mathrm{E}-07$ & $5.0 \mathrm{E}-07$ & $6.0 \mathrm{E}-07$ & $4.9 \mathrm{E}-07$ & $6.4 \mathrm{E}-07$ & $8.5 \mathrm{E}-07$ & 2.4 \\
\hline 4.0 & $4.7 \mathrm{E}-07$ & $1.4 \mathrm{E}-0$ & $7.5 \mathrm{E}-08$ & $6.4 \mathrm{E}$ & $1.8 \mathrm{E}-07$ & $3.2 \mathrm{E}$ & $4.6 \mathrm{E}$ & & $5.0 \mathrm{I}$ & & & & & & 07 & $3.9 \mathrm{E}-07$ & 4.0 \\
\hline 5.6 & $2.8 \mathrm{E}-07$ & $8.4 \mathrm{E}-08$ & $4.5 \mathrm{E}-08$ & $3.9 \mathrm{E}-$ & $1.1 \mathrm{E}-07$ & $1.9 \mathrm{E}-07$ & $2.8 \mathrm{E}-07$ & $2.3 \mathrm{E}$ & $3.0 \mathrm{E}-07$ & & $1.9 \mathrm{E}-07$ & & -07 & & $1.8 \mathrm{E}-07$ & $2.4 \mathrm{E}-07$ & 5.6 \\
\hline 7.2 & $2.0 \mathrm{E}-07$ & $5.8 \mathrm{E}-08$ & $3.1 \mathrm{E}-08$ & & 7.4E-08 & & & & & & & & & & & & \\
\hline 12.1 & $9.6 \mathrm{E}-08$ & $2.8 \mathrm{E}$ & $1.5 \mathrm{E}-08$ & & $3.6 \mathrm{E}-08$ & & $9.5 \mathrm{E}-$ & & 1.0E-07 & & & & & & & & 12.1 \\
\hline & $3.7 \mathrm{E}-08$ & & $5.7 \mathrm{E}-09$ & & & & & & & & & & & & & & \\
\hline 40.3 & $1.8 \mathrm{E}-08$ & $5.4 \mathrm{E}-$ & $2.9 \mathrm{E}-09$ & $2.4 \mathrm{E}$ & $6.9 \mathrm{E}-09$ & $1.3 \mathrm{E}-08$ & $1.8 \mathrm{E}-08$ & $1.5 \mathrm{E}$ & $2.0 \mathrm{E}-08$ & & $1.2 \mathrm{E}-$ & & & & & & 40.3 \\
\hline 56.3 & $1.2 \mathrm{E}-08$ & $3.4 \mathrm{E}-$ & $1.8 \mathrm{E}-09$ & & 4.4E-09 & & $1.2 \mathrm{E}-08$ & & & & & & & & & $9.7 \mathrm{E}-09$ & \\
\hline 72.4 & $8.4 \mathrm{E}-09$ & $2.5 \mathrm{E}-09$ & $1.3 \mathrm{E}-09$ & $1.1 \mathrm{E}-09$ & $3.2 \mathrm{E}-09$ & 5.7E-09 & $8.5 \mathrm{E}-09$ & $6.8 \mathrm{E}-09$ & $9.0 \mathrm{E}-09$ & $5.4 \mathrm{E}-09$ & $5.5 \mathrm{E}-09$ & $4.0 \mathrm{E}-09$ & 4.9E-09 & 4.1E-09 & $5.2 \mathrm{E}-09$ & $6.9 \mathrm{E}-09$ & 72.4 \\
\hline
\end{tabular}

Table 4.1-20. $\bar{\chi} / \mathrm{Q}^{\prime}$ Values $\left(\mathrm{sec} \mathrm{m}^{-3}\right)$ for Chronic 61-m Stack Releases from 300 Area Based on 1983 through 1991 Meteorological Information (Schreckhise et al. 1993)

\begin{tabular}{|c|c|c|c|c|c|c|c|c|c|c|c|c|c|c|c|c|c|}
\hline Distance & & & & & & & (Wind $\mathrm{fr}$ & $\mathrm{m} 300 \mathrm{Ar}$ & Toward & irection I & dicated) & & & Distance & & & \\
\hline$(\mathrm{km})$ & $\mathrm{S}$ & SSW & SW & WSW & W & WNW & NW & NNW & $\mathrm{N}$ & NNE & $\mathrm{NE}$ & ENE & E & ESE & SE & SSE & $(\mathrm{km})$ \\
\hline 0.1 & $6 . \overline{0 \mathrm{E}-10}$ & $5.6 \mathrm{E}-10$ & $5 . \overline{5 \mathrm{E}-10}$ & $4.7 \mathrm{E}-10$ & $1 . \overline{0 \mathrm{E}-09}$ & $6.2 \mathrm{E}-10$ & $6 . \overline{6 \mathrm{E}-10}$ & $5.4 \mathrm{E}-10$ & $5 . \overline{3 \mathrm{E}-10}$ & $5.5 \mathrm{E}-10$ & $6 . \overline{7 \mathrm{E}-10}$ & $5.2 \mathrm{E}-10$ & $2 . \overline{6 \mathrm{E}-10}$ & $1.2 \mathrm{E}-10$ & $2 . \overline{2 \mathrm{E}-10}$ & $3.2 \mathrm{E}-10$ & 0.1 \\
\hline 0.2 & $1.2 \mathrm{E}-07$ & $1.1 \mathrm{E}-07$ & $1 \mathrm{E}-07$ & $9.1 \mathrm{E}-08$ & 2.0E-07 & $1.2 \mathrm{E}-07$ & $1.3 \mathrm{E}-07$ & $1.1 \mathrm{E}-07$ & $1.1 \mathrm{E}-07$ & $1.1 \mathrm{E}-07$ & $1.3 \mathrm{E}-07$ & $1.0 \mathrm{E}-07$ & $5.3 \mathrm{E}-08$ & 2.6E-08 & 4.4E-08 & $6.5 \mathrm{E}-08$ & 0.2 \\
\hline 0.3 & $2.5 \mathrm{E}-07$ & $2.1 \mathrm{E}-07$ & $2.0 \mathrm{E}-07$ & $1.8 \mathrm{E}-07$ & 3.7E-07 & $2.3 \mathrm{E}-07$ & 2.6E-07 & $2.1 \mathrm{E}-07$ & 2.1E-07 & 2.1E-07 & $2.5 \mathrm{E}-07$ & $2.0 \mathrm{E}-07$ & $1.1 \mathrm{E}-07$ & $5.5 \mathrm{E}-08$ & $9.0 \mathrm{E}-08$ & $1.4 \mathrm{E}-07$ & 0.3 \\
\hline 0.4 & $2.3 \mathrm{E}-07$ & $1.9 \mathrm{E}-07$ & $1.7 \mathrm{E}-07$ & $1.5 \mathrm{E}-07$ & $3.1 \mathrm{E}-07$ & 2.0E-07 & $2.3 \mathrm{E}-07$ & $1.8 \mathrm{E}-07$ & $1.9 \mathrm{E}-07$ & $1.9 \mathrm{E}-07$ & $2.1 \mathrm{E}-07$ & 1.7E-07 & $1.1 \mathrm{E}-07$ & $5.4 \mathrm{E}-08$ & 8.4E-08 & $1.4 \mathrm{E}-07$ & 0.4 \\
\hline 0.6 & $1.8 \mathrm{E}-07$ & $1.3 \mathrm{E}-07$ & $1.0 \mathrm{E}-07$ & $9.9 \mathrm{E}-08$ & $2.0 \mathrm{E}-07$ & $1.4 \mathrm{E}-07$ & $1.7 \mathrm{E}-07$ & $1.3 \mathrm{E}-07$ & $1.5 \mathrm{E}-07$ & & $1.5 \mathrm{E}-07$ & & & & & & 0.6 \\
\hline 0.7 & $1.8 \mathrm{E}-07$ & $1.1 \mathrm{E}-07$ & 8.7E-08 & 8.7E-08 & $1.7 \mathrm{E}-07$ & $1.4 \mathrm{E}-07$ & $1.7 \mathrm{E}-07$ & $1.3 \mathrm{E}-07$ & $1.5 \mathrm{E}-07$ & & $1.4 \mathrm{E}-07$ & $.1 \mathrm{E}-07$ & & -08 & $7.9 \mathrm{E}-08$ & $1.3 \mathrm{E}-07$ & 0.7 \\
\hline 0.8 & $9 \mathrm{E}-07$ & $1.1 \mathrm{E}-07$ & 7.9E-08 & $8.0 \mathrm{E}-08$ & 1.6E-07 & $1.4 \mathrm{E}-07$ & & & & & & & & & & & 0.8 \\
\hline 0.9 & & $1.1 \mathrm{E}-0$ & $7.5 \mathrm{E}-08$ & & $1.5 \mathrm{E}-07$ & & & & & & & & & & & & 0.9 \\
\hline 1.0 & $2.0 \mathrm{E}-07$ & $1.0 \mathrm{E}-07$ & 7.1E-08 & 7.2E-08 & $1.5 \mathrm{E}-07$ & $1.4 \mathrm{E}-07$ & $1.8 \mathrm{E}-07$ & $1.4 \mathrm{E}-07$ & $1.7 \mathrm{E}-07$ & $1.5 \mathrm{E}-07$ & $1.5 \mathrm{E}-07$ & $1.1 \mathrm{E}-07$ & $9.9 \mathrm{E}-08$ & $6.1 \mathrm{E}$ & 1.0E-07 & $1.5 \mathrm{E}-07$ & 1.0 \\
\hline 4.0 & $9.5 \mathrm{E}-08$ & $3.7 \mathrm{E}-08$ & $2.3 \mathrm{E}-08$ & $2.1 \mathrm{E}-08$ & $4.8 \mathrm{E}-08$ & $6.7 \mathrm{E}-08$ & 8.3E-08 & 7.0E-08 & 9.6E-08 & $7.2 \mathrm{E}$ & 7.5E-08 & $5.4 \mathrm{E}-08$ & $5.7 \mathrm{E}-08$ & 4.11 & $5.9 \mathrm{E}-08$ & $8.2 \mathrm{E}-08$ & 4.0 \\
\hline 5.6 & & & & & & & & & & & & & & & 4.21 & & 5.6 \\
\hline 7.2 & $5.2 \mathrm{E}-08$ & $1.9 \mathrm{E}-08$ & $1.1 \mathrm{E}-08$ & $1.1 \mathrm{E}-08$ & $2.4 \mathrm{E}-08$ & 3.6E-08 & $4.6 \mathrm{E}-08$ & $3.9 \mathrm{E}-08$ & $5.4 \mathrm{E}-08$ & & 4.0E- 08 & 2.91 & $3.2 \mathrm{E}-08$ & -08 & $3.2 \mathrm{E}-08$ & 4.5E-08 & 7.2 \\
\hline 12.1 & $2.9 \mathrm{E}-08$ & & 6.1E-09 & & $1.3 \mathrm{E}-08$ & $2.0 \mathrm{E}-08$ & & & & & & & & & $1.8 \mathrm{E}-08$ & & 12.1 \\
\hline & $1.3 \mathrm{E}-08$ & $4.4 \mathrm{E}-0 \mathrm{c}$ & $2.6 \mathrm{E}-09$ & & & $9.0 \mathrm{E}-09$ & & $1.0 \mathrm{E}-08$ & & & & & & & $8.0 \mathrm{E}-09$ & & 24.1 \\
\hline & $6.9 \mathrm{E}-09$ & $2.3 \mathrm{E}-09$ & 1.4E-09 & & $0 \mathrm{E}-09$ & $4.8 \mathrm{E}-09$ & $6.6 \mathrm{E}$ & & $7.3 \mathrm{~F}$ & & & & & & 4.3E-09 & & 40.3 \\
\hline 56.3 & $4.5 \mathrm{E}-09$ & $1.5 \mathrm{E}-09$ & $8.9 \mathrm{E}-10$ & $7.9 \mathrm{E}-10$ & $2.0 \mathrm{E}-09$ & $3.2 \mathrm{E}-09$ & 4.4E-09 & 3.6E-09 & $4.9 \mathrm{E}-09$ & $3.2 \mathrm{E}-09$ & 3.3E-09 & 2.4E-09 & $2.7 \mathrm{E}-09$ & $2.1 \mathrm{E}-09$ & $2.8 \mathrm{E}-09$ & 3.8E-09 & 56.3 \\
\hline 72.4 & $3.3 \mathrm{E}-09$ & $1.1 \mathrm{E}-09$ & $6.5 \mathrm{E}-10$ & $5.8 \mathrm{E}-10$ & $1.5 \mathrm{E}-09$ & $2.3 \mathrm{E}-09$ & 3.2E-09 & $2.7 \mathrm{E}-09$ & 3.6E-09 & $2.3 \mathrm{E}-09$ & $2.4 \mathrm{E}-09$ & $1.8 \mathrm{E}-09$ & 2.0E-09 & 1.6E-09 & $2.1 \mathrm{E}-09$ & $2.8 \mathrm{E}-09$ & 72.4 \\
\hline
\end{tabular}


Table 4.1-21. $\bar{\chi} / \mathrm{Q}^{\prime}$ Values $\left(\mathrm{sec} \mathrm{m}^{-3}\right.$ ) for Chronic Ground-Level Releases from 400 Area Based on 1983 through 1991 Meteorological Information (Schreckhise et al. 1993)

\begin{tabular}{|c|c|c|c|c|c|c|c|c|c|c|c|c|c|c|c|c|c|}
\hline Distance & & & & & & $\underline{\operatorname{Sect}}$ & $\mathrm{r}$ (Wind $\mathrm{fr}$ & $\mathrm{m} 400 \mathrm{Ar}$ & a Toward & Direction I & dicated) & & & Distance & & & \\
\hline$(\mathrm{km})$ & $\mathrm{S}$ & SSW & SW & WSW & W & WNW & NW & NNW & $\mathrm{N}$ & NNE & $\mathrm{NE}$ & ENE & E & ESE & SE & SSE & $\frac{(\mathrm{km})}{\Omega}$ \\
\hline 0.1 & $2 . \overline{\mathrm{E}-04}$ & $\overline{1.4 \mathrm{E}-04}$ & $9 . \overline{6 \mathrm{E}-05}$ & $6.9 \mathrm{E}-05$ & $9 . \overline{7 \mathrm{E}-05}$ & $8.5 \mathrm{E}-05$ & $1 . \overline{2 \mathrm{E}-04}$ & $1.9 \mathrm{E}-04$ & $3 . \overline{1 \mathrm{E}-04}$ & $2.2 \mathrm{E}-04$ & $1 . \overline{5 \mathrm{E}-04}$ & $9 \overline{9 \mathrm{EE}-05}$ & $1 . \overline{5 \mathrm{E}-04}$ & $1 . \overline{5 \mathrm{E}-04}$ & $2 . \overline{1 \mathrm{E}-04}$ & $1 \overline{7 \mathrm{E}-04}$ & 0.1 \\
\hline 0.2 & $5.8 \mathrm{E}-05$ & $3.9 \mathrm{E}-05$ & $2.6 \mathrm{E}-05$ & $1.9 \mathrm{E}-05$ & $2.7 \mathrm{E}-05$ & 2.3E-05 & $3.1 \mathrm{E}-05$ & 5.1E- 05 & $8.5 \mathrm{E}-05$ & $6.0 \mathrm{E}-05$ & $4.0 \mathrm{E}-05$ & $2.7 \mathrm{E}-05$ & $4.2 \mathrm{E}-05$ & 4.2E- 05 & $5.8 \mathrm{E}-05$ & 4.8E- 05 & 0.2 \\
\hline 0.3 & $2.8 \mathrm{E}-05$ & $1.8 \mathrm{E}-05$ & $1.2 \mathrm{E}-05$ & $8.9 \mathrm{E}-06$ & $1.3 \mathrm{E}-05$ & $1.1 \mathrm{E}-05$ & $1.5 \mathrm{E}-05$ & $2.5 \mathrm{E}-05$ & $4.1 \mathrm{E}-05$ & $2.9 \mathrm{E}-05$ & $1.9 \mathrm{E}-05$ & $1.3 \mathrm{E}-05$ & $2.0 \mathrm{E}-05$ & $2.0 \mathrm{E}-05$ & $2.8 \mathrm{E}-05$ & $2.3 \mathrm{E}-05$ & 0.3 \\
\hline 0.4 & $1.7 \mathrm{E}-05$ & $1.1 \mathrm{E}-05$ & 7.4E-06 & $5.3 \mathrm{E}-06$ & 7.4E-06 & $6.5 \mathrm{E}-06$ & $8.9 \mathrm{E}-06$ & $1.5 \mathrm{E}-05$ & $2.4 \mathrm{E}-05$ & $1.7 \mathrm{E}-05$ & $1.1 \mathrm{E}-05$ & 7.7E-06 & $1.2 \mathrm{E}-05$ & $1.2 \mathrm{E}-05$ & $1.7 \mathrm{E}-05$ & $1.4 \mathrm{E}-05$ & 0.4 \\
\hline 0.5 & $1.1 \mathrm{E}-05$ & 7.3E-06 & $4.9 \mathrm{E}-06$ & $3.5 \mathrm{E}-06$ & $5.0 \mathrm{E}-06$ & 4.3E-06 & $5.9 \mathrm{E}-06$ & $9.8 \mathrm{E}-06$ & $1.6 \mathrm{E}-05$ & $1.2 \mathrm{E}-05$ & 7.6E-06 & $5.2 \mathrm{E}-06$ & $8.0 \mathrm{E}-06$ & $8.0 \mathrm{E}-06$ & $1.1 \mathrm{E}-05$ & $9.1 \mathrm{E}-06$ & 0.5 \\
\hline 0.6 & $8.0 \mathrm{E}-06$ & 5.3E-06 & 3.6E-06 & $2.5 \mathrm{E}-06$ & 3.6E-06 & 3.1E-06 & 4.3E-06 & 7.1E-06 & $1.2 \mathrm{E}-05$ & $8.3 \mathrm{E}-06$ & $5.5 \mathrm{E}-06$ & $3.8 \mathrm{E}-06$ & $5.8 \mathrm{E}-06$ & $5.8 \mathrm{E}-06$ & $8.1 \mathrm{E}-06$ & $6.6 \mathrm{E}-06$ & 0.6 \\
\hline 0.7 & $6.1 \mathrm{E}-06$ & $4.0 \mathrm{E}-06$ & $2.7 \mathrm{E}-06$ & $1.9 \mathrm{E}-06$ & $2.7 \mathrm{E}-06$ & $2.4 \mathrm{E}-06$ & $3.3 \mathrm{E}-06$ & $5.4 \mathrm{E}-06$ & $8.9 \mathrm{E}-06$ & $6.3 \mathrm{E}-06$ & $4.2 \mathrm{E}-06$ & $2.9 \mathrm{E}-06$ & $4.4 \mathrm{E}-06$ & $4.4 \mathrm{E}-06$ & $6.2 \mathrm{E}-06$ & $5.0 \mathrm{E}-06$ & 0.7 \\
\hline 0.8 & $4.8 \mathrm{E}-06$ & $3.2 \mathrm{E}-06$ & $2.1 \mathrm{E}-06$ & $1.5 \mathrm{E}-06$ & $2.1 \mathrm{E}-06$ & $1.9 \mathrm{E}-06$ & 2.6E-06 & 4.3E-06 & 7.0E-06 & $5.0 \mathrm{E}-06$ & $3.3 \mathrm{E}-06$ & $2.3 \mathrm{E}-06$ & $3.5 \mathrm{E}-06$ & $3.5 \mathrm{E}-06$ & $4.9 \mathrm{E}-06$ & $4.0 \mathrm{E}-06$ & 0.8 \\
\hline 0.9 & $3.9 \mathrm{E}-06$ & $2.6 \mathrm{E}-06$ & $1.7 \mathrm{E}-06$ & $1.2 \mathrm{E}-06$ & $1.7 \mathrm{E}-06$ & $1.5 \mathrm{E}-06$ & $2.1 \mathrm{E}-06$ & $3.5 \mathrm{E}-06$ & $5.7 \mathrm{E}-06$ & $4.0 \mathrm{E}-06$ & $2.7 \mathrm{E}-06$ & $1.8 \mathrm{E}-06$ & $2.8 \mathrm{E}-06$ & $2.8 \mathrm{E}-06$ & $4.0 \mathrm{E}-06$ & $3.2 \mathrm{E}-06$ & 0.9 \\
\hline 1.0 & 3.3E-06 & $2.1 \mathrm{E}-06$ & $1.4 \mathrm{E}-06$ & $1.0 \mathrm{E}-06$ & $1.4 \mathrm{E}-06$ & $1.3 \mathrm{E}-06$ & $1.7 \mathrm{E}-06$ & $2.9 \mathrm{E}-06$ & $4.7 \mathrm{E}-06$ & 3.4E-06 & $2.2 \mathrm{E}-06$ & $1.5 \mathrm{E}-06$ & $2.3 \mathrm{E}-06$ & $2.3 \mathrm{E}-06$ & $3.3 \mathrm{E}-06$ & $2.7 \mathrm{E}-06$ & 1.0 \\
\hline 2.4 & $7.5 \mathrm{E}-07$ & $4.9 \mathrm{E}-07$ & 3.3E-07 & $2.3 \mathrm{E}-07$ & 3.3E-07 & $2.9 \mathrm{E}-07$ & $4.0 \mathrm{E}-07$ & $6.7 \mathrm{E}-07$ & $1.1 \mathrm{E}-06$ & $7.8 \mathrm{E}-07$ & $5.2 \mathrm{E}-07$ & $3.5 \mathrm{E}-07$ & $5.4 \mathrm{E}-07$ & $5.4 \mathrm{E}-07$ & 7.6E-07 & $6.2 \mathrm{E}-07$ & 2.4 \\
\hline 4.0 & $3.4 \mathrm{E}-07$ & 2.2E-07 & $1.5 \mathrm{E}-07$ & $1.1 \mathrm{E}-07$ & $1.5 \mathrm{E}-07$ & $1.3 \mathrm{E}-07$ & $1.8 \mathrm{E}-07$ & $3.1 \mathrm{E}-07$ & $5.0 \mathrm{E}-07$ & $3.5 \mathrm{E}-07$ & $2.4 \mathrm{E}-07$ & $1.6 \mathrm{E}-07$ & $2.5 \mathrm{E}-07$ & $2.5 \mathrm{E}-07$ & $3.5 \mathrm{E}-07$ & $2.8 \mathrm{E}-07$ & 4.0 \\
\hline 5.6 & $2.1 \mathrm{E}-07$ & $1.4 \mathrm{E}-07$ & $9.1 \mathrm{E}-08$ & 6.4E-08 & $9.1 \mathrm{E}-08$ & $8.0 \mathrm{E}-08$ & $1.1 \mathrm{E}-07$ & $1.9 \mathrm{E}-07$ & $3.1 \mathrm{E}-07$ & $2.2 \mathrm{E}-07$ & 1.4E-07 & $9.8 \mathrm{E}-08$ & $1.5 \mathrm{E}-07$ & $1.5 \mathrm{E}-07$ & $2.1 \mathrm{E}-07$ & $1.7 \mathrm{E}-07$ & 5.6 \\
\hline 7.2 & $1.5 \mathrm{E}-07$ & $9.5 \mathrm{E}-08$ & $6.3 \mathrm{E}-08$ & $4.4 \mathrm{E}-08$ & $6.3 \mathrm{E}-08$ & $5.5 \mathrm{E}-08$ & 7.6E-08 & $1.3 \mathrm{E}-07$ & $2.1 \mathrm{E}-07$ & $1.5 \mathrm{E}-07$ & $1.0 \mathrm{E}-07$ & $6.8 \mathrm{E}-08$ & $1.0 \mathrm{E}-07$ & $1.0 \mathrm{E}-07$ & $1.5 \mathrm{E}-07$ & $1.2 \mathrm{E}-07$ & 7.2 \\
\hline 12.1 & $7.0 \mathrm{E}-08$ & $4.6 \mathrm{E}-08$ & $3.1 \mathrm{E}-08$ & $2.1 \mathrm{E}-08$ & $3.0 \mathrm{E}-08$ & $2.7 \mathrm{E}-08$ & $3.7 \mathrm{E}-08$ & $6.2 \mathrm{E}-08$ & $1.0 \mathrm{E}-07$ & $7.2 \mathrm{E}-08$ & $4.8 \mathrm{E}-08$ & 3.3E-08 & $5.0 \mathrm{E}-08$ & $5.0 \mathrm{E}-08$ & 7.1E-08 & $5.8 \mathrm{E}-08$ & 12.1 \\
\hline 24.1 & $2.7 \mathrm{E}-08$ & $1.8 \mathrm{E}-08$ & $1.2 \mathrm{E}-08$ & $8.2 \mathrm{E}-09$ & $1.2 \mathrm{E}-08$ & $1.0 \mathrm{E}-08$ & $1.4 \mathrm{E}-08$ & 2.4E-08 & $4.0 \mathrm{E}-08$ & $2.8 \mathrm{E}-08$ & $1.9 \mathrm{E}-08$ & $1.3 \mathrm{E}-08$ & $1.9 \mathrm{E}-08$ & $1.9 \mathrm{E}-08$ & $2.7 \mathrm{E}-08$ & $2.2 \mathrm{E}-08$ & 24.1 \\
\hline 40.3 & $1.4 \mathrm{E}-08$ & $8.9 \mathrm{E}-09$ & $5.8 \mathrm{E}-09$ & 4.1E-09 & $5.8 \mathrm{E}-09$ & $5.1 \mathrm{E}-09$ & 7.0E-09 & $1.2 \mathrm{E}-08$ & $2.0 \mathrm{E}-08$ & $1.4 \mathrm{E}-08$ & $9.3 \mathrm{E}-09$ & $6.3 \mathrm{E}-09$ & $9.6 \mathrm{E}-09$ & $9.5 \mathrm{E}-09$ & $1.4 \mathrm{E}-08$ & $1.1 \mathrm{E}-08$ & 40.3 \\
\hline 56.3 & $8.7 \mathrm{E}-09$ & $5.7 \mathrm{E}-09$ & $3.7 \mathrm{E}-09$ & $2.6 \mathrm{E}-09$ & $3.7 \mathrm{E}-09$ & 3.2E-09 & $4.5 \mathrm{E}-09$ & 7.6E-09 & $1.3 \mathrm{E}-08$ & $8.8 \mathrm{E}-09$ & $5.9 \mathrm{E}-09$ & $4.0 \mathrm{E}-09$ & $6.1 \mathrm{E}-09$ & $6.0 \mathrm{E}-09$ & $8.6 \mathrm{E}-09$ & 7.0E-09 & 56.3 \\
\hline 72.4 & $6.3 \mathrm{E}-09$ & $4.1 \mathrm{E}-09$ & $2.7 \mathrm{E}-09$ & $1.9 \mathrm{E}-09$ & $2.7 \mathrm{E}-09$ & 2.3E-09 & $3.2 \mathrm{E}-09$ & $5.5 \mathrm{E}-09$ & $9.1 \mathrm{E}-09$ & $6.3 \mathrm{E}-09$ & $4.2 \mathrm{E}-09$ & $2.9 \mathrm{E}-09$ & $4.4 \mathrm{E}-09$ & $4.3 \mathrm{E}-09$ & $6.1 \mathrm{E}-09$ & $5.0 \mathrm{E}-09$ & 72.4 \\
\hline
\end{tabular}

Table 4.1-22. $\bar{\chi} / \mathrm{Q}^{\prime}$ Values $\left(\mathrm{sec} \mathrm{m}^{-3}\right.$ ) for Chronic 30-m Stack Releases from 400 Area Based on 1983 through 1991 Meteorological Information (Schreckhise et al. 1993)

\begin{tabular}{|c|c|c|c|c|c|c|c|c|c|c|c|c|c|c|c|c|c|}
\hline istance & & & & & & & & & & & Inalus & & & Distance & & & \\
\hline$(\mathrm{km})$ & $\mathrm{S}$ & SSW & SW & WSW & $\mathrm{W}$ & WNW & NW & NNW & $\mathrm{N}$ & NNE & $\mathrm{NE}$ & ENE & E & ESE & SE & SSE & $(\mathrm{km})$ \\
\hline 0.1 & $6 . \overline{\mathrm{BE}-07}$ & $5.4 \mathrm{E}-07$ & $4 . \overline{3 \mathrm{E}-07}$ & $\overline{4.0 \mathrm{E}-07}$ & $5 . \overline{\mathrm{E}-07}$ & $5.2 \mathrm{E}-07$ & $4.6 \mathrm{E}-07$ & $4.2 \mathrm{E}-07$ & $9 . \overline{4 \mathrm{E}-07}$ & $7.5 \mathrm{E}-07$ & $3 . \overline{9 \mathrm{E}-07}$ & $3.0 \mathrm{E}-07$ & $4 . \overline{0 \mathrm{E}-07}$ & $2 . \overline{9 \mathrm{E}-07}$ & $3 . \overline{\mathrm{EE}-07}$ & $3 . \overline{\mathrm{E}-07}$ & 0.1 \\
\hline 0.2 & $1.1 \mathrm{E}-06$ & $9.4 \mathrm{E}-07$ & $.4 \mathrm{E}-07$ & $6.8 \mathrm{E}-07$ & $9.1 \mathrm{E}-07$ & $8.4 \mathrm{E}-07$ & 7.9E-07 & 7.5E-07 & $1.6 \mathrm{E}-06$ & $1.3 \mathrm{E}-06$ & $6.8 \mathrm{E}-07$ & $5.0 \mathrm{E}-07$ & $6.8 \mathrm{E}-07$ & $5.0 \mathrm{E}-07$ & $6.1 \mathrm{E}-07$ & $6.1 \mathrm{E}-07$ & 0.2 \\
\hline 0.3 & $9.7 \mathrm{E}-07$ & $8.0 \mathrm{E}-07$ & $3 \mathrm{E}-07$ & & $7.5 \mathrm{E}-07$ & & $7.0 \mathrm{E}-07$ & & & & & & & & $.7 \mathrm{E}-07$ & $1 \mathrm{E}-07$ & 0.3 \\
\hline 0.4 & $3 \mathrm{E}-07$ & $2 \mathrm{E}-07$ & $7 \mathrm{E}-07$ & $4.8 \mathrm{E}-07$ & $6.5 \mathrm{E}-07$ & $8 \mathrm{E}-07$ & & $7.4 \mathrm{E}$ & & $1.0 \mathrm{E}$ & & & & & & & 0.4 \\
\hline 0.5 & $0 \mathrm{E}-07$ & $6.6 \mathrm{E}-07$ & $3 \mathrm{E}-07$ & & $0 \mathrm{E}-07$ & $5.3 \mathrm{E}-07$ & & & & & & & & & & & 0.5 \\
\hline 0.6 & & $6.2 \mathrm{E}$ & $9 \mathrm{E}-\mathrm{C}$ & & & $4.9 \mathrm{E}-07$ & & & & & & & & & & & 0.6 \\
\hline 0.7 & & $5.7 \mathrm{E}-\mathrm{C}$ & & & & & & & & & & & & & & & 0.7 \\
\hline 0.8 & -07 & $5.3 \mathrm{E}-07$ & $4.2 \mathrm{~F}$ & $3.2 \mathrm{E}-$ & $4.5 \mathrm{E}-07$ & $4.1 \mathrm{E}-07$ & $5.0 \mathrm{I}$ & $6.8 \mathrm{E}$ & $1.1 \mathrm{E}$ & & & $3.9 \mathrm{I}$ & & & & 7 & 0.8 \\
\hline 0.9 & & $4.9 \mathrm{E}-07$ & $.9 \mathrm{E}-1$ & & & & & & & & & & & & & & 0.9 \\
\hline 1.0 & $6 \mathrm{E}-07$ & $4.5 \mathrm{E}-07$ & $.6 \mathrm{E}-07$ & & $3.8 \mathrm{I}$ & $3.4 \mathrm{E}-07$ & $4.3 \mathrm{E}$ & & & & & $3.4 \mathrm{E}$ & & & & $5.7 \mathrm{I}$ & 1.0 \\
\hline & & $1.9 \mathrm{E}-$ & & & & & & & & & & & & & & & 2.4 \\
\hline 4.0 & $6 \mathrm{E}-07$ & $1.1 \mathrm{E}-07$ & $7.6 \mathrm{E}-0$ & & $7.9 \mathrm{E}-$ & $7.0 \mathrm{E}-08$ & & $1.5 \mathrm{E}$ & & & & & & & -07 & $1.4 \mathrm{E}-07$ & 4.0 \\
\hline & & & & & & & & & & & & & & & & & \\
\hline 7.2 & & & & & & & & & & & & & & & & & 7.2 \\
\hline & & & & & & & & & & & & & & & & & 12.1 \\
\hline & & & & & & & & & & & & & & & & & 24.1 \\
\hline & & & & & & & & & & & & & & & & & 40.3 \\
\hline & & & & & & & & & & & & & & & & & 56.3 \\
\hline 72.4 & 9E-09 & $2.6 \mathrm{E}-09$ & $7 \mathrm{E}-09$ & $1.3 \mathrm{E}-09$ & $1.8 \mathrm{E}-09$ & $1.5 \mathrm{E}-09$ & $2.1 \mathrm{E}-09$ & $3.5 \mathrm{E}-09$ & $5.8 \mathrm{E}-09$ & $4.1 \mathrm{E}-09$ & 2.7E-09 & $1.9 \mathrm{E}-09$ & $2.8 \mathrm{E}-09$ & $2.8 \mathrm{E}-09$ & $3.9 \mathrm{E}-09$ & $3.2 \mathrm{E}-09$ & 72.4 \\
\hline
\end{tabular}


Table 4.1-23. 95th Percentile E/Q Values $\left(\mathrm{sec} \mathrm{m}^{-3}\right.$ ) for Acute Ground Level Releases from 100-N Area Based on 1983 through 1991 Meteorological Information (Schreckhise et al. 1993)

\begin{tabular}{|c|c|c|c|c|c|c|c|c|c|c|c|c|c|c|c|c|c|}
\hline Distance & & & & & & & r (Wind & om 100-1 & Toward D & rection In & cated) & & & Distance & & & \\
\hline$(\mathrm{km})$ & $\frac{\mathrm{S}}{S \Gamma(x)}$ & SSW & SW & WSW & W & WNW & NW & NNW & $\frac{\mathrm{N}}{1502}$ & NNE & $\mathrm{NE}$ & ENE & $\frac{E}{2}$ & ESE & SE & SSE & $\frac{(\mathrm{km})}{01}$ \\
\hline 0.1 & $6 . \overline{\mathrm{E}-02}$ & $5.1 \mathrm{E}-02$ & $6 . \overline{7 \mathrm{E}-02}$ & $7.3 \mathrm{E}-02$ & $6 . \overline{\mathrm{EE}-02}$ & $5.8 \mathrm{E}-02$ & $5 . \overline{5 \mathrm{E}-02}$ & $5.3 \mathrm{E}-02$ & $6 . \overline{1 \mathrm{E}-02}$ & $5.8 \mathrm{E}-02$ & $5 . \overline{5 \mathrm{E}-02}$ & $5.6 \mathrm{E}-02$ & $5 . \overline{\mathrm{E}-02}$ & $5 \overline{7 \mathrm{E}-02}$ & $6 . \overline{9 \mathrm{E}-02}$ & $7 \overline{5 \mathrm{E}-02}$ & 0.1 \\
\hline 0.2 & $1.9 \mathrm{E}-02$ & $1.5 \mathrm{E}-02$ & $2.0 \mathrm{E}-02$ & $2.2 \mathrm{E}-02$ & $2.1 \mathrm{E}-02$ & $1.8 \mathrm{E}-02$ & $1.7 \mathrm{E}-02$ & $1.6 \mathrm{E}-02$ & $1.8 \mathrm{E}-02$ & $1.8 \mathrm{E}-02$ & $1.6 \mathrm{E}-02$ & $1.7 \mathrm{E}-02$ & $1.6 \mathrm{E}-02$ & $1.7 \mathrm{E}-02$ & $2.1 \mathrm{E}-02$ & $2.3 \mathrm{E}-02$ & 0.2 \\
\hline 0.3 & $9.5 \mathrm{E}-03$ & 7.7E-03 & $1.0 \mathrm{E}-02$ & $1.1 \mathrm{E}-02$ & $1.0 \mathrm{E}-02$ & $8.8 \mathrm{E}-03$ & $8.3 \mathrm{E}-03$ & $8.0 \mathrm{E}-03$ & $9.2 \mathrm{E}-03$ & $8.8 \mathrm{E}-03$ & $8.3 \mathrm{E}-03$ & $8.5 \mathrm{E}-03$ & $8.0 \mathrm{E}-03$ & $8.6 \mathrm{E}-03$ & $1.0 \mathrm{E}-02$ & $1.1 \mathrm{E}-02$ & 0.3 \\
\hline 0.4 & $5.8 \mathrm{E}-03$ & $4.8 \mathrm{E}-03$ & $6.3 \mathrm{E}-03$ & $6.8 \mathrm{E}-03$ & $6.5 \mathrm{E}-03$ & $5.4 \mathrm{E}-03$ & $5.1 \mathrm{E}-03$ & $4.9 \mathrm{E}-03$ & $5.7 \mathrm{E}-03$ & $5.4 \mathrm{E}-03$ & $5.1 \mathrm{E}-03$ & $5.2 \mathrm{E}-03$ & $4.9 \mathrm{E}-03$ & $5.3 \mathrm{E}-03$ & $6.4 \mathrm{E}-03$ & $7.0 \mathrm{E}-03$ & 0.4 \\
\hline 0.5 & $4.0 \mathrm{E}-03$ & $3.3 \mathrm{E}-03$ & $4.3 \mathrm{E}-03$ & 4.7E-03 & $4.4 \mathrm{E}-03$ & $3.7 \mathrm{E}-03$ & $3.5 \mathrm{E}-03$ & & $3.9 \mathrm{E}-03$ & $3.7 \mathrm{E}-03$ & $3.5 \mathrm{E}-03$ & $3.6 \mathrm{E}-03$ & & & $4.4 \mathrm{E}-03$ & & 0.5 \\
\hline 0.6 & $3.0 \mathrm{E}-03$ & $2.4 \mathrm{E}-03$ & $3.2 \mathrm{E}-03$ & $3.4 \mathrm{E}-03$ & $3.3 \mathrm{E}-03$ & $2.8 \mathrm{E}-03$ & $2.6 \mathrm{E}-03$ & & $2.9 \mathrm{E}-03$ & $2.8 \mathrm{E}-03$ & $2.6 \mathrm{E}-03$ & $2.6 \mathrm{E}-03$ & $2.5 \mathrm{E}-03$ & & $3.2 \mathrm{E}-03$ & $3.5 \mathrm{E}-03$ & 0.6 \\
\hline 0.7 & $2.3 \mathrm{E}-03$ & $1.9 \mathrm{E}-03$ & $2.5 \mathrm{E}-03$ & 2.7E-03 & & $2.1 \mathrm{E}-03$ & $2.0 \mathrm{E}-03$ & & & & & & & & $2.5 \mathrm{E}-03$ & $2.7 \mathrm{E}-03$ & 0.7 \\
\hline 0.8 & $1.8 \mathrm{E}-03$ & $1.5 \mathrm{E}-03$ & $2.0 \mathrm{E}-03$ & $2.1 \mathrm{E}-$ & $2.0 \mathrm{E}-03$ & $1.7 \mathrm{E}-03$ & & & $1.8 \mathrm{E}-03$ & & & & & & & & 0.8 \\
\hline 0.9 & $1.5 \mathrm{E}-03$ & $1.2 \mathrm{E}-03$ & $1.6 \mathrm{E}-03$ & $1.8 \mathrm{E}-03$ & $1.7 \mathrm{E}-03$ & $1.4 \mathrm{E}-03$ & $1.3 \mathrm{E}-03$ & $1.3 \mathrm{E}-03$ & $1.5 \mathrm{E}-03$ & $1.4 \mathrm{E}-03$ & $1.3 \mathrm{E}-03$ & & $1.3 \mathrm{E}-03$ & & $1.6 \mathrm{E}-03$ & $1.8 \mathrm{E}-03$ & 0.9 \\
\hline 1.0 & & & & & & & & & & & & & & & & & 1.0 \\
\hline 2.4 & $3.2 \mathrm{E}-04$ & $2.6 \mathrm{E}-04$ & $3.5 \mathrm{E}-04$ & $3.8 \mathrm{E}-04$ & $3.6 \mathrm{E}-04$ & $3.0 \mathrm{E}-04$ & $2.8 \mathrm{E}-04$ & $2.7 \mathrm{E}-04$ & $3.2 \mathrm{E}-04$ & $3.0 \mathrm{E}-04$ & $2.8 \mathrm{E}-04$ & $2.9 \mathrm{E}-04$ & $2.7 \mathrm{E}-04$ & $2.9 \mathrm{E}$ & $3.5 \mathrm{E}-04$ & $3.9 \mathrm{E}-04$ & 2.4 \\
\hline 4.0 & & & & & & & & & & & & & & & & & 4.0 \\
\hline 5.6 & $1.0 \mathrm{E}-04$ & $8.2 \mathrm{E}-05$ & $1.1 \mathrm{E}-04$ & $1.2 \mathrm{E}-04$ & $1.1 \mathrm{E}-04$ & $9.4 \mathrm{E}-05$ & $8.8 \mathrm{E}-05$ & $8.5 \mathrm{E}-05$ & $9.9 \mathrm{E}-05$ & $9.4 \mathrm{E}-05$ & $8.8 \mathrm{E}-05$ & $9.0 \mathrm{E}-05$ & E-05 & -05 & $1.1 \mathrm{E}-04$ & $1.2 \mathrm{E}-04$ & 5.6 \\
\hline 7.2 & 7.3E-05 & $5.9 \mathrm{E}-$ & $7.8 \mathrm{E}-05$ & $8.5 \mathrm{E}$ & & $6.8 \mathrm{E}-05$ & & & & & & & & & & & \\
\hline 12.1 & $3.8 \mathrm{E}-05$ & $3.1 \mathrm{E}-\mathrm{C}$ & $4.1 \mathrm{E}-05$ & $4.4 \mathrm{E}-$ & $4.2 \mathrm{E}-05$ & $3.5 \mathrm{E}-05$ & & & $3.7 \mathrm{E}-05$ & $3.5 \mathrm{E}-05$ & 3.3E-05 & & & & $4.1 \mathrm{E}-05$ & $4.5 \mathrm{E}-05$ & 12.1 \\
\hline & $1.6 \mathrm{E}-05$ & $1.3 \mathrm{E}-$ & $1.7 \mathrm{E}-05$ & & & $1.5 \mathrm{E}-05$ & & & E-05 & & $1.4 \mathrm{E}-05$ & & & & E-05 & $1.9 \mathrm{E}-05$ & \\
\hline 40.3 & $8.6 \mathrm{E}-06$ & $7.0 \mathrm{E}-0$ & $9.2 \mathrm{E}-06$ & $1.0 \mathrm{E}-$ & $9.5 \mathrm{E}-06$ & $8.0 \mathrm{E}-06$ & $7.5 \mathrm{E}-06$ & $7.2 \mathrm{E}-06$ & 8.4E-06 & $8.0 \mathrm{E}-\mathrm{C}$ & & & & & $9.4 \mathrm{E}-06$ & $1.0 \mathrm{E}-05$ & 40.3 \\
\hline 56.3 & & $4.7 \mathrm{E}-0$ & $6.2 \mathrm{E}-06$ & $6.7 \mathrm{E}-$ & & $5.4 \mathrm{E}-06$ & & & & & & & & & $6.3 \mathrm{E}-06$ & & 56.3 \\
\hline 72.4 & $4.3 \mathrm{E}-06$ & $3.5 \mathrm{E}-06$ & $4.6 \mathrm{E}-06$ & $5.0 \mathrm{E}-06$ & $4.7 \mathrm{E}-06$ & $4.0 \mathrm{E}-06$ & $3.7 \mathrm{E}-06$ & $3.6 \mathrm{E}-06$ & $4.2 \mathrm{E}-06$ & $4.0 \mathrm{E}-06$ & $3.7 \mathrm{E}-06$ & $3.8 \mathrm{E}-06$ & $3.6 \mathrm{E}-06$ & $3.9 \mathrm{E}-06$ & $4.7 \mathrm{E}-06$ & $5.1 \mathrm{E}-06$ & 72.4 \\
\hline
\end{tabular}

Table 4.1-24. 95th Percentile E/Q Values $\left(\mathrm{sec} \mathrm{m}^{-3}\right.$ ) for Acute 61-m Stack Releases from 100-N Area Based on 1983 through 1991 Meteorological Information (Schreckhise et al. 1993)

\begin{tabular}{|c|c|c|c|c|c|c|c|c|c|c|c|c|c|c|c|c|c|}
\hline Distance & & & & & & & or (Wind & om 100-1 & Toward & ection In & icated) & & & Distance & & & \\
\hline$(\mathrm{km})$ & $\mathrm{S}$ & SSW & SW & WSW & W & WNW & NW & NNW & $\mathrm{N}$ & NNE & $\mathrm{NE}$ & ENE & E & ESE & SE & SSE & $(\mathrm{km})$ \\
\hline 0.1 & $1 . \overline{\mathrm{E}-07}$ & $8.4 \mathrm{E}-08$ & $9 . \overline{0 \mathrm{E}-08}$ & $5.3 \mathrm{E}-08$ & $7 . \overline{4 \mathrm{E}-08}$ & $7.5 \mathrm{E}-08$ & $5.4 \mathrm{E}-08$ & $5.8 \mathrm{E}-08$ & $8 . \overline{4 \mathrm{E}-08}$ & $5.1 \mathrm{E}-08$ & $5 . \overline{0 \mathrm{E}-08}$ & $4.7 \mathrm{E}-08$ & $4 . \overline{7 \mathrm{E}-08}$ & $5 . \overline{7 \mathrm{E}-08}$ & $1 . \overline{2 \mathrm{E}-07}$ & $1.4 \mathrm{E}-07$ & 0.1 \\
\hline 0.2 & $2.5 \mathrm{E}-05$ & $1.6 \mathrm{E}-05$ & $1.8 \mathrm{E}-05$ & $1.0 \mathrm{E}-05$ & $1.5 \mathrm{E}-05$ & $1.5 \mathrm{E}-05$ & $1.1 \mathrm{E}-05$ & $1.1 \mathrm{E}-05$ & $1.7 \mathrm{E}-05$ & $9.9 \mathrm{E}-06$ & $9.7 \mathrm{E}-06$ & $8.9 \mathrm{E}-06$ & $8.8 \mathrm{E}-06$ & $1.1 \mathrm{E}-05$ & $2.3 \mathrm{E}-05$ & $2.7 \mathrm{E}-05$ & 0.2 \\
\hline 0.3 & $4.1 \mathrm{E}-05$ & $2.8 \mathrm{E}-05$ & $3.1 \mathrm{E}-05$ & $2.6 \mathrm{E}-05$ & $3.1 \mathrm{E}-05$ & $3.1 \mathrm{E}-05$ & $2.7 \mathrm{E}-05$ & $3.0 \mathrm{E}-05$ & $3.5 \mathrm{E}-05$ & $2.5 \mathrm{E}-05$ & $2.2 \mathrm{E}-05$ & $1.8 \mathrm{E}-05$ & $1.8 \mathrm{E}-05$ & $2.5 \mathrm{E}-05$ & 4.1E-05 & 4.6E-05 & 0.3 \\
\hline 0.4 & $3.7 \mathrm{E}-05$ & $1 \mathrm{E}-05$ & $2.9 \mathrm{E}-05$ & $2.5 \mathrm{E}-05$ & $3.0 \mathrm{E}-05$ & $3.0 \mathrm{E}-05$ & $2.5 \mathrm{E}-05$ & $3.1 \mathrm{E}-05$ & $3.5 \mathrm{E}-05$ & $2.4 \mathrm{E}-05$ & $1.6 \mathrm{E}-05$ & $1.5 \mathrm{E}-05$ & $1.5 \mathrm{E}-05$ & $1.9 \mathrm{E}-05$ & $3.7 \mathrm{E}-05$ & $4.1 \mathrm{E}-05$ & 0.4 \\
\hline 0.6 & $2.8 \mathrm{E}-05$ & $5 \mathrm{~F}_{-}-05$ & & & & $2.0 \mathrm{E}-05$ & & & $2.9 \mathrm{E}-05$ & & & & & & & & 0.6 \\
\hline 0.7 & $4 \mathrm{E}-05$ & $3 \mathrm{E}-$ & $1.4 \mathrm{E}-\mathrm{C}$ & $1.4 \mathrm{E}-$ & & $1.4 \mathrm{E}-05$ & & $1.5 \mathrm{E}-05$ & & & & & & & & & 0.7 \\
\hline 0.8 & $4 \mathrm{E}-05$ & $2.0 \mathrm{E}-05$ & $2.2 \mathrm{E}-05$ & $2.3 \mathrm{E}-05$ & $2.4 \mathrm{E}-05$ & $2.5 \mathrm{E}-05$ & $2.5 \mathrm{E}-05$ & $2.5 \mathrm{E}-05$ & $2.6 \mathrm{E}-05$ & $2.3 \mathrm{E}-05$ & $2.0 \mathrm{E}-05$ & $1.6 \mathrm{E}-05$ & $1.5 \mathrm{E}-05$ & & $2.2 \mathrm{E}-05$ & $2.4 \mathrm{E}-05$ & 0.8 \\
\hline 0 . & & & & & & & & & & & & & & & & & 0.9 \\
\hline 1.0 & & & & & & $2.9 \mathrm{E}-05$ & & & $3.0 \mathrm{E}-05$ & & $2.5 \mathrm{E}-05$ & & E-05 & & $2.5 \mathrm{E}-05$ & -05 & 1.0 \\
\hline 4.0 & $1.7 \mathrm{E}-05$ & $1.7 \mathrm{E}-05$ & $1.8 \mathrm{E}-05$ & $1.8 \mathrm{E}$ & & $1.8 \mathrm{E}-05$ & $1.8 \mathrm{E}-05$ & $1.8 \mathrm{E}-05$ & $1.9 \mathrm{E}-05$ & $1.8 \mathrm{E}-05$ & $1.7 \mathrm{E}-05$ & $1.6 \mathrm{E}-05$ & $1.4 \mathrm{E}-05$ & -05 & $1.7 \mathrm{E}-05$ & E-05 & 4.0 \\
\hline & & $1.4 \mathrm{E}$ & & & & & & & & & & & & & & & \\
\hline 7.2 & $1.3 \mathrm{E}-05$ & $1.2 \mathrm{E}-$ & $1.3 \mathrm{E}$ & $1.3 \mathrm{E}-05$ & $1.3 \mathrm{E}$ & $1.3 \mathrm{E}-05$ & & $1.3 \mathrm{E}-05$ & $1.3 \mathrm{E}-05$ & $1.3 \mathrm{E}-$ & $1.2 \mathrm{E}-05$ & $1.2 \mathrm{E}-05$ & E-05 & -05 & E-05 & $1.3 \mathrm{E}-05$ & 7.2 \\
\hline 12.1 & & & & & & & & & & & & & & & & & 12.1 \\
\hline 24.1 & $5.6 \mathrm{E}-06$ & $5.5 \mathrm{E}-06$ & $5.7 \mathrm{E}-06$ & $5.9 \mathrm{E}-06$ & & $5.6 \mathrm{E}-06$ & & $5.5 \mathrm{E}-06$ & $5.7 \mathrm{E}-06$ & $5.6 \mathrm{E}-06$ & & $5.4 \mathrm{E}-06$ & E-06 & & $5.7 \mathrm{E}-06$ & E-06 & 24.1 \\
\hline 40.3 & $3.6 \mathrm{E}-06$ & & $3.8 \mathrm{E}-06$ & & & 3.7E-06 & & & $3.8 \mathrm{E}-06$ & & & & & & $3.9 \mathrm{E}-06$ & & 40.3 \\
\hline & 2. & & & & & & & & $2.8 \mathrm{E}-06$ & $2.7 \mathrm{E}-06$ & $2.4 \mathrm{E}-$ & & & & $2.9 \mathrm{E}-06$ & & 56.3 \\
\hline 72.4 & $2.1 \mathrm{E}-06$ & $1.9 \mathrm{E}-06$ & $2.2 \mathrm{E}-06$ & $2.6 \mathrm{E}-06$ & $2.5 \mathrm{E}-06$ & $2.1 \mathrm{E}-06$ & $2.0 \mathrm{E}-06$ & $1.9 \mathrm{E}-06$ & $2.2 \mathrm{E}-06$ & $2.1 \mathrm{E}-06$ & $1.9 \mathrm{E}-06$ & $1.8 \mathrm{E}-06$ & $1.7 \mathrm{E}-06$ & $1.8 \mathrm{E}-06$ & $2.3 \mathrm{E}-06$ & $2.6 \mathrm{E}-06$ & 72.4 \\
\hline
\end{tabular}


Table 4.1-25. 95th Percentile E/Q Values $\left(\mathrm{sec} \mathrm{m}^{-3}\right.$ ) for Acute Ground-Level Releases from 200 Areas Based on 1983 through 1991 Meteorological Information (Schreckhise et al. 1993)

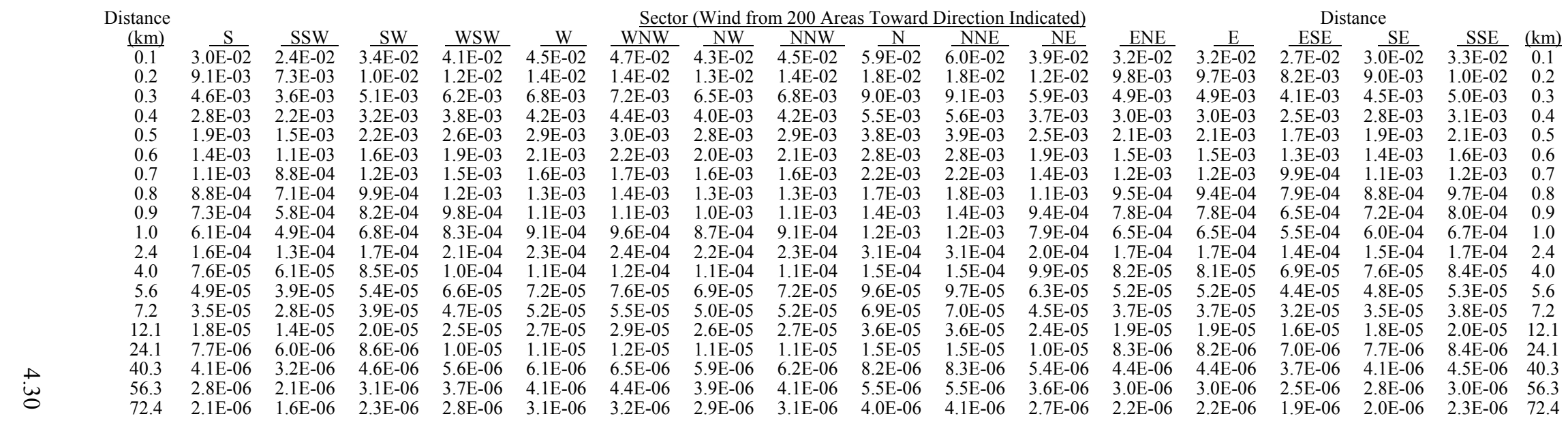

Table 4.1-26. 95th Percentile E/Q Values $\left(\mathrm{sec}^{-3}\right.$ ) for Acute 61-m Stack Releases from 200 Areas Based on 1983 through 1991 Meteorological Information (Schreckhise et al. 1993)

\begin{tabular}{|c|c|c|c|c|c|c|c|c|c|c|c|c|c|c|c|c|c|}
\hline istance & & & & & & & (Wind $\mathrm{f}$ & $\mathrm{n} 200 \mathrm{Ar}$ & as Toward & Direction & ndicated) & & & & & & \\
\hline$(\mathrm{km})$ & $\mathrm{S}$ & SSW & SW & WSW & W & WNW & $\mathrm{NW}$ & NNW & $\mathrm{N}$ & NNE & $\mathrm{NE}$ & ENE & $\mathrm{E}$ & ESE & SE & SSF & $(\mathrm{km})$ \\
\hline 0.1 & $1 . \overline{3 \mathrm{E}-07}$ & $\overline{1.1 \mathrm{E}-07}$ & $1 . \overline{4 \mathrm{E}-07}$ & $1.6 \mathrm{E}-07$ & $1 . \overline{5 \mathrm{E}-07}$ & $1.4 \mathrm{E}-07$ & $1 . \overline{1 \mathrm{E}-07}$ & $5.2 \mathrm{E}-08$ & $5 . \overline{2 \mathrm{E}-08}$ & $5.2 \mathrm{E}-08$ & $4 . \overline{1 \mathrm{E}-08}$ & $3 \overline{3 \mathrm{E}-08}$ & $2 . \overline{0 \mathrm{E}-08}$ & $1 . \overline{5 \mathrm{E}-08}$ & $3 . \overline{6 \mathrm{E}-08}$ & $1 \overline{1 \mathrm{E}-07}$ & 0.1 \\
\hline 0.2 & $2.6 \mathrm{E}-05$ & $2.2 \mathrm{E}-05$ & $2.8 \mathrm{E}-05$ & $3.1 \mathrm{E}-05$ & $3.0 \mathrm{E}-05$ & $2.7 \mathrm{E}-05$ & $2.1 \mathrm{E}-05$ & $1.0 \mathrm{E}-05$ & $1.0 \mathrm{E}-05$ & $1.0 \mathrm{E}-05$ & $6.3 \mathrm{E}-06$ & $6.1 \mathrm{E}-06$ & 4.3E-06 & $3.2 \mathrm{E}-06$ & $6.2 \mathrm{E}-06$ & $2.1 \mathrm{E}-05$ & 0.2 \\
\hline 0.3 & $4.4 \mathrm{E}-05$ & $3.6 \mathrm{E}-05$ & $5.0 \mathrm{E}-05$ & $5.5 \mathrm{E}-05$ & $5.3 \mathrm{E}-05$ & $4.5 \mathrm{E}-05$ & $3.2 \mathrm{E}-05$ & $2.0 \mathrm{E}-05$ & $2.3 \mathrm{E}-05$ & $2.1 \mathrm{E}-05$ & $1.3 \mathrm{E}-05$ & 1.1E-05 & $8.3 \mathrm{E}-06$ & $6.8 \mathrm{E}-06$ & $1.3 \mathrm{E}-05$ & $3.3 \mathrm{E}-05$ & 0.3 \\
\hline 0.4 & $4.0 \mathrm{E}-05$ & $3.7 \mathrm{E}-05$ & $4.3 \mathrm{E}-05$ & $4.5 \mathrm{E}-05$ & 4.3E-05 & 4.1E-05 & $2.8 \mathrm{E}-05$ & $1.6 \mathrm{E}-05$ & $1.6 \mathrm{E}-05$ & $1.6 \mathrm{E}-05$ & $1.2 \mathrm{E}-05$ & $9.2 \mathrm{E}-06$ & 7.6E-06 & $7.0 \mathrm{E}-06$ & $1.4 \mathrm{E}-05$ & $3.1 \mathrm{E}-05$ & 0.4 \\
\hline 0.5 & $3.0 \mathrm{E}-05$ & 0 E-05 & $3.4 \mathrm{E}-05$ & & & 3 F-05 & $2.5 \mathrm{E}-05$ & $14 \mathrm{E}-05$ & $1.6 \mathrm{E}-05$ & & $8.7 \mathrm{E}-06$ & & & $5.0 \mathrm{E}-06$ & & & 0.5 \\
\hline 0. & 7E-05 & $6 \mathrm{E}-$ & $3.7 \mathrm{E}-\mathrm{C}$ & $.1 \mathrm{E}$ & & $3.1 \mathrm{E}-05$ & $1.6 \mathrm{E}-05$ & $1.4 \mathrm{E}$ & $1.4 \mathrm{E}-05$ & $1.4 \mathrm{E}$ & & & & & $1.1 \mathrm{E}-05$ & $1.9 \mathrm{E}-05$ & 0.6 \\
\hline 0.7 & $6 \mathrm{E}-05$ & $.7 \mathrm{E}-05$ & $2.7 \mathrm{E}-0$ & $6 \mathrm{E}$ & $2.9 \mathrm{E}-05$ & $2.3 \mathrm{E}-05$ & & & $1.2 \mathrm{E}-05$ & & & & & $4.4 \mathrm{E}-06$ & & & 0.7 \\
\hline 0.8 & 05 & & $2.7 \mathrm{E}$ & & & & & & & & & & & & & & 0.8 \\
\hline 0.9 & $3 \mathrm{E}-05$ & $2.3 \mathrm{E}-05$ & $2.3 \mathrm{E}-05$ & $2.7 \mathrm{E}$ & 2.3E-05 & 2.3E-05 & $2.2 \mathrm{E}-05$ & $2.0 \mathrm{E}$ & $1.9 \mathrm{E}-05$ & $2.1 \mathrm{E}$ & $1.4 \mathrm{E}-05$ & & E-06 & 7.1E-06 & $5 \mathrm{E}-05$ & $2.2 \mathrm{E}-05$ & 0.9 \\
\hline 1.0 & & & & & & & & & & & & & & & & & 1.0 \\
\hline 4.0 & $1.5 \mathrm{E}-05$ & $1.4 \mathrm{E}-05$ & $1.7 \mathrm{E}-05$ & & & & & & & & & & & & & & 4.0 \\
\hline 5.6 & $1.3 \mathrm{E}-05$ & $1.3 \mathrm{E}-05$ & $1.4 \mathrm{E}-05$ & $1.4 \mathrm{E}$ & $1.4 \mathrm{E}-05$ & $1.4 \mathrm{E}-05$ & $1.4 \mathrm{E}-05$ & $1.5 \mathrm{E}$ & $1.5 \mathrm{E}$ & & & & & & E-05 & $1.4 \mathrm{E}-05$ & 5.6 \\
\hline & $1.0 \mathrm{E}-05$ & $1.0 \mathrm{E}-05$ & $1.2 \mathrm{E}-05$ & & $1.2 \mathrm{E}-05$ & & & & & & & & & & & & 7.2 \\
\hline & & & & & & & & & & & & & & & & & 12.1 \\
\hline & & & & & & & & & & & & & & & & & 24.1 \\
\hline & & $.6 \mathrm{E}$ & & & & & & $4.0 \mathrm{E}$ & & & & & & & & & 40.3 \\
\hline 56.3 & & & $1.3 \mathrm{E}$ & & & & & & & & & & & & & & 56.3 \\
\hline 72.4 & $8.6 \mathrm{E}-07$ & 7.6E-07 & $1.1 \mathrm{E}-06$ & $1.4 \mathrm{E}-06$ & $1.8 \mathrm{E}-06$ & $2.0 \mathrm{E}-06$ & $2.2 \mathrm{E}-06$ & $2.5 \mathrm{E}-06$ & $2.5 \mathrm{E}-06$ & $2.3 \mathrm{E}-06$ & $1.6 \mathrm{E}-06$ & $1.3 \mathrm{E}-06$ & $1.3 \mathrm{E}-06$ & $7.2 \mathrm{E}-07$ & $8.5 \mathrm{E}-07$ & $1.1 \mathrm{E}-06$ & 72.4 \\
\hline
\end{tabular}


Table 4.1-27. 95th Percentile E/Q Values $\left(\mathrm{sec}^{-3}\right.$ ) for Acute Ground-Level Releases from 300 Area Based on 1983 through 1991 Meteorological Information (Schreckhise et al. 1993)

\begin{tabular}{|c|c|c|c|c|c|c|c|c|c|c|c|c|c|c|c|c|c|}
\hline $\begin{array}{c}\text { Distance } \\
(\mathrm{km})\end{array}$ & $\mathrm{S}$ & & CW & WCW & W & Sect & $\mathrm{r}$ (Wind $\mathrm{f}$ & $\mathrm{m} 300 \mathrm{Ar}$ & $\frac{\text { Toward }}{\mathrm{N}}$ & irection I & dicated) & & & $\begin{array}{c}\text { Distance } \\
\text { ESE }\end{array}$ & & & $(\mathrm{km})$ \\
\hline$\frac{(\mathrm{INII1})}{0.1}$ & $3 . \overline{0 \mathrm{E}-02}$ & $2.4 \mathrm{E}-02$ & $3 . \overline{0 \mathrm{E}-02}$ & $2.9 \mathrm{E}-02$ & $3 . \overline{4 \mathrm{E}-02}$ & $3.2 \mathrm{E}-02$ & $3 . \frac{1}{3 \mathrm{E}-02}$ & $5.1 \mathrm{E}-02$ & $4 . \overline{9 \mathrm{E}-02}$ & $3.1 \mathrm{E}-02$ & $3 . \overline{0 \mathrm{E}-02}$ & $3.3 \mathrm{E}-02$ & $6 . \overline{3 \mathrm{E}-02}$ & $\frac{\mathrm{LEL}}{7.4 \mathrm{E}-02}$ & $5 . \overline{6 \mathrm{E}-02}$ & $3.4 \mathrm{E}-02$ & $\frac{(\mathrm{kini})}{0.1}$ \\
\hline 0.2 & $9.2 \mathrm{E}-03$ & $7.3 \mathrm{E}-03$ & $9.2 \mathrm{E}-03$ & $8.7 \mathrm{E}-03$ & $1.0 \mathrm{E}-02$ & $9.8 \mathrm{E}-03$ & $1.0 \mathrm{E}-02$ & $1.5 \mathrm{E}-02$ & $1.5 \mathrm{E}-02$ & $9.3 \mathrm{E}-03$ & $9.0 \mathrm{E}-03$ & $9.9 \mathrm{E}-03$ & $1.9 \mathrm{E}-02$ & $2.2 \mathrm{E}-02$ & $1.7 \mathrm{E}-02$ & $1.0 \mathrm{E}-02$ & 0.2 \\
\hline 0.3 & $4.6 \mathrm{E}-03$ & $3.6 \mathrm{E}-03$ & $4.6 \mathrm{E}-03$ & $4.4 \mathrm{E}-03$ & $5.1 \mathrm{E}-03$ & $4.9 \mathrm{E}-03$ & $5.1 \mathrm{E}-03$ & $7.7 \mathrm{E}-03$ & $7.5 \mathrm{E}-03$ & $4.7 \mathrm{E}-03$ & $4.5 \mathrm{E}-03$ & $5.0 \mathrm{E}-03$ & $9.5 \mathrm{E}-03$ & $1.1 \mathrm{E}-02$ & $8.4 \mathrm{E}-03$ & $5.2 \mathrm{E}-03$ & 0.3 \\
\hline 0.4 & $2.8 \mathrm{E}-03$ & $2.2 \mathrm{E}-03$ & $2.8 \mathrm{E}-03$ & $2.7 \mathrm{E}-03$ & $3.2 \mathrm{E}-03$ & $3.0 \mathrm{E}-03$ & $3.1 \mathrm{E}-03$ & $4.7 \mathrm{E}-03$ & $4.6 \mathrm{E}-03$ & $2.9 \mathrm{E}-03$ & $2.8 \mathrm{E}-03$ & $3.1 \mathrm{E}-03$ & $5.9 \mathrm{E}-03$ & $6.9 \mathrm{E}-03$ & $5.2 \mathrm{E}-03$ & $3.2 \mathrm{E}-03$ & 0.4 \\
\hline 0.5 & $2.0 \mathrm{E}-03$ & $1.5 \mathrm{E}-03$ & $2.0 \mathrm{E}-03$ & $1.8 \mathrm{E}-03$ & $2.2 \mathrm{E}-03$ & $2.1 \mathrm{E}-03$ & $2.1 \mathrm{E}-03$ & $3.3 \mathrm{E}-03$ & $3.2 \mathrm{E}-03$ & $2.0 \mathrm{E}-03$ & $1.9 \mathrm{E}-03$ & $2.1 \mathrm{E}-03$ & $4.0 \mathrm{E}-03$ & & $3.6 \mathrm{E}-03$ & $2.2 \mathrm{E}-03$ & 0.5 \\
\hline 0.6 & $1.4 \mathrm{E}-03$ & $1.1 \mathrm{E}-03$ & $1.4 \mathrm{E}-03$ & $1.4 \mathrm{E}-03$ & $1.6 \mathrm{E}-03$ & $1.5 \mathrm{E}-03$ & $1.6 \mathrm{E}-03$ & $2.4 \mathrm{E}-03$ & $2.3 \mathrm{E}-03$ & $1.5 \mathrm{E}-03$ & $1.4 \mathrm{E}-03$ & $1.6 \mathrm{E}-03$ & $3.0 \mathrm{E}-03$ & $3.5 \mathrm{E}-03$ & $2.6 \mathrm{E}-03$ & $1.6 \mathrm{E}-03$ & 0.6 \\
\hline 0.7 & $1.1 \mathrm{E}-03$ & $8.8 \mathrm{E}-04$ & $1.1 \mathrm{E}-03$ & $1.1 \mathrm{E}-03$ & $1.2 \mathrm{E}-03$ & $1.2 \mathrm{E}-03$ & $1.2 \mathrm{E}-03$ & $1.9 \mathrm{E}-03$ & $1.8 \mathrm{E}-03$ & $1.1 \mathrm{E}-03$ & $1.1 \mathrm{E}-03$ & $1.2 \mathrm{E}-03$ & $2.3 \mathrm{E}-03$ & $2.7 \mathrm{E}-03$ & $2.0 \mathrm{E}-03$ & $1.2 \mathrm{E}-03$ & 0.7 \\
\hline 0.8 & $8.9 \mathrm{E}-04$ & 7.0E-04 & $8.9 \mathrm{E}-04$ & $8.5 \mathrm{E}-04$ & $9.9 \mathrm{E}-04$ & $9.5 \mathrm{E}-04$ & $9.8 \mathrm{E}-04$ & $1.5 \mathrm{E}-03$ & $1.4 \mathrm{E}-03$ & $9.0 \mathrm{E}-04$ & 8.7E-04 & $9.6 \mathrm{E}-04$ & $1.8 \mathrm{E}-03$ & & 1.6E-03 & 1.0E-03 & 0.8 \\
\hline 0.9 & 7.3E-04 & $5.8 \mathrm{E}-04$ & 7.3E-04 & $7.0 \mathrm{E}-04$ & $8.2 \mathrm{E}-04$ & $7.8 \mathrm{E}-04$ & $8.1 \mathrm{E}-04$ & $1.2 \mathrm{E}-03$ & $1.2 \mathrm{E}-03$ & 7.4E-04 & 7.2E-04 & $7.9 \mathrm{E}-04$ & $1.5 \mathrm{E}-03$ & $1.8 \mathrm{E}-03$ & $1.3 \mathrm{E}-03$ & $8.2 \mathrm{E}-04$ & 0.9 \\
\hline 1.0 & $6.2 \mathrm{E}-04$ & $4.9 \mathrm{E}-04$ & $6.2 \mathrm{E}-04$ & $5.8 \mathrm{E}-04$ & $6.9 \mathrm{E}-04$ & $6.5 \mathrm{E}-04$ & & $1.0 \mathrm{E}-03$ & $1.0 \mathrm{E}-03$ & $6.2 \mathrm{E}-04$ & & & & & & $6.9 \mathrm{E}-04$ & 1.0 \\
\hline 2.4 & $1.6 \mathrm{E}-04$ & $1.2 \mathrm{E}-04$ & 1.6E-04 & $1.5 \mathrm{E}-04$ & $1.7 \mathrm{E}-04$ & $1.7 \mathrm{E}-04$ & $1.7 \mathrm{E}-04$ & $2.6 \mathrm{E}-04$ & $2.5 \mathrm{E}-04$ & $1.6 \mathrm{E}-04$ & $1.5 \mathrm{E}-04$ & $1.7 \mathrm{E}-04$ & $3.3 \mathrm{E}-04$ & $3.8 \mathrm{E}-04$ & $2.9 \mathrm{E}-04$ & $1.7 \mathrm{E}-04$ & 2.4 \\
\hline 4.0 & $7.7 \mathrm{E}-05$ & $6.1 \mathrm{E}-05$ & $7.7 \mathrm{E}-05$ & 7.2E-05 & $8.5 \mathrm{E}-05$ & $8.2 \mathrm{E}-05$ & $8.4 \mathrm{E}-05$ & $1.3 \mathrm{E}-04$ & $1.3 \mathrm{E}-04$ & $7.8 \mathrm{E}-05$ & 7.6E-05 & $8.3 \mathrm{E}-$ & & & $1.4 \mathrm{E}-04$ & $8.6 \mathrm{E}-05$ & 4.0 \\
\hline 5.6 & $4.9 \mathrm{E}-05$ & $3.9 \mathrm{E}-05$ & $4.9 \mathrm{E}-05$ & $4.6 \mathrm{E}-05$ & $5.4 \mathrm{E}-05$ & $5.2 \mathrm{E}-05$ & $5.4 \mathrm{E}-05$ & $8.2 \mathrm{E}-05$ & $8.0 \mathrm{E}-05$ & $5.0 \mathrm{E}-05$ & $4.8 \mathrm{E}-05$ & $5.3 \mathrm{E}-05$ & $1.0 \mathrm{E}-04$ & $1.2 \mathrm{E}-04$ & $9.0 \mathrm{E}-05$ & $5.5 \mathrm{E}-05$ & 5.6 \\
\hline 7.2 & $3.5 \mathrm{E}-05$ & $2.8 \mathrm{E}-05$ & $3.5 \mathrm{E}-$ & $3.3 \mathrm{E}$ & $3.9 \mathrm{E}-$ & $3.7 \mathrm{E}-05$ & $3.9 \mathrm{E}$ & $5.9 \mathrm{E}$ & 5.7 & & & & & & & $3.9 \mathrm{E}$ & 7.2 \\
\hline 12.1 & $1.8 \mathrm{E}-05$ & $1.5 \mathrm{E}-05$ & & $1.7 \mathrm{E}-05$ & $2.0 \mathrm{E}-05$ & $2.0 \mathrm{E}-05$ & $2.0 \mathrm{E}-05$ & $3.1 \mathrm{E}-$ & $3.0 \mathrm{E}-05$ & $1.9 \mathrm{E}$ & & $2.0 \mathrm{E}$ & & & 3.4E-05 & $2.0 \mathrm{E}-05$ & 12.1 \\
\hline & $7.8 \mathrm{E}-06$ & $6.2 \mathrm{E}-06$ & $7.8 \mathrm{E}-06$ & $7.2 \mathrm{E}-06$ & $8.6 \mathrm{E}-06$ & $8.3 \mathrm{E}-06$ & & $1.3 \mathrm{E}-$ & $1.3 \mathrm{E}-05$ & $7.9 \mathrm{E}-$ & & $8.4 \mathrm{E}$ & & & & $8.7 \mathrm{E}-06$ & 24.1 \\
\hline 40.3 & $4.2 \mathrm{E}-06$ & 3.3E-06 & $4.2 \mathrm{E}-06$ & $3.8 \mathrm{E}-$ & 4.6E- & 4.4E-06 & $4.6 \mathrm{E}$ & $7.0 \mathrm{E}$ & $6.8 \mathrm{E}-06$ & 4.2E-06 & $4.1 \mathrm{E}$ & $4.5 \mathrm{E}$ & & & 7.7E-06 & $4.7 \mathrm{E}-06$ & 40.3 \\
\hline 56.3 & $2.8 \mathrm{E}-06$ & $2.2 \mathrm{E}-06$ & $2.8 \mathrm{E}-\mathrm{C}$ & $2.6 \mathrm{E}-06$ & $3.1 \mathrm{E}-$ & 3.0E-06 & $3.1 \mathrm{E}-$ & $4.7 \mathrm{E}-\mathrm{C}$ & $4.5 \mathrm{E}-06$ & $2.8 \mathrm{E}-$ & $2.7 \mathrm{E}-06$ & $3.0 \mathrm{E}-$ & $5.8 \mathrm{E}-06$ & & $5.1 \mathrm{E}-06$ & $3.1 \mathrm{E}-06$ & 56.3 \\
\hline 72.4 & $2.1 \mathrm{E}-06$ & $1.6 \mathrm{E}-06$ & $2.1 \mathrm{E}-06$ & $1.9 \mathrm{E}-06$ & $2.3 \mathrm{E}-06$ & $2.2 \mathrm{E}-06$ & $2.3 \mathrm{E}-06$ & $3.5 \mathrm{E}-06$ & $3.4 \mathrm{E}-06$ & $2.1 \mathrm{E}-06$ & 2.0E-06 & $2.2 \mathrm{E}-06$ & 4.3E-06 & $5.0 \mathrm{E}-06$ & $3.8 \mathrm{E}-06$ & $2.3 \mathrm{E}-06$ & 72.4 \\
\hline
\end{tabular}

Table 4.1-28. 95th Percentile E/Q Values $\left(\mathrm{sec} \mathrm{m}^{-3}\right.$ ) for Acute 61-m Stack Releases from 300 Area Based on 1983 through 1991 Meteorological Information (Schreckhise et al. 1993)

\begin{tabular}{|c|c|c|c|c|c|c|c|c|c|c|c|c|c|c|c|c|c|}
\hline Distance & & & & & & Sect & (rWind fo & n $300-4$ & Toward & irection I & dicated) & & & Distance & & & \\
\hline$\underline{(\mathrm{km})}$ & $\mathrm{S}$ & SSW & SW & WSW & $\mathrm{W}$ & WNW & NW & NNW & $\mathrm{N}$ & NNE & $\mathrm{NE}$ & ENE & $\mathrm{E}$ & ESE & SE & SSE & $(\mathrm{km})$ \\
\hline$\frac{1.1}{0.1}$ & $2 . \overline{5 \mathrm{E}-08}$ & $4.7 \mathrm{E}-08$ & $1 . \overline{0 \mathrm{E}-07}$ & $1.2 \mathrm{E}-07$ & $1 . \overline{2 \mathrm{E}-07}$ & $5.0 \mathrm{E}-08$ & $4 \overline{2 \mathrm{E}-08}$ & $4.5 \mathrm{E}-08$ & $2 . \overline{9 \mathrm{E}-08}$ & $2.9 \mathrm{E}-08$ & $3 . \overline{0 \mathrm{E}-08}$ & $\overline{3.1 \mathrm{E}-08}$ & $2 . \overline{5 \mathrm{E}-08}$ & $1 . \overline{8 \mathrm{E}-08}$ & $1 . \overline{4 \mathrm{E}-08}$ & $6 . \overline{3 \mathrm{E}-09}$ & $\frac{1}{0.1}$ \\
\hline 0.2 & $5.4 \mathrm{E}-06$ & 7.3E-06 & $2.1 \mathrm{E}-05$ & $2.4 \mathrm{E}-05$ & $2.5 \mathrm{E}-05$ & $9.6 \mathrm{E}-06$ & 7.4E-06 & $8.2 \mathrm{E}-06$ & $6.0 \mathrm{E}-06$ & $6.0 \mathrm{E}-06$ & $6.0 \mathrm{E}-06$ & $6.1 \mathrm{E}-06$ & $5.6 \mathrm{E}-06$ & $4.7 \mathrm{E}-06$ & $3.1 \mathrm{E}-06$ & 3.4E-06 & 0.2 \\
\hline 0.3 & $1.1 \mathrm{E}-05$ & $1.6 \mathrm{E}-05$ & 3.0E-05 & 3.3E-05 & 3.3E-05 & $1.8 \mathrm{E}-05$ & 1.6E-05 & $1.8 \mathrm{E}-05$ & $1.1 \mathrm{E}-05$ & $1.1 \mathrm{E}-05$ & $1.1 \mathrm{E}-05$ & $1.1 \mathrm{E}-05$ & $1.1 \mathrm{E}-05$ & $9.0 \mathrm{E}-06$ & $6.7 \mathrm{E}-06$ & $8.5 \mathrm{E}-06$ & 0.3 \\
\hline 0.4 & $1.0 \mathrm{E}-05$ & $1.5 \mathrm{E}-05$ & 2.1E-05 & 3.1E-05 & $2.9 \mathrm{E}-05$ & $1.5 \mathrm{E}-05$ & $1.5 \mathrm{E}-05$ & 1.6E-05 & $1.2 \mathrm{E}-05$ & $9.4 \mathrm{E}-06$ & 8.7E-06 & $1.0 \mathrm{E}-05$ & $1.1 \mathrm{E}-05$ & $9.0 \mathrm{E}-06$ & $7.5 \mathrm{E}-06$ & $8.8 \mathrm{E}-06$ & 0.4 \\
\hline 0.6 & 7.7E-06 & $1.4 \mathrm{E}-05$ & $1.5 \mathrm{E}-05$ & $2.2 \mathrm{E}-05$ & $1.6 \mathrm{E}-05$ & $1.4 \mathrm{E}-05$ & $1.4 \mathrm{E}-05$ & $1.4 \mathrm{E}-05$ & 7.7E-06 & 7.7E-06 & 7.3E-06 & 7.7E-06 & 7.7E-06 & $7.5 \mathrm{E}-06$ & 7.4E-06 & 7.7E-06 & 0.6 \\
\hline 0.7 & $1.2 \mathrm{E}-05$ & $1.2 \mathrm{E}-05$ & $1.3 \mathrm{E}-05$ & $2.0 \mathrm{E}-05$ & $1.4 \mathrm{E}-05$ & $1.2 \mathrm{E}-05$ & $1.2 \mathrm{E}-05$ & $1.2 \mathrm{E}-05$ & $1.2 \mathrm{E}-05$ & $1.1 \mathrm{E}-05$ & $1.1 \mathrm{E}-05$ & $1.2 \mathrm{E}-05$ & $1.2 \mathrm{E}-05$ & $1.2 \mathrm{E}-05$ & $1.2 \mathrm{E}-05$ & $1.2 \mathrm{E}-05$ & 0.7 \\
\hline 0.8 & $1.2 \mathrm{E}-05$ & $1.4 \mathrm{E}-05$ & $1.6 \mathrm{E}-05$ & $2.3 \mathrm{E}-05$ & $2.0 \mathrm{E}-05$ & $1.6 \mathrm{E}-05$ & $1.5 \mathrm{E}-05$ & $1.7 \mathrm{E}-05$ & $1.5 \mathrm{E}-05$ & $1.0 \mathrm{E}-05$ & $8.4 \mathrm{E}-06$ & $1.2 \mathrm{E}-05$ & $1.7 \mathrm{E}-05$ & $1.7 \mathrm{E}-05$ & $1.5 \mathrm{E}-05$ & $1.5 \mathrm{E}-05$ & 0.8 \\
\hline 0.9 & $1.1 \mathrm{E}-05$ & $1.4 \mathrm{E}-$ & $2.0 \mathrm{E}-$ & $2.3 \mathrm{E}$ & $2.2 \mathrm{E}-05$ & $1.8 \mathrm{E}-05$ & $1.7 \mathrm{E}-05$ & $2.0 \mathrm{E}-05$ & $1.7 \mathrm{E}-05$ & & & & & & $1.7 \mathrm{E}-05$ & & 0.9 \\
\hline 1.0 & $1.1 \mathrm{E}-05$ & $1.4 \mathrm{E}$ & & $2.6 \mathrm{E}$ & $2.4 \mathrm{E}-05$ & $2.2 \mathrm{E}$ & $2.0 \mathrm{E}-05$ & & & & & & & & & & 1.0 \\
\hline 2.4 & $1.2 \mathrm{E}-05$ & $1.4 \mathrm{E}-05$ & 1.6E-05 & $2.3 \mathrm{E}-05$ & $2.0 \mathrm{E}-05$ & $1.6 \mathrm{E}-05$ & $1.5 \mathrm{E}-05$ & $1.7 \mathrm{E}-05$ & $1.5 \mathrm{E}-05$ & $1.0 \mathrm{E}-05$ & 8.3E-06 & & $1.7 \mathrm{E}-05$ & -05 & $1.5 \mathrm{E}-05$ & $1.5 \mathrm{E}-05$ & 2.4 \\
\hline 4.0 & $1.2 \mathrm{E}-05$ & & & & & & & & & & & & & & & & 4.0 \\
\hline 5.6 & $1.3 \mathrm{E}-05$ & $1.3 \mathrm{E}-05$ & 1.4E-05 & $1.4 \mathrm{E}-05$ & 1.6E-05 & 1.6E-05 & $1.6 \mathrm{E}-05$ & $1.7 \mathrm{E}-05$ & 1.6E-05 & & & & $1.8 \mathrm{E}-05$ & 1.8 & $1.6 \mathrm{E}-05$ & $1.5 \mathrm{E}-05$ & 5.6 \\
\hline 7.2 & & & & & & & & & & & & & & & & & 7.2 \\
\hline 12.1 & $7.3 \mathrm{E}-06$ & $7.1 \mathrm{E}-$ & $9.5 \mathrm{E}-06$ & $1.0 \mathrm{E}-05$ & $1.1 \mathrm{E}-05$ & $1.2 \mathrm{E}-05$ & $1.2 \mathrm{E}-05$ & $1.3 \mathrm{E}-05$ & $1.2 \mathrm{E}-05$ & $9.9 \mathrm{E}-06$ & 8.7E-06 & $1.1 \mathrm{E}-05$ & $1.3 \mathrm{E}-05$ & $1.3 \mathrm{E}-05$ & $1.2 \mathrm{E}-05$ & $1.1 \mathrm{E}-05$ & 12.1 \\
\hline 24.1 & $5.7 \mathrm{E}-06$ & $5.0 \mathrm{E}-$ & & $6.5 \mathrm{E}$ & $6.6 \mathrm{E}-06$ & 7.6E-06 & 7.7E-06 & $8.5 \mathrm{E}-06$ & $7.8 \mathrm{E}-06$ & & & & $8.2 \mathrm{E}-06$ & & 7.6E-06 & & 24.1 \\
\hline 40.3 & 2.6E-06 & $2.3 \mathrm{E}-$ & $2.9 \mathrm{E}-06$ & $3.2 \mathrm{E}$ & $4.5 \mathrm{E}-06$ & $5.5 \mathrm{E}-06$ & $5.6 \mathrm{E}-06$ & $5.7 \mathrm{E}-06$ & $5.6 \mathrm{E}-06$ & $3.6 \mathrm{E}$ & $2.9 \mathrm{E}-06$ & $3.9 \mathrm{E}-$ & $5.7 \mathrm{E}-06$ & $5.8 \mathrm{E}-06$ & $5.5 \mathrm{E}-06$ & 4.4E-06 & 40.3 \\
\hline 56.3 & $1.6 \mathrm{E}-06$ & $1.6 \mathrm{E}-06$ & $1.6 \mathrm{E}-06$ & $1.8 \mathrm{E}-0$ & $2.7 \mathrm{E}-06$ & $3.4 \mathrm{E}-06$ & $3.5 \mathrm{E}-06$ & $3.8 \mathrm{E}-06$ & & & & & & & $3.4 \mathrm{E}-06$ & & 56.3 \\
\hline 72.4 & $1.1 \mathrm{E}-06$ & $1.0 \mathrm{E}-06$ & $1.1 \mathrm{E}-06$ & $1.1 \mathrm{E}-06$ & $1.6 \mathrm{E}-06$ & $2.4 \mathrm{E}-06$ & $2.6 \mathrm{E}-06$ & $2.8 \mathrm{E}-06$ & $2.6 \mathrm{E}-06$ & $1.2 \mathrm{E}-06$ & $1.1 \mathrm{E}-06$ & $1.2 \mathrm{E}-06$ & $2.8 \mathrm{E}-06$ & $3.0 \mathrm{E}-06$ & $2.4 \mathrm{E}-06$ & $1.8 \mathrm{E}-06$ & 72.4 \\
\hline
\end{tabular}


Table 4.1-29. 95th Percentile E/Q Values $\left(\mathrm{sec}^{-3}\right.$ ) for Acute Ground-Level Releases from 400 Area Based on 1983 through 1991 Meteorological Information (Schreckhise et al. 1993)

\begin{tabular}{|c|c|c|c|c|c|c|c|c|c|c|c|c|c|c|c|c|c|c|}
\hline & $\begin{array}{c}\text { Distance } \\
(\mathrm{km})\end{array}$ & & & & & & $\frac{\text { Sect }}{\text { NW }}$ & $\mathrm{r}$ (Wind fi & $\frac{\mathrm{m} 400 \mathrm{Ar}}{\mathrm{NNW}}$ & a Toward & $\frac{\text { irection I }}{\text { NNF }}$ & $\frac{\text { dicated) }}{\mathrm{NF}}$ & & & $\begin{array}{c}\text { Distance } \\
\text { FSF }\end{array}$ & & & \\
\hline & $\frac{(\mathrm{km})}{0.1}$ & $3 . \frac{\mathrm{S}}{4 \mathrm{E}-02}$ & $\frac{\mathrm{SSW}}{3.2 \mathrm{E}-02}$ & $3 . \frac{\mathrm{SW}}{3 \mathrm{E}-02}$ & $\frac{\text { WSW }}{3.7 \mathrm{E}-02}$ & $4 \frac{W}{6 \mathrm{E}-02}$ & $\frac{\text { WNW }}{3.6 \mathrm{E}-02}$ & $\frac{\mathrm{NW}}{32 \mathrm{E}-02}$ & $\frac{\mathrm{NNW}}{29 \mathrm{E}-02}$ & $2 \frac{\mathrm{N}}{9 \mathrm{E}-02}$ & $\frac{\text { NNE }}{19 \mathrm{E}-02}$ & $\frac{\mathrm{NE}}{1 \mathrm{E}-02}$ & $\frac{\mathrm{ENE}}{33 \mathrm{E}-02}$ & $\frac{E}{4 \mathrm{E}-02}$ & $\frac{\mathrm{ESE}}{30 \mathrm{E}-02}$ & $\frac{S E}{7 \mathrm{E}-02}$ & $\frac{\mathrm{SSE}}{3 \mathrm{E}-02}$ & $\frac{(\mathrm{km})}{0.1}$ \\
\hline & 0.2 & $1.0 \mathrm{E}-02$ & $9.7 \mathrm{E}-03$ & $9.9 \mathrm{E}-03$ & $1.1 \mathrm{E}-02$ & $1.4 \mathrm{E}-02$ & $1.1 \mathrm{E}-02$ & $9.7 \mathrm{E}-03$ & $8.6 \mathrm{E}-03$ & $8.9 \mathrm{E}-03$ & $5.9 \mathrm{E}-03$ & $9.2 \mathrm{E}-03$ & $1.0 \mathrm{E}-02$ & $1.0 \mathrm{E}-02$ & $9.0 \mathrm{E}-03$ & $8.2 \mathrm{E}-03$ & $9.5 \mathrm{E}-03$ & 0.2 \\
\hline & 0.3 & $5.1 \mathrm{E}-03$ & $4.9 \mathrm{E}-03$ & $5.0 \mathrm{E}-03$ & $5.6 \mathrm{E}-03$ & $7.0 \mathrm{E}-03$ & $5.4 \mathrm{E}-03$ & $4.9 \mathrm{E}-03$ & $4.3 \mathrm{E}-03$ & $4.5 \mathrm{E}-03$ & $2.9 \mathrm{E}-03$ & 4.6E-03 & $5.0 \mathrm{E}-03$ & $5.2 \mathrm{E}-03$ & $4.5 \mathrm{E}-03$ & 4.1E-03 & $4.8 \mathrm{E}-03$ & 0.3 \\
\hline & 0.4 & $3.2 \mathrm{E}-03$ & $3.0 \mathrm{E}-03$ & $3.1 \mathrm{E}-03$ & $3.4 \mathrm{E}-03$ & $4.3 \mathrm{E}-03$ & $3.3 \mathrm{E}-03$ & $3.0 \mathrm{E}-03$ & $2.7 \mathrm{E}-03$ & $2.7 \mathrm{E}-03$ & $1.8 \mathrm{E}-03$ & $2.8 \mathrm{E}-03$ & $3.1 \mathrm{E}-03$ & $3.2 \mathrm{E}-03$ & $2.8 \mathrm{E}-03$ & $2.5 \mathrm{E}-03$ & $3.0 \mathrm{E}-03$ & 0.4 \\
\hline & 0.5 & $2.2 \mathrm{E}-03$ & $2.1 \mathrm{E}-03$ & $2.1 \mathrm{E}-03$ & $2.4 \mathrm{E}-03$ & $3.0 \mathrm{E}-03$ & $2.3 \mathrm{E}-03$ & $2.1 \mathrm{E}-03$ & $1.8 \mathrm{E}-03$ & $1.9 \mathrm{E}-03$ & $1.2 \mathrm{E}-03$ & $2.0 \mathrm{E}-03$ & $2.1 \mathrm{E}-03$ & $2.2 \mathrm{E}-03$ & $1.9 \mathrm{E}-03$ & $1.7 \mathrm{E}-03$ & $2.0 \mathrm{E}-03$ & 0.5 \\
\hline & 0.6 & $1.6 \mathrm{E}-03$ & $1.5 \mathrm{E}-03$ & $1.6 \mathrm{E}-03$ & $1.7 \mathrm{E}-03$ & $2.2 \mathrm{E}-03$ & $1.7 \mathrm{E}-03$ & $1.5 \mathrm{E}-03$ & $1.3 \mathrm{E}-03$ & $1.4 \mathrm{E}-03$ & $9.2 \mathrm{E}-04$ & $1.4 \mathrm{E}-03$ & $1.6 \mathrm{E}-03$ & $1.6 \mathrm{E}-03$ & $1.4 \mathrm{E}-03$ & $1.3 \mathrm{E}-03$ & $1.5 \mathrm{E}-03$ & 0.6 \\
\hline & 0.7 & $1.2 \mathrm{E}-03$ & $1.2 \mathrm{E}-03$ & $1.2 \mathrm{E}-03$ & $1.3 \mathrm{E}-03$ & $1.7 \mathrm{E}-03$ & $1.3 \mathrm{E}-03$ & $1.2 \mathrm{E}-03$ & $1.0 \mathrm{E}-03$ & $1.1 \mathrm{E}-03$ & 7.1E-04 & $1.1 \mathrm{E}-03$ & $1.2 \mathrm{E}-03$ & $1.3 \mathrm{E}-03$ & $1.1 \mathrm{E}-03$ & $9.9 \mathrm{E}-04$ & $1.2 \mathrm{E}-03$ & 0.7 \\
\hline & 0.8 & $9.9 \mathrm{E}-04$ & $9.4 \mathrm{E}-04$ & $9.6 \mathrm{E}-04$ & $1.1 \mathrm{E}-03$ & $1.4 \mathrm{E}-03$ & $1.0 \mathrm{E}-03$ & $9.4 \mathrm{E}-04$ & $8.3 \mathrm{E}-04$ & $8.6 \mathrm{E}-04$ & $5.7 \mathrm{E}-04$ & $8.9 \mathrm{E}-04$ & $9.7 \mathrm{E}-04$ & $1.0 \mathrm{E}-03$ & $8.8 \mathrm{E}-04$ & 7.9E-04 & $9.3 \mathrm{E}-04$ & 0.8 \\
\hline & 0.9 & $8.2 \mathrm{E}-04$ & 7.7E-04 & $7.9 \mathrm{E}-04$ & $8.8 \mathrm{E}-04$ & $1.1 \mathrm{E}-03$ & $8.6 \mathrm{E}-04$ & 7.7E-04 & $6.9 \mathrm{E}-04$ & 7.1E-04 & $4.7 \mathrm{E}-04$ & 7.3E-04 & $7.9 \mathrm{E}-04$ & 8.3E-04 & $7.2 \mathrm{E}-04$ & $6.5 \mathrm{E}-04$ & 7.6E-04 & 0.9 \\
\hline & 1.0 & $6.9 \mathrm{E}-04$ & $6.5 \mathrm{E}-04$ & $6.7 \mathrm{E}-04$ & & $9.4 \mathrm{E}-\mathrm{C}$ & $7.2 \mathrm{E}-04$ & $6.5 \mathrm{E}-$ & $5.8 \mathrm{E}-04$ & $5.9 \mathrm{E}-04$ & $3.9 \mathrm{E}-04$ & & $6.7 \mathrm{E}-04$ & $6.9 \mathrm{E}-04$ & & $5.5 \mathrm{E}-04$ & $6.4 \mathrm{E}$ & 1.0 \\
\hline & 2.4 & $1.7 \mathrm{E}-04$ & $1.6 \mathrm{E}-04$ & $1.7 \mathrm{E}-04$ & $1.9 \mathrm{E}-04$ & $2.4 \mathrm{E}-04$ & $1.8 \mathrm{E}-04$ & $1.7 \mathrm{E}-04$ & $1.5 \mathrm{E}-04$ & $1.5 \mathrm{E}-04$ & $1.0 \mathrm{E}-04$ & $1.6 \mathrm{E}-04$ & $1.7 \mathrm{E}-04$ & $1.8 \mathrm{E}-04$ & $1.5 \mathrm{E}-04$ & $1.4 \mathrm{E}-04$ & $1.6 \mathrm{E}-04$ & 2.4 \\
\hline & 4.0 & $8.5 \mathrm{E}-05$ & & $8.3 \mathrm{E}-05$ & & & & & & & & & & & & & & 4.0 \\
\hline & 5.6 & $5.4 \mathrm{E}-05$ & $5.2 \mathrm{E}-05$ & $5.3 \mathrm{E}-05$ & $5.9 \mathrm{E}-05$ & $7.5 \mathrm{E}-05$ & $5.7 \mathrm{E}-05$ & $5.2 \mathrm{E}-05$ & $4.6 \mathrm{E}-05$ & $4.8 \mathrm{E}-05$ & $3.1 \mathrm{E}-05$ & $4.9 \mathrm{E}-05$ & $5.3 \mathrm{E}-05$ & $5.5 \mathrm{E}-05$ & $4.8 \mathrm{E}-05$ & $4.4 \mathrm{E}-05$ & $5.1 \mathrm{E}-05$ & 5.6 \\
\hline & 7.2 & $3.9 \mathrm{E}-05$ & $3.7 \mathrm{E}-05$ & $3.8 \mathrm{E}-05$ & & $5.4 \mathrm{E}-05$ & & & $3.3 \mathrm{E}-$ & & & & & & & & & 7.2 \\
\hline & 12.1 & $2.0 \mathrm{E}-05$ & $1.9 \mathrm{E}-05$ & $2.0 \mathrm{E}-05$ & $2.2 \mathrm{E}-05$ & $2.8 \mathrm{E}-05$ & $2.1 \mathrm{E}-05$ & $1.9 \mathrm{E}-05$ & $1.7 \mathrm{E}-05$ & $1.8 \mathrm{E}-05$ & $1.2 \mathrm{E}-05$ & $1.8 \mathrm{E}-05$ & $2.0 \mathrm{E}-05$ & $2.1 \mathrm{E}-05$ & $1.8 \mathrm{E}-05$ & $1.6 \mathrm{E}-05$ & $1.9 \mathrm{E}-05$ & 12.1 \\
\hline & 24.1 & $8.6 \mathrm{E}-06$ & $8.2 \mathrm{E}-06$ & $8.4 \mathrm{E}-06$ & $9.3 \mathrm{E}-06$ & & $9.1 \mathrm{E}-06$ & $8.2 \mathrm{E}-06$ & 7.3E-06 & & & & & & & & & 24.1 \\
\hline & 40.3 & $4.6 \mathrm{E}-06$ & $4.4 \mathrm{E}-06$ & $4.5 \mathrm{E}-06$ & $5.0 \mathrm{E}-06$ & $6.4 \mathrm{E}-$ & $4.9 \mathrm{E}-06$ & $4.4 \mathrm{E}-$ & $3.9 \mathrm{E}-06$ & $4.1 \mathrm{E}-06$ & $2.7 \mathrm{E}-$ & $4.2 \mathrm{E}-06$ & $4.5 \mathrm{E}-06$ & $4.7 \mathrm{E}-06$ & $4.1 \mathrm{E}-06$ & $3.7 \mathrm{E}-06$ & $4.3 \mathrm{E}-06$ & 40.3 \\
\hline & 56.3 & $3.1 \mathrm{E}-06$ & $2.9 \mathrm{E}-06$ & $3.0 \mathrm{E}-06$ & $3.4 \mathrm{E}-06$ & $4.3 \mathrm{E}-$ & $3.3 \mathrm{E}-06$ & $2.9 \mathrm{E}-$ & 2.6E-06 & $2.7 \mathrm{E}-06$ & $1.8 \mathrm{E}-$ & & 3.0E-06 & $3.1 \mathrm{E}-06$ & & $2.5 \mathrm{E}-06$ & $2.9 \mathrm{E}-06$ & 56.3 \\
\hline & 72.4 & $2.3 \mathrm{E}-06$ & $2.2 \mathrm{E}-06$ & $2.2 \mathrm{E}-06$ & $2.5 \mathrm{E}-06$ & $3.2 \mathrm{E}-06$ & $2.4 \mathrm{E}-06$ & 2.2E-06 & $1.9 \mathrm{E}-06$ & $2.0 \mathrm{E}-06$ & $1.3 \mathrm{E}-06$ & $2.1 \mathrm{E}-06$ & $2.2 \mathrm{E}-06$ & $2.3 \mathrm{E}-06$ & $2.0 \mathrm{E}-06$ & $1.9 \mathrm{E}-06$ & $2.2 \mathrm{E}-06$ & 72.4 \\
\hline
\end{tabular}

Table 4.1-30. 95th Percentile E/Q Values $\left(\mathrm{sec} \mathrm{m}^{-3}\right.$ ) for Acute 30-m Stack Releases from 400 Area Based on 1983 through 1991 Meteorological Information (Schreckhise et al. 1993)

\begin{tabular}{|c|c|c|c|c|c|c|c|c|c|c|c|c|c|c|c|c|c|}
\hline Distance & & & & & & & (Wind $\mathrm{fr}$ & $\mathrm{n} 400 \mathrm{Ar}$ & Toward & irection I & dicated) & & & Distance & & & \\
\hline$(\mathrm{km})$ & $\mathrm{S}$ & SSW & SW & WSW & $\mathrm{W}$ & WNW & NW & NNW & $\mathrm{N}$ & NNE & $\mathrm{NE}$ & ENE & $\mathrm{E}$ & ESE & & SSE & $(\mathrm{km})$ \\
\hline$\frac{1}{0.1}$ & $3 . \overline{6 \mathrm{E}-05}$ & $3.7 \mathrm{E}-05$ & $3 . \overline{7 \mathrm{E}-05}$ & $5.9 \mathrm{E}-05$ & $5 . \overline{9 \mathrm{E}-05}$ & $6.8 \mathrm{E}-05$ & $3.7 \mathrm{E}-05$ & $3.2 \mathrm{E}-05$ & $3 . \overline{3 \mathrm{E}-05}$ & $2.7 \mathrm{E}-05$ & $2 . \overline{3 \mathrm{E}-05}$ & $3.0 \mathrm{E}-05$ & $3 . \overline{2 \mathrm{E}-05}$ & $2.1 \mathrm{E}-05$ & $1 . \overline{6 \mathrm{E}-05}$ & 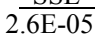 & $\frac{1}{0.1}$ \\
\hline 0.2 & $5.3 \mathrm{E}-05$ & $5.4 \mathrm{E}-05$ & $8.3 \mathrm{E}-05$ & $1.5 \mathrm{E}-04$ & $1.5 \mathrm{E}-04$ & $1.5 \mathrm{E}-04$ & 8.1E-05 & $5.2 \mathrm{E}-05$ & $5.1 \mathrm{E}-05$ & 4.0E-05 & 3.6E-05 & $5.1 \mathrm{E}-05$ & $5.2 \mathrm{E}-05$ & 3.6E-05 & 3.1E-05 & $5.0 \mathrm{E}-05$ & 0.2 \\
\hline 0.3 & $4.9 \mathrm{E}-05$ & $4.9 \mathrm{E}-05$ & $6.9 \mathrm{E}-05$ & $1.1 \mathrm{E}-04$ & $1.2 \mathrm{E}-04$ & $1.1 \mathrm{E}-04$ & $7.0 \mathrm{E}-05$ & 4.8E-05 & 4.7E-05 & 4.6E-05 & 4.7E-05 & 4.8E-05 & 4.8E-05 & 4.7E-05 & 4.6E-05 & $4.8 \mathrm{E}-05$ & 0.3 \\
\hline 0.4 & $8.5 \mathrm{E}-05$ & $8.1 \mathrm{E}-05$ & $9.2 \mathrm{E}-05$ & $9.6 \mathrm{E}-05$ & $9.7 \mathrm{E}-05$ & $9.5 \mathrm{E}-05$ & $9.2 \mathrm{E}-05$ & $6.3 \mathrm{E}-05$ & $4.5 \mathrm{E}-05$ & $3.5 \mathrm{E}-05$ & $4.8 \mathrm{E}-05$ & $6.7 \mathrm{E}-05$ & 7.7E-05 & $5.3 \mathrm{E}-05$ & $4.5 \mathrm{E}-05$ & 7.4E-05 & 0.4 \\
\hline 0.6 & $9.9 \mathrm{E}-05$ & $9.4 \mathrm{E}-05$ & $1.1 \mathrm{E}-04$ & $1.1 \mathrm{E}-04$ & $1.1 \mathrm{E}-04$ & $1.1 \mathrm{E}-04$ & $1.0 \mathrm{E}-04$ & $8.5 \mathrm{E}-05$ & 7.0E-05 & $5.9 \mathrm{E}-05$ & $8.2 \mathrm{E}-05$ & $9.3 \mathrm{E}-05$ & $9.6 \mathrm{E}-05$ & $8.4 \mathrm{E}-05$ & 7.4E-05 & $9.5 \mathrm{E}-05$ & 0.6 \\
\hline 0.7 & $9.6 \mathrm{E}-05$ & $9.0 \mathrm{E}-05$ & $1.0 \mathrm{E}-04$ & $1.0 \mathrm{E}-04$ & $1.0 \mathrm{E}-04$ & $1.0 \mathrm{E}-04$ & $9.9 \mathrm{E}-05$ & $8.6 \mathrm{E}-05$ & $8.0 \mathrm{E}-05$ & 7.6E-05 & $8.6 \mathrm{E}-05$ & $9.5 \mathrm{E}-05$ & $9.6 \mathrm{E}-05$ & $8.8 \mathrm{E}-05$ & $8.1 \mathrm{E}-05$ & $9.5 \mathrm{E}-05$ & 0.7 \\
\hline 0.8 & $9.3 \mathrm{E}-05$ & $9.1 \mathrm{E}-05$ & $9.6 \mathrm{E}-05$ & $9.7 \mathrm{E}-05$ & $9.7 \mathrm{E}-05$ & $9.7 \mathrm{E}-05$ & $9.5 \mathrm{E}-05$ & $8.9 \mathrm{E}-05$ & $8.4 \mathrm{E}-05$ & & $8.9 \mathrm{E}-05$ & $9.3 \mathrm{E}-05$ & $9.3 \mathrm{E}-05$ & $9.0 \mathrm{E}-05$ & $8.6 \mathrm{E}-05$ & $9.3 \mathrm{E}-05$ & 0.8 \\
\hline 0.9 & $8.8 \mathrm{E}-05$ & $8.8 \mathrm{E}-05$ & 8.9E-05 & 8.9E-05 & $8.9 \mathrm{E}-05$ & $8.9 \mathrm{E}-05$ & $8.9 \mathrm{E}-05$ & $8.8 \mathrm{E}-05$ & $8.5 \mathrm{E}-05$ & $6.0 \mathrm{E}-05$ & $8.8 \mathrm{E}-05$ & $8.8 \mathrm{E}-05$ & $8.8 \mathrm{E}-05$ & $8.8 \mathrm{E}-05$ & $8.8 \mathrm{E}-05$ & $8.8 \mathrm{E}-05$ & 0.9 \\
\hline 1.0 & $8.4 \mathrm{E}-05$ & $8.2 \mathrm{E}-05$ & $8.5 \mathrm{E}-05$ & & & $8.7 \mathrm{E}-05$ & & & & & & & & & $7.7 \mathrm{E}-05$ & $8.4 \mathrm{E}-05$ & 1.0 \\
\hline 2.4 & 4.7E-05 & 4.4E-05 & 4.8E-05 & $5.1 \mathrm{E}-05$ & $5.2 \mathrm{E}-05$ & $5.0 \mathrm{E}-05$ & 4.6E-05 & 3.6E-05 & $3.9 \mathrm{E}-05$ & $3.1 \mathrm{E}$ & $4.5 \mathrm{E}-05$ & $4.9 \mathrm{E}-05$ & 4.9E-05 & 4.4E-05 & $3.4 \mathrm{E}-05$ & 4.5E-05 & 2.4 \\
\hline 4.0 & & & & & & & & & & & & & & & & & 4.0 \\
\hline 5.6 & $2.5 \mathrm{E}-05$ & $2.1 \mathrm{E}-05$ & 2.3E-05 & 2.6E-05 & 2.6E-05 & 2.6E-05 & 2.2E-05 & $1.5 \mathrm{E}-05$ & $1.7 \mathrm{E}-05$ & $1.3 \mathrm{E}-05$ & 2.0E-05 & 2.4E-05 & $2.5 \mathrm{E}-05$ & $2.0 \mathrm{E}-05$ & $1.5 \mathrm{E}-05$ & 2.1E-05 & 5.6 \\
\hline 7.2 & & & & & & 2.0E-05 & & & & & & & & & & & 7.2 \\
\hline 12.1 & 1.1E-05 & 8.6E-06 & 1.0E-05 & $1.1 \mathrm{E}-05$ & $1.2 \mathrm{E}-05$ & $1.1 \mathrm{E}-05$ & $9.5 \mathrm{E}-06$ & $5.8 \mathrm{E}-06$ & $6.4 \mathrm{E}-06$ & $5.8 \mathrm{E}-06$ & $8.5 \mathrm{E}-06$ & $1.0 \mathrm{E}-05$ & 1.1E-05 & 8.0E-06 & $5.8 \mathrm{E}-06$ & $8.8 \mathrm{E}-06$ & 12.1 \\
\hline & $4.8 \mathrm{E}-06$ & & $4.5 \mathrm{E}-06$ & & $5.9 \mathrm{E}-06$ & $5.1 \mathrm{E}-06$ & $4.2 \mathrm{E}-06$ & & $3.2 \mathrm{E}-06$ & & & & & & $2.8 \mathrm{E}-06$ & & 24.1 \\
\hline .3 & $2.7 \mathrm{E}-06$ & $2.3 \mathrm{E}-06$ & $2.5 \mathrm{E}-06$ & $2.9 \mathrm{E}-06$ & $3.3 \mathrm{E}-06$ & $2.8 \mathrm{E}-06$ & $2.4 \mathrm{E}-06$ & $1.7 \mathrm{E}-06$ & $1.8 \mathrm{E}-06$ & $1.5 \mathrm{E}-06$ & $2.1 \mathrm{E}-06$ & $2.5 \mathrm{E}-06$ & $2.8 \mathrm{E}-06$ & E-06 & $1.6 \mathrm{E}-06$ & $2.3 \mathrm{E}-06$ & 40.3 \\
\hline 56.3 & $1.8 \mathrm{E}-06$ & $1.6 \mathrm{E}-06$ & $1.7 \mathrm{E}-06$ & $2.0 \mathrm{E}-\mathrm{C}$ & $2.3 \mathrm{E}-06$ & $1.9 \mathrm{E}-06$ & $1.6 \mathrm{E}-06$ & $1.2 \mathrm{E}-06$ & $1.3 \mathrm{E}-06$ & $9.9 \mathrm{E}-$ & $1.4 \mathrm{E}-06$ & & $1.9 \mathrm{E}-06$ & & $1.1 \mathrm{E}-06$ & $1.6 \mathrm{E}-06$ & 56.3 \\
\hline 72.4 & $1.4 \mathrm{E}-06$ & $1.2 \mathrm{E}-06$ & $1.3 \mathrm{E}-06$ & $1.5 \mathrm{E}-06$ & $1.7 \mathrm{E}-06$ & $1.4 \mathrm{E}-06$ & $1.2 \mathrm{E}-06$ & $9.0 \mathrm{E}-07$ & $9.7 \mathrm{E}-07$ & 7.4E-07 & $1.1 \mathrm{E}-06$ & $1.3 \mathrm{E}-06$ & 1.4E-06 & $1.0 \mathrm{E}-06$ & 8.7E-07 & $1.2 \mathrm{E}-06$ & 72.4 \\
\hline
\end{tabular}




\subsubsection{Nonradiological Air Quality}

The Clean Air Act (CAA) is the basis for federal regulation of air quality in the United States. The CAA was first passed in 1967 and had comprehensive amendments in 1970, 1977, and 1990. Section 108 of the CAA calls for the U.S. Environmental Protection Agency (EPA) to promulgate a list of air pollutants that are emitted by numerous or diverse sources and whose presence in the atmosphere may reasonably be anticipated to endanger public health or welfare. In response to this mandate, EPA has issued regulations in 40 CFR 50 setting national ambient air quality standards. These standards are not directly enforceable, but other enforceable regulations are based on these standards. The states have primary responsibility for ensuring that air quality within the state meets the national ambient air quality standards through state implementation plans (SIPs) that are approved by EPA. Areas that meet ambient air quality standards are said to be "in attainment". Areas that do not meet one or more ambient air standards are designated as "nonattainment areas". The CAA also establishes a permitting program for construction or modification of large sources of air pollutants in both attainment and nonattainment areas and an operating permit program.

Section 176 of the CAA states that federal agencies are not to engage in, support in any way, provide financial assistance for, license, permit, or approve any activity that does not conform to an applicable SIP. The DOE has guidance (DOE 2000a) on how to apply the CAA conformity requirements and associated EPA regulations in a NEPA document and how to coordinate the CAA and NEPA public participation requirements.

Ambient air quality standards define levels of air quality that are necessary, with an adequate margin of safety, to protect the public health (primary standards) and the public welfare (secondary standards). "Ambient air" is that portion of the atmosphere, external to buildings, to which the general public has access (40 CFR 50.1). EPA has issued ambient air standards for sulfur oxides (measured as sulfur dioxide), nitrogen dioxide, carbon monoxide, particulates with an aerodynamic diameter less than or equal to a nominal 10 micrometers $\left(\mathrm{PM}_{10}\right)$ and 2.5 micrometers $\left(\mathrm{PM}_{2.5}\right)$, lead, and ozone. The standards specify the maximum pollutant concentrations and frequencies of occurrence that are allowed for specific averaging periods. The averaging periods vary from 1 hour to 1 year, depending on the pollutant.

State and local governments have the authority to impose standards for ambient air quality that are stricter than the national standards. Washington State has established more stringent standards for sulfur dioxide. In addition, Washington has established standards for total suspended particulates (WAC 173470), radionuclides (WAC 173-480), and fluorides (WAC 173-481) that are not covered by national standards. The state standards for carbon monoxide, nitrogen dioxide, $\mathrm{PM}_{10}$, and lead are identical to the national standards. Table 4.1-31 summarizes the relevant air quality standards (federal and supplemental state standards).

On July 18, 1997, the EPA issued new air quality standards for particulate matter with a diameter of $2.5 \mu \mathrm{m}$ or less $\left(\mathrm{PM}_{2.5}\right)$ and an 8-hr ozone standard. Decisions on violations of the new particulate matter and ozone standard were to be delayed for 5 to 8 years to give states time to set up monitoring networks and obtain 3 years of data (Ecology 1997). 
Table 4.1-31. National and Washington State Ambient Air Quality Standards ${ }^{(a)}$

\begin{tabular}{|c|c|c|c|}
\hline Pollutant & National Primary & National Secondary & Washington State \\
\hline \multicolumn{4}{|c|}{ Total Suspended Particulates } \\
\hline Annual geometric mean & $\mathrm{NS}^{(\mathrm{b})}$ & NS & $60 \mu \mathrm{g} / \mathrm{m}^{3}$ \\
\hline 24-hr average & NS & NS & $150 \mu \mathrm{g} / \mathrm{m}^{3}$ \\
\hline \multicolumn{4}{|l|}{$\mathbf{P M}_{-10}$} \\
\hline Annual arithmetic mean & $50 \mu \mathrm{g} / \mathrm{m}^{3}$ & $50 \mu \mathrm{g} / \mathrm{m}^{3}$ & $50 \mu \mathrm{g} / \mathrm{m}^{3}$ \\
\hline 24-hr average & $150 \mu \mathrm{g} / \mathrm{m}^{3}$ & $150 \mu \mathrm{g} / \mathrm{m}^{3}$ & $150 \mu \mathrm{g} / \mathrm{m}^{3}$ \\
\hline \multicolumn{4}{|l|}{$\mathbf{P M}_{2.5}$} \\
\hline Annual arithmetic mean & $15 \mu \mathrm{g} / \mathrm{m}^{3}$ & $15 \mu \mathrm{g} / \mathrm{m}^{3}$ & NS \\
\hline 24-hr average & $65 \mu \mathrm{g} / \mathrm{m}^{3}$ & $65 \mu \mathrm{g} / \mathrm{m}^{3}$ & \\
\hline \multicolumn{4}{|l|}{ Sulfur Dioxide } \\
\hline Annual average & $\begin{array}{c}0.03 \mathrm{ppm} \\
\left(\cong 80 \mu \mathrm{g} / \mathrm{m}^{3}\right)\end{array}$ & NS & $\begin{array}{c}0.02 \mathrm{ppm} \\
\left(\cong 50 \mu \mathrm{g} / \mathrm{m}^{3}\right)\end{array}$ \\
\hline 24-hr average & $\begin{array}{c}0.14 \mathrm{ppm} \\
\left(\cong 365 \mu \mathrm{g} / \mathrm{m}^{3}\right)\end{array}$ & NS & $\begin{array}{c}0.10 \mathrm{ppm} \\
\left(\cong 260 \mu \mathrm{g} / \mathrm{m}^{3}\right)\end{array}$ \\
\hline 3-hr average & NS & $\begin{array}{c}0.50 \mathrm{ppm} \\
\left(\cong 1.3 \mathrm{mg} / \mathrm{m}^{3}\right)\end{array}$ & NS \\
\hline 1-hr average & NS & NS & $\begin{array}{c}0.40 \mathrm{ppm} \\
\left(\cong 1.0 \mathrm{mg} / \mathrm{m}^{3}\right)^{(\mathrm{c})}\end{array}$ \\
\hline \multicolumn{4}{|l|}{ Carbon Monoxide } \\
\hline 8-hr average & $\begin{array}{c}9 \mathrm{ppm} \\
\left(\cong 10 \mathrm{mg} / \mathrm{m}^{3}\right)\end{array}$ & $\begin{array}{c}9 \mathrm{ppm} \\
\left(\cong 10 \mathrm{mg} / \mathrm{m}^{3}\right)\end{array}$ & $\begin{array}{c}9 \mathrm{ppm} \\
\left(\cong 10 \mathrm{mg} / \mathrm{m}^{3}\right)\end{array}$ \\
\hline 1-hr average & $\begin{array}{c}35 \mathrm{ppm} \\
\left(\cong 40 \mathrm{mg} / \mathrm{m}^{3}\right)\end{array}$ & $\begin{array}{c}35 \mathrm{ppm} \\
\left(\cong 40 \mathrm{mg} / \mathrm{m}^{3}\right)\end{array}$ & $\begin{array}{c}35 \mathrm{ppm} \\
\left(\cong 40 \mathrm{mg} / \mathrm{m}^{3}\right)\end{array}$ \\
\hline \multicolumn{4}{|l|}{ Ozone } \\
\hline 8-hr average & $\begin{array}{c}0.08 \mathrm{ppm} \\
\left(\sim 157 \mu \mathrm{g} / \mathrm{m}^{3}\right)\end{array}$ & $\begin{array}{c}0.08 \mathrm{ppm} \\
\left(\sim 157 \mu \mathrm{g} / \mathrm{m}^{3}\right)\end{array}$ & NS \\
\hline 1-hr average & $\begin{array}{c}0.12 \mathrm{ppm} \\
\left(\cong 235 \mu \mathrm{g} / \mathrm{m}^{3}\right)\end{array}$ & $\begin{array}{c}0.12 \mathrm{ppm} \\
\left(\cong 235 \mu \mathrm{g} / \mathrm{m}^{3}\right)\end{array}$ & $\begin{array}{c}0.12 \mathrm{ppm} \\
\left(\cong 235 \mu \mathrm{g} / \mathrm{m}^{3}\right)\end{array}$ \\
\hline \multicolumn{4}{|l|}{ Nitrogen Dioxide } \\
\hline Annual average & $\begin{array}{c}0.053 \mathrm{ppm} \\
\left(\cong 100 \mu \mathrm{g} / \mathrm{m}^{3}\right)\end{array}$ & $\begin{array}{c}0.053 \mathrm{ppm} \\
\left(\cong 100 \mu \mathrm{g} / \mathrm{m}^{3}\right)\end{array}$ & $\begin{array}{c}0.053 \mathrm{ppm} \\
\left(\cong 100 \mu \mathrm{g} / \mathrm{m}^{3}\right)\end{array}$ \\
\hline \multicolumn{4}{|l|}{ Lead } \\
\hline Quarterly average & $1.5 \mu \mathrm{g} / \mathrm{m}^{3}$ & $1.5 \mu \mathrm{g} / \mathrm{m}^{3}$ & $1.5 \mu \mathrm{g} / \mathrm{m}^{3}$ \\
\hline Radionuclides & NS & NS & (d) \\
\hline \multicolumn{4}{|l|}{ Fluorides } \\
\hline \multirow{4}{*}{$\begin{array}{l}\text { 12-hr average } \\
\text { 24-hr average } \\
7 \text { day average } \\
30 \text { day average }\end{array}$} & NS & NS & $3.7 \mu \mathrm{g} / \mathrm{m}^{3}$ \\
\hline & & & $2.9 \mu \mathrm{g} / \mathrm{m}^{3}$ \\
\hline & & & $1.7 \mu \mathrm{g} / \mathrm{m}^{3}$ \\
\hline & & & $0.84 \mu \mathrm{g} / \mathrm{m}^{3}$ \\
\hline \multicolumn{4}{|c|}{$\begin{array}{l}\text { Abbreviations: } \mathrm{ppm}=\text { parts per million; } \mu \mathrm{g} / \mathrm{m}^{3}=\text { micrograms per cubic meter; } \mathrm{mg} / \mathrm{m}^{3}=\text { milligrams per cubic meter. } \\
\text { (a) Source: } 40 \mathrm{CFR} 50 \text { and WAC } 173-470-173-481 \text {. Annual standards are never to be exceeded; short-term standards are not to be } \\
\text { exceeded more than once per year unless otherwise noted. Particulate pollutants are in micrograms per cubic meter. Gaseous } \\
\text { pollutants are in parts per million and equivalent microgram (or milligram) per cubic meter. } \\
\text { (b) } \mathrm{NS}=\text { no standard. } \\
\text { (c) } 0.25 \text { ppm not to be exceeded more than twice in any } 7 \text { consecutive days. } \\
\text { (d) Emissions of radionuclides in the air shall not cause a maximum accumulated dose equivalent of more than } 25 \mathrm{mrem} / \mathrm{yr} \text { to the who } \\
\text { body or } 75 \mathrm{mrem} / \mathrm{yr} \text { to a critical organ of any member of the public. Doses due to radon- } 220 \text {, radon- } 222 \text {, and their respective dec }\end{array}$} \\
\hline
\end{tabular}




\subsubsection{Prevention of Significant Deterioration}

Prevention of significant deterioration (PSD) permits are issued to large sources of pollutants subject to ambient air standards in attainment areas. The Plutonium-Uranium Extraction (PUREX) and Uranium Trioxide $\left(\mathrm{UO}_{3}\right)$ facilities were issued a PSD permit for nitrogen oxide emissions in 1980. These facilities were permanently shut down in the late 1980s and deactivated in the 1990s. None of the currently operating Hanford facilities have nonradiological emissions of sufficient magnitude to warrant consideration under PSD regulations.

\subsubsection{Emissions of Nonradiological Pollutants}

Nonradiological pollutants are mainly emitted from power-generating and chemical-processing facilities located on the Hanford Site. Table 4.1-32 summarizes the 1999 emission rates of nonradiological constituents from these facilities. The 100, 400, and 600 Areas have no nonradioactive emission sources of concern (Poston et al. 2000).

Table 4.1-32. Non-radioactive Constituents Discharged to the Atmosphere, $1999^{(a)}$ (Poston et al. 2000)

\begin{tabular}{|c|c|c|}
\hline \multirow[b]{2}{*}{ Constituent } & \multicolumn{2}{|c|}{ Release (kg) } \\
\hline & 200 Areas & 300 Area \\
\hline Particulate matter & 886 & 596 \\
\hline Nitrogen oxides & 24,000 & 3,680 \\
\hline Sulfur oxides & 3,370 & 40 \\
\hline Carbon monoxide & 17,700 & 12,700 \\
\hline Lead & 53 & 0 \\
\hline Volatile organic compounds ${ }^{(b)}$ & 6,920 & 809 \\
\hline Ammonia $^{(\mathrm{c})}$ & 9,810 & $\mathrm{NE}^{(\mathrm{d})}$ \\
\hline Other toxic air pollutants ${ }^{(\mathrm{e})}$ & 2,970 & NE \\
\hline \multicolumn{3}{|c|}{$\begin{array}{l}\text { (a) The estimate of volatile organic compound emissions does not include emissions from certain laboratory operations. } \\
\text { (b) Produced from burning fossil fuels for steam generation and electrical generators, calculated estimates from the 200- } \\
\text { East and } 200 \text {-West Area tank farms, and operation of the } 242-\mathrm{A} \text { Evaporator and the } 200 \text { Areas Effluent Treatment } \\
\text { Facility. } \\
\text { (c) Ammonia releases are from the 200-East Area tank farms, 200-West Area tank farms, and operation of the 242-A } \\
\text { Evaporator, and the } 200 \text { Areas Effluent Treatment Facility. } \\
\text { (d) } \mathrm{NE} \mathrm{=} \mathrm{no} \mathrm{emissions.} \\
\text { (c) Releases are a composite of calculated estimates of toxic air pollutants, excluding ammonia, from the 200-East and 200- } \\
\text { West Area tank farms, and operation of the 242-A Evaporator and the } 200 \text { Areas Effluent Treatment Facility. }\end{array}$} \\
\hline
\end{tabular}

\subsubsection{Offsite Monitoring}

In 1998, the Washington State Department of Ecology conducted offsite monitoring near the Hanford Site for $\mathrm{PM}_{10}$ (Ecology 1999, 2000). $\mathrm{PM}_{10}$ was monitored at one location in Benton County, the Tri-Tech Vocational Center near the Hanford network's Vista Field meteorological monitoring site in Kennewick. During 1998, the 24-hr and annual $\mathrm{PM}_{10}$ standards established by the state of Washington were not exceeded. The highest and second highest 24-hr PM $\mathrm{PM}_{10}$ concentrations recorded in 1998 were $123 \mu \mathrm{g} / \mathrm{m}^{3}$ and $90 \mu \mathrm{g} / \mathrm{m}^{3}$, respectively. The arithmetic mean for 1998 was $18 \mu \mathrm{g} / \mathrm{m}^{3}$ (Ecology 2000). 


\subsubsection{Background Monitoring}

During the last 10 years, carbon monoxide, sulfur dioxide, and nitrogen dioxide have been monitored periodically in communities and commercial areas southeast of Hanford. These urban measurements are typically used to estimate the maximum background pollutant concentrations for the Hanford Site because of the lack of specific onsite monitoring.

Particulate concentrations can reach relatively high levels in eastern Washington because of exceptional natural events (i.e., dust storms and large brushfires) that occur in the region. In June 1996, EPA adopted the policy that allows dust storms to be treated as uncontrollable natural events ${ }^{(a)}$. This means that the EPA will not designate areas affected by dust storms as nonattainment. However, states are required to develop and implement a natural events action plan.

Areas that require more strict controls on air quality impacts are nonattainment areas and certain national parks and wilderness areas called Federal Class I areas. Actions on the Hanford Site are unlikely to produce air quality impacts that significantly affect these areas. The nearest nonattainment area to the Hanford Site is the Wallula area (located approximately $30 \mathrm{~km}$ [20 mi] southeast of the Site), which is a serious nonattainment area for $\mathrm{PM}_{10}$ (40 CFR 81.348, 66 FR 9663). The major source of $\mathrm{PM}_{10}$ in the Wallula area is from windblown dust. In making the nonattainment determination, EPA found that even if some of the data from the Wallula monitoring site are considered uncontrollable natural events and excluded from consideration in determining the air quality status of the area, the remaining data still show that the Wallula area has not attained the $\mathrm{PM}_{10}$ national ambient air quality standard (66 FR 9666).

The nearest Federal Class I areas to the Hanford Site are Mount Rainer National Park, located $160 \mathrm{~km}$ (100 mi) west of the Site; Goat Rocks Wilderness Area, located approximately $145 \mathrm{~km}$ (90 mi) west of the Site; Mount Adams Wilderness Area, located approximately $150 \mathrm{~km}$ (95 mi) southwest of the Site; and Alpine Lakes Wilderness Area, located approximately $175 \mathrm{~km}(110 \mathrm{mi})$ northwest of the Site (40 CFR 81.434). Operations at the Hanford Site have minimal effects on these Class I areas because of their distance from the Site and because topography and prevailing winds tend to keep emissions from sources on the Hanford Site away from the Class 1 areas.

\subsubsection{Radiological Air Quality}

Airborne effluents that may contain radioactive constituents are continually monitored at the Hanford Site. Samples are analyzed for gross alpha and gross beta activity as well as selected radionuclides.

Radioactive emissions during 1999 (the most recent year for which data are published) originated in the 100, 200, 300, and 400 Areas. 100 Area emissions originated from the deactivation of N Reactor, K Basins (irradiated fuel stored in two water-filled storage basins), a re-circulation facility designed to filter radioactive water from the $\mathrm{N}$ Reactor basin, and a radiochemistry laboratory. 200 Area emissions originated from the PUREX Plant, Plutonium Finishing Plant, T Plant, 222-S Laboratory, underground storage tanks, and waste evaporators. Emissions from the 300 Area originated from the 324 Waste Technology Engineering Laboratory, 325 Applied Chemistry Laboratory, 327 Post-Irradiation Laboratory, and 340 Vault and Tanks. 400 Area emissions originated at the Fast Flux Test Facility (FFTF) and Maintenance and Storage Facility (Poston et al. 2000). A summary of radiological air emissions is provided in Table 4.1-33.

(a) "EPA's Natural Events Policy for Particulate Matter," June 6, 1996. Available URL: http://www.epa.gov/ttncaaa1/t1/fact_sheets/nefact.pdf 
Table 4.1-33. Radionuclides Discharged to the Atmosphere at the Hanford Site, 1999 (Poston et al. 2000)

\begin{tabular}{|c|c|c|c|c|c|c|}
\hline \multirow[b]{2}{*}{ Radionuclide } & \multirow[b]{2}{*}{ Half-Life } & \multicolumn{5}{|c|}{ Release, $\mathbf{C i}{ }^{(a)}$} \\
\hline & & 100 Areas & 200 East Area & 200 West Area & 300 Area & 400 Area \\
\hline Tritium (as HTO) $^{(\mathrm{b})}$ & $12.3 \mathrm{yr}$ & $\mathrm{NM}^{(\mathrm{a})}$ & NM & NM & $1.5 \mathrm{E}+02$ & $1.4 \mathrm{E}+00$ \\
\hline Tritium (as HT) ${ }^{(\mathrm{b})}$ & $12.3 \mathrm{yr}$ & NM & NM & NM & $4.0 \mathrm{E}+01$ & NM \\
\hline Cobalt- 60 & $5.3 \mathrm{yr}$ & $3.9 \mathrm{E}-08$ & $1.6 \mathrm{E}-09$ & $\mathrm{ND}^{(\mathrm{a})}$ & ND & NM \\
\hline Strontium-90 & $29.1 \mathrm{yr}$ & $1.9 \mathrm{E}-05$ & $9.6 \mathrm{E}-05^{(\mathrm{c})}$ & $2.9 \mathrm{E}-04$ & $1.0 \mathrm{E}-05^{(\mathrm{c})}$ & NM \\
\hline Ruthenium-106 & $368 \mathrm{~d}$ & ND & ND & NM & ND & NM \\
\hline Antimony-125 & $2.77 \mathrm{yr}$ & $5.0 \mathrm{E}-08$ & ND & NM & $1.1 \mathrm{E}-07$ & NM \\
\hline Iodine-129 & $1.6 \times 10^{7} \mathrm{yr}$ & NM & $1.9 \mathrm{E}-04$ & NM & NM & NM \\
\hline Cesium-134 & $2.1 \mathrm{yr}$ & ND & ND & ND & ND & NM \\
\hline Cesium-137 & $30 \mathrm{yr}$ & $4.5 \mathrm{E}-05$ & $3.9 \mathrm{E}-05$ & $2.5 \mathrm{E}-09$ & $4.2 \mathrm{E}-07$ & $1.9 \mathrm{E}-06^{(\mathrm{d})}$ \\
\hline Plutonium-238 & $87.7 \mathrm{yr}$ & $5.8 \mathrm{E}-07$ & $1.9 \mathrm{E}-08$ & $4.9 \mathrm{E}-06$ & ND & NM \\
\hline Plutonium-239, 240 & $2.4 \times 10^{4} \mathrm{yr}$ & $4.2 \mathrm{E}-06^{(\mathrm{e})}$ & $6.9 \mathrm{E}-07^{(\mathrm{e})}$ & $2.1 \mathrm{E}-04^{(\mathrm{e})}$ & $1.1 \mathrm{E}-06^{(\mathrm{e})}$ & $3.0 \mathrm{E}-07^{(\mathrm{e})}$ \\
\hline Plutonium-241 & $14.4 \mathrm{yr}$ & $5.1 \mathrm{E}-05$ & $1.2 \mathrm{E}-06$ & $1.2 \mathrm{E}-04$ & 7.9E-08 & NM \\
\hline Americium-241 & $432 \mathrm{yr}$ & $2.4 \mathrm{E}-06$ & $5.6 \mathrm{E}-07$ & $4.5 \mathrm{E}-05$ & $1.4 \mathrm{E}-07$ & NM \\
\hline Europium-154 & $8.8 \mathrm{yr}$ & $4.8 \mathrm{E}-08$ & ND & ND & ND & NM \\
\hline \multicolumn{7}{|c|}{$\begin{array}{l}\text { (a) } 1 \mathrm{Ci}=3.7 \mathrm{E} 10 \mathrm{~Bq} ; \mathrm{NM}=\text { not measured; } \mathrm{ND}=\text { not detected (i.e., either the radionuclide was not detected in any sample during the year or } \\
\text { the average of all the measurements for that given radionuclide or type of radioactivity made during the year was below background levels). } \\
\text { (b) } \mathrm{HTO}=\text { tritiated water vapor; } \mathrm{HT}=\text { elemental tritium. } \\
\text { (c) This value includes gross beta release data. Gross beta and unspecified beta results assumed to be strontium- } 90 \text { for dose calculations. } \\
\text { (d) This value includes gross beta release data. Gross beta results assumed to be cesium-137 for dose calculations from Fast Flux Test Facility } \\
\text { emissions. } \\
\text { (c) This value includes gross alpha release data. Gross alpha and unspecified alpha results assumed to be plutonium-239/240 for dose } \\
\text { calculations. }\end{array}$} \\
\hline
\end{tabular}

\subsection{Geology}

\section{S. M. Goodwin and A. C. Rohay}

Geologic considerations for the Hanford Site include physiography, stratigraphy, structural geology, soil characteristics, and seismicity.

\subsubsection{Physiography}

The Hanford Site lies within the Columbia Basin subprovince of the Columbia Intermontane Province (Figure 4.2-1). The Columbia Intermontane Province is the product of Miocene flood basalt volcanism and regional deformation that occurred over the last 17 million years (17 Ma). The Columbia Plateau is that portion of the Columbia Intermontane Province that is underlain by the Columbia River Basalt Group (CRBG) (Thornbury 1965). 


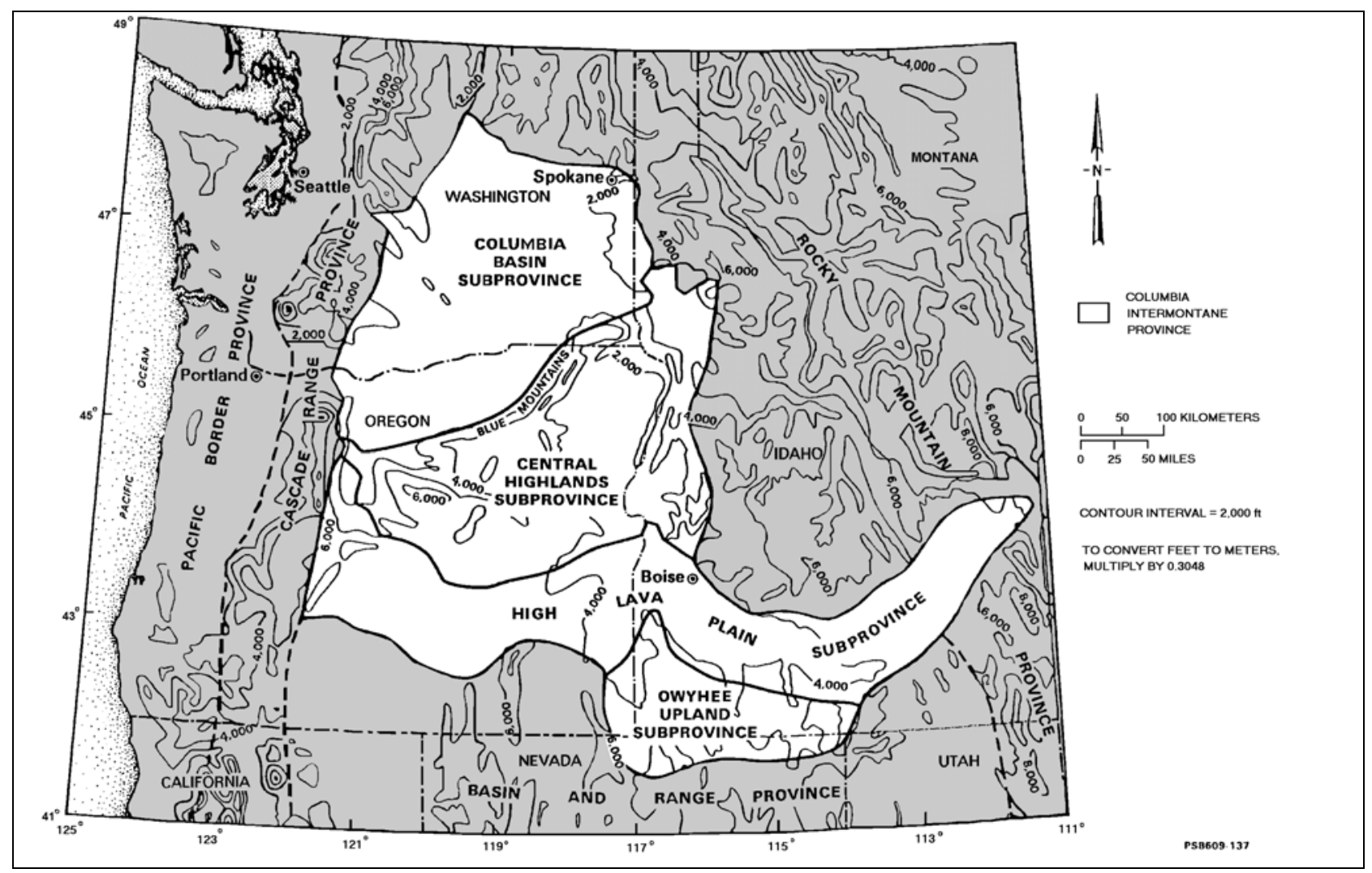

Figure 4.2-1. Physiographic Provinces of the Pacific Northwest, with Columbia Intermontane Province Shown in White (DOE 1988)

The low-relief plains of the Central Plains subprovince and anticlinal ridges of the Yakima Folds physiographic section dominate the physiography of the Hanford Site (DOE 1988). The surface topography has been modified within the past several million years by several geomorphic processes: 1 ) Pleistocene cataclysmic flooding, 2) Holocene eolian activity, and 3) landsliding. Cataclysmic flooding occurred when ice dams in western Montana and northern Idaho were breached, allowing large volumes of water to spill across eastern and central Washington forming the channeled scablands and depositing sediments in the Pasco Basin. The last major flood occurred approximately 13,000 years ago, during the late Pleistocene Epoch. Anastomosing flood channels, giant current ripples, bergmounds, and giant flood bars are among the landforms created by the floods. Waste management facilities in the 200 Area are located on one prominent flood bar, the Cold Creek bar (Figure 4.2-2) (DOE 1988).

Since the end of the Pleistocene, winds have locally reworked the flood sediments, depositing dune sands in the lower elevations and loess (windblown silt) around the margins of the Pasco Basin. Anchoring vegetation has stabilized many sand dunes. Where human activity has disturbed this vegetation, dunes have been reactivated. More recently, dunes have been reactivated by the removal of vegetation resulting from the July 2000 Hanford wildfire.

Landslides occur along the north limbs of some Yakima Folds and along steep river embankments such as the White Bluffs. Landslides on the Yakima Folds occur along contacts between basalt flows or sedimentary units intercalated with the basalt, whereas active landslides at White Bluffs occur in suprabasalt sediments. The active landslides at White Bluffs are principally the result of irrigation activity east of the Columbia River. 


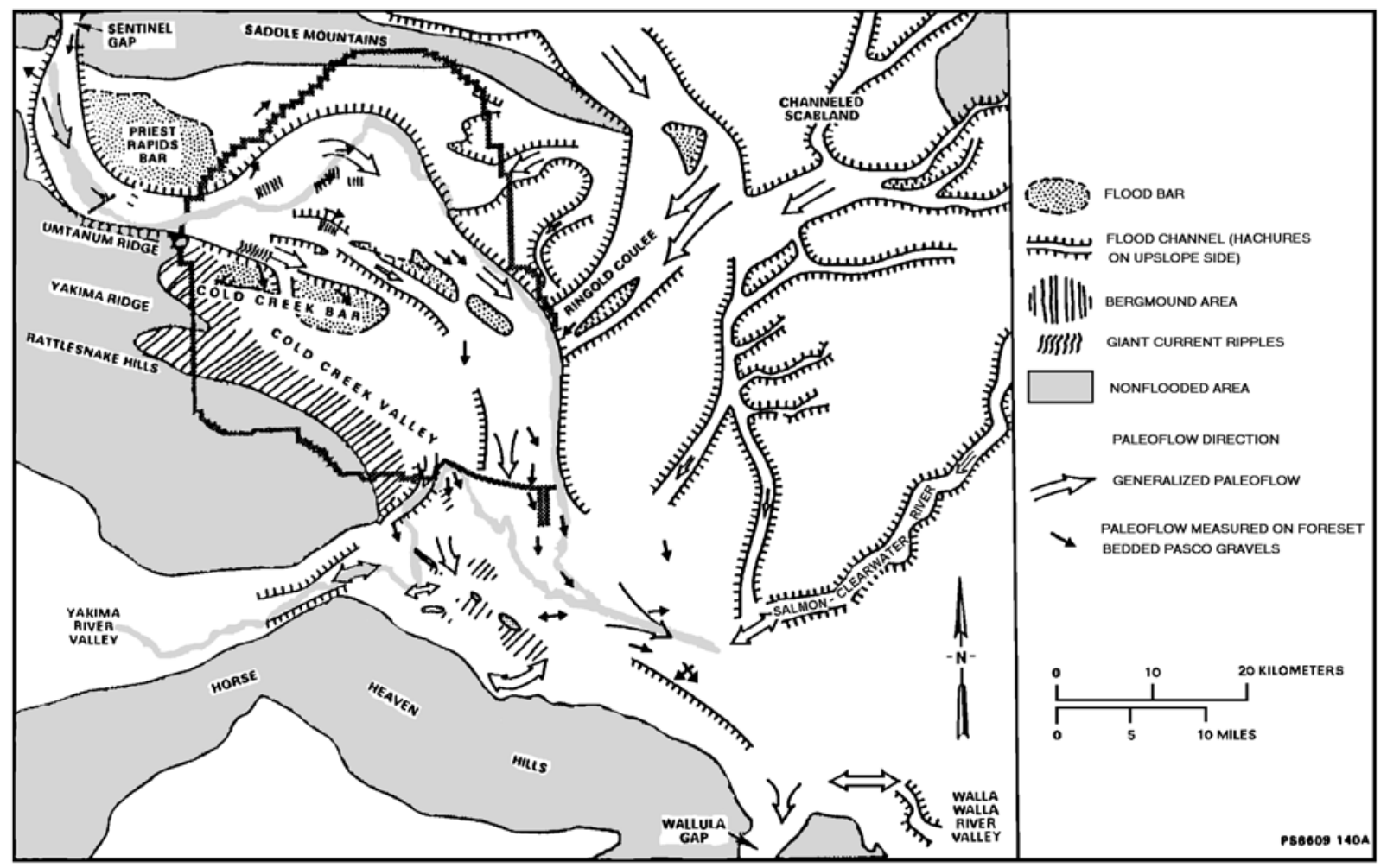

Figure 4.2-2. Paleoflow Directions and Landforms Associated with Cataclysmic Flooding in the Central Columbia Plateau (DOE 1988)

\subsubsection{Stratigraphy}

The stratigraphy of the Hanford Site consists of Miocene-age and younger rocks. Older Cenozoic sedimentary and volcaniclastic rock underlie the Miocene and younger rocks but are not exposed at the surface. The Hanford Site stratigraphy is summarized in Figure 4.2-3 and described in the following subsections. A more detailed discussion of the Hanford Site stratigraphy is given by DOE (1988); Delaney et al. (1991); Reidel et al. (1992); and Lindsey et al. (1994a).

\subsubsection{Columbia River Basalt Group (CRBG)}

The CRBG (Figure 4.2-3) consists of an assemblage of tholeiitic, continental flood basalts of Miocene age. These flows cover an area of more than $164,000 \mathrm{~km}^{2}\left(63,000 \mathrm{mi}^{2}\right)$ in Washington, Oregon, and Idaho and have an estimated volume of about $174,000 \mathrm{~km}^{3}\left(67,200 \mathrm{mi}^{3}\right)$ (Tolan et al. 1989). Isotopic age determinations suggest flows of the CRBG were erupted during a period from approximately 17 to 6 million years ago, with more than $98 \%$ by volume being erupted in a 2.5 -million-year period (17 to 14.5 million years ago). A new model for emplacement of the CRBG suggests rapid emplacement for the main part of the flow and slower emplacement along flow margins (Reidel 1998).

Columbia River basalt flows were erupted from north-northwest-trending fissures or linear vent systems in north-central and northeastern Oregon, eastern Washington, and western Idaho (Swanson et al. 1979a,b; Waters 1961). The CRBG is formally divided into five formations, from oldest to youngest: 


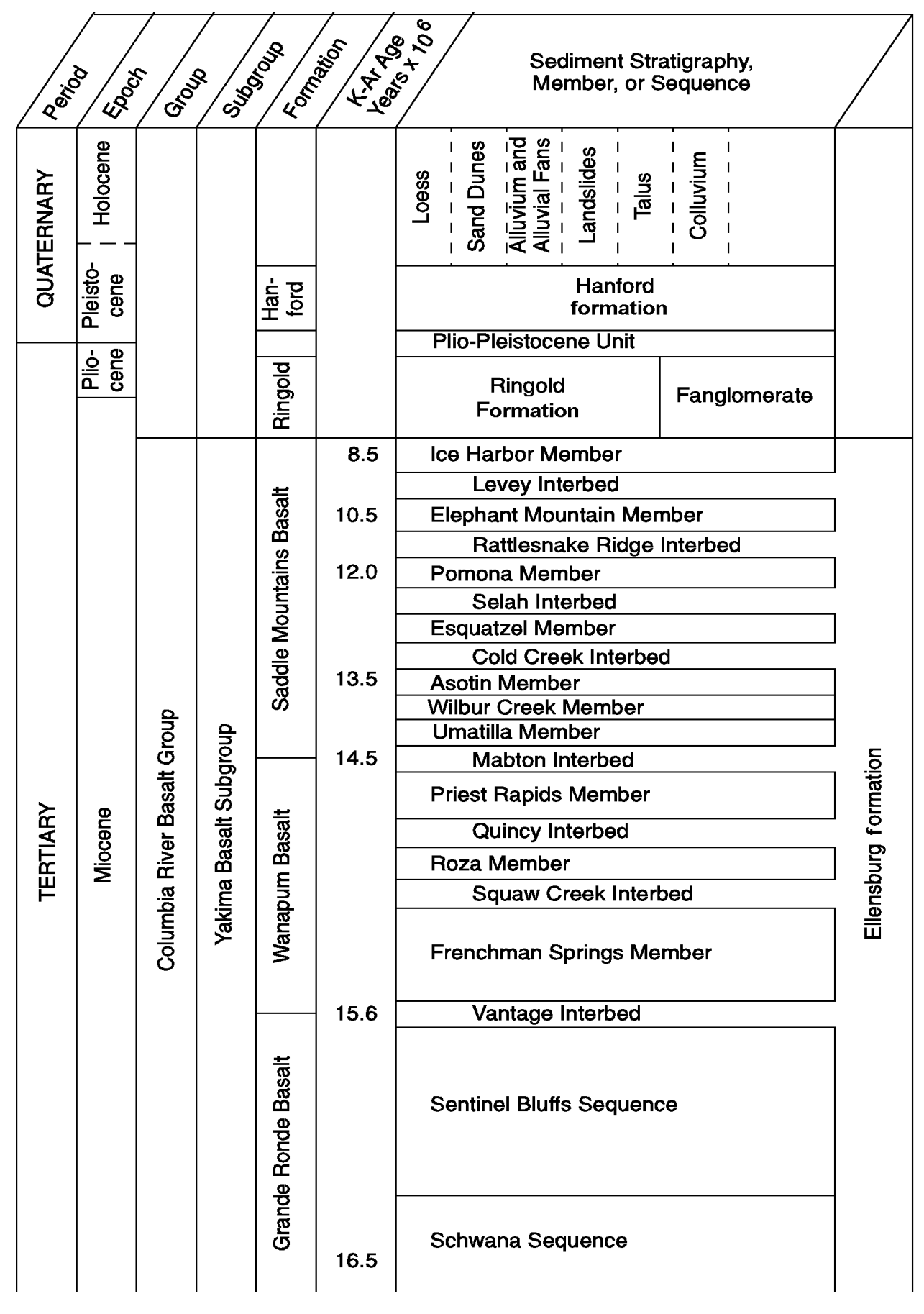

59508017.2

Figure 4.2-3. Stratigraphic Column for the Pasco Basin 
Imnaha Basalt, Picture Gorge Basalt, Grande Ronde Basalt, Wanapum Basalt, and Saddle Mountains Basalt. Beneath the Hanford Site, there is a minimum of 50 basalt flows with a combined thickness of greater than $3000 \mathrm{~m}$ (DOE 1988). The most recent basalt flow underlying the Hanford Site is the Elephant Mountain Member of the Saddle Mountains Basalt. However, the younger Ice Harbor Member is found in the southern portion of the site near the 300 Area (DOE 1988; Hartman 2000).

\subsubsection{Ellensburg Formation}

The Ellensburg Formation (Figure 4.2-3) includes epiclastic and volcaniclastic sedimentary rocks interbedded with the CRBG in the central and western part of the Columbia Plateau (Schmincke 1964; Smith 1988; Swanson et al. 1979a,b). The age of the Ellensburg Formation is principally Miocene, although locally it may be equivalent to early Pliocene. The thickest accumulations of the Ellensburg Formation lie along the western margin of the Columbia Plateau where Cascade Range volcanic and volcaniclastic materials interfinger with the CRBG. Within the Pasco Basin, individual interbeds, primarily in the Wanapum and Saddle Mountains Basalts, have been named (i.e., Mabton, Selah, and Cold Creek). The lateral extent and thickness of interbedded sediments generally increase upward in the section (Reidel and Fecht 1981). Two major facies, volcaniclastic and fluvial, are present either as distinct or mixed deposits. Deposition along the western margin of the plateau was primarily by volcanic debris flows (lahars) and related stream and sheet floods. Some airfall and pyroclastic-flow deposits are present. Airfall tuff is the dominant volcaniclastic material at the Hanford Site (Reidel et al. 1992). No volcanic debris flows have been identified at the Hanford Site (Lindsey et al. 1994a).

\subsubsection{Suprabasalt Sediments}

The suprabasalt sediments within and adjacent to the Hanford Site (Figure 4.2-3) are dominated by the fluvial-lacustrine Ringold Formation and glaciofluvial Hanford formation, with minor eolian and colluvium deposits (Baker et al. 1991; DOE 1988; Tallman et al. 1981).

Ringold Formation. Late Miocene to Pliocene deposits younger than the CRBG are represented by the Ringold Formation within the Pasco Basin (Grolier and Bingham 1978; Gustafson 1973; Newcomb et al. 1972; Rigby and Othberg 1979; Lindsey 1996). The Ringold Formation consists of fluvial gravel and sand, overbank deposits, and lacustrine silty sand, silt, and clay deposited by the ancestral Columbia River system (Tallman et al. 1981; DOE 1988; Lindsey 1996). The course of the ancestral Columbia River was restricted to the structural lows of the Pasco Basin and lower Yakima Valley and was controlled by the structural topography of rising ridges and constructional topography of the Ice Harbor Member of the Saddle Mountain basalt (Fecht et al. 1987). The ancestral Columbia River and its tributaries, in response to this structural restriction, deposited the Ringold Formation in generally eastwest trending valleys.

Although exposures of the Ringold Formation are limited to White Bluffs within the central Pasco Basin and to Smyrna and Taunton Benches north of the Pasco Basin, extensive data on the Ringold Formation are available from boreholes. The Ringold Formation at the Hanford Site is up to $185 \mathrm{~m}$ thick. Regionally, it has a cumulative thickness of approximately $285 \mathrm{~m}$ (Lindsey et al. 1994a). Newcomb (1958) used well logs to extend the Ringold Formation to include subsurface sediments down to the underlying basalt bedrock based on lithologic similarity and continuity of strata exposed at the surface. Newcomb was the first to divide the Ringold Formation into lithostratigraphic units, a lower "blue clay" unit composed of silt, clay, sand, and gravel; a middle gravel and sand unit known as the "conglomerate member"; and an upper unit composed of silt, sand, clay, volcanic ash, and gravel.

Continued studies of the Ringold Formation at the Hanford Site expanded the number of lithostratigraphic units (Myers et al. 1979; Tallman et al. 1979; Bjornstad 1984, 1985; DOE 1988). Other 
studies divided the Ringold into lithofacies (Grolier and Bingham 1978; Grolier 1978; Tallman et al. 1981) and a series of fining-upward sequences (PSPL 1982). These studies have proven to be of limited use in that they either over generalized the stratigraphic variation in the Ringold Formation for widespread use or are valid only within specific study areas on the Hanford Site.

Recent investigations (Lindsey and Gaylord 1990; Lindsey 1991, 1996) indicate that Ringold strata are best described and interpreted on the basis of facies associations. These studies demonstrate that the Ringold Formation can be divided into several stratigraphic packages defined on the basis of dominant facies associations. Facies associations are each defined on the basis of lithology, stratification, and facies architecture. The following facies are defined for the Ringold Formation on the basis of sediment characteristics and depositional environments. A more detailed description of the Ringold facies associations and their characteristics can be found in Lindsey (1996). Stratigraphic columns for the Hanford Site showing geologic correlations among various authors are exhibited in Figure 4.2-4.

Facies Association I: Clast- and matrix-supported pebble-to-cobble gravel in a fine-to-coarse sand matrix. Intercalated lenticular sand and silt lenses may also be present. Cementation varies throughout the facies from none to well developed. Primary cements include calcium carbonate, iron oxides, and silica. Clast composition is variable with basalt, quartzite, porphyritic volcanics, and greenstone, the most common rock types. Less typical are silicic plutonic rocks, gneisses, and volcanic breccias. Matrix sands are predominantly quartzo-feldspathic with a subordinate basalt lithic fraction. Stratification includes crudely defined massive bedding and low angle trough cross bedding. Planar cross beds may be well developed locally. Deposition of facies association I was characterized by alternating periods of high and low flow in a gravely fluvial braidplain with wide, shallow, shifting channels (Reidel et al. 1992; Lindsey et al. 1994a; Lindsey 1996).

Facies Association II: Fine-to-coarse quartzo-feldspathic sand similar in composition to sand in facies association I. Sands are typically light tan to buff, but may include brown, red-brown, yellow-brown, or salt-and-pepper colors. Intercalated silt and pebble beds may be present. Stratification is primarily composed of planar and trough cross-bedded sand lenses overlying scoured bases (Lindsey 1996). Facies association II is interpreted to have been bedload deposition in low sinuosity braided channels.

Facies Association III: Laminated to massive silt, silty fine-grained sand, and paleosols displaying medium to strongly developed blocky beds. Colors range from light gray to brown, green, and black. Red-brown massive sand may be found intercalated with the silts and clays as thin interbeds. Calcium carbonate and silica precipitates are present throughout the unit commonly as stringers, nodules, and concretions. Also present are filamentous, branching root, and burrow casts. Silcrete may be found locally. Facies association III formed as overbank, levee, and crevasse splay deposits in a floodplain environment where pedogenic alteration occurred (Lindsey 1996).

Facies Association IV: Plane laminated to massive clay with thin silt and silty sand interbeds dominate this facies. Colors range from gray, tan, and brown in outcrop, to gray and blue-gray in the subsurface (Lindsey 1996). Thin calcium carbonate and iron oxide cemented intervals are found in outcrop, along with evidence of soft sediment deformation. Facies association IV was deposited in a lake under standing water to deltaic conditions (Reidel et al. 1992; Lindsey et al. 1994a). A laterally continuous white diatomaceous clay present within the association records a period of deposition into a clear body of water relatively distant from fluvial distributaries (Lindsey 1996).

Facies Association V: Massive matrix-supported basaltic gravels forming sheet-like tabular bodies dominate facies association V (Lindsey 1996). These deposits are generally found around the periphery of the basin and record alluvial fan debris flows and side streams draining into the Pasco Basin (Reidel et al. 1992). 


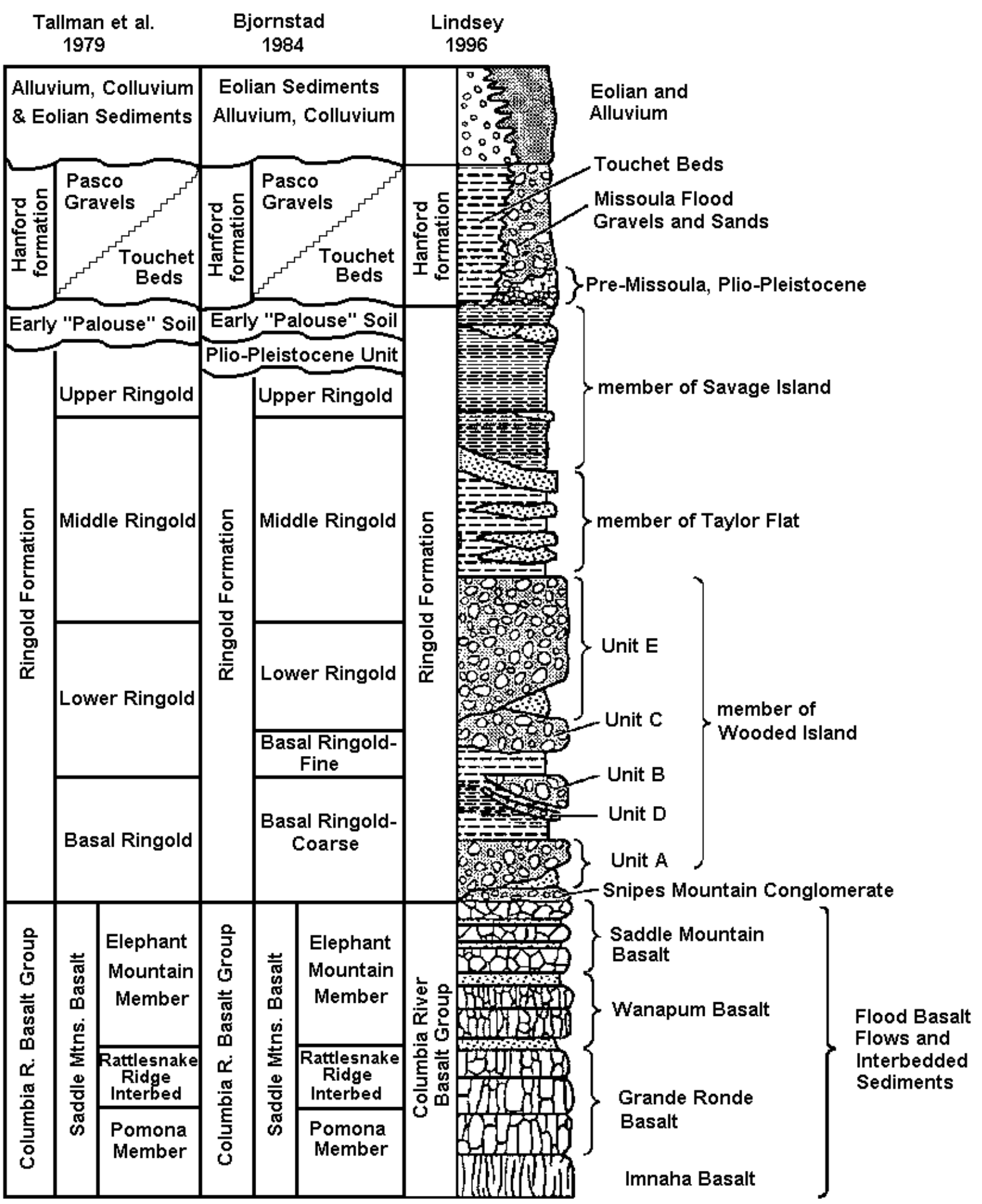

Figure 4.2-4. Stratigraphic Column for the Hanford Site Showing Correlations Among Various Authors 
Ringold Formation Facies Association Distribution. The Ringold Formation is divided into three informal members that are designated as the member of Wooded Island, the member of Taylor Flats, and the member of Savage Island. Each member contains characteristic facies associations. The member of Wooded Island is dominated by fluvial gravel (facies association I) and forms most of the lower half of the Ringold Formation. The member of Taylor Flats forms the middle part of the Ringold Formation and is dominated by fluvial sands (facies association II) and overbank-paleosol deposits (facies association III). The member of Taylor Flats interfingers with the member of Wooded Island in the northern portion of Pasco Basin where fluvial gravels pinch out. Lacustrine deposits (facies association IV) dominate the upper member, the member of Savage Island (Lindsey 1996). The following is a brief description of each informal member as defined by Lindsey. The reader should refer to Lindsey (1996) for a more detailed description of Ringold stratigraphy.

Informal Member of Wooded Island: The lower half of the Ringold Formation is designated as the informal member of Wooded Island and is characterized by five separate stratigraphic gravel-rich intervals. These gravels are designated units A, B, C, D, and E, and are separated by deposits typical of facies associations III and IV (laminated to massive silts, clays, and paleosols). Unit A is the lowermost gravel unit in the Ringold Formation. Unit A was deposited in a Columbia River braidplain from Sentinel Gap southeast into the Cold Creek syncline and marks the initial deposition of the Ringold Formation within Pasco Basin. Overlying unit A is a relatively extensive fine-grained deposit known as the lower mud unit. The lower mud unit was deposited in a lake that filled most of the Pasco Basin. Overlying the lower mud unit are two fluvial gravel-dominated units, B and D. Associated with units B and D are intercalated overbank-paleosol deposits. As the ancestral Columbia River and its tributaries traveled back and forth across the Pasco Basin, unit B was deposited in the eastern to east-central Pasco Basin, and unit $\mathrm{D}$ was deposited in the southwestern Pasco Basin. Where units B and D are absent, overbank and paleosols of facies association III overlie the lower mud unit. Units B and D are differentiated from overlying units $\mathrm{C}$ and $\mathrm{E}$ by a locally thick $(>10 \mathrm{~m})$ paleosol sequence typical of facies association III referred to as the sub $\mathrm{C}+\mathrm{E}$ interval. Where the sub $\mathrm{C}+\mathrm{E}$ interval is absent, units $\mathrm{B}$ and $\mathrm{D}$ are not differentiated from overlying gravel units $\mathrm{C}$ and $\mathrm{E}$.

Uppermost gravel units $\mathrm{C}$ and $\mathrm{E}$ are separated in the eastern Pasco Basin by an unnamed but widespread paleosol sequence similar in character to the paleosol sequence overlying units $\mathrm{B}$ and $\mathrm{D}$ and referred to as the sub E interval. In the western Pasco Basin, the sub E interval is absent, and units $\mathrm{C}$ and $\mathrm{E}$ are not differentiated. Combined, units $\mathrm{C}$ and $\mathrm{E}$ form a northwest-to-southeast-oriented linear body as much as $100 \mathrm{~m}$ thick stretching from Sentinel Gap to Wallula Gap in the subsurface. Units $\mathrm{C}$ and $\mathrm{E}$ interfinger with muddy paleosols around the fringe of the Pasco Basin, especially to the north where units $\mathrm{C}$ and $\mathrm{E}$ pinch out.

Informal Member of Taylor Flats: Approximately $90 \mathrm{~m}$ of interbedded fluvial sand (facies association II) and overbank fines (facies association III) form the member of Taylor Flats. Outcrops of the member extend the length of the White Bluffs. In the central to western portion of the Pasco Basin, most of this member has been removed by post-Ringold erosion and only a thin, discontinuous section remains. This thin erosional remnant has previously been referred to as the Upper Ringold Unit (Myers et al. 1979; Tallman et al. 1979, 1981; Lindsey et al. 1992). Although the member is now absent from much of the Pasco Basin, the distribution of erosional remnants indicates the member once extended across the entire basin.

Informal Member of Savage Island: Lacustrine deposits (facies association IV) dominate the uppermost Ringold Formation, the 90-m-thick member of Savage Island. Three successive lake-fill sequences are present in the member in the east central Pasco Basin. Each of the sequences has a basal diatomaceous interval that grade upward into interstratified silt and sand. The member has been almost 
completely removed by post-Ringold erosion from the central and western Pasco Basin. Small outcrops remain locally in shallow ravines along the northwest base of Rattlesnake Mountain.

Deposition of the Ringold Formation was followed by a period of regional incision in the late Pliocene to early Pleistocene. Within the Pasco Basin, this is reflected by the abrupt termination and eroded nature of the top of the Ringold Formation (Bjornstad 1985; Brown 1960; Newcomb et al. 1972). The exact timing and duration of incision are unknown; however, the incision probably occurred between 1 and 3.4 million years ago.

Plio-Pleistocene Unit. Unconformably overlying the Ringold Formation in the vicinity of 200 West is the laterally discontinuous Plio-Pleistocene unit (Reidel et al. 1992). This subunit is found in the westcentral limb of the Cold Creek syncline in the subsurface west, southwest, and within the 200 West Area. Distribution of the Pliocene-Pleistocene unit depends in part on erosion of the underlying Ringold Formation and post-depositional erosion by catastrophic Missoula floods (Slate 1996). Thickness of the Plio-Pleistocene deposits ranges from 0 to $20 \mathrm{~m}$. The finer and more massive carbonate horizons influence contaminant migration by slowing its rate of downward movement and potentially diverting contaminants laterally (Slate 1996).

The Plio-Pleistocene unit as defined by Slate (2000) includes all material overlying the Ringold Formation and underlying the Hanford formation. Historically, the Plio-Pleistocene unit has been divided into three subunits: the Plio-Pleistocene subunit, the "early" Palouse soil, and the Pre-Missoula gravels (Meyers et al. 1979; Tallman et al. 1981; Bjornstad 1984, 1985; DOE 1988; Last et al. 1989; Lindsey et al. 1991; Lindsey 1996; and Reidel et al. 1992). Slate (2000) proposes a unified Plio-Pleistocene unit consisting of two subunits (locally derived and distantly derived) to better represent this interval due to stratigraphic uncertainties and the lack of evidence in defining a separate eolian subunit of the early "Palouse" soil.

Distantly Derived Subunit: The predominantly felsic gravel with a quartzo-feldspathic sand matrix that composes this subunit displays fluvial sedimentary structure indicating a Columbia River source (Fecht et al. 1987; DOE 1988; Slate 2000). Sand and gravel river sediments, referred to informally as the preMissoula gravels (PSPL 1982), were deposited after incision of the Ringold and before deposition of the cataclysmic flood deposits. The distantly derived subunit, up to $25 \mathrm{~m}$ thick, contains less basalt than the underlying Ringold gravels and overlying Hanford deposits, and has a distinctive white or bleached color (Reidel et al. 1992). These sediments appear to occur in a swath that runs from the Old Hanford Townsite on the eastern side of the Hanford Site across the Site toward Horn Rapids on the Yakima River. Magnetic polarity data indicate that the pre-Missoula gravel unit is no younger than early Pleistocene in age (> $1 \mathrm{Ma}$ ) (Reidel et al. 1992). The stratigraphic relationship with other Plio-Pleistocene mainstream and sidestream deposits is not clear, nor is contact with the overlying Hanford formation (Slate 2000).

Locally Derived Subunit: The poorly sorted basalt-dominated gravel that composes this subunit indicates a local source (Slate 2000). This gravel is interbedded with sand and silt that varies from bedded or laminated to massive. Various forms of secondary carbonate accumulations have developed within these sediments including disseminated, filaments, nodules, massive, and partial to complete coating on clasts (Slate 2000). Sediments in this subunit were deposited in a former sidestream alluvial channel (ancestral Cold Creek). Deposition was episodic, and carbonate soils developed during hiatuses or in a cumulic regime primarily through pedogenic processes (Slate 2000).

In addition to the pedogenic carbonates in the subsurface, carbonates overlie and truncate the Ringold Formation member of Savage Island along the length of the White Bluffs. These carbonates are interpreted to be correlative to Plio-Pleistocene unit (Lindsey 1996). 
Hanford Plio-Pleistocene (H/PP) unit. While Slate (2000) suggests there is insufficient evidence to define a later, separate eolian unit (the "early" Palouse soil), recent studies continue to refer to a distinctive silt-rich unit formerly known as the "early" Palouse soil (Wood et al. 2001; Swanson et al. 1999). Recent investigations include what is currently referred to as H/PP deposits (Lindsey et al. 1994a; Wood et al. 2001). While the lower boundary of the unit is easily defined as the top of the underlying Plio-Pleistocene calcrete, the upper contact with the Hanford formation can be difficult to identify due to its gradational contact with the Hanford formation (Wood et al. 2001). Recent studies indicate that in addition to eolian sand and fine silt (Lindsey et al. 1994a; Slate 1996), H/PP deposits also contain intercalated layers of fine sand and silt more characteristic of alluvial deposits (Lindsey et al. 2000).

Hanford Formation. Cataclysmic floods inundated the Pasco Basin a number of times during the Pleistocene, beginning as early as 1 million years ago (Bjornstad and Fecht 1989); the last major flood sequence is dated at about 13,000 years ago by the presence of Mount St. Helen's "S" tephra (Mullineaux et al. 1978) interbedded with the flood deposits. The number and timing of cataclysmic floods continues to be debated. Baker et al. (1991) documented as many as 10 flood events during the last ice age. The largest and most frequent floods came from glacial Lake Missoula in northwestern Montana; however, smaller floods may have escaped down-valley from glacial lakes, Clark and Columbia, along the northern margin of the Columbia Plateau (Waitt 1980), or down the Snake River from glacial Lake Bonneville (Malde 1968). The flood deposits, informally called the Hanford formation, blanket low-lying areas over most of the central Pasco Basin.

Cataclysmic floodwaters entering the Pasco Basin quickly became impounded behind Wallula Gap, which was too restrictive for the volume of water involved. Floodwaters formed temporary lakes with a shoreline up to $381 \mathrm{~m}(1250 \mathrm{ft})$ in elevation, which lasted only a few weeks or less (Baker 1978). The Hanford formation is thickest in the vicinity of the 200 Areas where it is up to $65 \mathrm{~m}$ (Reidel et al. 1992).

The Hanford formation is divided into three facies: gravel-dominated, sand-dominated, and silty (Reidel et al. 1992). These facies are referred to as coarse-grained deposits, plane-laminated sands facies, and rhythmite facies in Baker et al. (1991). Locally, the gravel-dominated facies is commonly referred to as the "Pasco Gravels" and the silty facies is often designated as "Touchet Beds." Facies of the Hanford formation are commonly described as laterally interfingering. The relative proportion of each facies at any given location is related to distance from main high-energy flows at the time of deposition (Wood et al. 2001).

Gravel-Dominated Facies: These facies are generally coarse-grained basaltic sand and granule-toboulder gravel. Deposits display massive bedding, plane to low-angle bedding, and large-scale planar cross bedding in outcrop. The gravels usually are matrix-poor and display an open-framework texture. Lenticular sand and silt beds are intercalated throughout the facies. Gravel clasts are generally dominated by basalt (50 to $80 \%$ ). The gravel-dominated facies was deposited by high-energy floodwaters in or immediately adjacent to the main channel cataclysmic floodways (Reidel et al. 1992).

Sand-Dominated Facies: These facies are fine- to coarse-grained sand and granule gravel displaying plane lamination and bedding and less commonly, plane bedding and channel-fill sequences. Silt content is variable and sands may contain small pebbles and rip-up clasts. The sands are typically basaltic and are commonly referred to as "salt and pepper" in appearance. The laminated sand facies was deposited adjacent to main flood channelways during the waning stages of flooding and is transitional between the gravel-dominated and silty facies (Reidel et al. 1992). 
Silty Facies: Silty facies are thinly bedded plane-laminated and ripple cross-laminated silt, and fineto coarse-grained sand (Reidel et al. 1992). This facies commonly displays normally graded rhythimites a few centimeters to several tens of centimeters thick (Bjornstad et al. 1987; DOE 1988). These sediments were deposited under slackwater conditions and in back-flooded areas (DOE 1998).

Clastic Dikes. Clastic dikes are commonly associated with, but not restricted to, cataclysmic flood deposits on the Columbia Plateau. Dikes consist of fissures filled with sand, silt, clay, and minor coarser debris. Many dikes occur as sharp-walled, near-vertical tabular bodies filled with multiple layers of unconsolidated sediments. Thin clay/silt linings separate the margins of dikes and internal layers (Fecht et al 1999; Hartman et al. 2000). Dikes vary in width from less than $1 \mathrm{~mm}$ to greater than $2 \mathrm{~m}$. Vertical extents range from less than $1 \mathrm{~m}$ to greater than $50 \mathrm{~m}$, with a large number greater than $20 \mathrm{~m}$ (Fecht et al. 1999; Hartman et al. 2000). In plan view, clastic dikes often appear as a network of 4- to 8-sided polygons. Although there is general agreement that clastic dikes formed during cataclysmic flooding, a primary mechanism to satisfactorily explain the formation of all dikes has not been identified (Supply System 1981).

Previous investigations have proposed that clastic dikes may provide preferential pathways for contaminated water leaking from waste tanks through the thick unsaturated zone to the unconfined aquifer. At this time, there is insufficient evidence to determine if this speculation is accurate (Hartman et al. 2000).

Holocene Deposits. Alluvium is present, not only as a surficial deposit along major river and stream courses (Figure 4.2-5), but also in the subsurface, where it is found underlying and interbedded with proglacial flood deposits. Two types of alluvium are recognized in the Pasco Basin: quartzitic mainstream and basalt-rich sidestream alluvium. Colluvium (talus and slopewash) is a common Holocene deposit in moderate-to-high relief areas. Colluvium, like the dune sand that is found locally in the Pasco Basin, is not commonly preserved in the stratigraphic record. Varying thicknesses of loess or sand mantle much of the Columbia Plateau. Active and stabilized sand dunes are widespread over the Pasco Basin (Figure 4.2-5).

\subsubsection{100 Areas Stratigraphy}

The 100 Areas are spread out along the Columbia River in the northern portion of the Pasco Basin (Figure 4.0-1). All the 100 Areas, except the 100-B/C Area, lie on the north limb of the Wahluke syncline. The $100-\mathrm{B} / \mathrm{C}$ Area lies over the axis of the syncline. The top of basalt in the 100 Areas ranges in elevation from $46 \mathrm{~m}(150 \mathrm{ft})$ near the $100-\mathrm{H}$ Area to $-64 \mathrm{~m}(-210 \mathrm{ft})$ below sea level near the $100-\mathrm{B} / \mathrm{C}$ Area. The Ringold Formation and Hanford formation occur throughout this area; the distantly derived Plio-Pleistocene unit may be present near the $100-\mathrm{B} / \mathrm{C}$ and $100-\mathrm{K}$ Areas but is not readily distinguished from Ringold and Hanford sediments. The locally derived Plio-Pleistocene unit and H/PP deposits have not been recognized in the 100 Areas.

The Ringold Formation shows a marked west-to-east variation in the 100 Areas (Lindsey 1992). The main channel of the ancestral Columbia River flowed along the front of Umtanum Ridge and through the $100-\mathrm{B} / \mathrm{C}$ and 100-K Areas, before turning south to flow along the front of Gable Mountain and/or through the Gable Mountain-Gable Butte gap. This main channel deposited coarse-grained sand and gravel facies of the Ringold Formation (Units A, B, C, and E). Farther to the north and east, however, the Ringold sediments gradually become dominated by the lacustrine and overbank deposits and associated paleosols (Ringold Lower Mud Unit of the member of Wooded Island), with the 100-H Area showing almost none of the gravel facies. In the 100 Areas, the Hanford formation consists primarily of the gravel-dominated 


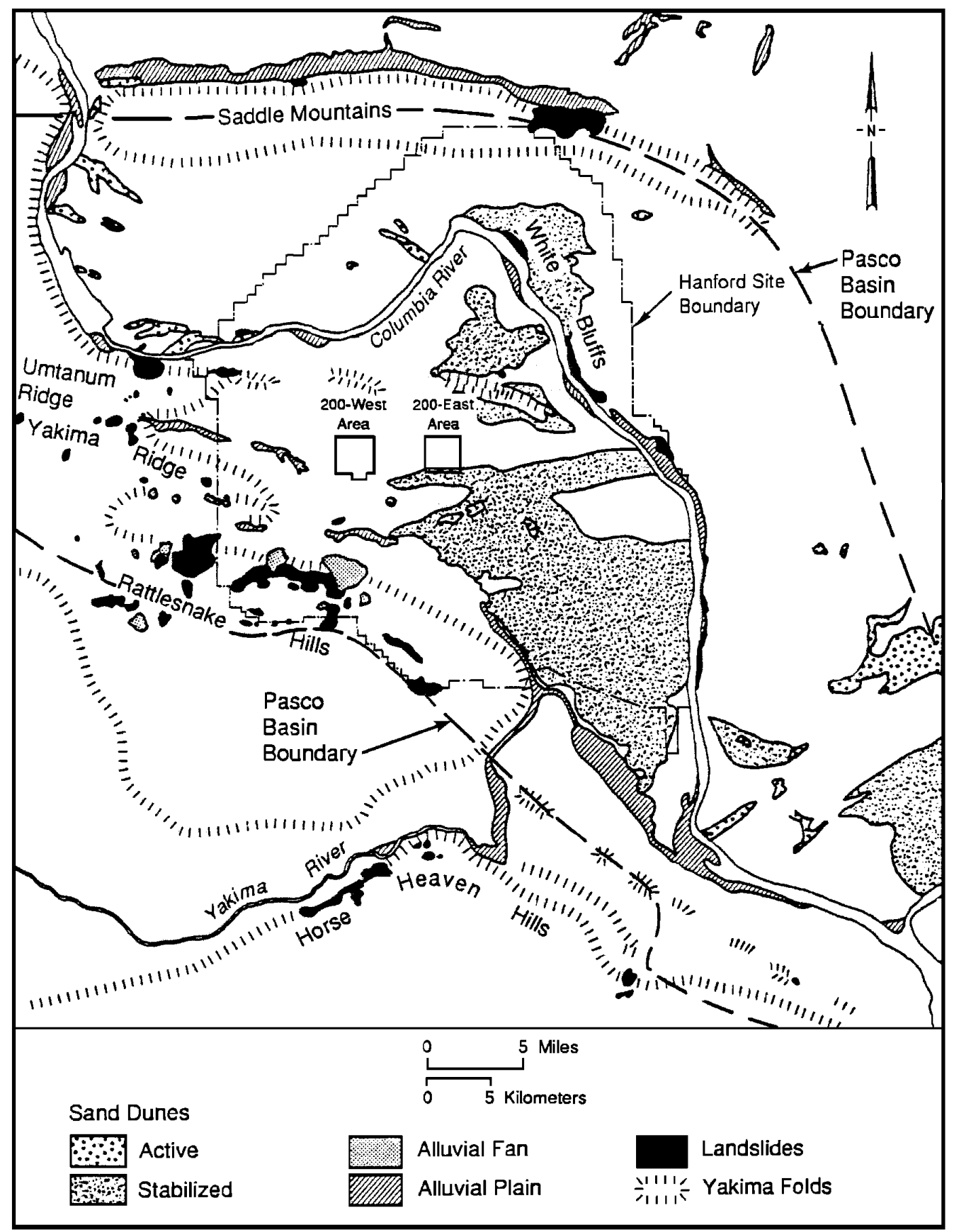

Figure 4.2-5. Location of Surficial Features (DOE 1988) 
facies, with local occurrences of the sand-dominated or silty facies. Hydrogeologic reports providing specific information have been written for each of the 100 Areas. These are as follows: 100-B/C Area Lindberg (1993a); 100-D Area - Lindsey and Jaeger (1993); 100-F Area - Lindsey et al. (1992); 100-H Area - Lindsey and Jaeger (1993); 100-K Area - Lindberg (1993b); and 100-N Area - Hartman and Lindsey (1993).

\subsubsection{200 Areas Stratigraphy}

The geology in the 200 West Area is notably different from that in the 200 East Area, considering they are separated by a distance of only $6 \mathrm{~km}(4 \mathrm{mi})$ (Figure 4.0-1). One of the most complete suprabasalt stratigraphic sections on the Hanford Site, with most of Lindsey's (1996) Ringold units, as well as the Plio-Pleistocene unit and the Hanford formation, is found in the 200 West Area. There are numerous reports on the geology of the 200 West Area, including Connelly et al. (1992a); Lindsey et al. (1991, 1994b); and Reidel et al. (1992).

In the 200 East Area, most of the Ringold Formation units are present in the southern part but have been eroded in a complex pattern to the north. On the north side of the 200 East Area, the Hanford formation rests directly on the basalt, and there are no Ringold sediments present. Erosion by the ancestral Columbia River and catastrophic flooding are believed to have removed the Ringold Formation from this area. A unit of questionable origin locally overlies basalt within the B-BX-BY Waste Management Area (WMA)(Wood et al. 2000). This unit may be equivalent or partially equivalent to the Plio-Pleistocene unit or it may represent the earliest ice-age flood deposits overlain by a locally thick sequence of fine-grained non-flood deposits. This unit is referred to informally as H/PP deposits. Reports on the geology of the 200 East Area include Wood et al. (2000); Connelly et al. (1992b); Lindsey et al. (1992, 1994b); and Tallman et al. (1979).

\subsubsection{300 Area Stratigraphy}

The 300 Area is located in the southeastern portion of the Hanford Site (Figure 4.0-1). The 300 Area lies above a gentle syncline formed by the intersection of the Palouse Slope and the western side of the Pasco Basin. Over most of the Hanford Site, the uppermost basalt flows belong to the Elephant Mountain Member, but near the 300 Area younger flows belonging to the Ice Harbor Member are present, causing a relative high in the top of basalt surface (Schalla et al. 1988) (Figure 4.2-4). Both Ringold Formation and Hanford formation sediments are found in the 300 Area. Swanson (1992) describes the geology in more detail.

\subsubsection{Structural Geology of the Region}

The Hanford Site is located near the junction of the Yakima Fold Belt and the Palouse structural subprovinces (DOE 1988). These structural subprovinces are defined on the basis of their structural fabric, unlike the physiographic provinces that are defined on the basis of landforms. The Palouse subprovince is primarily a regional paleoslope that dips gently toward the central Columbia Basin and exhibits only relatively mild structural deformation. A wedge of Columbia River basalt that overlies the Paleozoic North American craton underlies the Palouse Slope, thinning gradually toward the east and north and lapping onto the adjacent highlands.

The principal characteristics of the Yakima Fold Belt are a series of segmented, narrow, asymmetric anticlines that have wavelengths between 5 and $31 \mathrm{~km}(3$ and $19 \mathrm{mi})$ and amplitudes commonly $<1 \mathrm{~km}$ (0.6 mi) (Reidel 1984; Reidel et al. 1989, 1994). Broad synclines or basins that, in many cases, contain thick accumulations of Neogene- to Quaternary-age sediments separate these anticlinal ridges. The 
deformation of the Yakima Folds occurred under north-south compression. The fold belt was growing during the eruption of the Columbia River Basalt Group and continued to grow into the Pleistocene and probably into the present (Reidel 1984; Reidel et al. 1994).

Thrust or high-angle reverse faults with fault planes that strike parallel or subparallel to the axial trends are principally found along the limbs of the anticlines (Bentley et al. 1980; Hagood 1985; Reidel 1984; Reidel et al. 1994; Reidel and Fecht 1994a, b; Swanson et al. 1979a, b, 1981). The amount of vertical stratigraphic offset associated with these faults varies but commonly exceeds hundreds of meters.

The Saddle Mountains uplift is a segmented anticlinal ridge extending from near Ellensburg to the western edge of the Palouse Slope. This ridge forms the northern boundary of the Pasco Basin and the Wahluke syncline (Figure 4.2-6). It is generally steepest on the north, with a gently dipping southern limb. A major thrust or high-angle reverse fault occurs on the north side (Reidel 1984; Reidel et al. 1994).

The Umtanum Ridge-Gable Mountain uplift is a segmented, asymmetrical anticlinal ridge extending $137 \mathrm{~km}(85 \mathrm{mi})$ in an east-west direction, passing north of the 200 Areas (Figure 4.2-6), and forming the northern boundary of the Cold Creek syncline and the southern boundary of the Wahluke syncline. Three of this structure's segments are located on or adjacent to the Hanford Site. From the west, Umtanum Ridge plunges eastward toward the Pasco Basin and merges with the Gable Mountain-Gable Butte segment. The latter segment then merges with the southeast anticline, which trends southeast before dying out near the Columbia River eastern boundary of the Gable Mountain-Gable Butte segment.

There is a major thrust-to-high-angle reverse fault on the north side of the Umtanum Ridge structure (PSPL 1982; Reidel and Fecht 1994b) that dies out as it plunges eastward past the Gable Mountain-Gable Butte segment. Gable Mountain and Gable Butte are two topographically isolated anticlinal ridges composed of a series of northwest trending, doubly plunging echelon anticlines, synclines, and associated faults. The potential for present-day faulting has been identified on Gable Mountain (PSPL 1982).

The Yakima Ridge uplift extends from west of Yakima to the center of the Pasco Basin, where it forms the southern boundary of the Cold Creek syncline (DOE 1988; Reidel and Fecht 1994a) (Figure 4.2-6). The Yakima Ridge anticline plunges eastward into the Pasco Basin, where it continues on a southeastern trend mostly buried beneath sediments. A thrust-to-high-angle reverse fault is thought to be present on the north side of the anticline, dying out as the fold extends to the east.

Rattlesnake Mountain is an asymmetrical anticline with a steeply dipping and faulted northern unit that forms the southern boundary of the Pasco Basin (Figure 4.2-6). It extends from the structurally complex Snively Basin area southeast to the Yakima River, where the uplift continues as a series of doubly plunging anticlines (Fecht et al. 1984; Reidel and Fecht 1994a). At Snively Basin, the Rattlesnake Mountain structure intersects the Rattlesnake Hills anticline, which extends beyond Yakima and has an east-west trend.

The Cold Creek syncline (Figure 4.2-6) lies between the Umtanum Ridge-Gable Mountain uplift and the Yakima Ridge uplift. The Cold Creek syncline is an asymmetric and relatively flat-bottomed structure (DOE 1988; Reidel and Fecht 1994a). The Wahluke syncline lies between the Saddle Mountains and the Umtanum Ridge-Gable Mountain uplifts. It too is asymmetric and relatively flatbottomed, and it is broader than the Cold Creek syncline (Myers et al. 1979; Reidel and Fecht 1994b). 


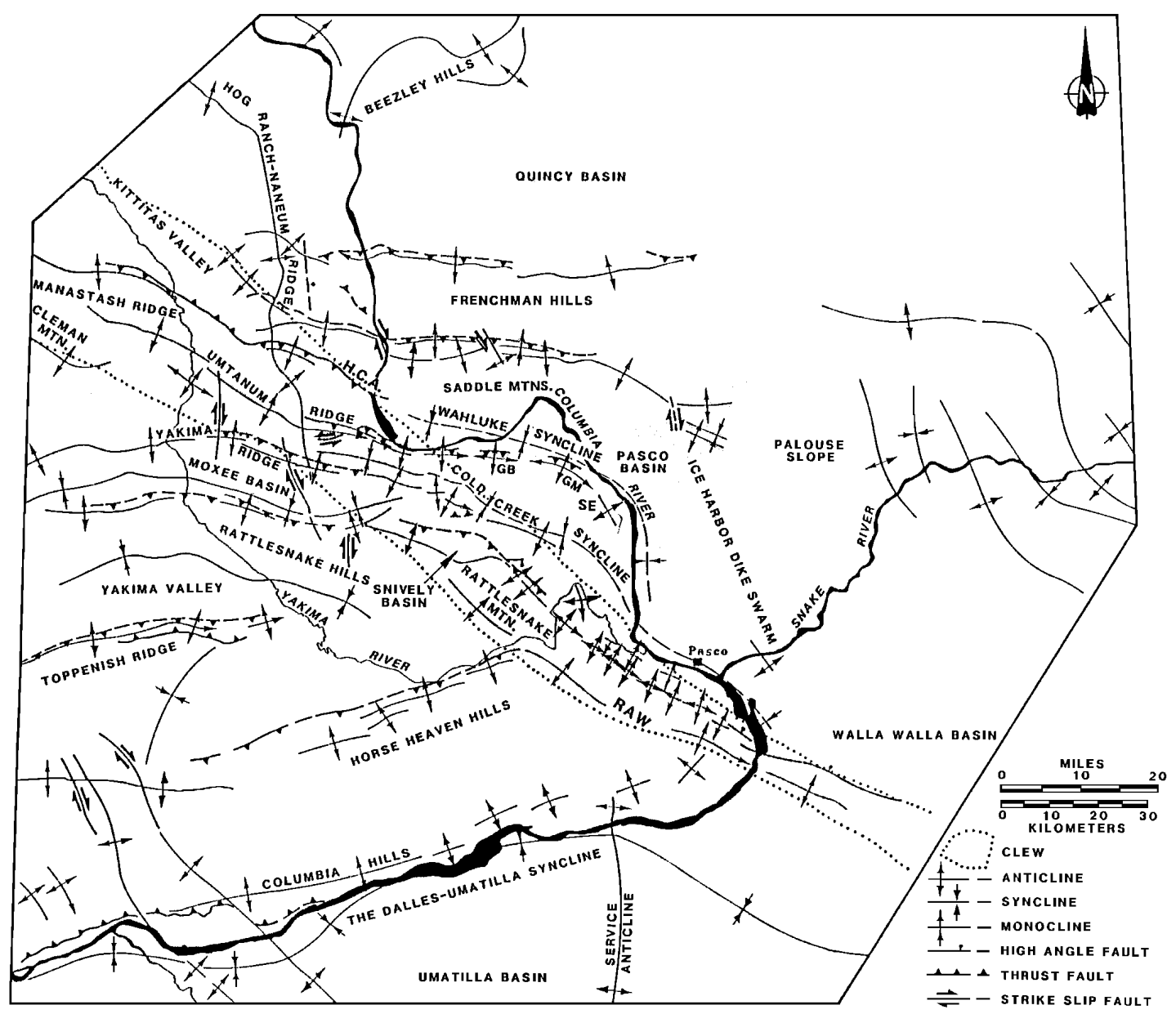

Figure 4.2-6. Location of Structural Features (Reidel et al. 1989)

The Cold Creek fault (Reidel and Fecht 1994a) occurs on the west end of the Cold Creek syncline and coincides with a west-to-east change in hydraulic gradient (Figure 4.2-6). The data suggest that this feature is a high-angle fault that has faulted the basalts and, at least, the older Ringold units (Johnson et al. 1993). This fault apparently has not affected younger Ringold units or the Hanford formation.

Another fault, informally called the May Junction fault (Reidel and Fecht 1994a), is located nearly $4.5 \mathrm{~km}(3 \mathrm{mi})$ east of the 200 East Area. Like the Cold Creek fault, this fault is thought to be a high-angle fault that has offset the basalts and the older Ringold units. It does not appear to have affected the younger Ringold units or the Hanford formation.

\subsubsection{Soils}

Hajek (1966) describes 15 different soil types on the Hanford Site, varying from sand to silty and sandy loam. These are shown in Figure 4.2-7 and briefly described in Table 4.2-1. Various classifications, including land use, are also given in Hajek (1966). The soil classifications given in Hajek (1966) have not been updated to reflect current reinterpretations of soil classifications. Until soils on the Hanford Site are resurveyed, the descriptions presented in Hajek (1966) will continue to be used. 


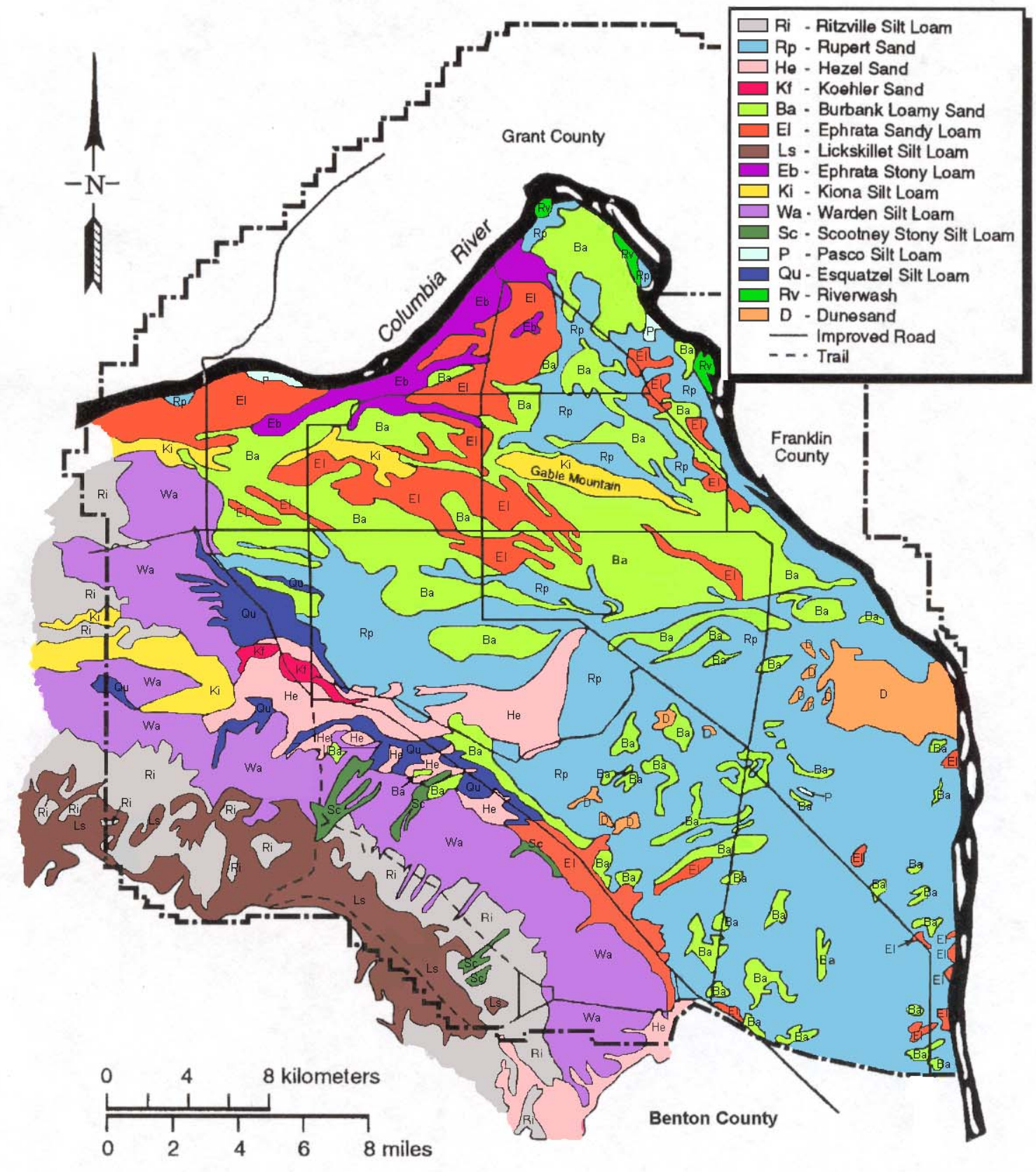

Figure 4.2-7. Soil Map of the Hanford Site (modified from Hajak 1966) 
Table 4.2-1. Soil Types on the Hanford Site (Hajek 1966)

Name (symbol)

Description

Ritzville Silt Loam (Ri)

Rupert Sand (Rp)

Hezel Sand (He)

Koehler Sand (Kf)

Burbank Loamy Sand (Ba)

Ephrata Sandy Loam (El)

Lickskillet Silt Loam (Ls)

Ephrata Stony Loam (Eb)
Dark-colored silt loam soils midway up the slopes of the Rattlesnake Hills. Developed under bunch grass from silty windlaid deposits mixed with small amounts of volcanic ash. Characteristically $>150 \mathrm{~cm}$ (60 in.) deep, but bedrock may occur between 75 and $150 \mathrm{~cm}$ (30 and $60 \mathrm{in}$.).

One of the most extensive soils on the Hanford Site. Brown-tograyish-brown coarse sand grading to dark grayish-brown at $90 \mathrm{~cm}$ (35 in.). Developed under grass, sagebrush, and hopsage in coarse sandy alluvial deposits that were mantled by wind-blown sand. Hummocky terraces and dune-like ridges.

Similar to Rupert sands; however, a laminated grayish-brown strongly calcareous silt loam subsoil is usually encountered within $100 \mathrm{~cm}$ (39 in.) of the surface. Surface soil is very dark brown and was formed in wind-blown sands that mantled lake-laid sediments.

Similar to other sandy soils on the Hanford Site. Developed in a wind-blown sand mantle. Differs from other sands in that the sand mantles a lime-silica cemented "Hardpan" layer. Very dark grayish-brown surface layer is somewhat darker than Rupert Sands. Calcareous subsoil is usually dark grayish-brown at about $45 \mathrm{~cm}$ (18 in.).

Dark-colored, coarse-textured soil underlain by gravel. Surface soil is usually about $40 \mathrm{~cm}$ (16 in.) thick but can be $75 \mathrm{~cm}(30 \mathrm{in}$.) thick. Gravel content of subsoil ranges from $20 \%$ to $80 \%$.

Surface is dark colored and subsoil is dark grayish-brown mediumtextured soil underlain by gravelly material, which may continue for many feet. Level topography.

Occupies ridge slopes of Rattlesnake Hills and slopes $>765 \mathrm{~m}$ (2509 ft) elevation. Similar to Kiona series except surface soils are darker. Shallow over basalt bedrock, with numerous basalt fragments throughout the profile.

Similar to Ephrata sandy loam. Differs in that many large hummocky ridges are made up of debris released from melting glaciers. Areas between hummocks contain many boulders several feet in diameter. 
Kiona Silt Loam (Ki)

Warden Silt Loam (Wa)

Scootney Stony Silt Loam (Sc)

Pasco Silt Loam (P)

Esquatzel Silt Loam (Qu)

Riverwash (Rv)

Dune Sand (D)
Occupies steep slopes and ridges. Surface soil is very dark grayishbrown and about $10 \mathrm{~cm}$ (4 in.) thick. Dark-brown subsoil contains basalt fragments $30 \mathrm{~cm}$ (12 in.) and larger in diameter. Many basalt fragments found in surface layer. Basalt rock outcrops present. A shallow stony soil normally occurring in association with Ritzville and Warden soils.

Dark grayish-brown soil with a surface layer usually $23 \mathrm{~cm}(9 \mathrm{in}$. thick. Silt loam subsoil becomes strongly calcareous at about $50 \mathrm{~cm}$ (20 in.) and becomes lighter colored. Granitic boulders are found in many areas. Usually $>150 \mathrm{~cm}$ (60 in.) deep.

Developed along the north slope of Rattlesnake Hills; usually confined to floors of narrow draws or small fan-shaped areas where draws open onto plains. Severely eroded with numerous basaltic boulders and fragments exposed. Surface soil is usually dark grayish-brown grading to grayish-brown in the subsoil.

Poorly drained very dark grayish-brown soil formed in recent alluvial material. Subsoil is variable, consisting of stratified layers. Only small areas found on the Hanford Site, located in low areas adjacent to the Columbia River.

Deep dark-brown soil formed in recent alluvium derived from loess and lake sediments. Subsoil grades to dark grayish-brown in many areas, but color and texture of the subsoil are variable because of the stratified nature of the alluvial deposits.

Wet, periodically flooded areas of sand, gravel, and boulder deposits that make up overflowed islands in the Columbia River and adjacent land.

Miscellaneous land type that consists of hills or ridges of sand-sized particles drifted and piled up by wind and are either actively shifted or so recently fixed or stabilized that no soil horizons have developed. 


\subsubsection{Seismicity}

The historic record of earthquakes in the Pacific Northwest dates from about 1840. The early part of this record is based on newspaper reports of human perception of the shaking and structural damage as classified by the Modified Mercalli Intensity (MMI) scale, and is probably incomplete because the region was sparsely populated. The historical record appears to be complete since 1905 for MMI V and since 1890 for MMI VI (Rohay 1989). Seismograph networks did not start providing earthquake locations and magnitudes of earthquakes in the Pacific Northwest until about 1960. A comprehensive network of seismic stations that provides accurate locating information for most earthquakes of magnitude $>2.5$ was installed in eastern Washington in 1969. DOE (1988) provides a summary of the seismicity of the Pacific Northwest, a detailed review of the seismicity in the Columbia Plateau region and the Hanford Site, and a description of the seismic networks used to collect the data.

Large earthquakes (magnitude $M \geq 7$ ) in the Pacific Northwest have occurred near Puget Sound, Washington, and near the Rocky Mountains in eastern Idaho and western Montana. Two large earthquakes occurred beneath Vancouver Island. The first occurred in 1918 and had a maximum MMI VII (estimated magnitude M 7.0). The second earthquake occurred in 1946 and had a maximum MMI VII (over a wider area) and magnitude $M=7.3$. The depth of these early, large earthquakes beneath Vancouver Island is uncertain. Another large earthquake occurred at a depth of $53 \mathrm{~km}$ near Olympia, Washington, in 1949 that had a maximum MMI VIII and a magnitude $\mathrm{M}=7.1$. A smaller $(\mathrm{M}=6.5)$, deep $(63 \mathrm{~km})$ earthquake occurred in 1965 between Seattle and Tacoma. These events may all be related to deformation within the subducting Juan de Fuca Plate at depth beneath the Vancouver Island/Puget Sound region.

Two large events occurred on the eastern boundary of the Pacific Northwest, in the Rocky Mountains. These were the 1959 Hebgen Lake earthquake in western Montana, which had a Richter magnitude of 7.5 and an MMI X, and the 1983 Borah Peak earthquake in eastern Idaho, which had a Richter magnitude of 7.3 and an MMI IX.

Closer to the Hanford Site, a significant large earthquake of uncertain location occurred in northcentral Washington in 1872. This event had an estimated maximum MMI ranging from VIII to IX and an estimated Richter magnitude of approximately 7.4. The distribution of intensities suggests a location within a broad region between Lake Chelan, Washington, and the British Columbia border. Evidence of landslides near Lake Chelan suggests a location near there.

On February 28, 2001, there was a moderate $(\mathrm{M}<7)$, deep earthquake near Olympia (termed the Nisqually earthquake). This earthquake was located at a depth of $52 \mathrm{~km}$ and had a magnitude of 6.8 ; reported ground shaking effects reached MMI VIII. This event is similar to those in 1949 and 1965 described above. Actual ground motions from this earthquake measured at the Hanford Site are reported at the end of this section.

Seismicity of the Columbia Plateau, as determined by the rate of earthquakes per area and the historical magnitude of these events, is relatively low when compared with other regions of the Pacific Northwest, the Puget Sound area, and western Montana/eastern Idaho. Figure 4.2-8 shows the locations of all earthquakes that occurred in the Columbia Plateau before 1969 with an MMI of $\geq \mathrm{V}$ and at Richter magnitude $\geq 4$, and Figure 4.2-9 shows the locations of all earthquakes that occurred from 1969 to 2000 at Richter magnitudes $\geq 3$. The largest known earthquake in the Columbia Plateau occurred in 1936 near Milton-Freewater, Oregon. This earthquake had a Richter magnitude of 5.75 and a maximum MMI of VII, and was followed by a number of aftershocks indicating a northeast-trending fault plane. 


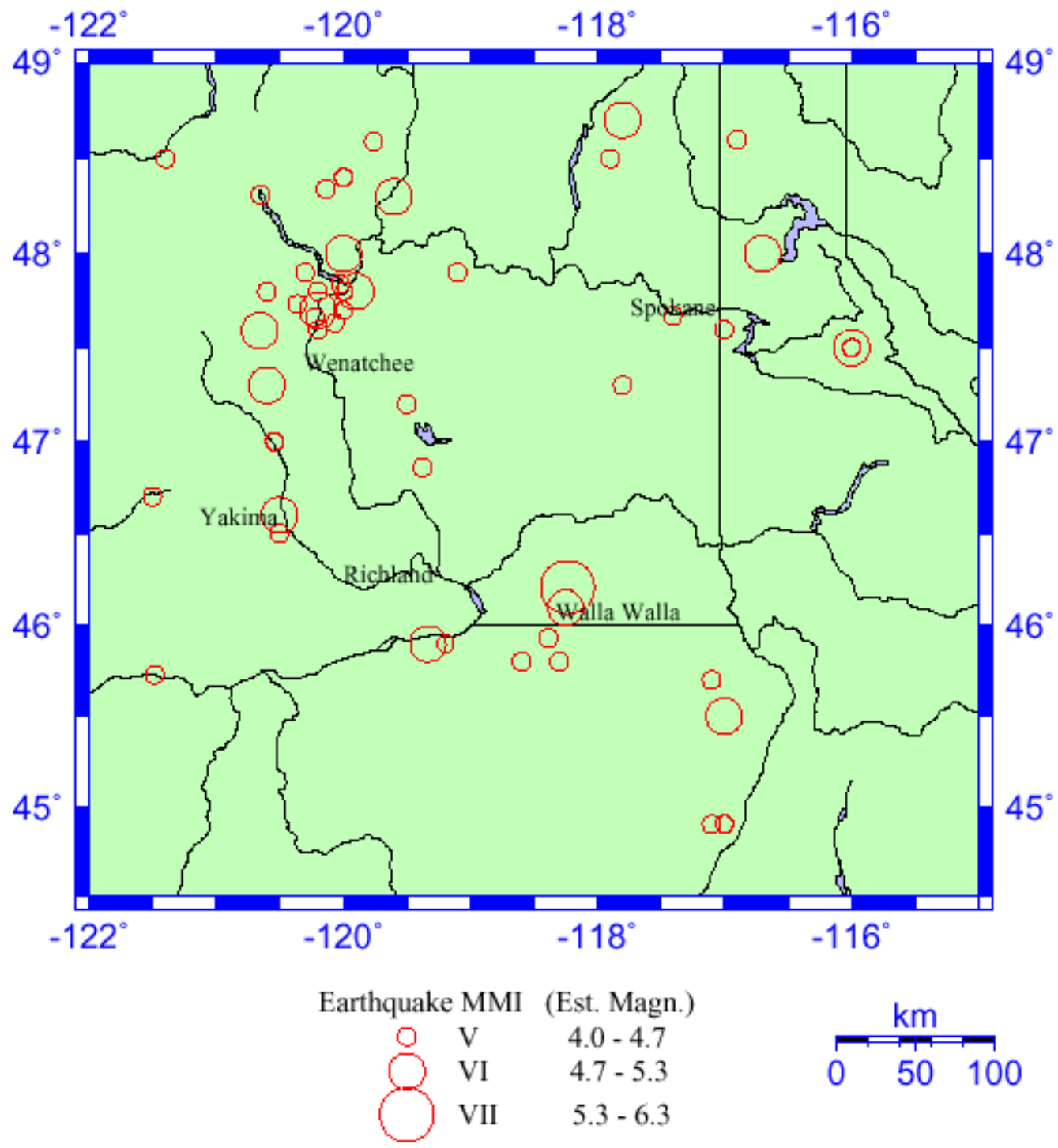

Figure 4.2-8. Historical Seismicity of the Columbia Plateau and Surrounding Areas. All earthquakes between 1850 and March 20, 1969, with a Modified Mercalli Intensity of $\mathrm{V}$ or larger or a Richter magnitude of 4 or larger are shown (Rohay 1989) 


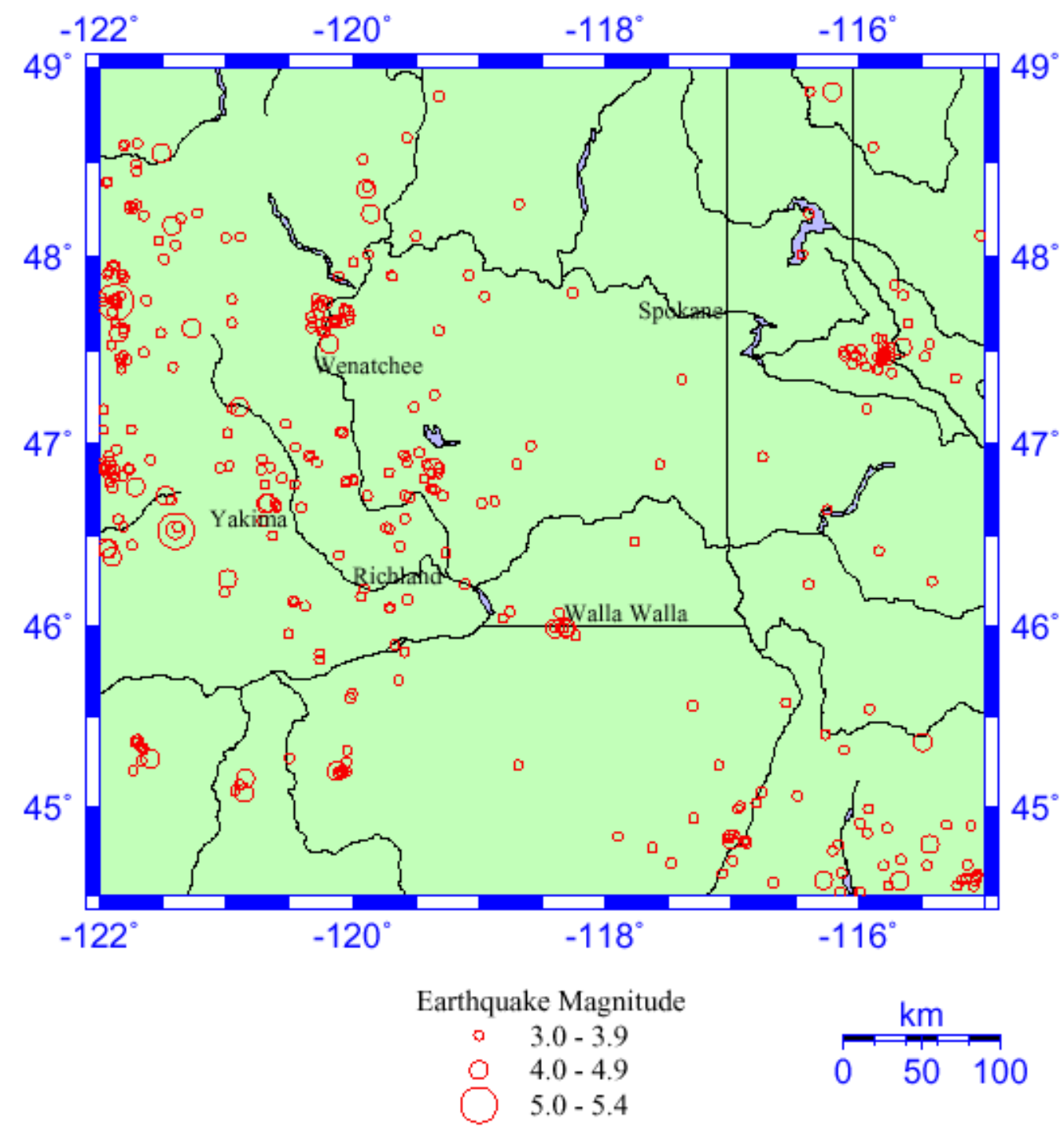

Figure 4.2-9. Seismicity of the Columbia Plateau and Surrounding Areas as Measured by Seismographs. All earthquakes from 3/20/1969 to 12/31/2000 with Richter magnitude 3 or larger are shown. Data sources UWGP (2001) and CNSS (2001) 
Other earthquakes with Richter magnitudes $\geq 5$ and/or MMIs of VI occurred along the boundaries of the Columbia Plateau in a cluster near Lake Chelan extending into the northern Cascade Range, in northern Idaho and Washington, and along the boundary between the western Columbia Plateau and the Cascade Range. Three MMI VI earthquakes have occurred within the Columbia Plateau, including one event in the Milton-Freewater, Oregon, region in 1921; one near Yakima, Washington, in 1892; and one near Umatilla, Oregon, in 1893. In the central portion of the Columbia Plateau, the largest earthquakes near the Hanford Site are two earthquakes that occurred in 1918 and 1973. These two events were magnitude 4.4 and intensity V, and were located north of the Hanford Site near Othello.

Earthquakes often occur in spatial and temporal clusters in the central Columbia Plateau, and are termed "earthquake swarms." The region north and east of the Hanford Site is a region of concentrated earthquake swarm activity, but earthquake swarms have also occurred in several locations within the Hanford Site. The frequency of earthquakes in a swarm tends to gradually increase and decay with no one outstanding large event within the sequence. Roughly $90 \%$ of the earthquakes in swarms have Richter magnitudes of 2 or less. These earthquake swarms generally occur at shallow depths, with $75 \%$ of the events located at depths $<4 \mathrm{~km}$. Each earthquake swarm typically lasts several weeks to months, consists of several to 100 or more earthquakes, and the locations are clustered in an area 5 to $10 \mathrm{~km}$ in lateral dimension. Often, the longest dimension of the swarm area is elongated in an east-west direction. However, detailed locations of swarm earthquakes indicate that the events occur on fault planes of variable orientation, and not on a single, throughgoing fault plane.

Earthquakes in the central Columbia Plateau also occur to depths of about $30 \mathrm{~km}$. These deeper earthquakes are less clustered and occur more often as single, isolated events. Based on seismic refraction surveys in the region, the shallow earthquake swarms are occurring in the Columbia River Basalts and the deeper earthquakes are occurring in crustal layers below the basalts.

The spatial pattern of seismicity in the central Columbia Plateau suggests an association of the shallow swarm activity with the east-west oriented Saddle Mountains anticline. However, this association is complex, and the earthquakes do not delineate a throughgoing fault plane that would be consistent with the faulting observed on this structure.

Earthquake focal mechanisms in the central Columbia Plateau generally indicate reverse faulting on east-west planes, consistent with a north-south-directed maximum compressive stress and with the formation of the east-west-oriented anticlinal folds of the Yakima Fold Belt (Rohay 1987). However, earthquake focal mechanisms indicate faulting on a variety of fault plane orientations.

Earthquake focal mechanisms along the western margin of the Columbia Plateau also indicate northsouth compression, but here the minimum compressive stress is oriented east to west, resulting in strikeslip faulting (Rohay 1987). Geologic studies indicate an increased component of strike-slip faulting in the western portion of the Yakima Fold Belt. Earthquake focal mechanisms in the Milton-Freewater region to the southeast indicate a different stress field, one with maximum compression directed east-west instead of north-south.

Estimates for the earthquake potential of structures and zones in the central Columbia Plateau have been developed during the licensing of nuclear power plants at the Hanford Site. In reviewing the operating license application for the Washington Public Power Supply System (now Energy Northwest) Project Columbia Generating Station (formerly WNP-2), the U.S. Nuclear Regulatory Commission (NRC) concluded that four earthquake sources should be considered for seismic design: the RattlesnakeWallula alignment, Gable Mountain, a floating earthquake in the tectonic province, and a swarm area (NRC 1982). 
For the Rattlesnake-Wallula alignment, which passes along the southwest boundary of the Hanford Site, the NRC estimated a maximum Richter magnitude of 6.5; for Gable Mountain, an east-west structure that passes through the northern portion of the Hanford Site, a maximum Richter magnitude of 5.0 was estimated. These estimates were based upon the inferred sense of slip, the fault length, and/or the fault area. The floating earthquake for the tectonic province was developed from the largest event located in the Columbia Plateau, the Richter magnitude 5.75 Milton-Freewater earthquake. The maximum swarm earthquake for the purpose of Columbia Generating Station seismic design was a Richter magnitude 4.0 event, based on the maximum swarm earthquake in 1973. (The NRC concluded that the actual magnitude of this event was smaller than estimated previously.)

Probabilistic seismic hazard analyses have been used to determine the seismic ground motions expected from multiple earthquake sources, and these are used to design or evaluate facilities on the Hanford Site. The most recent Hanford Site-specific hazard analysis (Geomatrix 1994, 1996) estimated that $0.10 \mathrm{~g}$ ( $1 \mathrm{~g}$ is the acceleration of gravity) horizontal acceleration would be experienced on average every $500 \mathrm{yr}$ (or with a $10 \%$ chance every $50 \mathrm{yr}$ ). This study also estimated that $0.2 \mathrm{~g}$ would be experienced on average every $2500 \mathrm{yr}$ (or with a $2 \%$ chance in $50 \mathrm{yr}$ ). These estimates are in approximate agreement with the results of national seismic hazard maps produced by the U.S. Geological Survey (USGS 1996).

The February 28, 2001, Nisqually earthquake in Puget Sound was recorded by a network of strong motion accelerographs at the Hanford Site. Peak horizontal accelerations measured ranged from 0.0016 to $0.0055 \mathrm{~g}$. These levels of ground shaking are considerably less than the design and evaluation values described above (PNNL Seismic Monitoring Team 2001).

\subsection{Hydrology}

\section{P. D. Thorne, D.G. Horton, and G. V. Last}

Hydrology considerations at the Hanford Site include surface water, the vadose zone, and groundwater. The vadose zone is the unsaturated or partially saturated region between ground surface and the saturated zone. Water in the vadose zone is called soil moisture. Groundwater refers to water within the saturated zone. Permeable saturated units in the subsurface are called aquifers.

The Hanford Site Groundwater/Vadose Zone Integration Project (Integration Project) was established in 1997 to help determine the potential effects that contaminants in Hanford Site soil and groundwater could have on people and the environment. The goal of this project is to provide a sound technical basis to inform and influence cleanup decisions. It integrates and coordinates related science and technology, modeling, monitoring and characterization work with a sitewide approach. The Integration Project is concerned with all work underway at Hanford that involves the Columbia River, groundwater, and the vadose zone.

\subsubsection{Surface Water}

Surface water at Hanford includes the Columbia River, Columbia riverbank seepage, springs, and ponds. Intermittent surface streams, such as Cold Creek, may also contain water after large precipitation or snowmelt events. In addition, the Yakima River flows along a short section of the southern boundary of the Hanford Site (Figure 4.3-1), and there is surface water associated with irrigation east and north of the Site. 


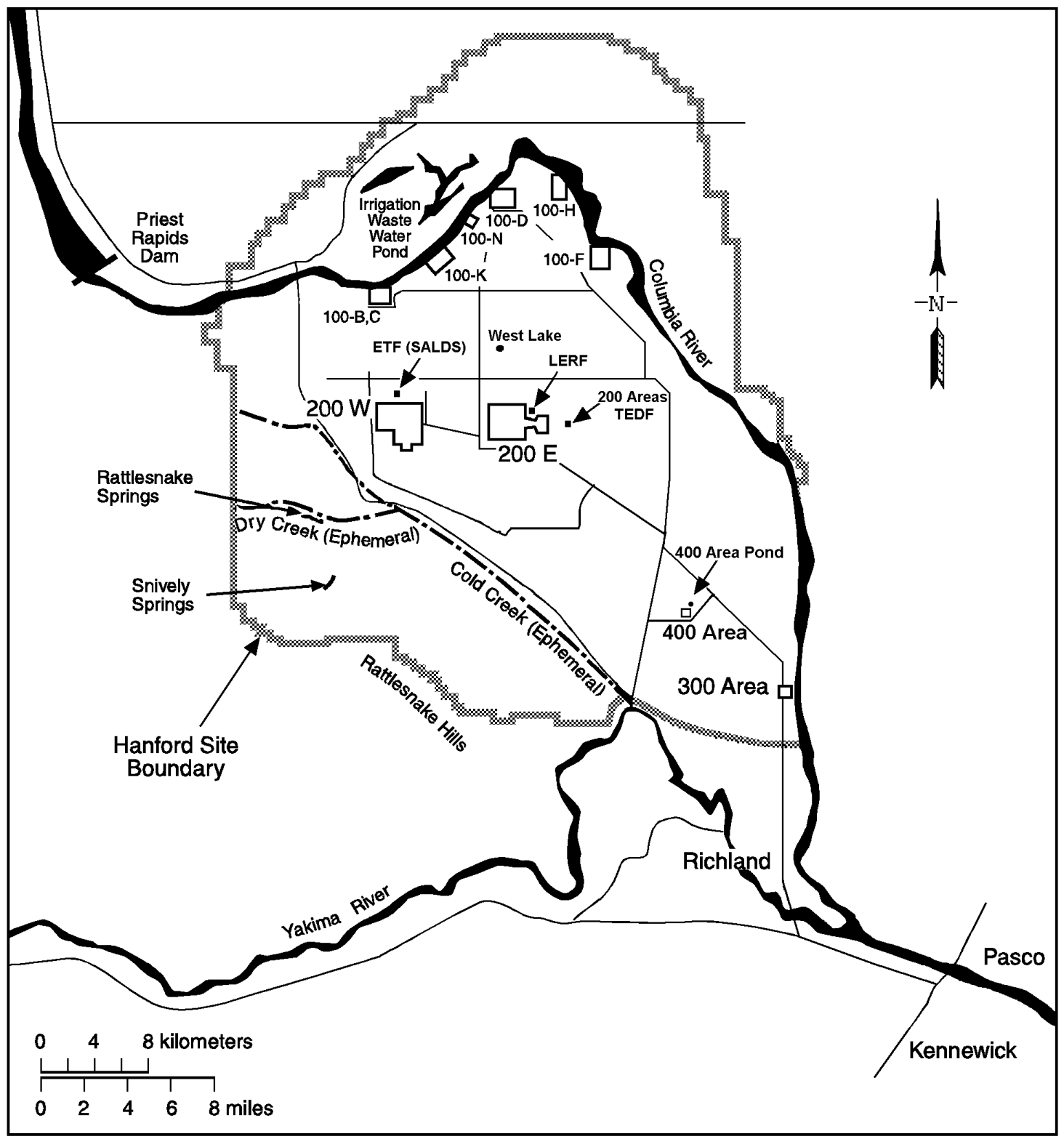

S9508017.4

Figure 4.3-1. Surface Water Features Including Rivers, Ponds, Major Springs, and Ephemeral Streams on the Hanford Site. (ETF = Effluent Treatment Facility; SALDS = State-approved land disposal structure; LERF $=$ Liquid Effluent Retention Facility; TEDF $=$ Treated Effluent Disposal Facility) 


\subsubsection{Columbia River}

The Columbia River is the second largest river in the contiguous United States in terms of total flow and is the dominant surface-water body on the Hanford Site. The original selection of the Hanford Site for plutonium production and processing was based, in part, on the abundant water provided by the Columbia River. The existence of the Hanford Site has precluded development of this section of the river for irrigation and power, and the Hanford Reach of the Columbia River was recently incorporated into the land area established as the Hanford Reach National Monument.

Originating in the Canadian Rockies of southeastern British Columbia, Canada, the Columbia River drains a total area of approximately $680,000 \mathrm{~km}^{2}\left(262,480 \mathrm{mi}^{2}\right)$ en route to the Pacific Ocean. Flow of the Columbia River is regulated by 11 dams within the United States: 7 upstream and 4 downstream of the Hanford Site. Priest Rapids is the nearest upstream dam, and McNary is the nearest downstream dam. Lake Wallula, the impoundment created by McNary Dam, extends upstream past Richland, Washington, to the southern part of the Hanford Site. Except for the Columbia River estuary, the only unimpounded stretch of the river in the United States is the Hanford Reach, which extends from Priest Rapids Dam downstream approximately 51 miles to the McNary Pool, north of Richland, Washington.

Flows through the Hanford Reach fluctuate significantly and are controlled primarily by releases from three upstream storage dams: Grand Coulee, Mica, and Keenleyside. Flows in the Hanford Reach are directly affected by releases from Priest Rapids Dam. Priest Rapids operates as a run-of-the-river dam rather than a storage dam. Columbia River flow rates near Priest Rapids during the 83-year period from 1917 to 2000 averaged nearly $3360 \mathrm{~m}^{3} / \mathrm{s}\left(120,000 \mathrm{ft}^{3} / \mathrm{s}\right)$. Daily average flows during this period ranged from 570 to $19,500 \mathrm{~m}^{3} / \mathrm{s}\left(20,000\right.$ to $\left.690,000 \mathrm{ft}^{3} / \mathrm{s}\right)$. The lowest and highest flows occurred before the construction of upstream dams. During the 10-year period from 1991 through 2000, the average flow rate was also about $3360 \mathrm{~m}^{3} / \mathrm{s}\left(120,000 \mathrm{ft}^{3} / \mathrm{s}\right)$. Daily average flows for this period are plotted in Figure $4.3-2$.

During 1996 and 1997, exceptionally high spring runoff resulted from larger than normal snowpacks. The highest daily average flow rate during 1997 was nearly $11,750 \mathrm{~m}^{3} / \mathrm{s}\left(415,000 \mathrm{ft}^{3} / \mathrm{s}\right)$ (USGS 2001a). Peak daily average flow during 2000 was $6476 \mathrm{~m}^{3} / \mathrm{s}\left(228,700 \mathrm{ft}^{3} / \mathrm{s}\right)$. Average daily flows for 2000 are plotted in Figure 4.3-3. As noted, both Figures 4.3-2 and 4.3-3 include provisional data from the U.S. Geological Survey that have not yet been reviewed and are subject to change. Columbia River flows typically peak from April through June during spring runoff from snowmelt, and are lowest from September through October. As a result of daily fluctuations in discharges from Priest Rapids Dam, the

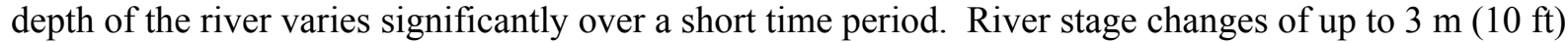
during a 24-hr period may occur along the Hanford Reach (Poston et al. 2000). The width of the river

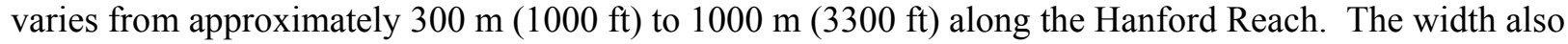
varies temporally as the flow rate changes, which causes repeated wetting and drying of an area along the shoreline.

The primary uses of the Columbia River include the production of hydroelectric power, irrigation of cropland in the Columbia Basin, and transportation of materials by barge. The Hanford Reach is the upstream limit of barge traffic on the mainstem Columbia River. Barges are used to transport reactor

vessels from decommissioned nuclear submarines to Hanford for disposal. Several communities located on the Columbia River rely on the river as their source of drinking water. The Columbia River is also used as a source of both drinking water and industrial water for several Hanford Site facilities (Dirkes 1993). In addition, the Columbia River is used extensively for recreation, which includes fishing, hunting, boating, sailboarding, water-skiing, diving, and swimming. 


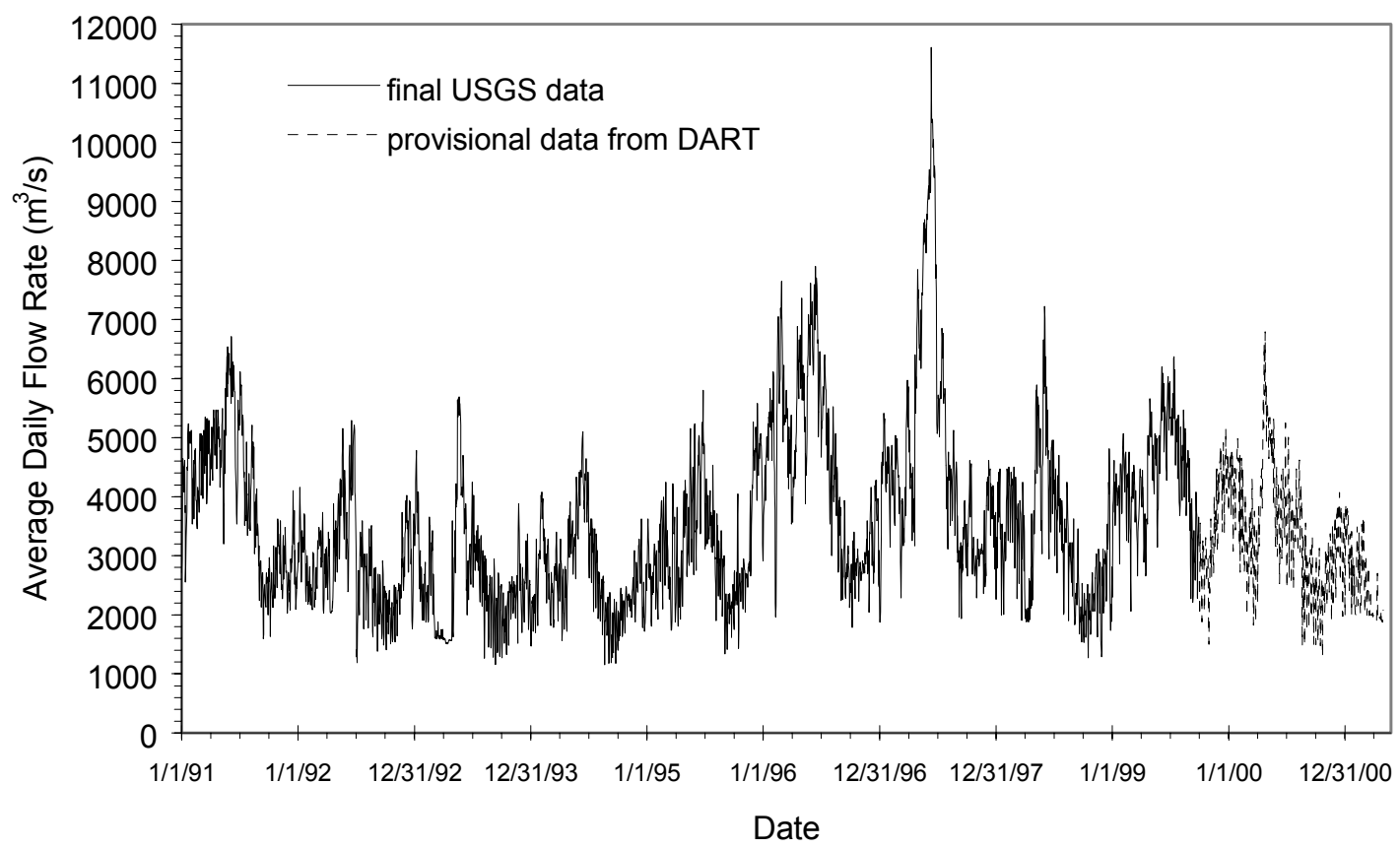

Figure 4.3-2. Average daily flow for the Columbia River below Priest Rapids Dam from 1990 through April 2001 (data from USGS 2001a and DART 2001)

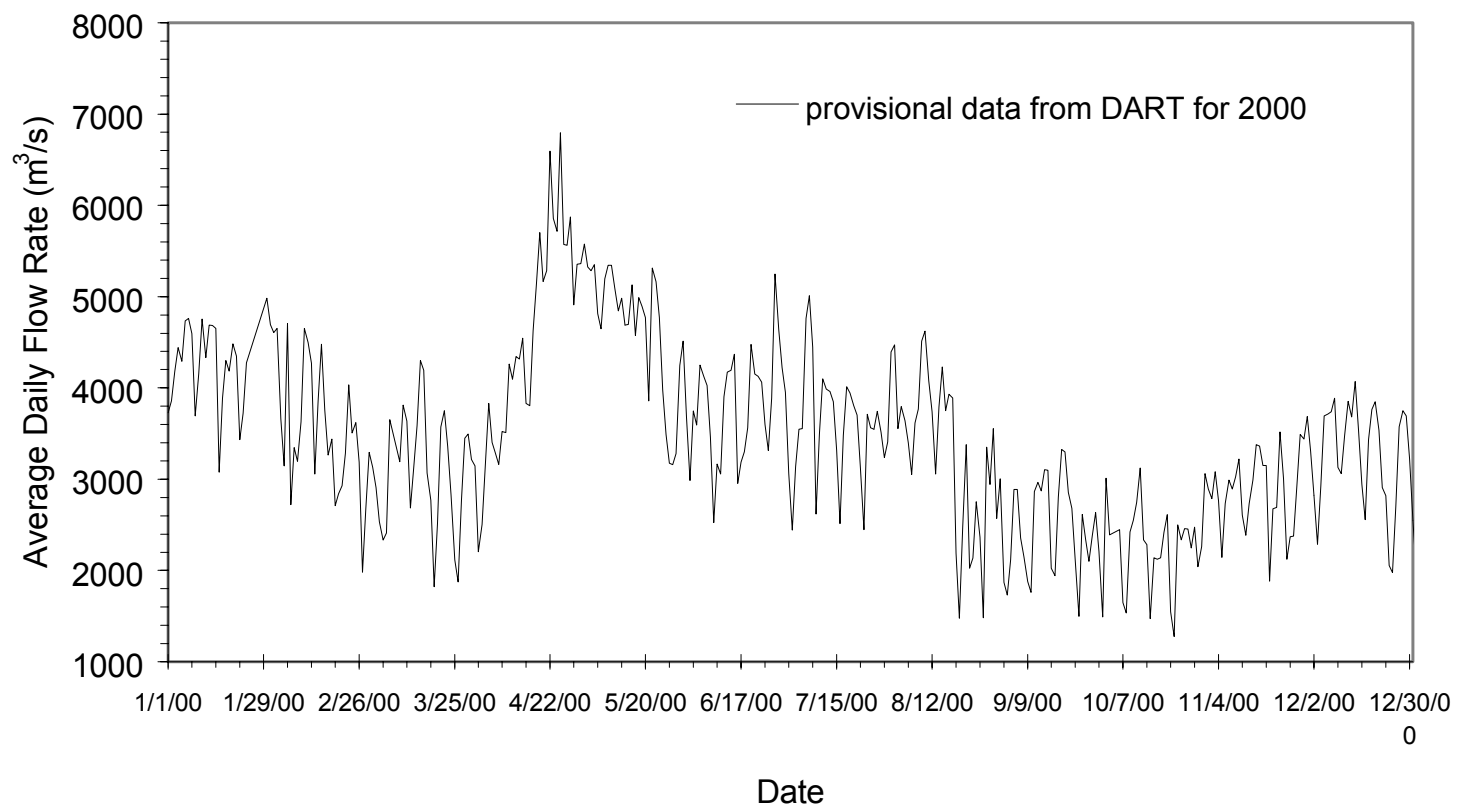

Figure 4.3-3. Average daily flow for the Columbia River During 2000 (data from USGS 2001a and DART 2001) 


\subsubsection{Water Quality of the Columbia River}

Water quality of the Columbia River is relatively good and meets U.S. Geological Survey (USGS) standards for a Class-A surface-water body (Poston et al. 2000). Class-A waters are to be suitable for essentially all uses, including raw drinking water, recreation, and wildlife habitat. State and federal drinking water standards apply to the Columbia River and are currently being met (see Section 6.2.2).

During 1999, the USGS measured several water quality parameters at Vernita Bridge, upstream of Hanford Site operations areas, and at the Richland pumphouse, which is downstream of the Hanford Site. Sample locations are shown in Figure 4.3-4. Total dissolved solids measured near the Hanford Site during 1999 ranged from 71 to $99 \mathrm{mg} / \mathrm{L}$ and total dissolved nitrogen ranged from 0.16 to $0.37 \mathrm{mg} / \mathrm{L}$. Dissolved oxygen ranged from 10 to $14 \mathrm{mg} / \mathrm{L}$ and $\mathrm{pH}$ was 7.7 to 8.2. There were no significant differences between upstream and downstream samples for these parameters. The results are presented in Poston et al. (2000).

Pacific Northwest National Laboratory (PNNL) measured both radiological and nonradiological constituents at Priest Rapids Dam and at the Richland pumphouse. Additional samples were taken at transects of the river and at near shore locations at Vernita Bridge, 100-F Area, 100-N Area, the Old Hanford Townsite, and the 300 Area. Results are presented in Bisping (2000) and summarized in Poston et al. (2000). Sample locations are shown in Figure 4.3-4. These data show an increase in tritium, nitrate, strontium-90, uranium, and iodine-129 along the Hanford Reach. All these constituents are known to be entering the river from contaminated groundwater beneath the Hanford Site (see Section 4.3.4.2). Measurements of tritium along transects showed higher concentrations near the Benton County shoreline (where Hanford operations are located) for samples at the 100-N Area, the Old Hanford Townsite, the 300 Area, and the Richland pumphouse.

Other sources of pollutants entering the river are irrigation return flows and groundwater seepage associated with irrigated agriculture. The USGS (1995) documented nitrate groundwater contamination in Franklin County, which also seeps into the river along the Hanford Reach. However, in spite of pollutants introduced from both the Hanford Site and other sources, dilution in the river results in contaminant concentrations that are well below drinking water standards (Poston et al. 2000).

\subsubsection{Yakima River}

The Yakima River, which follows a small length of the southwestern boundary of the Hanford Site, has much lower flows than the Columbia River. The average flow, based on nearly 60 years of daily flow records, is about $104 \mathrm{~m}^{3} / \mathrm{s}\left(3712 \mathrm{ft}^{3} / \mathrm{s}\right)$, with an average monthly maximum of $490 \mathrm{~m}^{3} / \mathrm{s}\left(17,500 \mathrm{ft}^{3} / \mathrm{s}\right)$ and minimum of $4.6 \mathrm{~m}^{3} / \mathrm{s}\left(165 \mathrm{ft}^{3} / \mathrm{s}\right)$. Exceptionally high flows were observed during 1996 and 1997 . The highest average daily flow rate during 1997 was nearly $1300 \mathrm{~m}^{3} / \mathrm{s}\left(45,900 \mathrm{ft}^{3} / \mathrm{s}\right)$. Average flow during 2000 was $89.9 \mathrm{~m}^{3} / \mathrm{s}\left(3176 \mathrm{ft}^{3} / \mathrm{s}\right)$. Average daily flows from 1991 through April 2001 are plotted in Figure 4.3-5. This figure includes provisional data from the USGS that have not yet been reviewed and are subject to change. The Yakima River System drains surface runoff from approximately one-third of the Hanford Site. Contaminant plumes from the Hanford Site do not reach the Yakima River and, because the elevation of the river surface is higher than the adjacent water table (based on well water-level measurements), groundwater is expected to flow from the Yakima River into the aquifer underlying the Site rather than from the aquifer into the river (Thorne et al. 1994). 


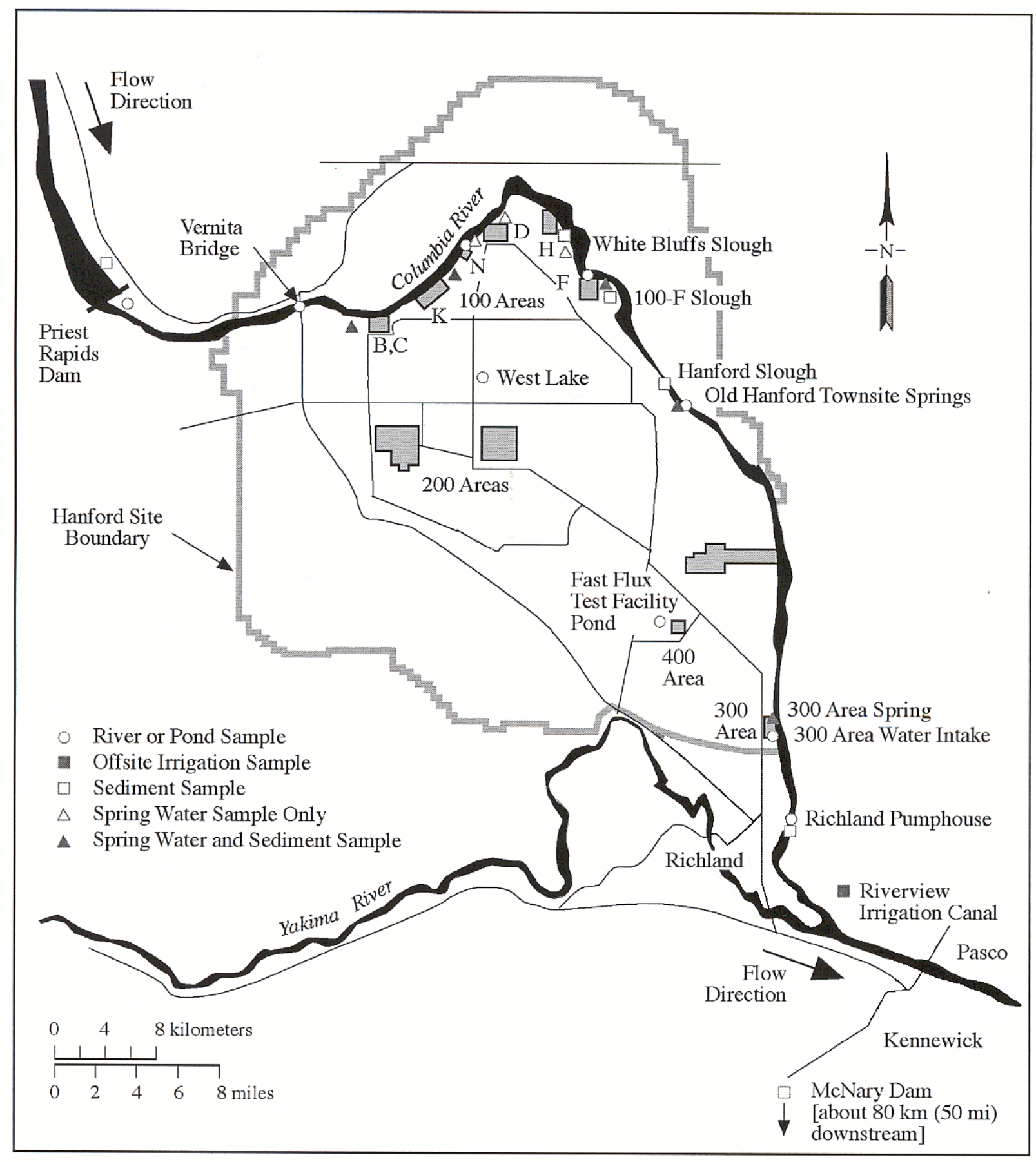

Figure 4.3-4. Columbia River Monitoring Locations (Poston et al. 2000) 


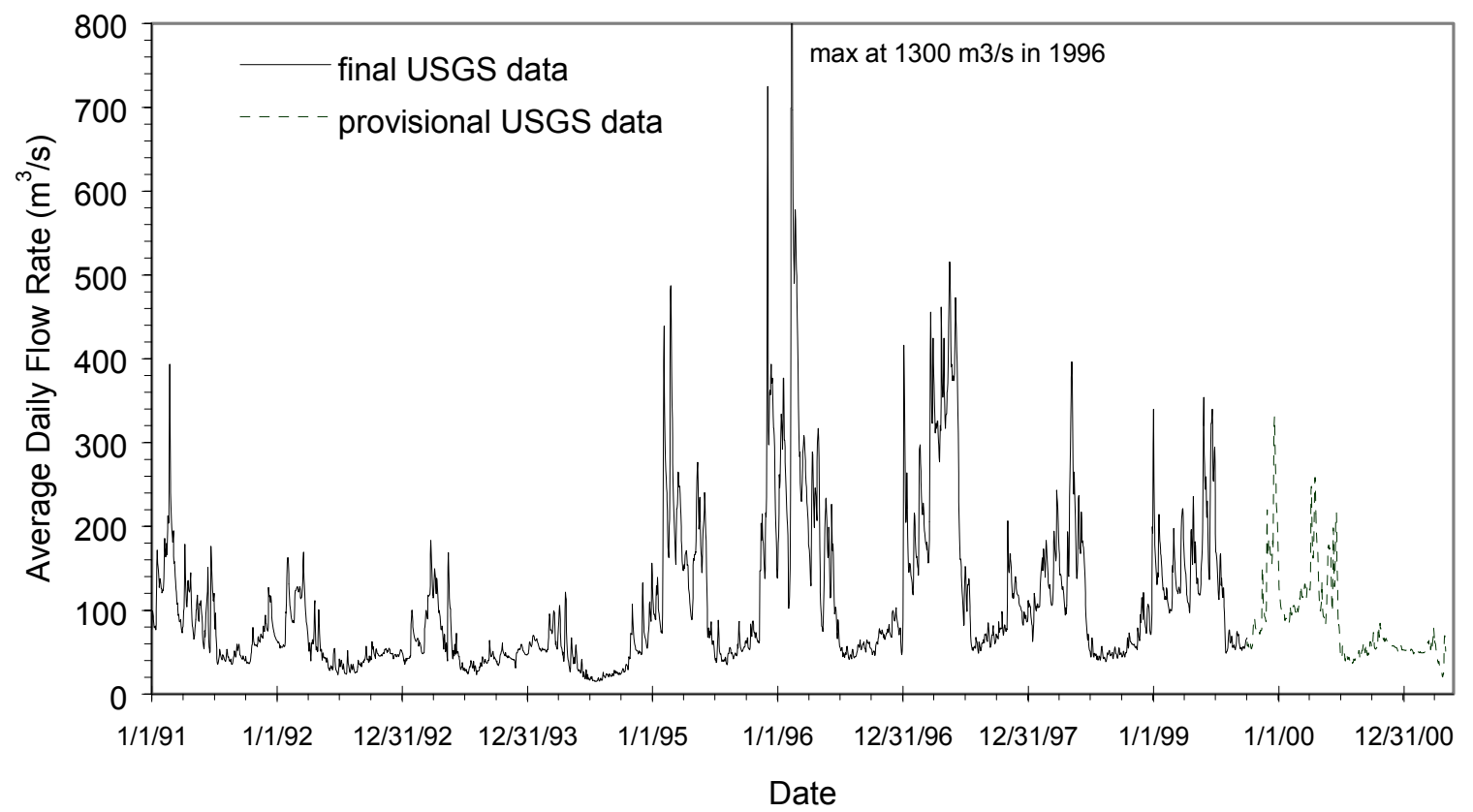

Figure 4.3-5. Average daily flow for the Yakima River from 1991 through April 2001 (data from USGS 2001a and USGS 2001b)

\subsubsection{Springs and Streams}

Several springs are found on the slopes of the Rattlesnake Hills along the western edge of the Hanford Site (DOE 1988). The Nature Conservancy of Washington, in its Biodiversity Inventory and Analysis of the Hanford Site - 1997 Annual Report (Hall 1998), documented an alkaline spring at the east end of Umtanum Ridge. Rattlesnake and Snively springs form small surface streams. Water discharged from Rattlesnake Springs flows down Dry Creek for about $3 \mathrm{~km}(1.6 \mathrm{mi})$ before disappearing into the ground (Figure 4.3-1). Cold Creek and its tributary, Dry Creek, are ephemeral streams within the Yakima River drainage system in the southwestern portion of the Hanford Site. These streams drain areas to the west of the Hanford Site and cross the southwestern part of the Site toward the Yakima River. When surface flow occurs, it infiltrates rapidly and disappears into the surface sediments in the western part of the Site. The ecological characteristics of these systems are described in Section 4.4.2.2.

\subsubsection{Columbia Riverbank Seepage}

The seepage of groundwater into the Columbia River has been known to occur for many years. Riverbank seeps were documented along the Hanford Reach long before Hanford operations began (Jenkins 1922). In the early 1980s, researchers identified 115 springs along the Benton County shoreline of the Hanford Reach (McCormack and Carlile 1984). Seepage occurs both below the river surface and on the exposed riverbank, particularly at low-river stage. The seeps flow intermittently, apparently influenced primarily by changes in river level. In many areas, water flows from the river into the aquifer at high river stage and then returns to the river at low river stage. This "bank-storage" phenomenon has been numerically modeled for the $100 \mathrm{H}$ Area (Hartman et al. 2001). 
In areas of contaminated groundwater, seeps and springs are also generally contaminated. However, the concentrations in seeping water along the riverbank may be lower than groundwater because of the bank-storage phenomenon. Elevated levels of contaminants have also been detected in near shore samples downstream from riverbank seeps (Poston et al. 2000). Riverbank seeps are monitored for radionuclides at the 100-N Area, the Old Hanford Townsite, and the 300 Area. Hanford-origin contaminants have been documented in some of these seeps (Peterson and Johnson 1992, Poston et al. 2000). Detected radionuclides include strontium-90, technetium-99, iodine-129, uranium-234, -235, and -238, and tritium. Detected metals include antimony, arsenic, cadmium, chromium, copper, lead, mercury, nickel, selenium, thallium, zinc, and occasionally silver. Chloride, fluoride, nitrate, and sulfate were also detected. Volatile organic compounds were near or below the detection limits. Results of these samples are listed in Bisping (2000) and summarized in Poston et al. (2000). Water samples are also collected along the 100-N shoreline at monitoring well 199-N-46 and at shoreline seepage wells. A strontium-90 concentration higher than the $1,000 \mathrm{pCi} / \mathrm{L}$ derived concentration guide was reported in 1999 for one of these seepage-monitoring wells (Poston et al. 2000). There were no visible riverbank seeps in the vicinity of this well. Concentrations of radionuclides including tritium, technetium-99, and iodine129 in riverbank seeps near the Old Hanford Townsite have generally been increasing since 1994. This is an area where a major groundwater plume from the 200 East Area intercepts the river. However, tritium concentration has declined since 1997. This decline may be due to the effects of radioactive decay and/or less wastewater disposal, resulting in the groundwater tritium plume moving at a slower velocity.

\subsubsection{Runoff and Net Infiltration}

Total estimated precipitation over the Pasco Basin is about $9 \times 10^{8} \mathrm{~m}^{3}\left(3.2 \times 10^{10} \mathrm{ft}^{3}\right)$ annually (DOE 1988). This was calculated by multiplying the average annual precipitation averaged over the Pasco Basin by the $4900 \mathrm{~km}^{2}\left(1900 \mathrm{mi}^{2}\right)$ basin area. Precipitation varies both spatially and temporally with higher amounts generally falling at higher elevations. As noted in Section 4.1.3, annual precipitation measured at the Hanford Meteorology Station (HMS) has varied from $7.6 \mathrm{~cm}(3$ in.) to $31.3 \mathrm{~cm}(12.3$ in.) since 1945. Most precipitation occurs during the late autumn and winter, with more than half of the annual amount occurring from November through February. Mean annual runoff from the Pasco Basin is estimated at $<3.1 \times 10^{7} \mathrm{~m}^{3} / \mathrm{yr}\left(1.1 \times 10^{9} \mathrm{ft}^{3} / \mathrm{yr}\right)$, or approximately $3 \%$ of the total precipitation (DOE 1988). Most of the remaining precipitation is lost through evapotranspiration. However, some precipitation that infiltrates the soil is not lost to evaporation or transpiration and eventually recharges the groundwater flow system.

\subsubsection{Flooding}

Large Columbia River floods have occurred in the past (DOE 1987), but the likelihood of recurrence of large-scale flooding has been reduced by the construction of several flood control/water-storage dams upstream of the Hanford Site. Major floods on the Columbia River are typically the result of rapid melting of the winter snowpack over a wide area augmented by above-normal precipitation. The maximum historical flood on record occurred June 7, 1894, with a peak discharge at the Hanford Site of $21,000 \mathrm{~m}^{3} / \mathrm{s}\left(742,000 \mathrm{ft}^{3} / \mathrm{s}\right)$. The floodplain associated with the 1894 flood is shown in Figure 4.3-6. This floodplain area was calculated by modeling based on topographic cross-sections across the river channel (ERDA 1976). The largest recent flood took place in 1948 with an observed peak discharge of 20,000 $\mathrm{m}^{3} / \mathrm{s}\left(700,000 \mathrm{ft}^{3} / \mathrm{s}\right)$ at the Hanford Site. The probability of flooding at the magnitude of the 1894 and 1948 floods has been greatly reduced because of upstream regulation by dams (Figure 4.3-7). The exceptionally high runoff during the spring of 1996 resulted in a maximum discharge of nearly 11,750 $\mathrm{m}^{3} / \mathrm{s}\left(415,000 \mathrm{ft}^{3} / \mathrm{s}\right)$ (USGS 2001a). 


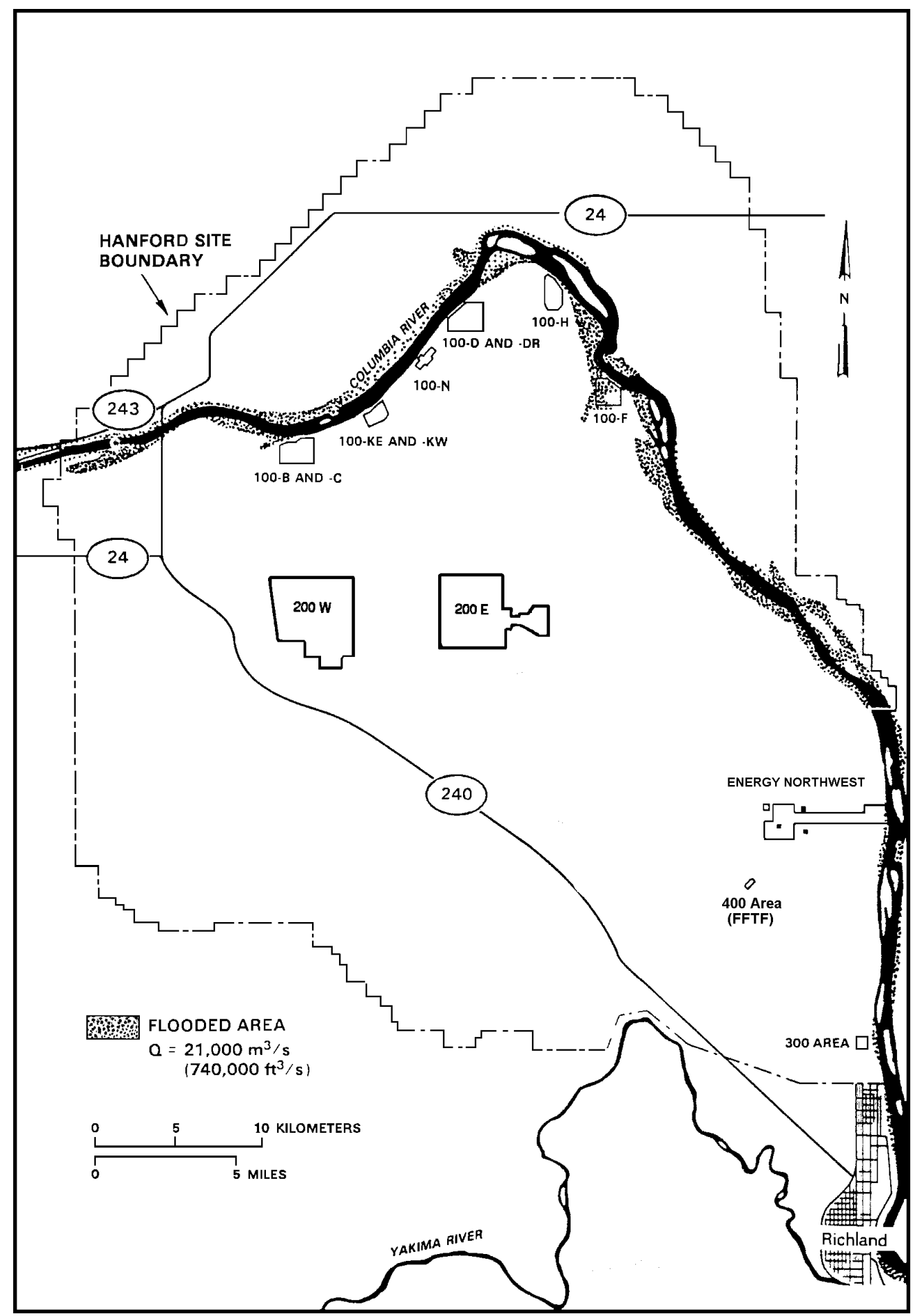

Figure 4.3-6. Flood Area During the 1894 Flood (DOE 1986) 


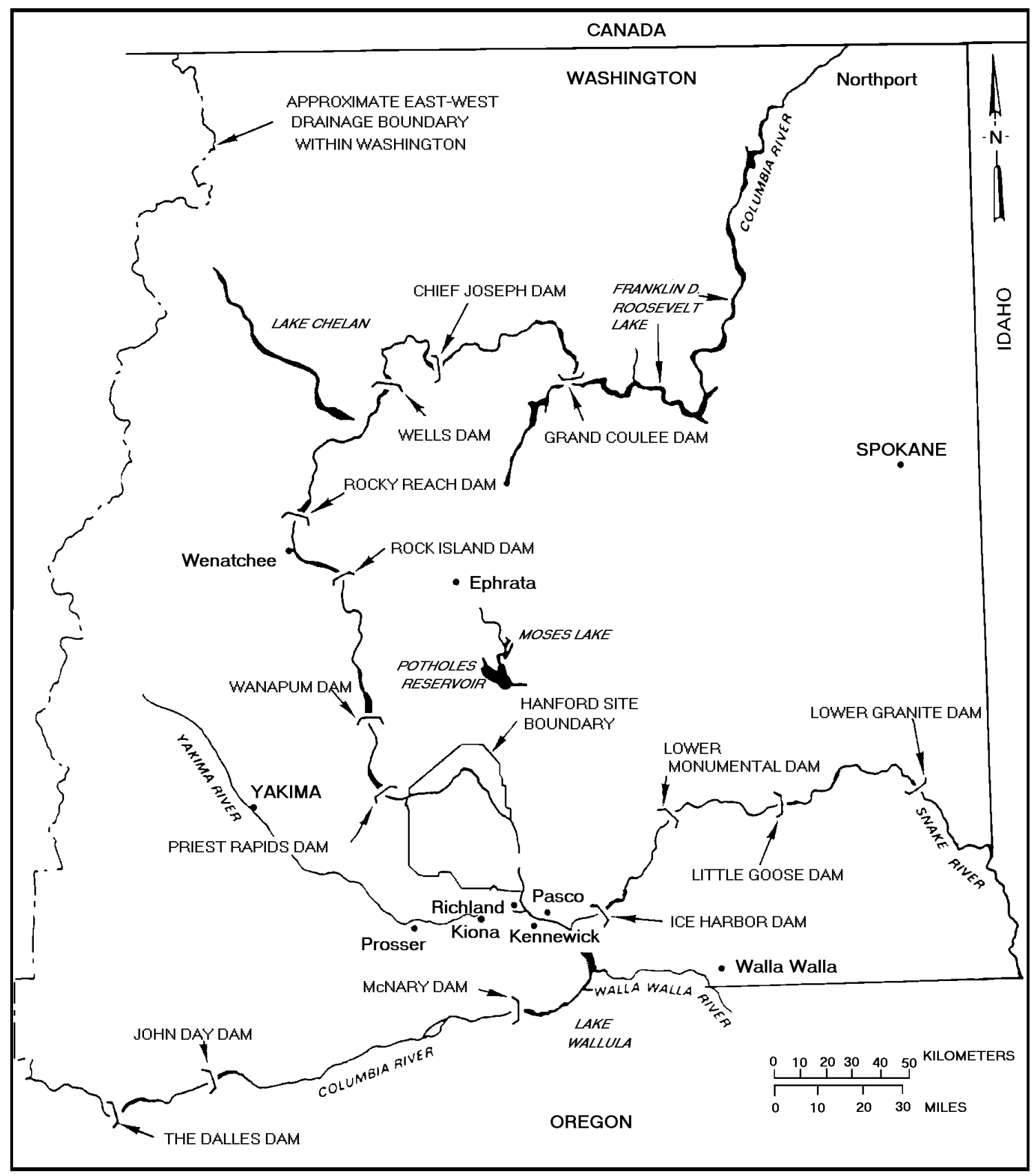

Figure 4.3-7. Locations of Principal Dams Within the Columbia Plateau (DOE 1988) 
There are no Federal Emergency Management Agency (FEMA) floodplain maps for the Hanford Reach of the Columbia River. FEMA only maps developing areas, and the Hanford Reach has been specifically excluded because the adjacent land is primarily under federal control.

Evaluation of flood potential is conducted in part through the concept of the probable maximum flood, which is determined from the upper limit of precipitation falling on a drainage area and other hydrologic factors, such as antecedent moisture conditions, snowmelt, and tributary conditions, that could result in maximum runoff. The probable maximum flood for the Columbia River downstream of Priest Rapids Dam has been calculated to be $40,000 \mathrm{~m}^{3} / \mathrm{s}\left(1.4\right.$ million $\left.\mathrm{ft}^{3} / \mathrm{s}\right)$ and is greater than the 500 -year flood. The floodplain associated with the probable maximum flood is shown in Figure 4.3-8. This flood would inundate parts of the 100 Areas located adjacent to the Columbia River, but the central portion of the Hanford Site would remain unaffected (DOE 1986).

The U.S. Army Corps of Engineers (Corps) (1989) has derived the Standard Project Flood with both regulated and unregulated peak discharges given for the Columbia River downstream of Priest Rapids Dam. Frequency curves for both natural (unregulated) and regulated peak discharges are also given for the same portion of the Columbia River. The regulated Standard Project Flood for this part of the river is given as $15,200 \mathrm{~m}^{3} / \mathrm{s}\left(54,000 \mathrm{ft}^{3} / \mathrm{s}\right)$ and the 100 -year regulated flood as $12,400 \mathrm{~m}^{3} / \mathrm{s}\left(440,000 \mathrm{ft}^{3} / \mathrm{s}\right)$. A map for the 100-yr flood area is available (DOE-RL 1998) but impacts to the Site are negligible and would be less than the probable maximum flood (Figure 4.3-8).

Potential dam failures on the Columbia River have been evaluated. Upstream failures could arise from a number of causes, with the magnitude of the resulting flood depending on the degree of breaching at the dam. The Corps evaluated a number of scenarios on the effects of failures of Grand Coulee Dam, assuming flow conditions of $11,000 \mathrm{~m}^{3} / \mathrm{s}\left(400,000 \mathrm{ft}^{3} / \mathrm{s}\right)$. For emergency planning, they hypothesized that $25 \%$ and $50 \%$ breaches, the "instantaneous" disappearance of $25 \%$ or $50 \%$ of the center section of the dam, would result from the detonation of nuclear explosives in sabotage or war. The discharge or floodwave resulting from such an instantaneous $50 \%$ breach at the outfall of the Grand Coulee Dam was determined to be $600,000 \mathrm{~m}^{3} / \mathrm{s}\left(21\right.$ million $\left.\mathrm{ft}^{3} / \mathrm{s}\right)$. In addition to the areas inundated by the probable maximum flood (Figure 4.3-8), the remainder of the 100 Areas, the 300 Area, and nearly all of Richland, Washington, would be flooded (DOE 1986; see also ERDA 1976). No determinations were made for failures of dams upstream, for associated failures downstream of Grand Coulee, or for breaches $>50 \%$ of Grand Coulee, for two principal reasons:

1. The $50 \%$ scenario was believed to represent the largest realistically conceivable flow resulting from either a natural or human-induced breach (DOE 1986), i.e., it was hard to imagine that a structure as large as Grand Coulee Dam would be $100 \%$ destroyed instantaneously.

2. It was also assumed that a scenario such as the $50 \%$ breach would occur only as the result of direct explosive detonation, and not because of a natural event such as an earthquake, and that even a $50 \%$ breach under these conditions would indicate an emergency situation in which there might be other overriding major concerns. 


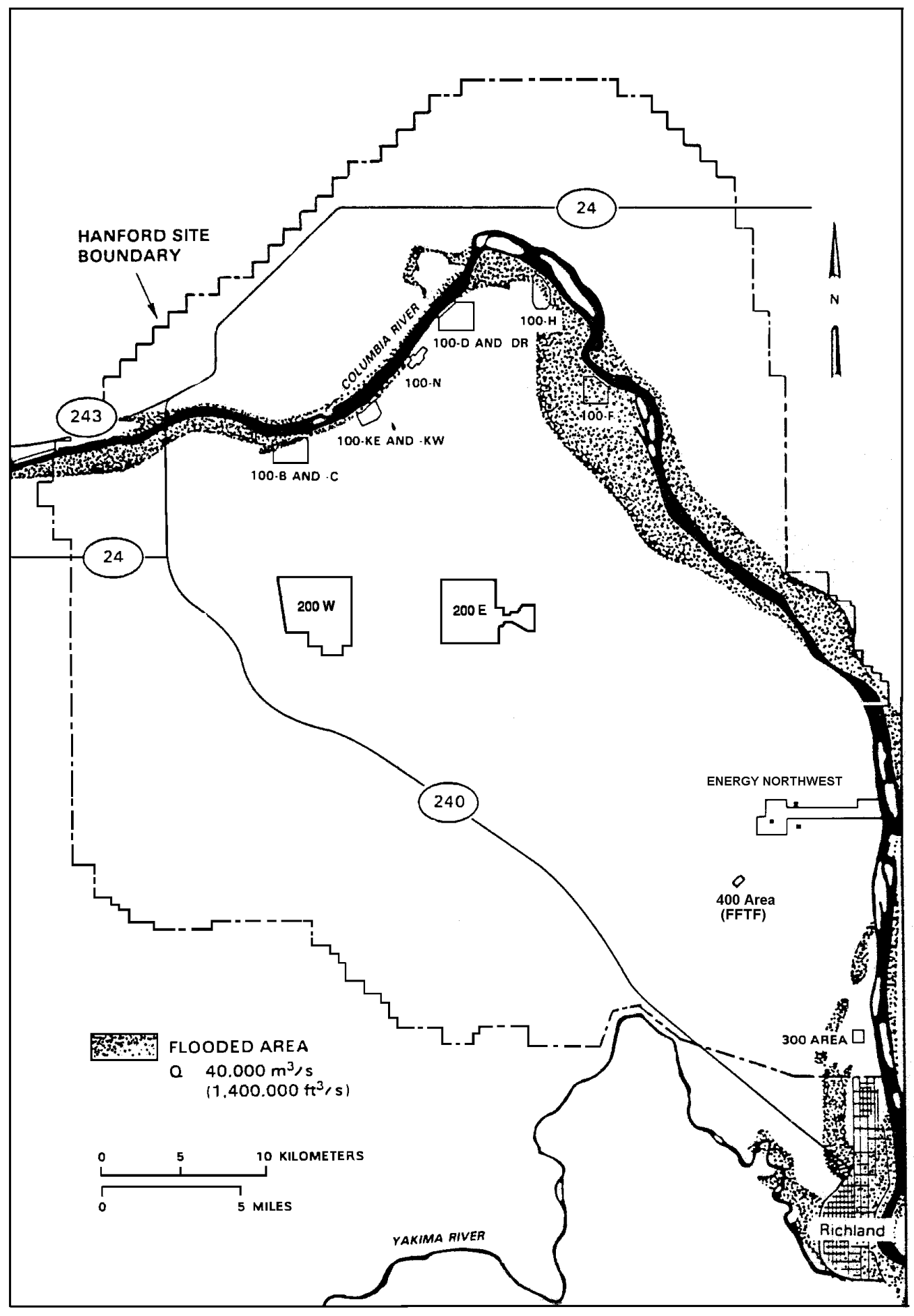

Figure 4.3-8. Flood Area for the Probable Maximum Flood (DOE 1986) 
The possibility of a landslide resulting in river blockage and flooding along the Columbia River has been examined for an area bordering the east side of the river upstream of the city of Richland. The possible landslide area considered was the $75-\mathrm{m}-(250-\mathrm{ft}-)$ high bluff generally known as White Bluffs. Calculations were made for an $8 \times 10^{5} \mathrm{~m}^{3}\left(1 \times 10^{6} \mathrm{yd}^{3}\right)$ landslide volume with a concurrent flood flow of

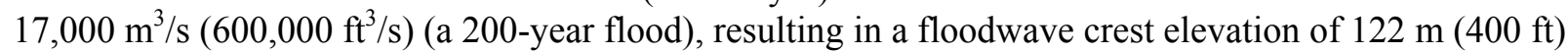
above mean sea level. Areas inundated upstream of such a landslide event would be similar to those shown in Figure 4.3-8 (DOE 1986).

There have been fewer than 20 major floods on the Yakima River since 1862 (DOE 1986). The most severe occurred in November 1906, December 1933, May 1948, and February 1996; discharge magnitudes at Kiona, Washington, were 1870, 1900, 1050, and $1300 \mathrm{~m}^{3} / \mathrm{s}(66,000,67,000,37,000$, and $\left.45,900 \mathrm{ft}^{3} / \mathrm{s}\right)$, respectively. Average flow of the Yakima River is $104 \mathrm{~m}^{3} / \mathrm{s}\left(165 \mathrm{ft}^{3} / \mathrm{s}\right)$, and the average monthly maximum is $490 \mathrm{~m}^{3} / \mathrm{s}\left(17,500 \mathrm{ft}^{3} / \mathrm{s}\right)$. The recurrence intervals for the 1933 and 1948 floods are estimated at 170 and 33 years, respectively. The development of irrigation reservoirs within the Yakima River Basin has considerably reduced the flood potential of the river. The southern border of the Hanford Site could be susceptible to a 100-year flood on the Yakima River (Figure 4.3-9).

In 1980, a flood risk analysis of Cold Creek was conducted as part of the characterization of a basaltic geologic repository for high-level radioactive waste. Such design work is usually done according to the criteria of Standard Project Flood or probable maximum flood, rather than the worst-case or 100-year flood scenario. Therefore, in lieu of 100- and 500-year floodplain studies, a probable maximum flood evaluation was performed (Skaggs and Walters 1981). The probable maximum flood discharge rate for the lower Cold Creek Valley was $2265 \mathrm{~m}^{3} / \mathrm{s}(80,000 \mathrm{ft} / \mathrm{s})$ compared to $564 \mathrm{~m}^{3} / \mathrm{s}\left(19,900 \mathrm{ft}^{3} / \mathrm{s}\right)$ for the 100 year flood. Modeling indicated that access to the reference repository would be unimpaired but that State Route (SR) 240 along the southwestern and western areas would not be usable (Figure 4.3-10). This area was delineated using the U.S. Army Corps of Engineers' HEC-2 Water Surface Profiles model.

\subsubsection{Onsite Ponds and Ditches}

Currently active ponds on the Hanford Site are shown in Figure 4.3-1. There are no currently active ditches on the Hanford Site. Ponds include West Lake, the 200 Areas Treated Effluent Disposal Facility (TEDF) disposal ponds, and a 400 Area wastewater disposal pond.

West Lake is north of the 200 East Area and is a natural feature recharged from groundwater (Gephardt et al. 1976; Poston et al. 1991). West Lake has not received direct effluent discharges from Site facilities; rather, its existence is caused by the intersection of the elevated water table with the land surface in the topographically low area. Water levels of West Lake fluctuate with water table elevation, which is influenced by wastewater discharge in the 200 Areas. The water level and size of the lake has been decreasing over the past several years because of reduced wastewater discharge (see Section 4.3.3.1). There is unsubstantiated information that sewage sludge may have been dumped in the vicinity of West Lake in the 1940s, and this has been cited as the reason for elevated dissolved solids and nitrate in the lake water (Emery and McShane 1978; Meinhardt and Frostenson 1979). However, it is possible that the concentration of salts resulted from evaporation of groundwater at the lake, which has no outlet. Total dissolved solids are approximately $15,000 \mathrm{mg} / \mathrm{L}$, and $\mathrm{pH}$ is over 9 . Nitrate and ammonia concentrations of about 1.8 and $2.6 \mathrm{mg} / \mathrm{L}$, respectively, have been reported, which are higher than freshwater lakes, but lower than other alkaline lakes in Washington such as Soap Lake and Lake Lenore. West Lake contains relatively high levels of uranium that are thought to be from natural sources concentrated by evaporation in the lake (Poston et al. 1991). Recent sampling results for West Lake are presented in Poston et al. (2000). 


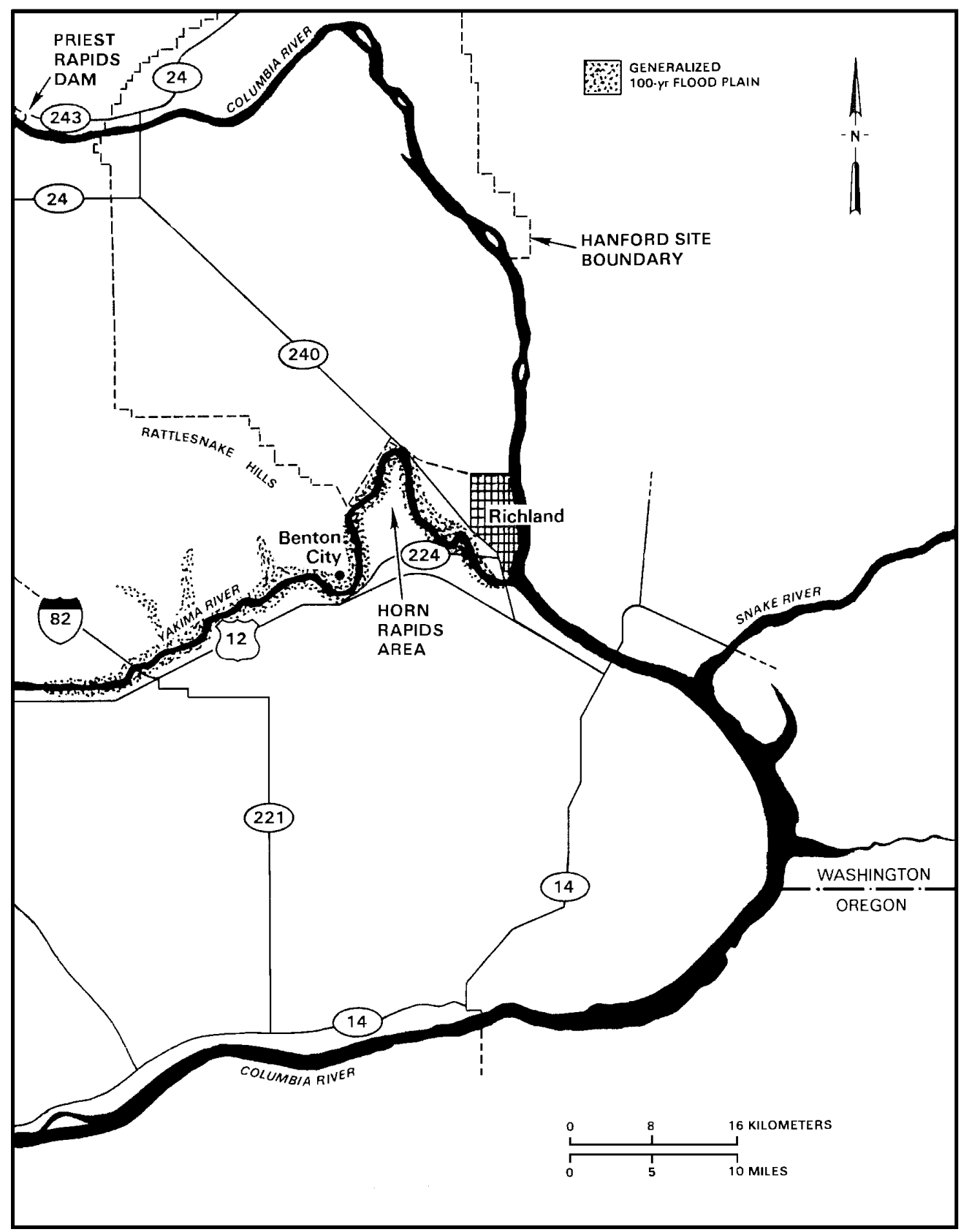

Figure 4.3-9. Flood Area from a 100-Year Flood of the Yakima River near the Hanford Site (DOE 1986) 


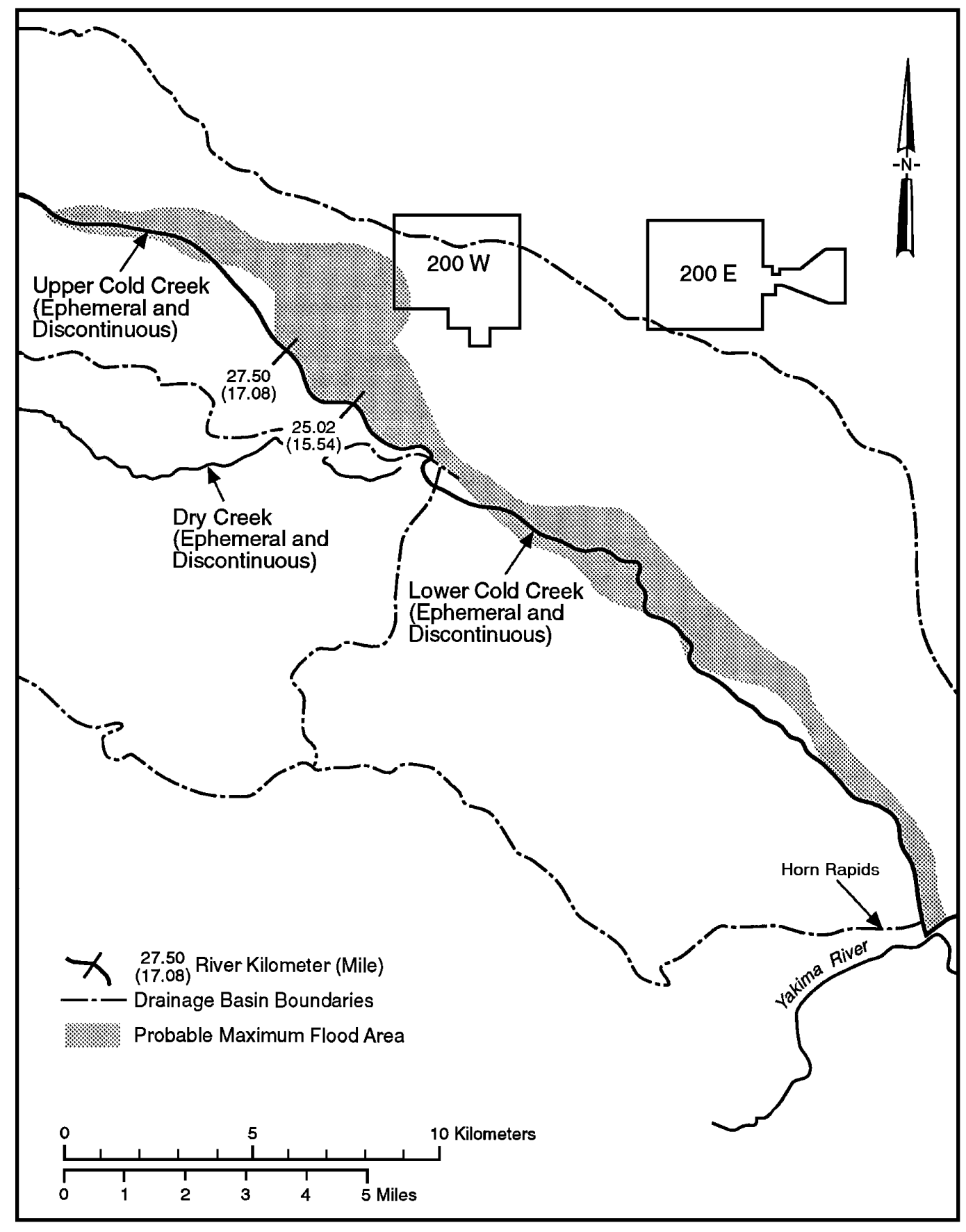

Figure 4.3-10. Extent of Probable Maximum Flood in Cold Creek Area (Skaggs and Waters 1981) 
The 200 Areas TEDF consists of two disposal ponds. These ponds are each $0.02 \mathrm{~km}^{2}\left(0.008 \mathrm{mi}^{2}\right)$ in size and receive industrial wastewater permitted in accordance with Ecology's State Waste Discharge Permit Program (WAC 173-216). The wastewater percolates into the ground from the disposal ponds. The 400 Area Pond is located near the 400 Area and is used for the disposal of process water (primarily cooling tower water) from the Fast Flux Test Facility (Dirkes and Hanf 1998). The pond water shows elevated levels of tritium because the groundwater in the 400 Area, which is the source of this water, is affected by a plume from the 200 East Area. The TEDF and 400 Area ponds are not accessible to the public. However, they are accessible to migratory waterfowl, creating a potential pathway for the dispersion of contaminants.

The Nature Conservancy (Hall 1998) documented the existence of several naturally occurring vernal ponds near Gable Mountain and Gable Butte. These ponds appear to occur where a depression is present in a relatively shallow buried basalt surface. Water collects within the depression over the winter resulting in a shallow pond that dries during the summer months. The formation of these ponds in any particular year depends on the amount and temporal distribution of precipitation and snowmelt events. The vernal ponds range in size from about $20 \mathrm{ft} \times 20 \mathrm{ft}$ to $150 \mathrm{ft} \times 100 \mathrm{ft}(6.1 \mathrm{~m} \times 6.1 \mathrm{~m}$ to $45.73 \mathrm{~m} \times 30.5$ $\mathrm{m}$ ), and were found in three clusters. Approximately 10 were documented at the eastern end of Umtanum Ridge, 6 or 7 were observed in the central part of Gable Butte, and 3 were found at the eastern end of Gable Mountain. (See Figure 4.0-1 for Gable Mountain, Gable Butte, and Umtanum Ridge locations.)

\subsubsection{Offsite Surface Water}

Other than rivers and springs, there are no naturally occurring bodies of surface water adjacent to the Hanford Site. However, there are artificial wetlands, caused by irrigation, on the east and west sides of the Wahluke Slope, which lies north of the Columbia River. Hatcheries and irrigation canals constitute the only other artificial surface water in the Hanford Site vicinity. The Ringold Hatchery, just south of the Hanford Site boundary on the east side of the Columbia River, is the only hatchery.

\subsubsection{Hanford Site Vadose Zone}

The vadose zone is that part of the subsurface found between the ground surface and the top of the water table. At the Hanford Site, the thickness of the vadose zone ranges from $0 \mathrm{~m}(0 \mathrm{ft})$ near the Columbia River to greater than $100 \mathrm{~m}$ (328 ft) beneath parts of the central plateau (Hartman 2000). Unconsolidated glacio-fluvial sands and gravels of the Hanford formation make up most of the vadose zone. In some areas, however, such as west and south of 200 East Area and in some of the 100 Areas, the fluvial-lacustrine sediments of the Ringold Formation make up the lower part of the vadose zone.

Moisture movement through the vadose zone is important at the Hanford Site because it is the driving force for migration of most contaminants. Radioactive and hazardous wastes in the soil column from past intentional liquid-waste disposals, unplanned leaks, solid waste burial grounds, and underground tanks are potential sources of continuing and future vadose zone and groundwater contamination. Contaminants may continue to move downward for long periods (tens to hundreds of years depending on recharge rates) after termination of liquid waste disposal.

Except for the State Approved Land Disposal Site (the 200 Area Treated Effluent Disposal Facility ponds), and septic drain fields, artificial recharge to the vadose zone ended in the mid-1990s. Currently, the major source of recharge is natural precipitation. Natural infiltration in the vadose zone causes older preexisting water to be displaced downward by newly infiltrated water. The amount of recharge at any particular site is highly dependent on the soil type and the presence of vegetation. Usually, vegetation reduces the amount of infiltration through the biological process of transpiration. 
Although most natural recharge is probably uniform flow (Jones et al. 1998), the vadose zone stratigraphy influences the movement of liquid through the soil column. Where conditions are favorable, lateral spreading of liquid effluent and/or local perched water zones may develop. Perched water zones form where downward moving moisture accumulates on top of low-permeability soil lenses or highly cemented horizons.

Preferential flow may also occur along discontinuities, such as clastic dikes and fractures. Clastic dikes are a common geologic feature in the suprabasalt sediments at the Hanford Site. Their most important feature is their potential to either enhance or inhibit vertical and lateral movement of contaminants in the subsurface, depending on textural relationships. Fecht et al. (1998) give the most recent compilation of information known about clastic dikes in the Pasco Basin.

Subsurface source characterization, sediment sampling and characterization, and vadose zone monitoring are employed to describe the current and future configuration of contamination in the vadose zone.

\subsubsection{Vadose Zone Contamination}

The Hanford Site has more than 800 past-practice liquid-disposal facilities. Radioactive liquid waste was discharged to the vadose zone through reverse (injection) wells, French drains, cribs, ponds, trenches, and ditches. Over the last 56 years, 1.5 to 1.7 billion $\mathrm{m}^{3}$ (396 to 449 billion gal) of effluent were disposed to the soils (Gephart 1999). Most effluent was released in the 200 Areas. The major groundwater contaminant plumes emanating from the 200 Areas are tritium and nitrate. The major source for both was discharges resulting from chemical processing.

Also of concern are technetium-99 and iodine-129 which, like tritium and nitrate, are mobile in groundwater. The major sources of technetium-99 and iodine-129 were discharges to liquid disposal facilities. Vadose zone sources for these contaminants almost certainly remain beneath many pastpractice disposal facilities. However, other than physical sampling and laboratory analysis, there are no currently available monitoring techniques for tritium, nitrate, technetium-99 and iodine-129 in the vadose zone.

Approximately 280 unplanned releases in the 200 Areas also contributed contaminants to the vadose zone (DOE 1997a). Many of these were from underground tanks and have contributed significant contamination to the vadose zone. In addition, approximately 50 active and inactive septic tanks and drain fields and numerous radioactive and non-radioactive landfills and dumps have impacted the vadose zone (DOE 1997a). The landfills are and were used to dispose of solid wastes, which, in most instances, are easier to locate, retrieve, and remediate than are liquid wastes.

One hundred forty-nine single-shell tanks and 28 double-shell tanks have been used to store highlevel radioactive and mixed wastes in the 200 Areas. The wastes resulted from uranium and plutonium recovery processes and, to a lesser extent, from strontium and cesium recovery processes. Sixty-seven of the single-shell tanks are assumed to have leaked an estimated total of 2839 to $3975 \mathrm{~m}^{3}(750,000$ to $1,050,000 \mathrm{gal}$ ) of contaminated liquid to the vadose zone (Hanlon 2001). The three largest tank leaks were $435,320 \mathrm{~L}$ (115,000 gal), 37,850 to $1,048,560 \mathrm{~L}$ (10,000 to 277,000 gal), and 265,980 L (70,365 gal). The average tank leak was between 41,640 and 60,565 L (11,000 and 16,000 gal)(Hanlon 2001). 
Cooling water from the single-pass reactors along the Columbia River was routinely routed to retention basins prior to return to the river. Thermal shock from the hot cooling water cracked the basins so that much of the cooling water leaked into the vadose zone. In addition, trenches were used for disposal of cooling water from 100-KE, 100-KW, and 100-N Reactors. The disposed cooling waters contained fission and neutron activation products and some chemicals and actinides. Of biggest concern are the impacts of tritium, strontium-90, nitrate, and chromium migrating through the vadose zone to groundwater, and ultimately, to the Columbia River. Chromium is actively being remediated at the $100-\mathrm{K}$ and $100-\mathrm{H}$ Areas by pump-and-treat methods and in the 100-D/DR Area by pump-and-treat and in situ redox methods (Hartman et al. 2001).

Highly contaminated cooling water, such as water that had contacted broken fuel rods, was routed to trenches rather than being directly returned to the river. These fluids contained large quantities of fission and neutron activation products.

Leakage from fuel-storage basins in the 100-K Area also contributes potential significant inventories of fission products, transuranics, and carbon-14 to the soil column (Johnson et al. 1995). Thus, both pastpractice sites and fuel-storage basin leakage are potential vadose zone sources of contaminants in the 100 Areas.

The amount of contamination remaining in the vadose zone is uncertain. Several compilations of vadose zone contamination have been formulated through the past years. DOE (1997a) and Kincaid et al. (1998) contain the most recent inventories of contaminants disposed to past-practice liquid disposal facilities in the 200 Areas. Dorian and Richards (1978) list contaminant inventories disposed to most 100 Area past-practice facilities. Agnew (1997) and Anderson (1990) list inventories of effluents sent to single-shell tanks. Most recently, MacTec-ERS has issued a series of reports that estimate the curies of gamma emitting radionuclides and the volumes of contaminated soil associated with each single-shell tank farm. (See the series of online reports at the Hanford Tank Farm Vadose Zone Project. Available URL: http://www.doegjpo.com/programs/hanf/HTFVZ.html ) Their estimates for all locations for the three most widespread contaminants are $8901 \mathrm{Ci}$ of Cesium-137 in $395,550 \mathrm{~m}^{3}$ of soil, $0.8611 \mathrm{Ci}$ of Europium-154 in $30,133 \mathrm{~m}^{3}$ of soil, and $0.7424 \mathrm{Ci}$ of Cobalt- 60 in $74,369 \mathrm{~m}^{3}$ of soil.

The quantities of contaminants remaining in the vadose zone beneath both tanks and past-practice liquid disposal sites depend on the amounts flushed through the vadose zone to groundwater, which is unknown. However, significant inventories of contaminants are expected to remain in the vadose zone especially beneath facilities that received small amounts of liquid but large amounts of contaminants.

Further information on vadose zone characterization and monitoring activities on the Hanford Site is available online at http://etd.pnl.gov:2080/vadose and http://www.bhi-erc.com/projects/vadose/ as well as in Ward and Gee (2000), Horton and Randall (2000), Hartman et al. (2001) and Serne et al. (2001a, b, c).

\subsubsection{Groundwater}

Groundwater is one component of the hydrologic cycle. Groundwater beneath the Hanford Site originated as either natural recharge from rain and snowmelt, or as artificial recharge from excess irrigation, canal seepage, and wastewater disposal. The groundwater will eventually return to the surface environment as discharge to springs and seepage into rivers and streams, or through evaporation from shallow water table areas. Or it may be brought to the surface through wells or excavations. However, it may take many thousands of years for groundwater in deeper aquifers to reach the surface. 


\subsubsection{Hanford Site Aquifer System}

Groundwater beneath the Hanford Site is found in both an upper unconfined aquifer system and deeper basalt-confined aquifers. The unconfined aquifer system is also referred to as the suprabasalt aquifer system because it is within the sediments that overlie the basalt bedrock. See Figure 4.2-4 for a stratigraphic column showing the relative positions of the basalts and overlying sediments. Portions of the suprabasalt aquifer system are locally confined by low-permeability layers of fine-grained sediment. However, because the entire suprabasalt aquifer system is interconnected on a site-wide scale, it is referred to in this report as the Hanford unconfined aquifer system.

Basalt-Confined Aquifer System. Relatively permeable sedimentary interbeds and the more porous tops and bottoms of basalt flows form confined aquifers within the Columbia River Basalts. The horizontal hydraulic conductivities of most of these aquifers fall in the range of $10^{-10}$ to $10^{-4} \mathrm{~m} / \mathrm{s}\left(3 \times 10^{-10}\right.$ to $3 \times 10^{-4} \mathrm{ft} / \mathrm{s}$ ). Saturated but relatively impermeable dense interior sections of the basalt flows have horizontal hydraulic conductivities ranging from $10^{-15}$ to $10^{-9} \mathrm{~m} / \mathrm{s}\left(3 \times 10^{-15}\right.$ to $\left.3 \times 10^{-9} \mathrm{ft} / \mathrm{s}\right)$, about five orders of magnitude lower than some of the confined aquifers that lie between these basalt flows (DOE 1988). Hydraulic-head information indicates that groundwater in the basalt-confined aquifers generally flows toward the Columbia River and, in some places, toward areas of enhanced vertical communication with the unconfined aquifer system (Hartman et al. 2001; DOE 1988; Spane 1987). The basalt-confined aquifer system is important because there is a potential for significant groundwater movement between the two systems. Head relationships presented in previous reports (DOE 1988) demonstrate the potential for such communication. In addition, limited water chemistry data indicate that interaquifer leakage has taken place in an area of increased vertical communication near the Gable Mountain anticlinal structure, north of the 200 East Area (Graham et al. 1984; Jensen 1987).

Unconfined Aquifer System. The unconfined aquifer system is composed primarily of the Ringold Formation and overlying Hanford formation described in Section 4.2. In some areas, pre-Missoula gravels (distantly derived subunit) of the Plio-Pleistocene unit lie between these formations and below the water table. The other subunits of the Plio-Pleistocene unit are generally above the water table.

Groundwater in the unconfined aquifer at Hanford generally flows from recharge areas in the elevated region near the western boundary of the Hanford Site toward the Columbia River on the eastern and northern boundaries. The Columbia River is the primary discharge area for the unconfined aquifer. A map showing water table elevations for the Hanford Site and adjacent areas across the Columbia River is shown in Figure 4.3-11. The Yakima River borders the Hanford Site on the southwest and is generally regarded as a source of recharge. Along the Columbia River shoreline, daily river level fluctuations may result in water table elevation changes of up to $3 \mathrm{~m}(10 \mathrm{ft})$. During the high river stage periods of 1996 and 1997, some wells near the Columbia River showed water level changes of more than $3 \mathrm{~m} \mathrm{(10} \mathrm{ft).} \mathrm{As}$ the river stage rises, a pressure wave is transmitted inland through the groundwater. The longer the duration of the higher river stage, the farther inland the effect is propagated. The pressure wave is observed farther inland than the water actually moves. For the river water to flow inland, the river level must be higher than the groundwater surface and must remain high long enough for the water to flow through the sediments. Typically, this inland flow of river water is restricted to within several hundred feet of the shoreline (McMahon and Peterson 1992).

Gee et al. (1992) and Fayer et al. (1996) estimate that recharge rates from precipitation range from near zero to over $100 \mathrm{~mm} /$ year. Recharge is highly variable both spatially and temporally. It is highest for coarse-textured soils bare of deep-rooted vegetation and in years with rapid snowmelt events and precipitation during cool months. The magnitude of recharge at a particular location is influenced by five 


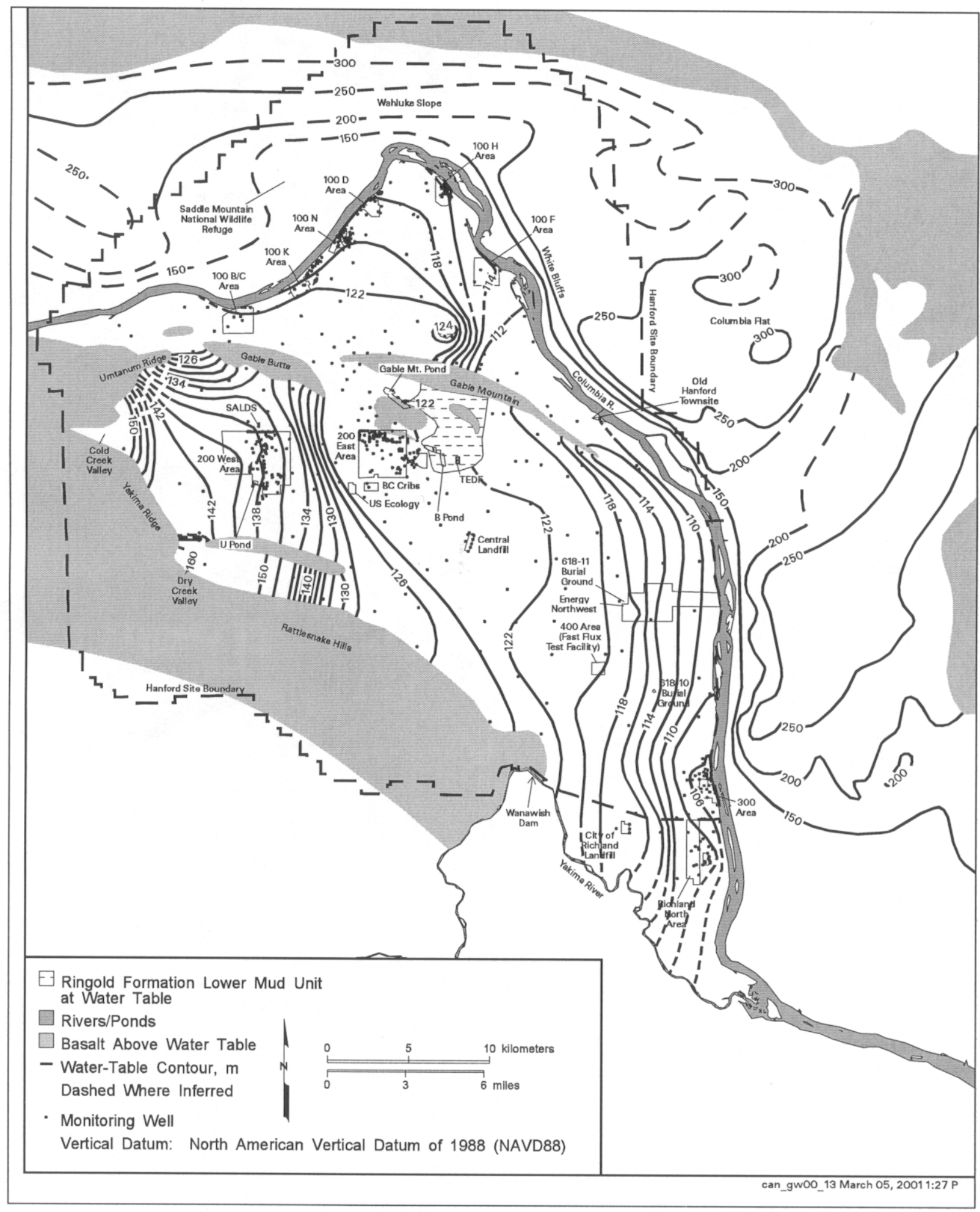

Figure 4.3-11. Water Table Elevations for the Unconfined Aquifer at Hanford, March/April 2000 (Hartman et al. 2001) 
main factors: climate, soils, vegetation, topography, and springs and streams. Events such as the 24 Command Fire that burned vegetation from a large portion of the Hanford Site during the summer of 2000 also affect recharge rates. Fayer et al. (1996) used several types of field data and computer modeling to estimate the areal distribution of mean recharge rates for the soil and vegetation conditions at the Hanford Site, including any disturbance by Hanford operations.

Between 1944 and the mid-1990s, the volume of artificial recharge from Hanford wastewater disposal was significantly greater than the natural recharge. An estimated $1.68 \times 10^{12} \mathrm{~L}\left(4.44 \times 10^{11}\right.$ gal) of liquid was discharged to disposal ponds, trenches, and cribs during this period. Wastewater discharge has decreased since 1984 and currently contributes a volume of recharge in the same range as the estimated natural recharge from precipitation. Because of the reduction in discharges, groundwater levels are falling, particularly around the operational areas (Hartman 2000).

After the beginning of Hanford operations in 1943, the water table rose about $27 \mathrm{~m}(89 \mathrm{ft})$ under the U Pond disposal area in the 200 West Area and about $9 \mathrm{~m} \mathrm{(30} \mathrm{ft)} \mathrm{under} \mathrm{disposal} \mathrm{ponds} \mathrm{near} \mathrm{the} 200$ East Area. The volume of water that was discharged to the ground at the 200 West Area was actually less than that discharged at the 200 East Area. However, the lower conductivity of the aquifer near the 200 West Area inhibited groundwater movement in this area resulting in a higher groundwater mound. The presence of the groundwater mounds locally affected the direction of groundwater movement, causing radial flow from the discharge areas. Zimmerman et al. (1986) documented changes in water table elevations between 1950 and 1980. Until about 1980, the edge of the mounds migrated outward from the sources over time. Water levels have declined over most of the Hanford Site since 1984 because of decreased wastewater discharges (Hartman 2000). Although the reduction of wastewater discharges has caused water levels to drop significantly, a residual groundwater mound beneath the 200 West Area is still shown by the curved water table contours near this area (Figure 4.3-11) and small groundwater mounds exist near the 200 Areas Treated Effluent Disposal Facility and the State-approved land disposal structure wastewater disposal sites. The contour interval in Figure 4.3-11 is too large to show these groundwater mounds.

Horizontal hydraulic conductivities of sand and gravel facies within the Ringold Formation generally range from about $10^{-5}$ to $10^{-4} \mathrm{~m} / \mathrm{s}(0.9$ to $9 \mathrm{ft} / \mathrm{d})$, compared to $10^{-2}$ to $10^{-3} \mathrm{~m} / \mathrm{s}(1000$ to $10,000 \mathrm{ft} / \mathrm{d})$ for the Hanford formation (DOE 1988). Because the Ringold sediments are more consolidated and partially cemented, they are about 10 to 100 times less permeable than the sediments of the overlying Hanford formation. Before wastewater disposal operations at the Hanford Site, the uppermost aquifer was mainly within the Ringold Formation, and the water table extended into the Hanford formation at only a few locations (Newcomb et al. 1972). However, wastewater discharges raised the water table elevation across the Site. The general increase in groundwater elevation caused the unconfined aquifer to extend upward into the Hanford formation over a larger area, particularly near the 200 East Area. This resulted in an increase in groundwater velocity because of both the greater volume of groundwater and the higher permeability of the newly saturated Hanford sediments.

Limitations of Hydrogeologic Information. The sedimentary architecture of the unconfined aquifer is very complex because of repeated deposition and erosion. Although hundreds of wells have been drilled on the Hanford Site, many penetrate only a small percentage of the total unconfined aquifer thickness, and there are a limited number of useful wells for defining the deeper sediment facies. A number of relatively deep wells were drilled in the early 1980s as part of a study for a proposed nuclear power plant (PSPL 1982), and these data are helpful in defining facies architecture. For most of the thinner and less extensive sedimentary units, correlation between wells is either not possible or uncertain. Coarse-grained units of the Ringold Formation (e.g., Units A, B, C, D, and E) are more permeable than are the fine-grained units, which generally act as aquitards that locally confine groundwater in deeper permeable sediments. 
A limited amount of hydraulic property data is available from testing of wells. Hydraulic test results from wells on the Hanford Site have been compiled for the Hanford Groundwater Monitoring Project and for environmental restoration efforts (Connelly et al. 1992a,b; Kipp and Mudd 1973; Thorne and Newcomer 1992; Thorne et al. 1993; Thorne et al. 1994). Depths of the tested intervals have been correlated with the top of the unconfined aquifer as defined by the water table elevations presented in Newcomer et al. (1991). Most hydraulic tests were done within the upper $15 \mathrm{~m}$ (49 ft) of the aquifer, and many were open to more than one geologic unit. In some cases, changes in water table elevation may have significantly changed the unconfined aquifer transmissivity at a well since the time of the hydraulic test. Few hydraulic tests within the Hanford Site unconfined aquifer system have yielded reliable estimates of aquifer-specific yield.

Groundwater Residence Times. Tritium and carbon-14 measurements indicate that residence or recharge time (length of time required to replace the groundwater) takes tens to hundreds of years for spring waters. Recharge takes from hundreds to thousands of years for the unconfined aquifer and more than 10,000 years for groundwater in the shallow confined aquifer (Johnson et al. 1992). However, groundwater travel time from the 200 East Area to the Columbia River has been shown to be much faster, in the range of 10 to 30 years (USGS 1987; Freshley and Graham 1988). This is because of large volumes of recharge from wastewater that was disposed in the 200 Areas between 1944 and the mid1990s and the relatively high permeability of Hanford formation sediments, which are below the water table between the 200 Areas and the Columbia River. Residence times in this portion of the aquifer are expected to increase because of the reduction in wastewater recharge in the 200 Areas. Chlorine-36 and noble gas isotope data suggest groundwater ages greater than 100,000 years in the deeper confined systems (Johnson et al. 1992). These relatively long residence times are consistent with semiarid-site recharge conditions.

Hydrology East and North of the Columbia River. The Hanford Site boundary extends east and north of the Columbia River to provide a buffer zone for non-Hanford activities such as recreation and agriculture. Hanford Site activities in these areas have not impacted the groundwater. However, the groundwater in this area is impacted by high artificial recharge from irrigation and canal leakage. Areas east and north of the Columbia River are irrigated by the South Columbia Basin Irrigation District. Artificial recharge has increased water table elevations in large areas of the Pasco Basin, in some places by as much as $92 \mathrm{~m}$ (300 ft) (Drost et al. 1989).

There are two general hydrologic areas that impinge upon the Hanford Site boundaries to the east and north of the river. The eastern area extends from north to south between the lower slope of the Saddle Mountains and the Esquatzel Diversion canal and includes the Ringold Coulee, White Bluffs area, and Esquatzel Coulee. The water table occurs in the Pasco gravels of the Hanford formation in both Ringold and Esquatzel Coulees. Brown (1979) reported that runoff from spring discharge at the mouth of Ringold Coulee is $>37,850 \mathrm{~L} / \mathrm{min}(10,000 \mathrm{gal} / \mathrm{min})$. Elsewhere in this area, the unconfined aquifer is in the lesstransmissive Ringold Formation. Irrigation has also created perched aquifers and resulted in a series of springs issuing from perched water along the White Bluffs. The increased hydraulic pressure in these sediments has caused subsequent slumping and landslides (Brown 1979; Newcomer et al. 1991).

The other principal irrigated area is the northern part of the Pasco Basin on the Wahluke Slope, which lies between the Columbia River and the Saddle Mountain anticline. Irrigation on Wahluke Slope has created ponds and seeps in the Saddle Mountain Wildlife Refuge. The direction of unconfined groundwater flow is southward from the basalt ridges toward the Columbia River. Bauer et al. (1985) reported that lateral water table gradients are essentially equal to or slightly less than the structural gradients on the flanks of the anticlinal fold mountains where the basalt dips steeply. 


\subsubsection{Groundwater Quality}

\subsubsection{Natural Groundwater Quality}

The natural quality of groundwater at the Hanford Site varies depending on the aquifer system and depth, which generally is related to residence time in the aquifer. Background water quality (i.e., unaffected by Hanford discharges) for the unconfined aquifer is discussed in DOE (1992), DOE (1997b), and Hartman (2000). The DOE (1997b) study involved examination of historical data and new data from wells in areas not affected by Hanford Site contaminants.

Groundwater chemistry in the basalt-confined aquifers displays a range depending on depth and residence time (DOE 1988). The chemical type varies from a calcium and magnesium-carbonate water to a sodium- and chloride-carbonate water. Some of the shallower basalt-confined aquifers in the region (e.g., the Wanapum basalt aquifer) have exceptionally good water quality characteristics: $<300 \mathrm{mg} / \mathrm{L}$ dissolved solids; $<0.1 \mathrm{mg} / \mathrm{L}$ iron and magnesium; $<20 \mathrm{mg} / \mathrm{L}$ sodium, sulfate, and chloride; and $<10 \mathrm{ppb}$ heavy metals (Johnson et al. 1992). However, deeper basalt-confined aquifers typically have high dissolved solids content and some have fluoride concentrations over the drinking water standard of 5 $\mathrm{mg} / \mathrm{L}$ (DOE 1988).

\subsubsection{Groundwater Contamination and Monitoring}

Groundwater beneath large areas of the Hanford Site has been impacted by radiological and chemical contaminants resulting from past Hanford Site operations. These contaminants were primarily introduced through wastewater discharged to cribs, ditches, injection wells, trenches, and ponds (Kincaid et al. 1998). Additional contaminants from spills, leaking waste tanks, and burial grounds (landfills) have also impacted groundwater in some areas. Contaminant concentrations in the existing groundwater plumes are expected to decline through radioactive decay, chemical degradation, and dispersion. However, contaminants also exist within the vadose zone beneath waste sites (see Section 4.3.2) as well as in waste storage and disposal facilities. These contaminants have a potential to continue to move downward into the aquifer. Some contaminants, such as tritium, move with the groundwater while the movement of other contaminants is slower because they react with or are sorbed on the surface of minerals within the aquifer or the vadose zone. Groundwater contamination is monitored and is being actively remediated in several areas through pump-and-treat operations. These are summarized in Hartman et al. (2001).

Monitoring of radiological and chemical constituents in groundwater at the Hanford Site is performed to characterize physical and chemical trends in the flow system, establish groundwater quality baselines, assess groundwater remediation, and identify new or existing groundwater problems. Groundwater monitoring is also performed to verify compliance with applicable environmental laws and regulations. Samples were collected from approximately 700 wells during fiscal year (FY) 2000 to determine the distributions of radiological and chemical constituents in Hanford Site groundwater. Detailed results and interpretations are presented in Hartman et al. (2001).

To assess the quality of groundwater, concentrations measured in samples were compared with maximum contaminant levels (MCL) or interim Drinking Water Standards (DWS) and DOE's Derived Concentration Guides (DCG). The MCL or DWS standards are legal limits for contaminant concentrations in public drinking water supplies enforceable by the Washington State Department of Health or the EPA. Although these standards are only applicable at the point of consumption of the groundwater, they provide a useful indicator of negative impacts to the groundwater resource. The DCG applies only to radionuclides and is based on the concentration that would result in a dose exposure of $100-\mathrm{mrem} /$ year through ingestion under specified intake scenarios. 
Radiological constituents including carbon-14, cesium-137, cobalt-60, iodine-129, strontium-90, technetium-99, total alpha, total beta, tritium, uranium, and plutonium were detected at levels greater than the DWS in one or more onsite wells. Concentrations of strontium-90, tritium, uranium, and plutonium were detected at levels greater than DOE's DCG. Certain nonradioactive chemicals regulated by the EPA and the state of Washington (nitrate, fluoride, chromium, cyanide, carbon tetrachloride, chloroform, trichloroethylene, and tetrachloroethylene) were also present in Hanford Site groundwater. Table 4.3-1 shows maximum concentrations of groundwater contamination detected at Hanford for 2000. Figure 4.312 shows the extent of radiological contamination in Hanford Site groundwater above the applicable DWS, and Figure 4.3-13 shows the extent of chemical constituents above the applicable DWS. The area of contaminant plumes on the Hanford Site with concentrations exceeding drinking water standards was estimated to be $231 \mathrm{~km}^{2}\left(89.2 \mathrm{mi}^{2}\right)$ in fiscal year 2000 . This is $\sim 9 \%$ smaller than the estimate for fiscal year 1999. The decrease is primarily due to shrinkage of tritium plume from 200 East Area, which was caused primarily by radioactive decay.

\subsubsection{Areas Hydrology}

The hydrology of the 100 Areas is unique because of their location adjacent to the Columbia River. The water table ranges in depth from near $0 \mathrm{~m}(0 \mathrm{ft})$ at the river edge to $30 \mathrm{~m}(107 \mathrm{ft})$. The groundwater flow direction is generally toward the river. However, during high river stage, the flow direction may reverse immediately adjacent to the river. The unconfined aquifer in the 100 Areas is composed of either the Ringold Unit E gravels or a combination of the Unit E gravels and the Hanford formation. As shown in Figure 4.3-14, there are two large areas where the water table is within the Ringold Formation (Lindsey 1992), and the Hanford formation is unsaturated. In the 100-H and 100-F Areas, the Ringold Unit $\mathrm{E}$ gravels are missing, and the Hanford formation lies directly over the fine-grained Ringold lower-mud unit. In most of the 100 Areas, the lower Ringold mud forms an aquitard, and the Ringold gravels below the mud are locally confined. Additional information on the hydrology of the 100 Areas is available in Hartman and Peterson (1992) and Peterson et al. (1996). A number of studies of various sites in the 100 Areas present specific hydrologic information. These include: 100-B/C Area - Lindberg (1993a); 100-D Area - Lindsey and Jaeger (1993); 100-F Area - Lindsey (1992), Peterson (1992); 100-H Area - Liikala et al. (1988), Lindsey and Jaeger (1993); 100-K Area - Lindberg (1993b); and 100-N Area - Gilmore et al. (1992), Hartman and Lindsey (1993).

\subsubsection{Areas Hydrology}

In the 200 West Area, the water table occurs almost entirely in the Ringold Unit E gravels, while in the 200 East Area, it occurs primarily in the Hanford formation and in the Ringold Unit A gravels. Along the southern edge of the 200 East Area, the water table is in the Ringold Unit E gravels. The upper Ringold facies were eroded in most of the 200 East Area by the Missoula floods that subsequently deposited Hanford gravels and sands on what was left of the Ringold Formation. Because the Hanford formation sand and gravel deposits are much more permeable than the Ringold gravels, the water table is relatively flat in the 200 East Area, but groundwater flow velocities are higher. On the north side of the 200 East Area, there is evidence of erosional channels that may allow communication between the unconfined and uppermost basalt-confined aquifer (Graham et al. 1984; Jensen 1987). 
Table 4.3-1. Maximum Concentrations of Groundwater Contaminants in Fiscal Year 2000 (Hartman et al. 2001)

\begin{tabular}{|c|c|c|c|c|c|c|c|c|c|c|c|c|c|c|c|}
\hline \multirow{2}{*}{$\begin{array}{c}\begin{array}{c}\text { Contaminant } \\
\text { (alphabetical order) }\end{array} \\
\end{array}$} & \multirow{2}{*}{$\begin{array}{c}\text { DWS or MCL } \\
{[\mathrm{DCG}]^{(a)}}\end{array}$} & \multirow[b]{2}{*}{ Units } & \multicolumn{2}{|c|}{$100-\mathrm{B} / \mathrm{C}$} & \multicolumn{2}{|c|}{ 100-K } & \multicolumn{2}{|c|}{$100-\mathrm{N}$} & \multicolumn{2}{|c|}{ 100-D } & \multicolumn{2}{|c|}{$100-H$} & \multicolumn{2}{|c|}{ 100-F } & \multirow{2}{*}{$\begin{array}{c}200 \text { West } \\
\text { Wells } \\
\end{array}$} \\
\hline & & & Wells & Shore ${ }^{(b)}$ & Wells & Shore $^{(b)}$ & Wells & Shore $^{(b)}$ & Wells & Shore ${ }^{(b)}$ & Wells & Shore ${ }^{(b)}$ & Wells & Shore & \\
\hline Carbon tetrachloride & 5 & $\mu \mathrm{g} / \mathrm{L}$ & & & & & & & & & & & & & 7,900 \\
\hline Carbon-14 & $2,000[70,000]$ & $\mathrm{pCi} / \mathrm{L}$ & & & 35,600 & 639 & & & & & & & & & \\
\hline Cesium-137 & $200[3,000]$ & $\mathrm{pCi} / \mathrm{L}$ & & & & & & & & & & & & & undetected \\
\hline Chloroform & 100 & $\mu \mathrm{g} / \mathrm{L}$ & & & & & & & & & & & & & 250 \\
\hline Chromium (filtered) & 100 & $\mu \mathrm{g} / \mathrm{L}$ & 77 & 115 & 474 & 76 & 122 & 34 & 2,260 & 641 & 178 & 49 & 208 & 23 & 542 \\
\hline Cobalt-60 & $100[5,000]$ & $\mathrm{pCi} / \mathrm{L}$ & & & & & & & & & & & & & \\
\hline Cyanide & 200 & $\mu \mathrm{g} / \mathrm{L}$ & & & & & & & & & & & & & \\
\hline cis-1,2 Dichloroethene & 70 & $\mu \mathrm{g} / \mathrm{L}$ & & & & & & & & & & & & & \\
\hline Fluoride & 4 & $\mathrm{mg} / \mathrm{L}$ & & & & & & & & & 0.25 & & & & 9.8 \\
\hline Gross alpha & 15 & $\mathrm{pCi} / \mathrm{L}$ & & & & & & & & & 131 & & & & 692 \\
\hline Gross beta & 50 & $\mathrm{pCi} / \mathrm{L}$ & 133 & 67.1 & 22,300 & 4.4 & 21,500 & 3,680 & & 10.5 & 593 & 21 & 553 & 15.8 & 23,000 \\
\hline Iodine-129 & $1[500]$ & $\mathrm{pCi} / \mathrm{L}$ & & & & & & & & & & & & & 52 \\
\hline Manganese (filtered) & 50 & $\mu \mathrm{g} / \mathrm{L}$ & & & & & 5,320 & & & & & & & & \\
\hline Nitrate (as $\mathrm{NO}_{3}^{-}$) & 45 & $\mathrm{mg} / \mathrm{L}$ & 30 & 39 & 98 & 22 & 104 & 18 & 100 & 18 & 387 & 60 & 144 & 54 & 1,213 \\
\hline Nitrite (as $\mathrm{NO}_{2}$ ) & 3.3 & $\mathrm{mg} / \mathrm{L}$ & & & & & & & 2.3 & & & & & & 36 \\
\hline Plutonium & NA [30] & $\mathrm{pCi} / \mathrm{L}$ & & & & & & & & & & & & & undetected \\
\hline Strontium-90 & $8[1,000]$ & $\mathrm{pCi} / \mathrm{L}$ & 66 & 17 & 6,970 & ND & 17,700 & 14,700 & 8.5 & 4.8 & 55 & 9.6 & 265 & 1.5 & 74.3 \\
\hline Sulfate & 250 & $\mathrm{mg} / \mathrm{L}$ & & & & & 370 & 38 & 744 & & & & & & \\
\hline Technetium-99 & $900[100,000]$ & $\mathrm{pCi} / \mathrm{L}$ & & & & & & & & & 1,070 & & & & 63,700 \\
\hline Tetrachloroethene & 5 & $\mu \mathrm{g} / \mathrm{L}$ & & & & & & & & & & & & & \\
\hline Trichloroethene & 5 & $\mu \mathrm{g} / \mathrm{L}$ & & & 8 & & & & & & & & 18 & & 32 \\
\hline Tritium & $20,000[2,000,000]$ & $\mathrm{pCi} / \mathrm{L}$ & 39,900 & 36,600 & $1,360,000$ & 6,660 & 45,000 & 29,100 & 20,000 & 15,700 & 5,580 & 978 & 36,900 & 1,450 & $2,940,000$ \\
\hline Uranium & $20[790]$ & $\mu \mathrm{g} / \mathrm{L}$ & & & & & & & & & 157 & & 20 & & 2,100 \\
\hline
\end{tabular}


Table 4.3-1. (cont'd)

\begin{tabular}{|c|c|c|c|c|c|c|c|c|c|c|c|}
\hline \multirow{2}{*}{$\begin{array}{c}\begin{array}{c}\text { Contaminant } \\
\text { (alphabetical order) }\end{array} \\
\end{array}$} & \multirow{2}{*}{$\begin{array}{c}\text { DWS or MCL } \\
{[\text { DCG }]^{(\mathrm{a})}}\end{array}$} & \multirow[b]{2}{*}{ Units } & \multicolumn{2}{|c|}{200 East } & \multirow{2}{*}{$\begin{array}{c}400 \\
------ \\
\text { Wells } \\
\end{array}$} & \multirow{2}{*}{$\begin{array}{c}600 \\
----- \\
\text { Wells } \\
\end{array}$} & \multicolumn{2}{|c|}{300} & \multirow{2}{*}{$\begin{array}{c}\text { 618-11 } \\
------ \\
\text { Wells } \\
\end{array}$} & \multirow{2}{*}{$\begin{array}{c}\text { Richland North } \\
--{ }^{-} \text {Wells } \\
\end{array}$} & \multirow{2}{*}{\begin{tabular}{c} 
Basalt-Confined \\
- \hdashline$-{ }^{-}$Wells \\
\end{tabular}} \\
\hline & & & Wells & Shore $^{(b)}$ & & & Wells & Shore $^{(b)}$ & & & \\
\hline Carbon tetrachloride & 5 & $\mu \mathrm{g} / \mathrm{L}$ & & & & 1 & & & & & \\
\hline Carbon-14 & $2,000[70,000]$ & $\mathrm{pCi} / \mathrm{L}$ & & & & & & & & & \\
\hline Cesium-137 & $200[3,000]$ & $\mathrm{pCi} / \mathrm{L}$ & $122^{(\mathrm{c})}$ & & & & & & & & \\
\hline Chloroform & 100 & $\mu \mathrm{g} / \mathrm{L}$ & & & & 7.7 & & & & & \\
\hline Chromium (filtered) & 100 & $\mu \mathrm{g} / \mathrm{L}$ & 3,250 & & & 200 & & & & & \\
\hline Cobalt-60 & $100[5,000]$ & $\mathrm{pCi} / \mathrm{L}$ & 78.2 & & & & & & & & \\
\hline Cyanide & 200 & $\mu \mathrm{g} / \mathrm{L}$ & 411 & & & & & & & & \\
\hline cis-1,2 Dichloroethene & 70 & $\mu \mathrm{g} / \mathrm{L}$ & & & & & 170 & & & & \\
\hline Fluoride & 4 & $\mathrm{mg} / \mathrm{L}$ & & & & & & & & 4.8 & \\
\hline Gross alpha & 15 & $\mathrm{pCi} / \mathrm{L}$ & 240 & & & & 60.2 & 228 & 30 & 65.4 & \\
\hline Gross beta & 50 & $\mathrm{pCi} / \mathrm{L}$ & 10,300 & 48.6 & & & & 49 & 74 & 46 & \\
\hline Iodine-129 & $1[500]$ & $\mathrm{pCi} / \mathrm{L}$ & 12.1 & 0.41 & & & & & & & undetected \\
\hline Manganese (filtered) & 50 & $\mu \mathrm{g} / \mathrm{L}$ & 141 & & & 6.5 & & & & & \\
\hline Nitrate (as $\mathrm{NO}_{3}^{-}$) & 45 & $\mathrm{mg} / \mathrm{L}$ & 562 & 36 & 92 & 54 & 101 & 29 & 149 & 203 & 11 \\
\hline Nitrite (as $\mathrm{NO}_{2}$ ) & 3.3 & $\mathrm{mg} / \mathrm{L}$ & & & 0.43 & & & & 3.9 & & \\
\hline Plutonium & NA [30] & $\mathrm{pCi} / \mathrm{L}$ & $9.4^{(c)}$ & & & & & & & & \\
\hline Strontium-90 & $8[1,000]$ & $\mathrm{pCi} / \mathrm{L}$ & $1,210^{(\mathrm{c})}$ & & & & 4.1 & 0.28 & & & \\
\hline Sulfate & 250 & $\mathrm{mg} / \mathrm{L}$ & & & & 55 & & & & & 28 \\
\hline Technetium-99 & $900[100,000]$ & $\mathrm{pCi} / \mathrm{L}$ & 13,300 & 120 & & & & & & & \\
\hline Tetrachloroethene & 5 & $\mu \mathrm{g} / \mathrm{L}$ & & & & & 0.65 & & & & \\
\hline Trichloroethene & 5 & $\mu \mathrm{g} / \mathrm{L}$ & & & & 1.4 & 3.5 & & & 3.7 & \\
\hline Tritium & $20,000[2,000,000]$ & $\mathrm{pCi} / \mathrm{L}$ & $2,510,000$ & 106,000 & $\mathbf{5 8 , 8 0 0}$ & 65,900 & 11,600 & 11,300 & $8,380,000$ & 546 & 5,770 \\
\hline Uranium & 20 [790] & $\mu \mathrm{g} / \mathrm{L}$ & 353 & & & & 234 & 301 & 30.8 & 13.6 & \\
\hline $\begin{array}{l}\text { Note: Table lists highes } \\
\text { indicate the constituent } \\
\text { (a) DWS = drinking } \\
\text { (b) Shoreline samplin } \\
\text { (c) Well typically sho }\end{array}$ & $\begin{array}{l}\text { ncentration for fiscal } \\
\text { t of concern in the gi } \\
\text { r standard; } \mathrm{MCL}=\mathrm{m} \\
\text { cludes aquifer sampli } \\
\text { g highest concentratic }\end{array}$ & $\begin{array}{l}\text { year } 2000 \\
\text { ven area. } \\
\text { aximum } \\
\text { ng tubes, } \\
\text { ons near }\end{array}$ & $\begin{array}{l}\text { n each geogra } \\
\text { ntaminant lev } \\
\text { eeps, and shor } \\
\text { 6-B-5 injectic }\end{array}$ & $\begin{array}{l}\text { hic region. } \\
\text { l; DCG }=\mathrm{I} \\
\text { line wells } \\
\text { a well not } \mathrm{s}\end{array}$ & $\begin{array}{l}\text { Concentrations } \\
\text { OE derived con } \\
\text { rom fall } 1999 . \\
\text { ampled in fiscal }\end{array}$ & $\begin{array}{l}\text { ed drinking } \\
\text { ide. See } \mathrm{P} \\
\text { a plumes } \mathrm{m}\end{array}$ & $\begin{array}{l}\text { standards. } \\
3080 \text { for } n \\
\text { dat Old } \mathrm{H}\end{array}$ & $\begin{array}{l}\text { Concentrati } \\
\text { ore informat } \\
\text { nford Town }\end{array}$ & bold italic $\mathrm{ex}$ & DOE derived conce & on guides. Blank sp \\
\hline
\end{tabular}




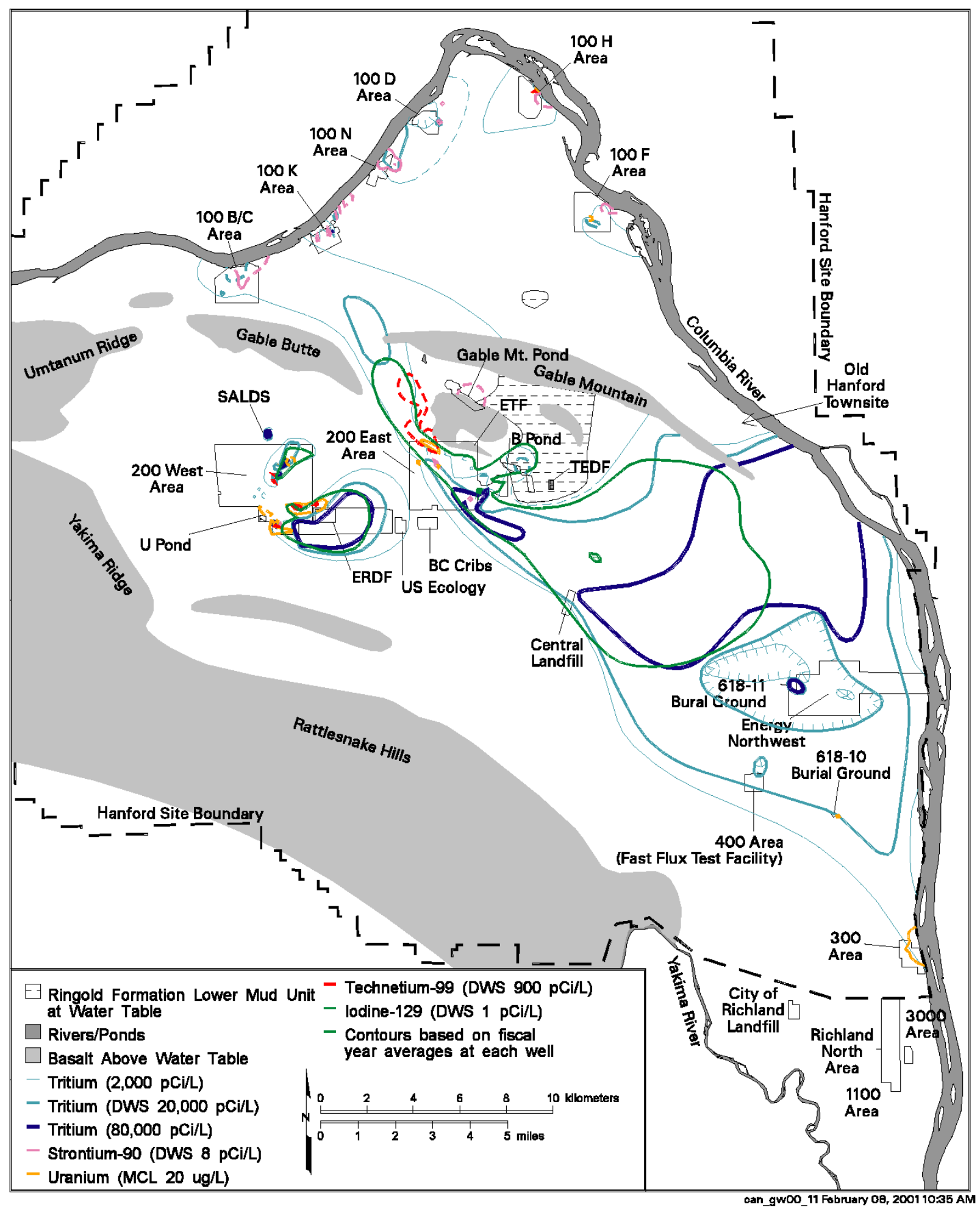

Figure 4.3-12. Distribution of Major Radionuclides in Groundwater at Concentrations Above the Maximum Contaminant Level or Interim Drinking Water Standard During Fiscal Year 2000 (Hartman et al. 2001) 


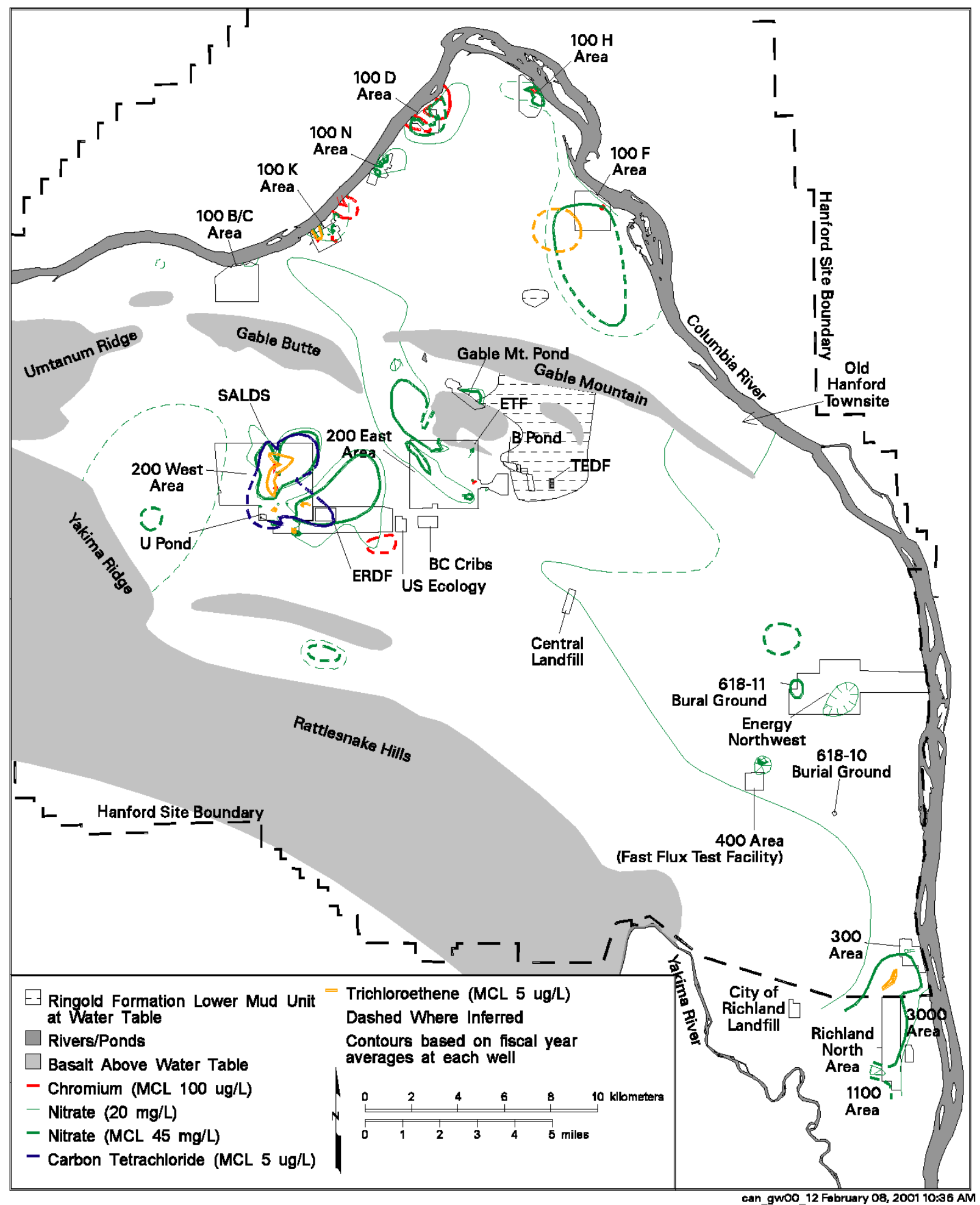

Figure 4.3-13. Distribution of Major Hazardous Chemicals in Groundwater at Concentrations Above the Maximum Contaminant Level or Interim Drinking Water Standard During Fiscal Year 2000 (Hartman et al. 2001) 


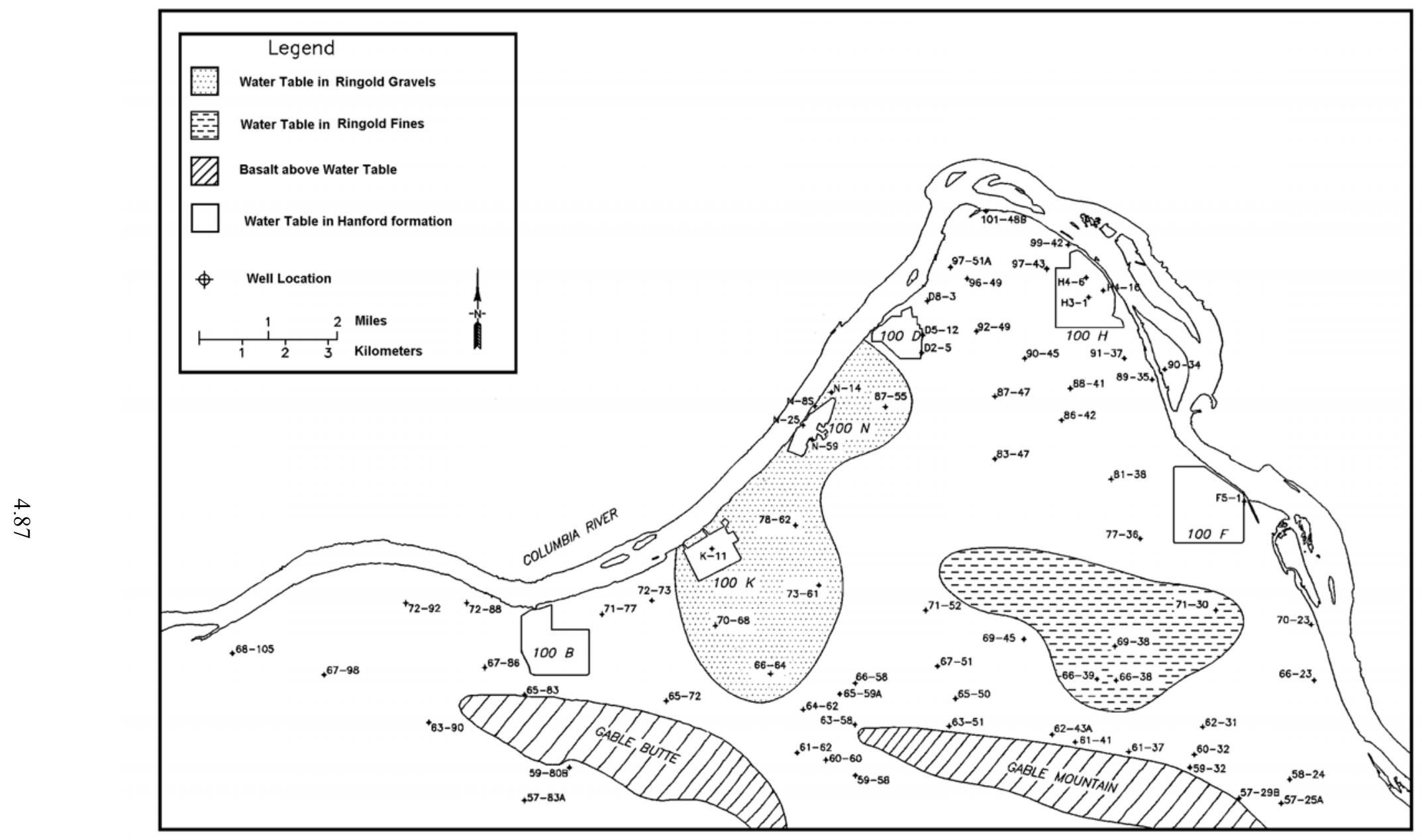

Figure 4.3-14. Geologic Units Intersected by the Water Table in the 100 Areas (modified from Lindsey 1992) 
The hydrology of the 200 Areas has been strongly influenced by the discharge of large quantities of wastewater to the ground over a 50-year period. Those discharges have caused elevated water levels across much of the Hanford Site resulting in a large groundwater mound beneath the former U Pond in the 200 West Area and a smaller mound beneath the former B Pond, east of the 200 East Area. Water table changes beneath 200 West Area have been greatest because of the lower transmissivity of the aquifer in this area. Discharges of water to the ground have been greatly reduced, and corresponding decreases in the elevation of the water table have been measured. The decline in part of the 200 West Area has been

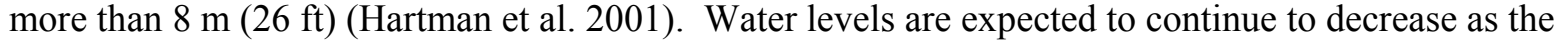
unconfined groundwater system reaches equilibrium with the new level of artificial recharge (Wurstner and Freshley 1994).

A number of reports dealing with the hydrogeology of the 200 Areas have been released including Graham et al. (1981); Last et al. (1989); and Connelly et al. (1992a,b). More detailed information is also provided in Hartman (2000).

\subsubsection{Area Hydrology}

The unconfined aquifer water table in the 300 Area is generally found in the Ringold Formation at a depth of 9 to $19 \mathrm{~m}$ (30 to $62 \mathrm{ft}$ ) below ground surface. Fluctuations in the river level strongly affect the groundwater levels and flow in the 300 Area, just as they do in the 100 Areas. Water table contours in the vicinity of the 300 Area are somewhat concentric, showing that this is a discharge area for the unconfined aquifer system. Groundwater flows from the northwest, west, and even the southwest to discharge into the Columbia River near the 300 Area. Schalla et al. (1988), Swanson (1992) and Hartman (2000) have provided more detailed information on the hydrogeology of the 300 Area.

\subsubsection{Richland North Areas Hydrology}

The Richland North Area is located in the southern part of the Hanford Site and includes the former 1100 Area, which was transferred from the DOE to the Port of Benton on October 1, 1998. The groundwater in this area is influenced by artificial recharge associated with the North Richland recharge basins and nearby irrigated farming. Water is pumped from the Columbia River to the recharge basins and subsequently pumped from nearby wells. This system is used by the city of Richland as a backup filtration system for city water. Because an excess of water is pumped into the recharge basins, a mound has been created in the water table, which helps to reduce the potential for groundwater flow from the Hanford Site into this area. The river stage elevation of Yakima River, which flows just west of the area, is high enough such that the river also acts as a recharge source for the groundwater system.

The southern portion of the tritium plume from the 200 Areas extends to the 300 Area, and there is concern that this plume could reach the Richland North Area and impact water supply wells. However, tritium concentrations decrease from greater than $10,000 \mathrm{pCi} / \mathrm{L}$ to less than $100 \mathrm{pCi} / \mathrm{L}$ across the 300 Area and the distribution across this area has changed little since fiscal year 1999 (Hartman et al. 2001). A few wells south of the 300 Area, in the vicinity of Richland's recharge ponds, have shown slightly elevated tritium levels, although well below the 20,000-pCi/L drinking water standard. These levels are consistent with tritium concentrations in the Columbia River water that is pumped into the ponds (Hartman et al. 2001). Nitrate contamination is also found in the Richland North Area. This is likely the result of industrial and agricultural sources off the Hanford Site. In fiscal year 2000, nitrate concentrations increased in a number of wells. The nitrate plume appears to be migrating east toward the Columbia River. Concentrations above the 45-mg/L maximum contaminant level are found over much of the Richland North Area (Hartman et al. 2001). 


\subsection{Ecology}

\section{T. M. Poston}

The Hanford Site encompasses about $1517 \mathrm{~km}^{2}$ (about $586 \mathrm{mi}^{2}$ ) of shrub-steppe habitat that is adapted to the region's mid-latitude semiarid climate (DOE 1999a). The Site encompasses undeveloped land interspersed with industrial development along the western shoreline of the Columbia River and at several locations in the interior of the Site. This land, with restricted public access, provides a buffer for the smaller areas currently used for storage of nuclear materials, waste storage, waste disposal, and some private activities such as the Energy Northwest Power Plant and LIGO.

The Columbia River borders the Hanford Site to the east. Operation of Priest Rapids Dam upstream of the Hanford Site accommodates maintenance of intakes at the Site and contributes to management of anadromous fish populations. The Columbia River and associated riparian zones provide habitat for numerous wildlife and plant species. The area known as the Hanford Reach, the Columbia River from Priest Rapids Dam (river mile 397) to McNary Pool (river mile 346), is the last free-flowing, non-tidal segment of the Columbia River in the United States. The National Park Service, in a record of decision issued on July 16, 1996, proposed that the Hanford Reach be designated as a recreational river in the national wild and scenic rivers system. On June 9, 2000, portions of the Hanford Site including the Hanford Reach and associated islands, wildlife management areas to the north, White Bluffs, Hanford Dunes, the Arid Lands Ecology Reserve (ALE), and the McGee Ranch and Riverland area were designated a National Monument (Figure 4.0-2) by the Clinton Administration.

The U.S. Fish and Wildlife Service (USFWS) manages the Hanford Reach National Monument/ Saddle Mountain National Wildlife Refuge, totaling $665 \mathrm{~km}^{2}\left(257 \mathrm{mi}^{2}\right)$. It includes the Fitzner/Eberhardt Arid Lands Ecology (ALE) Reserve Unit $\left(310 \mathrm{~km}^{2}\left[120 \mathrm{mi}^{2}\right]\right)$, the Saddle Mountain Unit $\left(130 \mathrm{~km}^{2}\right.$ [50 $\left.\left.\mathrm{mi}^{2}\right]\right)$ and the Wahluke Unit $\left(225 \mathrm{~km}^{2}\left[87 \mathrm{mi}^{2}\right]\right)$. The Saddle Mountain Unit and the Wahluke Unit are generally referred to as the North (or Wahluke) Slope.

Other descriptions of the ecology of the Hanford Site can be found in Cadwell (1994); Downs et al. (1993); ERDA (1975); Jamison (1982); Landeen (1996); Rogers and Rickard (1977); Sackschewsky et al. (1992); Watson et al. (1984); and Weiss and Mitchell (1992).

\subsubsection{Terrestrial Ecology}

The Hanford Site is characterized as a shrub-steppe ecosystem (Daubenmire 1970). Such ecosystems are typically dominated by a shrub overstory with a grass understory. In the early 1800 s, the dominant plants in the area were big sagebrush underlain by perennial Sandberg's bluegrass and bluebunch wheatgrass. With the advent of settlement, livestock grazing and agricultural production contributed to colonization by nonnative plant species that currently dominate the landscape. Although agriculture and livestock production were the primary subsistence activities at the turn of the century, these activities ceased when the Hanford Site was designated in 1943. Remnants of past agricultural practices are still evident.

Large areas of the Hanford Site have experienced range fires that have greatly influenced the vegetation canopy and distribution of wildlife. In 1984, a major fire burned across $800 \mathrm{~km}^{2}\left(310 \mathrm{mi}^{2}\right)$ of the Hanford Site (Price et al. 1986). From June 27 through July 2, 2000, the 24 Command Fire burned across the Hanford Site consuming most of the shrub-steppe habitat on the Arid Lands Ecology Reserve Unit, a small section of the McGee-Riverlands Unit, and other southwestern portions of the Site (Figure 4.0-3). The fire consumed a total of $655 \mathrm{~km}^{2}\left(250 \mathrm{mi}^{2}\right)$ of federal, state, and private lands before it was controlled (BAER 2000). 
These fires have radically altered the composition of the shrub-steppe habitat. Much of the 2000 burn was considered to be low severity, meaning that the soil structure and seed bank are intact, and the belowground portions of most perennial plants were unharmed and are expected to re-sprout as conditions permit. Most of the burned area is expected to recover to a configuration resembling the pre-fire conditions, except for the presence of shrubs, within 1 to 3 years. Sagebrush will take considerably longer to recover, depending on the availability of seed in the soil and the distance to other seed sources. It is likely to take at least 5 to 10 years, and potentially many decades, before sagebrush is once again an important feature of the landscape.

\subsubsection{Vegetation}

Natural plant communities have been altered by Euro-American activities that have resulted in the proliferation of nonnative species. Of the 590 species of vascular plants recorded for the Hanford Site, approximately $20 \%$ of all species are considered nonnative (Sackschewsky et al. 1992). The biodiversity inventories conducted by The Nature Conservancy of Washington (Soll et al. 1999) have identified 85 additional taxa, establishing the actual number of plant taxa on the Hanford Site at 675. Cheatgrass is the dominant nonnative species. It is an aggressive colonizer and has become well established across the Hanford Site (Rickard and Rogers 1983). Hanford Site plants are adapted to low annual precipitation (17 $\mathrm{cm}$ [6.8 in.]), low water-holding capacity of the rooting substrate (sand), dry summers, and cold winters.

The Nature Conservancy of Washington (Soll et al. 1999) conducted plant surveys on ALE, the Wahluke Slope, central Hanford, and riparian communities along the Columbia River shoreline from 1994 through 1997. These surveys tentatively identified 30 "potential" terrestrial plant communities. Designation as a potential community indicates the type of community that would exist in an area if it were free of disturbance. In addition to characterizing potential plant communities, the Conservancy found 112 populations/occurrences of 28 rare plant taxa on the Hanford Site (Soll et al. 1999). When combined with observations preceding the 1994-1999 inventory, a total of 127 populations of 30 rare species have been documented on the Hanford Site.

Range fires that historically burned through the area during the dry summers eliminate fire intolerant species (e.g., big sagebrush) and allow more opportunistic and fire-resistant species a chance to become established. The 24 Command Fire severely impacted vegetation on those areas burned in 2000 (see Figure 4.0-3). Existing vegetation and land use areas on the Hanford Site prior to the 24 Command Fire are illustrated in Figure 4.4-1. A much broader definition of these plant communities, including shrublands, grasslands, tree zones, riparian, and unique habitat follows. Recovery of burned areas is a slow process, and it will be many years before areas will re-establish the natural component of vegetation and associated animal life. A list of common plant species in shrub-steppe and riparian areas are presented in Appendix A, Table A-1.

Shrublands. Shrublands occupy the largest area in terms of acreage and comprise seven of the nine major plant communities on the Hanford Site. Of the shrubland types, sagebrush-dominated communities are predominant, with other shrub communities varying with changes in soil and elevation. About $80 \%$ of the mapped land on the Hanford Site had a big sagebrush plant community (Soll et al. 1999); however, much of this area was burned in the 24 Command Fire of 2000 . About $287 \mathrm{~km}^{2}\left(111 \mathrm{mi}^{2}\right)$ of shrub habitat dominated by big sagebrush was destroyed in the fire.

The areas botanically characterized as shrub-steppe include remnant native big sagebrush, threetip sagebrush, bitterbrush, gray rabbitbrush, and spiny hopsage. Remnant bluebunch wheatgrass, Sandberg's bluegrass, needle-and-thread grass, Indian ricegrass, and prairie junegrass also occur in this vegetation 


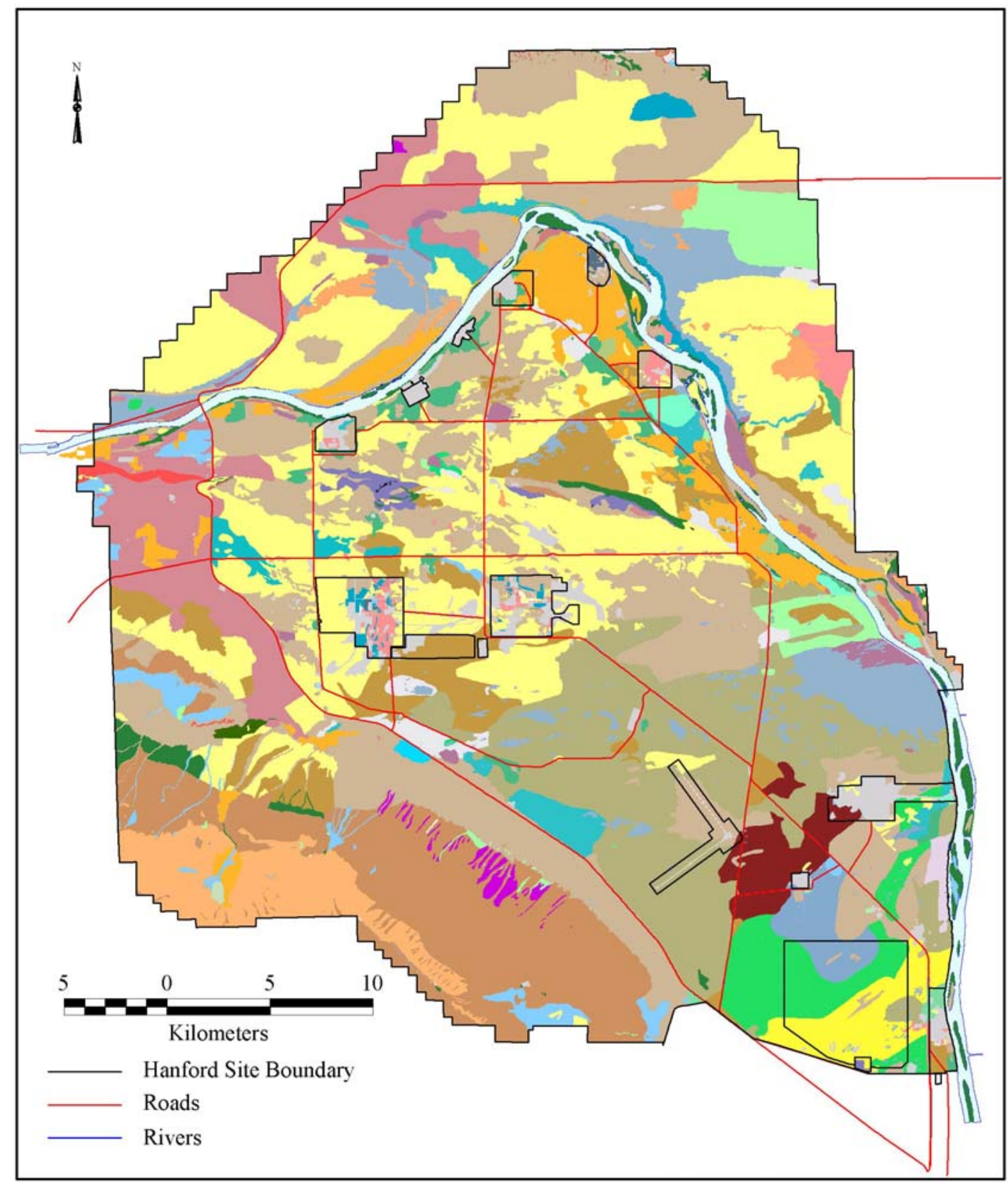

Data Collected: 1994, 1997/The Nature Conservancy

1991, 1999 Pacific Northwest National Laboratory

Map Created: September 1999/Pacific Northwest National Laboratory

Figure 4.4-1. Distribution of Vegetation Types and Land Use Areas on the Hanford Site Before the 24 Command Fire 


\section{LEGEND}

Abandoned Old Agricultural Fields

Alkali Saltgrass - Cheatgrass

Big Sagebrush - Bitterbrush / Bunchgrass

Big Sagebrush - Bitterbrush / Needle-and-Thread Grass

Big Sagebrush - Bitterbrush / Sandberg's Bluegrass

Big Sagebrush - Rigid Sagebrush / Bunchgrass

Big Sagebrush - Rock Buckwheat / Bunchgrass

Big Sagebrush - Spiny Hopsage / Bunchgrass

Big Sagebrush - Spiny Hopsage / Sandberg's Bluegrass - Cheatgrass

Big Sagebrush / Bluebunch Wheatgrass

Big Sagebrush / Bunchgrass

Big Sagebrush / Needle-and-Thread Grass

Big Sagebrush / Sand Dropseed

Big Sagebrush / Sandberg's Bluegrass - Cheatgrass

Bitterbrush / Bunchgrass

Bitterbrush / Indian Ricegrass

Bitterbrush / Needle-and-Thread Grass

Black Greasewood / Alkali Saltgrass

Bluebunch Wheatgrass - Needle-and-Thread Grass

Bluebunch Wheatgrass - Sandberg's Bluegrass

Bunchgrass - Cheatgrass

Crested Wheatgrass

Disturbed

Gray Rabbitbrush - Snow Buckwheat / Bunchgrass

Gray Rabbitbrush / Bunchgrass

Gray Rabbitbrush / Cheatgrass

Gray Rabbitbrush / Needle-and-Thread Grass

Gray Rabbitbrush / Sand Dropseed

Gray Rabbitbrush / Sandberg's Bluegrass - Cheatgrass

Needle-and-Thread Grass - Indian Ricegrass

Needle-and-Thread Grass -Sandberg's Bluegrass

Non-Riverine Wetlands and Associated Deepwater Habitats

Rabbitbrush / Bunchgrass

Rigid Sagebrush / Sandberg's Bluegrass

Riparian

Riverine Wetlands and Associated Deepwater Habitats

Sand Dropseed - Sandberg's Bluegrass - Cheatgrass

Sandberg's Bluegrass - Cheatgrass

Snow Buckwheat - Bitterbrush / Bunchgrass

Snow Buckwheat / Bunchgrass

Snow Buckwheat / Sandberg's Bluegrass - Cheatgrass

Spiny Hopsage / Sandberg's Bluegrass - Cheatgrass

Talus

Threetip Sagebrush / Bunchgrass

Thymeleaf Buckwheat / Sandberg's Bluegrass

Vernal Pool

White Bluffs

Winterfat / Bunchgrass

Figure 4.4-1. (cont'd) 
type. Heterogeneity of species composition varies with soil, slope, and elevation. Of the vegetation types depicted in Figure 4.4-1, those with a shrub component (i.e., big sagebrush, three-tip sagebrush, bitterbrush, spiny hopsage, rabbitbrush, winterfat, and snow-buckwheat) are considered shrub-steppe. Vegetation types with a significant cheatgrass component are generally of lower habitat quality than those with bunchgrass understories. Post-fire shrub-steppe on the Hanford Site refers to areas impacted by wildfire that are in the process of redeveloping shrub-steppe characteristics.

Grasslands. Most grasses occur as understory in shrub-dominated plant communities. Cheatgrass has replaced many native perennial grass species and is well established in many low-elevation $(<244 \mathrm{~m}$ [800 ft]) and/or disturbed areas (Rickard and Rogers 1983; Soll et al. 1999). Of the native grasses that occur on the Hanford Site, bluebunch wheatgrass occurs at higher elevations. Sandberg's bluegrass is more widely distributed and occurs within several plant communities. Needle-and-thread grass, Indian ricegrass, and thickspike wheatgrass occur in sandy soils and dune habitats. About $133 \mathrm{~km}^{2}\left(51 \mathrm{mi}^{2}\right)$ of shrubless habitat dominated by native grasses were destroyed in the 24 Command Fire. Species preferring more moist locations include bentgrass, meadow foxtail, lovegrasses, and reed canarygrass (DOE 1996a).

Trees. Before settlement, the Hanford Site landscape lacked trees, and the Columbia River shoreline supported a few scattered cottonwood or willows. Homesteaders planted trees in association with agricultural areas. Shade and ornamental trees were planted around former military installations and industrial areas on the Hanford Site. Currently, approximately 23 species of trees occur on the Site. The most commonly occurring species are black locust, Russian olive, cottonwood, mulberry, sycamore, and poplar. Many of these nonnative species are aggressive colonizers and have become established along the Columbia River (e.g., mulberry, cottonwood, poplar, Russian olive), serving as a functional component of the riparian zone (DOE 1996a). Trees provide nesting habitat and cover for many species of mammals and birds. The 24 Command Fire destroyed most of the shrubs and trees associated with streams on ALE.

Riparian (Wetland) Areas. Riparian habitat includes sloughs, backwaters, shorelines, islands, and palustrine areas associated with the Columbia River floodplain. Vegetation that occurs along the river shoreline includes water smartweed, pondweed, sedges, reed canary grass, and bulbous bluegrass. Trees include willow, mulberry, and Siberian elm. Other riparian vegetation occurs in association with perennial springs and seeps. Rattlesnake and Snively springs are highly diverse biologic communities (Cushing and Wolf 1984) that support bulrush, spike rush, and cattail. Watercress, which persists at these sites, is also abundant for a large portion of the year. In recent years, exotic trees and shrubs have become established in the riparian zone along these springs. The riparian transects associated with Snively and Rattlesnake springs were greatly impacted by the 24 Command Fire (BAER 2000).

Most wastewater ponds and ditches on the Hanford Site have been decommissioned and no longer support riparian vegetation. On the North Slope, several irrigation return ponds support riparian vegetation.

Riparian habitat that occurs in association with the Columbia River includes riffles, gravel bars, backwater sloughs, and cobble shorelines. These emergent habitats occur infrequently along the Hanford Reach and have acquired greater significance because of the net loss of wetland habitat elsewhere within the region. The Nature Conservancy identified 13 rare plant species (out of 19 total on the Hanford Site) residing along the Hanford Reach during surveys conducted in 1994 and 1995 (Soll and Soper 1996). The Conservancy (Soll et al. 1999) found four new species previously not listed at Hanford (Sackschewsky et al. 1992) in the 31 wetland areas surveyed. Noxious weeds are also becoming established along the riparian zones of the Hanford Reach. Purple loosestrife, yellow nutsedge, reed canary grass, knapweed, and yellow star thistle are some of the more common species found near or on 
wetlands. Common emergent species include reed canary grass, common witchgrass, and large barnyard grass. Rushes and sedges occur along the shorelines of the Columbia River and at several sloughs along the Hanford Reach at White Bluffs, below the 100-H Area, downstream of the 100-F Area, and at the Hanford Slough. Flow management practices at Priest Rapids Dam have facilitated the establishment of non-native trees along the riparian zones of the Hanford Reach.

Unique Habitats. Unique habitats on the Hanford Site include bluffs, dunes, and islands (DOE 1996a). The White Bluffs, Umtanum Ridge, and Gable Mountain on the Hanford Site include rock outcrops that occur infrequently on the Site. Plant communities dominated by buckwheat and Sandberg's bluegrass most often occupy basalt outcrops.

Snow buckwheat and Sandberg's bluegrass/cheatgrass communities dominate a large dune area north of the Energy Northwest complex along the Columbia River shoreline (Figure 4.4-1). The terrain of the dune habitat rises and falls between 3 and $5 \mathrm{~m} \mathrm{(10} \mathrm{and} 16 \mathrm{ft})$ above ground level, creating areas that range from 2.5 to several hundred acres in size (U.S. Department of the Army 1990). The dunes are vegetated by bitterbrush, scurfpea, and thickspike wheatgrass. Smaller dunes containing basalt grains that impart a dark color to the sand are found near the 100-F Area and westward across the site north of Gable Mountain.

Island habitat accounts for approximately $4.74 \mathrm{~km}^{2}\left(1.8 \mathrm{mi}^{2}\right)$ (Hanson and Browning 1959) and 64.3 $\mathrm{km}$ (39.9 mi) of river shoreline within the main channel of the Hanford Reach (Figure 4.4-2). Island ownership descriptions pertain to status prior to national monument designation and are subject to change. DOE owns and administers the upland portions of Locke Island (River mile [RM] 371-373.5) and Wooded Island (RM 348-351), and all of Island \# 7 (RM 367). Locke and Wooded islands fall under management by the U.S. Fish and Wildlife Service as part of the Hanford Reach National Monument designation. The Washington State Department of Natural Resources oversees the shorelines of Locke and Wooded islands. Recent landslides caused by rotational slumping in the White Bluffs area have resulted in accelerated erosion of Locke Island by the Columbia River. Shoreline riparian vegetation that characterizes the islands includes willow, poplar, Russian olive, and mulberry. Before regulation of river flows by dams, trees were not found along river shoreline habitat. Species occurring on the island interior include buckwheat, lupine, mugwort, thickspike wheatgrass, giant wildrye, yarrow, and cheatgrass (Warren 1980). Management of these islands is the responsibility of the island owners that include DOE, USFWS, and the U.S. Bureau of Land Management.

West Lake and its immediate basin represent a unique habitat that is characterized by highly saline conditions (Poston et al. 1991). These conditions occurred most likely from the evaporation of water from the pond and the accumulation of dissolved solids during the production years on the Site. West Lake is classified as a waste site under CERCLA. Water levels of the pond fluctuate with wastewater discharge levels in the 200 Areas. Predominant plants include salt grass, plantain, and rattle box. Threespine bulrush grows along the shoreline; however, the water in the pond is too saline to support aquatic macrophytes.

Operable Units. The Hanford Site encompasses numerous waste management units and groundwater contamination plumes that have been grouped into operable units under CERCLA. Each unit has complementary characteristics of such parameters as geography, waste content, type of facility, and relationship of contaminant plumes. In general, nonnative or invasive plants typify the operable units. Cheatgrass, Russian thistle, and tumble mustard are invasive species that have colonized many of the disturbed portions of these sites. The 100 Area operable units are characterized by a narrow band of riparian vegetation along the shoreline of the Columbia River, with much of the area shoreward consisting of old agricultural fields dominated by cheatgrass and tumble mustard. Scattered big sagebrush and gray 


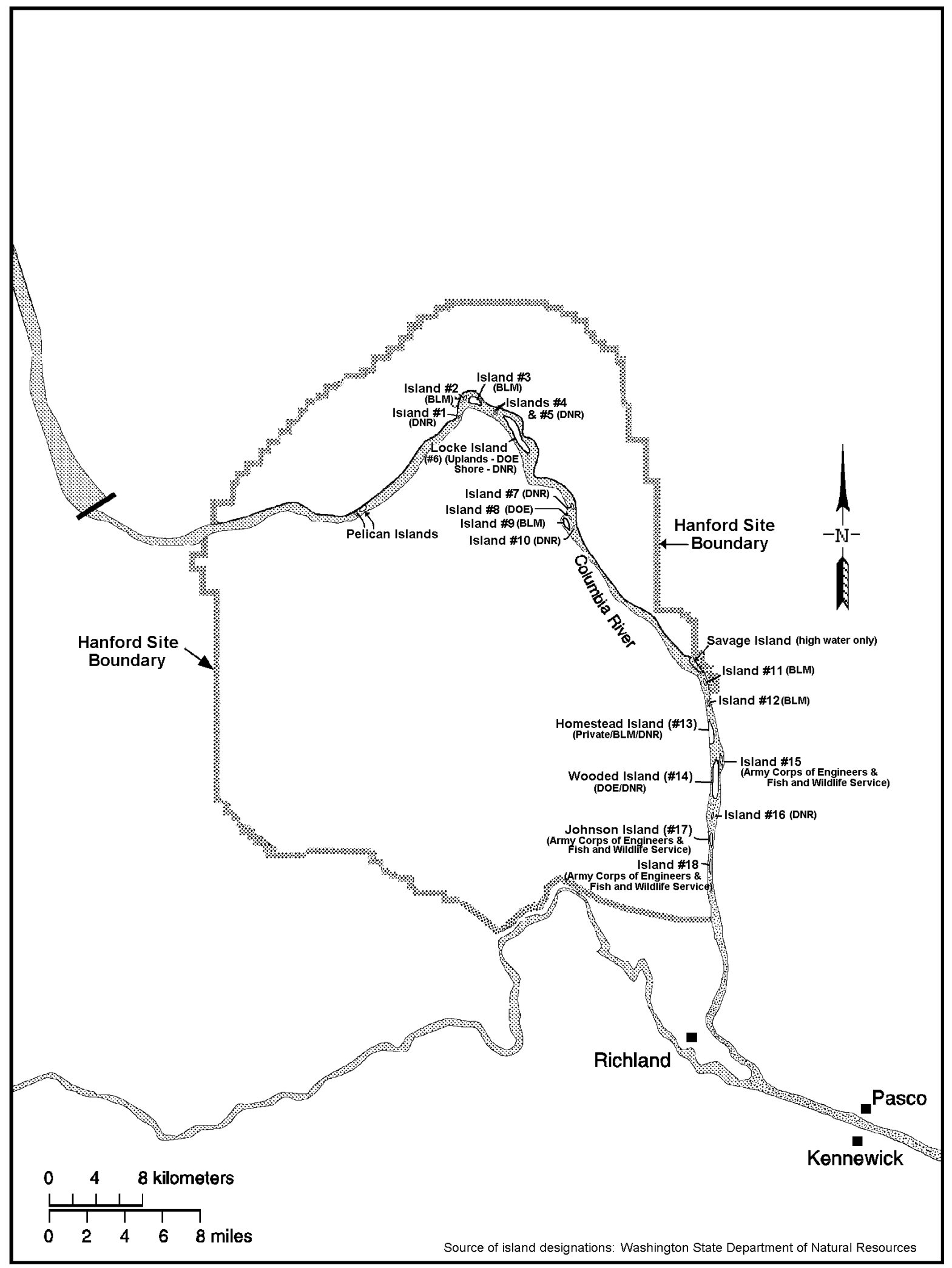

Figure 4.4-2. Columbia River Islands 
rabbitbrush also occur throughout the 100 Areas (Landeen et al. 1993). An area of natural big sagebrush habitat near the 100-D area has experienced significant and apparently natural decline in recent years (Cardenas et al. 1997). A total area encompassing $17.8 \mathrm{~km}^{2}\left(6.9 \mathrm{mi}^{2}\right)$ is in decline, and a central core area of $2.8 \mathrm{~km}^{2}\left(1.1 \mathrm{mi}^{2}\right)$ has experienced more than $80 \%$ mortality. State threatened, endangered, or species of concern that occur within the 100 Area operable units include persistent sepal yellowcress, southern mudwort, false pimpernel, shining flatsedge, gray cryptantha, and possibly, dense sedge (see Table 4.4-1 and Table 4.4-2 in Section 4.4.3) (Landeen et al. 1993; Soll et al. 1999).

Waste management areas, reactors, and crib sites are generally either barren or vegetated by invasive species, including Russian thistle, tumble mustard, and cheatgrass. Most of the waste disposal and storage sites are covered by nonnative vegetation or are kept in a vegetation-free condition with the use of herbicides, as the plants could potentially accumulate waste constituents. Russian thistle and gray rabbitbrush that occur in these areas are deep rooted and have the potential to accumulate radionuclides and other buried contaminants, functioning as a pathway to other parts of the ecosystem (Landeen et al. 1993). The undisturbed portions of the 200 Areas are characterized as sagebrush/cheatgrass or Sandberg's bluegrass communities of the 200 Area Plateau. The dominant plants on the 200 Area Plateau are big sagebrush, rabbitbrush, cheatgrass, and Sandberg's bluegrass. Cheatgrass provides half of the total plant cover. Most of the waste disposal and storage sites are planted with crested or Siberian wheatgrass to stabilize surface soil, control soil moisture, or displace more invasive deep-rooted species like Russian thistle.

Vegetation surveys were conducted at the 300-FF-5 Operable Unit during 1992. The shrub-steppe vegetation community in the unit is characterized as antelope bitterbrush/Sandberg's bluegrass with an overstory of bitterbrush and big sagebrush and an understory of cheatgrass and Sandberg's bluegrass (Brandt et al. 1993). Dominant riparian vegetation in the unit included white mulberry and shrub willow, reed canarygrass, bulbous bluegrass, sedges, and horsetail. Persistent sepal yellowcress, a state threatened species, was identified at 18 locations near this operable unit.

\subsubsection{Wildlife}

Approximately 300 species of terrestrial vertebrates have been observed on the Hanford Site. The species list includes approximately 42 species of mammals (Appendix A, Table A-2), 246 species of birds, 5 species of amphibians, and 12 species of reptiles (Appendix A, Table A-3) (Soll and Soper 1996; Brandt et al. 1993). From 1991 to 1993, surveys for birds, mammals, insects, and vegetation were conducted at several of the 100 and 300 Area operable units and the results documented in topical reports (Brandt et al. 1993; Landeen et al. 1993). The Nature Conservancy (Soll et al. 1999) recently summarized its findings for birds and mammal surveys. These surveys fall short of the number of species that have been documented historically on the Hanford Site. For example, 221 species of birds were observed in the bird surveys of The Nature Conservancy's biodiversity 4-year effort (Soll et al. 1999). This number falls short of the 238 species identified historically (Landeen et al. 1992). The Nature Conservancy identified 258 species of birds on Hanford based on their surveys and the historical record (Soll et al. 1999). There are 144 species considered common to the Hanford Site (Appendix A, Table A4). The Nature Conservancy did not conduct specific surveys for mammals, but encounters were documented and compared to historic lists.

Shrubland and Grassland Wildlife. The shrub and grassland habitat of the Hanford Site supports many groups of terrestrial wildlife. Species include large game animals like Rocky Mountain elk and mule deer; predators such as coyote, bobcat, and badger; and herbivores like deer mice, harvest mice, ground squirrels, voles, and black-tailed jackrabbits. The most abundant mammal on the Hanford Site is the Great Basin pocket mouse. 
Mule deer are reliant on shoreline vegetation and bitterbrush shrubs for browse (Tiller et al. 1997). Elk, which are more dependent on open grasslands for forage, seek the cover of sagebrush and other shrub species during the summer months. Elk first appeared on the Hanford Site in 1972 (Fitzner and Gray 1991), and have increased from approximately 8 animals in 1975 to approximately 900 in 1999. The Rattlesnake Hills herd of elk that inhabits the Hanford Site primarily occupies ALE and private lands that adjoin the reserve to the south and west. They are occasionally seen on the 200 Area plateau and have been sighted at the White Bluffs boat launch on the Hanford Site. The herd tends to congregate on ALE in the winter and disperses during the summer months to higher elevations on ALE, private land to the west of ALE, and the Yakima Training Center. Efforts were taken in March 2000 to remove and relocate about 200 elk from the ALE Reserve. Special hunts adjacent to the Hanford Site in 2000 accounted for removal of 207 additional elk. The 24 Command Fire in June 2000 destroyed nearly all the elk forage on ALE. The herd moved onto unburned private land west of the site, to unburned areas on central Hanford, and along the Columbia River near the 100-B/C and 100-K Areas. There was a reported sighting of a cougar on ALE during the elk relocation effort in March 2000, supplementing anecdotal accounts of other observations of the presence of the big cat on the Hanford Site.

Shrubland and grassland provide nesting and foraging habitat for many passerine bird species. Surveys conducted during 1993 (Cadwell 1994) reported the occurrence of western meadowlarks and horned larks more frequently in shrubland habitats than in other habitats on the Hanford Site. Soll et al. (1999) reported a total of 41 species that are considered steppe or shrub-steppe habitat dependent. Longbilled curlews and vesper sparrows were also noted as commonly occurring species in shrubland habitat. Species that are dependent on undisturbed shrub habitat include sage sparrow, sage thrasher, and loggerhead shrike. Both the sage sparrow and loggerhead shrike tend to roost and nest in sagebrush or bitterbrush that occurs at lower elevations (DOE 1996a). Ground-nesting species that occur in grasscovered uplands include long-billed curlews, western meadowlark, and burrowing owls.

Common upland gamebird species that occur in shrub and grassland habitat include chukar, partridge, California quail, and ring-necked pheasant. Chukars are most numerous in the Rattlesnake Hills, Yakima Ridge, Umtanum Ridge, Saddle Mountains, and Gable Mountain areas of the Hanford Site. Less common species include greater sage grouse, Hungarian partridge, and scaled quail. Greater sage grouse were historically abundant on the Hanford Site; however, populations have declined since the early $1800 \mathrm{~s}$ because of the conversion of sagebrush-steppe habitat. Although surveys conducted by the Washington Department of Fish and Wildlife and PNNL during late winter and early spring 1993, and biodiversity inventories conducted by The Nature Conservancy in 1997, did not observe greater sage grouse in sagebrush-steppe habitat at ALE, sage grouse have been observed in 1999 and 2000. (a) The 24 Command Fire in June 2000 destroyed potential sagegrouse habitat on ALE, and it is unlikely that sage grouse will return to ALE in the near future.

Among the more common raptor species that use shrub and grassland habitat are the ferruginous hawk, Swainson's hawk, and red-tailed hawk. Northern harriers, sharp-shinned hawks, rough-legged hawks, and golden eagles also occur in these habitats, although infrequently. In 1994, nesting by redtailed, Swainson's, and ferruginous hawks included 41 nests located across the Hanford Site on high voltage transmission towers, trees, cliffs, and basalt outcrops. In recent years, the number of breeding ferruginous hawks (a Washington State threatened species) on the Hanford Site has increased, as a result, in part, to their use of steel powerline towers in the open grass and shrubland habitats for nesting.

Many species of insects occur throughout all habitats on the Hanford Site. Butterflies, grasshoppers, and darkling beetles are among the most conspicuous of the approximately 1500 species of insects that have been identified from specimens collected on the Hanford Site (Soll et al. 1999). The actual number

(a) Source: Personal communication with B.L. Tiller, PNNL, April 2000. 
of insect species occurring on the Hanford Site may reach as high as 15,500. A total of 1509 specieslevel identifications were completed in 1999 and 500 more are expected. Recent surveys performed by The Nature Conservancy included the collection of 40,000 specimens and have resulted in the identification of 43 new taxa and 142 new findings in the state of Washington (Soll et al. 1999). The high diversity of insect species on the Hanford Site reflects the size, complexity, and relatively undisturbed quality of the shrub-steppe habitat.

The side-blotched lizard is the most abundant reptile species occurring on the Hanford Site. Shorthorned and sagebrush lizards are reportedly found on the Hanford Site, but occur infrequently. The most common snake species include gopher snake, yellow-bellied racer, and Pacific rattlesnake. The Great Basin spadefoot toad, Woodhouse's toad, Pacific tree frog, tiger salamander, and bullfrog are the only amphibians found on the Hanford Site (Soll et al. 1999; Brandt et al. 1993).

Riparian Wildlife. Riparian areas provide nesting and foraging habitat and escape cover for many species of birds and mammals. Shoreline riparian communities are seasonally important for a variety of species. Willows trap food for waterfowl (e.g., Canada geese) and birds that use shoreline habitat (e.g., Forster's tern) and provide nesting habitat for passerines (e.g., mourning doves). Terrestrial and aquatic insects are abundant in emergent grasses and provide food for fish, waterfowl, and shorebirds. Riparian areas provide nesting and foraging habitat and cover for many species of birds and mammals.

Mammals occurring primarily in riparian areas include rodents, bats, furbearers (e.g., mink and weasel), porcupine, raccoon, skunk, and mule deer. Beavers rely on shoreline habitat for dens and foraging. River otters have been observed infrequently in the Hanford Reach. During the summer, mule deer rely on riparian vegetation for foraging. Mule deer use Columbia River islands for fawning and nursery areas. Beaver and muskrat rely on shoreline habitat for dens and foraging. The Columbia River and Rattlesnake Springs provide foraging habitat for most species of bats including myotis, small-footed myotis, silver-haired bats, and pallid bats, which feed on emergent aquatic insects (Becker 1993).

Common bird species that occur in riparian habitats include American robin, black-billed magpie, song sparrow, and dark-eyed junco (Cadwell 1994). Upland gamebirds that use this habitat include ringnecked pheasants and California quail. Predatory birds include common barn owl and great horned owl. Species known or expected to nest in riparian habitat are Brewer's blackbird, mourning dove, black-billed magpie, northern oriole, lazuli bunting, eastern and western kingbird, and western wood peewee. Bald eagles have wintered on the Hanford Site since 1960. Great blue herons and black crowned night herons are associated with trees in riparian habitat along the Columbia River and use groves or individual trees for perching and nesting. On occasion, great blue herons have constructed nests in the large metal powerline towers that cross the Columbia River.

The Hanford Site is located in the Pacific Flyway, and the Hanford Reach serves as a resting area for neotropical migrant birds, migratory waterfowl, and shorebirds (Soll et al. 1999). During the fall and winter months, ducks (primarily mallards) and Canada geese rest on the shorelines and islands along the Hanford Reach. The area between the Old Hanford Townsite and Vernita Bridge is closed to recreational hunting, and large numbers of migratory waterfowl find refuge in this portion of the river. Other species observed during this period include American white pelicans, egrets, double-crested cormorants, coots, and common loons.

Wildlife Occurring in Unique Habitat. Bluffs provide perching, nesting, and escape habitat for several bird species on the Hanford Site. The White Bluffs and Umtanum Ridge provide nesting habitat for prairie falcons, red-tailed hawks, cliff swallows, bank swallows, and rough-winged swallows. In the past, Canada geese used the lower elevations of the White Bluffs for nesting and brooding. Bald eagles use the White Bluffs for roosting. Bluff areas provide habitat for sensitive species (i.e., Hoover's desert 
parsley and peregrine falcon) that otherwise may be subject to impact from frequent or repeated disturbance. The White Bluffs bladderpod is a newly discovered Washington State endangered species that grows on the White Bluffs. Trees that do not normally occur in arid steppe habitat supply nesting, perching, and roosting sites for many birds. Consequently, raptors, like ferruginous and Swainson's hawks, can use trees for breeding in areas that previously did not support breeding populations. Ferruginous hawks also nest on electrical transmission line towers.

Dune habitat is unique in its association with the surrounding shrub-steppe vegetation type. The uniqueness of the dunes is noted in its vegetation component as well as the geologic formation. The terrain of the Hanford dunes provides habitat for mule deer, burrowing owls, and coyotes as well as many transient species.

Islands afford a unique arrangement of upland and shoreline habitat for avian and terrestrial species. Islands vary in soil type and vegetation and range from narrow cobble beaches to extensive dune habitats. Except for several plant species, the islands accommodate many of the same species that occur in mainland habitats. Operation of Priest Rapids Dam upstream of the Hanford Reach creates daily and seasonal fluctuations in river levels, which may limit community structure and overall shoreline species viability along the shoreline interface.

Islands provide resting, nesting, and escape habitat for waterfowl and shorebirds. Use of islands for nesting by Canada geese has been monitored since 1950. The suitability of habitat for nesting Canada geese is attributed to restricted human use of islands during the nesting season, suitable substrate, and adequate forage and cover for broods (Eberhardt et al. 1989). The nesting population fluctuates annually. In recent years, geese have used the downstream islands in the Reach for nesting as a result of coyote predation in the upper Reach islands. Islands also accommodate colonial nesting species including California gulls, ring-billed gulls, and Forster's terns. Island areas ranging from 0.1 to $0.2 \mathrm{~km}^{2}(0.05$ to $0.08 \mathrm{mi}^{2}$ ) accommodate colonial nesting species that may range in population size of upward of 2000 individuals.

\subsubsection{Aquatic Ecology}

There are two types of natural aquatic habitats on the Hanford Site: the Columbia River which flows along the northern and eastern edges of the Hanford Site, and small spring-streams and seeps located mainly on ALE (Figure 4.4-2) in the Rattlesnake Hills. West Lake is a small saline pond created by a rise in the water table in the 200 Areas and is not fed by surface flow. Evaporation of groundwater and possible disposal of sewage during the early Hanford years created highly saline and alkaline conditions that greatly restricted the complement of biota in West Lake (Poston et al. 1991).

\subsubsection{Columbia River}

The Columbia River is the dominant aquatic ecosystem on the Hanford Site and supports a large and diverse community of plankton, benthic invertebrates, fish, and other communities. It has a drainage area of about $680,000 \mathrm{~km}^{2}\left(262,480 \mathrm{mi}^{2}\right)$, an estimated average annual discharge of $6600 \mathrm{~m}^{3} / \mathrm{s}\left(71,016 \mathrm{ft}^{3} / \mathrm{s}\right)$, and a total length of about $2000 \mathrm{~km}$ (about $1240 \mathrm{mi}$ ) from its origin in British Columbia to its mouth at the Pacific Ocean. The Columbia has been dammed both upstream and downstream of the Hanford Site, and the Reach flowing through the Site is the last free-flowing, but regulated, section of the Columbia River in the United States above Bonneville Dam. Plankton populations in the Hanford Reach are influenced by communities that develop in the reservoirs of upstream dams, particularly Priest Rapids Reservoir, and by manipulation of water levels below by dam operations in upstream and downstream reservoirs. Phytoplankton and zooplankton populations at the Hanford Site are largely transient, flowing 
from one reservoir to another. There is generally insufficient time for characteristic endemic groups of phytoplankton and zooplankton to develop in the Hanford Reach. No tributaries enter the Columbia during its passage through the Hanford Site; however, there are several irrigation water return canals that discharge into the Columbia River along the Franklin County shoreline. The presence of irrigation drainage ponds on the North Slope in Grant County indicates that groundwater seepage enters the river along the north shoreline opposite the 100-B/C to 100-D Areas as well as the eastern shoreline bordering Franklin County.

As a result of the Hanford Reach National Monument designation, the USFWS manages the Hanford Reach as a wildlife management unit, along with the other units associated with the Arid Lands National Wildlife Refuge Complex. The Department of the Interior's Record of Decision, Hanford Reach of the Columbia River: Final Environmental Impact Statement for Comprehensive River Conservation Study recommended that the Hanford Reach be designated a Wild and Scenic River (DOI 1996).

The Columbia River is a very complex ecosystem because of its size and biotic diversity. Streams in general, especially smaller ones usually depend on organic matter from outside sources (e.g., terrestrial plant debris) to provide energy for the ecosystem. Large rivers, particularly the Columbia River with its series of large reservoirs, contain significant populations of primary energy producers (e.g., algae and plants) that contribute to the basic energy requirements of the biota.

Phytoplankton (free-floating algae) and periphyton (sessile algae) are abundant in the Columbia River and provide food for herbivores such as immature insects, which in turn, are consumed by predaceous species.

Phytoplankton. Phytoplankton species identified from the Hanford Reach include diatoms, golden or yellow-brown algae, green algae, blue-green algae, red algae, and dinoflagellates. Studies show diatoms are the dominant algae in the Columbia River phytoplankton, usually representing more than $90 \%$ of the populations. The main genera included Asterionella, Cyclotella, Fragilaria, Melosira, Stephanodiscus, and Synedra (Neitzel et al. 1982a). These are typical of those forms found in lakes and ponds and originate in upstream reservoirs. A number of algae found as free-floating species in the Hanford Reach of the Columbia River are actually derived from the periphyton; they were detached and suspended by current and frequent fluctuations of the water level.

Cushing (1967a) found peak concentrations of phytoplankton occurred in April and May, with a secondary peak in late summer/early autumn. The spring pulse in phytoplankton density was probably related to increasing light and water temperature rather than to availability of nutrients, as phosphate and nitrate nutrient concentrations are never limiting. Minimum numbers were present in December and January. Green algae (Chlorophyta) and blue-green algae (Cyanophyta) occur in phytoplankton communities during warmer months but in substantially fewer numbers than diatoms. Diversity indices, carbon uptake, and chlorophyll-a concentrations for the phytoplankton at various times and locations can be found in Beak Consultants Inc. (1980), Neitzel et al. (1982a), and Wolf et al. (1976).

Periphyton. Communities of periphytic species ("benthic microflora") develop on suitable solid substrate wherever there is sufficient light for photosynthesis. Cushing (1967b) observed peaks of production to occur in spring and late summer. Dominant genera are the diatoms Achnanthes, Asterionella, Cocconeis, Fragilaria, Gomphonema, Melosira, Nitzchia, Stephanodiscus, and Synedra (Beak Consultants Inc. 1980; Neitzel et al. 1982a; Page and Neitzel 1978; Page et al. 1979). 
Macrophytes. Macrophytes are sparse in the Columbia River because of strong currents, rocky bottom, and frequently fluctuating water levels. Rushes (Juncus spp.) and sedges (Carex spp.) occur along shorelines of the slack-water areas such as White Bluffs Slough below the 100-H Area, the slough area downstream of the 100-F Area, and Hanford Slough. Reed canary grass is a common non-native species found along shoreline areas. Macrophytes are also present along gently sloping shorelines that are subject to flooding during the spring freshet and daily fluctuating river levels (below Coyote Rapids and the 100-D Area). Commonly found plants include duckweed (Lemna), and rooted pond weeds (Potamogeton sp. and Elodea). Where they exist, macrophytes have considerable ecological value. They provide food and shelter for juvenile fish and spawning areas for some species of warm water game fish. Milfoil (Myriophyllum), an exotic macrophyte, has increased to nuisance levels, and may encourage increased sedimentation of fine particulate matter. These changes could have a significant impact on trophic relationships in the Columbia River.

Zooplankton. The zooplankton populations in the Hanford Reach of the Columbia River are generally sparse. Studies by Neitzel et al. (1982b) indicate crustacean zooplankters were dominant in the open-water regions. Dominant genera were Bosmina, Diaptomus, and Cyclops. Densities were lowest in winter and highest in the summer, with summer peaks dominated by Bosmina, ranging up to 160,650 organisms $/ \mathrm{m}^{3}$ (4500 organisms $\left./ \mathrm{ft}^{3}\right)$. Winter densities were generally $<1785$ organisms $/ \mathrm{m}^{3}$ $\left(<50\right.$ organisms $\left./ \mathrm{ft}^{3}\right)$ (Brandt et al. 1993). Diaptomus and Cyclops dominated in winter and spring, respectively.

Benthic Organisms. Benthic organisms are found either attached to or closely associated with the substratum. All major freshwater benthic taxa are represented in the Columbia River. Insect larvae such as caddisflies (Trichoptera), midge flies (Chironomidae), and black flies (Simuliidae) are dominant. Dominant caddisfly species are Hydropsyche cockerelli, Cheumatopsyche campyla, and C. enonis. Other benthic organisms include clams, limpets, snails, sponges, and crayfish. Early Hanford studies found crayfish numbers in shallow water areas ranged from 0.2 to 1.1 individuals $/ \mathrm{ft}^{2}$ of river bottom, with a diet primarily of vegetation (Coopey 1953), while insect larvae numbers were sometimes as high as 2000/ $\mathrm{ft}^{2}$ (Davis and Cooper 1951). Peak larval insect densities are found in late fall and winter, and the major emergence is in spring and summer (Wolf 1976). Stomach contents of fish collected in the Hanford Reach from June 1973 through March 1980 revealed that benthic invertebrates were important food items for nearly all juvenile and adult fish. There was a close relationship between food organisms in the stomach contents and those in the benthic and invertebrate drift communities. A recent survey by The Nature Conservancy (Soll et al. 1999) identified 21 new taxa of aquatic invertebrates from the Hanford Reach bringing the total number of aquatic invertebrate taxa at Hanford to 151.

Invertebrate surveys at Rattlesnake Springs and Snively Springs on the ALE Reserve identified 30 and 12 new taxa at each spring, respectively (Soll et al. 1999). These recent findings bring the total number of taxa at each spring to 43 and 24, respectively.

Fish. Gray and Dauble (1977) listed 43 species of fish in the Hanford Reach of the Columbia River. The brown bullhead, collected since 1977, brings the total number of fish species identified in the Hanford Reach to 44 (Appendix A, Table A-5). Of these species, chinook salmon, sockeye salmon, coho salmon, and steelhead trout use the river as a migration route to and from upstream spawning areas and are of the greatest economic importance. Additionally, fall chinook salmon and steelhead trout spawn in the Hanford Reach. The relative contribution of up-river bright stocks to fall chinook salmon runs in the Columbia River increased from about $24 \%$ of the total in the early 1980 s, to $50 \%$ to $60 \%$ of the total by 1988 (Dauble and Watson 1990). Inundation of other mainstream Columbia spawning grounds by dams has increased the relative importance of the Hanford Reach to fall chinook salmon production in the Columbia and Snake rivers (Watson 1970, 1973, Dauble and Watson 1997). 
The steelhead fishery in the Hanford Reach (Highway 395 Bridge to Priest Rapids Dam) consists almost exclusively of summer run fish. The estimated sport catch for the 1998-1999 season was 1066 fish. The majority of these fish (98\%) were marked hatchery fish. About $90 \%$ of this harvest occurred from May through July (WDFW 2000).

American shad, another anadromous species, may also spawn in the Hanford Reach. The upstream range of the shad has been increasing since 1956 when $<10$ adult shad ascended McNary Dam. Since then, the number of shad ascending Priest Rapids Dam has risen to many thousands each year, and youngof-the-year have been collected in the Hanford Reach. Shad are not dependent on the same conditions that are required by the salmonids for spawning and apparently have found favorable conditions for reproduction throughout much of the Columbia and Snake rivers.

Other fish of importance to sport fishermen are mountain whitefish, white sturgeon, smallmouth bass, crappie, catfish, walleye, and yellow perch. Large populations of rough fish are also present, including carp, redside shiner, suckers, and northern pikeminnow (formerly known as "squawfish"). Because northern pikeminnow feed on juvenile salmon, the Washington State Department of Fish and Wildlife has established a bounty program on adult pikeminnow to bolster salmon runs. Northern pikeminnow removed from the Hanford Reach are usually turned in at bounty stations located at Columbia Point in Richland and at the Vernita Bridge rest stop.

\subsubsection{Spring Streams}

Small interrupted streams, such as Rattlesnake and Snively springs, contain diverse biotic communities and are extremely productive (Cushing and Wolf 1984). Dense blooms of watercress occur and are not lost until a major flash floods occurs. Aquatic insect production is fairly high as compared with mountain streams (Gaines 1987). The macrobenthic biota varies from site to site and is related to the proximity of colonizing insects and other factors. The 24 Command Fire of 2000 (BAER 2000) has had little direct impact on the stream ecology, even though the riparian transect along the lower two thirds of the stream was heavily damaged by the fire.

Rattlesnake Springs, on the western side of the Hanford Site, forms a small surface stream that flows for about $2.5 \mathrm{~km}(1.6 \mathrm{mi})$ before disappearing into the ground as a result of seepage and evapotranspiration. Base flow of this stream is about $0.01 \mathrm{~m}^{3} / \mathrm{s}\left(0.4 \mathrm{ft}^{3} / \mathrm{s}\right)$ (Cushing and Wolf 1982). Water temperature ranges from $2^{\circ}$ to $22^{\circ} \mathrm{C}\left(36^{\circ}\right.$ to $\left.72^{\circ} \mathrm{F}\right)$. Mean annual total alkalinity $\left(\right.$ as $\left.\mathrm{CaCO}_{3}\right)$, nitrate nitrogen, phosphate phosphorus, and total dissolved solids are $127,0.3,0.18$, and $217 \mathrm{mg} / \mathrm{L}$, respectively (Cushing and Wolf 1982; Cushing et al. 1980). The sodium content of the spring water is about $7 \mathrm{ppm}$ (Brown 1970). Rattlesnake Springs is of ecological importance because it provides a source of water to terrestrial animals in an otherwise arid part of the Hanford Site. Snively Springs, located farther west and at a higher elevation than Rattlesnake Springs, is also another source of drinking water for terrestrial animals. Both springs provide a valuable source of drinking water for the Rattlesnake Hills elk herd. The major rooted aquatic plant, which in places may cover the entire width of the stream, is watercress. Isolated patches of bulrush, spike rush, and cattail occupy $<5 \%$ of the streambed.

Primary productivity at Rattlesnake Springs is greatest during the spring and coincident with the maximum periphyton standing crop. Net primary productivity averaged $0.9-\mathrm{g} / \mathrm{cm}^{2} / \mathrm{d}$ organic matter during 1969 and 1970; the spring maximum was $2.2 \mathrm{~g} / \mathrm{cm}^{2} / \mathrm{d}$. Seasonal productivity and respiration rates are within the ranges reported for arid region streams. Although Rattlesnake Springs is a net exporter of organic matter during much of the growing season, it is subject to flash floods and severe scouring and denuding of the streambed during winter and early spring, making it an importer of organic materials on an annual basis (Cushing and Wolf 1984). 
Secondary production is dominated by detritus-feeding collector-gatherer insects (mostly Chironomidae and Simuliidae) that have multiple cohorts and short generation times (Gaines et al. 1992). Overall production is not high and is likely related to the low diversity found in these systems related to the winter spates that scour the spring-streams. Total secondary production in Rattlesnake and Snively springs is 16,356 and $14,154 \mathrm{~g}$ dry weight $/ \mathrm{m}^{2} / \mathrm{yr}$, respectively. There is an indication that insects in these spring-streams depend on both autochthonous (originating within the stream) and allochthonous (originating outside the stream) primary production as an energy source, despite significant shading by exotic species of trees and shrubs (Mize 1993).

Schwab et al. (1979) has published an inventory of the many springs occurring on the Rattlesnake Hills. Limited physical and chemical data are included for each site.

\subsubsection{Wetland Habitats}

Several habitats on the Hanford Site could be considered wetlands. The largest wetland habitat is the riparian zone bordering the Columbia River. The extent of this zone varies but includes extensive stands of willows, grasses, various aquatic macrophytes, and other plants. The zone is extensively impacted by both seasonal water-level fluctuations and daily variations related to power generation at Priest Rapids Dam immediately upstream of Hanford. There are also minor impacts to shoreline areas near the 300 Area, as a result of fluctuating water levels in Lake Wallula as established by operations at McNary Dam downstream of the Hanford Site.

Other wetland habitat can be found within the Saddle Mountain National Wildlife Refuge Unit and the Wahluke Unit. These two areas encompass all the lands extending from the north bank of the Columbia River northward to the Hanford Site boundary and east of the Columbia River down to Ringold Springs. Wetland habitat in these areas consists of fairly large pond habitat resulting from irrigation runoff (see Figure 4.3-1). These ponds have extensive stands of cattails and other emergent aquatic vegetation surrounding the open-water regions. They are extensively used as nesting sites by waterfowl.

With the cessation of nuclear materials production activities at the Hanford Site, the amount of water discharged to the ground in the 200 Area Plateau has significantly decreased. West Lake is a saline pond that is created by the elevated water table cause by surface water discharges in the 200 Areas (Poston et al. 1991). Over the past 10 years, the pond has decreased in size and currently consists of a group of small isolated pools and mud flats. Avocets, killdeer, and sandpipers still use the lake basin and feed on invertebrates that can tolerate the high salinity of the pond. The reduced pond does not support coots or other nesting waterfowl. The water is too saline for consumption by mammals.

Some wetland habitat exists in the riparian zones of some of the larger spring streams on the ALE Reserve. These are not extensive and usually amount to less than $0.01 \mathrm{~km}^{2}\left(0.004 \mathrm{mi}^{2}\right)$ in size, although the riparian zone along Rattlesnake Springs is probably about $2 \mathrm{~km}(1.2 \mathrm{mi})$ in length and consists of peach leaf willows, cattails, and other exotic plants. The 24 Command Fire impacted these riparian areas and killed many of the large trees found along the riparian zone.

The USFWS has published a series of 1:24,000 maps that show the locations of wetlands. An accompanying booklet describes how to use these maps. Four sets of these maps covering the Hanford Site and the instructional booklet for their use are available from D. A. Neitzel, Sigma 5 Building/Room 1105 (PNNL) or P. F. Dunigan, Federal Building/Room 576 (DOE). 


\subsubsection{Temporary Water Bodies}

Several artificial water bodies, both ponds and ditches, were formed as a result of wastewater disposal practices associated with operation of the reactors and separation facilities. Most of these have been taken out of service and have been backfilled with the cessation of activities (except West Lake). When present, however, they formed established aquatic ecosystems complete with representative flora and fauna (Emery and McShane 1980). The temporary wastewater ponds and ditches existed for as long as two decades and covered fairly large areas. Rickard et al. (1981) discusses the ecology of Gable Mountain Pond, one of the former major lentic sites. Emery and McShane (1980) present ecological characteristics of all the temporary water bodies. The ponds developed luxuriant riparian communities and became quite attractive to autumn and spring migrating birds. Several species have nested near the ponds. Section 4.3.1.8 describes those water bodies still active. These former sites have been decommissioned and are now covered with overburden and planted with grasses for stabilization.

\subsubsection{Threatened and Endangered Species}

Threatened and endangered plants and animals identified on the Hanford Site, as listed by the federal government (50 CFR 17) and Washington State (Washington Natural Heritage Program 2000), are shown in Table 4.4-1. No plants or mammals on the federal list of threatened and endangered wildlife and plants (50 CFR 17) are known to occur on the Hanford Site. However, the bald eagle and two species of fish (steelhead and spring-run chinook salmon), currently found on the federal list of threatened and endangered species, are present on the Hanford Site on a regular basis. Several species of both plants and animals are under consideration for formal listing by the federal government and Washington State. The USFWS reviews the status of candidate species for listing under the Endangered Species Act on an annual basis. The results of these reviews are posted on the USFWS homepage (http://www.fws.gov). Anadromous fish are reviewed and listed by the National Marine Fisheries Service (NMFS) (http://www.nwr.noaa.gov).

Washington State considers pristine shrub-steppe habitat priority habitat because of its relative scarcity in the state, and because of its requirement as nesting/breeding habitat by several state and federal species of concern. Designation and characterization of priority habitat serves to provide a basis for sound and defensible land management planning and assists the DOE in implementing sound stewardship activities into site management to protect regulated species. Several recent publications describing the distribution of threatened and endangered species on the Hanford Site have been prepared by Becker (1993), Cadwell (1994), Downs et al. (1993), Fitzner et al. (1994), Frest and Johannes (1993), Soll et al. (1999).

\subsubsection{Plants}

Eight species of Hanford Site plants are included in the Washington State listing as threatened or endangered (Washington Natural Heritage Program 1997). Columbia milkvetch, dwarf evening primrose, loeflingia, white eatonella, persistent sepal yellowcress, and Hoover's desert parsley are listed as threatened. Umtanum desert buckwheat and White Bluffs bladderpod are designated as endangered species. Columbia milkvetch occurs on dry-land beaches along the Columbia River near Priest Rapids Dam, Midway, and Vernita; it also has been found atop Umtanum Ridge, in Cold Creek Valley near the present vineyards, and on Yakima Ridge (on ALE). Dwarf evening primrose has been found north of Gable Mountain near the Vernita Bridge, Ringold, and on mechanically disturbed areas (e.g., the gravel pit near the Wye Barricade). Hoover's desert parsley grows on steep talus slopes near Priest Rapids Dam, Midway, and Vernita. Persistent sepal yellowcress occurs in the wetted zone of the water's edge along 
Table 4.4-1. Federal- or Washington State-Listed Threatened (T) and Endangered (E) Species Occurring on the Hanford Site

\begin{tabular}{|c|c|c|c|}
\hline Common Name & Scientific Name & Federal & State $^{(\mathbf{a})}$ \\
\hline \multicolumn{4}{|l|}{$\underline{\text { Plants }}$} \\
\hline Columbia milkvetch & Astragalus columbianus & $\mathrm{SC}^{(\mathrm{b})}$ & $\mathrm{T}$ \\
\hline Dwarf evening primrose & Camissonia (= Oenothera) pygmaea & & $\mathrm{T}$ \\
\hline Hoover's desert parsley & Lomatium tuberosum & $\mathrm{SC}$ & $\mathrm{T}$ \\
\hline Loeflingia & Loeflingia squarrosa var. squarrosa & & $\mathrm{T}$ \\
\hline Persistent sepal yellowcress & Rorippa columbiae & $\mathrm{SC}$ & $\mathrm{T}$ \\
\hline Umtanum desert buckwheat & Eriogonum codium & $\mathrm{C}^{(\mathrm{c})}$ & $\mathrm{E}$ \\
\hline White Bluffs bladderpod & Lesquerella tuplashensis & $\mathrm{C}$ & $\mathrm{E}$ \\
\hline White eatonella & Eatonella nivea & & $\mathrm{T}$ \\
\hline \multicolumn{4}{|l|}{$\underline{\text { Fish }}$} \\
\hline Spring-run chinook & Oncorhynchus tshawytscha & E & $\mathrm{C}$ \\
\hline Steelhead & Oncorhynchus mykiss & $\mathrm{E}$ & $\mathrm{C}$ \\
\hline \multicolumn{4}{|l|}{$\underline{\text { Birds }}$} \\
\hline American white pelican & Pelecanus erythrorhychos & & $\mathrm{E}$ \\
\hline Bald eagle $^{(d)}$ & Haliaeetus leucocephalus & $\mathrm{T}$ & $\mathrm{T}$ \\
\hline Ferruginous hawk & Buteo regalis & $\mathrm{SC}$ & $\mathrm{T}$ \\
\hline Sandhill crane & Grus canadensis & & $\mathrm{E}$ \\
\hline Greater sage grouse & Centrocercus urophasianus phaios & $\mathrm{SC}$ & $\mathrm{T}$ \\
\hline $\begin{array}{l}\text { (a) Washington Natural Heritage } \\
\text { (bttp://www.wa.gov/wdfw/hab/ } \\
\text { (b) } \mathrm{SC}=\text { Species of concern (h} \\
\text { (c) } \mathrm{C}=\text { Candidate, } 50 \text { CFR } 17 \\
\text { (d) Currently under review for cha }\end{array}$ & $\begin{array}{l}2000 \text { (http://www.nwr.noaa.gov); } \\
\text { al. } 1999 \text { ). } \\
\text { w.fws.gov). } \\
\text { www.fws.gov). } \\
\text { atus (http://www.fws.gov). }\end{array}$ & & \\
\hline
\end{tabular}


the Hanford Reach. Umtanum desert buckwheat (reported on central Hanford) and White Bluffs bladderpod (reported on the Wahluke Unit) occur only on the Hanford Site and nowhere else in the world (Soll et al. 1999).

Two species of listed plants have been considered as possible inhabitants of the Hanford Site in prior years. Northern wormwood is a federal candidate for listing and is a Washington State endangered species. It is known to occur near Beverly; however, surveys by The Nature Conservancy (Soll et al. 1999) did not find any occurrences along the northern shoreline of the Columbia River across from the 100 Areas. The Nature Conservancy believes the only remaining portions of the Hanford Site that have not been surveyed and could support northern wormwood are islands in the Hanford Reach. Similarly, Wanapum crazyweed is only found near the western end of the Saddle Mountains and could also be found on the Hanford Site. This plant is a federal species of concern and is listed as endangered by the state of Washington.

Table 4.4-2 lists Washington State plant species of concern that are currently listed as sensitive or are in one of three monitored groups (Washington Natural Heritage Program 2000; Soll et al. 1999).

\subsubsection{Animals}

The federal government lists the bald eagle as threatened, and the upper Columbia River spring-run chinook salmon and upper Columbia River steelhead as endangered. Washington State lists the American white pelican and sandhill crane, as endangered, and lists the ferruginous hawk, greater sage grouse, and the bald eagle as threatened. Sage grouse were sighted on ALE in 1999 and again in 2000. White pelicans have become residents of the Hanford Site, and sandhill cranes, on occasion, have been observed on the Reach during their spring migrations.

The bald eagle is a regular winter resident and forages on dead salmon and waterfowl along the Columbia River; it does not nest on the Hanford Site, although it has attempted to nest for the last several years. Access controls are in place along the river at certain times of the year to prevent the disturbance of eagles. Washington State Bald Eagle Protection Rules were issued in 1986 (Washington Administrative Code [WAC]-232-12-292). DOE developed a site management plan (Fitzner and Weiss 1994) to mitigate eagle disturbance. This document constitutes a biological assessment for those activities implemented in accordance with the plan and, unless there are extenuating circumstances associated with a given project, the document fulfills the requirements of Section 7 (a)(2) of the Endangered Species Act for bald eagles and peregrine falcons. Section 7 (a) of the Endangered Species Act also requires consultation with the U.S. Department of the Interior when any action is taken that may destroy, adversely modify, or jeopardize the existence of bald eagle or other endangered species' habitat. At this time, bald eagles are under consideration for de-listing; however, the species will require 5 years of post de-listing monitoring (50 CFR Part 17).

Steelhead and salmon are regulated as Evolutionary Significant Units (ESU) by the NMFS based on their historical geographic spawning areas. The upper Columbia River ESU steelhead was listed as endangered in August 1997. The upper Columbia River ESU spring-run chinook salmon was listed as endangered in March 1999. These adult steelhead and chinook salmon migrate upstream through the Hanford Reach to spawn in upriver tributaries and juveniles pass through the Hanford Reach on their outward migration to the sea. A salmon and steelhead management plan (DOE 2000b) for Hanford Reach steelhead and upriver Columbia River ESU spring-run chinook was developed as required by section 7 (a)(2) of the Endangered Species Act. 
Table 4.4-2. Washington State Plant Species of Concern Occurring on the Hanford Site

\begin{tabular}{|c|c|c|}
\hline Common Name & Scientific Name & State Listing $^{(\mathrm{a})}$ \\
\hline Annual paintbrush & Castilleja exilis & $\mathrm{R} 1$ \\
\hline Awned halfchaff sedge & Lipocarpha $(=$ Hemicarpha) aristulata & R1 \\
\hline Basalt milk-vetch & Astragalus conjunctus var. rickardii & $\mathrm{R} 1$ \\
\hline Bristly combseed & Pectocarya setosa & $\mathrm{W}$ \\
\hline Brittle prickly pear & Opuntia fragilis & $\mathrm{R} 1$ \\
\hline Canadian St. John's wort & Hypericum majus & $\mathrm{S}$ \\
\hline Chaffweed & Centunculus minimus & $\mathrm{R} 1$ \\
\hline Columbia River mugwort & Artemesia lindleyana & $\mathrm{W}$ \\
\hline Coyote tobacco & Nicotiana attenuata & $\mathrm{S}$ \\
\hline Crouching milkvetch & Astragalus succumbens & $\mathrm{W}$ \\
\hline Desert dodder & Cuscuta denticulata & $\mathrm{S}$ \\
\hline Desert evening-primrose & Oenothera caespitosa & $\mathrm{S}$ \\
\hline False pimpernel & Lindernia dubia anagallidea & $\mathrm{R} 2$ \\
\hline Fuzzytongue penstemon & Penstemon eriantherus whitedii & $\mathrm{R} 1$ \\
\hline Geyer's milkvetch & Astragalus geyeri & $\mathrm{S}$ \\
\hline Grand redstem & Ammannia robusta & $\mathrm{R} 1$ \\
\hline Gray cryptantha & Cryptantha leucophaea & $\mathrm{S}$ \\
\hline Great Basin gilia & Gilia leptomeria & $\mathrm{R} 1$ \\
\hline Hedge hog cactus & Pediocactus simpsonii var. robustior & $\mathrm{R} 1$ \\
\hline Kittitas larkspur & Delphinium multiplex & $\mathrm{W}$ \\
\hline Lowland toothcup & Rotala ramosior & $\mathrm{R} 1$ \\
\hline Miner's candle & Cryptantha scoparia & $\mathrm{R} 1$ \\
\hline Piper's daisy & Erigeron piperianus & $\mathrm{S}$ \\
\hline Robinson's onion & Allium robinsonii & $\mathrm{W}$ \\
\hline Rosy balsamroot & Balsamorhiza rosea & $\mathrm{W}$ \\
\hline Rosy pussypaws & Calyptridium roseum & $\mathrm{S}$ \\
\hline Scilla onion & Allium scilloides & $\mathrm{W}$ \\
\hline Shining flatsedge & Cyperus bipartitus (rivularis) & $\mathrm{S}$ \\
\hline Small-flowered evening-primrose & Camissonia (= Oenothera) minor & $\mathrm{R} 1$ \\
\hline Small-flowered nama & Nama densum var. parviflorum & $\mathrm{R} 1$ \\
\hline Smooth cliffbrake & Pellaea glabella simplex & $\mathrm{W}$ \\
\hline Snake River cryptantha & Cryptantha spiculifera $(=C$. interrupta $)$ & $\mathrm{S}$ \\
\hline Southern mudwort & Limosella acaulis & $\mathrm{W}$ \\
\hline Stalked-pod milkvetch & Astragalus sclerocarpus & $\mathrm{W}$ \\
\hline Suksdorf's monkey flower & Mimulus suksdorfii & $\mathrm{S}$ \\
\hline Winged combseed & Pectocarya linearis & $\mathrm{R} 1$ \\
\hline \multicolumn{3}{|c|}{$\begin{array}{l}\text { The following species have been reported as occurring on the Hanford Site, but the known collections an } \\
\text { questionable in terms of location or identification, and have not been recently collected on the Hanford } \\
\text { Site. }\end{array}$} \\
\hline Beaked spike-rush & Eleocharis rostellata & $\mathrm{S}$ \\
\hline Dense sedge & Carex densa & S \\
\hline Few-flowered collinsia & Collinsia sparsiflora var. bruciae & $\mathrm{S}$ \\
\hline Giant helleborine & Epipactis gigantea & $\mathrm{S}$ \\
\hline Medic milkvetch & Astragalus speirocarpus & $\mathrm{W}$ \\
\hline Orange balsam & Impatiens aurella & $\mathrm{R} 2$ \\
\hline
\end{tabular}


Table 4.4-2 (cont'd.)

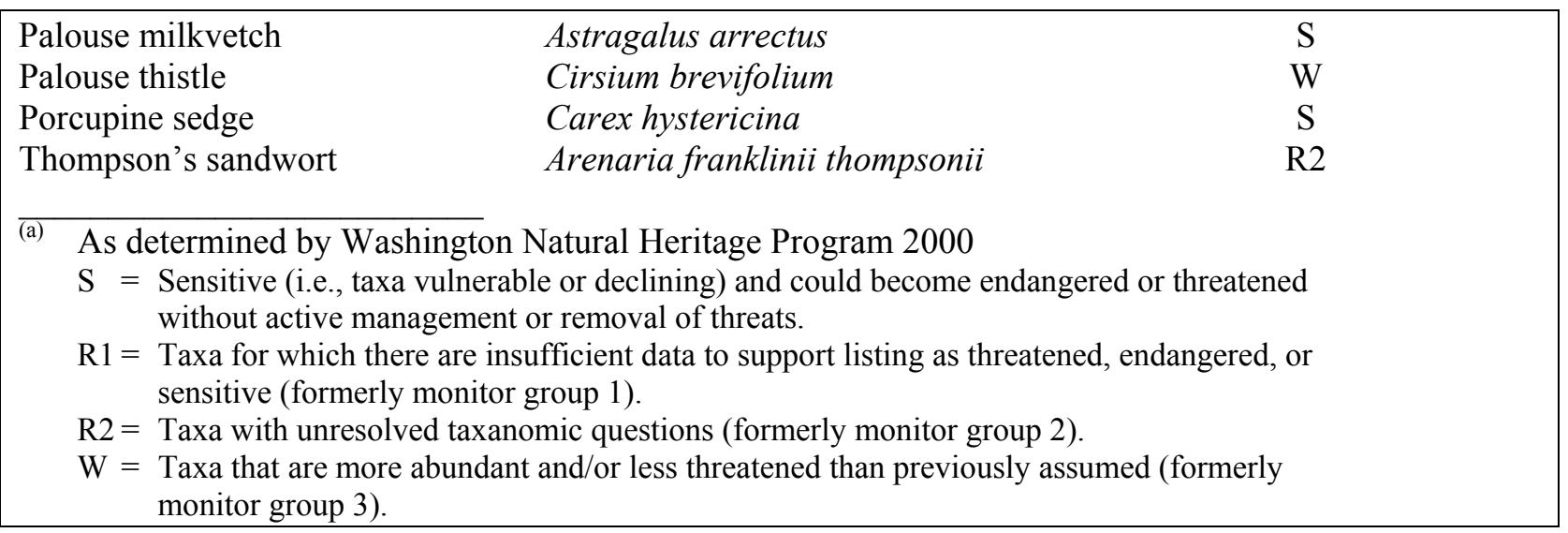

Several federal or state listed species have been reported on very rare instances on or near the Hanford Site. A banded Aleutian Canada goose was harvested in Franklin County in prior years. The bull trout, a state candidate species and federal threatened species, has been reported in the Hanford Reach, but its natural habitat is mountain streams; anecdotal accounts of bull trout in the Hanford Reach are likely individuals moved downstream during the spring freshet. Peregrine falcons occasionally are seen on the Hanford Site during migration, but are no longer listed as a state endangered species. The pygmy rabbit has been reported as residing on the ALE Reserve (Fitzner and Gray 1991). However, this observation is based on only one reported sighting in 1979. Their presence on the Hanford Site is unlikely, and has not been documented with additional sightings or physical evidence since that time despite intensive surveys.

There are several Washington State candidate species that have been reported on the Hanford Site (Table 4.4-3). The Columbia River tiger beetle is most likely extirpated from the Hanford Site, as the existing pattern of river flow regulation is not conducive to the insect's breeding behavior. Decline of steppe habitat statewide has resulted in the designation of black-tailed and white-tailed jackrabbits as state candidate species. Any number of species capable of flight could inadvertently be found onsite. Two candidate butterflies, the juniper hairstreak and the silver bordered bog fritillary, have been sighted in areas close to Hanford, but have never been observed on the Site. Similarly, Townsend's big eared bat has not been observed on the Hanford Site, but could migrate to the Site. Surveys of likely roosting areas in the 100 Area buildings have not documented its presence to date. Two candidate birds, the flammulated owl and Lewis' woodpecker, have been observed onsite but are considered rare visitors rather than resident species. 
Table 4.4-3. Washington State Candidate Animal Species Occurring on the Hanford Site

\begin{tabular}{|c|c|}
\hline Common Name & Scientific Name \\
\hline \multicolumn{2}{|l|}{ Molluses } \\
\hline Giant Columbia River spire snail $^{(\mathrm{a})}$ & Fluminicola (= Lithoglyphus) columbiana \\
\hline Giant Columbia River limpet & Fisherola $(=$ Lanx $)$ nuttalli \\
\hline \multicolumn{2}{|l|}{ Fish } \\
\hline$\overline{\text { Spring-run chinook }}{ }^{(b)}$ & Oncorhynchus tshawytscha \\
\hline Steelhead ${ }^{(b)}$ & Oncorhynchus mykiss \\
\hline \multicolumn{2}{|l|}{ Insects } \\
\hline Columbia River tiger beetle ${ }^{(c)}$ & Cicindela columbica \\
\hline \multicolumn{2}{|l|}{$\underline{\text { Birds }}$} \\
\hline Burrowing owl $^{(a)}$ & Athene cunicularia \\
\hline Golden eagle & Aquila chrysaetos \\
\hline Loggerhead shrike ${ }^{(a)}$ & Lanius ludovicianus \\
\hline Merlin & Falco columbarius \\
\hline Northern goshawk $^{(\mathrm{a}, \mathrm{d})}$ & Accipter gentilis \\
\hline Sage sparrow & Amphispiza belli \\
\hline Sage thrasher & Oreoscoptes montanus \\
\hline \multicolumn{2}{|l|}{ Reptiles } \\
\hline$\overline{\text { Striped whipsnake }}$ & Masticophis taeniatus \\
\hline \multicolumn{2}{|l|}{ Mammals } \\
\hline 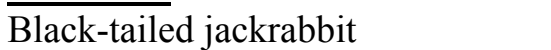 & Lepus californicus \\
\hline Merriam's shrew & Sorex merriami \\
\hline Washington ground squirrel ${ }^{(\mathrm{e})}$ & Spermophilus washingtoni \\
\hline White-tailed jackrabbit & Lepus townsendi \\
\hline \multicolumn{2}{|c|}{ Information from Washington Department of Fish and Wildlife ( http://www.wa.gov/wdfw/hab/) } \\
\hline \multicolumn{2}{|l|}{ (a) Federal species of concern. } \\
\hline \multicolumn{2}{|c|}{ (b) Federal endangered. } \\
\hline \multicolumn{2}{|c|}{ (c) Probable, but not observed on the Hanford Site. } \\
\hline \multicolumn{2}{|c|}{$\begin{array}{l}\text { (d) Reported, but seldom observed on the Hanford Site. } \\
\text { (e) Federal candidate. }\end{array}$} \\
\hline
\end{tabular}

\subsection{Cultural, Archaeological, and Historical Resources}

\section{E. L. Prendergast, D. W. Harvey, and T. O. Eschbach}

The Hanford Site is one of the richest cultural resource areas remaining in the western Columbia Plateau. The Site comprises a series of cultural landscapes containing the cumulative record of multiple occupations by both Native and non-Native Americans. These landscapes contain numerous wellpreserved archaeological sites representing prehistoric, ethnographic, and historic periods. Period resources include sites with cultural materials that are thousands of years old, traditional cultural places, and buildings and structures from the pre-Hanford, Manhattan Project, and Cold War eras. Sitewide management of Hanford's cultural resources follows the Hanford Cultural Resources Management Plan (DOE 2001a). Further information on the management of cultural resources as impacted by the National Monument land designation and the Memorandum of Understanding is located in Section 4.0. 
The Hanford Cultural Resources Laboratory (HCRL) holds records for approximately 1138 cultural resource sites and isolated finds as well as 531 buildings and structures that have been recorded on Washington State Historic Property Inventory forms. Of the 1138 recorded cultural resource sites, 121 have been evaluated for listing in the National Register of Historic Places (National Register). The remaining sites have not been evaluated.

Many sites were recorded during four archaeological reconnaissance projects conducted between 1926 and 1968 (Drucker 1948; Krieger 1928; Rice 1968a,b). Much of this early archaeological survey and reconnaissance activity concentrated on islands and on a strip of land approximately $400 \mathrm{~m}$ (1312 ft) wide on either side of the river (Rice 1980a). Reconnaissance of several project-specific areas and other selected locations conducted through the mid-1980s added to recorded Hanford Site inventories.

Systematic intensive archaeological surveys conducted from the mid-1980s through 1999 are responsible for much of the remainder of the inventories (Chatters 1989; Chatters and Cadoret 1990; Chatters and Gard 1992; Chatters et al. 1990, 1991, 1992; Last et al. 1993; Andrefsky et al. 1996). During his reconnaissance of the Hanford Site in 1968, Rice inspected portions of Gable Mountain, Gable Butte, Snively Canyon, Rattlesnake Mountain, and Rattlesnake Springs (Rice 1968b). Rice also inspected additional portions of Gable Mountain and part of Gable Butte in the late 1980s (Rice 1987). Other reconnaissance surveys include the Basalt Waste Isolation Project (BWIP), a proposed land exchange in T. 22 N., R. 27 E., Section 33 (Rice 1981), and three narrow transportation and utility corridors (ERTEC 1982; Morgan 1981; Smith et al. 1977). Other large-scale survey areas have been completed in recent years, including the 100 Areas from 1991 through 1993 (Chatters et al. 1992; Wright 1993), McGee Ranch (Gard and Poet 1992), the Laser Interferometer Gravitational Wave Observatory Project (O’Neil and Crist 1993), the Environmental Restoration Disposal Facility (Cadoret 1993), the 1995 WSU Archaeological Block Survey of the Hanford 600 Area (Andrefsky et al. 1996), and the Section 110 Vernita Survey ${ }^{(\mathrm{a}, \mathrm{b})}$. Over the past two years, Section 110 surveys have occurred predominantly in the 600 area of the Hanford Site. These include Rattlesnake Springs Archaeological Survey ${ }^{(c)}$, Dunes Survey ${ }^{(d)}$, White Bluffs Road Survey ${ }^{(\mathrm{e})}$, Gable Mountain Survey ${ }^{(\mathrm{f})}$, and West Vernita Cultural Resources Survey and Current Impacts Study ${ }^{(g)}$. The fire assessment also surveyed portions of the previously unsurveyed

\footnotetext{
(a) Unpublished report: Bard, J., and R. McClintock. August 16, 1998. Memorandum to Darby Stapp, PNNL. Block Survey Report: ca 8.26 sq. km (ca 2040 acres/3.19 sq miles) in the Hanford 600 Area near Vernita Bridge - Project 98-600-029. Copy on file at the Hanford Cultural Resources Laboratory, Pacific Northwest National Laboratory, Richland, Washington.

(b) Unpublished report: Hale, L.L., and R. McClintock. 1998. Cultural Resources Report Narrative \#98-0600-029, Vernita Block Survey. Report on file at the Hanford Cultural Resources Laboratory, Pacific Northwest National Laboratory, Richland, Washington.

(c) Unpublished report: Hale, L.L. September 1998. Cultural Resources Survey Narrative Report - Rattlesnake Springs Archaeological Survey (HCRC \#99-0600-001). Report prepared for the U.S. Department of Energy - Richland Operations. Copy on file at Pacific Northwest National Laboratory, Richland, Washington.

(d) Unpublished report: Hale, L.L. September 1999. Cultural Resources Survey Narrative Report - Dunes Archaeological Block Survey (HCRC \#99-0600-009). Report prepared for the U.S. Department of Energy - Richland Operations. Copy on file at Pacific Northwest National Laboratory, Richland, Washington.

(e) Unpublished report: Hale, L.L. September 2000. Cultural Resources Survey Narrative Report - White Bluffs Road Archaeological Survey (HCRC \#2000-0600-023). Report prepared for the U.S. Department of Energy - Richland Operations. Copy on file at Pacific Northwest National Laboratory, Richland, Washington.

(f) Unpublished report: Hale, L.L. September 2000. Cultural Resources Survey Narrative Report - Gable Mountain Block Survey (HCRC \#2000-0600-017). Report prepared for the U.S. Department of Energy - Richland Operations. Copy on file at Pacific Northwest National Laboratory, Richland, Washington.

${ }^{(g)}$ Unpublished report: Hazelbrook, R.E. September 2000. Draft Cultural Resources Survey Narrative Report - West Vernita Bridge Cultural Resources Survey and Current Impacts Report (HCRC \#2000-0600-019). Report prepared for the U.S. Department of Energy - Richland Operations. Copy on file at Pacific Northwest National Laboratory, Richland, Washington.
} 
lands in the 600 Area $^{(\text {a) }}$. To date, approximately $22 \%$ of the Hanford Site has been surveyed (Figure 4.5$1)$.

Evaluated cultural resource areas include 49 sites listed in the National Register (1 reactor building, 4 individual archaeological sites, and 44 archaeological sites in 6 archaeological districts) (Table 4.5-1).

Eleven individual archaeological sites and three historic districts comprised of 58 archaeological sites and 530 buildings/structures have been determined to be eligible for listing in the National Register (Table 4.5-2). In addition to the National Register sites and districts just described, 47 of Hanford's cultural resource sites (46 in three districts and one site) are listed in the Washington Heritage Register (Table 4.5-3). More information on sites listed and eligible for listing in the National Register and the Washington Heritage Register may be found by contacting the DOE Richland Operations Office, Hanford Cultural Resources Program.

The DOE identified a National Register-eligible Hanford Site Manhattan Project and Cold War Era Historic District that serves to organize and delineate the evaluation and mitigation of Hanford's plutonium production built environment (Table 4.5-2). Standards for evaluating and mitigating the built environment were established in accordance with National Register criteria, as well as historic contexts and themes associated with nuclear technology for national defense and non-military purposes, energy production, and human health and environmental protection. A programmatic agreement that addresses management of the built environment (buildings and structures) constructed during the Manhattan Project and Cold War periods was completed by the Department of Energy. The Advisory Council on Historic Preservation and Washington State Historic Preservation Officer accepted this programmatic agreement in 1996 (DOE 1996b).

Establishment of the Hanford Site Manhattan Project and Cold War Era Historic District resulted in the selection of 190 buildings, structures, and complexes as contributing properties within the historic district recommended for individual documentation. Certain property types, such as mobile trailers, modular buildings, storage tanks, towers, wells and structures with minimal or no visible surface manifestations, were exempt from the identification and evaluation requirements.

Approximately 900 buildings and structures were identified as either contributing properties with no individual documentation requirement (not selected for mitigation) or as non-contributing exempt properties, and will be documented in a DOE-maintained database (Marceau 1998).

Hanford Site projects that entail transfer or lease of property, disturbing ground, and/or altering or demolishing existing structures result in cultural resource reviews. These reviews ensure that prehistoric and historic sites, traditional use areas, and existing structures listed in or eligible for the National Register are considered before impacts by proposed projects. (For Manhattan Project/Cold War era properties, refer to Appendix A, Table A.5, Hanford Site Manhattan Project and Cold War Historic District Treatment Plan for the list of buildings/structures eligible for the National Register as contributing properties within the Historic District recommended for individual documentation. (Online report available at: http://www.hanford.gov/docs/r197-56/appa.htm\#table_a5 )(Marceau 1998).

\footnotetext{
${ }^{\text {(a) }}$ Unpublished report: Hale, L.L and Harvey, D.W. September 2000. Cultural Resources Report - FY01 Fire Assessment Task. (HCRC \#2001-0600-002). Report prepared for the U.S. Department of Energy - Richland Operations. Copy on file at Pacific Northwest National Laboratory, Richland, Washington.
} 


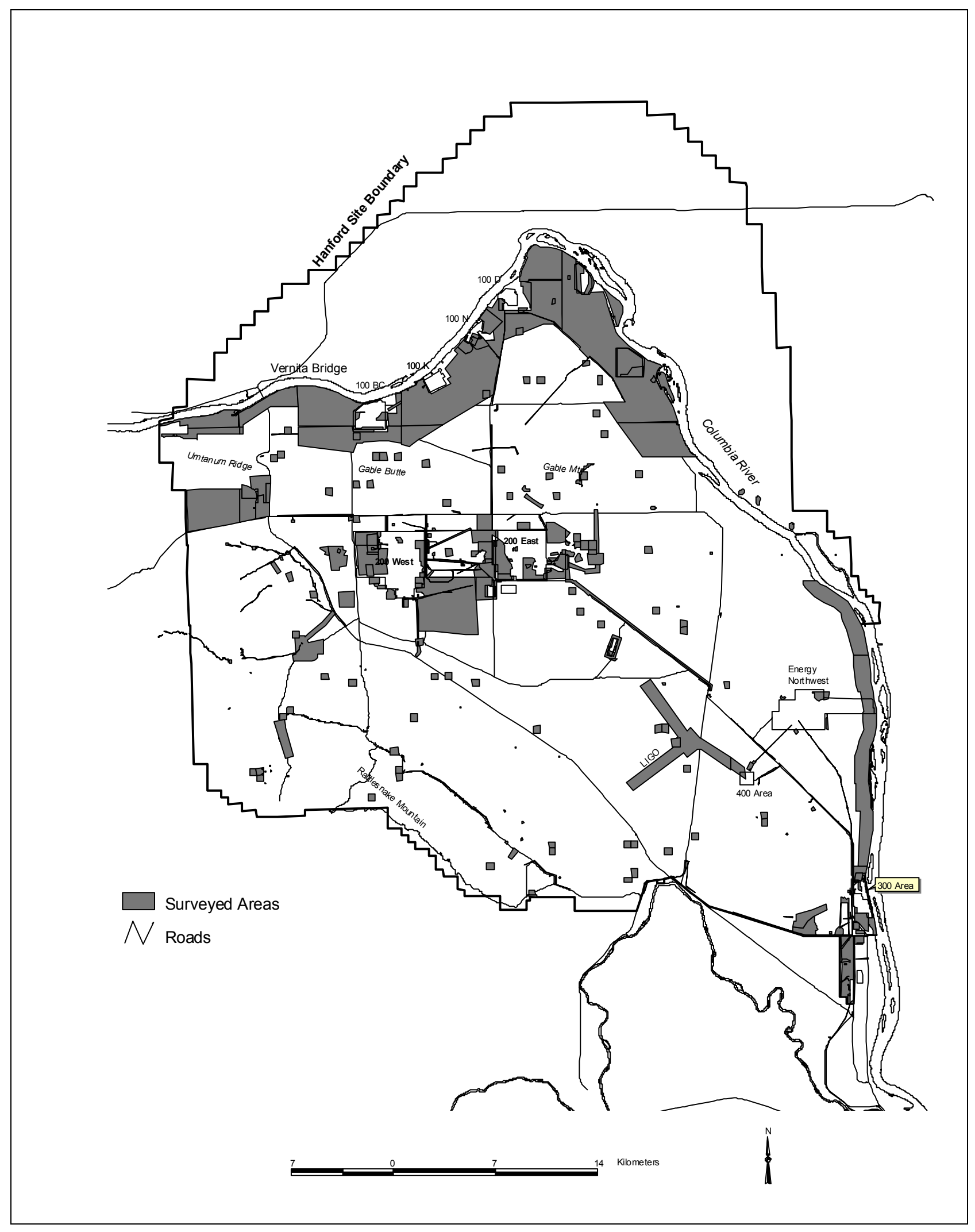

Figure 4.5-1. Cultural Resources Areas Surveyed on the Hanford Site 
Table 4.5-1. Historic Buildings, Archaeological Sites and Districts Listed in the National Register of Historic Places

\begin{tabular}{|ll|}
\hline Property Name & Date Listed by Keeper \\
\hline Districts: & \\
Hanford North Archaeological District & August 28, 1976 \\
Locke Island Archaeological District & August 28, 1976 \\
Ryegrass Archaeological District & January 31, 1976 \\
Savage Island Archaeological District & August 28, 1976 \\
Snively Canyon Archaeological District & August 28, 1976 \\
Wooded Island Archaeological District & July 19, 1976 \\
Sites: & \\
Hanford Island Archaeological Site & August 28, 1976 \\
Paris Archaeological Site & September 20, 1978 \\
Rattlesnake Springs Sites (2) & May 4, 1976 \\
Building: & \\
105-B Reactor & April 3, 1992 \\
& \\
\hline
\end{tabular}

\subsubsection{Native American Cultural Landscape}

Native Americans have lived in and around the present-day Hanford Site for thousands of years (Relander 1956; Spier 1936; Walker 1998). When Euro-Americans arrived in the 1800s, peoples presently referred to as the Wanapum inhabited villages and fishing camps. Neighboring groups known today as the Yakama, Umatilla, Cayuse, Walla Walla, Palus, Nez Perce, and Middle Columbia Salish frequented the area to trade, gather resources, and conduct other activities. Many descendants of these tribes and bands are affiliated with the Wanapum, Yakama Nation, Confederated Tribes of the Umatilla Reservation, Nez Perce Tribe, or the Confederated Tribes of the Colville Reservation, and they retain traditional, cultural, and religious ties to Hanford's places and resources. (See Section 6.5 for further information on the treaties associated with the Hanford Site).

\subsubsection{Traditional Cultural Places and Traditional Use Areas}

In 1990, the National Park Service developed the concept of traditional cultural property or traditional cultural place (TCP) as a means to identify and protect cultural landscapes, places, and objects that have special cultural significance to Native Americans and other ethnic groups (Parker and King 1990). A significant TCP is associated with "cultural practices or beliefs of a living community that are rooted in that community's history, and are important in maintaining the continuing cultural identity of the community" (Parker and King 1990).

The Hanford Reach and the greater Hanford Site, a geographic center for regional Native American religious belief, is central to the practice of Indian religion of the region, and many believe the creator made the first people here (DOI 1994). Indian religious leaders such as Smoholla, a prophet of Priest Rapids who brought the Washani religion to the Wanapum and others during the late $19^{\text {th }}$ century, began their teachings here. Native plant and animal foods, some of which can be found on the Hanford Site, are used in the ceremonies performed by tribal members. Certain landforms, especially Rattlesnake 
Table 4.5-2. Archaeological Sites and Historic Districts Determined Eligible for Listing in the National Register of Historic Places

\begin{tabular}{|ll|}
\hline Property Name & $\begin{array}{c}\text { Date Determined Eligible for Listing in the } \\
\text { National Register of Historic Places }\end{array}$ \\
\hline Districts: & \\
Gable Mountain Cultural District & February 12, 1990 \\
Hanford Site Manhattan Project and Cold War & August 21, 1996 \\
Era Historic District & \\
McGee Ranch/Cold Creek Valley District & December 23, 1994 \\
Archaeological Sites: & \\
HT-95-050 (Fry and Conforth Farm) & May 1, 1998 \\
H3-121 (White Bluffs Road) & January 6, 1994 \\
45BN423 & May 17, 1994 \\
45BN434 & May 31, 1995 \\
45BN446 & May 17, 1994 \\
45BN606 (HT-95-186) & December 6, 2000 \\
HT-94-028 & December 6, 1994 \\
HT-94-029 & December 6, 1994 \\
HT-94-030 & December 6, 1994 \\
HT-94-031 & December 6, 1994 \\
HT-99-007 & September 30, 2000 \\
(Hanford Atmospheric Dispersion Test Facility) & \\
Pre-Hanford Historic Structures: & \\
HT-95-231 (White Bluffs Bank) & \\
HT-98-039 (Bruggemann's Warehouse) & Pecembing 21, 2000 \\
\hline
\end{tabular}

Table 4.5-3. Archaeological Sites and Districts Listed in the Washington Heritage Register

\begin{tabular}{|ll|}
\hline \multicolumn{1}{|c|}{ Property Name } & \multicolumn{1}{c|}{$\begin{array}{c}\text { Date Listed by State Historic Preservation } \\
\text { Officer }\end{array}$} \\
\hline Districts: & \\
Coyote Rapids Archaeological District & May 23, 1975 \\
Hanford South Archaeological District & August 26, 1983 \\
Wahluke Archaeological District & May 23, 1975 \\
Site: & \\
Gable Mountain Archaeological Site & November 15, 1974 \\
\hline
\end{tabular}


Mountain, Gable Mountain, Gable Butte, and various sites along and including the Columbia River, remain sacred to them. The Gable Mountain Block Survey conducted by tribal members in 2000, recorded important attributes that contribute to the significance of Gable Mountain to Native Americans.

Native American traditional cultural places within the Hanford Site include but are not limited to a wide variety of places and landscapes: archaeological sites, cemeteries, trails and pathways, campsites and villages, fisheries, hunting grounds, plant gathering areas, holy lands, landmarks, important places in Indian history and culture, places of persistence and resistance, and landscapes of the heart (Bard 1997). Traditional cultural places of importance to Native Americans are determined through methods that are mutually agreed upon by DOE and the Native American community.

A historic context for the Prehistoric and Ethnographic/Contact Periods of the Hanford Site has been prepared as part of a National Register Multiple Property Documentation form to assist with the evaluation of the National Register eligibility of Native American ethnographic resources (Online report available at: http://www.hanford.gov/doe/culres/mpd/sec3.htm\#3.0)(DOE 1997c).

\subsubsection{Archaeological Resources}

People have inhabited the Middle Columbia River region since the end of the glacial period. More than 8000 years of prehistoric human activity in this largely arid environment have left extensive archaeological deposits along the river shores (Chatters 1989; Greengo 1982; Leonhardy and Rice 1970). Well-watered areas inland from the river also show evidence of concentrated human activity (Chatters 1982, 1989; Daugherty 1952; Greene 1975; Leonhardy and Rice 1970; Rice 1980a), and recent surveys have indicated extensive, although dispersed, use of arid lowlands for hunting. Throughout most of the region, hydroelectric development, agricultural activities, and domestic and industrial construction have destroyed or covered the majority of these deposits. Amateur artifact collectors have had an immeasurable impact on what remains at numerous sites. However, by virtue of their inclusion in the Hanford Site from which the public is restricted, archaeological deposits found in the Hanford Reach of the Columbia River and on adjacent plateaus and mountains have not been destroyed.

Four hundred thirty-nine archaeological sites and isolated finds associated with the prehistoric period have been recorded on Hanford; of these, approximately 68 contain historic components as well. Prehistoric period sites common to the Hanford Site include remains of numerous pit house villages, various types of open campsites, spirit quest monuments (rock cairns), hunting camps, game drive complexes, and quarries in nearby mountains and rocky bluffs (Rice 1968a,b; Rice 1980a); hunting/kill sites in lowland stabilized dunes; and small temporary camps near perennial sources of water located away from the river (Rice 1968b).

A historic context for the Prehistoric Period of the Hanford Site has been prepared as part of a National Register Multiple Property Documentation form to assist with the evaluation of the National Register eligibility of prehistoric archaeological resources (Online report available at: http://www.hanford.gov/doe/culres/mpd/sec2.htm\#2.0)(DOE 1997c).

\subsubsection{Non-Native American Cultural Landscape}

\subsubsection{Archaeological Resources}

Some of the first Euro-Americans who passed nearest the Hanford Site were Lewis and Clark, who traveled along the Columbia and Snake rivers during their 1803 to 1806 exploration of the Louisiana Territory. The first European explorer to cross the Hanford Site was David Thompson, who traveled 
along the Columbia River from Canada during his 1811 exploration of the Columbia River. Other visitors included fur trappers, military units, and miners who traveled through the Hanford Site on their way to lands up and down the Columbia River and across the Columbia Basin. It was not until the 1860s that merchants set up stores, a freight depot, and the White Bluffs Ferry on the Hanford Reach. Chinese miners soon began to work the gravel bars for gold. Cattle ranches were established in the 1880s, and farmers soon followed. Agricultural development, irrigation districts, and roads soon dotted the landscape, particularly in the eastern portion of the central Hanford Site. Several small thriving towns, including Hanford, White Bluffs, Richland, and Ringold, grew up along the riverbanks in the early 20th century. The communities' accessibility to outside markets grew with the arrival in 1913, of the Chicago, Milwaukee, St. Paul Railroad branch line (Priest Rapids-Hanford Line) from Beverly, Washington. Ferries were established at Richland, Hanford, Wahluke, and Richmond. The towns and nearly all other structures were razed in the years after the U.S. government acquired the land for the Hanford Engineer Works in 1943 (Chatters 1989; ERTEC 1981; Rice 1980a).

Six hundred fifty-five historic archaeological sites associated with the pre-Hanford (Euro-American) era and the Cold War era, including an assortment of farmsteads, corrals, dumps, and military sites, have been recorded by the HCRL since 1987. Fifty-six of these sites contain prehistoric components as well. Archaeological resources from the pre-Hanford period are scattered over the entire Hanford Site and include numerous areas of gold mining features along the riverbanks of the Columbia and remains of homesteads, building foundations, agricultural equipment and fields, ranches, and irrigation features. Properties from this period include former semi-subterranean structures near McGee Ranch; the Hanford Irrigation Ditch; former Hanford Townsite; Wahluke Ferry; White Bluffs Townsite; Richmond Ferry; Arrowsmith Townsite; White Bluffs road; and Chicago, Milwaukee, St. Paul Railroad (Priest RapidsHanford Line) and associated stops.

Historic archaeological military sites associated with the Cold War era are scattered throughout the Hanford Site's 600 Area. These archaeological resources are mainly located within the former Camp Hanford forward positions, the 16 anti-aircraft artillery sites that encircled the 100 and 200 Areas, and the three Nike missile installations on Wahluke Slope. (A fourth Nike position, in relatively intact condition, is located at the base of Rattlesnake Mountain on ALE.) The Nike position on ALE has been determined eligible for inclusion in the National Register as a contributing property within the Hanford Site Manhattan Project and Cold War Era Historic District. Five of the 16 anti-aircraft artillery sites have also been determined eligible for the National Register.

The anti-aircraft artillery and Nike sites were strategic components in Camp Hanford's military defense of the Site's plutonium production facilities during the 1950s. Potential archeological resources at these sites include former gun emplacements, launch and radar sites, concrete foundations and pads, pathways/sidewalks, and associated dumpsites, small arms firing ranges, and ammunition caches.

\subsubsection{Traditional Cultural Places}

Euro-American traditional cultural places found on the Hanford Site include structures and places that are important to descendents of pre-1943 settlers in the former White Bluffs, Hanford, Allard, Fruitvale, Vernita, and Cold Springs areas. These places are deeply rooted in the memories of local residents and include but are not limited to a former cemetery, numerous former homesites and townsites, orchards, fields, and places of former community activities, e.g., Hanford Grange Hall, churches, and schools. Former residents visit these areas annually with friends and family. 
A historic context for the Euro-American Resettlement Period (pre-Hanford era) has been prepared as part of a National Register Multiple Property Documentation form to assist with the evaluation of the National Register eligibility of historic archaeological resources, traditional cultural places, and traditional use areas (Online report available at: http://www.hanford.gov/doe/culres/mpd/sec4.htm\#4.0)(DOE 1997c).

\subsubsection{Historic Built Environment}

A number of buildings associated with the pre-Hanford Site era have been documented. They include the Hanford Irrigation and Power Company's pumping plant at Coyote Rapids, the high school and the electrical substation at the Hanford Townsite, White Bluffs bank, Bruggemann's fruit warehouse, and the blacksmith cabin at the East White Bluffs ferry landing.

Historic built resources documented from the Manhattan Project and Cold War eras include buildings and structures found in the 100, 200, 300, 400, 600, and 700 Areas. The most important of these are the plutonium production and test reactors, chemical separation and plutonium finishing buildings, and fuel fabrication/manufacturing facilities. The first reactors, 105-B, 105-D, and 105-F, were constructed during the Manhattan Project. Plutonium for the first atomic explosion and the bomb that destroyed Nagasaki to end World War II were produced at the Hanford Site. Additional reactors and processing facilities were constructed after World War II during the Cold War period. All reactor containment buildings still stand, although many ancillary structures have been removed, and C, DR, and F Reactors have been considerably modified.

DOE-RL will consider the retention of National Register-eligible buildings and structures that may qualify for adaptive reuse as interpretive centers, museums, industrial, or manufacturing facilities.

Historic contexts were completed for the Manhattan Project and Cold War eras as part of a National Register Multiple Property Documentation Form prepared for the Hanford Site to assist with the evaluation of National Register eligibility of buildings and structures sitewide (Online report available at: http://www.hanford.gov/doe/culres/mpd/sec4.htm\#5.0 )(DOE 1997c).

Additionally, historical narratives and individual building documentations have been completed for the draft History of the Plutonium Production Facilities at the Hanford Site Historic District, 1943-1990 and have been placed on the internet for public review and comment at www.hanford.gov/docs/rl-971047/index.htm. Five hundred, twenty-eight Manhattan Project and Cold War era buildings/structures and complexes have been determined eligible for the National Register as contributing properties within the Historic District. Of that number, 190 were recommended for individual documentation. DOE/RL is in the process of undertaking an assessment of the contents of the contributing buildings and structures to locate and identify any Manhattan and Cold War era artifacts that may have interpretive or educational value for museum exhibit purposes (see Appendix A, Table A.5, Hanford Site Manhattan Project and Cold War Era Historic Treatment Plan) (Marceau 1998).

\subsubsection{Cultural, Archaeological, and Historical Resources Impacts from the 24 Command Wildland Fire}

The HCRL along with the Confederated Tribes of the Umatilla Indian Reservation, the Nez Perce Tribe, the Yakama Nation, and the Wanapum Band conducted an assessment of the cultural, archaeological, and historical resources impacts as a result of the June 27 - July 2, 2000, 24 Command Fire that consumed over $655 \mathrm{~km}^{2}\left(250 \mathrm{mi}^{2}\right)$ of federal, state, and private lands on and near the Hanford Site. 
In most areas, the burn severity was low, and only minor wind erosion has occurred. However, west of the 200 West Area burn severity was high, and major wind erosion has occurred. The heaviest impacts were noted along the White Bluffs Road. Other impacts of the 24 Command Fire noted during the assessment include recreational impacts from dirt bikes near the ethnographic village of Wanawish across from Horn Rapids Dam within the Hanford Site; vehicle tracks and ruts near the 6652 Nike Missile launch installation and several Anti-Aircraft Artillery (AAA) installations; and bulldozer tracks along Highway 4 south. Only one site was recorded in the bulldozer tracks - a prehistoric lithic concentration. Bulldozer tracks were also noted on the western edge of 200 West, the western edge of Highway 4 southwest of the 200 East Area, and near the 6652 Nike Missile launch installation on the ALE Reserve. ${ }^{(a)}$ Vehicle and bulldozer tracks were the result of fighting the fire.

\subsubsection{Site Areas}

Cultural and historical resources are found in each of several areas on the Hanford Site, including the $100,200,300,400,600$, and 700 Areas. A brief synopsis of known resources found in these areas is presented in the following subsections.

\subsubsection{100 Areas}

Intensive field surveys were completed in the 100 Areas from 1991 to 1995 (Andrefsky et al. 1996; Chatters et al. 1992; Wright 1993). Much of the surface area within the 100 Area operable units has been disturbed by the industrial activities that have taken place during the past 50 years. However, Native American traditional use areas and traditional cultural properties as well as numerous prehistoric and historic archaeological sites have been encountered, and many are potentially eligible for the National Register. As remediation continues in the 100 Areas, the potential exists for inadvertent discoveries of either prehistoric or historic cultural resources. To understand impacts to cultural resources and to reduce the need to perform extensive reviews on highly disturbed areas, disturbance maps and reports have been completed for 100-B/C, 100-D/DR, and 100-F Areas. Contact the DOE, Richland Operations Office Hanford Cultural Resources Program for further information.

Nine plutonium production reactors and their ancillary and support facilities were located in the 100 Areas. The production reactors functioned to irradiate uranium fuel elements, the essential second step in the plutonium production process. A complete inventory of 100 Area buildings and structures was completed during FY 1995, and a National Register evaluation for each was finalized during 1996. To date, 146 buildings/structures have been inventoried in the 100 Areas. Of that number, 55 have been determined eligible for the National Register as contributing properties within the Historic District recommended for individual documentation (Marceau 1998).

100-B/C Area. Three archaeological sites can be identified from area literature (Rice 1968a; Rice 1980a,b); all lie partially within the 100-B/C Area. Thirty-five sites and isolated finds were recorded in the $\mathrm{B} / \mathrm{C}$ Area during archaeological surveys competed in 1995. The remains of Haven Station, a small stop on the former Chicago, Milwaukee, and St. Paul Railroad, is located to the west of the reactor compound. One archaeological site and the remains of the small community of Haven lie on the opposite bank of the Columbia River. Many sites related to hunting and religious activities are located at the westend of Gable Butte, due south of the 100-B/C Area. These sites are part of the proposed Gable Mountain/Gable Butte Cultural District nomination.

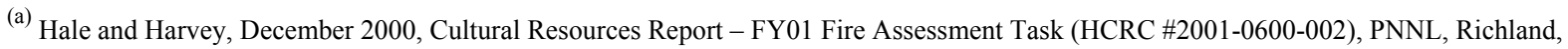
Washington.
} 
Two archaeological sites located near 100-B/C have been investigated. Test excavations conducted in 1991 at one hunting site revealed large quantities of deer and mountain sheep bone and projectile points dating from 500 to 1500 years old. The second archaeological site is considered to be eligible for listing in the National Register, in part, because it may contain new information about the Frenchman Springs and Cayuse Phases of prehistory.

The former Hanford Irrigation and Power Company pumping plant built near Coyote Rapids in 1908 is located east of the 100-B/C Area. The Hanford Irrigation Ditch, which carried water from the pumping plant to the former Hanford and White Bluffs townsites, is located adjacent and south of the plant.

The 105-B Reactor was the world's first full-scale plutonium production reactor and is designated as a National Historic Mechanical Engineering Landmark. It is also listed in the National Register, was recently named as a National Civil Engineering Landmark, and was given the Nuclear Historic Landmark Award. Historic American Engineering Record documentation of B Reactor was completed in 1999. A total of 14 buildings and structures within the reactor compound have been recorded on historic property inventory forms. Of that number, 10 properties have been determined eligible for the National Register as contributing properties within the Historic District recommended for individual documentation. These include 105-B Reactor, 181-B River Pumphouse, 104-B-1 Tritium Vault, 104-B-2 Tritium Laboratory, 105-B-Rod Tip Cave, 116-B Reactor Exhaust Stack, 117-B Exhaust Air Filter Building, 118-B-1 Solid Waste Burial Trench, and 182-B Reservoir and Pumphouse (Marceau 1998).

An assessment of the contents of 105-B was conducted to locate and identify Manhattan Project and Cold War era artifacts that may have interpretive or educational value in potential exhibits. Thirty-nine industrial artifacts were identified and tagged, located mainly in the fuel basin, exhaust fan room, and supply room. For the time being, these artifacts will be retained in place.

100-D/DR Area. One hundred and six known archaeological sites lie within $2 \mathrm{~km}(1.2 \mathrm{mi})$ of the $100-\mathrm{D} / \mathrm{DR}$ Reactor compound, three on the northern bank and the remainder on the southern bank of the Columbia River. The Wahluke Archaeological District is located north of the reactor compound area. Twenty-seven sites located south of the reactor compound may potentially be eligible for the National Register because of their association with a traditional cultural property. Most remaining sites represent early Euro-American settlement activities. The former community of Wahluke, which was at the landing of a ferry of the same name, is situated on the river's north bank.

All the buildings and structures in the 100-D/DR Area were built during the Manhattan Project and Cold War eras. Twenty buildings/structures have been inventoried, including the 105-D and 105-DR Reactor buildings. Both reactors were determined eligible for the National Register as contributing properties within the Historic District, but were not recommended for individual documentation. An assessment of the contents of 105-D was conducted to locate and identify Manhattan Project and Cold War era artifacts that may have interpretive or educational value in potential exhibits. Twenty-four industrial artifacts were identified and tagged, from control panels and a reactor curtain to lunch tables, benches, tools, and signs. An assessment of the contents of 105-DR was conducted to locate and identify any Cold War era artifacts that may have interpretive or educational value in potential museum exhibits.

Ten industrial artifacts were identified and tagged, which included a radiological worker procedures poster, instrument ladder, three metal signs, a lead sampling chamber "pig", control panel, vintage ceiling lights and graphite blocks. The 185/189-D buildings and adjoining facilities, all part of the 190-D complex, have been determined eligible for the National Register and were documented to Historic American Engineering Record standards (Marceau 1998). However, the 190-D Complex has been demolished. 
100-F Area. The 100-F Area is situated on a segment of the Columbia River that contains many cultural sites. According to Relander (1956), camps and villages of the Wanapum extended from the Old Hanford Townsite upstream to the former White Bluffs Townsite. Eighty-one archaeological sites have been recorded near the 100-F Area. Sites of particular importance include a site recently determined eligible to the National Register, a cemetery, a National Register site, and a site that appears to contain artifact deposits dating to at least 6000 years ago.

The principal historic site in the vicinity is the East White Bluffs ferry landing and former townsite. This location was the upriver terminus of shipping during the mid-19th century. It was at this point that supplies for trappers, traders, and miners were off-loaded, and commodities from the interior were transferred from pack trains and wagons to riverboats. The first store and ferry of the Mid-Columbia region were located at the ferry landing (ERTEC 1981). A log cabin, thought by some to have been a blacksmith shop in the mid-19th century, still stands there. Test excavations conducted at the cabin by the University of Idaho revealed historic and prehistoric cultural materials. The structure has been recorded according to standards of the Historic American Buildings Survey (Rice 1976). While a formal Determination of National Register eligibility for the East White Bluffs cabin has not been completed by the DOE-RL and USFWS and submitted to the State Historic Preservation Officer (SHPO) for concurrence, the two agencies are exploring options for restoration of the structure. The only remaining structure associated with the White Bluffs Townsite (near the railroad) is the White Bluffs bank. A restoration feasibility study was conducted of the White Bluffs bank that outlined emergency stabilization and restoration options and costs. A committee comprised of members of the interested public and staff of DOE-RL, PNNL and Bechtel Hanford, Inc., has been established to explore stabilization and restoration alternatives.

Three Manhattan Project/Cold War era buildings/structures have been inventoried in this area, including the 105-F Reactor building. An assessment of the contents of 105-F was conducted to identify any artifacts that may have value as potential museum exhibits. Eleven industrial artifacts were identified and tagged, which included a fuel scale, elevator control panel, two shop signs, four safety signs, hardhat, graphite blocks and vintage ceiling lights.

100-H Area. As of 2000, there have been 40 archaeological sites recorded within $2 \mathrm{~km}(1.2 \mathrm{mi})$ of the 100-H area. Included in this group are two historic Wanapum cemeteries, six camps (one with an associated cemetery), and three housepit villages. The largest village contains approximately 100 housepits and numerous storage caches. It appears to have been occupied from 2500 years ago to historic times (Rice 1968a). The cemeteries, camps, and villages are included in the Locke Island Archaeological District.

Historic sites recorded during 1992, 1993, and 1995 include 20th century farmsteads, household dumps, and military encampments. None have yet been evaluated for eligibility to the National Register.

Four Cold War era buildings/structures were inventoried in the 100-H Area. Of that number, only the 105-H Reactor was determined eligible for the National Register as a contributing property within the Historic District. The reactor, however, was not recommended for individual documentation (Marceau 1998). An assessment of the contents of $105-\mathrm{H}$ was conducted to locate and identify Cold War era artifacts that may have interpretive or educational value in potential exhibits. One artifact was identified and tagged - the "Blue Lady" drawing located on the air plenum.

100-K Area. Events took place at this locality in the mid-19th century that were of great significance to Native American people in the interior Northwest (Relander 1956). The origin of the Washani religion (also known as Seven Drums or Dreamer religion) began in this area, spreading to many neighboring tribes. 
An archaeological survey of the 100-K Area in 1991 revealed five previously unrecorded archaeological sites. Archaeological surveys conducted during 1995 of areas not surveyed in 1991 resulted in documentation of 31 additional prehistoric and historic sites. Two of these sites are believed to date to the Cascade Phase (9000 to 4000 years ago). More importantly, a group of pithouses with an associated long house and sweat lodge were identified that may have been the site of Smohalla's first Washat dance. Coyote Rapids, which is a short distance upstream, was called Moon, or Water Swirl Place. Two National Register Districts are located near the 100-K Area, the Coyote Rapids Archaeological District and the Ryegrass Archaeological District. Two individual archaeological sites near the 100-K Area have been determined to be eligible for listing in the National Register.

The Hanford Irrigation Ditch and the former Chicago, Milwaukee, and St. Paul Railroad, two important linear features are also present in the 100-K Area. Remnants of the Allard community and the Allard Pumphouse at Coyote Rapids are located west of the K Reactor compound. Historic farmstead sites are widely scattered throughout the nearby area.

Thirty-eight buildings/structures have been inventoried in the 100-K Reactor Area, including the 105$\mathrm{KE}$ and KW Reactor buildings. Of that number, 13 have been determined eligible for the National Register as contributing properties within the Historic District recommended for individual documentation. These include the 105-KW Reactor, 190-KW Main Pumphouse, 107-KW Retention Basin, 183-KW Filter Plant, and 181-KW River Pumphouse (Marceau 1998).

An assessment of the contents of $105-\mathrm{KE}$ and $\mathrm{KW}$ was conducted to identify any artifacts that may have educational or interpretive value as potential museum exhibits. Fourteen industrial artifacts were identified and tagged in 105-KE Reactor, including tools, signage, radiation monitor equipment, furniture, and a gas mask. Seven artifacts were identified and tagged from 105-KW Reactor, including furniture, a measurement scale, tools, and a floodlight. An assessment of the 109-KW Pumphouse was also conducted, and two artifacts were tagged, a phone booth with phone set and a wooden safety bulletin board.

100-N Area. Thirty-one archaeological sites have been recorded within $2 \mathrm{~km}(1.2 \mathrm{mi})$ of the $100-\mathrm{N}$ Area perimeter. Four of these sites are either listed, or considered eligible for listing, in the National Register. Three sites (two housepit villages and one cemetery) comprise the Ryegrass Archaeological District. Site 45BN179, once considered for a National Register nomination as the Hanford Generating Plant Site, has been found to be part of 45BN149, which is already listed in the National Register. Extant knowledge about the archaeology of the 100-N Area is based largely on reconnaissance-level archaeological surveys conducted during the late 1960s to late 1970s (Rice 1968b; see also Rice 1980a,b), which do not purport to produce complete inventories of the areas covered. Intensive surveys of areas surrounding 100-N were conducted during 1991 and 1995.

Three areas near the 100-N Area are known to have been of importance to the Wanapum. The knobs and kettles surrounding the area are called Mooli Mooli, which means Little Stacked Hills. Gable Mountain (called Nookshai or Otter) and Gable Butte, which lie to the south of the river, are sacred mountains where youths would go on overnight vigils seeking guardian spirits (Relander 1956). Sites of religious importance may also exist near the 100-N compound.

The most common evidence of pre-Hanford Site era activities now found near the 100-N Area consists of historic archaeological sites where farmhouses once stood and agricultural fields remain. The historic Hanford Ditch is adjacent to and south of the 100-N compound. 
Sixty-six Cold War era buildings and structures have been inventoried in the 100-N Area (Marceau 1998). The 100-N Reactor, completed in 1963, was the last of the plutonium production, graphitemoderated reactors. The design of $\mathrm{N}$ Reactor differed from the previous eight reactors in several ways to afford greater safety and to enable co-generation of electricity. Thirty $100-\mathrm{N}$ Area buildings/structures have been determined eligible for the National Register as contributing properties within the Historic District recommended for individual documentation. These include the 105-N Reactor, 109-N Heat Exchanger Building, 181-N River Water Pumphouse, 183-N Water Filter Plant, 184-N Plant Service Powerhouse, 185-N Export Powerhouse, and the 1112-N Guard Station (DOE 1997d).

An assessment of the contents of 185-N was conducted to locate and identify Cold War era artifacts that may have interpretive or educational value in potential exhibits. Six artifacts were identified and tagged, including control room panels, phone booths, a "hear-here" phone, metal cart, and a safety sign.

\subsubsection{200 Areas}

The HCRL conducted a comprehensive archaeological resources review for the fenced portions of the 200 Areas in 1987 and 1988. This review incorporated both an examination of the existing literature as well as "an intensive pedestrian survey of all undisturbed portions of the 200 East Area and a stratified random survey [of the undisturbed portions] of the 200 West Area" (Chatters and Cadoret 1990).

Two historic-archaeological sites (i.e., can and glass scatters), four isolated historic artifacts, one isolated cryptocrystalline flake, and an extensive linear feature (i.e., the White Bluffs Road) were the only materials greater than 50 years old discovered during the field survey. Only the White Bluffs Road, in its entirety, was determined eligible for listing in the National Register. This road, which passes diagonally southwest to northeast through the 200 West Area, originated as a Native American trail. It played a role in Euro-American immigration, development, agriculture, and Hanford Site operations. Segments of the White Bluffs Road that are located in the 200 West Area have been determined to be non-contributing. Such non-contributing segments of the White Bluffs Road are those that do not add to the historic significance of the road, but retain evidence of its contiguous bearing. The 2000 White Bluffs Road survey recorded an additional 54 historic isolated finds and 2 prehistoric isolated finds, as well as six can dump features.

The 200 Areas were the locations of the chemical separations (processing) plants and their ancillary and support facilities. The plants functioned to dissolve the irradiated fuel elements to separate out the plutonium, the essential third step in plutonium production. Historic property inventory forms have been completed for 72 buildings/structures in the 200 Area. Of that number, 58 have been determined eligible for the National Register as contributing properties within the Historic District recommended for individual documentation. These include the 202-A Purex Plant, 212-N Lag Storage Facility, 221-T Plant, 222-S Redox Plant, 225-B Encapsulation Building, 231-Z Plutonium Metallurgical Laboratory, 234-5Z Plutonium Finishing Plant, 236-Z Plutonium Reclamation Facility, 242-Z Water Treatment Facility, 282-E Pumphouse and Reservoir Building, 283-E Water Filtration Plant, and the 284-W Powerhouse and Steam Plant. The 232-Z Waste Incinerator Facility and the 233-S Plutonium Concentration Building, determined eligible for the National Register, have been documented to Historic American Engineering Record standards (Marceau 1998).

An assessment of the contents of six facilities in the Plutonium Finishing Plant complex was conducted during fiscal year 1998. These buildings/structures included the 234-5Z Plutonium Finishing Plant, 291-Z Exhaust Stack, 2704-Z Safeguards and Security Building, and the 2736-Z, ZA and ZB Plutonium Storage Facilities. Because of security/radiological exposure concerns and/or inaccessibility, a number of identified artifacts were not tagged. These included a radiation detection device, plutonium storage vaults, and a dry air glove box. In 234-5Z, the entire Remote Mechanical C line (gloveboxes) and 
control room, and the Remote Mechanical A line (gloveboxes) and control room, were identified and tagged. Ten additional Cold War era artifacts were identified and tagged as a result of a walk-through of the Analytical Laboratories in 234-5Z. An assessment was also conducted of the 2704-C Building in 200 East and three artifacts were identified but not tagged: classified documents vault, typology of "cans" poster, and vintage fluorescent light fixtures.

Thirty-two industrial artifacts were identified and tagged in chemical separations buildings located in 200 East and West. The following buildings were inspected for artifacts during the walkthroughs: 202A, 202-S, 221-T, 221-U, 224-U, 224-B, and 271-U. Types of artifacts selected included electrical equipment, control panels, tools, vintage lights, health and safety items, signage, and communications equipment.

\subsubsection{300 Area}

Much of the 300 Area has been highly disturbed by industrial activities. Five recorded archaeological sites, including campsites, housepits, and a historic trash scatter are located at least partially within the 300 Area; many more may be located in subsurface deposits. Twenty-seven archaeological sites and 13 isolated artifacts have been recorded within $2 \mathrm{~km}(1.2 \mathrm{mi})$ of the 300 Area fence. The historic archaeological sites contain debris scatters and roadbeds associated with farmsteads. One archaeological site has been tested and is recognized as eligible for listing in the National Register. Several archaeological sites in this area are in the Hanford South Archaeological District, which is listed in the Washington Heritage Register. Disturbance maps and reports have been prepared for the 300 Area. Contact the DOE, Richland Operations Office, Hanford Cultural Resources Program for further information.

One documented locality with great importance to the historic Wanapum is located near the 300 Area. Other areas near the 300 Area have been found to be of great importance to the Native Americans and are fenced.

The 300 Area, the location of the uranium fuel fabrication plants that manufactured fuel rods to be irradiated in the Hanford Site reactors, provided the first essential step in the plutonium production process. The 300 Area was also the location of most of the research and development laboratories. One hundred fifty-nine buildings/structures in the 300 Area have been documented on historic property inventory forms. Of that number, 47 buildings/structures have been determined eligible for the National Register as contributing properties within the Historic District recommended for individual documentation. This total includes the 305 Test Pile, 313 Fuels Fabrication Facility, 314 Metal Press/Extrusion Building, 318 High Temperature Lattice Test Reactor, 321 Separation Building, 325 Radiochemistry Laboratory, 333 Fuel Cladding Facility, 3706 Radiochemistry Laboratory, and the 3760 (former) Hanford Technical Library (Marceau 1998).

Assessments of the contents of former fuel manufacturing facilities in the 300 Area have been conducted including the 303-A Magazine Product Storage Building, 305 Test Pile, 306-W Materials Development Laboratory, 313 Fuels Fabrication Facility/Metal Fabrication Building, 314 Press Building, and the 333 Fuel Cladding Facility. The 11 Manhattan Project/Cold War era artifacts that were identified and tagged are mainly industrial in nature associated with the fuel manufacturing process. A second walkthrough of Building 333 resulted in an additional 12 artifacts being identified that included a selection of safety signs/posters, a control panel, a safety shower, protective worker clothes, and a sample uranium fuel element. An assessment of the contents of the 305 Test Pile resulted in the identification and tagging of six artifacts, which included a lead sampling chamber "pig", chairs, benches, safety sign, barbecue pit, and an electric shop supply cabinet. 
Other 300 Area buildings assessed include the 303-K Fresh Metal Storage Building, 304 Uranium Scrap Concentration Storage Facility, 324 Chemical Engineering Laboratory, 327 Post Irradiation Test Laboratory, 329 Biophysics Laboratory, 334 Chemical Handling Facility, 334-A Acid Pumphouse, 3701D (former) Hanford Patrol Building, 3707-G Change House, 3716 Fuels Manufacturing Storage/ Automotive Repair Shop, 3727 Classified Storage Facility, 3746 Radiological Physics Building, 3762 Technical Safety Building, and the 340 Waste Neutralization Complex. The artifacts identified and tagged in these facilities were Hanford Site Emergency Response Signs located in 3701-D and three artifacts identified and tagged in the 340 Building Complex. Two control panels were selected in the 340control room, and a Toledo scale was tagged in 340-B West. A facility model was identified in Building 324, a control panel and manipulator were identified in Building 327, and a neutron source drive assembly and lead cave were selected in Building 329.

\subsubsection{400 Area}

Most of the 400 Area has been so disrupted by construction activities that archaeologists surveying the site in 1978 were able to find only $0.12 \mathrm{~km}^{2}\left(0.047 \mathrm{mi}^{2}\right)$ that were undisturbed (Rice et al. 1978). They found no cultural resources in the undisturbed area. No archaeological sites are known to be located within $2 \mathrm{~km}(1.2 \mathrm{mi})$ of the 400 Area.

The 400 Area consists of the Fast Flux Test Facility (FFTF) complex. The 405 Reactor Containment Building includes a 400 megawatt, sodium-cooled test reactor designed primarily to test fuels and materials for advanced nuclear power plants. All the buildings and structures in the 400 Area were constructed during the Cold War era. Twenty-one building/structures have been recorded on historic property inventory forms. Of that number, six have been determined eligible for the National Register as contributing properties within the Historic District recommended for individual documentation. These include the 405 Reactor Containment Building, 436 Training Facility, 4621-W Auxiliary Equipment Facility, 4703 FFTF Control Building, 4710 Operation Support Building, and the 4790 Patrol Headquarters (Marceau 1998). An assessment of the contents of Building 427 was conducted to locate and identify Cold War era artifacts that may have interpretive or educational value in potential exhibits. Four artifacts were identified and tagged, including fuel assembly components.

\subsubsection{600 Area}

The 600 Area contains a diverse wealth of cultural resources and traditional cultural properties representing a full range of human activity across the Hanford Site. Project-driven surveys have been conducted throughout the area, but much of the 600 Area remains unsurveyed. Several National Register Districts are located within the 600 Area including the Hanford Archaeological Site, the Hanford North Archaeological District, the Paris Archaeological Site, Rattlesnake Springs Sites, Savage Island Archaeological District, Snively Basin Archaeological District, and the Wooded Island Archaeological District. The McGee Ranch/Cold Creek Valley District has been determined to be eligible for listing in the National Register, and the Gable Mountain Cultural District is pending nomination to the National Register. Areas of traditional cultural importance include Rattlesnake Mountain and foothills, the Columbia River, and Gable Mountain and Butte. Portions of the 600 Area were surveyed for the 2000 Fire Assessment. Five newly identified archaeological sites and two newly identified isolated finds were recorded. The fire consumed two historic structures at the 6652 Nike Missile launch installation: the 40foot wooden Crow's Nest and Observation Post and the concrete block Guard Shack. The fire destroyed all of the site's wooden light poles (except one), utility poles, and fence posts leaving only a few charred posts and boards remaining. The fire did not affect the main complex of historic buildings, constructed mainly of concrete. Five other 600 Area properties, 604 Yakima Patrol Checking Station, 604-A Sentry House, 607 Batch Plant, 618-10 Solid Waste Burial Trench, and the Hanford Site Railroad, have been 
determined eligible for the National Register as contributing properties within the Historic District recommended for individual documentation. Twenty-five railcars located at the $212-\mathrm{N}$ rail spur were designated Register-eligible as contributing features of the Hanford Site Railroad and recommended for mitigation. Documentation/mitigation of the 25 railcars was completed as an addendum (HCRC \#2000600-007) to the Hanford Site Railroad Expanded Historic Property Inventory Form (ExHPIF).

The 600 Area contains features that supplied more than one specific site area, including roads, railroads, and support structures. Former townsites, farmsteads, roads, and rail lines are widely scattered throughout the 600 Area. Pre-Hanford Site properties in the 600 Area include the former Hanford Townsite, high school, and electric substation; the former White Bluffs Townsite, ferry landing, East White Bluffs cabin, and White Bluffs bank building (which has been determined eligible to the National Register); the Chicago, Milwaukee and St. Paul Railroad line and associated whistle stops; Allard Pumphouse; and the Hanford Irrigation Ditch.

Fifteen Cold War era buildings/structures, including the underground missile storage facility, have been inventoried at the former 6652 Nike launch and control center in the Fitzner/Eberhardt ALE Reserve. The 622 Meteorological Complex, located near 200 West, includes seven inventoried properties. Both complexes have been determined eligible for the National Register as contributing properties within the Historic District recommended for mitigation. An assessment of the contents of $622-\mathrm{F}$ and the 6652 Nike site were conducted. No artifacts of interpretive or educational value were identified.

Five anti-aircraft artillery sites located in the 600 Area and associated with Camp Hanford's defense of the Hanford Site during the 1950s have been determined eligible for the National Register. The former Central Shops complex located in the 600 Area north of the 200 Areas was determined to be ineligible for the National Register (DOE 1997c). The burn severity of the 24 Command Fire upon the five antiaircraft artillery sites ranged from none, where sandbagged revetments and concrete pads and foundations were not affected, to high, where sandbagged revetments and wooden structures were completely consumed and ground surfaces were completely denuded of most vegetation. In a few limited areas, most notably at HT-94-032 on 200 East Hill, the fire had a positive effect by exposing several building foundations, pads, and sidewalks previously covered by thick vegetation.

Buildings 623 (Gable Mountain Relay Station) and 213 (Magazine/Waste Storage Vault) were originally designated as contributing properties with no individual documentation required. They were reevaluated and designated as contributing properties recommended for individual documentation.

The Hanford Atmospheric Dispersion Test Facility was identified/evaluated and selected as a contributing property recommended for individual documentation. Mitigation required the completion of an Expanded Historic Property Inventory Form for the Test Facility. Numerous artifacts were identified as having interpretive or educational value in potential exhibits. A selected, representative number of artifacts will be removed and curated at a later date.

A Request for Determination of Eligibility for the Bruggemann Agricultural Complex was completed and submitted by DOE to the Washington SHPO. Located approximately $3 \mathrm{mi}(1.6 \mathrm{~km})$ east of $100-\mathrm{B} / \mathrm{C}$, the Bruggemann Warehouse, a contributing feature of the complex built in 1922, was the focus of a restoration feasibility study that outlined stabilization and restoration efforts to assist the DOE in determining the future use of the former fruit warehouse. 


\subsubsection{700 Area}

The 700 Area was the location of the administrative functions of the early Hanford Site period. Most of the 700 Area has been highly disturbed by industrial activities. Of the seven Manhattan Project and Cold War era buildings/structures identified in this area, the 703 Administrative Building, 712 Records/Printing/Mail Office Facility, and the 748 Radiosurgery/Emergency Decontamination Facility have been determined eligible for listing in the National Register as contributing properties within the Historic District recommended for individual documentation (Marceau 1998).

\subsection{Socioeconomics}

\section{R. A. Fowler}

Activity on the Hanford Site plays a dominant role in the socioeconomics of the Tri-Cities and other parts of Benton and Franklin counties. The agricultural community also has a significant effect on the local economy. Any major changes in Hanford activity would potentially affect the Tri-Cities and other areas of Benton and Franklin counties.

\subsubsection{Local Economy}

Three major sectors have been the principal driving forces of the economy in the Tri-Cities since the early 1970s: 1) DOE and its contractors operating the Hanford Site; 2) Energy Northwest (formerly the Washington Public Power Supply System) in its construction and operation of nuclear power plants; and 3 ) the agricultural community, including a substantial food-processing component. With the exception of a minor amount of agricultural commodities sold to local-area consumers, the goods and services produced by these sectors are exported outside the Tri-Cities. In addition to the direct employment and payrolls, these major sectors also support a sizable number of jobs in the local economy through their procurement of equipment, supplies, and business services.

In addition to these three major employment sectors, three other components can be readily identified as contributors to the economic base of the Tri-Cities. The first of these, loosely termed "other major employers," includes the five major non-Hanford employers in the region. The second component is tourism. The Tri-Cities area has increased its convention business substantially in recent years as well as recreational travel. The final component in the economic base relates to the local purchasing power generated not from current employees, but from retired former employees. Government transfer payments, specifically retirement and disability insurance benefit payments, constitute a significant proportion of total spendable income in the local economy.

\subsubsection{DOE Contractors (Hanford)}

The Hanford Site is the largest single source of employment in the Tri-Cities. During fiscal year (FY) 2000, DOE Office of River Protection (ORP) and its prime contractors CH2M Hill Hanford Group, Inc. and Bechtel National, Inc.; DOE-RL and its prime contractors Fluor Hanford, Inc. (and its principal subcontractors); PNNL; Bechtel Hanford, Inc.; and the Hanford Environmental Health Foundation employed an average of 10,590 employees. Fiscal year 2000 year-end employment at Hanford was 10,870. In FY 1999, average employment was 10,290, compared to an average employment of 11,940 in 1996. The drop between FY 1996 and FY 1999 reflects both employment declines and reorganization of the DOE contractors under the Project Hanford Management Contract (PHMC), which was created in 1996. Under the PHMC, almost 2200 employees of the former management and operations contractor 
were moved into six "enterprise companies" and were no longer counted as official Hanford employees. The number of employees at Hanford is down considerably from a peak of 19,200 in FY 1994, but still represents nearly $12 \%$ of the total jobs in the economy $(89,500)$.

The impact of Hanford payrolls and other spending on the Tri-Cities economy is significant. A model created by PNNL indicates that in 1999 about 21,350 Tri-Cities jobs were supported directly or indirectly by the Hanford payroll, and about 6900 by procurements and affiliate company contracts, for a total of 28,250 jobs. This represents $32 \%$ of the non-farm jobs in the economy. About $35 \%$ of the non- farm wage and proprietor income in the economy ( $\$ 1.08$ billion out of $\$ 3.08$ billion) may depend directly or indirectly on Hanford payrolls and spending (DOE-RL 2000).

Based on employee residence records as of April 2001, 91\% of the direct employment of Hanford lives in Benton and Franklin counties. Approximately 74\% of Hanford employees reside in Richland, Pasco, or Kennewick. More than 36\% are Richland residents, 9\% are Pasco residents, and 29\% live in Kennewick. Residents of other areas of Benton and Franklin counties, including West Richland, Benton City, and Prosser, account for about $17 \%$ of total Hanford Site employment.

\subsubsection{Energy Northwest}

Although activity related to commercial nuclear power plant construction ceased with the completion of the WNP-2 reactor in 1983 (now named Columbia Generating Station), Energy Northwest continues to be a major employer in the Tri-Cities area. Headquarters personnel based in Richland oversee the operation of the Columbia Generating Station and perform a variety of functions related to Hanford Generating Project. Decommissioning of mothballed nuclear power plants (WNP-1 and WNP-3), which were never completed, began in 1995. In FY 1999, Energy Northwest employed around 29 people at the two plants, which was one-third of the 90 people who were employed in 1994 as a result of decommissioning activities. As part of an effort to reduce electricity production costs, Energy Northwest headquarters decreased the size of its workforce from over 1900 in 1994 to 1016 at the end of 1999 (Energy Northwest 2001). As part of a refueling and maintenance project, employment had grown to 1117 in April 2001.

\subsubsection{Agriculture}

In 1999, agricultural production in the bi-county area generated about 10,395 wage and salary jobs, or about $13 \%$ of the area's total employment, as represented by the employees covered by unemployment insurance (LMEA 2000a). Seasonal farm workers are not included in that total but are estimated by the U.S. Department of Labor (DOL) for the agricultural areas in the state of Washington. In 2000, there was an average of 5877 seasonal farm workers per month in Benton, Franklin, and Walla Walla counties, ranging from 1106 workers during the winter pruning season to 10,095 workers at the peak of harvest. An estimated average of 5432 seasonal workers were classified as local (ranging from 1102 to 10,074); an average of 30 were classified as intrastate (ranging from 0 to 172), and an average of 416 were classified as interstate (ranging from 0 to 1174). The weighted seasonal wage for 2000 ranged from $\$ 6.52 / \mathrm{hr}$ to $\$ 7.33 / \mathrm{hr}$, with an average wage of $\$ 6.82 / \mathrm{hr}$ (DOL 2000).

According to the U.S. Department of Commerce's Regional Economic Information System (REIS), about 2656 people were classified as farm proprietors in 1999. Farm proprietors' income, according to this same source, was estimated to be $\$ 18.5$ million (DOC 2000). 
The area's farms and ranches generate a sizable number of jobs in supporting activities, such as agricultural services (e.g., application of pesticides and fertilizers and irrigation system development) and wholesale trade (e.g. farm supply and equipment sales, and fruit packing). Although formally classified as a manufacturing activity, food processing is a natural extension of the farm sector. More than 20 food processors in Benton and Franklin counties produce such items as potato products, canned fruits, and vegetables, wine, and animal feed.

\subsubsection{Other Major Employers}

In 2000, the five largest non-Hanford Site employers employed approximately 5300 people in Benton and Franklin counties. These companies include: 1) Lamb Weston, which employed 1808; 2) Iowa Beef Processing Inc., which employed 1700; 3) Framatome ANP, Richland Inc. (formerly Siemens Power Corporation), which employed 750; 4) Boise Cascade Corporation, which employed 700, and 5) J.R. Simplot which employed 350. Both Boise Cascade and Iowa Beef are located in western Walla Walla County, but most of their workforce resides in the area (TRIDEC 2001).

\subsubsection{Tourism}

A significant rise in the number of visitors to the Tri-Cites over the last several years has resulted in tourism playing an increasing role in helping to diversify and stabilize the area economy. The Tri-Cities Visitors and Convention Bureau reported that 96,560 people attended conventions and sporting events, spending an estimated \$31.8 million in the Mid-Columbia in 2000. The number of people attending convention and group events has more than doubled since 1995 and more than tripled since 1991.

The importance of tourism is evidenced by the amount of money spent on local goods and services. Overall tourism expenditures in the Tri-Cities were roughly $\$ 204.7$ million in 1999 , up from $\$ 187.2$ million in 1998. Travel-generated employment in Benton and Franklin counties was about 4090 with an estimated \$44.7 million in payroll, up from an estimated 3180 employed and a \$37.1 million payroll in 1998. In addition, tourism generated $\$ 3.2$ million in local taxes and $\$ 14.4$ million in state taxes in 1999 (OTED 2000).

\subsubsection{Retirees}

Although Benton and Franklin counties have a relatively young population (approximately 53\% under the age of 35) 18,855 people over the age of 65 resided in Benton and Franklin counties in 2000. The portion of the total population 65 years and older in Benton and Franklin counties accounts for 9.8\% of the total population, which is below the $11.2 \%$ for the state of Washington (Census 2001a). This segment of the population supports the local economy on the basis of income received from government transfer payments and pensions, private pension benefits, and prior individual savings.

Although information on private pensions and savings is not available, data are available regarding the magnitude of government transfer payments. The U.S. Department of Commerce's REIS has estimated transfer payments by various programs at the county level. A summary of estimated major government transfer payments received by the residents of Benton and Franklin counties in 1999 is shown in Table 4.6-1. Nearly $45 \%$ of the payments are for retirement and disability insurance benefit payments, which provides over $\$ 263$ million of spendable income to the local economy. 
Table 4.6-1. Federal Government Transfer Payments in Benton and Franklin Counties, 1999 (millions of dollars) ${ }^{(a)}$

\begin{tabular}{|c|c|c|c|}
\hline Government Payments to Individuals & $\begin{array}{l}\text { Benton } \\
\text { County }\end{array}$ & $\begin{array}{c}\text { Franklin } \\
\text { County }\end{array}$ & Total \\
\hline Retirement \& disability insurance payments & 208.6 & 54.9 & 263.5 \\
\hline Medical payments & 140.0 & 75.2 & 215.2 \\
\hline Income maintenance benefit payments & 37.6 & 20.4 & 58.0 \\
\hline Unemployment insurance benefit payments & 25.0 & 13.5 & 38.5 \\
\hline Veterans benefit payments & 9.0 & 2.4 & 11.4 \\
\hline Federal education \& training assistance payments & 2.3 & 2.2 & 5.5 \\
\hline Other payments to individuals & 0.6 & 0.2 & 0.8 \\
\hline Total & 423.1 & 168.8 & 591.9 \\
\hline
\end{tabular}

\subsubsection{Employment and Income}

Nonagricultural employment in the Tri-Cities grew steadily from 1988 to 1994 . The total annual average employment fell in 1995 and 1996, but has grown every year since. In 2000, nonagricultural employment rose over 3\%. Table 4.6-2 provides a breakdown of nonagricultural wage and salary workers employed in Benton and Franklin counties in 1999 and 2000 (LMEA 2000a). There was an average of 76,200 jobs in the Tri-Cities in 2000, up approximately 2300 from 1999. Gains in employment ranged from 100 workers in the manufacturing and contract construction sectors to 900 in wholesale and retail trade, while the finance, insurance, and real estate sector lost about 100 workers (LMEA 2000b).

Three measures of area income are presented in this section: total personal income, per capita income, and median household income. Total personal income comprises all forms of income received by the populace, including wages, dividends, and other revenues. Per capita income is roughly equivalent to total personal income divided by the number of people residing in the area. Median household income is the point at which half of the households have an income greater than the median and half have less.

In 1999, the total personal income for Benton County was $\$ 3.4$ billion, Franklin County was $\$ 840$ million, and Washington was $\$ 174.9$ billion. Per capita income in 1999 for Benton County was $\$ 25,004$, Franklin County was $\$ 17,961$, and Washington was $\$ 30,380$ (DOC 2000). The preliminary estimate of median household income in 2000 for Benton County is $\$ 46,316$; Franklin County is estimated at $\$ 32,398$, and Washington is estimated at $\$ 50,152$ (OFM 2000a).

\subsubsection{Demography}

According to Census 2000, population totals for Benton and Franklin counties were 142,475 and 49,347, respectively (Census 2001a). Both Benton and Franklin counties grew at a faster pace than Washington as a whole in the $1990 \mathrm{~s}$. The population of Benton County grew $26.6 \%$, up from 112,560 in 1990. The population of Franklin County grew 31.7\%, up from 37,473 in 1990 (Census 2001a). 
Table 4.6-2. Nonagricultural Wage and Salary Workers in Benton and Franklin Counties, 1999 and $2000^{\text {(a) }}$

\begin{tabular}{|lccc|}
\hline \multicolumn{1}{|c}{ Industry } & $\begin{array}{c}\text { 1999 Annual } \\
\text { Average } \\
\text { (Revised) }\end{array}$ & $\begin{array}{c}\text { 2000 Annual } \\
\text { Average } \\
\text { (Preliminary) }\end{array}$ & $\begin{array}{c}\text { Change } \\
\text { 1998-1999 (\%) }\end{array}$ \\
\hline Nonagricultural wage and salary & 73,900 & 76,200 & 3.1 \\
workers & 6,100 & 6,200 & 1.6 \\
Manufacturing & 4,100 & 4,200 & 2.4 \\
Construction & 8,800 & 9,100 & 3.4 \\
Transportation and public & 16,700 & 17,600 & 5.4 \\
utilities & 2,400 & 2,300 & 4.2 \\
Wholesale and retail trade & 21,900 & 22,500 & 2.7 \\
Finance, insurance, and real & 14,000 & 14,200 & 1.4 \\
estate & & & \\
Services & & & \\
Government & & & \\
\hline (a) Source: Washington State Employment Security Department (LMEA 2000b). & \\
\hline
\end{tabular}

Within each county, census figures indicate the distribution of the Tri-Cities population by city as follows: Richland 38,708; Pasco 32,066; and Kennewick 54,693. The combined populations of Benton City, Prosser, and West Richland totaled 15,847 in 2000. The unincorporated population of Benton County was 33,227. In Franklin County, incorporated areas other than Pasco had a total population of 3595. The unincorporated population of Franklin County was 13,886 (Census 2001a).

The 2000 population figures by race and Hispanic origin indicate that in Benton and Franklin counties, Asians represent a lower proportion, and individuals of Hispanic origin represent a higher proportion of the racial distribution than those in the state of Washington. Countywide, Benton and Franklin counties exhibit varying racial distributions, as indicated by the data in Table 4.6-3.

In 2000, Benton and Franklin counties accounted for 3.3\% of Washington's population. The population demographics of Benton and Franklin counties are quite similar to those found within Washington. The population in Benton and Franklin counties under the age of 35 is $53.1 \%$, compared to $49.4 \%$ for Washington State. In general, the population of Benton and Franklin counties is somewhat younger than that of Washington. The 0 - to 14 -year old age group accounts for $25.6 \%$ of the total bicounty population as compared to $21.3 \%$ for Washington. In 2000, the 65 -year old and older age group constituted $9.8 \%$ of the population of Benton and Franklin counties compared to $11.2 \%$ for Washington (Census 2001a).

\subsubsection{Environmental Justice}

Executive Order 12898, "Federal Actions to Address Environmental Justice in Minority Populations and Low-income Populations," (59 FR 7629), directs federal agencies in the Executive Branch to consider environmental justice so that their programs will not have "...disproportionately high and adverse human health or environmental effects..." on minority and low-income populations. Executive Order 12898 further directed federal agencies to consider effects to "populations with differential patterns of subsistence consumption of fish and wildlife." The Executive Branch agencies also were directed 
Table 4.6-3. Population Estimates and Percentages by Race and Hispanic Origin, $2000^{\text {(a) }}$

\begin{tabular}{|c|c|c|c|c|c|c|}
\hline Subject & $\begin{array}{c}\text { WA } \\
\text { State }\end{array}$ & $\%$ & $\begin{array}{c}\text { Benton/ } \\
\text { Franklin }\end{array}$ & $\%$ & $\begin{array}{l}\text { Benton } \\
\text { County }\end{array}$ & $\begin{array}{c}\text { Franklin } \\
\text { County }\end{array}$ \\
\hline Total population & $5,894,121$ & $100.0 \%$ & 191,822 & $100.0 \%$ & 142,475 & 49,347 \\
\hline Single Race & $5,680,602$ & $96.4 \%$ & 185,948 & $96.9 \%$ & 138,646 & 47,302 \\
\hline White & $4,821,823$ & $81.8 \%$ & 153,432 & $80.0 \%$ & 122,879 & 30,553 \\
\hline Black or African American & 190,267 & $3.2 \%$ & 2,549 & $1.3 \%$ & 1,319 & 1,230 \\
\hline American Indian/Alaska Native & 93,301 & $1.6 \%$ & 1,527 & $0.8 \%$ & 1,165 & 362 \\
\hline Asian & 322,335 & $5.5 \%$ & 3,934 & $2.1 \%$ & 3,134 & 800 \\
\hline Native Hawaiian/Pacific Islander & 23,953 & $0.4 \%$ & 220 & $0.1 \%$ & 163 & 57 \\
\hline Other Race & 228,923 & $3.9 \%$ & 24,286 & $12.7 \%$ & 9,986 & 14,300 \\
\hline Two or More Races & 213,519 & $3.6 \%$ & 5,874 & $3.1 \%$ & 3,829 & 2,045 \\
\hline Hispanic Origin (of any race) ${ }^{(b)}$ & 441,509 & $7.5 \%$ & 40,838 & $21.3 \%$ & 17,806 & 23,032 \\
\hline \multicolumn{7}{|c|}{$\begin{array}{l}\text { (a) From Census 2000a - Population by Race and Hispanic Origin. } \\
\text { (b) Hispanic origin is not a racial category: it may be viewed as the ancestry, nationality group, lineage, or country of } \\
\text { birth of the person or person's parents or ancestors before arrival in the United States. Persons of Hispanic origin may be } \\
\text { of any race and are counted in the racial categories shown. }\end{array}$} \\
\hline
\end{tabular}

to develop plans for carrying out the order. The Council on Environmental Quality (CEQ) provided additional guidance later for integrating environmental justice (EJ) into the National Environmental Policy Act process in a December 1997 document, Environmental Justice Guidance under the National Environmental Policy Act (CEQ 1997).

Minority populations are defined as all nonwhite individuals, plus all individuals of Hispanic origin, as reported in the 2000 Census (Census 2001b). Low-income persons are defined as living in households that report an annual income less than the United States official poverty level, as reported by the Census Bureau. The poverty level varies by size and relationship of the members of the household. The year 2000 poverty level was $\$ 17,761$ for a family of four (Census 2001b). Nationally, in 1999, 29.9\% of all persons were minorities, and $11.8 \%$ of all persons lived in households that had incomes less than the poverty level (which was $\$ 17,029$ for a family of four in that year) (Census 2000a,b). The most recent available state and county area poverty estimates report that Washington had $10.3 \%$ of its population living in poverty in 1997, while Benton County and Franklin County had 9.3\% and 17.7\%, respectively (Census 2000c).

The year 2000 census data indicate that a total population of approximately 511,500 people resided within an $80-\mathrm{km}(50-\mathrm{mi})$ radius of the Hanford Site. Based on the 2000 census, the $80-\mathrm{km}(50-\mathrm{mi})$ area surrounding the Hanford Site had a total minority population of about 185,000. The ethnic composition of the minority population is primarily White Hispanic (24\%), self-designated "other" races (54\%), American Native (6\%), and two or more races (9\%). Asians and Pacific Islanders (4\%) and African American (3\%) make up the rest. The Hispanic population resides predominantly in Franklin, Yakima, Grant, and Adams counties. Native Americans within the $80-\mathrm{km}$ area reside primarily on the Yakama Reservation and upstream of the Hanford Site near the town of Beverly, Washington. 
Figure 4.6-1 shows the location of Census block groups from the 2000 Census that had either a majority of residents who were members of a minority group (racial minority or Hispanic), or whose percentage of residents belonging to any minority group was at least 20 percentage points greater than the corresponding percentage of the state population (Census 2001a,c).

The 1990 low-income population was approximately 70,440 or $17 \%$ of the total population residing in the $80-\mathrm{km}$ circle. Detailed area poverty statistics will not be available from the 2000 Census of Population until the summer of 2002. However, Table 4.6.4 shows the estimated numbers and percentages of people living below the poverty level in the counties touched by the $80-\mathrm{km}$ circle in Figure 4.6-2 for 1997 and 1990. The 1997 values are Census Bureau model-based estimates; therefore, the differences between 1989 and 1997 reflect differences in methods as well as actual changes in the incidence of poverty. Even so, it appears that the $80-\mathrm{km}$ region surrounding Hanford has a lower percentage of low-income persons than at the 1990 Census. The low-income population of the area is dispersed throughout this region with the highest concentrations occurring in Franklin, Yakima, Grant, and Adams counties. Figure 4.6-2 shows the location of Census block groups from the 1990 Census that had either a majority of residents who were low income (members of a household below the national poverty level), or a percentage of low income residents at least 20 percentage points greater than the corresponding percentage of the state population. Other unincorporated areas within 50 miles of the Hanford Site in Oregon and Washington with high proportions of Native Americans depend on salmon from the Columbia River and other natural resources in the Columbia Basin.

The CEQ guidance recognizes that many minority and low-income populations derive part of their sustenance from subsistence hunting, fishing, and gathering activities (sometimes for species unlike those consumed by the majority population) or are dependent on water supplies or other resources that are atypical or used at different rates than other groups. These differential patterns of resource use are to be identified where practical and appropriate. There are Native Americans of various tribal affiliations that live in the greater Columbia Basin that rely on natural resources for subsistence.

Table 4.6.4. Number and Percentages of Persons Living in Low-Income Households in Counties Near the Hanford Site, 1997 and 1989 (Census 1998a,b, 2000c,d)

\begin{tabular}{|c|c|c|c|c|}
\hline \multirow[b]{2}{*}{ Washington: } & \multicolumn{2}{|c|}{$\begin{array}{c}\text { Number of Persons in Low-Income } \\
\text { Households }\end{array}$} & \multicolumn{2}{|c|}{$\begin{array}{c}\text { Percentage of Persons in Low Income } \\
\text { Households }\end{array}$} \\
\hline & $\begin{array}{c}1997 \text { (Model- } \\
\text { Based Estimate) }\end{array}$ & $\begin{array}{l}1989(1990 \\
\text { Census })\end{array}$ & $\begin{array}{l}1997 \text { (Model- Based } \\
\text { Estimate) }\end{array}$ & $\begin{array}{c}1989(1990 \\
\text { Census })\end{array}$ \\
\hline Adams County & 2,388 & 2,360 & 15.4 & 17.5 \\
\hline Benton County & 12,859 & 12,402 & 9.3 & 11.1 \\
\hline Chelan County & 8,230 & 7,844 & 13.6 & 15.3 \\
\hline Columbia County & 514 & 757 & 12.5 & 19.4 \\
\hline Franklin County & 8,212 & 8,491 & 17.7 & 23.0 \\
\hline Grant County & 10,638 & 10,631 & 14.9 & 19.6 \\
\hline Kittitas County & 3,974 & 4,913 & 13.3 & 20.2 \\
\hline Klickitat County & 3,011 & 2,786 & 15.5 & 17.0 \\
\hline Walla Walla County & 7,220 & 7,144 & 14.5 & 16.0 \\
\hline Yakima County & 40,192 & 37,486 & 18.3 & 20.2 \\
\hline Oregon: & & & & \\
\hline Morrow County & 700 & 1,141 & 7.0 & 15.1 \\
\hline Umatilla County & 10,071 & 9,419 & 15.6 & 16.5 \\
\hline Union County & 3,437 & 3,627 & 13.9 & 15.8 \\
\hline Total & 111,446 & 109,001 & & \\
\hline
\end{tabular}


Asian, Black, Hispanic, Native American, Pacific Islander, Other Ethnic, and Overall Minority Populations

Gray denotes block groups with environmental justice concerns.

144 block groups highlighted
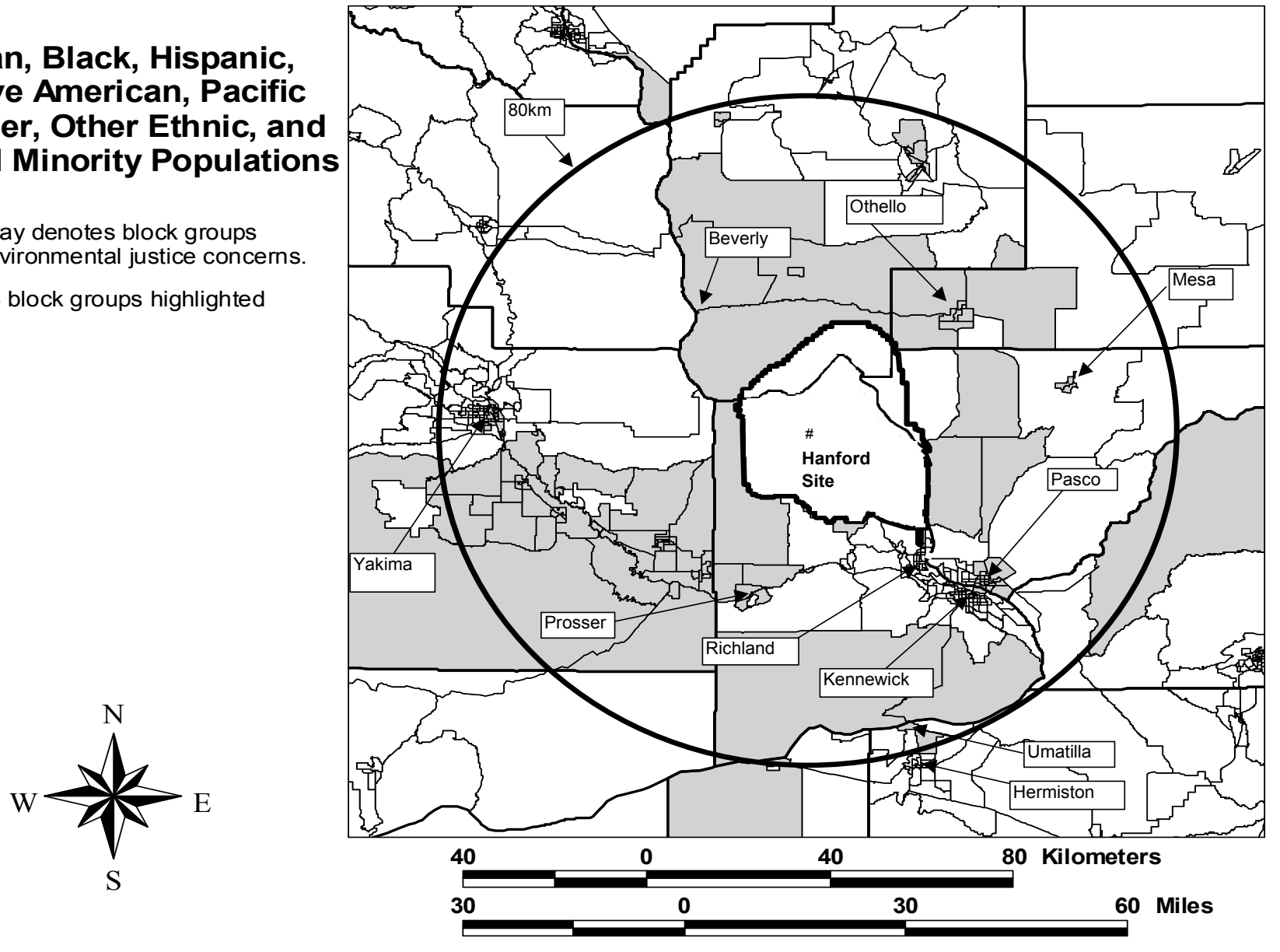

Figure 4.6-1. Location of Minority Populations Near the Hanford Site 


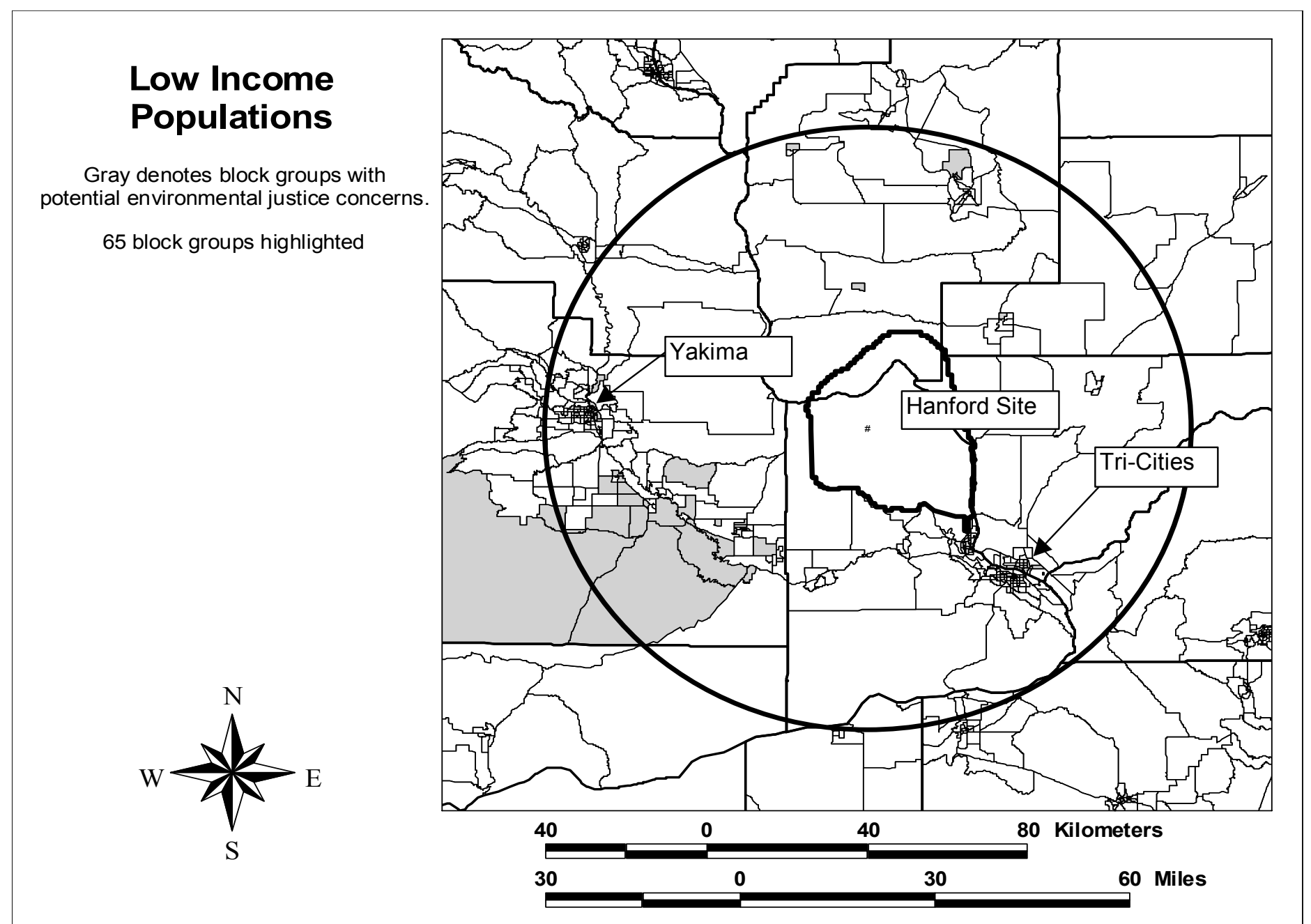

Figure 4.6-2. Location of Low-Income Populations Near the Hanford Site (Census 1991) 
There is dependence on natural resources for dietary subsistence for the Nez Perce Tribe, the Confederated Tribes of the Umatilla Indian Reservation, and the Yakama Nation (Harris and Harper 1997). The treaties of 1855 (DOE 1999a) maintain the rights of these tribes to fish, hunt, erect fishcuring structures, gather food, and graze stock in their usual and accustomed places on open/unclaimed portions of the lands ceded to the government. Some of this ceded territory is located on the Hanford Site. The Wanapum, a non-treaty tribe, historically lived on what is now the Hanford Site and continue to live adjacent to the Site. They fish on the Columbia River and gather food resources near the Hanford Site. The Confederated Tribes of the Colville Reservation, established by an Executive Order in 1872, traditionally fished and gathered food resources in the Hanford area. They are also recognized as having cultural and religious ties to the Hanford Site.

\subsubsection{Housing}

In FY 2000, 2195 houses were sold in the Tri-Cities at an average price of $\$ 128,928$, compared to 2176 houses sold at an average price of $\$ 123,428$ in 1999 (TCAR 2000). In FY 2000, 760 single-family houses were built, up 1.6\% from the 748 that were built in 1999, but down from a peak of 1117 in 1994 (WCRER 2000a).

As of April 1, 2000, there were 73,982 housing units in Benton and Franklin counties, 44,009 of which were single-units, 15,946 were two or more units, and 14,027 were manufactured homes. The number of total units has increased 26.4\% from 58,541 in 1990 (OFM 2000b). The number of apartments has increased from 8225 in 1990 to 10,226 in 2000. The vacancy rate of apartments in Benton and Franklin counties in September 2000 was $4.3 \%$, and the average rent was $\$ 530$. These figures are up from the 3.3\% vacancy rate and $\$ 489$ average rent in 1999 (WCRER 2000b).

\subsubsection{Transportation}

The Tri-Cities serves as a regional transportation and distribution center with major air, land, and river connections. The BNSF and Union Pacific provide direct rail service. Union Pacific operates the largest fleet of refrigerated rail cars in the United States and is essential to food processors, which ship frozen food from this area. Passenger rail service is provided by Amtrak, which has a station in Pasco. There is currently no rail service on the Hanford Site. Although the main Hanford rail line is still in place, it would require maintenance, upgrades, and trained crews to resume operation.

Docking facilities at the Ports of Benton, Kennewick, and Pasco are important aspects of this region's infrastructure. These facilities are located on the 525-km- (325.5-mi-) long commercial waterway, which includes the Snake and Columbia rivers, that extends from the Ports of Lewiston-Clarkston in Idaho to the deep-water ports of Portland, Oregon, and Vancouver, Washington. The average shipping time from the Tri-Cities to these deep-water ports by barge is $36 \mathrm{hr}$ (Evergreen Community Development Association 1986).

Daily air passenger and freight services connect the area with most major cities through the Tri-Cities Airport, located in Pasco. This modern commercial airport links the Tri-Cities to major hubs and access to destinations anywhere in the world. Delta Airlines, United Express, and Horizon Air offer 33 flights into and out of the Tri-Cities daily connecting to domestic and international flights through Salt Lake City, Seattle, and Portland. There are two runways, a main and minor crosswind. The main runway is equipped for precision instrumentation landings and takeoffs. Each runway is $2347 \mathrm{~m}(7700 \mathrm{ft})$ long and $46 \mathrm{~m}$ (150 ft) wide, and can accommodate landings and takeoffs by medium-range commercial aircraft, such as the Boeing 727-200 and Douglas DC-9. 
The Tri-Cities Airport set a record of 209,434 passengers (enplanements) in 2000, surpassing the 1999 total of 202,408, which exceeded 200,000 for the first time. Enplanements were up 3.5\% from 1999, and it was the sixth year in a row that the airport had an increase. Projections indicate that the terminal can serve almost 300,000 passengers annually.

The Tri-Cities region has three general aviation airports that serve private aircraft. The Richland Airport, owned by the Port of Benton, is located northwest of the Richland central business district, adjacent to the Richland by-pass highway (SR-240). Vista Field Airport, owned by the Port of Kennewick, is located at the intersection of Columbia Center Boulevard and Canal Drive, with easy access to SR-240, I-82 and I-182. The Prosser Airport, owned by the Port of Benton, is located one mile northwest of the business district of Prosser, and is adjacent to US-12. Airfreight shippers that service the region include Airborne from Richland, United Parcel Service from Kennewick, and Federal Express from the Tri-Cities Airport in Pasco.

Mass transit within the Tri-Cities is provided by the Ben Franklin Transit system. The system covers more than $110 \mathrm{mi}^{2}$ and provides frequent service to all four local communities. The Ben Franklin fleet consists of 54 buses, 31 Dial-a-Ride para-transit vehicles, and 75 VanPool vans. Two local taxi companies provide radio-dispatched taxicab service 24 hours per day: A-1 Tri-Cities Cab and AMR Transportation. Intercity bus transportation is provided by Greyhound Bus Lines. Buses to Seattle, Spokane, and Portland leave twice daily from the Pasco terminal.

The regional transportation network in the vicinity includes the areas in Benton and Franklin counties from which most of the commuter traffic associated with the Hanford Site originates. Interstate highways that serve the area are I-82, I-182, I-84, and I-90. Interstate- 82 is $8 \mathrm{~km} \mathrm{(5} \mathrm{mi)} \mathrm{south-southwest} \mathrm{of}$ Hanford. Interstate-182, a 24-km- (15-mi-) long urban connector route, located $8 \mathrm{~km}(5 \mathrm{mi})$ southsoutheast of the Hanford Site, provides an east-west corridor linking I-82 to the Tri-Cities area. I-90, located north of the Site, is the major link to Seattle and Spokane and extends to the East Coast; I-82 serves as a primary link between Hanford and I-90, as well as Interstate-84. I-84, located south of the Hanford Site in Oregon, is a major corridor leading to Portland. SR 224, south of Hanford, serves as a 16-km (10-mi) link between I-82 and SR 240. SR 24 enters the Hanford Site from the west, continues eastward across the northernmost portion of the Site, and intersects SR 17 approximately $24 \mathrm{~km}$ (15 mi) east of the Site boundary. SR 17 is a north-south route that links I-90 to the Tri-Cities and joins U.S. Route 395, which continues south through the Tri-Cities. SRs 240 and 24 traverse the Hanford Site and are maintained by Washington State. DOE maintains other roads within the Hanford Site.

\subsubsection{Educational Services}

The bulk of primary and secondary education in the Tri-Cities area is served by the Richland, Pasco, Kennewick, and Benton City School Districts. The total 2000 fall enrollment for all districts in Benton and Franklin counties was 39,702 students, an increase of 1.3\% from the 1999 total of 39,202 students. The 2000 totals include 9464 from the Richland School District, up from 9343 in 1999, 8850 students from the Pasco School District, up from 8537 in 1999, 13,629 students from the Kennewick School District, up from 13,535 in 1999, and 1673 from the Benton City School District, down from 1733 in 1999 (OSPI 2001).

There are several private elementary and secondary schools in the Tri-Cities, including Bethlehem Lutheran (K-8) and St. Josephs (K-8) in Kennewick, Christ the King (K-8) and Liberty Christian (K-12) in Richland, Faith Christian (K-12), Country Haven Academy, St. Patrick's (K-8), Tri-City Junior Academy (K-10), and Tri-Cities Prep Catholic High School in Pasco. Fall 2000 enrollment at these schools totaled 2312 students, an increase of 3\% from the 1999 total of 2244 (OSPI 2001). 
Post-secondary education in the Tri-Cities area is provided by a junior college, Columbia Basin College (CBC), City University, and Washington State University, Tri-Cities branch campus (WSU-TC). The 2000 fall/winter enrollment was approximately 7069 at CBC, 100 at City University, and 1157 at WSU-TC. Many of the programs offered by these three institutions are geared toward the vocational and technical needs of the area. In 1999-2000, CBC offered 25 Associate in Applied Science (AAS) degree programs. City University offers 2 associate degree programs, 4 undergraduate, and 3 graduate programs plus access to several more programs through Distance Learning. WSU-TC offers 10 undergraduate and 19 graduate programs, as well as access to 8 more graduate programs via satellite (OSPI 2001).

\subsubsection{Health Care and Human Services}

The Tri-Cities has three major hospitals and five minor emergency centers. All three hospitals offer general medical services and include a 24-hr emergency room, basic surgical services, intensive care, and neonatal care.

Kadlec Medical Center, located in Richland, has 124 beds and functioned at 58\% capacity with 6408 total admissions in 2000. Non-Medicare/Medicaid patients accounted for 52\% of Kadlec's annual admissions in 2000. An average stay of 3.7 days per admission was reported for 2000.

Kennewick General Hospital maintained a 63\% occupancy rate of its 71 beds with 5262 annual admissions in 2000. Non-Medicare/Medicaid patients represented $43.6 \%$ of its total admissions. An average stay of 3.1 days per admission was reported in 2000 .

Our Lady of Lourdes Hospital operates a 132-bed Health Center, located in Pasco, providing acute, sub-acute, skilled nursing and rehabilitation, and alcohol and chemical dependency services. Our Lady of Lourdes also operates the Carondolet Psychiatric Care Center, a 32-bed psychiatric hospital located in Richland. They also provide a significant amount of outpatient and home health services. For their calendar year 2000, Our Lady of Lourdes had a total of 4321 admissions, $29 \%$ of which were nonMedicare/Medicaid. Lourdes had an average acute care length of stay of 3.4 days, and the occupancy rate was $43.6 \%$ in 2000 .

The Tri-Cities offers a broad range of social services. State human service offices in the Tri-Cities include the Job Service Center within the Employment Security Department; food stamp offices; the Developmental Disabilities Division; financial and medical assistance; the Child Protective Service; emergency medical service; a senior companion program; and vocational rehabilitation.

The Tri-Cities is also served by a large number of private agencies and voluntary human service organizations. The United Way, an umbrella fund-raising organization, incorporates 21 participating agencies offering 38 programs. These member agencies had a cumulative budget total of \$27 million in 2000. In addition, there were 572 organizations that received funds as part of the United Way BentonFranklin County donor designation program (Davis 2000).

\subsubsection{Police and Fire Protection}

Benton and Franklin counties' sheriff departments, local municipal police departments, and the Washington State Patrol Division, with headquarters in Kennewick, provide police protection in Benton and Franklin counties. Table 4.6-5 shows the number of commissioned officers and patrol cars in each department in April 2001. The Kennewick Municipal Police Department maintains the largest staff of commissioned officers with 79 . 
Table 4.6-5. Police Personnel in the Tri-Cities, $2001^{\text {(a) }}$

\begin{tabular}{|lccc|}
\hline Area & Commissioned Officers & Reserve Officers & Patrol Cars \\
\hline Kennewick Municipal & 79 & 9 & 23 \\
Pasco Municipal & 46 & 13 & 17 \\
Richland Municipal & 49 & 12 & 13 \\
West Richland & 13 & 4 & 11 \\
Municipal & & 17 & 56 \\
Benton County Sheriff & 48 & $\underline{10}$ & $\underline{140}$ \\
Franklin County & $\underline{65}$ & & \\
Sheriff & 256 & & \\
Tri-Cities Totals & & & \\
(a) Source: Personal communication with each department office, April 2001. & \\
\hline
\end{tabular}

Fire protection is provided by the fire departments of the cities of Kennewick, Pasco, and Richland, and by Benton County Rural Fire Departments \#1, \#2, and \#4. Table 4.6-6 indicates the number of fire fighting personnel, both paid and unpaid, on the staffs of fire districts in the area.

The Hanford Fire Department currently has four fire stations strategically located on the Hanford Site. From these stations four pumper crews, staffed with at least three firefighters each, provide suppression response. Four ambulance crews (one in each fire station), staffed with two firefighters (Emergency Medical Technicians [EMT]- or paramedic-trained), provide emergency medical services $24 \mathrm{hr} /$ day, 7 days/week. A total of 40 emergency response vehicles, representing diverse capabilities, are maintained at the four fire stations. Some emergency equipment was specifically to control situations unique to the Hanford Site.

The Hanford Fire Department provides coverage to the entire Hanford Site and to SR 240 and SR 24. Coverage on the highways extends from the Vernita Bridge to the Silver Dollar Cafe on SR 24 and along SR 240 from the Yakima Barricade to the intersection with SR 225. Additionally, the Hanford Fire Department responds to mutual aid requests from 10 surrounding fire districts.

Table 4.6-6. Fire Protection Personnel in the Tri-Cities, $2001^{\text {(a) }}$

\begin{tabular}{|lcccl|}
\hline Fire Station & Fire Fighting Personnel & Volunteers & Total & Service Area \\
\hline Kennewick & 69 & 0 & 66 & City of Kennewick \\
Pasco & 39 & 0 & 42 & City of Pasco \\
Richland & 53 & 0 & 54 & City of Richland \\
BCRFD & (b) 1 & 115 & 125 & Kennewick Area \\
BCRFD 2 & 10 & 30 & 34 & Benton City \\
BCRFD 4 & $\underline{5}$ & $\underline{40}$ & $\underline{45}$ & West Richland \\
Tri-Cities Total & 180 & 185 & 365 & \\
(a) Source: Personal communication with each department office, April 2001. \\
(b) BCRFD = Benton County Rural Fire Department. \\
\hline
\end{tabular}




\subsubsection{Parks and Recreation}

The convergence of the Columbia, Snake, and Yakima rivers offers residents of the Tri-Cities a variety of recreational opportunities. The Lower Snake River Project includes Ice Harbor, Lower Monumental, Little Goose, and Lower Granite locks and dams, and a levee system and parkway at Clarkston and Lewiston. Although navigation capabilities and the electrical output are the major benefits of this project, recreational benefits have also resulted. The Lower Snake River Project provides boating, camping, and picnicking facilities in nearly a dozen areas along the Snake River. In 2000, nearly 2 million people visited the area and participated in activities along the river.

Similarly, the Columbia River provides ample water recreational opportunities on the lakes formed by the dams. Lake Wallula, formed by McNary Dam, offers a large variety of parks and activities, which attracted more than 4.2 million visitors in 2000. The Columbia River Basin is also a popular area for migratory waterfowl and upland game bird hunting.

Other opportunities for recreational activities in the Tri-Cities are accommodated by the indoor and outdoor facilities available, some of which are listed in Table 4.6-7. Numerous tennis courts, ball fields, and golf courses offer outdoor recreation to residents and tourists. Several privately owned health clubs in the area offer indoor tennis and racquetball courts, pools, and exercise programs. Bowling lanes and skating rinks also serve the Tri-Cities.

Table 4.6-7. Examples of Physical Recreational Facilities Available in the Tri-Cities

\begin{tabular}{|c|c|}
\hline Activity & Facilities \\
\hline Team sports & $\begin{array}{l}\text { Baseball fields and basketball courts are located throughout the Tri-Cities. } \\
\text { Soccer and football fields are also located in various areas. }\end{array}$ \\
\hline Bowling & $\begin{array}{l}\text { Lanes in each city, including Fiesta Bowling Center, Celebrity Bowl, Columbia } \\
\text { Lanes, and Go-Bowl. }\end{array}$ \\
\hline Camping & $\begin{array}{l}\text { Several hundred campsites within driving distance from the Tri-Cities area, } \\
\text { including Fishhook Park and Sun Lakes. }\end{array}$ \\
\hline Fishing & $\begin{array}{l}\text { Steelhead, sturgeon, trout, walleye, bass, and crappie fishing in the lakes and } \\
\text { rivers near the Tri-Cities. }\end{array}$ \\
\hline Golf & $\begin{array}{l}\text { Public courses include Canyon Lakes, Horn Rapids, and West Richland } \\
\text { Municipal, two private courses, and a number of driving ranges and pro shops } \\
\text { available. }\end{array}$ \\
\hline Hunting & $\begin{array}{l}\text { Duck, geese, pheasant, and quail hunting. Deer and elk hunting in the Blue } \\
\text { Mountains and the Cascade Range. }\end{array}$ \\
\hline Skating & $\begin{array}{l}\text { Roller skating in Richland, Kennewick, and Prosser; Junior professional ice } \\
\text { hockey arena available to the public in Kennewick. }\end{array}$ \\
\hline Water sports & $\begin{array}{l}\text { Private and public swimming pools in the area. Boating, sailing, windsurfing, } \\
\text { diving, water-skiing, swimming, etc. on the Columbia River. }\end{array}$ \\
\hline Tennis & $\begin{array}{l}\text { Several outdoor city courts, with additional outdoor courts located at area } \\
\text { schools. }\end{array}$ \\
\hline Walking/bicycling & Several miles of paved bike/hike paths. \\
\hline
\end{tabular}




\subsubsection{Utilities}

The principal source of water in the Tri-Cities and the Hanford Site is the Columbia River. The water systems of Richland, Pasco, and Kennewick drew a large portion of the 51.5-billion L (13.6-billion gal) used in 2000 from the Columbia River. Each city operates its own supply and treatment system. The Richland water supply system derives about $90 \%$ of its water directly from the Columbia River, while the remainder is split between a well field in North Richland (which is recharged from the river) and groundwater wells. The city of Richland's total usage in 2000 was 27.6 billion L ( 7.3 billion gal). The city of Pasco system also draws from the Columbia River for its water needs. In 2000, Pasco consumed 10.6 billion L ( 2.8 billion gal). The Kennewick system uses two wells and the Columbia River for its supply. These wells serve as the sole source of water between November and March and can provide approximately $40 \%$ of the total maximum supply of 30 billion L ( 8 billion gal). Total 2000 usage in Kennewick was 13.0 billion L (3.4 billion gal).

The major incorporated areas of Benton and Franklin counties are served by municipal wastewater treatment systems, whereas the unincorporated areas are served by onsite septic systems. Richland's wastewater treatment system is designed to treat a total capacity of $43.1 \mathrm{million} \mathrm{L} / \mathrm{d}$ (11.4 million gal/d) and processed an average flow of 23.4 million L/d (6.2 million gal/d) in 2000. Kennewick's waste treatment system processed an average 19.3 million L/d (5.1 million gal/d) in 2000 , while the system is capable of treating about $46.1 \mathrm{million} \mathrm{L} / \mathrm{d}$ (12.2 million gal/d). Pasco's waste treatment system processed an average 9.8 million $\mathrm{L} / \mathrm{d}(2.6$ million gal/d) while the system is capable of treating 18.9 million $\mathrm{L} / \mathrm{d}(5.0$ million gal/d).

In the Tri-Cities, the Benton County Public Utility District, Benton Rural Electrical Association, Franklin County Public Utility District, and city of Richland Energy Services Department provide electricity. All the power these utilities provide in the local area is purchased from the Bonneville Power Administration (BPA), a federal power-marketing agency. The average rate for residential customers served by the four local utilities was approximately $\$ 0.046 /$ kilowatt-hour $(\mathrm{kWh})$ in 2000 . Total electrical consumption in 2000 was 3.65 billion $\mathrm{kWh}$.

Electrical power for the Hanford Site is purchased wholesale from BPA. Energy requirements for the Hanford Site during FY 2000 were over 278 million $\mathrm{kWh}$ for a total cost of $\$ 6.7$ million. Additionally, the Site spends about $\$ 0.024 / \mathrm{kWh}$ for electrical transportation and distribution within the Hanford Site.

Natural gas, provided by the Cascade Natural Gas Corporation, serves a small portion of residents, with 7950 residential customers as of April 2001. The average annual gas bill for residential customers was $\$ 575$ in FY 2000. The Cascade Natural Gas Corporation also serves the Hanford Site 300 Area.

In the Pacific Northwest, hydropower, and to a lesser extent coal and nuclear power, constitute the bulk of the region's electrical generation system. In 2000, generating resources in the Northwest totaled more than 25,924 average megawatts (MW) of energy. The region's electrical power system, more than any other system in the nation, is dominated by hydropower, as $62.3 \%$ of the Pacific Northwest's regional generation came from the hydroelectric system in 2000. Coal resources were the next largest component, representing nearly $23 \%$ of all generating resources, followed by natural gas $(9.6 \%)$, nuclear $(3.3 \%)$, and biomass (3.2\%) resources (NPPC 2001).

The Pacific Northwest system's reliance on hydroelectric power means that it is more constrained by the seasonal variations in peak demand than in meeting momentary peak demand. The Columbia River hydroelectric system's installed capacity was 33,545 MW in 2000 (NPPC 2001), but limitations on the storage capacity of the system result in significant variations in the system's energy output from year to 
year, depending on annual rainfall and snowpack accumulation. In the driest years, the hydroelectric system produces only about 11,700 average MW of energy. In the wettest years, the hydroelectric system produces about 20,000 average MW. In average water years, the dams generate approximately 16,500 average MW (NPPC 1998).

Additional constraints on hydroelectric production include measures designed to protect and enhance the production of salmon, as many salmon runs have dwindled to the point of being threatened or endangered. These measures, outlined by the Northwest Power Planning Council's (NPPC) Columbia River Basin Fish and Wildlife Program, include minimum flow levels and a "water budget," which refers to water in the Columbia and Snake rivers that is released to speed the migration of young fish to the sea. Generation capacity of the hydroelectric system is decreased with these measures, as less water is available to pass through the turbines.

In addition to the hydroelectric system, other sources of bulk electric power in the Northwest include large coal-fired power plants, industrial cogeneration plants, small biomass plants, numerous small hydroelectric projects, simple-cycle and combined-cycle natural gas combustion turbines and the Energy Northwest Columbia Generating Station nuclear plant. The Columbia Generating Station (the only commercial nuclear power plant remaining in service in the Pacific Northwest), upgraded from its original peak capacity, can now serve about $1170 \mathrm{MW}$ of winter peak load. The plant produced 851 average MW of energy in fiscal year 2000.

\subsubsection{Land Use}

The DOE completed a Hanford Comprehensive Land-Use Plan EIS [HCP-EIS] (DOE 1999a) in September 1999, and a Record of Decision (ROD) was issued on November 2, 1999 (64 FR 61615). The purpose of this land-use plan and its implementing policies and procedures is to facilitate decision-making about the Hanford Site's uses and facilities over at least the next 50 years. The Preferred Alternative map shown in Figure 4.6-3 represents DOE's future land-management values, goals, and objectives. The landuse plan consists of several key elements that are included in the Department's Preferred Alternative in the Final HCP-EIS (DOE 1999a). These elements include a land-use map that addresses the Hanford Site as five geographic areas-Wahluke Slope, Columbia River Corridor, Central Plateau, All Other Areas of the Site, and the Fitzner/Eberhardt Arid Lands Ecology Reserve and depicts the planned future uses for each area; a set of 10 land-use designations that define the permissible uses for each area of the Hanford Site; and the planning and implementing policies and procedures that will govern the review and approval of future land uses. Together these four elements create the Hanford Comprehensive Land-Use Plan.

The key features of the Hanford Site that form the basis for the five geographic areas used in the environmental impact analysis and land-use plans are summarized as follows:

- The Wahluke Slope. The area north of the Columbia River and the Hanford Site proper encompasses approximately $357 \mathrm{~km}^{2}\left(138 \mathrm{mi}^{2}\right)$ of relatively undisturbed or recovering shrub-steppe habitat managed by the USFWS for the DOE. These lands consist of two wildlife management units within the Hanford Reach National Monument/Saddle Mountain National Wildlife Refuge, the 130 $\mathrm{km}^{2}\left(50 \mathrm{mi}^{2}\right)$ Saddle Mountain Unit and the $225 \mathrm{~km}^{2}\left(87 \mathrm{mi}^{2}\right)$ Wahluke Unit. Portions of the Saddle Mountain Unit, which is closed to public access, still serve as buffer areas for the Hanford Site. The Wahluke Unit is open to public recreational access. A small strip of land approximately $1.62 \mathrm{~km}^{2}$ $\left(0.63 \mathrm{mi}^{2}\right)$ located between SR 243 and the Columbia River west of SR 24 is managed by the Washington State Department of Fish and Wildlife and retains public access. 


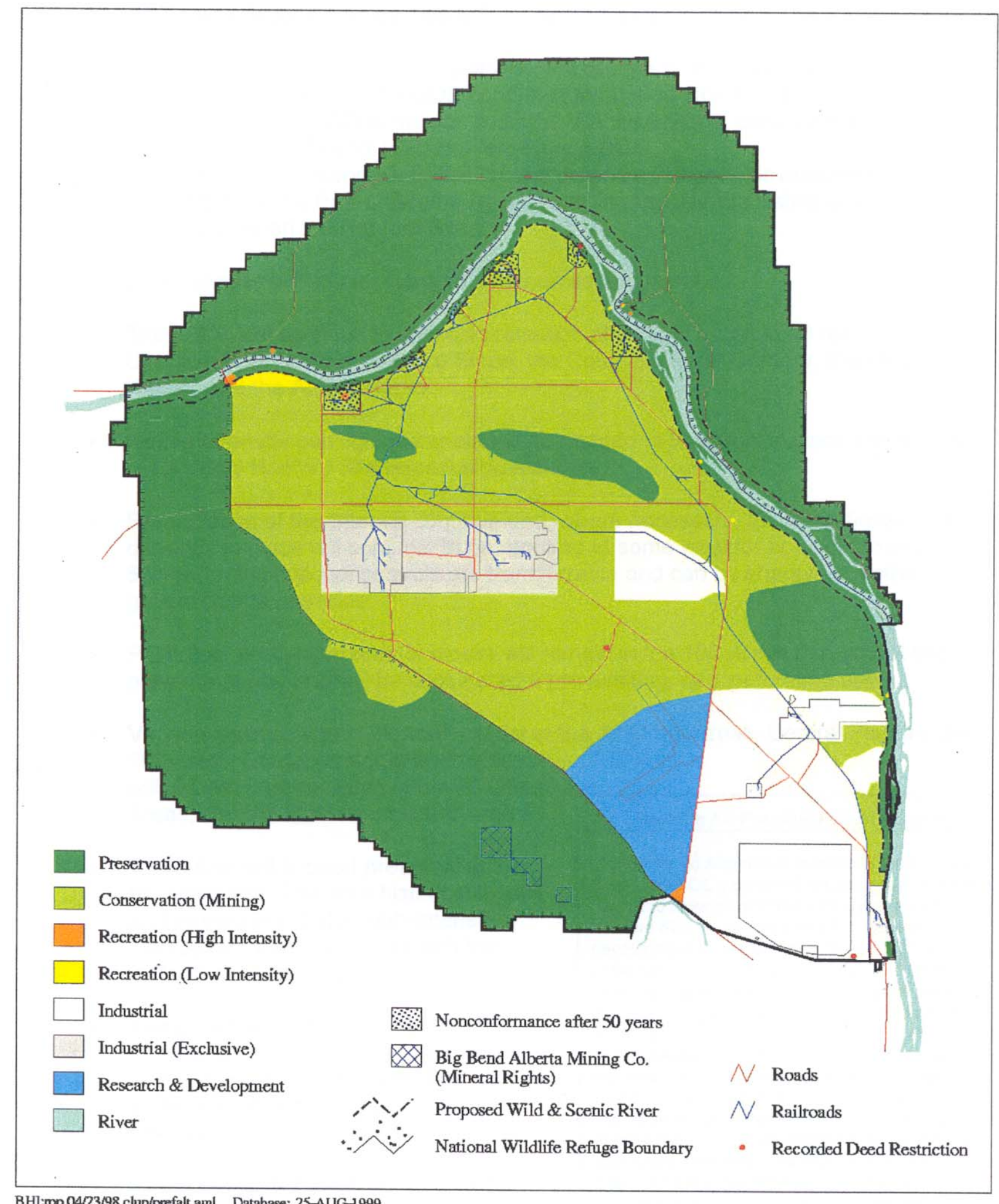

BHI:npp 04/23/98 clup/prefalt.aml Database: 25-AUG-1999

Figure 4.6-3. DOE's Preferred Alternative for Land Use on the Hanford Site (DOE 1999a) 
- Columbia River Corridor. The $111.6 \mathrm{~km}^{2}\left(43.1 \mathrm{mi}^{2}\right)$ Columbia River Corridor, which is adjacent to and runs through the Hanford Site, is used for boating, water skiing, fishing, and hunting of upland game birds and migratory waterfowl. Although public access is allowed on certain islands, access to other islands and adjacent areas is restricted because of unique habitats and the presence of cultural resources.

Along the southern shoreline of the Columbia River Corridor, the 100 Areas occupy approximately $68 \mathrm{~km}^{2}\left(26 \mathrm{mi}^{2}\right)$. The facilities in the 100 Areas include nine retired plutonium production reactors, associated facilities, and structures. Resource Conservation and Recovery Act of 1976 (RCRA) closure permit restrictions have been placed in the vicinity of the 100-H Area, which is associated with the 183-H Solar Evaporation Basins. Additional deed restrictions or covenants for activities that potentially extend more than $4.6 \mathrm{~m}(15 \mathrm{ft})$ below ground surface are expected for the Comprehensive Environmental Restoration, Compensation, and Liability Act of 1980 (CERCLA) remediation areas.

The area within the Columbia River Corridor known as the Hanford Reach includes an average of a $402 \mathrm{~m}$ (1320 ft) strip of public land on either side of the Columbia River.

- Central Plateau. The 200 East and 200 West Areas occupy approximately $51 \mathrm{~km}^{2}\left(19.5 \mathrm{mi}^{2}\right)$ in the Central Plateau of the Hanford Site. Facilities located in the Central Plateau were built to process irradiated fuel from the plutonium production reactors. The operation of these facilities resulted in the treatment, storage, disposal, and unplanned release of radioactive and nonradioactive waste. The Environmental Restoration Disposal Facility for CERCLA cleanup wastes is located in the Central Plateau. Other federal agencies, such as the Department of the Navy, also use Hanford nuclear waste treatment, storage, or disposal facilities. Deed restrictions or covenants for activities that potentially may extend more than $4.6 \mathrm{~m}(15 \mathrm{ft})$ below ground surface are expected for CERCLA remediation areas in the Central Plateau.

A commercial low-level radioactive waste disposal facility, run by U.S. Ecology, Inc., currently operates on $0.4 \mathrm{~km}^{2}\left(0.16 \mathrm{mi}^{2}\right)$ of the Central Plateau.

- All Other Areas. All Other Areas comprise $689 \mathrm{~km}^{2}\left(266 \mathrm{mi}^{2}\right)$ and contain the 300,400 , and 1100 Areas, Energy Northwest facilities, and a section of land currently owned by the state of Washington for the disposal of hazardous substances.

The Hanford 1100 Area and the Hanford railroad southern connection (from Horn Rapids Road to Columbia Center) have been transferred from DOE ownership to Port of Benton ownership to support future economic development. Although the 1100 Area is no longer under DOE control, it is included in the HCP EIS to support the local governments with their State Environmental Policy Act (SEPA) EIS analyses of the Hanford sub-area of Benton County under the state of Washington's Growth Management Act.

The 300 Area is located just north of the city of Richland and covers $1.5 \mathrm{~km}^{2}\left(0.6 \mathrm{mi}^{2}\right)$. The 300 Area is the site of former reactor fuel fabrication facilities and is also the principal location of nuclear research and development facilities serving the Hanford Site.

The 400 Area, located southeast of the 200 East Area, is the site of the Fast Flux Test Facility that is being evaluated in an ongoing EIS. The proposed mission for the 400 Area is reactor operations and irradiation services with attendant support functions including fuel and target fabrication, target processing, and interim storage. 
Energy Northwest currently operates Columbia Generating Station on leased land approximately 10 $\mathrm{km}(6 \mathrm{mi})$ north of Richland. Originally leased for the operation of three nuclear power plants, construction of two of the plants was halted; other industrial options are currently being considered.

In 1980, the federal government sold a $2.59 \mathrm{~km}^{2}\left(1 \mathrm{mi}^{2}\right)$ section of land (known as Section 1) south of the 200 East Area, near SR 240, to the state of Washington for the purpose of nonradioactive hazardous waste disposal. To date, this parcel has not been used for hazardous waste disposal, and it is undeveloped and uncontaminated (although the underlying groundwater is contaminated). The deed requires that if it were used for any purpose other than hazardous waste disposal, ownership would revert to the federal government.

Additional activities in the All Other Areas include:

(1) A specialized training center: The Hazardous Materials Management and Emergency Response (HAMMER) Volpentest Training and Education Center is used to train hazardous materials response personnel. It is located north of the former 1100 Area and covers about $0.3 \mathrm{~km}^{2}(0.12$ $\left.\mathrm{mi}^{2}\right)$.

(2) A regional law-enforcement training facility: The Hanford Patrol Training Academy provides a range of training environments including classrooms, library resources, practice shoot houses, an exercise gym, and an obstacle course.

(3) A national research facility: The Laser Interferometer Gravitational Wave Observatory (LIGO), built by the National Science Foundation for scientific research, is designed to detect cosmic gravitational waves. The facility consists of two optical tube arms, each $4 \mathrm{~km}(2.5 \mathrm{mi})$ long, arrayed in an "L" shape, and is extremely sensitive to vibrations.

- Fitzner/Eberhardt Arid Lands Ecology Reserve Unit (ALE Reserve). The $308.7 \mathrm{~km}^{2}$ (119.2 mi ${ }^{2}$ ALE Unit is part of the Hanford Reach National Monument and is managed by the USFWS for the DOE. The Unit is located in the southwestern portion of the Hanford Site and is managed as a wildlife reserve and environmental research center. The public is currently restricted from the site.

The Hanford Site facilities and activities are consolidated within the operating areas that occupy about $6 \%$ of the total available area of the Site (DOE 1999b). Some of the Hanford Site that is not involved with the current mission has been leased, disposed, or permitted to federal or state agencies, or private entities. Table 4.6-8 is a summary of those changes in land allocations.

\subsubsection{Visual Resources}

With the exception of Rattlesnake Mountain, the land near the Hanford Site is generally flat with little relief. Rattlesnake Mountain, rising to $1060 \mathrm{~m}$ (3477 ft) above mean sea level forms the western boundary of the Hanford Site, and Gable Mountain and Gable Butte are the highest landforms within the Site (Figure 4.6-4). The view toward Rattlesnake Mountain is visually pleasing, especially in the springtime when wildflowers are in bloom. Large rolling hills are located to the west and far north. The Columbia River, flowing across the northern part of the Hanford Site and forming the eastern boundary, is generally considered scenic, with its contrasting blue against a background of brown basaltic rocks and sagebrush. The White Bluffs, steep whitish-brown bluffs adjacent to the Columbia River and above the northern boundary of the river in this region, are a strong feature of the landscape. 


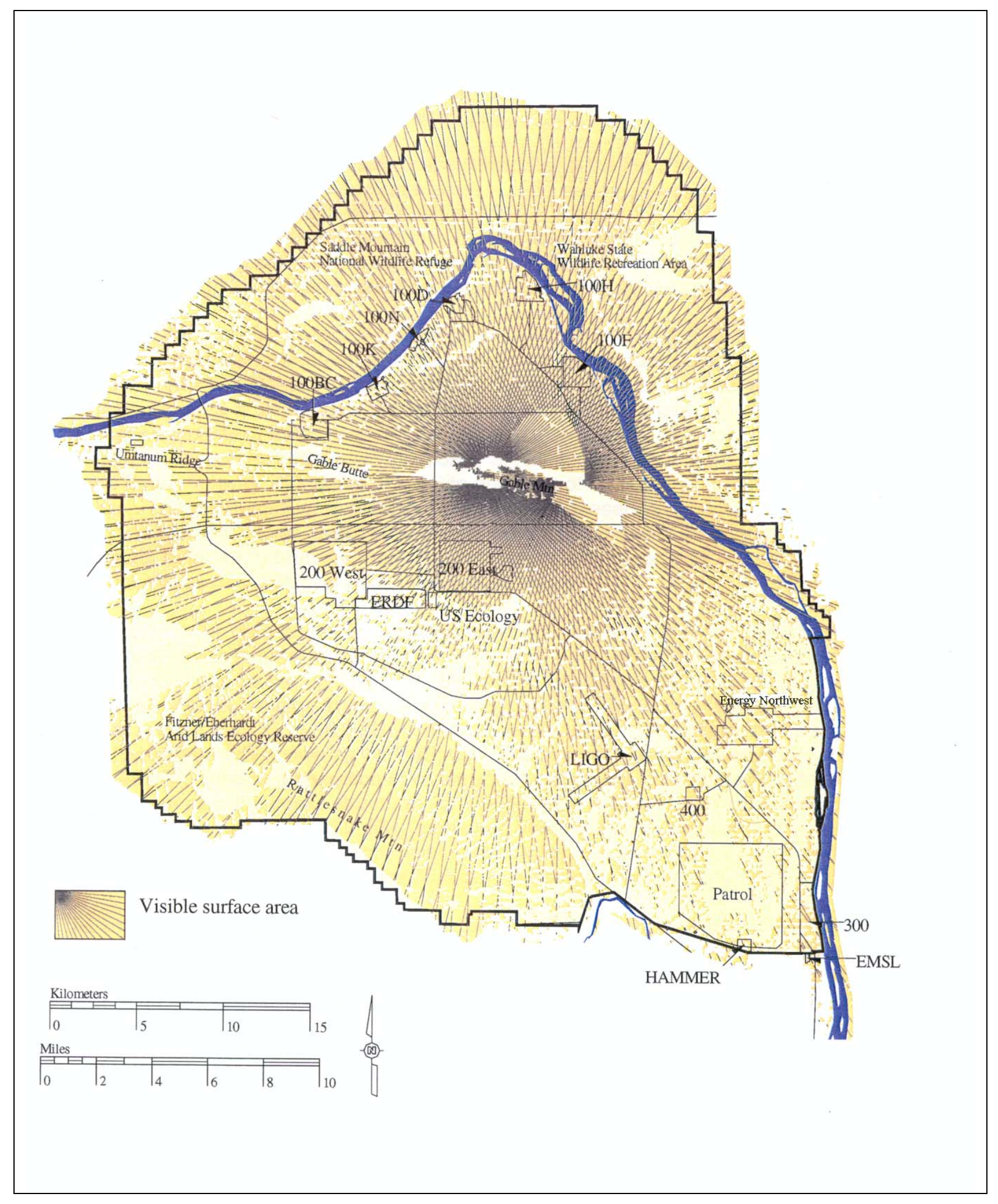

Figure 4.6-4. Viewshed From Gable Mountain (DOE 1999a) 
Table 4.6-8. Areas of the Hanford Site Outgranted/Released to Date ${ }^{(a)}$

\begin{tabular}{|c|c|c|c|c|}
\hline Area & Management & Use & Year & Controls \\
\hline $\begin{array}{l}\text { US Ecology Low- } \\
\text { Level Radioactive } \\
\text { Waste Disposal } \\
\text { Facility }\end{array}$ & $\begin{array}{l}\text { State of } \\
\text { Washington }\end{array}$ & $\begin{array}{l}\text { Radioactive } \\
\text { Waste } \\
\text { Disposal }\end{array}$ & 1964 & Leased \\
\hline Vernita Rest Area ${ }^{(b)}$ & $\begin{array}{l}\text { Washington } \\
\text { State } \\
\text { Department of } \\
\text { Transportation }\end{array}$ & Rest area & 1966 & Washington State Highway Patrol \\
\hline $\begin{array}{l}\text { Columbia } \\
\text { Generating Station }\end{array}$ & $\begin{array}{l}\text { Energy } \\
\text { Northwest }\end{array}$ & $\begin{array}{l}\text { Power } \\
\text { Production }\end{array}$ & 1971 & Leased \\
\hline $\begin{array}{l}\text { West End of } \\
\text { Wahluke Slope } \\
\text { (Saddle Mountain }_{\text {National Wildlife }} \\
\text { Refuge) } \\
\text { (b) }^{\text {(Superseded) }}\end{array}$ & $\begin{array}{l}\text { U.S. Fish and } \\
\text { Wildlife } \\
\text { Service }\end{array}$ & $\begin{array}{l}\text { Wildlife } \\
\text { Refuge }\end{array}$ & 1971 & $\begin{array}{l}\text { Permitted with the following } \\
\text { controls: } \\
\text { - No overnight camping } \\
\text { - Access control plans required } \\
\text { - No drilling of wells for } \\
\text { residential water }\end{array}$ \\
\hline $\begin{array}{l}\text { East End of } \\
\text { Wahluke Slope }^{(b)} \\
\text { (Superseded) }\end{array}$ & $\begin{array}{l}\text { Washington } \\
\text { State } \\
\text { Department of } \\
\text { Fish and } \\
\text { Wildlife } \\
\end{array}$ & $\begin{array}{l}\text { Wildlife \& } \\
\text { Recreational } \\
\text { Reserve } \\
\end{array}$ & $\begin{array}{l}1971- \\
1999 \\
\end{array}$ & $\begin{array}{l}\text { Permitted with same controls as } \\
\text { mentioned for Wahluke Slope } \\
\text { above. }\end{array}$ \\
\hline Section 1 & $\begin{array}{l}\text { State of } \\
\text { Washington }\end{array}$ & $\begin{array}{l}\text { Hazardous } \\
\text { Waste } \\
\text { Disposal } \\
\end{array}$ & 1980 & Disposed (Title Transfer) \\
\hline 3000 Area & Port of Benton & $\begin{array}{l}\text { Economic } \\
\text { Development }\end{array}$ & 1996 & Disposed (Title Transfer) \\
\hline $\begin{array}{l}\text { Fitzner/Eberhardt } \\
\text { Arid Lands Ecology } \\
\text { Reserve (ALE) }{ }^{(b)}\end{array}$ & $\begin{array}{l}\text { U.S. Fish and } \\
\text { Wildlife } \\
\text { Service }\end{array}$ & $\begin{array}{l}\text { Wildlife } \\
\text { Reserve }\end{array}$ & 1998 & $\begin{array}{l}\text { Permitted with same controls as } \\
\text { mentioned for Wahluke Slope } \\
\text { above. }\end{array}$ \\
\hline $\begin{array}{l}\text { Laser Interferometer } \\
\text { Gravitational Wave } \\
\text { Observatory (LIGO) }\end{array}$ & $\begin{array}{l}\text { The National } \\
\text { Science } \\
\text { Foundation }\end{array}$ & Research & 1998 & Permitted \\
\hline 1100 Area & Port of Benton & $\begin{array}{l}\text { Economic } \\
\text { Development }\end{array}$ & 1998 & Disposed (Title Transfer) \\
\hline $\begin{array}{l}\text { Wahluke Slope } \\
\text { (Remainder/all) }^{(\text {bem }}\end{array}$ & $\begin{array}{l}\text { U.S. Fish and } \\
\text { Wildlife } \\
\text { Service } \\
\end{array}$ & $\begin{array}{l}\text { Wildlife } \\
\text { Refuge }\end{array}$ & 1999 & $\begin{array}{l}\text { Permitted with same controls as } \\
\text { mentioned for Wahluke Slope } \\
\text { above. }\end{array}$ \\
\hline
\end{tabular}


Traditional Native American religion is manifest in the earth, water, sky, and all animate or inanimate beings that inhabit a given location. The National Historic Preservation Act, the Native American Graves Protection and Repatriation Act, the Archaeological Resources Protection Act, and DOE's American Indian Policy, among other legislation and guidelines, all require the identification and protection of areas and resources of concern to Native Americans.

The acquisition of spiritual guidance and assistance through personal vision quests is deeply rooted in the religious practices of the indigenous people of the Columbia Basin. High spots were selected because they afforded extensive views of the natural landscape and seclusion for quiet meditation.

\subsection{Noise}

\section{T. M. Poston}

Noise is technically defined as sound waves that are unwanted and perceived as a nuisance by humans. Sound waves are characterized by frequency, measured in Hertz $(\mathrm{Hz})$, and sound pressure expressed as decibels (dB). Humans have a perceptible hearing range of 31 to $20,000 \mathrm{~Hz}$. The decibel is a value equal to 10 times the logarithm of the ratio of a sound pressure squared to a standard reference sound-pressure level (20 micropascals) squared. The threshold of audibility ranges from about $60 \mathrm{~dB}$ at a frequency of $31 \mathrm{~Hz}$ to less than about $1 \mathrm{~dB}$ between 900 and $8000 \mathrm{~Hz}$. (For regulatory purposes, noise levels for perceptible frequencies are weighted to provide an A-weighted sound level [dBA] that correlates highly with individual community response to noise.) Sound levels outside the range of human hearing are not considered noise in a regulatory sense, even though wildlife may hear at these frequencies.

Noise levels are often reported as the equivalent sound level $\left(\mathrm{L}_{\mathrm{eq}}\right)$. The $\mathrm{L}_{\mathrm{eq}}$ is expressed in $\mathrm{dBA}$ over a specified period of time, usually 1 or $24 \mathrm{hr}$. The $\mathrm{L}_{\mathrm{eq}}$ is the equivalent steady sound level that, if continuous during a specified time period, would contain the same total energy as the actual time-varying sound over the monitored or modeled time period.

\subsubsection{Background Information}

Studies of the propagation of noise at Hanford have been concerned primarily with occupational noise at work sites. Environmental noise levels have not been extensively evaluated because of the remoteness of most Hanford activities and isolation from receptors that are covered by federal or state statutes. This discussion focuses on what few environmental noise data are available. The majority of available information consists of model predictions, which in many cases have not been verified because the predictions indicated that the potential to violate federal or state standards is remote or unrealistic.

\subsubsection{Environmental Noise Regulations}

The Noise Control Act of 1972 and its subsequent amendments (Quiet Communities Act of 1978 and 40 CFR 201-211) direct the regulation of environmental noise to the state. The state of Washington has adopted Revised Code of Washington (RCW) 70.107, which authorizes Ecology to implement rules consistent with federal noise control legislation. RCW 70.107 and the implementing regulations embodied in WAC 173-60 through 173-70 defined the regulation of environmental noise levels. Maximum noise levels are defined for the zoning of the area in accord with environmental designation for noise abatement (EDNA). The Hanford Site is classified as a Class C EDNA on the basis of industrial activities. Unoccupied areas are also classified as Class $\mathrm{C}$ areas by default because they are neither Class A (residential) nor Class B (commercial). Maximum noise levels are established based on the EDNA classification of the receiving area and the source area (Table 4.7-1). 
Table 4.7-1. Applicable State Noise Limitations for the Hanford Site Based on Source and Receptor EDNA Designation

\begin{tabular}{|l|ccc|}
\hline \multirow{2}{*}{$\begin{array}{c}\text { Source Hanford } \\
\text { Site }\end{array}$} & $\begin{array}{c}\text { Class A } \\
\text { Residential } \\
\text { (dBA) }\end{array}$ & $\begin{array}{c}\text { Class B } \\
\text { Commercial } \\
\text { (dBA) }\end{array}$ & $\begin{array}{c}\text { Class C } \\
\text { Industrial } \\
\text { (dBA) }\end{array}$ \\
\hline Class C - Day & 60 & 65 & 70 \\
Night & 50 & -- & -- \\
\hline
\end{tabular}

\subsubsection{Hanford Site Sound Levels}

Most industrial facilities on the Hanford Site are located far enough away from the Site boundary that noise levels at the boundary are not measurable or are barely distinguishable from background noise levels. Modeling of environmental noises has been performed for commercial reactors and SR 240 through the Hanford Site. These data are not concerned with background levels of noise and are not reviewed here. There are two sources of measured environmental noise at Hanford. Environmental noise measurements were made in 1981 during site characterization for the Skagit/Hanford Nuclear Power Plant Site (NRC 1982). Measurements were also made when the Hanford Site was considered for a geologic waste repository (Basalt Waste Isolation Project) for spent commercial nuclear fuel and other high-level nuclear waste. Hanford Site characterization studies performed in 1987 included measurement of background environmental noise levels at five locations. Additionally, certain activities such as well drilling and sampling have the potential for producing noise in the field apart from major permanent facilities.

\subsubsection{Skagit/Hanford Data}

Pre-construction measurements of environmental noise were taken in June 1981 on the Hanford Site during site characterization for the Skagit/Hanford Nuclear Power Plant (NRC 1982). Fifteen sites were monitored, and noise levels ranged from 30 to $60.5 \mathrm{dBA}\left(\mathrm{L}_{\mathrm{eq}}\right)$. The values for isolated areas ranged from 30 to $38.8 \mathrm{dBA}$. Measurements taken around the sites where Energy Northwest was constructing nuclear power plants (WNP-1, WNP-2 (now the Columbia Generating Station), and WNP-4) ranged from 50.6 to 64 dBA. Measurements taken along the Columbia River near the intake structures for the Columbia Generating Station were 47.7 and 52.1 dBA compared with more remote river noise levels of $45.9 \mathrm{dBA}$ (measured about $4.8 \mathrm{~km}$ [3 mi] upstream of the intake structures). Community noise levels in North Richland (Horn Rapids Road and SR 240) were 60.5 dBA.

\subsubsection{Basalt Waste Isolation Project (BWIP) Data}

Background noise levels were determined at five locations within the Hanford Site (Figure 4.7-1). Noise levels are expressed as $\mathrm{L}_{\mathrm{eq}}$ for $24 \mathrm{hr}\left(\mathrm{L}_{\mathrm{eq}-24}\right)$. Sample location, date, and $\mathrm{L}_{\mathrm{eq}-24}$ are listed in Table 4.72. Wind was identified as the primary contributor to background noise levels, with winds exceeding 19 $\mathrm{km} / \mathrm{hr}(12 \mathrm{mi} / \mathrm{hr})$ significantly affecting noise levels. Background noise levels in undeveloped areas at Hanford can best be described as a mean $\mathrm{L}_{\text {eq-24 }}$ of 24 to $36 \mathrm{dBA}$. Periods of high wind, which normally occur in the spring, would elevate background noise levels. 


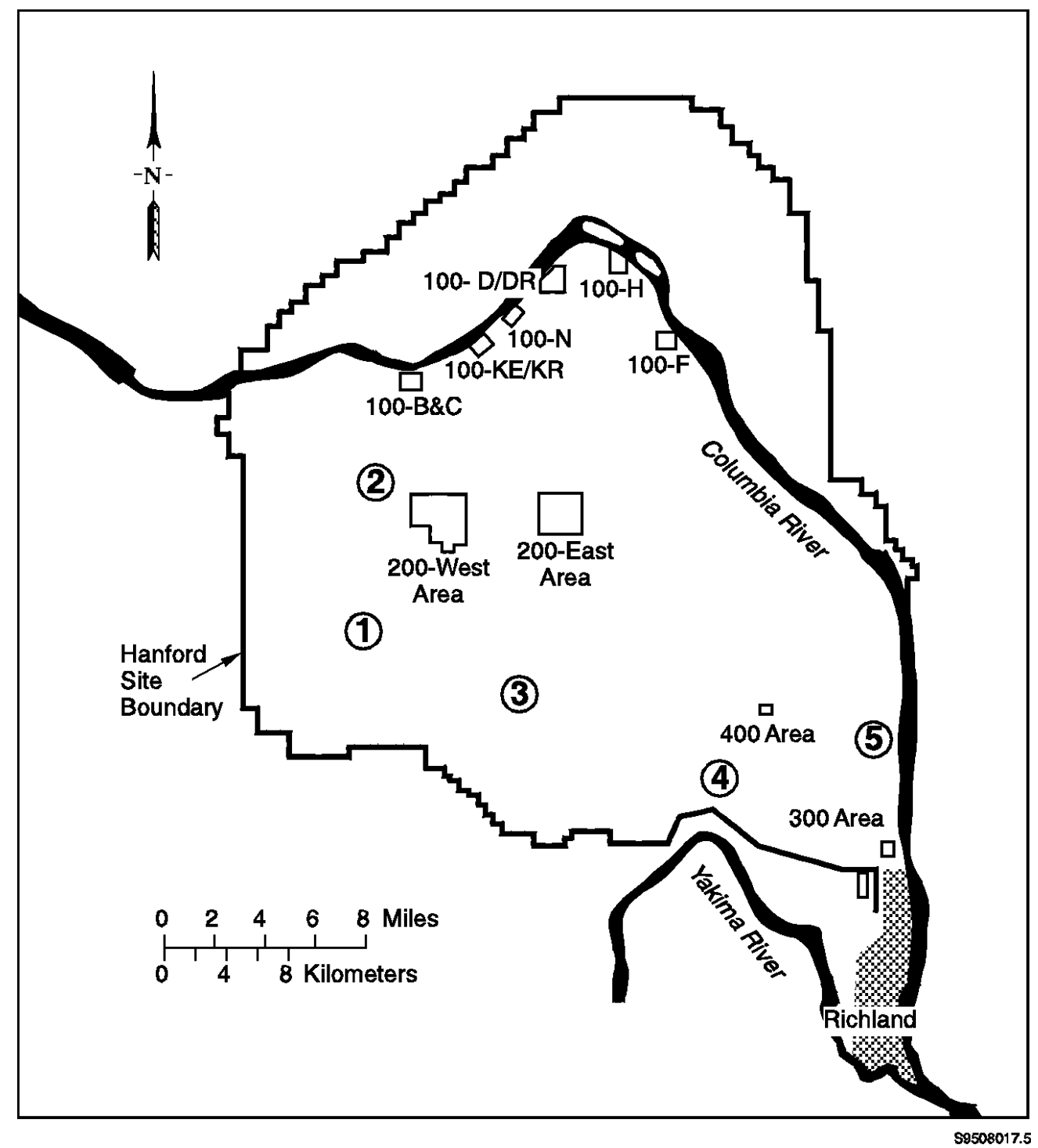

Figure 4.7-1. Location of Background Noise Measurements (see Table 4.7-2) 
Table 4.7-2. Background Noise Levels Measured at Isolated Areas

\begin{tabular}{|c|c|c|c|c|c|}
\hline \multirow[b]{2}{*}{ Site } & \multicolumn{3}{|c|}{ Location } & \multirow[b]{2}{*}{ Date } & \multirow[b]{2}{*}{$\mathrm{L}_{\mathrm{eq}-24}(\mathrm{dBA})$} \\
\hline & Section & Range & Township & & \\
\hline \multirow[t]{5}{*}{1} & 9 & $\mathrm{R} 25 \mathrm{E}$ & $\mathrm{T} 12 \mathrm{~N}$ & $07-10-87$ & 41.7 \\
\hline & & & & $07-11-87$ & 40.7 \\
\hline & & & & $07-12-87$ & 36.0 \\
\hline & & & & $07-13-87$ & 37.2 \\
\hline & & & & $07-14-87$ & 35.6 \\
\hline \multirow[t]{5}{*}{2} & 26 & R25E & $\mathrm{T} 13 \mathrm{~N}$ & $07-25-87$ & 43.9 \\
\hline & & & & $07-26-87$ & 38.8 \\
\hline & & & & $07-27-87$ & 43.8 \\
\hline & & & & $07-28-87$ & 37.7 \\
\hline & & & & $07-29-87$ & 43.2 \\
\hline \multirow[t]{5}{*}{3} & 18 & $\mathrm{R} 26 \mathrm{E}$ & $\mathrm{T} 12 \mathrm{~N}$ & $08-08-87$ & 39.0 \\
\hline & & & & $08-09-87$ & 35.4 \\
\hline & & & & $08-10-87$ & $51.4^{(\mathrm{a})}$ \\
\hline & & & & $08-11-87$ & $56.7^{(\mathrm{a})}$ \\
\hline & & & & $08-12-87$ & 36.0 \\
\hline \multirow[t]{5}{*}{4} & 34 & $\mathrm{R} 27 \mathrm{E}$ & $\mathrm{T} 11 \mathrm{~N}$ & 09-09-87 & 35.2 \\
\hline & & & & $09-10-87$ & 34.8 \\
\hline & & & & $09-11-87$ & 36.0 \\
\hline & & & & $09-12-87$ & 33.2 \\
\hline & & & & $09-13-87$ & 37.3 \\
\hline \multirow[t]{5}{*}{5} & 14 & $\mathrm{R} 28 \mathrm{E}$ & $\mathrm{T} 11 \mathrm{~N}$ & $10-15-87$ & 40.8 \\
\hline & & & & $10-16-87$ & 36.8 \\
\hline & & & & $10-17-87$ & 33.7 \\
\hline & & & & $10-18-87$ & 31.3 \\
\hline & & & & $10-19-87$ & 35.9 \\
\hline
\end{tabular}

\subsubsection{New Production Reactor (NPR) EIS}

Baseline noise estimates were determined for two locations: SR 24, leading from the Hanford Site west to Yakima, and SR 240, south of the Hanford Site and west of Richland where it handles maximum traffic volume (DOE 1991). Traffic volumes were predicted based on an operational work force and a construction work force. Both peak (rush hour) and off-peak hours were modeled. Noise levels were expressed in $\mathrm{L}_{\mathrm{eq}}$ for 1-hr periods in dBA at a receptor located $15 \mathrm{~m}(49 \mathrm{ft})$ from the road edge (Table 4.73). Adverse community responses would not be expected at increases of $5 \mathrm{dBA}$ over background noise levels. 
Table 4.7-3. Modeled Noise Resulting from Automobile Traffic at Hanford in Association with the New Production Reactor Environmental Impact Statement (DOE 1991) ${ }^{\text {(a) }}$

\begin{tabular}{|c|c|c|c|c|c|c|c|}
\hline \multirow{2}{*}{\multicolumn{2}{|c|}{ Location $^{(\mathbf{b})}$}} & \multirow[b]{2}{*}{ Scenario } & \multicolumn{2}{|c|}{$\begin{array}{c}\text { Traffic flow } \\
\text { (Vehicles/hr) }\end{array}$} & \multicolumn{2}{|c|}{$\begin{array}{c}\text { Noise levels } \\
\left(\mathrm{L}_{\mathrm{eq}}-\mathbf{1} \mathrm{hr} \text { in dBA }\right) \\
\end{array}$} & \multirow[b]{2}{*}{$\begin{array}{c}\text { Maximum } \\
\text { Increase } \\
(\text { dBA })\end{array}$} \\
\hline & & & Baseline & Maximum $^{(\mathrm{c})}$ & $\begin{array}{c}\text { Baseline } \\
\text { Noise } \\
\text { Levels }\end{array}$ & $\begin{array}{c}\text { Modeled } \\
\text { Noise } \\
\text { Levels }^{(\mathrm{c})}\end{array}$ & \\
\hline \multicolumn{8}{|c|}{$\begin{array}{l}\text { Construction } \\
\text { Phase }\end{array}$} \\
\hline & SR 24 & $\begin{array}{l}\text { Off- } \\
\text { Peak } \\
\text { Peak }\end{array}$ & $\begin{array}{l}91 \\
91\end{array}$ & $\begin{array}{r}91 \\
343\end{array}$ & $\begin{array}{l}62.0 \\
62.0\end{array}$ & 62.0 & 0.0 \\
\hline & SR 240 & $\begin{array}{l}\text { Off- } \\
\text { Peak } \\
\text { Peak }\end{array}$ & $\begin{array}{l}571 \\
571\end{array}$ & $\begin{array}{r}579 \\
2839\end{array}$ & $\begin{array}{l}70.2 \\
70.2\end{array}$ & $\begin{array}{l}70.6 \\
73.5\end{array}$ & $\begin{array}{l}0.4 \\
3.3\end{array}$ \\
\hline \multicolumn{8}{|c|}{ Operation Phase } \\
\hline & SR 24 & $\begin{array}{l}\text { Off- } \\
\text { Peak } \\
\text { Peak }\end{array}$ & $\begin{array}{r}91 \\
300\end{array}$ & $\begin{array}{r}91 \\
386\end{array}$ & $\begin{array}{l}62.0 \\
65.7\end{array}$ & $\begin{array}{l}62.0 \\
66.2\end{array}$ & $\begin{array}{l}0.0 \\
1.5\end{array}$ \\
\hline & SR 240 & $\begin{array}{l}\text { Off- } \\
\text { Peak } \\
\text { Peak }\end{array}$ & $\begin{array}{r}571 \\
2239\end{array}$ & $\begin{array}{r}582 \\
3009\end{array}$ & $\begin{array}{l}70.2 \\
74.1\end{array}$ & $\begin{array}{l}70.5 \\
74.7\end{array}$ & $\begin{array}{l}0.3 \\
0.6\end{array}$ \\
\hline $\begin{array}{l}\text { (a) } \\
\text { (b) } \\
\text { (c) }\end{array}$ & $\begin{array}{l}\text { Measured } 15 \\
\text { SR } 24 \text { leads t } \\
\text { Traffic flow } \\
\text { techniques a }\end{array}$ & $\begin{array}{l}9 \mathrm{ft} \text { ) from th } \\
\text { kima; SR } 24 \\
\text { hoise estimat } \\
\text { hown here. }\end{array}$ & $\begin{array}{l}\text { le road edge } \\
0 \text { leads to t } \\
\text { es varied w }\end{array}$ & $\begin{array}{l}\text { Tri-Cities area } \\
\text { th NPR technolo }\end{array}$ & $y$; the max & Im impacts & three NPR \\
\hline
\end{tabular}

\subsubsection{Noise Levels of Hanford Field Activities}

In the interest of protecting Hanford workers and complying with Occupational Safety and Health Administration (OSHA) standards for noise in the workplace, the Hanford Environmental Health Foundation (HEHF) has monitored noise levels resulting from several routine operations performed at Hanford. Occupational sources of noise propagated in the field have been summarized in Table 4.7-4. These levels are reported here because operations such as well sampling are conducted in the field away from established industrial areas and have the potential for disturbing sensitive wildlife. 
Table 4.7-4. Monitored Levels of Noise Propagated from Outdoor Activities at the Hanford Site ${ }^{\text {(a) }}$

\begin{tabular}{|c|c|c|c|c|}
\hline Activity & $\begin{array}{c}\text { Average } \\
\text { Noise } \\
\text { Level }\end{array}$ & $\begin{array}{l}\text { Maximum } \\
\text { Noise Level }\end{array}$ & $\begin{array}{c}\text { Year } \\
\text { Measured }\end{array}$ & Distance \\
\hline Water wagon operation $^{(a)}$ & 104.5 & 111.9 & 1984 & On staff member \\
\hline Well sampling $^{(a)}$ & $74.8-78.2$ & & 1987 & On staff member \\
\hline Truck $^{(\mathrm{a})}$ & $78-83$ & & 1989 & On staff member \\
\hline Compressor $^{(\mathrm{b})}$ & $88-90$ & & & $0.3 \mathrm{~m}(1 \mathrm{ft})$ from truck \\
\hline Generator $^{(b)}$ & $93-95$ & & & $0.3 \mathrm{~m}(1 \mathrm{ft})$ from truck \\
\hline Well drilling, Well 32-2(a) & $98-102$ & 102 & 1987 & $23 \mathrm{~m}(75 \mathrm{ft})$ \\
\hline Well drilling, Well 32-3 $3^{(a)}$ & $105-11$ & $120-125$ & 1987 & $15 \mathrm{~m}(49 \mathrm{ft})$ \\
\hline Well drilling, Well 33-29(a) & $89-91$ & & 1987 & $15 \mathrm{~m}(49 \mathrm{ft})$ \\
\hline Pile driver $^{(a)}$ & $118-119$ & & 1981 & $1.5 \mathrm{~m}(5 \mathrm{ft})$ \\
\hline Tank farm filter building ${ }^{(a)}$ & 86 & & 1976 & $9.0 \mathrm{~m}(30 \mathrm{ft})$ \\
\hline
\end{tabular}

\subsection{Occupational Safety}

\section{E. Antonio}

Total occupational work hours at the Hanford Site for the 5-year period, 1996 through 2000, were 121,552,993 hours, or about 63,975 worker-years (DOE 2001b). The DOE records occupational injuries and illnesses in four categories pertinent to NEPA analysis. Total Recordable Cases (TRC) are workrelated deaths, illnesses, or injuries that resulted in loss of consciousness, restriction of work or motion, transfer to another job, or required medical treatment beyond first aid. Lost Workday Cases (LWC) represent the number of recordable cases that resulted in days away from work or days of restricted work activity, or both, for affected employees. Lost Workdays (LWD) are the total number of workdays (consecutive or not), after the day of injury or onset of illness, during which employees were away from work or limited to restricted work activity because of an occupational injury or illness. Fatalities are the number of occupationally related deaths. Information on occupational safety used in this section is updated quarterly and is available at URL: http://tis.eh.doe.gov/cairs

Occupational injury and illness incidence rates at the Hanford Site have been decreasing since 1996. Figure 4.8-1 shows 4.4 TRC per 200,000 worker hours (100 worker years) in 1996. By 2000 the rate had decreased to 2.0 cases per 200,000 worker hours. Over the 5-year period from 1996 through 2000, the average Hanford Site incidence rate, 2.9 cases per 200,000 worker hours, was similar to the average incidence rate for the entire DOE complex, which was 3.1 cases per 200,000 worker hours. Both the Hanford Site and DOE-wide average TRC rates were well below the Bureau of Labor Statistics (BLS) rates for U.S. private industry during the same period. 


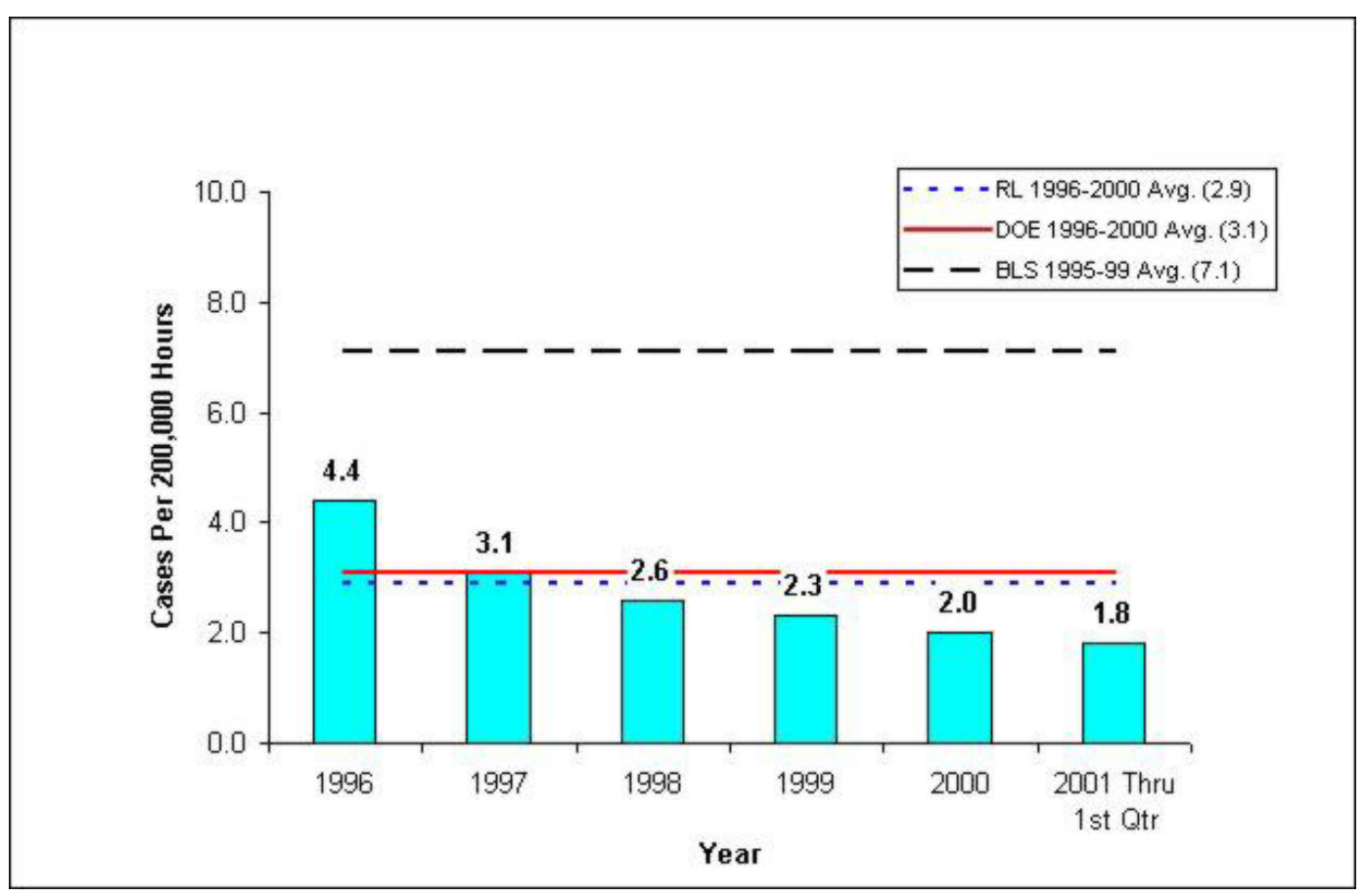

Figure 4.8-1. Occupational Injury and Illness Total Recordable Case Rates at the Hanford Site (DOE 2001b)

Table 4.8-1 shows occupational injury, illness, and fatality rates reported for the private sector by the Bureau of Labor Statistics (Department of Labor), and throughout the DOE complex including the DOERL (DOE 2001b). During the 5-year period from 1996-2000, the Hanford Site TRC, LWC, and LWD rates were slightly higher than those for the overall DOE complex and approximately $58 \%$ of comparable rates for the private sector. The fatality rate for DOE facilities was less than $30 \%$ of that for the private sector, and the Hanford Site had no occupational fatalities during the period.

Table 4.8-1. Occupational Injury, Illness, and Fatality Incidence Rates for Department of Energy Facilities and Private Industries, 1996-2000 Averages (DOE 2001b) ${ }^{\text {(a) }}$

\begin{tabular}{|c|c|c|c|c|}
\hline & $\begin{array}{l}\text { Total } \\
\text { Recordable } \\
\text { Cases }\end{array}$ & $\begin{array}{l}\text { Lost Work } \\
\text { Cases }\end{array}$ & $\begin{array}{c}\text { Lost Work } \\
\text { Days }\end{array}$ & Fatalities \\
\hline Bureau of Labor Statistics ${ }^{(b)}$ & 7.1 & 3.3 & $\mathrm{~N} / \mathrm{A}^{(\mathrm{c})}$ & 0.0048 \\
\hline U.S. Department of Energy ${ }^{(d)}$ & 3.1 & 1.4 & 42.8 & 0.0015 \\
\hline $\begin{array}{l}\text { DOE Richland Operations Office } \\
\text { (DOE-RL), Hanford Site }^{(d)}\end{array}$ & 4.1 & 1.8 & 66.8 & 0 \\
\hline \multicolumn{5}{|c|}{$\begin{array}{l}\text { (a) Per 200,000 worker hours ( } 100 \text { worker-years). } \\
\text { (b) BLS values are average rates for the private sector from } 1995 \text { to } 1999 . \\
\text { (c) N/A = data not available. } \\
\text { (d) DOE-wide and Hanford Site values are average rates from } 1996 \text { through }\end{array}$} \\
\hline
\end{tabular}




\section{References}

40 CFR 201-211. Subchapter G. Protection of Environment: Noise Abatement Programs. U.S. Code of Federal Regulations.

40 CFR 50. National Primary and Secondary Ambient Air Quality Standards. U.S. Code of Federal Regulations.

40 CFR 81.348. Designation of Areas for Air Quality Planning Purposes, Washington, Attainment Status Designations. U.S. Environmental Protection Agency.

40 CFR 81.434. Designation of Areas for Air Quality Planning Purposes, Washington, Identification of Mandatory Class I Federal Areas Where Visibility Is an Important Value. U.S. Environmental Protection Agency.

50 CFR 17 (1986). U.S. Department of the Interior, "Endangered and Threatened Wildlife and Plants." U.S. Code of Federal Regulations.

59 FR 7629. Environmental Justice. Executive Order 12898, "Federal Actions to Address Environmental Justice in Minority Populations and Low-income Populations." Federal Register, February 16, 1994. Washington, D.C.

64 FR 61615 (1999). Record of Decision on the Comprehensive Land Use Plan (CLUP) Environmental Impact Statement (EIS) for Hanford. Federal Register, Volume 64, pp 6161561625.

65 FR 37253. Proclamation 7319 of June 9, 2000, Establishment of the Hanford Reach National Monument. Presidential Documents, June 13, 2000.

66 FR 9663. Clean Air Act Reclassification; Wallula, Washington Particulate Matter (PM-10) Nonattainment Area. Environmental Protection Agency.

Agnew, S.F. 1997. Hanford Tank Chemical and Radionuclide Inventories: HDW Model, Rev. 4. LA-UR-96-3860, [Online Report]. Available URL: http://lib-www.lanl.gov/lapubs/00326181.pdf

Anderson, J.D. 1990. A History of the 200 Area Tank Farms. WHC-MR-0132, Westinghouse Hanford Company, Richland, Washington.

Andrefsky, Jr., W., L.L. Hale, and D.A. Harder. 1996. 1995 WSU Archaeological Block Survey of the Hanford 600 Area. Project Report No. 29. Center for Northwest Anthropology, Department of Anthropology, Washington State University, Pullman, Washington.

BAER 2000. 24 Command Fire Burn Area Emergency Rehabilitation (BAER) Plan. Northern States Burned Area Emergency Rehabilitation Team, U.S. Department of the Interior, Washington, D.C. 
Baker, V.R. 1978. "Large-Scale Erosional and Depositional Features of the Channeled Scabland." In: V.R. Baker and D. Nummedal, eds. The Channeled Scabland. National Aeronautics and Space Administration, Washington, D.C.

Baker, V.R., B.N. Bjornstad, A.J. Busacca, K.R. Fecht, U.L. Moody, J.G. Rigby, D.F. Stradling, and A.M. Tallman. 1991. "Quaternary Geology of the Columbia Plateau." In Quaternary Nonglacial Geology; Conterminous U.S., ed., R. B. Morrison, The Geology of North America, Geological Society of America, Vol. K-2, Boulder, Colorado.

Bard, J.C. 1997. "Ethnographic /Contact Period (Lewis and Clark 1805 - Hanford Engineer Works 1943) of the Hanford Site, Washington." In National Register of Historic Places Multiple Property Documentation Form - Historic, Archaeological and Traditional Cultural Properties of the Hanford Site, Washington. DOE/RL-97-02. Richland, Washington.

Bauer, H.H., J.J. Vaccaro, and R.C. Lane. 1985. Maps Showing Ground-Water Levels in the Columbia River Basalt and Overlying Materials, Spring 1983, Southeastern Washington. Water Resources Investigation Report 84-4360, U.S. Geological Survey, Tacoma, Washington.

Beak Consultants Inc. 1980. Aquatic Ecological Studies Near WNP-1, 2 and 4. August 1978March 1980. WPPSS Columbia River Ecology Studies, Vol. 7, prepared for Washington Public Power Supply System by Beak Consultants Inc., Portland, Oregon.

Becker, J.M. 1993. A Preliminary Survey of Selected Structures on the Hanford Site for Townsend's Big-Eared Bat (Plecotus townsendii). PNL-8916, Pacific Northwest Laboratory, Richland, Washington.

Bentley, R.D., J.L. Anderson, N.P. Campbell, and D.A. Swanson. 1980. Stratigraphy and Structure of the Yakima Reservation, with Emphasis on the Columbia River Basalt Group. OpenFile Report 80-200, U.S. Geological Survey, Washington, D.C.

Bisping, L.E. 2000. Hanford Site Environmental Surveillance Data Report for Calendar Year 1999. PNNL-13230, App. 1, Pacific Northwest National Laboratory, Richland, Washington.

Bjornstad, B.N. 1984. Suprabasalt Stratigraphy Within and Adjacent to the Reference Repository Location. SD-BWI-DP-039, Rockwell Hanford Operations, Richland, Washington.

Bjornstad, B.N. 1985. "Late-Cenozoic Stratigraphy and Tectonic Evolution Within a Subsiding Basin, South-Central Washington." Geological Society of America Abstracts with Programs 17(7): 524.

Bjornstad, B.N., and K.R. Fecht. 1989. "Pre-Wisconsin Glacial Outburst Floods: Pedogenic and Paleomagnetic Evidence from the Pasco Basin and Adjacent Channeled Scabland." Geological Society of America Abstracts with Programs 21(5): 58.

Bjornstad, B.N., K.R. Fecht, and A.M. Tallman. 1987. Quarternary Stratigraphy of the Pasco Basin Area, South-central Washington. RHO-BW-SA-563A, Rockwell Hanford Operations, Richland, Washington.

Brandt, C.A., C.E. Cushing, W.H. Rickard, N.A. Cadoret, and R. Mazaika. 1993. Biological Resources of the 300-FF-5 Operable Unit. WHC-SD-EN-TI-121, Westinghouse Hanford Company, Richland, Washington. 
Brown, D.J. 1960. An Eolian Deposit Beneath 200-West Area. HW- 67549, General Electric Company, Richland, Washington.

Brown, R.E. 1970. Interrelationships of Geologic Formations and Processes Affecting Ecology as Exposed at Rattlesnake Springs, Hanford Project. BNWL-B-29, Pacific Northwest Laboratories, Richland, Washington.

Brown, R.E. 1979. A Review of Water Well Data from the Unconfined Aquifer in the Eastern and Southern Parts of the Pasco Basin. RHO-BWI-C-56, Rockwell Hanford Operations, Richland, Washington.

Cadoret, N.A. 1993. Cultural Resources Report for the Environmental Restoration Disposal Facility. HCRC\# 93-200-001. Pacific Northwest Laboratory, Richland, Washington.

Cadwell, L.L. 1994. Wildlife Studies on the Hanford Site: 1993 Highlights Report. PNL-9380, Pacific Northwest Laboratory, Richland, Washington.

Cardenas, A., J. Lewinsohn, C. Auger, J.L. Downs, L.L. Cadwell, and R. Burrows. 1997. Characterization of a Sagebrush (Artemisia tridentata spp. wyomingensis) Die-Off on the Hanford Site. PNNL-11673, Pacific Northwest National Laboratory, Richland, Washington.

CEQ (see Council on Environmental Quality).

Chatters, J.C. 1982. "Prehistoric Settlement and Land Use in the Dry Columbia Basin." Northwest Anthropol. Res. Notes 16:125-147.

Chatters, J.C., ed. 1989. Hanford Cultural Resources Management Plan. PNL-6942, Pacific Northwest Laboratory, Richland, Washington.

Chatters, J.C., and N.A. Cadoret. 1990. Archeological Survey of the 200-East and 200-West Areas, Hanford Site, Washington. PNL-7264, Pacific Northwest Laboratory, Richland, Washington.

Chatters, J.C., and H.A. Gard. 1992. Hanford Cultural Resources Laboratory Annual Report for Fiscal Year 1991. PNL-8101, Pacific Northwest Laboratory, Richland, Washington.

Chatters, J.C., N.A. Cadoret, and P.E. Minthorn. 1990. Hanford Cultural Resources Laboratory Annual Report for Fiscal Year 1989. PNL-7362, Pacific Northwest Laboratory, Richland, Washington.

Chatters J.C., H.A. Gard, and P.E. Minthorn. 1991. Hanford Cultural Resources Laboratory Annual Report for Fiscal Year 1990. PNL-7853, Pacific Northwest Laboratory, Richland, Washington.

Chatters, J.C., H.A. Gard, and P.E. Minthorn. 1992. Fiscal Year 1991 Report on Archaeological Surveys of the 100 Areas, Hanford Site, Washington. PNL-8143, Pacific Northwest Laboratory, Richland, Washington.

CNSS (see Council of the National Seismic System). 
Connelly, M.P., B.H. Ford, and J.V. Borghese. 1992a. Hydrogeologic Model for the 200 West

Groundwater Aggregate Area. WHC-SD-EN-TI-014, Rev. 0, Westinghouse Hanford Company, Richland, Washington.

Connelly, M.P., B.H. Ford, J.W. Lindberg, S.J. Trent, C.D. Delaney, and J.V. Borghese. 1992b. Hydrogeologic Model for the 200 East Groundwater Aggregate Area. WHC-SD-EN-TI-019, Westinghouse Hanford Company, Richland, Washington.

Coopey, R.W. 1953. The Abundance of the Principal Crustacea of the Columbia River and the Radioactivity they Contain. HW-251, Hanford Atomic Products Operation, Richland, Washington.

Corps (see U.S. Army Corps of Engineers).

Council of the National Seismic System (CNSS). 2001. On-line Earthquake Catalog. Council of the National Seismic System. Available URL: http://www.quake.geo.berkeley.edu/cnss

Council on Environmental Quality (CEQ). 1997. Environmental Justice. Guidance Under the National Environmental Policy Act. Executive Office of the President, Washington, D.C.

Cushing, C.E., Jr. 1967a. "Concentration and Transport of ${ }^{32} \mathrm{P}$ and ${ }^{65} \mathrm{Z}$ by Columbia River Plankton." Limnol. Oceanogr. 12:330-332.

Cushing, C.E., Jr. 1967b. "Periphyton Productivity and Radionuclide Accumulation in the Columbia River, Washington, USA." Hydrobiologia 29:125-139.

Cushing, C.E., and E.G. Wolf. 1982. "Organic Energy Budget of Rattlesnake Springs, Washington." Am. Midl. Nat. 107(2): 404-407.

Cushing, C.E., and E.G. Wolf. 1984. "Primary Production in Rattlesnake Springs, a Cold Desert Spring-Stream." Hydrobiologia 114:229-236.

Cushing, C.E., C.D. McIntire, J.R. Sedell, K.W. Cummins, G.W. Minshall, R.C. Petersen, and R.L. Vannote. 1980. "Comparative Study of Physical-Chemical Variables of Streams Using Multivariate Analyses." Arch. Hydrobiologia 89(3):343-352.

DART (see Data Acquisition in Real Time).

Data Acquisition in Real Time (DART). 2001. Columbia Basin Research Project, Department of Fisheries, University of Washington, Seattle, Washington. Available URL:

http://www.cbr.washington.edu/dart/headwater com.html

Daubenmire, R. 1970. Steppe Vegetation of Washington. Technical Bulletin 62, Experimental Station, Washington State University, Pullman, Washington.

Dauble, D.D., and D.G. Watson. 1990. Spawning and Abundance of Fall Chinook Salmon (Oncorhynchus tshawytscha) in the Hanford Reach of the Columbia River, 1948-1988. PNL7289, Pacific Northwest Laboratory, Richland, Washington.

Dauble, D.D., and D.G. Watson. 1997. Status of Fall Chinook Salmon Populations in the Mid Columbia River, 1948-1992. North American Journal of Fisheries Management 17: 283-300. 
Daugherty, R.D. 1952. “Archaeological Investigations of O'Sullivan Reservoir, Grant County, Washington." American Antiquity 17: 274-278.

Davis, J.J., and C.L. Cooper. 1951. Effect of Hanford Pile Effluent upon Aquatic Invertebrates in the Columbia River. HW-20055, Hanford Works, Richland, Washington.

Davis, J. J., and C. L. Cooper. 1951. Effect of Hanford Pile Effluent upon Aquatic Invertebrates in the Columbia River. HW-20055, Hanford Works, Richland, Washington.

Davis, M. 2000. United Way of Benton and Franklin Counties, Tri-Cities, Washington.

Delaney, C.D., K.A. Lindsey, and S.P. Reidel. 1991. Geology and Hydrology of the Hanford Site: A Standardized Text for Use in Westinghouse Hanford Company Documents and Reports. WHC-SD-ER-TI-0003, Rev. 0, Westinghouse Hanford Company, Richland, Washington.

Dirkes, R.L. 1993. Columbia River Monitoring: Distribution of Tritium in Columbia River Water at the Richland Pumphouse. PNL-8531, Pacific Northwest Laboratory, Richland, Washington.

Dirkes, R.L., and R.W. Hanf. 1998. Hanford Site Environmental Report for Calendar Year 1997. PNNL-11795, Pacific Northwest National Laboratory, Richland, Washington.

DOC (see U.S. Department of Commerce).

DOI (see U.S Department of the Interior).

DOL (see U.S. Department of Labor Employment and Training Administration).

Dorian, J.J., and V.R. Richards. 1978. Radiological Characterization of the Retired 100 Areas. UNI-946, United Nuclear Industries, Inc., Richland, Washington.

Downs, J.L., W.H. Rickard, C.A. Brandt, L.L. Cadwell, C.E. Cushing, D.R. Geist, R.M. Mazaika, D.A. Neitzel, L.E. Rogers, M.R. Sackschewsky, and J.J. Nugent. 1993. Habitat Types on the Hanford Site: Wildlife and Plant Species of Concern. PNL-8942, Pacific Northwest Laboratory, Richland, Washington.

Drost, B.W., K.M. Schurr, and W.E. Lum II. 1989. Selected Ground-Water Information for the Pasco Basin and Adjacent Areas, Washington, 1986-1989. Open-File Report 89-228, U.S. Geological Survey, Tacoma, Washington.

Drucker, P. 1948. Appraisal of the Archaeological Resources of the McNary Reservoir, OregonWashington. Report on file, Columbia Basin Project, River Basin Survey, Smithsonian Institution, Washington, D.C.

Eberhardt, L.E., R.E. Anthony, and W.H. Rickard. 1989. "Survival of Juvenile Canada Geese during the Rearing Period." J. Wildlife Management 53:372-377.

Ecology (see Washington State Department of Ecology) 
Emery, R.M., and M.C. McShane. 1978. Comparative Ecology of Nuclear Waste Ponds and Streams on the Hanford Site. PNL-2499, Pacific Northwest Laboratory, Richland, Washington.

Emery, R.M., and M.C. McShane. 1980. "Nuclear Waste Ponds and Streams on the Hanford Site: An Ecological Search for Radiation Effects." Health Physics 38:787-809.

Energy Northwest. 2001. Energy Northwest Financial Information Web Page. Available URL: http://www.wnp2.com/CorpINFO/financia.htm

ERDA (see U.S. Energy Research and Development Administration).

ERTEC. 1981. Cultural Resources Survey and Exploratory Excavations for the Skagit-Hanford Nuclear Power Project. ERTEC Northwest, Seattle, Washington.

ERTEC. 1982. A Cultural Resources Overview and Scenic and Natural Resources Assessment for the Skagit-Hanford Nuclear Power Project. ERTEC Northwest, Seattle, Washington.

Evergreen Community Development Association. 1986. Tri-Cities Enterprise Center Business Development Plan. Evergreen Community Development Association, Richland, Washington.

Fayer M.J., G.W. Gee, M.L. Rockhold, M.D. Freshley, and T.B. Walters. 1996. "Estimating recharge rates for a groundwater model using a GIS. " Journal of Environmental Quality 25: 510-518.

Fecht, K.R., R.E. Gephart, D.L. Graham, S.P. Reidel, and A.C. Rohay. 1984. Summary of Geotechnical Information in the Rattlesnake Mountain Area. SD-BWI-TI-247, Rockwell Hanford Operations, Richland, Washington.

Fecht, K.R., S.P. Reidel, and A.M. Tallman. 1987. Paleodrainage of the Columbia River System on the Columbia Plateau of Washington State - A Summary. Washington Division of Geology and Earth Resources Bulletin 77.

Fecht, K.R., K.A. Lindsey, B.N. Bjornstad, D.G. Horton, G.V. Last, and S.P. Reidel. 1998. An Atlas of Clastic Injection Dikes of the Pasco Basin and Vicinity. BHI-01103, [Online Report]. Available URL: http://www.erc.rl.gov/ pgs/readroom/BHI/01103/Intro.pdf

Fecht, K.R., K.A. Lindsey, B.N. Bjornstad, D.G. Horton, G.V. Last, and S.P. Reidel. 1999. Clastic Injection Dikes of the Pasco Basin and Vicinity - Geologic Atlas Series. BHI-01103, Rev. 0, [Online Report]. Available URL: http://www.erc.rl.gov/ pgs/ClasticDikes.pdf

Fitzner, R.E., and R.H. Gray. 1991. "The Status, Distribution, and Ecology of Wildlife on the U.S. DOE Hanford Site: A Historical Overview of Research Activities." Environ. Monit. Assess. 18:173-202.

Fitzner, R.E., and S.G. Weiss. 1994. Bald Eagle Site Management Plan for the Hanford Site, South Central Washington. DOE/RL-94-150, U.S. Department of Energy, Richland, Washington.

Fitzner, R.E., S.G. Weiss, and J.A. Stegen. 1994. Threatened and Endangered Wildlife Species of the Hanford Site Related to CERCLA Characterization Activities. WHC-EP-0513, Westinghouse Hanford Company, Richland, Washington. 
Freshley, M.D., and M.J. Graham. 1988. Estimation of Ground-Water Travel Time at the Hanford Site: Description, Past Work, and Future Needs. PNL-6328, Pacific Northwest National Laboratory, Richland, Washington.

Frest, T.J., and E.J. Johannes. 1993. Mollusc Survey of the Hanford Site, Benton and Franklin Counties, Washington. PNL-8653, Pacific Northwest Laboratory, Richland, Washington.

Gaines, W.E. 1987. Secondary Production of Benthic Insects in Three Cold Desert Streams. PNL-6286, Pacific Northwest Laboratory, Richland, Washington.

Gaines, W.L., C.E. Cushing, and S.D. Smith. 1992. "Secondary Production Estimates of Benthic Insects in Three Cold Desert Streams." Great Basin Naturalist 52(1):11-24.

Gard, H.A., and R.M. Poet. 1992. Archaeological Survey of the McGee Ranch Vicinity, Hanford Site, Washington. PNL-8186, Pacific Northwest Laboratory, Richland, Washington.

Gee, G.W., M.J. Fayer, M.L. Rockhold, and M.D. Campbell. 1992. "Variations in Recharge at the Hanford Site." Northwest Science 66(4): 237.

Geomatrix. 1994. Probabilistic Seismic Hazard Assessment DOE Hanford Site, Washington. Prepared for Westinghouse Hanford Company. WHC-SD-W236A-TI-002, Westinghouse Hanford Company, Richland, Washington.

Geomatrix. 1996. Probabilistic Seismic Hazard Analysis DOE Hanford Site, Washington. Prepared for Westinghouse Hanford Company. WHC-SD-W236A-TI-002, Rev. 1, Westinghouse Hanford Company, Richland, Washington.

Gephart, R.E. 1999. A Short History of Plutonium Production and Nuclear Waste Generation, Storage, and Release at the Hanford Site. PNNL-SA-32152, Pacific Northwest National Laboratory, Richland, Washington.

Gephardt, R.E., P.A. Eddy, R.C. Arnett, and G.A. Robinson. 1976. Geohydrologic Study of the West Lake Basin. ARH-CD-775, Atlantic Richfield Hanford Company, Richland, Washington.

Gilmore, T.J., D.R. Newcomer, S.K. Wurstner, and F.A. Spane Jr. 1992. Calculation of Groundwater Discharge to the Columbia River in the 100-N Area. PNL-8057, Pacific Northwest Laboratory, Richland, Washington.

Glantz, C. S., and M. M. Islam. 1988. The Data Collection Component of the Hanford Meteorology Monitoring Program. PNL-6684, Pacific Northwest Laboratory, Richland, Washington.

Glantz, C. S., M. N. Schwartz, K. W. Burk, R. B. Kasper, M. W. Ligotke, and P. J. Perrault. 1990. Climatological Summary of Wind and Temperature Data for the Hanford Meteorology Monitoring Network. PNL-7471, Pacific Northwest Laboratory, Richland, Washington.

Graham, M.J., M.D. Hall, S.R. Straight, and W.R. Brown. 1981. Hydrology of the Separations Area. RHO-ST-42, Rockwell Hanford Operations, Richland, Washington. 
Graham, M.J., G.V. Last, and K.R. Fecht. 1984. An Assessment of Aquifer Intercommunication with B Pond-Gable Mountain Pond Area of the Hanford Site. RHO-RE-ST-12P, Rockwell Hanford Operations, Richland, Washington.

Gray, R.H., and D.D. Dauble. 1977. "Checklist and Relative Abundance of Fish Species from the Hanford Reach of the Columbia River.” Northwest Science 51:208-215.

Greene, G.S. 1975. Prehistoric Utilization of the Channeled Scablands of Eastern Washington. Ph.D. Dissertation, Department of Anthropology, Washington State University, Pullman, Washington.

Greengo, R.E. 1982. Studies in Prehistory: Priest Rapids and Wanapum Reservoir Areas, Columbia River, Washington. Department of Anthropology, University of Washington, Seattle, Washington.

Grolier, M.J. 1978. Geology of Parts of Grant, Adams, and Franklin Counties, East-Central Washington. Bulletin 71, Washington State Department of Natural Resources, Olympia, Washington.

Grolier, M.J., and J.W. Bingham. 1978. Geology of Parts of Grant, Adams, and Franklin Counties, East-Central Washington. Bulletin 71, Washington State Department of Natural Resources, Olympia, Washington.

Gustafson, E.P. 1973. The Vertebrate Fauna of the Late Pliocene Ringold Formation, SouthCentral Washington. Washington State University, Pullman, Washington.

Hagood, M.C. 1985. Structure and Evolution of the Horse Heaven Hills in South-Central Washington. RHO-BWI-SA-344 P, Rockwell Hanford Operations, Richland, Washington.

Hajek, B.F. 1966. Soil Survey: Hanford Project in Benton County, Washington. BNWL-243, Pacific Northwest Laboratories, Richland, Washington.

Hall, J.A. (ed.). 1998. Biodiversity Inventory and Analysis of the Hanford Site, 1997 Annual Report. The Nature Conservancy of Washington, Seattle, Washington.

Hanlon, B.M. 2001. Waste Tank summary Report for Month Ending January 31, 2001. HNFEP-0182-154, [Online Report]. Available URL: http://www.doe.gov/bridge

Hanson, W.C., and R.L. Browning. 1959. "Nesting Studies of Canada Geese on the Hanford Reservation, 1953-1956." J. Wildlife Manage. 23:129-137.

Harris, S., and B.L. Harper. 1997. "A Native American Exposure Scenario" Society for Risk Analysis 17(6): 789-795.

Hartman, M.J., ed. 2000. Hanford Site Groundwater: Setting, Sources, and Methods. PNNL13080, [Online Report]. Available URL: http://hanford-

site.pnl.gov/groundwater/reports/gwset $99 / \mathrm{html} / \mathrm{start} 1 . \mathrm{htm}$

Hartman, M.J., and K.A. Lindsey. 1993. Hydrogeology of the 100-N Area, Hanford Site, Washington. WHC-SD-EN-EV-027, Rev. 0, Westinghouse Hanford Company, Richland, Washington. 
Hartman, M.J., and R.E. Peterson. 1992. Hydrologic Information Summary for the Northern Hanford Site. WHC-SD-EN-TI-023, Westinghouse Hanford Company, Richland, Washington.

Hartman, M.J., L.F. Morasch, and W.D. Webber, eds. 2000. Hanford Site Groundwater Monitoring for Fiscal Year 1999, PNNL-13116, Pacific Northwest National Laboratory, Richland Washington.

Hartman, M.J., L.F. Morasch, and W.D. Webber, eds. 2001. Hanford Site Groundwater Monitoring for Fiscal Year 2000. PNNL-13404, [Online Report]. Available URL: http://hanford-site.pnl.gov/groundwater/reports/gwrep00/start.htm

Hoitink, D.J., K.W. Burk, and J.V. Ramsdell, Jr. 2001. Hanford Site Climatological Data Summary 2000 With Historical Data. PNNL-13469, Pacific Northwest National Laboratory, Richland, Washington.

Horton, D.G., and R.R. Randall. 2000. Results of 1999 Spectral Gamma-Ray and Neutron Moisture Monitoring of Boreholes at Specific Retention Facilities In the 200 East Area, Hanford Site. PNNL-13077, [Online Report]. Available URL:

http://www.osti.gov/bridge/product.biblio.jsp?osti id $=750263$

Jamison, J.D. 1982. Standardized Input for Hanford Environmental Impact Statements Part II: Site Descriptions. PNL-3509, Pt. 2, Pacific Northwest Laboratory, Richland, Washington.

Jenkins, O.P. 1922. Underground Water Supply of the Region About White Bluffs and Hanford. Bulletin No. 26, Division of Geology, State of Washington Department of Conservation and Development, Olympia, Washington.

Jensen, E.J. 1987. An Evaluation of Aquifer Intercommunication Between the Unconfined and Rattlesnake Ridge Aquifers on the Hanford Site. PNL-6313, Pacific Northwest Laboratory, Richland, Washington.

Johnson, V.G., F.N. Hodges, and S.P. Reidel. 1992. "Arid Environment Aquifers: An Example from South Central Washington.” Geological Society of America (ABS) 24(5):36.

Johnson, V.G., D.L. Graham, and S.P. Reidel. 1993. "Methane in Columbia River Basalt Aquifers: Isotopic and Geohydrologic Evidence for a Deep Coal-Bed Gas Source in the Columbia Basin, Washington." American Association of Petroleum Geologists Bulletin 77(7): 1192-1207.

Johnson, V.G., C.J. Chou, and J.W. Lindberg. 1995. Groundwater Monitoring and Assessment Plan for the 100-K Area Fuel Storage Basins. WHC-SD-EN-AP-174, Westinghouse Hanford Company, Richland, WA.

Jones, T.E., R. Khaleel, D.A. Myers, J.W. Shade, and M.I. Wood. 1998. Summary and Evaluation of Hanford Site Tank Farm Subsurface Contamination. HNF-2603, Rev. 0, Lockheed Martin Hanford, Richland, Washington. 
Kincaid, C.T., M.P. Bergeron, C.R. Cole, M.D. Freshley, N.L. Hassig, V.G. Johnson, D.I. Kaplan, R.J. Serne, G.P. Streile, D.L. Strenge, P.D. Thorne, L.W. Vail, G.A. Whyatt, and S.K. Wurstner. 1998. Composite Analysis for Low-Level Waste Disposal in the 200 Areas Plateau of the Hanford Site. PNNL-11800, Pacific Northwest National Laboratory, Richland, Washington.

Kipp, K.L., and R.D. Mudd. 1973. Collection and Analysis of Pump Test Data for

Transmissivity Values. BNWL-1709, Pacific Northwest Laboratories, Richland, Washington.

Krieger, H.W. 1928. "A Prehistoric Pithouse Village Site at Wahluke, Grant County, Washington.” Proc. U.S. Natl. Mus. 73:1-29.

Landeen, D. (ed.). 1996. Wetes pe m'e wes - I am of this land. [Wildlife of the Hanford Site]. Confluence Press, Lewiston, Idaho.

Landeen, D.S., A.R. Johnson, and R.M. Mitchell. 1992. Status of Birds at the Hanford Site in Southeastern Washington. WHC-EP-0402, Westinghouse Hanford Company, Richland, Washington.

Landeen, D.S., M.R. Sackschewsky, and S. Weiss. 1993. 100 Areas CERCLA Ecological Investigations. WHC-EP-0620, Westinghouse Hanford Company, Richland, Washington.

Last, G.V., B.N. Bjornstad, M.P. Bergeron, D.W. Wallace, D.R. Newcomer, J.A. Schremke, M.A. Chamness, C.S. Cline, S.P. Airhart, and J.S. Wilbur. 1989. Hydrogeology of the 200 Areas Low Level Burial Grounds - An Interim Report. PNL-6820, Pacific Northwest Laboratory, Richland, Washington.

Last, G.V., M.K. Wright, M.E. Crist, N.A. Cadoret, M.V. Dawson, K.A. Simmons, D.W. Harvey, and J.G. Longenecker. 1993. Hanford Cultural Resources Laboratory Annual Report for Fiscal Year 1993. PNL-10077, Pacific Northwest Laboratory, Richland, Washington.

Leonhardy, F.C., and D.G. Rice. 1970. "A Proposed Culture Typology for the Lower Snake River Region, Southeastern Washington.” Northwest Anthropol. Res. Notes 4:1-29.

Liikala, T.L., R.L. Aaberg, N.J. Aimo, D.J. Bates, T.J. Gilmore, E.J. Jensen, G.V. Last, P.L. Oberlander, K.B. Olsen, K.R. Oster, L.R. Roome, J.C. Simpson, S.S. Teel, and J. Westergard. 1988. Geohydrologic Characterization of the Area Surrounding the 183-H Solar Evaporation Basins. PNL-6728, Pacific Northwest Laboratory, Richland, Washington.

Lindberg, J.W. 1993a. Geology of the 100-B/C Area, Hanford Site, South-Central Washington. WHC-SD-EN-TI-133, Westinghouse Hanford Company, Richland, Washington.

Lindberg, J.W. 1993b. Geology of the 100-K Area, Hanford Site, South-Central Washington. WHC-SD-EN-TI-155, Westinghouse Hanford Company, Richland, Washington.

Lindsey, K.A. 1991. Revised Stratigraphy for the Ringold Formation, Hanford Site, South-

Central Washington. WHC-SD-EN-EE-004, Rev. 0, Westinghouse Hanford Company, Richland, Washington.

Lindsey, K.A. 1992. Geology of the Northern Part of the Hanford Site: An Outline of Data Sources and the Geologic Setting of the 100 Areas. WHC-SD-EN-TI-011, Rev. 0, Westinghouse Hanford Company, Richland, Washington. 
Lindsey, K.A. 1996. The Miocene to Pliocene Ringold Formation and Associated Deposits of the Ancestral Columbia River System, South-central Washington and North-central Oregon. Washington Division of Geology and Earth Resources Open File Report 96-8, Washington State Department of Natural Resources, Olympia, Washington.

Lindsey, K.A., and D.R. Gaylord. 1990. Sedimentology and Stratigraphy of the MiocenePliocene Ringold Formation, Hanford Site, South-central Washington. Northwest Science 64: 165-180.

Lindsey, K.A., and G.K. Jaeger. 1993. Geologic Setting of the 100-HR-3 Operable Unit, Hanford Site, South-Central Washington. WHC-SD-EN-TI-132, Westinghouse Hanford Company, Richland, Washington.

Lindsey, K.A., M.P. Connelly, and B.N. Bjornstad. 1991. Geologic Setting of the 200 West Area: An Update. WHC-SD-EN-TI-008, Rev. 0. Westinghouse Hanford Company, Richland, Washington.

Lindsey, K.A., B.N. Bjornstad, J.W. Lindberg, and K.M. Hoffman. 1992. Geologic Setting of the 200 East Area: An Update. WHC-SD-EN-TI-012, Westinghouse Hanford Company, Richland, Washington.

Lindsey, K.A., and G.K. Jaeger. 1993. Geologic Setting of the 100-HR-3 Operable Unit, Hanford Site, South-Central Washington. WHC-SD-EN-TI-132, Westinghouse Hanford Company, Richland, Washington.

Lindsey, K.A., S.P. Reidel, K.R. Fecht, J.L. Slate, A.G. Law, and A.M. Tallman. 1994a. "Geohydrologic Setting of the Hanford Site, South-Central Washington." In: Swanson, D.A. and Haugerud, R.A., eds. Geologic Field Trips in the Pacific Northwest: 1994 Geologic Society of America Annual Meeting.

Lindsey, K.A., J.L. Slate, G.K. Jaeger, K.J. Swett, and R.B. Mercer. 1994b. Geologic Setting of the Low-Level Burial Grounds. WHC-SD-EN-TI-290, Rev. 0., Westinghouse Hanford Company, Richland, Washington.

Lindsey, K.A., S.E. Kos, and K.D. Reynolds. 2000. Vadose Zone Geology of Boreholes 299W22-50 and 299-W23-19, S-SX Waste Management Area, Hanford Site, South-Central

Washington. RPP-6149, Rev. 0. WMTS Sampling and Well Services, Richland, Washington.

LMEA (see Washington State Labor Market and Economic Analysis).

Malde, H.E. 1968. The Catastrophic Late Pleistocene Bonneville Flood in the Snake River Plain, Idaho. Professional Paper 596, U.S. Geological Survey, Washington, D.C.

Marceau, T.E. 1998. Hanford Site Manhattan Project and Cold War Era Historic Treatment Plan. DOE/RL-97-56 Rev. 1 [Online report]. Available URL:

http://www.hanford.gov/docs/r197-56/r197-56.htm

McCormack, W.D., and J.M.V. Carlile. 1984. Investigation of Ground-Water Seepage from the Hanford Shoreline of the Columbia River. PNL-5289, Pacific Northwest Laboratory, Richland, Washington. 
McMahon, W.J., and R.E. Peterson. 1992. Estimating Aquifer Hydraulic Properties Using the Ferris Method, Hanford Site, Washington. DOE/RL-92-64, U.S. Department of Energy, Richland, Washington.

Meinhardt, C.C., and J.C. Frostenson. 1979. Current Status of the 200 Area Ponds. RHO-CD798, Rockwell Hanford Operations, Richland, Washington.

Mize, A.L. 1993. Differential Utilization of Allochthonous and Autochthonous Carbon By Aquatic Insects of Two Shrub-Steppe Desert Spring-Streams: A Stable Carbon Isotope Analysis and Critique of the Method. PNL-8684, Pacific Northwest Laboratory, Richland, Washington.

Morgan, V. 1981. Archaeological Reconnaissance of the North Richland Toll Bridge and Associated Access Roads (L6909). Archaeological and Historical Services, Eastern Washington University, Cheney, Washington.

Mullineaux, D.R., R.E. Wilcox, W.F. Ebaugh, R. Fryxell, and M. Rubin. 1978. "Age of the Last Major Scabland Flood of the Columbia Plateau in Eastern Washington." Quaternary Research 10: $171-180$.

Myers, C.W., S.M. Price, J.A. Caggiano, M.P. Cochran, W.J. Czimer, N.J. Davidson, R.C. Edwards, K.R. Fecht, G.E. Holmes, M.G. Jones, J.R. Kunk, R.D. Landon, K. Ledgerwood, J.T. Lillie, P.E. Long, T.H. Mitchell, E.H. Price, S.P. Reidel, and A.M. Tallman. 1979. Geologic Studies of the Columbia Plateau Status Report. RHO-BWI-ST-4, Rockwell Hanford Operations, Richland, Washington.

Napier, B.A., R.A. Peloquin, D.L. Strenge, and J.V. Ramsdell. 1988. GENII - The Hanford Environmental Dosimetry Software System. Volume I - Conceptual Representation. PNL-6584, Pacific Northwest Laboratory, Richland, Washington.

Neitzel, D.A., T.L. Page, and R.W. Hanf, Jr. 1982a. “Mid-Columbia River Microflora.” J. Freshwater Ecology 1(5):495-505.

Neitzel, D.A., T.L. Page, and R.W. Hanf, Jr. 1982b. "Mid-Columbia River Zooplankton." Northwest Science 57:112-118.

Newcomb, R.C. 1958. Ringold Formation of Pleistocene age in type locality, the White Bluffs, Washington. American Journal of Science 256: 328-340.

Newcomb, R.C., J.R. Strand, and F.J. Frank. 1972. Geology and Ground-Water Characteristics of the Hanford Reservation of the U.S. Atomic Energy Commission, Washington. Professional Paper 717, U.S. Geological Survey, Washington, D.C.

Newcomer, D.R., K.D. Pohlod, and J.P. McDonald. 1991. Water-Table Elevations on the Hanford Site, 1990. PNL-7693, Pacific Northwest Laboratory, Richland, Washington. NRC (see U.S. Nuclear Regulatory Commission).

Northwest Power Planning Council (NPPC). 1998. Revised Fourth Northwest Conservation and Electric Power Plan, document 98-22. July 1998, [Online report]. Available URL: http://www.nwcouncil.org/library/1998/98-22/index.htm 
Northwest Power Planning Council (NPPC). 2001. Existing Generating Projects. April 2001. Northwest Power Planning Council, Portland Oregon. Available URL:

http://www.nwcouncil.org/energy/powersupply/index.htm

NPPC (see Northwest Power Planning Council).

NPS (see National Park Service).

NRC (see U.S. Nuclear Regulatory Commission).

Office of Financial Management (OFM). 2000a. Median Household Income by County: 1989 to 1999 and Forecast for 2000. Office of Financial Management, Forecasting Division, Olympia, Washington. Available URL: http://www.ofm.wa.gov/poptrends/poptrendtoc.htm

Office of Financial Management (OFM). 2000b. Washington State April 1 Housing Estimates for Cities, Towns, and Counties. Office of Financial Management, Forecasting Division, Olympia, Washington. Available URL: http://www.ofm.wa.gov/housing/housingtoc.htm

Office of Superintendent of Public Instruction (OSPI). 2001. 2000-2001 Public and Private School Enrollment Summary (Report 1345A-by county, district, building, and ethnicity). Office of Superintendent of Public Instruction, Olympia, Washington. Available URL:

http://www.k12.wa.us/dataadmin/

OFM (see Office of Financial Management).

O’Neil, T.K., and M.E. Crist. 1993. Laser Interferometer Gravitational Wave Observatory (LIGO) Project - - A Cultural Resources Inventory Report. Pacific Northwest Laboratory, Richland, Washington.

OSPI (see Office of Superintendent of Public Instruction).

OTED (see Washington State Office of Trade and Economic Development).

Page, T.L., and D.A. Neitzel. 1978. "Columbia River Benthic Macrofauna and Microfauna Near WNP 1, 2, and 4: January through December 1977." In Aquatic Ecological Studies Near WNP 1, 2, and 4, January through December 1977, WPPSS Columbia River Ecology Studies, Vol. 5, Section 4. Battelle, Pacific Northwest Laboratories, Richland, Washington.

Page, T.L., D.A. Neitzel, and R.W. Hanf. 1979. "Columbia River Benthic Macrofauna and Microflora Near WNP 1, 2, and 4: January through August 1978." In Aquatic Ecological Studies Near WNP 1, 2, and 4, January through August 1978, WPPSS Columbia River Ecology Studies, Vol. 6, Section 4. Battelle, Pacific Northwest Laboratories, Richland, Washington.

Parker, P.L., and T.F. King. 1990. Guidelines for Evaluating and Documenting Traditional Cultural Properties. National Register Bulletin 38, National Park Service, Washington, D.C.

Peterson, R.E. 1992. Hydrologic and Geologic Data Available for the Region North of Gable Mountain, Hanford Site, Washington. WHC-SD-EN-TI-006, Westinghouse Hanford Company, Richland, Washington. 
Peterson, R.E., and V.G. Johnson. 1992. Riverbank Seepage of Groundwater Along the 100 Areas Shoreline, Hanford Site. WHC-EP-0609, Westinghouse Hanford Company, Richland, Washington.

Peterson, R.E., R.F. Raidl, and C.W. Denslow. 1996. Conceptual Site Models for Groundwater Contamination at 100-BC-5, 100-KR-4, 100-HR-3, and 100-FR-3 Operable Units. BHI-00917, Bechtel Hanford Incorporated, Richland, Washington.

PNNL Seismic Monitoring Team. 2001. Second and Third Quarter Hanford Seismic Report for Fiscal Year 2001. PNNL-11557-18, Pacific Northwest National Laboratory, Richland, Washington.

Poston, T.M., K.R. Price, and D.R. Newcomer. 1991. An Evaluation of the Chemical, Radiological, and Ecological Conditions of West Lake on the Hanford Site. PNL-7662, Pacific Northwest Laboratory, Richland, Washington.

Poston, T.M., R.W. Hanf and R.L. Dirkes. 2000. Hanford Site Environmental Report for Calendar Year 1999. PNNL13230, Pacific Northwest National Laboratory, Richland, Washington.

Price, K.R., J.M. Carlile, R.L. Dirkes, R.E. Jaquish, M.S. Trevathan, and R.K. Woodruff. 1986. Environmental Monitoring at Hanford for 1984. Supplement. PNL-5407 Suppl. Pacific Northwest Laboratory, Richland, Washington.

PSPL (see Puget Sound Power and Light Company).

Puget Sound Power and Light Company (PSPL). 1982. Preliminary Safety Analysis for Skagit/Hanford Nuclear Project. Amendment 29, Puget Sound Power and Light Company, Bellevue, Washington.

Ramsdell, J.V., and G.L. Andrews. 1986. Tornado Climatology of the Contiguous United States. NUREG/CR-4461, U.S. Nuclear Regulatory Commission, Washington, D.C.

Reidel, S.P. 1984. "The Saddle Mountains: The Evolution of an Anticline in the Yakima Fold Belt." American Journal of Science 284:942-978.

Reidel, S.P. 1998. Emplacement of Columbia River Flood Basalt. Journal of Geophysical Research, Vol. 103, No. B11, p. 27,393-27,410.

Reidel, S.P., and K.R. Fecht. 1981. "Wanapum and Saddle Mountains Basalts of the Cold Creek Syncline Area." In Subsurface Geology of the Cold Creek Syncline, eds. C.W. Myers and S.M. Price. RHO-BWI-ST-14, Rockwell Hanford Operations, Richland, Washington.

Reidel, S.P., and K.R. Fecht. 1994a. Geologic map of the Richland 1:100,000 quadrangle, Washington. Open File Report 94-8, 21 pp., 1 plate, Washington Division of Geology and Earth Resources, Olympia, Washington.

Reidel, S.P., and K.R. Fecht. 1994b. Geologic map of the Priest Rapids 1:100,000 quadrangle, Washington. Open File Rep. 94-13, 22 pp. 1 plate, Washington Division of Geology and Earth Resources, Olympia, Washington. 
Reidel, S.P., K.R. Fecht, M.C. Hagood, and T.L. Tolan. 1989. "The Geologic Evolution of the Central Columbia Plateau." In Volcanism and Tectonism in the Columbia River Flood-Basalt Province, Special Paper 239, pp. 247-264, eds. S.P. Reidel and P.R. Hooper, Geological Society of America, Boulder, Colorado.

Reidel, S.P., K.A. Lindsey, and K.R. Fecht. 1992. Field Trip Guide to the Hanford Site. WHCMR-0391. Westinghouse Hanford Company, Richland, Washington.

Reidel, S.P., N.P. Campbell, K.R. Fecht, and K.A. Lindsey. 1994. Late Cenozoic Structure and Stratigraphy of South-central Washington. In: Lasmanis, R. and E.S. Cheney, convenors, Regional Geology of Washington State. Washington Division of Geology and Earth Resources Bulletin 80: 159-180.

Relander, C. 1956. Drummers and Dreamers. Caxton Printers, Caldwell, Idaho.

Rice, D.G. 1968a. Archaeological Reconnaissance: Ben Franklin Reservoir Area, 1968. Washington State University, Laboratory of Anthropology, Pullman, Washington.

Rice, D.G. 1968b. Archaeological Reconnaissance: Hanford Atomic Works. U.S. Atomic Energy Commission, National Park Service and Washington State University, Pullman, Washington.

Rice, D.G. 1976. The Log Structure at White Bluffs Landing, Franklin County, Washington. Anthropological Research Manuscript Series No. 25, University of Idaho, Moscow, Idaho.

Rice, D.G. 1980a. Overview of Cultural Resources on the Hanford Reservation in South Central Washington State. Report submitted to U.S. Department of Energy, Richland Operations, Richland, Washington.

Rice, D.G. 1980b. Cultural Resources Assessment of the Hanford Reach of the Columbia River, State of Washington. U.S. Army Corps of Engineers, Seattle District, Seattle, Washington.

Rice, D.G. 1981. Archaeological Transects Through Interior Dunes on the Hanford Reservation, Washington. U.S. Department of Energy, Richland, Washington.

Rice, D.G. 1987. Archaeological Reconnaissance of Gable Butte and Gable Mountain on the Hanford Site, Washington. Westinghouse Hanford Company, Richland, Washington.

Rice, H.S., D.H. Stratton, and G.W. Lundeman. 1978. An Archaeological and Historic Survey of the 400 Area, Hanford Reservation. National Heritage, Inc., Pullman, Washington.

Rickard, W.H., and L.E. Rogers. 1983. "Industrial Land Use and the Conservation of Native Biota in the Shrub-Steppe Region of Western North America." Environmental Conservation 10:205-211.

Rickard, W.H., R.E. Fitzner, and C.E. Cushing. 1981. "Biological Colonization of an Industrial Pond." Environ. Conserv. 8:241-247. 
Rigby, J.G., and K. Othberg. 1979. Reconnaissance Surficial Geologic Mapping of the Late Cenozoic Sediments of the Columbia Basin, Washington. Open-File Report 79-3, Washington State Department of Natural Resources, Division of Geology and Earth Resources, Olympia, Washington.

Rogers, L.E., and W.H. Rickard. 1977. Ecology of the 200 Area Plateau Waste Management Environs: A Status Report. PNL-2253, Pacific Northwest Laboratory, Richland, Washington.

Rohay, A.C. 1987. Earthquake Focal Mechanisms, Recurrence Rates and Deformation in the Columbia River Basalts. RHO-BW-SA-666 P, Rockwell Hanford Operations, Richland, Washington.

Rohay, A.C. 1989. "Earthquake Recurrence Rate Estimates for Eastern Washington and the Hanford Site." In Proceedings, Second DOE Natural Phenomena Hazards Mitigation Conference, CONF-8910192, October 3-5, 1989, Knoxville, Tennessee, sponsored by U.S. Department of Energy Headquarters, Office of Nuclear Safety, NTIS, Springfield, Virginia.

Sackschewsky, M.R., D.S. Landeen, G.I. Baird, W.H. Rickard, and J.L. Downs. 1992. Vascular Plants of the Hanford Site. WHC-EP-0554, Westinghouse Hanford Company, Richland, Washington.

Schalla, R., R.W. Wallace, A.L. Aaberg, S.P. Arihart, D.J. Bates, J.V.M. Carlile, C.S. Cline, I. Dennison, M.D. Freshley, P.R. Heller, E.J. Jensen, K.B. Olsen, R.G. Parkhurst, J.T. Rieger, and E.J. Westergard. 1988. Interim, Characterization Report for the 300 Area Process Trenches. PNL-6716, Pacific Northwest Laboratory, Richland, Washington.

Schmincke, H. 1964. Petrology, Paleocurrents, and Stratigraphy of the Ellensburg Formation. Johns Hopkins University, Baltimore, Maryland.

Schreckhise, R.G., K. Rhoads, J.S. Davis, B.A. Napier, and J.V. Ramsdell. 1993. Recommended Environmental Dose Calculation Methods and Hanford-Specific Parameters. PNL-3777, Rev. 2, Pacific Northwest Laboratory, Richland, Washington.

Schwab, G.E., R.M. Colpitts Jr., and D.A. Schwab. 1979. Spring Inventory of the Rattlesnake Hills. W.K. Summers and Associates, Inc., Socorro, New Mexico.

Serne, R.J., H.T. Schaef, B.N. Bjornstad, B.A. Williams, D.C. Lanigan, D.G. Horton, R.E. Clayton, V.L. LeGore, M.J. O'Hara, C.F. Brown, D.E. Parker, I.V. Kutnyakov, J.N. Serne, A.V. Mitroshkov, G.V. Last, S.C. Smith, C.W. Lindenmeier, J.M. Zachara, and D.B. Burke. 2001 a. Characterization of Uncontaminated Sediments from the Hanford Reservation - RCRA Borehole Core Samples and Composite Samples. PNNL-2001-1, Pacific Northwest National Laboratory, Richland, Washington.

Serne, R.J., G.V. Last, G.W. Gee, H.T. Schaef, D.C. Lanigan, C.W. Lindenmeier, R.E. Clayton, V.L. LeGore, R.D. Orr, M.J. O'Hara, C.F. Brown, D.B. Burke, A.T. Owen, I.V. Kutnyakov, T.C. Wilson, and D.A. Myers. 2001b. Geologic and Geochemical Data Collected from Vadose Zone Sediments from Borehole SX 41-09-39 in the S/SX Waste Management Area and Preliminary Interpretations. PNNL-2001-2, Pacific Northwest National Laboratory, Richland, Washington. 
Serne, R.J., H.T. Schaef, B.N. Bjornstad, D.C. Lanigan, G.W. Gee, C.W. Lindenmeier, R.E. Clayton, V.L. Legore, R.D. Orr, M.J. O'Hara, C.F. Brown, G.V. Last, I.V. Kutnyakov, D.B. Burke, T.C. Wilson, and B.A. Williams. 2001c. Geologic and Geochemical Data Collected from Vadose Zone Sediments from Borehole 299-W23-19 [SX-115] in the S/SX Waste Management Area and Preliminary Interpretations. PNNL-2001-3, Pacific Northwest National Laboratory, Richland, Washington.

Skaggs, R.L., and W.H. Walters. 1981. Flood Risk Analysis of Cold Creek Near the Hanford Site. RHO-BWI-C-120/PNL-4219, Rockwell Hanford Operations, Richland, Washington.

Slate, J.L. 1996. Buried Carbonate Paleosols Developed in Pliocene-Pleistocene Deposits of the Pasco Basin, South-central Washington, U.S.A. Quarternary International 34-36: 191-196.

Published by Elsevier Science Ltd., Great Britain.

Slate, J.L. 2000. Nature and Variability of the Plio-Pleistocene Unit in the 200 West Area of the Hanford Site. BHI-01203, Rev. 0, [Online Report]. Available URL:

http://www.erc.rl.gov/ pgs/readroom/BHI/bhi01203.pdf

Smith, G.A. 1988. "Neogene Synvolcanic and Syntectonic Sedimentation in Central Washington." Geologic Society of America Bulletin 100: 1479-1492.

Smith, W.C., M.L. Uebelacker, T.E. Eckert, and L.J. Nickel. 1977. An Archaeological Historical Survey of the Proposed Transmission Power Line Corridor from Ashe Substation, Washington to Pebble Springs Substation, Oregon. Washington Archaeological Research Center Project Report 42, Washington State University, Pullman, Washington.

Soll, J.A., and C. Soper, eds. 1996. Biodiversity Inventory and Analysis of the Hanford Site, 1995 Annual Report. The Nature Conservancy of Washington, Seattle, Washington.

Soll, J.A., Hall, R. Pabst, and C. Soper. 1999. Biodiversity Inventory and Analysis of the Hanford Site Final Report 1994 - 1999. The Nature Conservancy of Washington. Seattle, Washington.

Spane, F.A., Jr. 1987. Fresh-Water Potentiometric Map and Inferred Flow Direction of Ground Water Within the Mabton Interbed, Hanford Site, Washington State - January 1987. SD-BWI-TI335, Rockwell Hanford Operations, Richland, Washington.

Spier, L. 1936. Tribal Distribution in Washington. General Services in Anthropology No. 3, George Banta Publishing Co., Menasha, Wisconsin.

Stone, W.A., D.E. Jenne, and J.M. Thorp. 1972. Climatography of the Hanford Area. BNWL-1605, Pacific Northwest Laboratories, Richland, Washington.

Stone, W.A., J.M. Thorp, O.P. Gifford, and D.J. Hoitink. 1983. Climatological Summary for the Hanford Area. PNL-4622, Pacific Northwest Laboratory, Richland, Washington.

Supply System (see Washington Public Power Supply System).

Swanson, D.A., J.L. Anderson, R.D. Bentley, V.E. Camp, J.N. Gardner, and T.L. Wright. 1979a. Reconnaissance Geologic Map of the Columbia River Basalt Group in Eastern Washington and Northern Idaho. Open-File Report 79-1363, U.S. Geological Survey, Washington, D.C. 
Swanson, D.A., T.L. Wright, P.R. Hooper, and R.D. Bentley. 1979b. Revisions in Stratigraphic Nomenclature of the Columbia River Basalt Group. USGS Bulletin 1457, U.S. Geological Survey, Boulder, Colorado.

Swanson, D.A., J.L. Anderson, V.E. Camp, P.R. Hooper, W.H. Taubeneck, and T.L. Wright. 1981. Reconnaissance Geologic Map of the Columbia River Basalt Group, Northern Oregon and Western Idaho. Open-File Report 81-0797, U.S. Geological Survey, Washington, D.C.

Swanson, L.C. 1992. Phase 1 Hydrogeologic Summary of the 300-FF-5 Operable Unit, 300 Area. WHC-SD-EN-TI-052, Rev. 0, Westinghouse Hanford Company, Richland, Washington.

Swanson, L.C., V.J. Rohay, and J.M. Faurote. 1999. Hydrogeologic Conceptual Model for the Carbon Tetrachloride and Uranium/Technetium Plumes in the 200 West Area: 1994 through 1999 Update. BHI-01311, Rev. 0, [Online Report]. Available URL:

http://www.erc.rl.gov/ pgs/readroom/BHI/bhi01311.pdf

Tallman, A.M., K.R. Fecht, M.C. Marratt, and G.V. Last. 1979. Geology of the Separation Areas Hanford Site, South-Central Washington. RHO-ST-23, Rockwell Hanford Operations, Richland, Washington.

Tallman, A.M., J.T. Lillie, and K.R. Fecht. 1981. "Suprabasalt Sediments of the Cold Creek Syncline Area." In Subsurface Geology of the Cold Creek Syncline, eds. C.W. Myers and S.M. Price. RHO-BW1-ST-14, Rockwell Hanford Operations, Richland, Washington.

TCAR (see Tri-City Association of Realtors).

Thornbury, W.D. 1965. Regional Geomorphology of the United States. John Wiley and Sons, Inc., New York.

Thorne, P.D., and D.R. Newcomer. 1992. Summary and Evaluation of Available Hydraulic Property Data for the Hanford Site Unconfined Aquifer System. PNL-8337, Pacific Northwest Laboratory, Richland, Washington.

Thorne, P.D., M.A. Chamness, F.A. Spane Jr., V.R. Vermeul, and W.D. Webber. 1993. ThreeDimensional Conceptual Model for the Hanford Site Unconfined Aquifer System, FY 1993 Status Report. PNL-8971, Pacific Northwest Laboratory, Richland, Washington.

Thorne, P.D., M.A. Chamness, V.R. Vermeul, Q.C. MacDonald, and S.E. Schubert. 1994. ThreeDimensional Conceptual Model for the Hanford Site Unconfined Aquifer System: FY 1994 Status Report. PNL-10195, Pacific Northwest National Laboratory, Richland, Washington.

Tiller, B.L., G.E. Dagle, L.L. Cadwell, T.M. Poston, and A. Oganesian. 1997. Investigation of Anatomical Anomalies in Hanford Site Mule Deer. PNL-11518, Pacific Northwest National Laboratory, Richland, Washington.

Tolan, T.L., S.P. Reidel, M.H. Beeson, J.L. Anderson, K.R. Fecht, and D.A. Swanson. 1989. "Revisions to the Estimates of the Areal Extent and Volume of the Columbia River Basalt Group", In: Volcanism and Tectonism in the Columbia River Flood Basalt Province, edited by S.P. Reidel and P.R. Hooper. Spec. Paper Geol. Soc. Am., 239: 1-20. 
Tri-City Association of Realtors (TCAR). 2000. Tri-City Area Housing Statistics. Tri-City Association of Realtors, Kennewick, Washington. Available URL:

http://www.tricityrealtors.com/

Tri-Cities Industrial Development Council (TRIDEC). 2001. Industry and Major Employers Directory. Tri-Cities Industrial Development Council, Kennewick, Washington.

TRIDEC (see Tri-Cities Industrial Development Council)

U.S. Army Corps of Engineers (Corps). 1989. Water Control Manual for McNary Lock and Dam, Columbia River, Oregon and Washington. U.S. Army Corps of Engineers, Walla Walla District, Walla Walla, Washington.

U.S. Census Bureau (Census). 1991. 1990 U.S. Census of Population and Housing, State and County Profiles, Washington (Summary Tape File 1A for Washington State). Bureau of the Census, U.S. Department of Commerce, Washington, D.C.

U.S. Census Bureau (Census). 1998a. County Estimates for People of All Ages in Poverty for Oregon: Census 1989. Table ACN-41. Estimated Number and Percent People of all Ages in Poverty by County: Oregon Census 1989. January 1998. Bureau of the Census, U.S. Department of Commerce, Washington, D.C. Online URL: http://www.census.gov

U.S. Census Bureau (Census). 1998b. County Estimates for People of All Ages in Poverty for Washington: Census 1989. Table ACN-53. Estimated Number and Percent People of all Ages in Poverty by County: Washington Census 1989. January 1998. Bureau of the Census, U.S. Department of Commerce, Washington, D.C. Online URL: http://www.census.gov

U.S. Census Bureau (Census). 2000a. "People and Families in Poverty by Selected Characteristics; 1999 and 2000. Data from the U.S. Census Bureau, March 1999 and 2000 Current Population Surveys. Bureau of the Census, U.S. Department of Commerce, Washington, D.C. Online URL: http://www.census.gov

U.S. Census Bureau (Census). 2000b. Poverty 1999. Poverty Thresholds in 1999, by Size of Family and Number of Related Children Under 18 Years. Last revised September 26, 2000. Bureau of the Census, U.S. Department of Commerce, Washington, D.C. Online URL: http://www.census.gov

U.S. Census Bureau (Census). 2000c. County Estimates for People of All Ages in Poverty for Washington: 1997. Table A97-53. Estimated Number and Percent People of all Ages in Poverty by County: Washington 1997. November 2000. Bureau of the Census, U.S. Department of Commerce, Washington, D.C. Online URL: http://www.census.gov

U.S. Census Bureau (Census). 2000d. County Estimates for People of All Ages in Poverty for Oregon: 1997. Table A97-41. Estimated Number and Percent People of all Ages in Poverty by County: Oregon 1997. November 2000. Bureau of the Census, U.S. Department of Commerce, Washington, D.C. Online URL: http://www.census.gov

U.S. Census Bureau (Census). 2001a. Census 2000 Redistricting Data (P.L. 94-171) Summary File - Washington. U.S. Bureau of the Census, U.S. Department of Commerce, Washington, D.C. 
U.S. Census Bureau. 2001b. Poverty Thresholds in 2000, by Size of Family and Number of Related Children Under 18 Years. Last revised January 29, 2001. Bureau of the Census, U.S. Department of Commerce, Washington, D.C. Online URL: http://www.census.gov

U.S. Census Bureau (Census). 2001c. Census 2000 Redistricting Data (P.L. 94-171) Summary File - Oregon. . U.S. Bureau of the Census, U.S. Department of Commerce, Washington, D.C.

U.S. Department of Commerce (DOC). 2000. Regional Economic Information System (REIS). Bureau of Economic Analysis, U.S. Department of Commerce, Washington, D.C. Available URL: http://www.bea.doc.gov/bea/regional/reis/

U.S. Department of Energy (DOE). 1986. Environmental Assessment, Reference Repository Location, Hanford Site, Washington. DOE/RW-0070, U.S. Department of Energy, Washington, D.C.

U.S. Department of Energy (DOE). 1987. Final Environmental Impact Statement, Disposal of Hanford Defense High-Level, Transuranic and Tank Wastes, Hanford Site, Richland, Washington. DOE/EIS-0113, Vol. I-III, U.S. Department of Energy, Washington, D.C.

U.S. Department of Energy (DOE). 1988. Consultation Draft: Site Characterization Plan, Reference Repository Location, Hanford Site, Washington. DOE/RW-0164, U.S. Department of Energy, Washington, D.C.

U.S. Department of Energy (DOE). 1991. Draft Environmental Impact Statement for the Siting, Construction, and Operation of New Production Reactor Capacity. DOE/EIS-0144D, Vol. 4, Appendix E, U.S. Department of Energy, Washington, D.C.

U.S. Department of Energy (DOE). 1992. Hanford Site Groundwater Background. DOE/RL92-23, U.S. Department of Energy, Richland Field Office, Richland, Washington.

U.S. Department of Energy (DOE). 1996a. Hanford Site Biological Resources Management Plan. Draft. DOE/RL-96-32, Rev. 0. U.S. Department of Energy, Richland, Washington.

U.S. Department of Energy (DOE). 1996b. Programmatic Agreement Among the U.S. Department of Energy Richland Operations Office, the Advisory Council on Historic Preservation, and the Washington State Historic Preservation Office for the Maintenance, Deactivation, Alteration, and Demolition of the Built Environment on the Hanford Site, Washington. DOE/RL-96-77, [Online report]. Available URL: http://www.hanford.gov/docs/96eap154/96eap154.htm

U.S. Department of Energy (DOE). 1997a. Waste Site Grouping for 200 Areas Soil Investigations. DOE/RL-96-81, Rev 0, [Online Report]. Available URL: http://www.erc.rl.gov/ pgs/READROOM/DOE_RL.HTM

U.S. Department of Energy (DOE). 1997b. Hanford Site Background: Part 3, Groundwater Background. DOE/RL-96-61. U.S. Department of Energy, Richland, Washington.

U.S. Department of Energy (DOE). 1997c. National Register of Historic Places Multiple Property Documentation Form - Historic, Archaeological and Traditional Cultural Properties of the Hanford Site, Washington. DOE/RL-97-02, [Online report]. Available URL: http://www.hanford.gov/doe/culres/mpd/toc.htm 
U.S. Department of Energy (DOE). 1997d. N Reactor Comprehensive Treatment Report, Hanford Site, Washington. DOE/RL-96-91, Draft A, Volumes I, II, III. U.S. Department of Energy, Richland, Washington.

U.S. Department of Energy (DOE). 1998. Hanford Site Manhattan Project and Cold War Era Historic District Treatment Plan. DOE/RL-97-56, Rev. 1. U.S. Department of Energy, Richland, Washington.

U.S. Department of Energy (DOE). 1999a. Final Hanford Comprehensive Land-Use Plan Environmental Impact Statement. DOE/EIS-0222, U.S. Department of Energy, Washington, D.C.

U.S. Department of Energy (DOE). 1999b. Site Service Division, Site Engineering Property. [Online Report]. Available URL: http://www.hanford.gov/infrastructure/sid/property.html

U.S. Department of Energy (DOE). 2000a. Clean Air Act General Conformity Requirements and the National Environmental Policy Act Process. [Online Report]. Available URL: http://tis.eh.doe.gov/nepa/tools/guidance/caaguidance.pdf

U.S. Department of Energy (DOE). 2000b. Threatened and Endangered Species Management Plan: Salmon \& Steelhead. DOE/RL-2000-27, Rev. 0. U.S. Department of Energy, Richland, Washington.

U.S. Department of Energy (DOE). 2001a. Hanford Cultural Resources Management Plan. DOE/RL-98-10, Rev. 1. U.S. Department of Energy, Richland Operations Office, Richland, Washington.

U.S. Department of Energy (DOE). 2001b. DOE Computerized Accident/Incident reporting System (CAIRS). [Online Report]. Available URL: http://tis.eh.doe.gov/cairs (Data Downloaded August 2001).

U.S. Department of Energy, Richland Operations Office (DOE-RL). 1998. Hanford Facility Dangerous Waste Permit Application, General Information Portion. DOE/RL-91-28, Rev. 4. [Online Report]. Available URL: http://www.hanford.gov/docs/rl-91-28/r19128chp 02f.htm\#f2-9

U.S. Department of Energy, Richland Operations Office (DOE-RL). 2000. Hanford, Diversification, and the Tri-Cities Economy, FY 1999. U.S. Department of Energy, Richland Operations Office, Richland, Washington.

U.S. Department of the Army. 1990. Final Environmental Impact Statement Yakima Firing Center Proposed Land Acquisition, Yakima Firing Center, Washington. Department of Army, I Corps, Fort Lewis, Washington.

U.S. Department of the Interior (DOI). 1994. Hanford Reach of the Columbia River: Comprehensive River Conservation Study and Environmental Impact Statement - Final. Volumes II and I. U.S. Department of Interior, Washington, D.C.

U.S. Department of the Interior (DOI). 1996. United States Department of the Interior Record of Decision - Hanford Reach of the Columbia River: Final Environmental Impact Statement for Comprehensive River Conservation Study. U.S. Department of the Interior, Washington D.C. 
U.S. Department of Labor, Employment and Training Administration (DOL). 2000. Domestic Agricultural In-Season Farm Labor Report. Monthly Farm Labor Abstracts, January-December 2000. Washington State Employment Security Department for the U.S. Department of Labor, Olympia, Washington.

U.S. Energy Research and Development Administration (ERDA). 1975. Final Environmental Impact Statement of Waste Management Operations, Hanford Reservation, Richland, Washington, 2 vol. ERDA-1538, U.S. Energy Research and Development Administration, Washington, D.C.

U.S. Energy Research and Development Administration (ERDA). 1976. Evaluation of Impact of Potential Flooding Criteria on the Hanford Project. RLO-76-4, U.S. Energy Research and Development Administration, Richland, Washington.

USGS (see U.S Geological Survey)

U.S. Geological Survey (USGS). 1987. Subsurface Transport of Radionuclides in Shallow Deposits of the Hanford Nuclear Reservation, Washington - Review of Selected Previous Work and Suggestions for Further Study. USGS Open File Report 87-222, Tacoma, Washington.

U.S. Geological Survey (USGS). 1995. Nitrate Concentrations in Ground Water of the Central Columbia Plateau. Open File Report 95-445, U.S. Geological Survey, Tacoma, Washington.

U.S. Geological Survey (USGS). 1996. National Seismic-Hazard Maps: Documentation June 1996. U.S. Geological Survey Open-File Report 96-532, Denver, Colorado.

U.S. Geological Survey (USGS). 2001a. Washington NWIS-W Data Retrieval. May 2001. Available URL: http://water.usgs.gov/wa/nwis/discharge

U.S. Geological Survey (USGS). 2001b. Provisional Yakima River Discharge Data (provided by Gregory Ruppert), U.S. Geological Survey, Pasco, Washington.

U.S. Nuclear Regulatory Commission (NRC). 1982. Draft Environmental Statement Related to the Construction of Skagit/Hanford Nuclear Project, Units 1 and 2. Prepared by Puget Sound Power \& Light Company, Pacific Power and Light Company, the Washington Water Power Company, and Portland General Electric Company. NUREG-0894, U.S. Nuclear Regulatory Commission, Washington, D.C.

University of Washington Geophysics Program (UWGP). 2001. On-line Earthquake Catalog. University of Washington Geophysics Program, Seattle, Washington. Available URL: http://www.geophys.washington.edu/SEIS

UWGP (see University of Washington Geophysics Program).

WAC 173-470. Ambient Air Quality Standards for Particulate Matter. Washington Administrative Code, Olympia, Washington.

WAC 173-480. Ambient Air Quality Standards and Emission Limits for Radionuclides. Washington Administrative Code, Olympia, Washington. 
WAC 173-481. Ambient Air Quality and Environmental Standards for Fluorides. Washington Administrative Code, Olympia, Washington.

Waitt, R.B. 1980. "About Forty Last-Glacial Lake Missoula Jokulhlaups Through Southern Washington.” Journal of Geology 88: 653-679.

Walker, D.E., Jr. 1998. Handbook of North American Indians. Volume 12:Plateau. Smithsonian Institute, Washington D.C.

Ward, A.L., and G.W. Gee. 2000. Vadose Zone Transport Field Study: Detailed Test Plan for Simulated Leak Tests. PNNL-13263, [Online Report]. Available URL:

http://etd.pnl.gov:2080/vadose

Warren, J.L. 1980. Vegetation Maps of the Hanford Reach, Columbia River. Prepared for the U.S. Army Corps of Engineers, Seattle District, Seattle, Washington, by Pacific Northwest Laboratory, Richland, Washington.

Washington Center for Real Estate Research (WCRER). 2000a. Washington State Housing Market. Washington State University, Pullman, Washington. Available URL: http://www.cbe.wsu.edu/ wcrer/market/HousingMarket.asp

Washington Center for Real Estate Research (WCRER). 2000b. WCRER Apartment Market Survey, Fall 2000. Washington State University, Pullman, Washington. Available at http://www.cbe.wsu.edu/ wcrer/market/apt_intro.htm

Washington Natural Heritage Program. 1997. Rare Plant Species County List. Washington Natural Heritage Program, Washington Department of Natural Resources, Olympia, Washington. Available URL: http://www.wa.gov/dnr/htdocs/fr/nhp/inforeq.html

Washington Natural Heritage Program. 2000. Rare Plant Species With Ranks. Washington Department of Natural Resources, Olympia, Washington. Available URL:

http://www.wa.gov/dnr/htdocs/fr/nhp/wanhp.html

Washington Public Power Supply System (Supply System). 1981. Final Safety Analysis Report, Washington Nuclear Power Plant No. 2. Amendment 18, Richland, Washington.

Washington State Department of Ecology (Ecology). 1997. 1997 Air Quality Annual Report for Washington State. \#97-208, Washington State Department of Ecology, Olympia, Washington.

Washington State Department of Ecology (Ecology) Air Quality Program. 1999. 1998 Washington State Air Quality Report. \#98-213, [Online Report]. Available URL: http://www.wa.gov/ecology/air/98rptbea.pdf

Washington State Department of Ecology (Ecology), Air Quality Program. 2000. 1998 Air Quality Data Summary. \#00-02-005, [Online Report]. Available URL: http://www.wa.gov/ecology/air/98-final1.pdf

Washington State Department of Fish and Wildlife (WDFW). 2000. Steelhead Harvest Summary. WDFW, Olympia Washington. Available URL: http://www.wa.gov/wdfw/fish/harvest/98-99/99shs-a.htm 
Washington State Labor Market and Economic Analysis (LMEA). 2000a. Washington State Labor Summaries, February 2001. Olympia, Washington. Available URL: http://www.wa.gov/esd/lmea/pubs/labrarea/lasmain.htm

Washington State Labor Market and Economic Analysis (LMEA). 2000b. Covered Employment and Wages by Industry, 1999. Olympia, Washington. Available URL: http://www.wa.gov/esd/lmea/labrmrkt/emp/emphome.htm

Washington State Office of Trade and Economic Development (OTED). 2000. Washington State Travel Impacts \& Visitor Volume 1991-2000p. Washington State Tourism Division, Olympia, Washington. Available URL: http://198.239.32.121/researchframes.asp

Waters, A.C. 1961. "Stratigraphic and Lithologic Variations in the Columbia River Basalt." American Journal of Science 259: 583-611.

Watson, D.G. 1970. Fall Chinook Salmon Spawning in the Columbia River Near Hanford 19471969. BNWL-1515, Pacific Northwest Laboratories, Richland, Washington.

Watson, D.G. 1973. Fall Chinook Salmon Population Census. BNWL-1750, Pacific Northwest Laboratories, Richland, Washington.

Watson, E.C., C.D. Becker, R.E. Fitzner, K.A. Gano, C.L. Imhoff, R.F. McCallum, A. Myers, T.L. Page, K.R. Price, J.V. Ramsdell, D.G. Rice, D.L. Schreiber, L.A. Skumatz, D.J. Sommer, J.J. Tawil, R.W. Wallace, and D.G. Watson. 1984. Environmental Characterization of Two Potential Locations at Hanford for a New Production Reactor. PNL-5275, Pacific Northwest Laboratory, Richland, Washington.

WCRER (see Washington Center for Real Estate Research).

WDFW (see Washington State Department of Fish and Wildlife).

Weiss, S.G., and R.M. Mitchell. 1992. A Synthesis of Ecological Data from the 100 Areas of the Hanford Site. WHC-EP-0601, Westinghouse Hanford Company, Richland, Washington.

Westinghouse Hanford Company (WHC). 1989. Primary Operable Units Designation Project. WHC-EP-0216, Environmental Engineering Group, Westinghouse Hanford Company, Richland, Washington.

WHC (see Westinghouse Hanford Company).

Wolf, E.G. 1976. "Characterization of the Benthos Community." In Final Report on Aquatic Ecological Studies Conducted at the Hanford Generating Project, prepared by Battelle, Pacific Northwest Laboratories for United Engineers and Constructors, Inc. for Washington Public Power Supply System under Contract No. 2311201335, Richland, Washington.

Wolf, E.G., T.L. Page, and D.A. Neitzel. 1976. "Phytoplankton Community: Primary Productivity, Pigment Concentration, Species Composition and Relative Abundance of Phytoplankton and Physicochemical Analysis," Section 2. In Final Report on Aquatic Ecological Studies Conducted at the Hanford Generating Project, 1973-1974, WPPSS Columbia River Ecological Studies, Vol. 1. Prepared by Battelle, Pacific Northwest Laboratories for Washington Public Power Supply System, Richland, Washington. 
Wood, M.I., R. Schalla, B.N. Bjornstad, and S.M. Narbutovskih. 2000. Subsurface Conditions Description of the B-BX-BY Waste Management Area. HNF-5507, Rev. 0. CH2MHill Hanford Group, Incorporated, Richland, Washington.

Wood, M.I., T.E. Jones, R. Schalla, B.N. Bjornstad, and F.N. Hodges. 2001. Surface Conditions Description of the T-TX-TY Waste Management Area. RPP-7123, Rev. 0. CH2MHill Hanford Group, Incorporated, Richland, Washington.

Wright, M.K. 1993. Fiscal Year 1992 Report on Archaeological Surveys of the 100 Areas, Hanford Site, Washington. PNL-8819, Pacific Northwest Laboratory, Richland, Washington.

Wurstner, S.K., and M.D. Freshley. 1994. Predicted Impacts of Future Water Level Decline on Monitoring Wells Using a Ground-Water Model of the Hanford Site. PNL-10196, Pacific Northwest Laboratory, Richland, Washington.

Zimmerman, D.A., A.E. Reisenauer, G.D. Black, and M.A. Young. 1986. Hanford Site Water Table Changes, 1950 Through 1980 - Data Observations and Evaluation. PNL-5506, Pacific Northwest Laboratory, Richland, Washington. 


\subsection{Statutory and Regulatory Requirements \\ P.L. Hendrickson}

The Hanford Site is owned by the U.S. government and is managed by the U.S. Department of Energy (DOE). It is the policy of the DOE to carry out its operations in compliance with all applicable federal, state, and local laws and regulations, presidential executive orders, DOE directives, and treaty rights. Environmental regulatory authority over the Hanford Site is vested both in federal agencies, primarily the U.S. Environmental Protection Agency (EPA), and in Washington State agencies, primarily the Washington State Department of Ecology (Ecology) and the Washington State Department of Health $(\mathrm{DOH})$. In addition, the Benton Clean Air Authority (BCAA) has certain regulatory authority over Hanford activities, including open burning, asbestos removal, and fugitive dust control. Significant environmental laws, regulations, and other requirements are discussed in this chapter in the following order:

- Major federal environmental laws

- Significant applicable federal and state regulations

- Presidential executive orders

- DOE directives

- Existing environmental permits covering activities at the Hanford Site.

There are a number of sources of information available concerning statutory and regulatory requirements as they relate to the National Environmental Policy Act (NEPA) process. Sources available over the Internet include the following:

- Links to Hanford NEPA documents at URL: http://www.hanford.gov/rl/resource.asp

- DOE's NEPA web site at URL: http://www.eh.doe.gov/nepa/

- Council on Environmental Quality's (CEQ's) web site at URL: http://www.whitehouse.gov/CEQ/

- EPA's links to federal agencies' NEPA web sites at URL: http://es.epa.gov/oeca/ofa/nepaweb.html

The National Environmental Policy Act Compliance Guide (DOE 1998) issued by the DOE Office of Environment, Safety, and Health, contains useful information including regulations, DOE and CEQ guidance, copies of relevant executive orders, as well as other preparation assistance documents such as checklists.

(The following introduction [boxed text] is intended to be explanatory for persons writing the chapter of a Hanford Site environmental impact statement [EIS] or environmental assessment [EA] covering regulatory requirements, but is not intended to be included in the EIS or EA.) The material following the boxed text can be adapted, as appropriate, for use in an EIS or EA at the discretion of the authors.

Normally, additional specificity should be added to the material to reflect the particular circumstances and facts that are the subject of the EA or EIS. 


\section{Introduction}

The CEQ regulations in the Code of Federal Regulations (CFR) at 40 CFR 1500-1508 implement NEPA and set forth requirements for the preparation of environmental documentation by federal agencies that satisfies NEPA. DOE has adopted the CEQ regulations as part of its NEPA implementing procedures (10 CFR 1021.103). The CEQ regulations identify the types of actions proposed by a federal agency that require preparation of an EIS, prescribe the content of an EIS, and identify actions and other environmental reviews that must or should be undertaken by the federal agency in preparing and circulating an EIS. In general, an EIS must be prepared by a federal agency for any major federal action significantly affecting the quality of the human environment (40 CFR 1502.3). The regulations also state reasons why an agency may want to prepare an EA instead of an EIS (40 CFR 1508.9).

A specific requirement in the CEQ regulations (40 CFR 1502.25) is that the draft EIS must list "all Federal permits, licenses, and other entitlements which must be obtained in implementing the proposal." If it is uncertain whether a federal permit or license is needed, the draft EIS is to so indicate. There is, however, no requirement in the CEQ regulations or in the DOE NEPA implementing procedures at 10 CFR Part 1021 that the EIS must list or discuss applicable environmental laws and regulations. Nevertheless, applicable environmental laws and regulations (federal, state, and local) have been discussed in recent Hanford Site EISs and EAs in a chapter usually captioned "Statutory and Regulatory Requirements." The discussion below assumes this chapter is chapter 6 of the EIS or EA, but another chapter number is possible. Given the large number of applicable environmental regulations, the rapidly changing character of environmental regulation, and the public's interest in environmental regulation, this practice is likely to continue.

Chapter 6 of Hanford Site EISs and EAs should include the list called for by 40 CFR 1502.25(b). The list should also include significant permits that will be needed from state and local government agencies. Chapter 6 should not normally include information on environmental impacts associated with any of the requirements. For example, Executive Order (E.O.) 12962 requires federal agencies to evaluate the effects of their actions on aquatic systems and recreational fisheries. Although E.O. 12962 should be mentioned in Chapter 6 in appropriate cases, the actual impacts of the alternatives on aquatic systems and recreational fisheries should be discussed in the Environmental Consequences chapter (normally Chapter 5) of the EIS or EA and any recreational fisheries aspects of the affected environment should be discussed in the Affected Environment chapter (normally Chapter 4) of the EIS or EA. Chapter 6 can refer the reader to the portion of the EIS or EA where the environmental impacts associated with a particular environmental requirement are discussed.

The purpose, then, of Chapter 6 in this document is to present a "reference" that can be used as the basis for the preparation of future Hanford Site EISs and EAs. The intent is to present a reasonably complete discussion of federal, state, and local environmental laws, regulations, and permit requirements that are applicable to activities at the Hanford Site. The information in this chapter can then be adapted to any future Hanford Site EIS/EA by deleting irrelevant parts and by adding some specificity with respect to the proposed action and the alternatives being considered.

It should be noted that environmental standards and permit requirements usually appear in regulations and not in the laws themselves. Thus, more emphasis is placed on regulations and less on laws in this chapter. 


\section{Federal and State Environmental Laws}

Federal law governs environmental regulation of federal facilities. Most major federal environmental laws now include provisions for regulation of federal activities that impact the environment. The activity to be regulated is usually an activity being carried out by an agency of the executive branch. The federal environmental law will also typically designate a specific agency, such as the EPA or the U.S. Nuclear Regulatory Commission (NRC), as the regulator. In addition, federal laws may provide for the delegation of the environmental regulation of federal facilities to the states or may directly authorize the environmental regulation of federal facilities by the states through waivers of sovereign immunity. At Hanford, all these situations apply in varying degrees. The EPA has regulatory authority over Hanford facilities and has delegated regulatory authority to, shares regulatory authority with, or is in the process of delegating regulatory authority to the state of Washington. The state of Washington also asserts its own independent regulatory authority over Hanford facilities under federal waivers of sovereign immunity and state legislation. Ecology has also delegated various air compliance responsibilities to the BCAA.

As a legal matter at Hanford, applicable federal and state environmental standards must be met. As a practical matter, differences in language between federal and state laws and regulations may result in some differences in applicability and interpretation. Guidance on specific applicability should be obtained from the Office of Chief Counsel of the DOE Richland Operations Office (DOE-RL) or the Office of River Protection (DOE-ORP).

\section{Citation of Laws and Regulations}

Laws and regulations may be cited both by their common name and by their location in the appropriate document. Federal laws are most often cited by their common name (e.g., Clean Water Act [CWA]), by their public law (Pub. L. or PL) number, or by their location in the United States Code (USC). Section numbers differ between laws as enacted and as codified in the USC, so it must be understood which is being cited. Federal regulations appear in the CFR. Washington State laws are most often cited by their location in the Revised Code of Washington (RCW). Washington State regulations are cited by their location in the Washington Administrative Code (WAC). Links to the RCW and WAC are available at: http://www.wolfenet.com/ dhillis/statewa.htm. Announcements of proposed and final federal regulations appear in the Federal Register (FR). Announcements of proposed and final Washington State regulations appear in the Washington State Register (WSR).

\section{Specific Federal Laws Cited in the CEQ Regulations}

Four federal laws are specifically cited in the CEQ regulations [40 CFR 1502.25(a) and 1504.1(b)]:

- $\quad$ Section 309 of the Clean Air Act (CAA) (42 USC 7609)

- Fish and Wildlife Coordination Act (16 USC 661 et seq.)

- National Historic Preservation Act (NHPA) (16 USC 470 et seq.)

- Endangered Species Act (16 USC 1531 et seq.). 
Section 309 of the CAA directs the EPA to review and comment in writing on the environmental impacts of any matter relating to EPA's authority contained in proposed legislation, federal construction projects, other federal actions requiring EISs, and new regulations. In addition to commenting on EISs, EPA rates every draft EIS prepared by a federal agency under its Section 309 authority. Ratings are made for the environmental impact of the proposed action and the adequacy of the impact statement. Rating categories for environmental impact are: LO - lack of objections, EC environmental concern, EO - environmental objections, and EU - environmentally unsatisfactory. Rating categories for adequacy are: Category 1 - adequate, Category 2 - insufficient information, and Category 3 - inadequate. A summary of the EPA rating definitions is available at: http://es.epa.gov/oeca/ofa/rating.html . EPA's comments on a draft EIS are answered in the final EIS.

The CEQ regulations (40 CFR 1502.25[a]) direct federal agencies to prepare draft EISs concurrently with and integrated with environmental impact analyses and related surveys required by the Fish and Wildlife Coordination Act, the NHPA, the Endangered Species Act, and other environmental review laws and executive orders. The three preceding statutes should be cited in Chapter 6. Environmental impacts associated with the laws should be discussed in Chapter 5.

\subsection{Federal Environmental Laws}

Significant federal environmental laws applicable to the Hanford Site include the following:

- American Antiquities Act (16 USC 431 to 433)

- American Indian Religious Freedom Act (42 USC 1996)

- Archaeological and Historic Preservation Act (16 USC 469 to 469c)

- Archaeological Resources Protection Act (ARPA) (16 USC 470aa to 470mm)

- Bald and Golden Eagle Protection Act (16 USC 668 to 668c)

- Clean Air Act (CAA) (42 USC 7401 to 7671q)

- Clean Water Act (CWA) (33 USC 1251 to 1387) (the CWA is also known as the Federal Water Pollution Control Act)

- Comprehensive Environmental Response, Compensation, and Liability Act (CERCLA) as amended by the Superfund Amendments and Reauthorization Act (SARA) (42 USC 9601 to 9675)

- Emergency Planning and Community Right-To-Know Act (EPCRA) (42 USC 11001 to 11050)

- Endangered Species Act (16 USC 1531 to 1544)

- Federal Facilities Compliance Act (FFCA) (42 USC 6901) 
- Fish and Wildlife Coordination Act (16 USC 661 to 667c)

- Hanford Reach Act (PL 100-605), as amended by PL 104-333

- $\quad$ Migratory Bird Treaty Act (16 USC 703 to 712)

- National Historic Preservation Act (NHPA) (16 USC 470 to 470w-6)

- Native American Graves Protection and Repatriation Act (25 USC 3001 to 3013)

- National Environmental Policy Act (NEPA) (42 USC 4321 to 4370e)

- Pollution Prevention Act (42 USC 13101 to 13109)

- Resource Conservation and Recovery Act (RCRA) of 1976 as amended by the Hazardous and Solid Waste Amendments (42 USC 6901 to 6992k) of 1984 (RCRA is also known as the Solid Waste Disposal Act)

- $\quad$ Safe Drinking Water Act (SDWA) (42 USC 300f to 300j-26)

- Toxic Substances Control Act (TSCA) (15 USC 2601 to 2692)

In addition, the Atomic Energy Act (AEA) (42 USC 2011 to 2286), the Low-Level Radioactive Waste Policy Act (LLWPA) (42 USC 2021b to 2021j), and the Nuclear Waste Policy Act (NWPA) (42 USC 10101 to 10270), while not environmental laws per se, contain provisions under which environmental regulations applicable to the Hanford Site may be or have been promulgated.

\subsection{Federal and State Environmental Regulations}

Under the Supremacy Clause of the U.S. Constitution (Article VI, Clause 2), activities of the federal government are ordinarily not subject to regulation by the states unless Congress creates specific exceptions. Congress has created exceptions with respect to environmental regulation and provisions in several federal laws give specific authority to the states to regulate federal activities affecting the environment. These waivers (or partial waivers) of sovereign immunity appear in Section 118 of the CAA, Section 313 of the CWA, Section 1447 of the SDWA, Section 6001 of RCRA, and Section 120 of CERCLA/SARA. The FFCA is an amendment to RCRA that makes the RCRA waiver of sovereign immunity more explicit. Many Washington State programs with respect to the environmental regulation of Hanford Site facilities under the preceding statutes are coordinated with the U.S. Environmental Protection Agency (EPA) Region 10 office.

Federal and state environmental regulations that may apply to operations at the Hanford Site have been promulgated under the CAA, CWA, SDWA, RCRA, CERCLA, SARA, AEA, LLWPA, NWPA, under other federal statutes, and under relevant state statutes. The CAA amendments of 1990 have resulted in extensive revisions of federal and state air quality regulations. Federal and state regulations relating to hazardous waste management continue to be promulgated under RCRA at a rapid rate.

Several of the more important existing federal and state environmental regulations are discussed briefly below. These regulations are grouped according to environmental media. 


\subsubsection{Air Quality}

The 1990 federal Clean Air Act and the 1991 Washington Clean Air Act provide the statutory basis for air quality regulation of Hanford Site activities. The federal CAA establishes a floor or minimum level of requirements. State requirements can exceed, i.e., be more stringent than, federal requirements.

- 40 Code of Federal Regulations (CFR) 50, "National Primary and Secondary Ambient Air Quality Standards." EPA regulations in 40 CFR 50 set national ambient air quality standards for sulfur oxides, particulate matter, carbon monoxide, ozone, nitrogen dioxide, and lead. These standards are not directly enforceable, but other enforceable regulations are based on these standards. Washington's ambient air standards are at Washington Administrative Code (WAC) 173-470 through 173-481 and include standards for radionuclides and fluorides.

- 40 CFR 51-52, State Implementation Plans (SIPs). EPA regulations in 40 CFR 51-52 establish the requirements for SIPs and record the approved plans. The SIPs are directed at the control of emissions for which federal ambient air standards exist. Information on the Washington SIP is available at:

http://yosemite.epa.gov/r10/airpage.nsf/ee8a9f190103579b882564610001b99c/be3d13447b9c42c088 256516006b2c64?OpenDocument

- 40 CFR 60, "Standards of Performance for New Stationary Sources." EPA regulations in 40 CFR 60 provide standards for the control of the emission of pollutants to the atmosphere. Construction or modification of an emissions source in an attainment area such as Hanford can require a prevention of significant deterioration (PSD) of air quality permit under 40 CFR 52.21 and WAC 173-400-141.

- 40 CFR 61, "National Emission Standards for Hazardous Air Pollutants," (NESHAP); 40 CFR 63, National Emission Standards for Hazardous Air Pollutants for Source Categories." EPA hazardous emission standards in 40 CFR 61 provide for the control of the emission of hazardous pollutants to the atmosphere. Standards in 40 CFR 61 Subpart H apply specifically to the emission of radionuclides from DOE facilities. Emissions of radionuclides (other than radon-220 and radon-222) to the ambient air from DOE facilities are not to exceed those amounts that would cause any member of the public to receive in any year an effective dose equivalent of $10 \mathrm{mrem} / \mathrm{yr}$ (40 CFR 61.92). Approval to construct a new facility or to modify an existing one may be required under 40 CFR 61.07. EPA has delegated interim authority to the state of Washington to implement and enforce 40 CFR 61 Subpart H, but has not yet delegated the construction approval authority (60 FR [Federal Register] 39263, August 2, 1995). Emission standards for sources of hazardous air pollutants designated in the 1990 CAA amendments appear at 40 CFR 63.

- 40 CFR 70, "State Operating Permit Programs." These regulations provide for the establishment of comprehensive state air quality permitting programs. All major sources of air pollutants including hazardous air pollutants are covered. EPA granted interim approval to Washington's operating permit program in January 2001 (66 FR 16). Washington's operating permit regulations appear at WAC 173-401.

- 40 CFR 93 Subpart B, "Determining Conformity of General Federal Actions to State or Federal Implementation Plans." The general conformity requirements require that actions of Federal agencies are to comply with state implementation plans designed to achieve national ambient air quality standards. 
- WAC 173-400 through 173-495, Washington State Air Pollution Control Regulations. Ecology air pollution control regulations, promulgated under the Washington CAA (Revised Code of Washington [RCW] 70.94), appear in WAC 173-400 through 173-495 and are available at:

http://www.ecy.wa.gov/laws-rules/ecywac.html\#air These regulations include emission standards, ambient air quality standards, and the standards in WAC 173-460, "Controls for New Sources of Toxic Air Pollutants." The state of Washington has delegated much of its authority under the Washington CAA to the BCAA. However, except for certain air pollution sources (e.g., asbestos removal, fugitive dust, and open burning) administered by the BCAA, Ecology continues to administer air pollution control requirements for the Hanford Site.

- WAC 246-247, "Radiation Protection--Air Emissions." Washington DOH regulations in WAC 246247 contain standards and permit requirements for the emission of radionuclides to the atmosphere.

- Regulation 1 of the Benton Clean Air Authority can be accessed at URL: http://www.bcaa.net/RegPol.htm

\subsubsection{Water Quality}

The CWA and the Washington Water Pollution Control Act provide the statutory basis for the regulation of water quality in Washington State. The CWA established the National Pollutant Discharge Elimination System (NPDES) to limit the amount of pollutants that could be discharged.

- 40 CFR 121, "State Certification of Activities Requiring a Federal License or Permit." These regulations provide for state certification that any activity requiring a federal water permit, i.e., a National Pollutant Discharge Elimination System (NPDES) permit or a discharge of dredged or fill material permit, will not violate state water quality standards.

- 40 CFR 122, "EPA Administered Permit Programs: The National Pollutant Discharge Elimination System." EPA regulations in 40 CFR 122 (and also in 40 CFR 125 and 129) apply to the discharge of pollutants from any point source into waters of the United States. These regulations also apply to the discharge of storm waters ( 40 CFR 122.26) and the discharge of runoff waters from construction areas over $0.02 \mathrm{~km}^{2}\left(0.008 \mathrm{mi}^{2}\right)$ in size into waters of the United States. NPDES permits may be required by 40 CFR 122. EPA has not delegated to the state of Washington the authority to issue NPDES permits at the Hanford Site.

- 40 CFR 141, "National Primary Drinking Water Regulations." EPA drinking water standards in 40 CFR 141 apply to Columbia River water at community water supply intakes downstream of the Hanford Site. Standards in 40 CFR 141.16 apply indirectly to releases of radionuclides from DOE facilities (and also non-DOE facilities) to the extent that the releases impact community water systems. The average annual concentration of beta particle and photon radioactivity from man-made radionuclides in drinking water are not to produce an annual dose equivalent to the body or any internal organ greater than $4 \mathrm{mrem} /$ year. Maximum contaminant levels in community water systems of $5 \mathrm{pCi} / \mathrm{L}$ of combined radium-226 and radium $-228 ; 15 \mathrm{pCi} / \mathrm{L}$ of gross alpha particle activity, including radium-226 but excluding radon and uranium; and $30 \mu \mathrm{g} / \mathrm{L}$ for uranium are specified in 40 CFR 141.66. The average annual concentration of beta particle and photon radioactivity from manmade radionuclides in drinking water must not produce an annual dose equivalent to the total body or any internal organ greater than $4 \mathrm{mrem} / \mathrm{yr}$ [40 CFR 141.66(d)].

- 40 CFR 144-147, Underground Injection Control Program. EPA regulations in 40 CFR 144-147 apply to the underground injection of liquids and wastes and may require a permit for any 
underground injection. In Washington State, the EPA has approved Ecology regulations in WAC 173-218, "Underground Injection Control Program," to operate in lieu of the EPA program. The Ecology regulations provide standards and permit requirements for the disposal of fluids by well injection.

- 10 CFR 1022, "Compliance with Floodplain/Wetlands Environmental Review Requirements.” DOE regulations in 10 CFR 1022 implement Executive Orders 11988 and 11990 and apply to DOE activities that are proposed to take place either in wetlands or in floodplains.

- $\quad 33$ CFR 322-323, 40 CFR 230-233. Structures in the Columbia River and work in the Columbia River, as well as the discharge of dredged or fill material into the Columbia River, require permits under these U.S. Army Corps of Engineers and EPA regulations.

- WAC 173-160. Under WAC 173-160, DOE provides notification to Ecology for water-well drilling on the Hanford Site.

- WAC 173-216, “State Waste Discharge Permit Program.” Ecology regulations in WAC 173-216 establish a state permit program for the discharge of waste materials from industrial, commercial, and municipal operations into ground and surface waters of the state. Discharges covered by NPDES or WAC 173-218 permits are excluded from the WAC 173-216 program. DOE has agreed to meet the requirements of this program at the Hanford Site for discharges of liquids to the ground.

- RCW 75.20.100, "Construction Projects in State Waters." WAC 220-110. As a matter of comity, DOE will obtain hydraulic project approval from the Washington State Department of Fish and Wildlife to construct any form of hydraulic project or perform work that will divert, obstruct, or change the natural flow of the Columbia River.

- WAC 332-30, "Aquatic Land Management." Where applicable, DOE will obtain an aquatic land use lease or permit from the Washington Department of Natural Resources for the placement of structures in the Columbia River on lands owned by the state of Washington. DOE owns most of the riverbed along the Hanford Site to the line of navigation.

- WAC 246-272-08001 and 246-272-09001. These regulations, administered by the Washington DOH, contain permit requirements for onsite sewage systems.

- WAC 246-290. These regulations, administered by the Washington DOH, contain requirements applicable to water systems providing piped water for human consumption.

\subsubsection{Hazardous Waste Management}

Regulation of hazardous wastes at Hanford is conducted under RCRA, CERCLA, the Tri-Party Agreement, and the Washington State Hazardous Waste Management Act.

- 40 CFR 300, "National Oil and Hazardous Substances Pollution Contingency Plan.” EPA CERCLA regulations in 40 CFR 300 apply to the cleanup of inactive hazardous waste disposal sites, the cleanup of hazardous substances released into the environment, the reporting of hazardous substances released into the environment, and natural resource damage assessments. On November 3, 1989, (54 FR 41015) the Hanford Site was placed on the EPA's National Priorities List (NPL). Placement on the list requires DOE, in consultation with EPA and Washington State, to conduct remedial investigations and feasibility studies leading to a record of decision on the cleanup of inactive waste disposal sites at 
Hanford. Standards for cleanup under CERCLA are "applicable or relevant and appropriate requirements" (ARARs), which may include both federal and state laws and regulations. In anticipation of Hanford's being placed on the NPL, DOE, EPA, and Ecology signed the Hanford Federal Facility Agreement and Consent Order (Tri-Party Agreement) on May 15, 1989. This agreement describes the cleanup responsibilities and authorities of the three parties under CERCLA (and RCRA), and also provides for permitting of the treatment, storage, and disposal of hazardous wastes under RCRA. The Tri-Party Agreement has been amended a number of times. The agreement is available at: http://www.hanford.gov/tpa/tpahome.htm

- 40 CFR 260-268 and 270-272, Hazardous Waste Management. EPA RCRA regulations in 40 CFR 260-268 and 270-272 apply to the generation, transport, treatment, storage, and disposal of hazardous wastes (but not to source, by-product, or special nuclear material [i.e., not in general to radioactive wastes]), and apply to the hazardous component of hazardous radioactive mixed wastes (but not to the radioactive component) owned by DOE. RCRA regulations (40 CFR 268) require treatment of many hazardous wastes before they can be disposed of in landfills (land disposal restrictions). RCRA permits are required for the treatment, storage, or disposal of hazardous wastes. The regulations also require cleanup (corrective action) of any RCRA facility from which there is an unauthorized release before a RCRA permit is granted. Ecology has been authorized by EPA to administer the RCRA program and all but the land disposal restriction and waste minimization provisions of the Hazardous and Solid Waste Amendments. Ecology has oversight authority for RCRA corrective actions at Hanford under the Tri-Party Agreement.

- 40 CFR 280-281, Underground Storage Tanks. EPA has regulations in 40 CFR 280-281 issued under RCRA Subtitle IX that apply to new and existing underground storage tanks containing petroleum or substances regulated under CERCLA (except for hazardous wastes regulated under RCRA). New tanks must meet strict design and operating standards. Owners of new tanks must notify the applicable regulatory agency and certify compliance with the regulations. The regulations require the reporting, investigation, and cleanup of releases from underground tanks. EPA has authorized Washington State to administer the underground storage tank program. Washington's requirements are in RCW 90.76 and WAC 173-360.

- WAC 173-303, "Dangerous Waste Regulations." The EPA has authorized the state of Washington through Ecology to conduct its own dangerous waste regulation program in lieu of major portions of the RCRA interim and final permit program for the treatment, storage, and disposal of hazardous wastes. Ecology is also authorized to conduct its own program for the hazardous portion of radioactive-mixed wastes. The state regulations include both standards and permit requirements, as well as a larger universe of covered materials than the federal hazardous waste program.

\subsubsection{Species Protection}

- 50 CFR 10-24, 222, 402, and 450-453, Species Protection Regulations. Regulations under the Endangered Species Act, the Bald and Golden Eagle Protection Act, and the Migratory Bird Treaty Act in 50 CFR 10-24 apply to the protection of plant and animal species on the Hanford Site. Regulations in 50 CFR 17, 81, 222, 223, 402, and 450-453 apply to endangered or threatened species. In addition, the Fish and Wildlife Coordination Act [16 U.S.C. 662(a, h)] requires that a federal agency consult with the U.S. Fish and Wildlife Service and the state agency exercising administration over wildlife if any body of water over $0.04 \mathrm{~km}^{2}\left(0.015 \mathrm{mi}^{2}\right)$ in size is to be modified by a federal agency, or a licensee or permitee of the agency, for any purpose. The purpose of this consultation is to prevent loss and damage to wildlife resources. 


\subsubsection{Historic and Cultural Resource Preservation}

- 25 CFR 261; 36 CFR 60, 79, 800; 43 CFR 3, 7, 10; Historic Preservation Regulations. Regulations implementing the NHPA in 36 CFR 60 and 36 CFR 800; the American Antiquities Act in 25 CFR 261 and 43 CFR 3; the ARPA and the American Indian Religious Freedom Act in 43 CFR 7; and the Native American Graves Protection and Repatriation Act in 43 CFR 10 apply to the protection of historic and cultural properties, including both existing properties and those discovered during excavation and construction. Regulations in 36 CFR 79 establish procedures and guidelines to be followed by federal agencies to preserve collections of historical material, remains, and records. Additional information on these statutes and regulations may be found by contacting the Department of Energy, Richland Operations Office, Hanford Cultural Resources Program or by accessing the Hanford website at http://www.hanford.gov/doe/culres/index.htm

- DOE's policy statement on management of Cultural Resources states that DOE will uphold cultural resource laws and consult with affected stakeholders early in the planning process of proposed undertakings (DOE 2001).

\subsubsection{Land Use}

The Hanford Reach National Monument was created on June 9, 2000 by a proclamation ${ }^{(a)}$ signed by President Clinton under the authority of the Antiquities Act of 1906. The Monument includes $792.6 \mathrm{~km}^{2}$ $\left(306 \mathrm{mi}^{2}\right)$ of federally owned land making up a portion of the Hanford Site. The principal components of the monument are the Fitzner/Eberhardt Arid Lands Ecology (ALE) Reserve, the McGee Ranch and Riverlands area, the Saddle Mountain National Wildlife Refuge, land along the south and west sides of the Columbia River corridor (excluding the reactor areas), the federally owned islands within the portion of the Columbia River included in the Monument, and the Hanford sand dune field. The U.S. Fish and Wildlife Service (USFWS) manages lands within the ALE reserve, the McGee Ranch, the Riverlands area, and the Saddle Mountain Wildlife Refuge. DOE manages the remainder of the Monument in consultation with the Department of the Interior. On June 14, 2001, DOE and USFWS signed a Memorandum of Understanding covering management responsibilities for the Monument. The June 9, 2000, proclamation provides that it does not affect the responsibilities and authority of DOE on Hanford Site lands nor does it affect DOE activities on lands not included within the Monument boundaries. In a separate memorandum ${ }^{(b)}$ to the Secretary of Energy, DOE is directed by the President to protect the natural values of the Hanford Site land not included within the monument. DOE is to consult with the Department of the Interior in providing this protection including the possibility of adding additional Hanford Site land to the Monument as the lands are remediated.

The Hanford Reach Act (PL 100-605), as amended by section 404 of the Omnibus Parks and Public Lands Management Act of 1996 (PL 104-333), requires the Secretary of the Interior, in consultation with the Secretary of Energy, to conduct a study ${ }^{(\mathrm{c})}$ of the Hanford Reach of the Columbia River defined to extend from one mile below Priest Rapids Dam downstream approximately 51 miles to the McNary Pool north of Richland. In addition, the amended Act requires that federal and non-federal entities planning

\footnotetext{
(a) Proclamation 7319 of June 9, 2000, "Establishment of the Hanford Reach National Monument," 65 FR 37253, June 13, 2000.

(b) The memorandum is available at: http://clinton6.nara.gov/2000/06/2000-06-09-memorandum-on-hanford-reachnational-monument.html

(c) The study was published in 1994 (DOI 1994).
} 
new projects within the study area consult and coordinate with the Secretary of the Interior ${ }^{(a)}$ to minimize and provide mitigation for any direct and adverse effects on the values for which the river is under study. The 1994 study states that the lateral boundaries of the study area are one-quarter mile on either side of the Columbia River (DOI 1994, p. 9).

In September 1999, DOE issued the Final Hanford Comprehensive Land-Use Plan Environmental Impact Statement (DOE 1999). The Record of Decision issued in November 1999 (64 FR 61615) states that the purpose of the land-use plan and its implementing policies is to facilitate decision making about the Hanford Site's uses and facilities over at least the next 50 years.

\subsubsection{Other}

- 40 CFR 191, "Environmental Radiation Protection Standards for Management and Disposal of Spent Nuclear Fuel, High-Level and Transuranic Radioactive Wastes.” EPA regulations in 40 CFR 191 provide environmental standards for the management, storage, and disposal of spent nuclear fuel, high-level radioactive wastes, and transuranic radioactive wastes at high-level or transuranic waste disposal sites.

- 40 CFR 355, 370, and 372. These regulations implement the federal Emergency Planning and Community Right-to-Know Act (EPCRA). EPCRA was signed into law in October 1986 as part of the Superfund Amendments and Reauthorization Act.

- 40 CFR 700-799, TSCA Regulations. EPA's regulations in 40 CFR 700-799 implement TSCA and, in particular, regulate polychlorinated biphenyls (PCBs) and dioxins and partially regulate asbestos.

- $\quad 40$ CFR 1500-1508, Council on Environmental Quality. The CEQ regulations in 40 CFR 1500-1508 implement NEPA.

- 10 CFR 830, "Nuclear Safety Management.” Part 830 contains nuclear safety management requirements applicable to DOE contractors.

- 10 CFR 835, "Occupational Radiation Protection.” These DOE rules establish radiation protection standards, limits, and program requirements for protecting individuals from ionizing radiation resulting from DOE activities.

- 10 CFR 1021, "National Environmental Policy Act Implementing Procedures." DOE regulations in 10 CFR 1021 set out procedures that DOE uses to comply with section 102(2) of NEPA and the CEQ regulations for implementing the procedural provisions of NEPA (40 CFR parts 1500-1508). The DOE regulations supplement, and are to be used in conjunction with, the CEQ regulations.

- 49 CFR 171-179, Hazardous Materials Regulations. These Department of Transportation regulations apply to the handling, packaging, labeling, and shipment of hazardous materials offsite, including radioactive materials and wastes.

\footnotetext{
(a) Secretary of the Interior Bruce Babbitt designated the Fish and Wildlife Service as the agency for such consultation and coordination in a July 26, 2000, memorandum to the Director of the Fish and Wildlife Service.
} 


\subsection{Executive Orders}

DOE is subject to a number of presidential executive orders (E.O.s) concerning environmental matters. Some of these orders may be appropriately considered in a Hanford EIS. Potentially relevant E.O.s include:

E.O. 11288 Prevention, Control, and Abatement of Water Pollution by Federal Activities

E.O. 11514 Protection and Enhancement of Environmental Quality

E.O. 11593 Protection and Enhancement of the Cultural Environment

E.O. 11738 Providing for Administration of the Clean Air Act and the Federal Water Pollution Control Act with Respect to Federal Contracts, Grants, or Loans

E.O. 11987 Exotic Organisms

E.O. $11988 \quad$ Floodplain Management

E.O. 11990 Protection of Wetlands

E.O. 12088 Federal Compliance with Pollution Control Standards

E.O. 12144 Environmental Effects Abroad of Major Federal Actions

E.O. 12196 Occupational Safety and Health Programs for Federal Employees

E.O. 12580 Superfund Implementation (as amended by E.O. 13016)

E.O. 12843 Procurement Requirements and Policies for Federal Agencies for Ozone Depleting Substances

E.O. 12856 Federal Compliance with Right-to-Know Laws and Pollution Prevention Requirements

E.O. 12898 Federal Actions to Address Environmental Justice in Minority Populations and LowIncome Populations

E.O. 12962 Recreational Fisheries

E.O. 12969 Federal Acquisition and Community Right-to-Know

E.O. 13007 Indian Sacred Sites

E.O. 13045 Protection of Children from Environmental Health Risks and Safety Risks

E.O. 13084 Consultation and Coordination with Indian Tribal Governments

E.O. 13101 Greening the Government through Waste Prevention, Recycling, and Federal Acquisition

E.O. 13112 Invasive Species 
E.O. 13123 Greening the Government Through Energy Efficient Management

E.O. 13134 Developing and Promoting Biobased Products and Bioenergy

E.O. 13148 Greening the Government Through Leadership in Environmental Management

E.O. 13149 Greening the Government Through Federal Fleet and Transportation Efficiency

E.O. $13150 \quad$ Federal Workforce Transportation

E.O. 13186 Responsibilities of Federal Agencies to Protect Migratory Birds

E.O. 13195 Trails for America in the $21^{\text {st }}$ Century

The preceding E.O.s can be accessed at the following URL:

http://www.denix.osd.mil/denix/Public/Legislation/EO/toc.html

\subsection{DOE Directives}

Categories of DOE directives include orders, policy statements, standards, notices, manuals, and contractor requirements documents.

DOE directives can be accessed at the following URL:

\section{http://www.directives.doe.gov/}

Directives with particular application to DOE's environmental activities are found in the 400 series of the new series directives and the 5000 series (particularly the 5400 and 5800 series) under the old series directives.

DOE directives cover environmental protection, safety, and health protection standards; hazardous and radioactive-mixed waste management; cleanup of retired facilities; safety requirements for the packaging and transportation of hazardous materials; safety of nuclear facilities; radiation protection; and other standards for the safety and protection of workers and the public. Regulations and standards of other federal agencies and regulatory bodies are incorporated by reference into DOE directives. 


\subsection{Treaties of the United States with American Indian Tribes of the Hanford Region}

In June 1855 at Camp Stevens in the Walla Walla Valley, representatives of the United States negotiated treaties with leaders of various Columbia Plateau American Indian Tribes and Bands. The negotiations resulted in three treaties, one with the 14 tribes and bands of what would become the Confederated Tribes and Bands of the Yakama Nation, one with the three tribes that would become the Confederated Tribes of the Umatilla Indian Reservation, and one with the Nez Perce Tribe. The U.S. Senate ratified the treaties in $1859^{(\mathrm{a})}$. The negotiated treaties are as follows:

1. Treaty with the Walla Walla, Cayuse, etc. (June 9, 1855; 12 Stats. 945)

2. Treaty with the Yakama (June 9, 1855; 12 Stats. 951)

3. Treaty with the Nez Perce (June 11, 1855; 12 Stats. 957).

The Yakama Nation, the Confederated Tribes of the Umatilla Reservation, and the Nez Perce Tribe of Idaho are federally recognized tribes which have the immunities and privileges available to other federally acknowledged Indian tribes by virtue of their government-to-government relationship with the United States as well as the responsibilities, powers, limitations and obligations of such tribes (65 FR 13298; March 13, 2000).

The terms of the three preceding treaties are similar. Each of the three Tribes agreed to cede large blocks of land to the United States. The Hanford Site is within the ceded lands. The Tribes retained certain lands for their exclusive use (the three reservations) and also retained certain rights and privileges to continue traditional activities outside the reservations. These included 1) the right to fish (and erect temporary fish-curing facilities) at usual and accustomed places in common with citizens of the United States, and 2) the privileges of hunting, gathering roots and berries, and pasturing horses and cattle on open and unclaimed lands.

DOE-RL interacts and consults on a direct basis with the three federally recognized tribes affected by Hanford operations, i.e., the Nez Perce, Umatilla, and Yakama tribes. In addition, the Wanapum people, who still live adjacent to the Hanford Site, are a non-federally recognized tribe with strong cultural ties to the site. The Wanapum are also consulted on cultural resource issues in accordance with DOE policy and relevant legislation. The Hanford area was also used by groups whose descendants are now enrolled members of the Confederated Tribes of the Colville Reservation.

\subsection{Permits}

Information on the status of environmental permits at Hanford is included in DOE (2000). Included are information on current and anticipated environmental permitting required by RCRA, TSCA, CAA, CWA, the State Waste Discharge and Underground Injection Control Programs, the Offsite Sewage System Program, and the Petroleum Underground Storage Tank Program.

\footnotetext{
(a) The text of the three treaties can be viewed in Appendix A of the Final Hanford Comprehensive Land-Use Plan Environmental Impact Statement and Comprehensive Land-Use Plan (HRA-EIS) Revised Draft (DOE 1999). The treaties are also available at: http://www.rootsweb.com/ usgenweb/wa/indians/treaties.htm 
The Hanford Site is considered a single facility for purposes of RCRA and the Washington State Hazardous Waste Management Act. The Site has been issued the EPA/State identification number WA7890008967. The Hanford RCRA Permit governs all final status treatment, storage, and/or disposal (TSD) activities at Hanford (DOE 2000). The initial permit was issued in 1994 for less than the entire Hanford Site because all TSD units cannot be permitted at once. Through permit revisions, all Hanford TSD units will be incorporated into the permit (DOE 2000).

Clean Air Act compliance requires both facility and sitewide compliance. DOE (2000) identifies existing facility-specific and sitewide compliance activities and requirements. The air operating permit for the Hanford Site became effective in July 2001 (permit number 00-05-006).

The Sitewide National Pollutant Discharge Elimination System Permit (WA-002591-7) governs liquid process effluent discharges to the Columbia River (DOE 2000).

DOE has asserted a federally reserved water withdrawal right with respect to its Hanford operations. Current Hanford activities use water withdrawn under the DOE's federally reserved water rights. 


\section{References}

U.S. Department of Energy (DOE). 1998. National Environmental Policy Act Compliance Guide Volume II (Reference Book). Washington, D.C. Available URL:

http://tis.eh.doe.gov/nepa/tools/tools.htm

U.S. Department of Energy (DOE). 1999. Final Hanford Comprehensive Land-Use Plan Environmental Impact Statement and Comprehensive Land-Use Plan. DOE/EIS-0222F. U.S. Department of Energy, Richland, Washington. Available URL:

http://www.hanford.gov/netlib/eis.html

U.S. Department of Energy (DOE). 2000. Annual Hanford Site Environmental Permitting Status Report. DOE/RL-96-63, Revision 4, U.S. Department of Energy, Richland, Washington. Available URL: http://www.hanford.gov/docs/rl-96-63/r196-63 contents1.html

U.S. Department of Energy (DOE). 2001. Department of Energy Management of Cultural Resources. DOE P 141.1, Washington, D.C. Available URL: http://www.directives.doe.gov

U.S. Department of the Interior (DOI). 1994. Hanford Reach of the Columbia River: Comprehensive River Conservation Study and Environmental Impact Statement - Final. Volumes I and II. U.S. Department of Interior, Washington, D.C. 


\section{APPENDIX A}

Hanford Site Species Lists 
Table A-1. Common Vascular Plants on the Hanford Site (Taxonomy follows Hitchcock and Cronquist 1973).

\section{A. Shrub-Steppe Species}

\section{Shrub}

Big sagebrush

Bitterbrush

Gray rabbitbrush

Green rabbitbrush

Snow buckwheat

Spiny hopsage

Threetip sagebrush

\section{Perennial Grasses}

Bluebunch wheatgrass

Bottlebrush squirreltail

Crested wheatgrass

Indian ricegrass

Needle-and-thread grass

Prairie junegrass

Sand dropseed

Sandberg's bluegrass

Thickspike wheatgrass

\section{Perennial Forbs}

Bastard toad flax

Buckwheat milkvetch

Carey's balsamroot

Cusick's sunflower

Cutleaf ladysfoot mustard

Douglas' clusterlily

Dune scurfpea

Franklin's sandwort

Gray's desertparsley

Hoary aster

Hoary falseyarrow

Longleaf phlox

Munro's globemallow

Pale eveningprimrose

Sand beardtongue

Stalked-pod milkvetch

Threadleaf fleabane

\section{Scientific Name}

Artemisia tridentata

Purshia tridentata

Chrysothamnus nauseosus

Chrysothamnus viscidiflorus

Eriogonum niveum

Grayia (Atriplex) spinosa

Artemisia tripartita

Agropyron spicatum

Sitanion hystrix

Agropyron desertorum (cristatum) ${ }^{(a)}$

Oryzopsis hymenoides

Stipa comata

Koeleria cristata

Sporobolus cryptandrus

Poa sandbergii (secunda)

Agropyron dasytachyum

Comandra umbellata

Astragalus caricinus

Balsamorhiza careyana

Helianthus cusickii

Thelypodium laciniatum

Brodiaea douglasii

Psoralea lanceolata

Arenaria franklinii

Lomatium grayi

Machaeranthera canescens

Chaenactis douglasii

Phlox longifolia

Sphaeralcea munroana

Oenothera pallida

Penstemon acuminatus

Astragalus sclerocarpus

Erigeron filifolius 


\begin{tabular}{|c|c|}
\hline A. Shrub-Steppe Species (cont'd.) & Scientific Name \\
\hline Turpentine spring parsley & Cymopteris terebinthinus \\
\hline Winged dock & Rumex venosus \\
\hline Yarrow & Achillea millefolium \\
\hline Yellow bell & Fritillaria pudica \\
\hline \multicolumn{2}{|l|}{ Annual Forbs } \\
\hline Annual Jacob's ladder & Polemonium micranthum \\
\hline Blue mustard & Chorispora tenella $^{(\mathrm{a})}$ \\
\hline Bur ragweed & Ambrosia acanthicarpa \\
\hline Clasping pepperweed & Lepidium perfoliatum \\
\hline Indian wheat & Plantago patagonica \\
\hline Jagged chickweed & Holosteum umbellatum $^{(\mathrm{a})}$ \\
\hline Jim Hill's tumblemustard & Sisymbrium altissimum $^{(a)}$ \\
\hline Matted cryptantha & Cryptantha circumscissa \\
\hline Pink microsteris & Microsteris gracilis \\
\hline Prickly lettuce & Lactuca serriola $^{(\mathrm{a})}$ \\
\hline Rough wallflower & Erysimum asperum \\
\hline Russian thistle (tumbleweed) & Salsola kali ${ }^{(\mathrm{a})}$ \\
\hline Slender hawksbeard & Crepis atrabarba \\
\hline Spring whitlowgrass & Draba verna ${ }^{(a)}$ \\
\hline Storksbill & 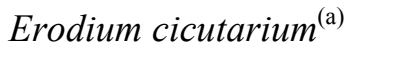 \\
\hline Tall willowherb & Epilobium paniculatum \\
\hline Tarweed fiddleneck & Amsinckia lycopsoides \\
\hline Threadleaf scorpion weed & Phacelia linearis \\
\hline Western tansymustard & Descurainia pinnata \\
\hline White cupseed & Plectritis macrocera \\
\hline Whitestem stickleaf & Mentzelia albicaulis \\
\hline Winged cryptantha & Cryptantha pterocarya \\
\hline Yellow salsify & Tragopogon dubius ${ }^{(\mathrm{a})}$ \\
\hline \multicolumn{2}{|l|}{ Annual Grasses } \\
\hline Cheatgrass & Bromus tectorum $^{(\mathrm{a})}$ \\
\hline Slender sixweeks & Festuca octoflora \\
\hline Small sixweeks & Festuca microstachys \\
\hline
\end{tabular}




\section{B. Riparian Species}

\section{Trees and Shrubs}

Black cottonwood

Black locust

Coyote willow

Dogbane

Peach, apricot, cherry

Peachleaf willow

Willow

White mulberry

\section{Perennial Grasses and Forbs}

Bentgrass

Blanket flower

Bulrushes

Cattail

Columbia River gumweed

Hairy golden aster

Heartweed

Horsetails

Horseweed tickseed

Lovegrass

Lupine

Meadow foxtail

Pacific sage

Prairie sagebrush

Reed canary grass

Rushes

Russian knapweed

Sedge

Water speedwell

Western goldenrod

Wild onion

Wiregrass spikerush

\section{Aquatic Vascular}

Canadian waterweed

Columbia yellowcress

Duckweed
Scientific Name

Populus trichocarpa

Robinia pseudo-acacia

Salix exigua

Apocynum cannabinum

Prunus spp.

Salix amygdaloides ${ }^{(a)}$

Salix spp.

Morus alba ${ }^{(\mathrm{a})}$

Agrostis spp. ${ }^{\text {(b) }}$

Gaillardia aristata

Scirpus spp. ${ }^{(\mathrm{b})}$

Typha latifolia ${ }^{(\mathrm{b})}$

Grindelia columbiana

Heterotheca villosa

Polygonum persicaria

Equisetum spp.

Coreopsis atkinsoniana

Eragrostis spp. ${ }^{(b)}$

Lupinus spp.

Alopecurus aequalis ${ }^{(b)}$

Artemisia campestris

Artemisia ludoviciana

Phalaris arundinacea ${ }^{(\mathrm{a}, \mathrm{b})}$

Juncus spp.

Centaurea repens $^{(\mathrm{a})}$

Carex spp. ${ }^{(b)}$

Veronica anagallis-aquatica

Solidago occidentalis

Allium spp.

Eleocharis spp. ${ }^{(\mathrm{b})}$

Elodea canadensis

Rorippa columbiae

Lemna minor 
B. Riparian Species

Aquatic Vascular (cont'd.)

Pondweed

Spiked water milfoil

Watercress

Introduced

(b) Perennial grasses and graminoids.
Scientific Name

Potamogeton spp.

Myriophyllum spicatum

Rorippa nasturtium-aquaticum 
Table A-2. Mammals That Have Been Observed on the Hanford Site

\begin{tabular}{|c|c|}
\hline Species & Scientific Name \\
\hline Merriam shrew & Sorex merriami \\
\hline Vagrant shrew & Sorex vagrans \\
\hline Pallid bat & Antrozous pallidus \\
\hline Big brown bat & Eptesicus fuscus \\
\hline Silver-haired bat & Lasionycteris octivagans \\
\hline Hoary bat & Lasiurus cinereus \\
\hline California myotis & Myotis californicus \\
\hline Little brown myotis & Myotis lucifugus \\
\hline Small-footed myotis & Myotis subulatus \\
\hline Long-legged myotis & Myotis volans \\
\hline Yuma myotis & Myotis yumanensis \\
\hline Western pipistrel & Pipistrellus hesperus \\
\hline Black-tailed jackrabbit & Lepus californicus \\
\hline White-tailed jackrabbit & Lepus townsendi \\
\hline Mountain cottontail & Sylvilagus nutalli \\
\hline Townsend ground squirrel & Citellus townsendi \\
\hline Washington ground squirrel & Citellus washingtoni \\
\hline Least chipmunk & Eutamius minimus \\
\hline Porcupine & Erithizon dorsatum \\
\hline Sagebrush vole & Lagurus curtatus \\
\hline Meadow vole & Microtus pennsylvanicus \\
\hline Yellowbelly marmot & Marmota flaviventris \\
\hline Bushytail woodrat & Neotoma cinerea \\
\hline Muskrat & Ondatra zibethica \\
\hline Northern grasshopper mouse & Onychomys leucogaster \\
\hline Great basin pocket mouse & Perognathus parvus \\
\hline Deer mouse & Peromyscus maniculatus \\
\hline House mouse & Mus musculus \\
\hline Western harvest mouse & Riethrodontonomys megalotis \\
\hline Northern pocket gopher & Thomomys talpoides \\
\hline River otter & Lutra canadensis \\
\hline Striped skunk & Mephitis mephitis \\
\hline Shorttail weasel & Mustela erminea \\
\hline Longtail weasel & Mustela frenata \\
\hline Mink & Mustela vison \\
\hline Badger & Taxidea taxus \\
\hline Raccoon & Procyon lotor \\
\hline Coyote & Canis latrans \\
\hline Bobcat & Lynx rufus \\
\hline Mountain lion & Felis concolor \\
\hline Elk & Cervus elaphus \\
\hline Mule deer & Odocoileus hemionus \\
\hline White-tailed deer & Odocoileus virginianus \\
\hline
\end{tabular}


Table A-3. Reptiles and Amphibians found on the Hanford Site

\begin{tabular}{|ll|}
\hline Common Name & Scientific Name \\
\hline Reptiles & \\
\hline Northern sagebrush lizard & Sceloporus graciosus \\
Side-blotched lizard & Uta stansburiana \\
Short-horned lizard & Phrynosoma douglassii \\
Rocky Mountain rubber boa & Charina bottae \\
Western yellow-bellied racer & Coluber constrictor \\
Western rattlesnake & Crotalus viridis \\
Night snake & Hupsiglena torquata \\
Striped whipsnake & Masticophis taeniatus \\
Great Basin gopher snake & Pituiphis melanoleucus \\
Common garter snake & Thamnophis sirtalis \\
Western terrestrial garter snake & Thamnophis elegans \\
Painted turtle & Chrysemys picta \\
& \\
Amphibians & \\
\hline Woodhouse toad & Bufo woodhousei \\
Great Basin spadefoot & Scaphiopus intermontanus \\
Bullfrog & Rana catesbeiana \\
Pacific Treefrog & Hyla regilla \\
Tiger Salamander & Ambystoma tigrinum \\
\hline
\end{tabular}


Table A-4. Common bird species known to occur annually on the Hanford Site (Fitzner and Gray 1991; Landeen et al. 1992; Duberstein 1997). Season Code: $\mathrm{Yr}=$ all year, W = winter, $\mathrm{B}=$ Breeding, $\mathrm{M}=$ Migration

\begin{tabular}{|c|c|c|}
\hline Common Name & Scientific Name & $\begin{array}{c}\text { Season of } \\
\text { highest } \\
\text { abundance }\end{array}$ \\
\hline Common loon & Gavia immer & $\mathrm{Yr}$ \\
\hline Eared grebe & Podiceps nigricollis & $\mathrm{W}$ \\
\hline Pied-billed grebe & Podilymbus podiceps & $\mathrm{Yr}$ \\
\hline Western grebe & Aechmophorus occidentalis & $\mathrm{W}$ \\
\hline Horned grebe & Podiceps auritus & $\mathrm{W}$ \\
\hline American white pelican & Pelecanus erythrorhynchos & Yr \\
\hline Double-crested cormorant & Phalacrocorax auritus & $\mathrm{Yr}$ \\
\hline Canada goose & Branta canadensis & $\mathrm{Yr}$ \\
\hline American wigeon & Anas americana & $\mathrm{W}$ \\
\hline Gadwall & Anas strepera & $\mathrm{Yr}$ \\
\hline Northern pintail & Anas acuta & Yr \\
\hline American green-winged teal & Anas crecca & $\mathrm{Yr}$ \\
\hline Mallard & Anas platyrhynchos & $\mathrm{Yr}$ \\
\hline Blue-winged teal & Anas discors & $\mathrm{B}$ \\
\hline Cinnamon teal & Anas cyanoptera & $\mathrm{B}$ \\
\hline Northern shoveler & Anas clypeata & $\mathrm{Yr}$ \\
\hline Common goldeneye & Bucephala clangula & $\mathrm{W}$ \\
\hline Bufflehead & Bucephala albeola & $\mathrm{W}$ \\
\hline Redhead & Aythya americana & $\mathrm{W}$ \\
\hline Ruddy duck & Oxyura jamaicensis & $\mathrm{Yr}$ \\
\hline Barrow's goldeneye & Bucephala islandica & $\mathrm{W}$ \\
\hline Common merganser & Mergus merganser & $\mathrm{Yr}$ \\
\hline Hooded merganser & Lophodytes cucullatus & $\mathrm{W}$ \\
\hline American coot & Fulica americana & Yr \\
\hline Virginia rail & Rallus limicola & $\mathrm{B}$ \\
\hline Sora & Porzana carolina & $\mathrm{B}$ \\
\hline California gull & Larus californicus & $\mathrm{Yr}$ \\
\hline Forster's tern & Sterna forsteri & $\mathrm{B}$ \\
\hline Ring-billed gull & Larus delawarensis & $\mathrm{Yr}$ \\
\hline Caspian tern & Sterna caspia & $\mathrm{B}$ \\
\hline Glaucous-winged gull & Larus glaucescens & $\mathrm{Yr}$ \\
\hline Herring gull & Larus argentatus & $\mathrm{W}$ \\
\hline Great blue heron & Ardea herodias & $\mathrm{Yr}$ \\
\hline Great egret & Casmerodius albus & $\mathrm{B}$ \\
\hline Black-crowned night-heron & Nycticorax nycticorax & $\mathrm{B}$ \\
\hline Sandhill crane & Grus canadensis & M \\
\hline American avocet & Recurvirostra americana & $\mathrm{B}$ \\
\hline Common snipe & Gallinago gallinago & $\mathrm{B}$ \\
\hline Killdeer & Charadrius viociferus & $\mathrm{B}$ \\
\hline Greater yellowlegs & Tringa melanoleuca & M \\
\hline Lesser yellowlegs & Tringa flavipes & $\mathrm{M}$ \\
\hline Dunlin & Calidris alpinis & M \\
\hline
\end{tabular}


Table A-4 (cont'd)

\begin{tabular}{|c|c|c|}
\hline Common Name & Scientific Name & $\begin{array}{c}\text { Season of } \\
\text { highest } \\
\text { abundance }\end{array}$ \\
\hline Long-billed dowitcher & Limnodromus scolopaceus & M \\
\hline Western sandpiper & Calidris mauri & M \\
\hline Red-necked phalarope & Phalaropus lobatus & M \\
\hline Long-billed curlew & Numenius americanus & $\mathrm{B}$ \\
\hline Spotted sandpiper & Actitis macularia & $\mathrm{B}$ \\
\hline Solitary sandpiper & Tringa solitaria & M \\
\hline California quail & Callipepla californica & Yr \\
\hline Ring-necked pheasant & Phasianus colchicus & $\mathrm{Yr}$ \\
\hline Chukar & Alectoris chukar & $\mathrm{Yr}$ \\
\hline Grey partridge & Perdix perdix & Yr \\
\hline Northern harrier & Circus cyaneus & Yr \\
\hline Sharp-shinned hawk & Accipiter striatus & $\mathrm{W}$ \\
\hline Cooper's hawk & Accipiter cooperii & $\mathrm{W}$ \\
\hline Swainson's hawk & Buteo swainsoni & $\mathrm{B}$ \\
\hline Red-tailed hawk & Buteo jamaicensis & $\mathrm{Yr}$ \\
\hline Northern rough-legged hawk & Buteo lagopus & $\mathrm{W}$ \\
\hline Ferruginous hawk & Buteo regalis & $\mathrm{B}$ \\
\hline Bald eagle & Haliaeetus leucocephalus & $\mathrm{W}$ \\
\hline Golden eagle & Aquila chrysaetos & $\mathrm{Yr}$ \\
\hline Osprey & Pandion haliaetus & $\mathrm{B}$ \\
\hline American kestrel & Falco sparverius & $\mathrm{Yr}$ \\
\hline Prairie falcon & Falco mexicanus & $\mathrm{Yr}$ \\
\hline Merlin & Falco columbarius & M \\
\hline Burrowing owl & Athene cunicularia & $\mathrm{B}$ \\
\hline Common barn-owl & Tyto alba & $\mathrm{Yr}$ \\
\hline Great horned owl & Bubo virginianus & $\mathrm{Yr}$ \\
\hline Long-eared owl & Asio otus & $\mathrm{Yr}$ \\
\hline Short-eared owl & Asio flammeus & $\mathrm{Yr}$ \\
\hline Belted kingfisher & Cerle alcyon & $\mathrm{Yr}$ \\
\hline Rock dove & Columba livia & $\mathrm{Yr}$ \\
\hline Mourning dove & Zenaida macroura & $\mathrm{Yr}$ \\
\hline Common nighthawk & Chordeiles minor & $\mathrm{B}$ \\
\hline Common poorwill & Pahalaenoptilus nuttallii & $\mathrm{B}$ \\
\hline Rufous hummingbird & Selasphorus rufus & $\mathrm{M}$ \\
\hline Northern flicker & Colaptes auratus & $\mathrm{Yr}$ \\
\hline Eastern kingbird & Tyrannus tyrannus & $\mathrm{B}$ \\
\hline Western kingbird & Tyrannus verticalis & $\mathrm{B}$ \\
\hline Western wood-pewee & Contopus sordidulus & M \\
\hline Say's phoebe & Sayornis saya & $\mathrm{B}$ \\
\hline Pacific-slope flycatcher & Empidonax difficilis & M \\
\hline Hammond's flycatcher & Empidonax hammondii & M \\
\hline Horned lark & Eremophila alpestris & $\mathrm{Yr}$ \\
\hline Barn swallow & Hirundo rustica & $\mathrm{B}$ \\
\hline
\end{tabular}


Table A-4 (cont'd)

\begin{tabular}{|c|c|c|}
\hline Common Name & Scientific Name & $\begin{array}{c}\text { Season of } \\
\text { highest } \\
\text { abundance }\end{array}$ \\
\hline Cliff swallow & Hirundo pyrrhonota & $\mathrm{B}$ \\
\hline Bank swallow & Riparia riparia & $\mathrm{B}$ \\
\hline Violet-green swallow & Tachycineta thalassina & M \\
\hline Tree swallow & Tachycineta bicolor & M \\
\hline Northern rough-winged swallow & Stelgidopteryx serripennis & $\mathrm{B}$ \\
\hline Black-billed magpie & Pica pica & Yr \\
\hline Common raven & Corvus corax & Yr \\
\hline American crow & Corvus brachyrhynchos & Yr \\
\hline Red-breasted nuthatch & Sitta canadensis & $\mathrm{W}$ \\
\hline Rock wren & Salpinctes obsoletus & $\mathrm{B}$ \\
\hline Marsh wren & Cistothorus palustris & $\mathrm{B}$ \\
\hline Winter wren & Troglodytes troglodytes & $\mathrm{W}$ \\
\hline Bewick's wren & Thryomanes bewickii & $\mathrm{B}$ \\
\hline Canyon wren & Catherpes mexicanus & $\mathrm{B}$ \\
\hline House wren & Troglodytes aedon & $\mathrm{B}$ \\
\hline Golden-crowned kinglet & Regulus satrapa & $\mathrm{M}$ \\
\hline Ruby-crowned kinglet & Regulus calendula & M \\
\hline Sage thrasher & Oreoscoptes montanus & $\mathrm{B}$ \\
\hline American robin & Turdus migratorius & Yr \\
\hline Varied thrush & Ixoreus naevius & $\mathrm{W}$ \\
\hline Townsend's solitaire & Myadestes townsendi & M \\
\hline Loggerhead shrike & Lanius ludovicianus & Yr \\
\hline European starling & Sturnus vulgaris & Yr \\
\hline Cedar waxwing & Bombycilla cedrorum & M \\
\hline Warbling vireo & Vireo gilvus & M \\
\hline Solitary vireo & Vireo solitarius & M \\
\hline Yellow-rumped warbler & Dendroica coronata & M \\
\hline Nashville warbler & Vermivora ruficapilla & M \\
\hline Wilson's warbler & Wilsonia pusilla & M \\
\hline MacGillivray's warbler & Oporornis tolmiei & $\mathrm{B}$ \\
\hline Townsend's warbler & Dendroica townsendi & $\mathrm{M}$ \\
\hline Orange-crowned warbler & Vermivora celata & M \\
\hline Yellow warbler & Dendroica petechia & M \\
\hline Yellow-breasted chat & Icteria virens & $\mathrm{B}$ \\
\hline Red-winged blackbird & Agelaius phoeniceus & $\mathrm{B}$ \\
\hline Western meadowlark & Sturnella neglecta & Yr \\
\hline Yellow-headed blackbird & Xanthocephalus xanthocephalus & $\mathrm{B}$ \\
\hline Bullock's oriole & Icterus galbula & $\mathrm{B}$ \\
\hline Brewer's blackbird & Euphagus cyanocephalus & $\mathrm{B}$ \\
\hline Brown-headed cowbird & Molothrus ater & $\mathrm{B}$ \\
\hline Western tanager & Piranga ludoviciana & M \\
\hline Dark-eyed junco & Junco hyemalis & Yr \\
\hline White-crowned sparrow & Zonotrichia leucophrys & $\mathrm{W}$ \\
\hline
\end{tabular}


Table A-4 (cont'd)

\begin{tabular}{|llc|}
\hline Common Name & Scientific Name & $\begin{array}{c}\text { Season of } \\
\text { highest } \\
\text { abundance }\end{array}$ \\
\hline House sparrow & Passer domesticus & $\mathrm{Yr}$ \\
Vesper sparrow & Pooecetes gramineus & $\mathrm{B}$ \\
Sage sparrow & Amphispiza belli & $\mathrm{B}$ \\
Song sparrow & Melospiza melodia & $\mathrm{Yr}$ \\
Brewer's sparrow & Spizella breweri & $\mathrm{B}$ \\
Savannah sparrow & Passerculus sandwichensis & $\mathrm{B}$ \\
Lark sparrow & Chondestes grammacus & $\mathrm{B}$ \\
Rufous-sided towhee & Pipilo erythrophthalmus & $\mathrm{B}$ \\
Golden-crowned sparrow & Zonotrichia atricapilla & $\mathrm{M}$ \\
Grasshopper sparrow & Ammodramus savannarum & $\mathrm{B}$ \\
Lincoln's sparrow & Melospiza lincolnii & $\mathrm{M}$ \\
Chipping sparrow & Spizella passerina & $\mathrm{M}$ \\
Black-headed grosbeak & Pheucticus melanocephalus & $\mathrm{B}$ \\
Lazuli bunting & Passerina amoena & $\mathrm{B}$ \\
Rosy finch & Leucosticte arctoa & $\mathrm{M}$ \\
American goldfinch & Carduelis tristis & $\mathrm{Yr}$ \\
House finch & Carpodacus mexicanus & $\mathrm{Yr}$ \\
\hline
\end{tabular}


Table A-5. Fish Species in the Hanford Reach of the Columbia River

\begin{tabular}{|c|c|}
\hline Common Name & Scientific Name \\
\hline American shad & Alosa sapidissima \\
\hline Black bullhead & Ameiurus melas \\
\hline Black crappie & Pomoxis nigromaculatus \\
\hline Bluegill & Lepomis macrochirus \\
\hline Bridgelip sucker & Catostomus columbianus \\
\hline Brown bullhead & Ictalurus nebulosus \\
\hline Burbot & Lota lota \\
\hline Carp & Cyprinus carpio \\
\hline Channel catfish & Ictalurus punctatus \\
\hline Chinook salmon & Oncorhynchus tshawytscha \\
\hline Chiselmouth & Acrocheilus alutaceus \\
\hline Coho salmon & Oncorhynchus kisutch \\
\hline Cutthroat trout & Oncorhynchus clarki \\
\hline Dolly Varden & Salvelinus malma \\
\hline Lake whitefish & Coregonus clupeaformis \\
\hline Largemouth bass & Micropterus salmoides \\
\hline Largescale sucker & Catostomus macrocheilus \\
\hline Leopard dace & Rhinichthys falcatus \\
\hline Longnose dace & Rhinichthys cataractae \\
\hline Mottled sculpin & Cottus bairdi \\
\hline Mountain sucker & Catostomus platyrhynchus \\
\hline Mountain whitefish & Prosopium williamsoni \\
\hline Northern pikeminnow (aka squawfish) & Ptychocheilus oregonensis \\
\hline Pacific lamprey & Entosphenus tridentatus \\
\hline Peamouth & Mylocheilus caurinus \\
\hline Paiute sculpin & Cottus beldingi \\
\hline Prickley sculpin & Cottus asper \\
\hline Pumpkinseed & Lepomis gibbosus \\
\hline Rainbow trout (steelhead) & Oncorhynchus mykiss \\
\hline Redside shiner & Richardsonius balteatus \\
\hline Reticulate sculpin & Cottus perplexus \\
\hline River lamprey & Lampetra ayresi \\
\hline Sandroller & Percopsis transmontana \\
\hline Smallmouth bass & Micropterus dolomieui \\
\hline Sockeye salmon & Oncorhynchus nerka \\
\hline Speckled dace & Rhinichthys osculus \\
\hline Tench & Tinca tinca \\
\hline Threespine stickleback & Gasterosteus aculeatus \\
\hline Torrent sculpin & Cottus rotheus \\
\hline Walleye & Stizostedion vitreum \\
\hline White crappie & Pomoxis annularis \\
\hline White sturgeon & Acipenser transmontanus \\
\hline Yellow perch & Perca flavescens \\
\hline Yellow bullhead & Ameiuruss natalis \\
\hline
\end{tabular}




\section{Distribution}

No. of

Copies

\section{OFFSITE}

1 C. Borgstrom

U.S. Department of Energy (EH-42)

1000 Independence Avenue SW

Washington, D.C. 20585-0119

1 A. J. Dvorak

Environmental Assessment Division Argonne National Laboratory 9700 S. Cass Avenue Bldg 900

Argonne, IL 60439-4832

2 Gary Ballew

Sustainable Development Manager

Benton County Planning Dept.

P.O. Box 910

Prosser, WA 99350

1 S. Frank

U.S. Department of Energy (EM-1)

1000 Independence Avenue SW

Washington, D.C. 20585

1 C. Hickey

NEPA Compliance Officer

U.S. Department of Energy

Office of Science (SC-83)

19901 Germantown Road

Germantown, MD 20874-1290

$1 \quad$ N. Lane

Lane Environmental, Inc.

P. O. Box 925

Richland, WA 99352

1 J. E. Loving

U.S. Department of Energy (EH-42)

Office Of NEPA Policy \&Compliance

1000 Independence Avenue SW

Washington, D.C. 20585
No. of

$\underline{\text { Copies }}$

1 R. Sharma

NEPA Compliance Officer

U.S. Department of Energy (NE-47)

19901 Germantown Road

Germantown, MD 20874-1290

1 E. Williams

Jason Associates Corporation 3250 Port of Benton Blvd.

Richland, WA 99352

7 G. Hughes

U.S. Fish and Wildlife Service

3250 Port of Benton Blvd

Richland, WA 99352

$1 \quad$ Lloyd Lorenzi

NEPA Compliance Officer

National Energy Technology Lab

P.O. Box 10940

Pittsburgh, PA 15236

$\underline{\text { Native American Tribes }}$

3 Mr. William Burke

Confederated Tribes of the Umatilla

Indian Reservation

P.O. Box 638

Pendleton, OR 97801

2 Mr. Russell-Jim, Manager

Environmental Restoration/

Waste Management Program

Yakama Nation

2808 Main Street

Union Gap, WA 98903

2 Mr. Patrick Sobotta

Environmental Restoration and

Waste Management

Nez Perce Tribe

P.O. Box 365

Lapwai, ID 83540

Distr. 1 
No. of

$\underline{\text { Copies }}$

3 Ms. Leanora Seelatsee

Wanapum Band

Grant County P.U.D.

30 "C" Street, S.W.

P.O. Box 878

Ephrata, WA 98823

$1 \quad$ Ms. Adeline Fredin

Colville Tribal Historic Preservation

Officer

P.O. Box 150

Nespelem WA 99155

\section{ONSITE}

47 DOE Richland Operations

Office/Office of River Protection

T. L. Aldridge, LOD

K8-50

D. W. Bowser, EMD

H6-60

M. E. Burandt, EMD

B. F. Burton, OOD

N2-36

R. M. Carosino, OCC

A4-52

D. H. Chapin, FFTF

C. E. Clark, RCA

S. S. Clark, RCA

K. V. Clarke, IPI

M. S. Collins, WMD

J. L. Daily, MPD

P. F. Dunigan, RCA (20)

W. F. Edwards, OTS

T. W. Ferns, EMT

H. B. Hathaway, OTS

R. D. Hildebrand, GWVZ

E. V. Hiskes, OCC

R. A. Holten, MPD

L. A. Huffman, EMD

D. W. Lloyd, RCA

R. R. McNulty, ORP

G. M. Neath, EMD

S. M. Ortiz, OSS

J. E. Rasmussen, EMD

A. L. Rodriguez, RCA

C. V. Smith, OCC

D. C. Ward, OSS

B. D. Williamson, OCC

J. H. Zeisloft, OSS

A7-75

H0-12

A5-58

A5-58

A2- 45

K8-50

A2-45

A5-13

A4-52

A5-16

H6-60

A5-58

H6-60

H6-60

A2-15

H6-60

A5-58

A $4-52$

A5-15

A $4-52$

A2-15
H6-60

No. of

$\underline{\text { Copies }}$

$2 \quad \underline{\text { CH2M HILL Hanford Group, Inc. }}$

F. M. Mann

$\mathrm{H} 0-22$

K. S. Tollefson

R1-51

$3 \quad$ CH2M Hill Hanford, Inc.

E. T. Coenenberg

H9-03

L. M. Johnson

H9-01

R. W. Ovink

H9-01

13 Bechtel Hanford, Inc.

R. A. Carlson

$\mathrm{H} 0-17$

K. R. Fecht

$\mathrm{H} 0-02$

G. E. Fitzgibbon

H0-09

K. A. Gano

$\mathrm{H} 0-23$

R. J. Landon

$\mathrm{H} 0-02$

T. J. Lazarski

$\mathrm{H} 0-16$

T. E. Marceau

$\mathrm{H} 0-23$

G. B. Mitchem

$\mathrm{H} 0-17$

D. D. Teel

$\mathrm{H} 0-23$

B. L. Vedder

$\mathrm{H} 0-02$

J. G. Woolard

$\mathrm{H} 0-02$

S. G. Weiss

$\mathrm{H} 0-20$

R. H. Wyer

H0-09
8 Fluor Hanford, Inc.

G. D. Cummins

A1-14

D. P. Demitruk

S3-96

A. M. Hopkins

H5-24

K. A. Hadley

B. B. Nelson-Maki

T5-57

R. L. Newell

T5-54

F. A. Ruck, III

H8-66

D. J. Watson

G1-37

X3-79

1 Fluor Federal Services, Inc.

P. D. Rittmann

B4-43

2 DynCorp Tri-Cities Services, Inc.

B. J. Dixon

G3-26

C. J. Grando

G3-26 
No. of

$\underline{\text { Copies }}$

$1 \quad$ Washington State University

R. G. Schreckhise H2-52

5 Duratek Federal Services of Hanford, Inc.

R. H. Engelmann G1-30

2 Duratek Federal Services Northwest Operations

J. J. Dorian

H1-11

R. M. Mitchell

H1-11

2 Jacobs Engineering, Inc.

M. R. Harker

B2-05

D. Nichols

B2-05

74 Pacific Northwest National Laboratory

E. J. Antonio

M. P. Bergeron

K3-54

G. R. Bilyard

C. A. Brandt

R. W. Bryce

A. L. Bunn

L. L. Cadwell

C. R. Cole

P. J. Cowley

P. M. Daling

D. D. Dauble

R. L. Dirkes (5)

J. P. Duncan (15)

T. O. Eschbach

M. J. Fayer

R. A. Fowler

S. W. Gajewski

D. R. Geist

S. M. Goodwin

G. R. Guensch

D. L. Hadley

J. C. Hail

R. W. Hanf

D. W. Harvey

P. L. Hendrickson

D. J. Hoitink

K6-85

K6-85

K9-36

K7-22

K6-04

K6-84

K6-75

K6-85

K3-54

K9-33

K6-05

K9-18

K6-85

P7-07

K9-33

K5-16

K5-16

K6-75

K6-75

K6-04

K9-30
No. of

Copies

$\underline{\text { Pacific NW Natl. Laboratory (cont'd) }}$

D. G. Horton

K6-81

D. J. Hostick

K6-05

U. P. Jenquin

K8-34

R. A. Kennedy

K7-63

C. T. Kincaid

K9-33

W. W. Laity

K8-02

G. V. Last

K6-81

B. A. Napier K3-54

D. A. Neitzel (5) K6-85

W. E. Nichols K9-33

G. P. O'Connor K6-86

T. L. Page K9-18

K. A. Poston P7-79

T. M. Poston K6-75

E. Prendergast K6-75

J. V. Ramsdell, Jr. K9-30

K. Rhoads K3-54

A. C. Rohay K6-81

M. R. Sackschewsky K6-85

M. J. Scott K6-05

D.C. Stapp K6-75

D. L. Strenge K3-54

P. D. Thorne K9-33

K. M. Tominey K7-97

M. D. White K9-36

R. S. Weeks P7-79 